\author{
Universidade de São Paulo \\ Instituto de Astronomia, Geofísica e Ciências Atmosféricas
}

Departamento de Astronomia

Rafael Alves de Souza Ribeiro

\title{
Optical Conceptual Design for the Giant Magellan Telescope Multi-Object Astronomical and Cosmological
} Spectrograph

São Paulo 



\section{Optical Conceptual Design for the Giant Magellan Telescope Multi-Object Astronomical and Cosmological Spectrograph}

Tese apresentada ao Departamento de Astronomia do Instituto de Astronomia, Geofísica e Ciências Atmosféricas da Universidade de São Paulo como requisito parcial para a obtenção do título de Doutor em Ciências.

Área de Concentração: Astronomia (Astronomia Instrumental)

Orientadora: Prof ${ }^{a}$. Dr ${ }^{\mathrm{a}}$. Claudia Lucia Mendes de Oliveira

Versão Corrigida. O original encontra-se disponível na Unidade.

São Paulo 

In memory of my father, Nelson. 



\section{Acknowledgements}

Firstly, my sincere gratitude goes to my dear adviser, Dr. Claudia Lucia Mendes de Oliveira, for inviting me to join the recently formed Institute of Astronomy, Geophysics and Atmospheric Sciences (IAG) Instrumentation Group to work on the GMACS optical design research in mid-2015, for trusting and supporting my work. I feel honored to have had the chance to work with and learn from her.

The interest in participating in the development of the GMACS optical system is a direct consequence of my professional journey in optical instrumentation. Mainly due to my bachelor's degree in physics with emphasis on optics, and photonics and master's

degree in applied physics from the Institute of Physics of São Carlos at the University of São Paulo (USP) I had the opportunity to work for many years in optical design, modeling, simulations and electro-optical tests in several projects in the aerospace, military, medical and scientific areas at private companies.

I am grateful for the opportunity to contribute to the development of Brazilian astronomical instrumentation. A thesis that covers the proposed topics is, to our best knowledge, a unique initiative in Brazil and will be, among many other works that the group of astronomical instrumentation of the IAG has carried out since its conception, a manner to return to society the investment that FAPESP made in the research project number 11/51680-6 "Exploring the universe: from the formation of galaxies to earth-type planets with the Giant Magellan Telescope" (Agência FAPESP, 2015).

Not less important, I would also like to express a special thanks

- to my dear colleagues, Dr. Keith Taylor, Dr. Luke Schmidt, and Dr. Damien Jones, for their friendship and the valuable exchange of experiences in astronomical optical instrumentation over the past five years. I could not have imagined having better 
mentors for this work;

- to to my colleagues from the Astronomical Instrumentation Lab at Texas A\&M University colleagues; particularly to Prof. Dr. Darren Depoy and Dr. Jennifer Marshall, the GMACS principal and co-principal investigator, respectively, for giving me a vote of confidence and motivation to work in the GMACS optical design research;

- to my Ph.D. thesis committee, Dr. Jacques Lepine (who, together with Dr. Jane Hetem, were my reviewers throughout the Ph.D. research period), Dr. Andrew Szentgyorgyi, Dr. Roderik Overzier, Dr. Bruno Vaz Castilho, Dr. Antonio Kanaan Neto, Dr. Alex Carciofi, Dr. Alessandro Ederoclite, Dr. Steve Eikenberry, Dr. Mikiya Muramatsu, Dr. Lino Misoguti and Dr. Bruno Correa Quint for generously offering their time and expertise for this research;

- to Dr. Rebecca Bernstein and Dr. Daniel Fabricant for providing me with helpful discussions and lessons about optical spectrograph design for ELF instrumentation;

- to Dr. Alessandro Ederoclite and Dr. Roderick Overzier for their contribution to my introduction to GMACS-like spectrographs and sciences cases with GMT and GMACS;

- to the GMT Brazilian Office members for our valuable technical discussions and friendship, particularly the late Dr. João Steiner, for trusting and supporting my work;

- to Antonio Braulio and Dr. Tárcio Vieira for their contribution in the thesis review;

- to IAG-USP and the GMT Brazilian Office, for the financial support during the research period;

- to CNPq, for the financial support under the Ph.D.-fellowship n ${ }^{\circ}$ : 870199/1997-8;

- to Deywhildson de Oliveira, for his careful final review of the thesis;

- to my mother and brother, Sueli and Júnior, for always trust, support, and encourage me to continue on my scientific career; and

- to Helena, my dear longtime companion.

This thesis was written in $\mathrm{AT}_{\mathrm{E} X} \mathrm{x}$ with the class "IAGTESE", for thesis and dissertation of IAG, with minor modifications by the author. 
"Dans ses écrits un sage Italien Dit que le mieux est l'ennemi du bien"

[In his writings a wise Italian Said that the best is the enemy of good] Voltaire, Contes "La Begueule" (1772)

"Would you tell me, please, which way I ought to go from here? That depends a good deal on where you want to get to. I don't much care where. Then it doesn't matter which way you go." Lewis Carroll, Alice in Wonderland (1865) 



\section{Abstract}

This thesis is being submitted to the area of optical astronomical instrumentation applied to Extreme Large Telescopes (ELTs). It presents the development of the conceptual design for the optics of the Giant Magellan Telescope Multi-object Astronomical and Cosmological Spectrograph (GMACS) for the period 2015 to 2020.

ELTs will be the next generation of telescopes, scheduled for the end of the 2020s or early in the following decade. Together with the James Webb Space Telescope (JWST) and major Survey Telescopes, such as the Vera C. Rubin Observatory, they will provide the scientific community with a set of instruments which will have unprecedented capabilities for application in many areas of astronomy, such as studies of the formation and evolution of planetary systems, galaxy assembly and evolution, exoplanets characterizations, and first light and reionization exploration. The Giant Magellan Telescope (GMT) will be the first ELT in operation, and it is planned for commissioning in the late 2020s.

GMT is a Gregorian optical telescope with a collecting area of $368 \mathrm{~m}^{2}$ and is currently under construction in north-northeast of La Serena, Chile, at the Las Campanas Observatory. As part of the GMT first light instruments, a wide-field optical Multi-Object Spectrograph (MOS) with a moderate resolution is foreseen to be essential to satisfying the diverse GMT scientific goals.

This research is based on the optical activities for the conceptual development of this instrument, GMACS, a general-purpose wide-field spectrograph for GMT. These activities consisted of: (i) a conceptual review of low to medium resolution Volume Phase Holographic (VPH) grating optical spectrographs for ELTs, from the perspective of the optical design; (ii) a review of pre-existing MOS with similar GMACS specifications to correlate the design solutions adopted with their technical requirements and design challenges; (iii) a 
detailed description of the methodology and tools developed for the conceptual optical design, modeling, and analysis; (iv) the results, presented in the conceptual design review held in September, 2019, based on the 2016 project de-scope requested by the GMTO through its Statement of Work and attachments (GMT-SOW-01091); and (v) the conclusions and the descriptions of the future stages of the optical project.

The proposed GMACS optical concept is a multi-object, two-channel, VPH transmission grating optical spectrograph with spectral coverage spanning from $320 \mathrm{~nm}$ to 1,000 $\mathrm{nm}$, the highest practical throughput over the entire spectral range (including the deep UV-Blue $320 \mathrm{~nm}$ to $350 \mathrm{~nm}$ ), a wide-field of $\approx 7.5^{\prime}$ in diameter, spectral resolving powers from 500 to 6,000 and resolutions from $\approx 8.5 \AA$ to $0.7 \AA$ for a $0.7^{\prime \prime}$ slit width baseline. The optical design is composed of a 2,200 $\mathrm{mm} \mathrm{f} / 8.2$ refractive split collimator $(270 \mathrm{~mm}$ diameter exit pupil) and two $594 \mathrm{~mm} \mathrm{f} / 2.2$ refractive cameras optimized for the $320 \mathrm{~nm}$ to $600 \mathrm{~nm}$ and $550 \mathrm{~nm}$ to 1,000 $\mathrm{nm}$ spectral ranges, resulting in the current largest étendue for a single optical MOS.

As part of the optomechanical performance results, we emphasize the solution found for mechanical deformations generated by the variation of the gravity vector of the current GMACS structure that affect the spectral stability and the image quality. The methodology developed in this research for the integration of finite element analysis and the Zemax optical design software revealed that two synchronized active compensators for each of the GMACS channels (one located in the collimator group, for focusing, and the other in the camera group, for fine focusing) could satisfactorily compensate these effects and meet the image stabilization requirements.

In conclusion, the research results were crucial to direct the development of other GMACS engineering areas and decisive for the success of the instrument. 


\section{Resumo}

Esta tese está inserida no contexto da área de instrumentação óptica aplicada aos Telescópios Extremamente Grandes (Extreme Large Telescopes, ELTs). Ela apresenta o desenvolvimento do design óptico referente à fase conceitual do Espectrógrafo Astronômico e Cosmológico de Múltiplos Objetos do Telescópio Gigante de Magalhães (Giant Magellan Telescope Multi-Object Astronomical and Cosmological Spectrograph, GMACS), realizada durante os anos de 2015 a 2020.

A próxima geração de telescópios, os ELTs, prevista para o final da década de 2020 e o início da próxima década, fornecerá à comunidade científica, juntamente com o Telescópio Espacial James Webb (James Webb Space Telescope, JWST) e Telescópios de Survey, como o Observatório Vera C. Rubin, um conjunto de instrumentos com recursos sem precedentes para aplicação em diversas áreas da astronomia, tais como estudos da formação e evolução de sistemas planetários, dinâmica e evolução de galáxias, caracterização de exoplanetas e reionização. Entre os ELTs em desenvolvimento, o primeiro será o Telescópio Gigante de Magalhães (Giant Magellan Telescope, GMT), previsto para o final da década de 2020.

O GMT é um telescópio óptico gregoriano com área de coleta efetiva de $368 \mathrm{~m}^{2}$ atualmente em construção no nordeste de La Serena, Chile, no Observatório de Las Campanas. Como integrante dos instrumentos selecionados para primeira luz, um Espectrógrafo MultiObjeto (Multi-Object Spectrograph, MOS) óptico de amplo campo de visão e resolução moderada demonstrou ser essencial para atender a objetivos como o estudo da formação de estrelas, de populações estelares e da maioria das ciências extragalácticas.

Esta pesquisa está inserida no contexto das atividades de desenvolvimento do sistema óptico desse instrumento, o GMACS. Elas consistiram em: (i) revisão conceitual de espectrógrafos ópticos baseados em rede de difração de fase no volume (Volume Phase Ho- 
lographic, VPH) de baixa a média resolução para ELTs, da perspectiva do design óptico; (ii) revisão dos atuais MOS com especificações similares ao GMACS, a fim de correlacionar as soluções de design adotadas com seus requisitos e desafios técnicos; (iii) descrição detalhada da metodologia e das ferramentas que desenvolvemos para o design, a modelagem e a análise óptica; (iv) apresentação dos resultados na revisão conceitual realizada em setembro de 2019, baseado no escopo referente ao redesign solicitado pelo GMTO por meio da proposta GMT-SOW-01091 de 2016 e seu anexo; e (v) apresentação de conclusões e direcionamentos para as etapas futuras do projeto óptico.

O desenho óptico conceitual proposto é um espectrógrafo de dois canais, baseado em redes VPH transmissão como elemento dispersivo, cobertura espectral de $320 \mathrm{~nm}$ a 1.000 nm com o alta eficiência (incluindo na região ultravioleta de $320 \mathrm{~nm}$ a $350 \mathrm{~nm}$ ), campo de visada relativamente amplo de aproximadamente $7.5^{\prime}$ em diâmetro, poder resolvente de 500 a 6.000 e resolução de aproximadamente $8.5 \AA$ a $0.7 \AA$ para uma fenda padrão de $0.7^{\prime \prime}$ de largura. O sistema óptico é composto por um colimador $2200 \mathrm{~mm} \mathrm{f/8.2} \mathrm{refrativo}$ (com pupila de saída de $270 \mathrm{~mm}$ de diâmetro) e por duas câmeras $594 \mathrm{~mm} \mathrm{f/2.2} \mathrm{refrativas,}$ otimizadas nas regiões de $320 \mathrm{~nm}$ a $600 \mathrm{~nm}$ e $550 \mathrm{~nm}$ a $1000 \mathrm{~nm}$, resultando no maior étendue para um único MOS óptico da atualidade.

Dentre os dados de desempenho optomecânicos obtidos, destaca-se a solução alcançada para as deformações mecânicas geradas pela variação do vetor de gravidade da estrutura atual do GMACS, que afetam a estabilidade espectral e a qualidade da imagem. A metodologia desenvolvida nesta pesquisa para a integração da análise de elementos finitos e o software de design óptico Zemax mostrou que a utilização sincronizada de dois compensadores ativos em cada canal do GMACS (um localizado no grupo colimador, para ajuste de foco, e outro no grupo da câmera, para foco fino) pode compensar satisfatoriamente esses efeitos, satisfazendo os requisitos de estabilidade da imagem.

Por fim, cabe ressaltar que os resultados desta pesquisa foram cruciais para direcionar o progresso de outras áreas do projeto e decisivos para o sucesso do instrumento. 


\section{Author's declaration}

The work in this thesis is based on research carried out at the Department of Astronomy of the Institute of Astronomy, Geophysics, and Atmospheric Sciences at the University of São Paulo (Brazil) in collaboration with the Department of Physics and Astronomy at the Texas A\&M University (United States), and the private companies Prime Optics (Australia) and Instruments4 (United States). This thesis or any part of it has not been submitted for any degrees. The presented results, images, diagrams, and optical drawings are the result of my research, except as explicitly described in the text. As expected for a large project, several parts of this work have been shared to the internal members of the GMACS development team by employing reports, slides presentation, e-mails, and internal discussion; through the deliverable documents to GMTO; and at three SPIE conferences: 2016 and 2018 "SPIE Astronomical Instrumentation + Telescopes", and 2018 "SPIE Optical System Design". These publication are:

1. Ribeiro R., Jones D., Schmidt L., Taylor K., Cook E., DePoy D., et al. Optical design for the Giant Magellan Telescope Multi-object Astronomical and Cosmological Spectrograph (GMACS): design methodology, issues, and trade-offs. In: Proc SPIE 10690, Optical Design and Engineering VII. SPIE; 2018

2. Ribeiro R., Jones D., Schmidt L., Taylor K., Souza A., Papovich C., et al. The optical design for the Giant Magellan Telescope Multi-object Astronomical and Cosmological Spectrograph (GMACS). In: Proc SPIE 10702, Ground-based and Airborne Instrumentation for Astronomy VII. SPIE; 2018

3. Schmidt L., Ribeiro R., Taylor K., Jones D., Prochaska T., DePoy DL, et al. Optical design concept for the Giant Magellan Telescope Multi-object Astronomical and Cos- 
mological Spectrograph (GMACS). In: Proc SPIE 9908, Ground-based and Airborne Instrumentation for Astronomy VI. SPIE; 2016 


\section{List of Figures}

1.1 Simms' apparatus for measuring refractive and dispersive powers . . . . . . 37

1.2 GMACS mounted in GMT, as presented in CoDR 2019 . . . . . . . . . . 43

1.3 Digitized Sky Survey image of the region around Abell 2744 with overlay instrument fields of view . . . . . . . . . . . . . . . 45

2.1 Optical layout of a slit transmissive grating spectrograph . . . . . . . . . 55

2.2 Collimator exit pupil ray angle of incidence as a function of the telescope field of view with and without the small telescope angle approximation . . 60

2.3 Spectrograph Field of View . . . . . . . . . . . . . . . . . 63

2.4 Inscribed and circumscribed camera FoV projection at the detector plane . 66

2.5 Detector's principal parameters . . . . . . . . . . . . . . . 68

2.6 Diagram of a VPH grating . . . . . . . . . . . . . . . . 70

2.7 Fringe structures for VPH gratings . . . . . . . . . . . . . . . . 71

2.8 Variation of diffraction efficiency versus grating thickness for two different Bragg angles . . . . . . . . . . . . . . . . . . . . . 74

2.9 Grating Magnification . . . . . . . . . . . . . . . 75

2.10 Variables for defining the maximum value for the size of the dispersive element. 76

2.11 Optical parameters for radiometry in a finite conjugate imaging system . . 88

2.12 Depth of focus and detector tilt first-order tolerance . . . . . . . . . . . . 89

2.13 Example of detector footprint for high-resolution mode . . . . . . . . . . . 92

2.14 Distortion of the image spectra for several objects . . . . . . . . . . . 93

2.15 Spectral vignetting caused by the camera aperture . . . . . . . . . . . . . . 94

2.16 Slit transmission factor . . . . . . . . . . . . . . . . . . . . . . 95

2.17 Thin lens shape factor f . . . . . . . . . . . . . . . . . . . . . 98 
2.18 Position factor . . . . . . . . . . . . . . . . . . . . 99

2.19 Primary spherical aberration and coma of a thin lens as a function of the lens shape factor $q$ and position factor $p \ldots \ldots \ldots$. . . . . . . 100

2.20 Window aberration as a function of the tilt to the optical axis . . . . . . 102

2.21 Tilted plate in a converging beam . . . . . . . . . . . . . . . . 104

3.1 Layout of each individual channel of GMACS shown from two orthogonal orientations. . . . . . . . . . . . . . . . . . 109

3.22012 GMACS collimator cell and structure . . . . . . . . . . . . . 110

3.32012 GMACS collimator cell and structure . . . . . . . . . . . . . 111

3.4 GMOS optical layout. . . . . . . . . . . . . . . . . . 112

3.5 GMOS Collimator Design . . . . . . . . . . . . . . . . 112

3.6 GMOS Camera Design . . . . . . . . . . . . . . . . 113

3.7 DEIMOS major optical and mechanical components . . . . . . . . . . . . 114

3.8 DEIMOS camera . . . . . . . . . . . . . . . . . . 115

3.9 IMACS optical system layout . . . . . . . . . . . . . . . 116

3.10 IMACS fast camera layout . . . . . . . . . . . . . . . . 117

3.11 OSIRIS optical layout . . . . . . . . . . . . . . . . . 117

3.12 OSIRIS camera optical layout . . . . . . . . . . . . . . 118

3.13 A 3D solid model of one of Binospec's two beams. . . . . . . . . . . . . . 119

3.14 Binospec optical layout . . . . . . . . . . . . . . . . . . . . . 119

3.15 FORS's three main sections: the top or VLT's focal plane section, the central collimating section with the two collimator designs and the filter/grisms/camera section . . . . . . . . . . . . . . . 121

3.16 FORS optical layout . . . . . . . . . . . . . . . . . . . . . 121

3.17 (left) VIMOS optical layout . . . . . . . . . . . . . . . . . 122

3.18 VIMOS collimator layout . . . . . . . . . . . . . . . . 123

3.19 VIMOS' camera layout . . . . . . . . . . . . . . . . . . . 123

3.20 Overview of FOCAS and structure. The Subaru Telescope is to the left. . . 124

3.21 FOCAS optical layout . . . . . . . . . . . . . . . . . 125 
4.1 Graphical User Interface for the modeling of an optical spectrograph principal parameters. The yellowish banks are user defined and the white ones are calculated in real time by the software. . . . . . . . . . . . . 131

4.2 Optical Design Configuration Manager . . . . . . . . . . . . . . 133

4.3 GMACS Requirements cyclical flow diagram . . . . . . . . . . . . . . . 141

4.4 Flow diagram of the methodology for the GMACS optical system design 144

4.5 Flowchart for Tolerancing Process . . . . . . . . . . . . . . . . . 149

4.6 Sensitivity Diagram example generated by the Sensitivity Diagram Script. 151

4.7 Zemax coordinate breaks to move from local to global coordinates. . . . . . 153

4.8 Example of the LDE with three nodes after applying the local-to-global macro.154

4.9 FEA to Zemax integration and spectral stability analysis diagram process. 156

5.1 General optical layout of the GMT Wide Field Optical Spectrograph design 160

5.2 Grating size, $L_{\text {grat }}$, as a function of the collimator EFL . . . . . . . . . . . 164

5.3 Collimator EFL as a function of the camera EFL for telescope f/8.16 and several camera speeds. . . . . . . . . . . . . . . . 165

5.4 Resolving power as a function of the $f_{c o l}$ for three grating grooves density, four blaze wavelengths, $0.7^{\prime \prime}$ slit width and first diffraction order. . . . . . . 166

5.5 Camera FoV as a function of the camera EFL for several detector aspect ratio167

5.6 Lens Data Editor and Multi-Configuration Editor for the paraxial model . 170

5.7 Optical layout for the paraxial model . . . . . . . . . . . . . . . . . . . 171

5.8 Detector footprint diagram for the imaging, low- and high-resolution mode paraxial models . . . . . . . . . . . . . . . . . . . 171

5.9 Aperture and field size for classical refractive design-form . . . . . . . . 173

5.10 Aperture and field size for classical reflective design-form . . . . . . . . . . 174

5.11 Abbe Diagram for GMACS glasses and the corresponding CTEs $\left[1 \cdot K^{-1}\right] . \quad 176$

5.12 Collimator concepts, architectures and design examples . . . . . . . . . 179

5.13 TMA Collimator . . . . . . . . . . . . . . . . 180

5.14 General optical layouts for the refractive collimator architectures. . . . . . 182

5.15 GMACS collimator refractive architectures explored in this work . . . . . . 183

5.16 Finite element results due to gravity for two field lens shape factors . . . . 184

$5.17 \mathrm{KoM}$ and 2017 Baseline collimator optical layout . . . . . . . . . . . 185 
5.18 Baseline Panchromatic collimator spot diagram . . . . . . . . . . . . 187

5.19 Baseline Panchromatic collimator geometric ensquared energy . . . . . . 188

5.20 Panchromatic collimator chromatic focal shift. . . . . . . . . . . . . . . . 188

5.21 Broadband Collimator Optical Layout. . . . . . . . . . . . . . . . . . . . . 189

5.22 Split Collimator general layout and the variables to define the axial distance between the channels. . . . . . . . . . . . . . . . . . . . . . . . . 191

5.23 Wedged plano-spherical dichroic acting as a beamsplitter and an astigmatism compensator. . . . . . . . . . . . . . . . . . . . . . . 194

5.24 CoD Split Collimator 2,200 $\mathrm{mm} \mathrm{f} / 8.2$, FoV $7.4^{\prime}$ in diameter, exit pupil of $270 \mathrm{~mm}$ in diameter, with paraxial cameras. . . . . . . . . . . . . . 195

5.25 Spot size diagram for the Split Collimator . . . . . . . . . . . . . . 196

5.26 The PV and RMS profile for the Split Collimator in the blue channel. . . . 196

5.27 The PV and RMS profile for the Split Collimator in the red channel. . . . 197

5.28 Transverse Chromatic Aberrations for the Split Collimator. . . . . . . . . . 197

5.29 Comparison between hexagonal and circular FoV for low-resolution mode projected on the detector plane . . . . . . . . . . . . . . . . . . . . 198

5.30 3D Layout of an alternative Squared FoV Split Collimator . . . . . . . . . 199

5.31 An example of the eyepiece optical layout _ . . . . . . . . . . . . . . . 201

5.32 An example of the layout of a flat-field microscope objective in which the Petzval curvature is corrected by a thick negative achromatic placed at the entrance aperture . . . . . . . . . . . . . . . . . . 201

5.33 Examples of Petzval projection lenses . . . . . . . . . . . . . . . . . 202

$5.34 \mathrm{CoDR} 594 \mathrm{~mm} \mathrm{f} / 2.2$ blue camera with entrance pupil of $270 \mathrm{~mm}$ in diameter.204

$5.35 \mathrm{CoDR} 594 \mathrm{~mm} \mathrm{f} / 2.2$ red camera with entrance pupil of $270 \mathrm{~mm}$ in diameter. 205

5.36 Alternative CoD red camera designs. . . . . . . . . . . . . . . . . 207

5.37 GMACS CoDR Blue Arm with a low- and high-resolution modes superposed 208

5.38 GMACS CoDR blue arm spot size diagram. . . . . . . . . . . . . . . . . 209

5.39 (a) Detector footprint diagram for a low-resolution mode and (b) vignetting

5.40 (a) Detector footprint diagram for a high-resolution mode and (b) vignetting heat map. . . . . . . . . . . . . . . . . . . . . 211 
5.41 Source of asymmetry of the vignetting. Source: GMACS Team (2019) (Damien Jone $\ldots \ldots \ldots$. . . . . . . . . . . . . . . . . . . 212

5.42 Transversal Chromatic Aberrations for the GMACS Blue Arm in the image mode. . . . . . . . . . . . . . . . . . . 213

5.43 Matrix spot diagram and spot diagram for the blue arm image mode . . . 214

5.44 Polychromatic diffraction MTF for the blue arm in image mode . . . . . . 214

5.45 GMACS CoDR Red Arm with a low- and high-resolution modes superposed 215

5.46 GMACS CoDR red arm spot size diagram . . . . . . . . . . . . . . 216

5.47 Transversal Chromatic Aberrations for the GMACS Red Arm in the image

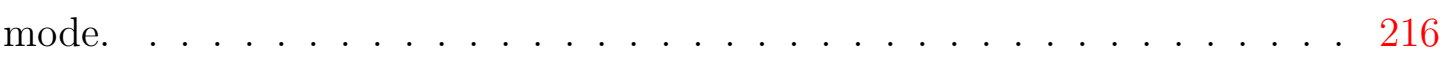

5.48 Optomechanical Conceptual Design . . . . . . . . . . . . . 219

5.49 Details of the articulation mechanisms for the blue camera . . . . . . . . . 220

5.50 Image quality sensitivity diagram for the blue arm at low-resolution mode. 223

5.51 Image quality sensitivity diagram for the red arm at low-resolution mode. . 225

5.52 Detector Assy tolerance for assembling. . . . . . . . . . . . . . . . 228

5.53 Groups definition for the flexure analysis. . . . . . . . . . . . 230

5.54 Displacement histogram and quiver plot at the focal plane of the perturbed system for the $250 \mu \mathrm{m}$ transverse displacement of the collimator group. . . 231

5.55 Displacement histogram and quiver plot at the focal plane of the perturbed system for $0.5^{\circ}$ tilt of the VPHG in the spectral direction . . . . . . . . . 232

5.56 Displacement histogram and quiver plot at the focal plane of the perturbed system for $0.5^{\circ}$ tilt of the VPHG in the spatial direction . . . . . . . . . . 233

5.57 Example FEA Reference Plane in the Blue Collimator Group. . . . . . . . 233

5.58 EED80 Histogram before and after flexure . . . . . . . . . . . . 234

5.59 Displacement histogram and quiver plot at the focal plane of the perturbed

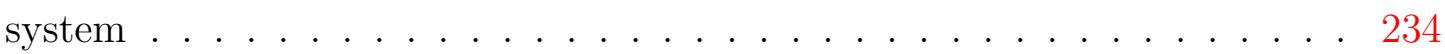

5.60 EED and SS results for the FL as a compensator when the IQ is optimized in the blue arm. . . . . . . . . . . . . . . . . . . 235

5.61 EED and SS results for the FL as a compensator when the SS is optimized in the blue arm. . . . . . . . . . . . . . . . . . . . . 236

5.62 EED and SS results for the collimator groups as a compensator when the IQ is optimized in the blue arm. . . . . . . . . . . . . . 236 
5.63 EED and SS results for the collimator groups as a compensator when the SS is optimized in the blue arm. . . . . . . . . . . . . . . . 237

5.64 EED and SS results for the camera group as a compensator when the IQ is optimized in the blue arm. . . . . . . . . . . . . . . . 237

5.65 EED and SS results for the camera groups as a compensator when the SS is optimized in the blue arm. . . . . . . . . . . . . . . . . 238

5.66 Current strategy flexure compensator . . . . . . . . . . . . . 238

5.67 EED and SS results for the collimator in the axial position ( $\mathrm{Z}$ axis) and camera groups in the transversal directions ( $\mathrm{X}$ and $\mathrm{Y}$ axis) as a compensator when the SS is optimized in the blue arm. . . . . . . . . . . . . 239

A.1 The founding members of the GMT partnership are an international consortium of universities and research institutions. . . . . . . . . . . 265

A.2 GMT optical layout, fast-steering mirror system, and an adaptive secondary mirror system . . . . . . . . . . . . . . . . . . . 270

A.3 The GMT illustrative design within the dome . . . . . . . . . . . . 270

A.4 The field curvature for Ritchey-Chrétien and Gregorian telescopes . . . . . 272

A.5 Available instrument mounting for GMT . . . . . . . . . . . . . . . . . . 274

A.6 GMT prescription data . . . . . . . . . . . . . . . . 274

A.7 GMT DGNF non-segmented spot diagram . . . . . . . . . . . . . . 275

A.8 GMT DGNF non-segmented encircled energy diagram . . . . . . . . . . 275

A.9 GMT DGNF segmented spot diagram . . . . . . . . . . . . 276

A.10 Center field PSF at the reference wavelength of $2.18 \mu \mathrm{m} \ldots \ldots$. . . . . 276

C.1 Split collimator blue arm PV wavefront performance at the exit pupil. . . . 298

C.2 Split collimator blue arm RMS wavefront performance at the exit pupil. . . 300

C.3 Split collimator red arm PV wavefront performance at the exit pupil. . . . 302

C.4 Split collimator red arm RMS wavefront performance at the exit pupil. . . 304 


\section{List of Tables}

2.1 Astronomical spectrograph parameters from the perspective of optical design 56

2.2 Spectrograph design top-level requirements . . . . . . . . . . . . . . . . . 83

2.3 Specification and requirements for a spectrograph's optical system basic

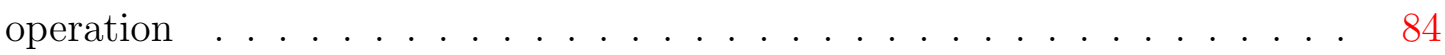

2.4 Seidel Coefficients for a plano-plano window . . . . . . . . . . . . . 103

3.1 Principal parameters of the selected instruments . . . . . . . . . . . 108

$4.1 \quad$ FEA results data example . . . . . . . . . . . . . . . . . . . . 153

5.1 Summary of the principal functional parameters . . . . . . . . . . 163

5.2 Simulation of the system parameters as a function of the camera speed . . 167

5.3 General characteristics of refractive and reflective optical designs for ELT instruments. . . . . . . . . . . . . . . . . . . 172

5.4 List of large optical glass blanks . . . . . . . . . . . . . . . . . . 175

5.5 Nikon NIGS i-line glass family blank dimensions . . . . . . . . . . . 176

5.6 Lens Data for the KoM and 2017 Baseline Collimators. . . . . . . . . . . . 186

5.7 Performance of the single on-axis collimators. . . . . . . . . . . . . . . . 190

5.8 Aspheric surface parameters of the blue and red CoDR Split Collimator arms.194

5.9 Blue camera aspheric surface parameters. . . . . . . . . . . . . . . . 204

5.10 Red camera aspheric surface parameters. . . . . . . . . . . . . 206

5.11 GMACS CoDR Blue Arm spectrograph parameters . . . . . . . . . . . 210

5.12 GMACS CoDR Encircled Energy for Blue Arm Low-Resolution Mode. . 210

5.13 GMACS CoDR Red Arm spectrograph configuration parameters . . . . . . 215

5.14 GMACS CoDR Encircled Energy for Red Arm Low-Resolution Mode. . . . 215 
5.15 Examples of spectrograph configurations for a $0.7^{\prime \prime}$ slit width. . . . . . . 218

5.16 Adapted Zemax tolerance acronyms used for the sensitivity analysis. . . . . 221

5.17 Tightest tolerances for the blue arm for $3 \%$ of image quality degradation for each parameter considering axial back focus adjustment (maximum range

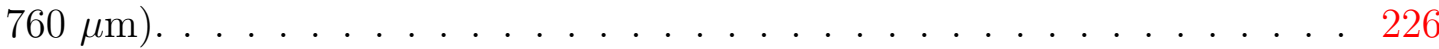

5.18 The optical groups that were tracked in the sensitivity analysis and FEA and had resulting motions input into Zemax for image quality and spectral stability analysis. . . . . . . . . . . . . . . . . . . . . 229

5.19 Inverse Sensitivity analysis for the GMACS blue arm in low-resolution mode for 0.3 spectral resolution displacement at the focal plane $(\approx 57 \mu m) \ldots$. . . 230

5.20 Inverse Sensitivity analysis for the GMACS red arm in low-resolution mode for 0.3 spectral resolution displacement at the focal plane $(\approx 57 \mu m) \ldots$. . . 231

A.1 Technical specifications of the new generation of Extreme Large Telescopes,

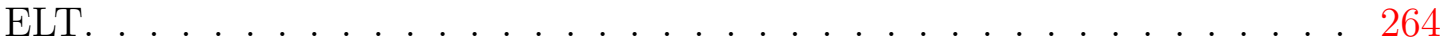

A.2 Basic GMT properties . . . . . . . . . . . . . . . . . . . 269

A.3 GMT Optical Prescription . . . . . . . . . . . . . . . . . 271

A.4 GMT's first generation instruments . . . . . . . . . . . . . . . . 273 


\section{Contents}

Symbols and Acronyms . . . . . . . . . . . . . . . . . . . . . . . . . . . 29

1. Introduction . . . . . . . . . . . . . . . . . . 35

1.1 Brief Historical Development of Astronomical Spectroscopes . . . . . . . . 35

1.2 Optical Multi-Object Spectrograph . . . . . . . . . . . . . . 38

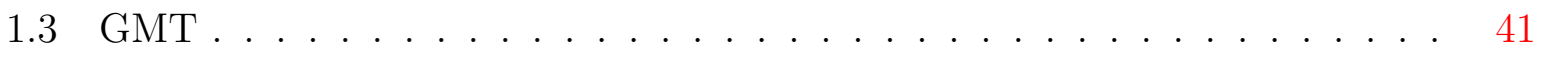

1.3 .1 MOS in ELTs . . . . . . . . . . . . . . . . 41

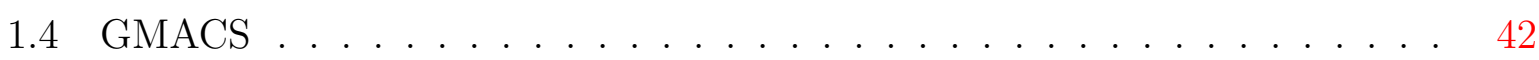

1.4.1 Integration with other instruments . . . . . . . . . . . . . 44

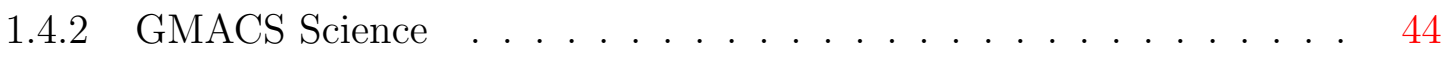

1.5 GMT Brazil Office . . . . . . . . . . . . . . . . . 48

1.6 Thesis' Objectives and Outline . . . . . . . . . . . . . . 49

2. Low- and Mid-Resolution Multi-Object Transmissive Grating Spectrographs . . 53

2.1 General Principles of a Multi-Object Optical Spectrograph . . . . . . . . . 54

2.1.1 Spectrograph Layout . . . . . . . . . . . . . . . 54

2.1.2 Figure of Merit . . . . . . . . . . . . . . . 57

2.2 Spectrograph Optical Relationships . . . . . . . . . . . . . . 58

2.2.1 Spectrograph Optical Principles . . . . . . . . . . . 58

2.2.2 Components' Principal Parameters . . . . . . . . . . . . . . 64

2.3 General VPH Transmission Grating Equations . . . . . . . . . . . . . . . 68

2.3.1 Blaze grating and Littrow condition . . . . . . . . . . . . 71

2.3.2 First-Order Diffraction Efficiencies . . . . . . . . . . . . . 72

2.3.3 Spectrograph Principal Parameters . . . . . . . . . . . . . . . . . . 74 
2.3.4 Spectrograph System and Subsystems Requirements . . . . . . . . . 82

2.3.5 Spectrograph Performance Criteria . . . . . . . . . . . . . 83

2.3.6 Degradation of the Spectra Quality . . . . . . . . . . . . 90

2.3.7 Opto-mechanical Influences on the Spectrograph Quality . . . . . . 95

2.4 Spectrograph Subsystems Optical Design . . . . . . . . . . . . . . . . 97

2.4.1 Collimator . . . . . . . . . . . . . . . . . 97

2.4.2 Cameras ........................... 106

3. Review of MOS Optical Designs for Large Telescope Instrumentation . . . . . . 107

3.1 GMACS 2011 CoDR . . . . . . . . . . . . . . . . 108

3.2 GMACS-like Multi-object spectrographs . . . . . . . . . . . . . . . . . . 110

3.2.1 Gemini Observatory . . . . . . . . . . . . . . . 110

$3.2 .2 \quad$ Keck II . . . . . . . . . . . . . . . . . . . . . 113

3.2.3 Las Campanas Observatory, Magellan 1 . . . . . . . . . . . . . . . 114

3.2.4 Gran Telescopio Canarias, GTC . . . . . . . . . . . . . . 116

3.2.5 Multiple Mirror Telescope, MMT . . . . . . . . . . . . . 118

3.2.6 Very Large Telescope, VLT . . . . . . . . . . . . . . . . . . . 120

3.2.7 Subaru . . . . . . . . . . . . . . . . . . . 124

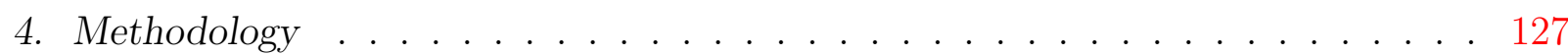

4.1 Optical Design as Part of the Project . . . . . . . . . . . . . 127

4.2 Design Tools . . . . . . . . . . . . . . . . . . . . . . . . . . . 129

4.2 .1 Optical Design Software . . . . . . . . . . . . . . . . . . 129

4.2.2 Graphical User Interface for Grating Spectrographs Simulator . . . 130

4.2.3 Computational Infrastructure . . . . . . . . . . . . . . . 132

4.2.4 Optical Design Configuration Manager . . . . . . . . . . . . . . 132

4.2.5 Communication, Meetings, Data sharing and Reviews . . . . . . . . 134

4.2 .6 Literature Resources . . . . . . . . . . . . . . . . . . 135

4.3 System Engineering and Management Tools . . . . . . . . . . . . . . 136

4.3.1 Product Breakdown Structure . . . . . . . . . . . . . . . . 136

4.3 .2 Process Flowchart . . . . . . . . . . . . . . . 137

4.3.3 Decision Analysis - Tradeoff Studies . . . . . . . . . . . . . . . . . 137

4.4 Optical Spectrograph Design . . . . . . . . . . . . . . . . . . 138 
4.4.1 Basic Principles for General Optical Design _ . . . . . . . . . . . 138

4.4.2 Glass Selection for ELT Instrumentation . . . . . . . . . . . . . . 139

4.4.3 Requirement Definition and Flow Diagram . . . . . . . . . . . . . 140

4.4.4 Preliminary Phase . . . . . . . . . . . . . . . . . . 141

4.4.5 GMACS Optical Design Methodology . . . . . . . . . . . . 143

4.4.6 Tolerancing . . . . . . . . . . . . . . . . . . . . . . 148

4.4.7 Flexure Analysis . . . . . . . . . . . . . . . . . 150

5. GMACS Optical System Design . . . . . . . . . . . . . . . . . . . . . . 159

5.1 Design Chronology . . . . . . . . . . . . . . . . . 159

5.2 GMACS Requirements . . . . . . . . . . . . . . . . . . 162

5.3 Preliminary Design Trade Study . . . . . . . . . . . . . . . . . 162

5.3.1 Principal Parameters Trade Study Results . . . . . . . . . . . . 163

5.3.2 GMACS Paraxial Modeling . . . . . . . . . . . . . . 169

5.4 Definition of Optical Concepts and Architectures . . . . . . . . . . . . 171

5.4.1 Glass Selection for ELT Instrumentation . . . . . . . . . . . . 173

5.5 Initial Considerations for the Optical Design . . . . . . . . . . . . . . 177

5.5.1 Telescope Optical Model . . . . . . . . . . . . . . . . . . . 177

5.5.2 Collimator Optical Quality Metrics . . . . . . . . . . . 177

5.6 Collimator Concept Tradeoff Analysis . . . . . . . . . . . . . . . . . . . . 178

5.7 Refractive Collimator Architecture Tradeoff Analysis . . . . . . . . . . . . 180

5.7 .1 Common Elements . . . . . . . . . . . . . . . 183

5.7 .2 Single On-axis Collimators . . . . . . . . . . . . . 185

5.7 .3 Split collimators . . . . . . . . . . . . . . . . . . . . . 189

5.7.4 Collimator Architecture Trade Conclusion . . . . . . . . . . . . . . 199

5.8 Camera Design . . . . . . . . . . . . . . . . . 200

5.8 .1 Petzval Lens . . . . . . . . . . . . . . . . . . . . 202

5.8.2 Conceptual Design Cameras . . . . . . . . . . . . . . 203

5.9 GMACS Conceptual Design . . . . . . . . . . . . . . 208

5.9.1 CoDR GMACS Blue Arm Performance . . . . . . . . . . . 208

5.9.2 CoDR GMACS Red Arm Performance . . . . . . . . . . . . . . 213

5.9.3 GMACS Configurations . . . . . . . . . . . . 217 
5.9.4 GMACS Optomechanical Design . . . . . . . . . . . 217

5.10 Tolerance Analysis . . . . . . . . . . . . . . . . . . . 217

5.10 .1 Sensitivity . . . . . . . . . . . . . . . . 220

5.10 .2 Tolerance . . . . . . . . . . . . . . . 226

5.10 .3 Detector Assy . . . . . . . . . . . . . . . . . 227

5.11 Spectral Stability Analysis . . . . . . . . . . . . . . . . 228

5.11 .1 Flexure Sensitivity Analysis . . . . . . . . . . . . . 228

5.11 .2 Flexure Tolerance Analysis . . . . . . . . . . . . . . . . 232

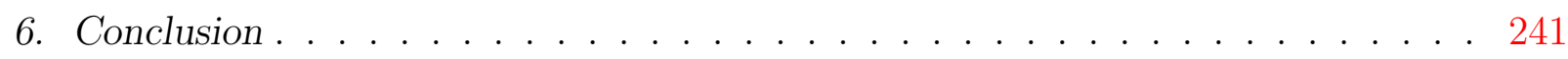

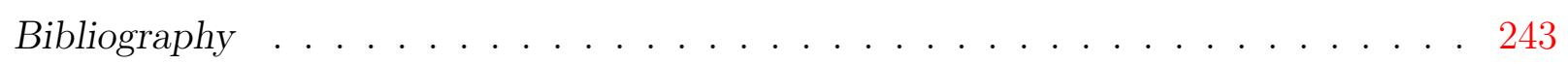

Appendix 261

A. GMT Optical Design . . . . . . . . . . . . . . . . . . . 263

A.1 Introduction to the Extreme Large Telescopes, ELTs . . . . . . . . . . . 263

A.2 The Giant Magellan Telescope, GMT . . . . . . . . . . . . . . . . 264

A.2.1 GMT Science Goals . . . . . . . . . . . . . . 265

A.2.2 GMT Optical Design . . . . . . . . . . . . . 267

A.2.3 GMT's First Generation Instruments . . . . . . . . . . . . . . 272

A.3 GMT Design and Optical Performance . . . . . . . . . . . . . . 274

B. List of Zemax Macros and Python Scripts . . . . . . . . . . . . . . . . . . 277

B.1 Optical Design . . . . . . . . . . . . . . . . 278

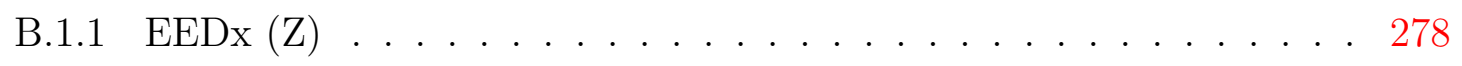

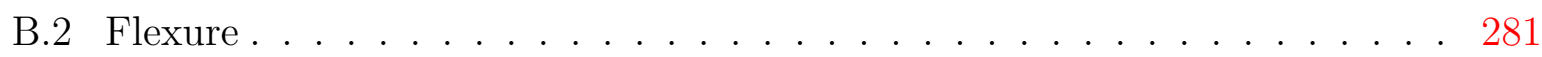

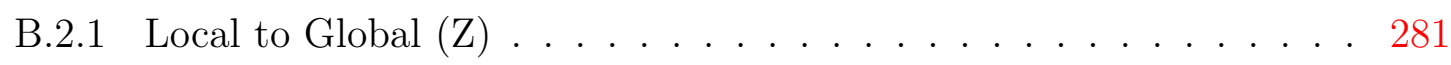

B.2.2 Local to Global - Remove $(\mathrm{Z})$. . . . . . . . . . . . . . 285

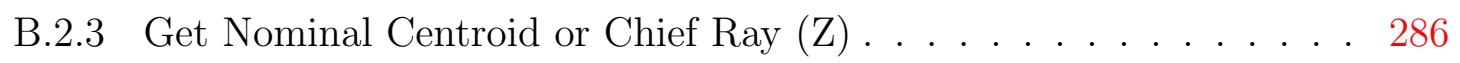

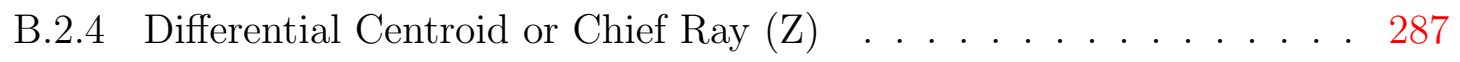

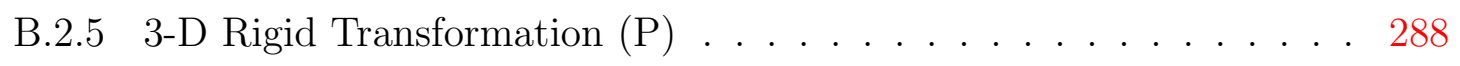

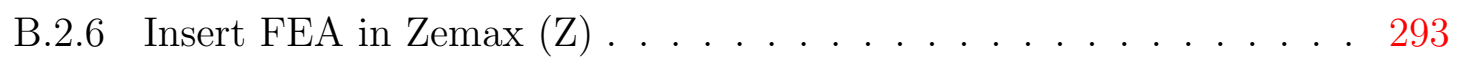


B.2.7 Create Merit Function for Spectral Stability $(\mathrm{Z}) \ldots . . . . . .295$

C. CoD Split Collimator Wavefront Performance . . . . . . . . . . . . . . . 297 



\section{Symbols and Acronyms}

The units or the most commonly used units are shown in brackets.

\begin{tabular}{|c|c|c|}
\hline Term & Description & Unit \\
\hline$\alpha$ & $\begin{array}{l}\text { Incident beam angle on grating from the normal } \\
\text { vector }\end{array}$ & [angle] \\
\hline$\beta$ & $\begin{array}{l}\text { Diffracted ray angle from grating to the surface } \\
\text { normal vector }\end{array}$ & [angle] \\
\hline$\Gamma$ & Plate scale & [angle length ${ }^{-1}$ ] \\
\hline$\gamma$ & $\begin{array}{l}\text { Angle between the incident light path and the } \\
\text { plane perpendicular to the grooves at the grating } \\
\text { center (off-plane angle) }\end{array}$ & [angle] \\
\hline$\delta$ & Depth of Focus (see DoF) & {$[$ length $]$} \\
\hline$\delta \lambda$ & Bandwidth of the resolution element & [length] \\
\hline$\delta \lambda_{\text {slit }}$ & Bandwidth per slit width & [length] \\
\hline$\Delta \alpha_{\mathrm{FWHM}}$ & Angular Bragg envelope & [angle] \\
\hline$\Delta \lambda_{\mathrm{FWHM}}$ & Spectral Bragg envelope & {$[$ length] } \\
\hline$\Delta \lambda$ & Spectral Coverage & {$[$ length] } \\
\hline$\Theta$ & Collimator-camera angle & [angle] \\
\hline$\theta_{\text {seeing }}$ & Observatory site seeing & [angle] \\
\hline$\theta_{\text {slit }}$ & Angular size of the slit projected back onto the sky & [angle] \\
\hline$\theta_{B}$ & Blaze angle & [angle] \\
\hline$\lambda_{B}$ & Blaze wavelength & {$[$ length $]$} \\
\hline$\nu$ & Grating groove density $(=1 / d)$ & {$\left[\right.$ length $\left.^{-1}\right]$} \\
\hline$\Omega_{p}$ & Projected Solid Angle in cosine space weighted & [angle] \\
\hline
\end{tabular}




\begin{tabular}{|c|c|c|}
\hline$A_{d}$ & Detector area & {$\left[\right.$ length $\left.^{2}\right]$} \\
\hline$B_{\lambda}$ & Spectral brightness (see Spectral Radiance) & {$\left[\mathrm{W} \mathrm{\textrm {m } ^ { - 2 }} \mathrm{\mu m}^{-1} \mathrm{sr}^{-1}\right]$} \\
\hline$B_{x}$ & Binning Factor & \\
\hline$D_{\theta}$ & Angular dispersion & {$[$ angle $/ \AA]$} \\
\hline$D_{c o l}$ & Collimator exit pupil diameter & [length] \\
\hline$D_{t e l}$ & Telescope entrance pupil diameter & [length] \\
\hline$D_{x}$ & Linear dispersion & {$\left[\right.$ length $\left.\AA^{-1}\right]$} \\
\hline$D_{x_{p}}$ & Linear dispersion in units of pixel size & {$\left[\right.$ pixels $\left.\AA^{-1}\right]$} \\
\hline$d$ & $\begin{array}{l}\text { Separation between fringes in a grating with uns- } \\
\text { lanted fringes }\end{array}$ & [length] \\
\hline$d_{g}$ & $\begin{array}{l}\text { Projected separation between the fringes in the } \\
\text { plane of the grating }\end{array}$ & [length] \\
\hline$F_{i}$ & f-number (speed) of the $i$ optical group & \\
\hline$f_{\#}$ & $\begin{array}{l}\text { f-number (speed) - } X \mathrm{~mm} \mathrm{f} / y \text { means ELF of } X \mathrm{~mm} \\
\text { at speed } Y\end{array}$ & \\
\hline$G_{\lambda}$ & Grating length along the spectral direction & [length] \\
\hline$G_{x}$ & Grating length along the spatial direction & [length] \\
\hline$H$ & Lagrange invariant & [length] \\
\hline$I^{\prime}$ & Image size on detector plane & [length] \\
\hline$L_{\lambda}$ & Spectral radiance (radiometric definition - see & \\
\hline & Brightness) & \\
\hline$L_{\text {grat }}$ & Length of the grating illuminated by the collimated & [length] \\
\hline & beam & \\
\hline$m$ & Diffraction order & \\
\hline M & Transverse (or lateral) magnification & \\
\hline$M_{\text {pupil }}$ & Pupil (de)magnification & \\
\hline$n$ & Index of refraction & \\
\hline$N_{\lambda}$ & Number of pixel in the detector spectral direction & \\
\hline$N_{x}$ & Number of pixel in the detector field direction & \\
\hline$P_{\theta}$ & Angular reciprocal dispersion & {$\left[\right.$ length angle $\left.{ }^{-1}\right]$} \\
\hline$P_{x_{p}}$ & Linear reciprocal dispersion in units of pixels & [length pixels $\left.{ }^{-1}\right]$ \\
\hline$P_{x}$ & Linear reciprocal dispersion & {$\left[\right.$ length length $\left.{ }^{-1}\right]$} \\
\hline
\end{tabular}




\begin{tabular}{|c|c|c|}
\hline$p$ & Lens Conjugate Factor & \\
\hline$p_{x}$ & Pixel size (pixel pitch) & [length] \\
\hline$q$ & Lens Shape Factor & \\
\hline$r_{a n}$ & Anamorphic factor & \\
\hline$r_{\text {ele }}$ & Resolution element & [length] \\
\hline$r_{\text {elep }}$ & Resolution element in pixel units & [number of pixels] \\
\hline$t$ & DCG thickness & [length] \\
\hline$u$ & Marginal ray angle & [angle] \\
\hline$u_{p}$ & Principal (or Chief) ray angle & [angle] \\
\hline$v$ & Abbe number & \\
\hline$w_{s}$ & Slit width along the spectral direction & [length] \\
\hline$w_{s}^{\prime}$ & Image size of the slit along the spectral direction & [length] \\
\hline$y$ & Marginal ray height & [length] \\
\hline$y_{p}$ & Principal (or Chief) ray height & [length] \\
\hline $\mathrm{ADC}$ & Atmospheric Dispersion Corrector & \\
\hline $\mathrm{AGN}$ & Active Galactic Nuclei & \\
\hline $\mathrm{AO}$ & Adaptive Optics & \\
\hline AOI & Angle of Incidence & [angle] \\
\hline $\mathrm{AR}$ & Anti-Reflection (coatings) & \\
\hline $\mathrm{ASM}$ & Adaptive Secondary Mirror & \\
\hline $\mathrm{BFD}$ & Back Focal Distance & [length] \\
\hline BFSD & Best Fit Spherical Deviation & [length] \\
\hline BOSS & Baryon Oscillation Spectroscopic Survey & \\
\hline BTA-6 & Large Altazimuth Telescope (alternative name) & \\
\hline $\mathrm{CBK}$ & Coordinate Break & \\
\hline $\mathrm{CCD}$ & Charge-Coupled Device & \\
\hline CfA & Center for Astrophysics & \\
\hline CGM & Circumgalactic medium & \\
\hline $\mathrm{CoD}$ & Conceptual Design & \\
\hline CoDR & Conceptual Design Review & \\
\hline $\mathrm{CR}$ & Chief Ray & \\
\hline DCG & Dichromated Gelatin & \\
\hline
\end{tabular}


DEIMOS Deep Extragalactic Imaging Multi-Object Spectrograph

DGNF Direct Gregorian Narrow Field

DGWF Direct Gregorian Wide Field

DoF Depth of Focus (see $\delta$ )

[length]

EEDx $\quad \mathrm{x} \%$ Encircled Energy Diameter

[angle or length]

E-ELT European Extreme Large Telescope

EFL Effective Focal Length

[length]

ELT Extreme Large Telescope

ENPD Entrance Pupil Diameter

EPO European Patent Search

ETC Expusure Time Calculator

EXPD Exit Pupil Diameter [length]

FAPESP Fundação de Amparo à Pesquisa do Estado de São

Paulo (São Paulo Research Foundation)

FEA Finite Element Analysis

FF Field Flatter

FFa Fill Factor

FL Field Lens

FOCAS Faint Object Camera and Spectrograph on the Subaru Telescope

FORS Focal Reducer and Low Dispersion Spectrograph

FoV Field of View [angle]

FP Folded Ports

FSM Fast-steering Secondary Mirror

FWHM Full Width at Half Maximum

G-CLEF GMT-Consortium Large Earth Finder

GIS Gravity-invariant Instrument Station

GMACS Giant Magellan Telescope Astronomical and Cosmological Multi-Object Spectrograph

GMOS Gemini Multi-Object Spectrograph

GMT Giant Magellan Telescope 


\begin{tabular}{|c|c|c|}
\hline GMTO & Giant Magellan Telescope Cooperation & \\
\hline GTC & Gran Telescopio Canarias & \\
\hline HST & Hubble Space Telescope & \\
\hline IAG & Institute of Astronomy, Geophysics and Atmosphe- & \\
\hline & ric Sciences & \\
\hline IGM & Intergalactic Medium & \\
\hline IMACS & Inamori-Magellan Areal Camera and Spectrograph & \\
\hline IMF & Instrument Mounting Frame & \\
\hline INPI & National Institute of Industrial Property (Brazil) & \\
\hline IQ & Image Quality & \\
\hline JWST & James Webb Space Telescope & \\
\hline LBT & Large Binocular Telescope & \\
\hline $\mathrm{LCO}$ & Las Campanas Observatory & \\
\hline LDE & Lens Data Editor & \\
\hline LSST & The Large Synoptic Survey Telescope & \\
\hline $\mathrm{LyC}$ & Lyman Continuum & \\
\hline MANIFEST & MANy Instrument FibEr SysTem & \\
\hline $\mathrm{MCE}$ & Multi-Configuration Editor & \\
\hline $\mathrm{MF}_{\text {tel }}$ & Telescope Merit Function & {$\left[\right.$ length $^{2}$ angle $\left.^{-1}\right]$} \\
\hline MMT & Multiple Mirror Telescope & \\
\hline MoEs & Measures of Effectiveness & \\
\hline MOS & Multi-object Spectroscopy & \\
\hline OSS & Optical Support Structure & \\
\hline $\mathrm{R} \& \mathrm{D}$ & Research and Development & \\
\hline RCWA & Rigorous Coupled-Wave Analysis & \\
\hline RMS & Root Mean Square & \\
\hline $\mathrm{RoC}$ & Radius of curvature & [length] \\
\hline $\mathrm{SMBH}$ & Supermassive Black-Holes & \\
\hline SNR & Signal-to-Noise Ratio & {$[\mathrm{dB}$ or none $]$} \\
\hline SOW & Statement of Work & \\
\hline SS & Spectral Stability & \\
\hline SVD & Singular Value Decomposition & \\
\hline
\end{tabular}


STD Standard Deviation

TAMU Texas A\&M University

TCA Transverse Chromatic Aberration (Lateral Colour) [length or angle]

TMT Third Meter Telescope

UDO User-Defined Object

UDS User-Defined Surface

UFD Ultra-Faint Dwarf

USP University of São Paulo

USPTO United States Patent and Trademark Office

VLT Very Large Telescopes

VPHG Volume Phase Holographic Grating

WFC Wide Field Corrector

WFIRST Wide Field Infrared Survey Telescope

WFOS Wide Field Optical Spectrograph (TMT)

ZOS-API Zemax OpticStudio Application Programming Interface

ZPL Zemax Programming Language

ZPLM Zemax Programming Language Macro 
Chapter 1

\section{Introduction}

This thesis presents a compilation of the research carried out regarding part of the development of the GMACS optical project for the conceptual phase during the years 2015 to 2020. In this chapter, we make a brief introduction to multi-object optical spectrographs for ground-based astronomy and the principal technical challenges behind the development of such instruments for the Extreme Large Telescopes (ELTs) in the context of the optical design for GMACS, the GMT Multi-Object Astronomical and Cosmological Spectrograph. Therefore, we outline the primary and secondary objectives of this research and describe the organization of the chapters.

\subsection{Brief Historical Development of Astronomical Spectroscopes}

The astronomical spectrograph has played a unique role in the development of modern astrophysics and astronomy. Among all the variety of instruments other than the telescope itself, "none other can compete with the spectrograph for the range of new astronomical knowledge it has provided, and for the insights it has given on the physical nature of the celestial bodies in the Universe" (Hearnshaw, 2009). The spectrograph and its predecessor, the visual spectroscope, have revolutionized the study of the Sun, the planets, stars, gaseous nebulae, the interstellar medium, galaxies and quasars.

The origin of the spectroscopy theory and its subsequent instrumentation are identified from the discovery of the law of refraction in the early seventeenth century by Willebrord Snell (1951-1626) in 1621 and later by René Descartes (1596-1650) in his treatise Dioptrics of 1637 (van Berkel et al., 1998). The Snell-Descartes law of refraction was used to account for the formation of rainbows, although it did not explicitly explain the colors produced. 
However, in his first paper published by the Royal Society in 1672, Issac Newton (16421726) presented the experimental results with glass prisms and sunlight, as well as his (corrected) conclusions about the relationship between the refraction and colors (Newton, 1672). Despite the primitive apparatus, which consisted of a small hole in his window shutters, a glass prism, and a wall 6 meters from the prism used as a screen onto which the spectrum was projected, these experiments were the fundamental starting point for the science of spectroscopy. Newton optimized the experimental setup by adding lenses to improve the quality of the projected image and employing different density prisms since he correctly believed that the dispersive power of prisms was related to the refractive index of the glass. By observing the continuum spectrum formed by the planet Venus and bright stars, he concluded that using a telescope would both increase the quantity of light as well as reduce the undesirable effects of the atmosphere scintillation. Willian Herchel (17381822), in 1800, used similar prism-based apparatus to measure the temperature of the sunlight spectrum employing thermometers with blackened bulbs (to absorb heat better). He noticed the temperature rises in the direction of the red portion of the spectral, even beyond the visible spectrum (Herschel, 1800)

The diffraction grating, introduced by Thomas Young (1773-1829) in 1801, demonstrates the wave nature of light and shows that the wavelength could be obtained from the groove spacing of a grating. His first gratings comprised a series of parallel grooves ruled on glass at the spacing of about 20 grooves per millimeter (Young, 1802). Young's work was continued by glass maker Joseph Fraunhofer (1787-1826) in the early 1820s, whose first gratings were coarse transmission gratings made by stretching fine parallel wires between the threads of two screws - typically with spacings of up to 20 wires per millimeter. In later experiments, Fraunhofer produced 130 grooves per millimeter gratings by ruling with a diamond directly onto glass, which resulted in a higher dispersion. His primary interest was the determination of the dispersion of different glasses (the relationship of the material's index of refraction with the wavelength). He employed a simple diffraction grating monochromator to select the wavelengths and used the Snell's law of refraction to a prism spectroscope for the index measurement. Such experiments were the basis for the future of spectroscopy and spectroscope design, as well as the design of telescopes employing achromatic doublets for the objective lenses (Hearnshaw, 2009).

The early 1820s spectroscopes were comprised of a slit in a window-shutter, a dispersive 
element, a lens objective and a projection plane. The collimator in prism spectroscopes was introduced by Jacques Babinet (1794-1872) and presented to the Académie des Sciences in 1839 by François Arago (1786-1853), together with the announcement of the Daguerreotype process for photography. Arago predicted the use of the photographic technique in the field of selenography, photometry and spectroscopy (Hearnshaw, 2009; Hughes, 2012). One year later, the famous optical instrument maker William Simms (1793-1860) described in details the slit and collimator of his solar spectroscope components, see figure 1.1 (Simms, 1839). His design became a model for many laboratory spectroscopes of the mid nineteenth century. In the following years, collimators became increasingly common and by 1860 the collimator was essentially a universal feature of all astronomical and laboratory spectroscopes.

Fig. 1.

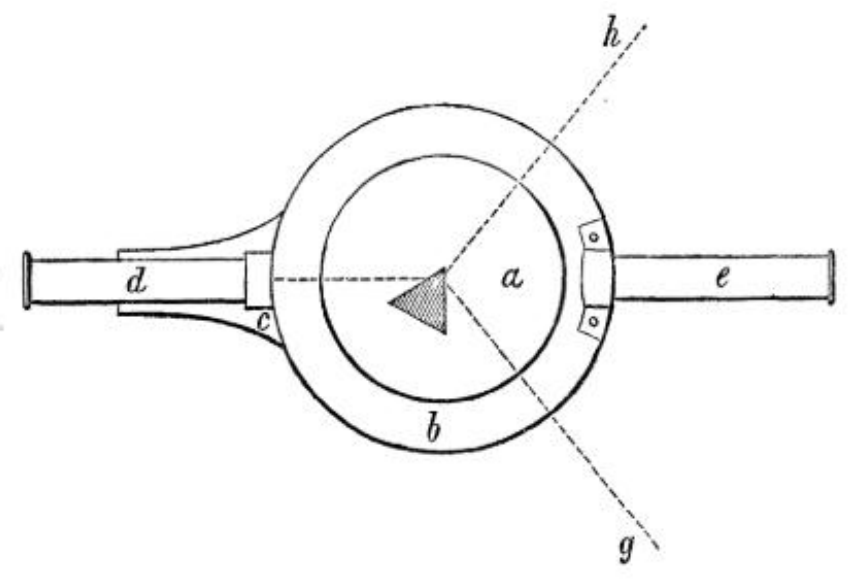

Fig. 2.

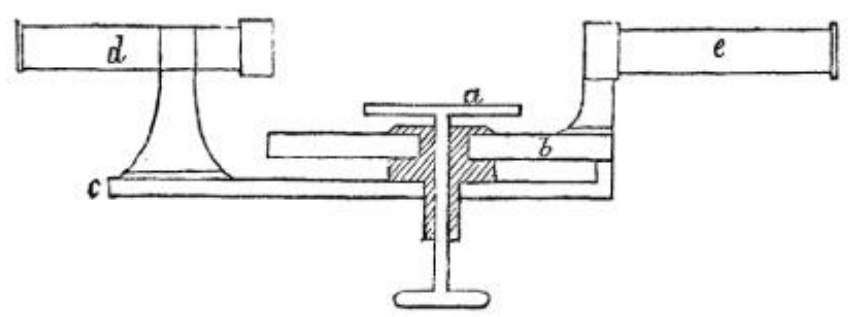

Figure 1.1: Simms' apparatus for measuring refractive and dispersive powers that employs two telescopes, one as collimator, and the other for making observations ("d" and "e", respectively), and two independent rotation tables for the observations telescope and the dispersive element ("b" and "a", respectively). Source: Simms (1839). 


\subsection{Optical Multi-Object Spectrograph}

According to Watson (1983), the spectrograph basic optical components have changed a little from a slit, a collimator, some kind of dispersive element, an imaging system for the resulting spectrum, and a detector. Even considering the advances in optical design, improvements in manufacturing and testing of precise components, the development of antireflection coatings and the inclusion of a photo-counting system from the advent of digital array detectors, the underlying instrument principles remain the same. However, since the fundamental weakness of earlier spectrographs is the general inability to observe more than one object at a time, except in circumstances in which the objects are aligned on the slit, it is highly desired that spectrographs incorporate the capability of having simultaneous recording of a number of spectra of astronomical objects in a single observation, known as Multi-Objects Spectroscopy (MOS), to increase the efficiency of telescope use. Prism spectroscopy was the only MOS solution available to astronomers until the year 1885; however, this solution has observational limitations due to overlapping spectra, sky brightness, and the dependence on the resolving power as a function of the seeing (Hearnshaw, 2009).

Alternative for increasing the efficiency of MOS emerged in the 1980's with the use of optical fibers (Hill et al., 1982; Tubbs et al., 1982) or aperture plate (Butcher, 1982; Fort et al., 1986), and have led to remarkable progress in our understanding of many fields of astrophysics, including galaxy and large-scale structure evolution, clusters of galaxies, the dynamics of our galaxy, and identifying the most distant galaxies. In fiber-fed MOS, fibers are inserted either manually or automatically (computer-controlled by robotic arms) below the telescope focal plane to couple the light of the objects and conduct it to the spectrograph aperture. An advantage of fiber-fed MOS is that the spectrograph can be installed in the Nasmith or another invariant gravity mount of the telescope to allow for a high stability, and high-resolution spectrograph. Fiber-optic MOS systems can accept more spectra on the available CCD area, as the spectra are aligned for maximum packing efficiency on the available area (Hearnshaw, 2009). The efficiency is measured by the resolving power-throughput product. It is a function of the fibers focal ratio degradation, losses due to reflection and internal absorption, uncorrected fiber positioning, among others.

In MOS, the aperture plate consists of a black anodized aluminum sheet at the teles- 
cope focal plane with slits drilled in it, also known as a slit mask. The slits are effectively the entrance slit of the spectrometer, whose size defines the resolution and spectral resolving power of the spectrograph (see chapter 2). Aperture plate systems do not make as efficient use of the CCD area as fiber-fed ones do, given that the spectra are not aligned; consequently, they have fewer objects in a given field and are generally limited to a smaller field of view. Therefore, the slits must be chosen so that the resulting spectra do not overlap in the detector. Nevertheless, the sky-background measurements in MOS systems are more effective than for fiber-fed instruments.

Just as the MOS capability increases the efficiency of using the telescope by allowing simultaneous recording of a number of spectra of astronomical objects in a single observation, increasing the field of view of the spectrograph also maximizes the amount of available objects. This characteristic is limited by the optical performance of the telescope and requires a physical increase in the components of the spectrograph. Therefore, an instrument that offers optical MOS capabilities combined with a wide-field, wide wavelength coverage and low to mid-resolution is a multi-purpose and versatile astronomical instrument. Such instruments are therefore "popular" and almost every large telescope has one, since they are suitable to a broad range of applications. In the following, we give some examples of visible, wide-field, low and mid-resolution MOS widely used in the largest ground-based telescope available today. We discuss the optical design details of some of them in chapter 3.

- The Gemini Multi-Object Spectrographs (GMOS), at the Gemini Observatory, are 0.36-0.94 $\mu \mathrm{m}$ long-slit and multi-slit spectroscopy and imaging over a $5.5 \operatorname{arcmin}^{2}$ FoV (Davies et al., 1997);

- The Deep Imaging Multi-Object Spectrograph (DEIMOS), at the Keck II, is a faintobject, multi-slit imaging spectrograph with slit length spanning 16.6' on sky (Cowley et al., 1997);

- The Inamori-Magellan Areal Camera \& Spectrograph (IMACS), at the Magellan, is a highly-versatile wide field imager $\left(15.5^{\prime}\right.$ or $27.2^{\prime}$ squared FoV with two camera f/2 and f/4 option), longslit, and multislit spectrograph ${ }^{1}$ (Dressler et al., 2011);

\footnotetext{
${ }^{1}$ Available at http://www.Ico.cl/lco/telescopes-information/magellan/instruments/imacs/. Accessed on 2020-05-21.
} 
- The Focal Reducer and low dispersion Spectrograph for the Very Large Telescope (FORS 2), at the Very large Telescope (VLT), is a 330-1,100 nm, imaging, polarimetry, long slit and multi-object spectroscopy, with $6.8^{\prime}$ and $4.25^{\prime}$ arcmin square $\mathrm{FoV}^{2}$

- The Visible Multi-Object Spectrograph (VIMOS), also at the VLT, is a wide-field imager, Integral Field Unit, IFU, and spectral resolving power of 200-2,500, providing $224 \operatorname{arcmin}^{2}=4 \times\left(7 \times 8 \operatorname{arcmin}^{2}\right)$ (LeFevre et al., 2003a);

- The Low Resolution Imaging Spectrometer (LRIS), at the Keck II, is a $6^{\prime}$ x $7.8^{\prime}$ FoV, imaging and MOS with spectral resolving power spanning from $\mathrm{R}=300-5,000$;

- The Binospec, at the Multiple Mirror Telescope (MMT), is a multi-slit imaging spectrograph with two $8^{\prime} \times 15^{\prime}$ fields of view, 390 to $1,000 \mathrm{~nm}$ wavelength coverage, high efficiency and a very compact layout for excellent stability (Fabricant et al., 1998). Binospec has $5,000 \AA$ of spectral coverage at $6 \AA$ resolution to $2,100 \AA$ of spectral coverage at $2 \AA$ resolution ${ }^{3}$.

- The multi-object fiber spectrographs for the Sloan Digital Sky Survey (SDSS) and their upgrade for the Baryon Oscillation Spectroscopic Survey (BOSS) are transmitting grism and Volume Phase Holographic Grating (VPHG) fiber-fed spectrographs, respectively, with spectral resolving power from $\approx 2,000$ over a $360-1,000 \mathrm{~nm}$, collecting 640 spectra over the $3^{\circ}$ diameter field in one exposure (Smee et al., 2013).

Ultraviolet, visible and infrared spectrograph and imaging spectrometer are also widely used in space missions. Some important examples are the Space Telescope Imaging Spectrograph (STIS) installed in the Hubble Space Telescope; the Alice UV spectrograph in the European Space Agency's Rosetta comet mission and the SwRI-led New Horizons mission to Pluto; the Lyman Alpha Mapping Project on the Lunar Reconnaissance Orbiter mission, and the Ultraviolet Spectrograph (UVS) in the Juno mission.

\footnotetext{
${ }^{2}$ Available at http://www.eso.org/sci/facilities/paranal/instruments/fors/overview.html. Accessed on 2020-05-13.

${ }^{3}$ Available at https://www.cfa.harvard.edu/mmti/binospec.html. Accessed on 2020-05-25.
} 


\subsection{GMT}

The Giant Magellan Telescope (GMT), one of the three planned ELTs for the next decade, is managed by the GMT Corporation (GMTO), an independent nonprofit organization formed by an international consortium of universities and research institutions responsible for the development, construction, and operation of the GMT.

The GMT is an aplanatic Gregorian optical telescope to be installed at the Las Campanas Observatory. It has a segmented primary mirror totaling an effective collecting area of 21.6 meters in diameter (seven monolith segments of 8.4 meters each). At the edge of a $20^{\prime}$ diameter field of view, the GMT forms images with RMS diameter of $0.6^{\prime \prime}$, improving to $<0.1^{\prime \prime}$ with corrective lenses and adaptive secondary mirrors. More technical details related to the optical design, operation modes, and the first light instruments are described in Appendix A. The GMT Multi-object Astronomical and Cosmological Spectrograph (GMACS), a low to mid-resolution spectrograph operating in the visible spectral range whose optical design is the subject of this research, is among the planned first light instruments, as described below in Section 1.4.

\subsubsection{MOS in ELTS}

Optical wide-field MOS is a highly desirable instrument for the new generation of ELTs due to the large variety of science cases they can address. Each of the three planned ELTs have its own planned optical MOS spectrograph: in the case of the TMT it is the Wide Field Optical Spectrograph, WFOS (Kupke et al., 2018; TMT International Observatory, 2020), for the E-ELT it is the MOSAIC (Fèvre et al., 2010; Hammer et al., 2016; Morris et al., 2018), and for the GMT it is GMACS.

There are a number of challenges that impact directly or indirectly the optical design of an ELT MOS:(i) scientific, such as high throughput, simultaneous wide wavelength coverage, wide-field, accurate and precise sky subtraction, and high spectral stability; (ii) technical, for instance, the challenges of mechanical envelope constraints for large and heavy instruments, which in turn require solutions to suppress significant structural flexure due to gravity and thermal flexure, and metrology; and (iii) manufacturing, such as the availability of large and high homogeneity glass blanks with suitable transmission at wavelengths blueward of $370 \mathrm{~nm}$, high-precision optical surfaces including aspherics with 
deep spherical deviation and slope, large dichroic and beamsplitter efficiency, and large and high-efficiency VPH gratings.

Besides the scientific and technical challenges, the management of long-term, and global projects involving members from different organizations, spread in locations across the planet with different time zones and native languages, and considerably high budgets requires the use of dedicated project management and the application of System Engineering methodologies and concepts applied to astronomical instrumentation (Faes et al., 2018). The optical design, which is part of the optical project, must contemplate and incorporate these methodologies and processes together with the usual tasks of exploring different solutions and alternative designs in order to meet the requirements and design constraints.

\subsection{GMACS}

Wide-field imaging, multi-object, moderate resolution, optical spectrographs are considered as "workhorse" instruments for ground-based astronomy (DePoy et al., 2012). Indeed, the original GMT Science Requirements document states: "A spectrometer operating in the visible spectrum $(0.32 \mu \mathrm{m}$ to $1 \mu \mathrm{m})$ with the capability to observe multiple targets simultaneously is critical to our goals in the areas of star formation, stellar populations and most extragalactic science (...) Maximizing sensitivity should be the highest priority for this instrument. Secondary priorities include optimizing field of view, multiplex factors, and simultaneous wavelength coverage." (GMTO Coorporation, 2013a, p. 4-11). The ability to obtain moderate resolution spectra of astronomical targets at optical wavelengths has been a fundamental scientific capability for more than a century. According to GMTO Coorporation (2013d), the scientific usefulness of this information remains high and this capability is unlikely to become obsolete over the expected lifetime of the GMT.

The wide-field optical spectrograph for the GMT is called GMACS by analogy with IMACS, the wide field optical spectrograph for Magellan. The meaning of the acronym GMACS has changed from "GMT Areal Camera and Spectrograph", which recalls the IMACS acronym (Johns, 2008), to "GMT Multi-Object Astronomical and Cosmological Spectrograph" (DePoy et al., 2012), the acronym used since 2012.

Similar to IMACS, which shares scientific capabilities similar to those proposed to GMACS and is responsible for $\approx 50 \%$ of the total scientific impact on Magellan instruments 
(GMTO Coorporation, 2013d), GMACS is a critical first-generation instrument for the observatory, meeting many of the high priorities GMT science goals.

GMACS will be mounted, through the Instrument Mounting Frame (IMF), in the GMT Gregorian Instrument Rotator (GIR) and installed at the Direct Gregorian (DG). According to the GMTO Coorporation (2016, p. 27), "a Direct Gregorian (DG) instrument is a science instrument designed to be mounted and perform observations at one of the designated Direct Gregorian Port locations". The IMF is a mechanical envelope of dimensions of $5.76 \mathrm{~m} \times 2.73 \mathrm{~m} \times 2.73 \mathrm{~m}(\mathrm{HxWxL})$ that provides the structure that mounts to the GIR and holds the telescope instrument subsystems in place. Figure 1.2 shows GMACS in the GMT, as presented in the Conceptual Design Review in 2019.

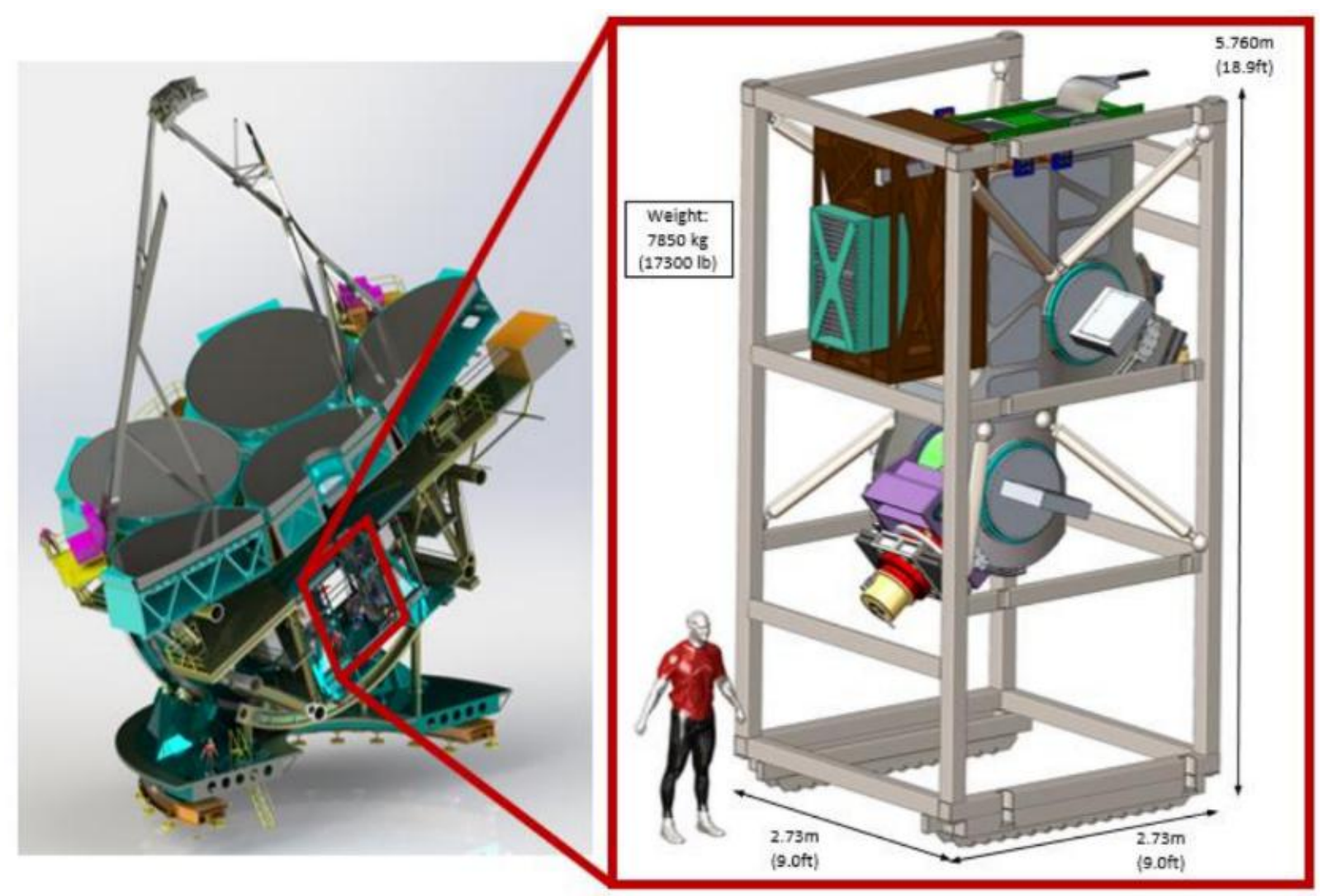

Figure 1.2: GMACS being mounted in the GMT Gregorian Instrument Rotator (GIR) and installed at the Direct Gregorian (DG) location on the telescope mount. Source: GMACS Team (2019). 


\subsubsection{Integration with other instruments}

\subsubsection{Many Instrument Fiber System, MANIFEST}

Although GMACS is designed to operate with slit masks, positionable optical fibers on the telescope focal plane offer unique characteristics for observation of multiple objects and extended targets. In particular, the GMT's Wide Field Corrector and Atmospheric Dispersion Corrector (WFC/ADC) combined with the Many Instrument Fiber System (MANIFEST) provide GMACS and the GMT-Consortium Large Earth Finder (G-CLEF) access to the full field of view of the GMT 20' (Colless, 2018). MANIFEST will also increase the observable target density via an IFU or other dense fiber configuration whose outputs are appropriately arranged into GMACS to make the most use of the CCD area without spectral overlap. Finally, the MANIFEST fibers will provide higher spectral resolution modes for GMACS by sampling the focal plane at 2-3 times the resolution of the slit-fed instrument. Such capabilities will increase the efficiency of telescope use (more targets simultaneously observed) and will greatly enhance scientific cases that are sensitive to large fields of view, extended targets, high target density, and increased spectral resolution (DePoy et al., 2014, 2015; Colless, 2018). The use of MANIFEST can also enhance the potential for facility parallel observing modes in which GMACS can target objects in the surrounding field during pointed observations with other instruments, such as G-CLEF. Figure 1.3 shows a Digitized Sky Survey image of the region around Abell 2744.

\subsubsection{GMACS Science}

A seeing limited spectrograph, such as GMACS, is suitable for a large number of projects, from nearby stars to cosmology. According to the GMT Science Book (2018), the science drivers include (i) time domain astrophysics including gamma ray bursts (GRBs), gravitational wave counterparts, supernovae (SNe), and exoplanet transits; (ii) atmospheres of brown dwarfs and exoplanets; (iii) dynamics of dwarf and ultra-faint dwarf galaxies; (iv) redshift surveys; (v) formation and assembly of galaxies; and (vi) Intergalactic medium (IGM) and circumgalactic medium (CGM) tomography of distant galaxies.

Open questions in a variety of areas will be tackled with this instrument. A few examples, divided in those addressed by GMACS and GMACS+MANIFEST, are given below. 


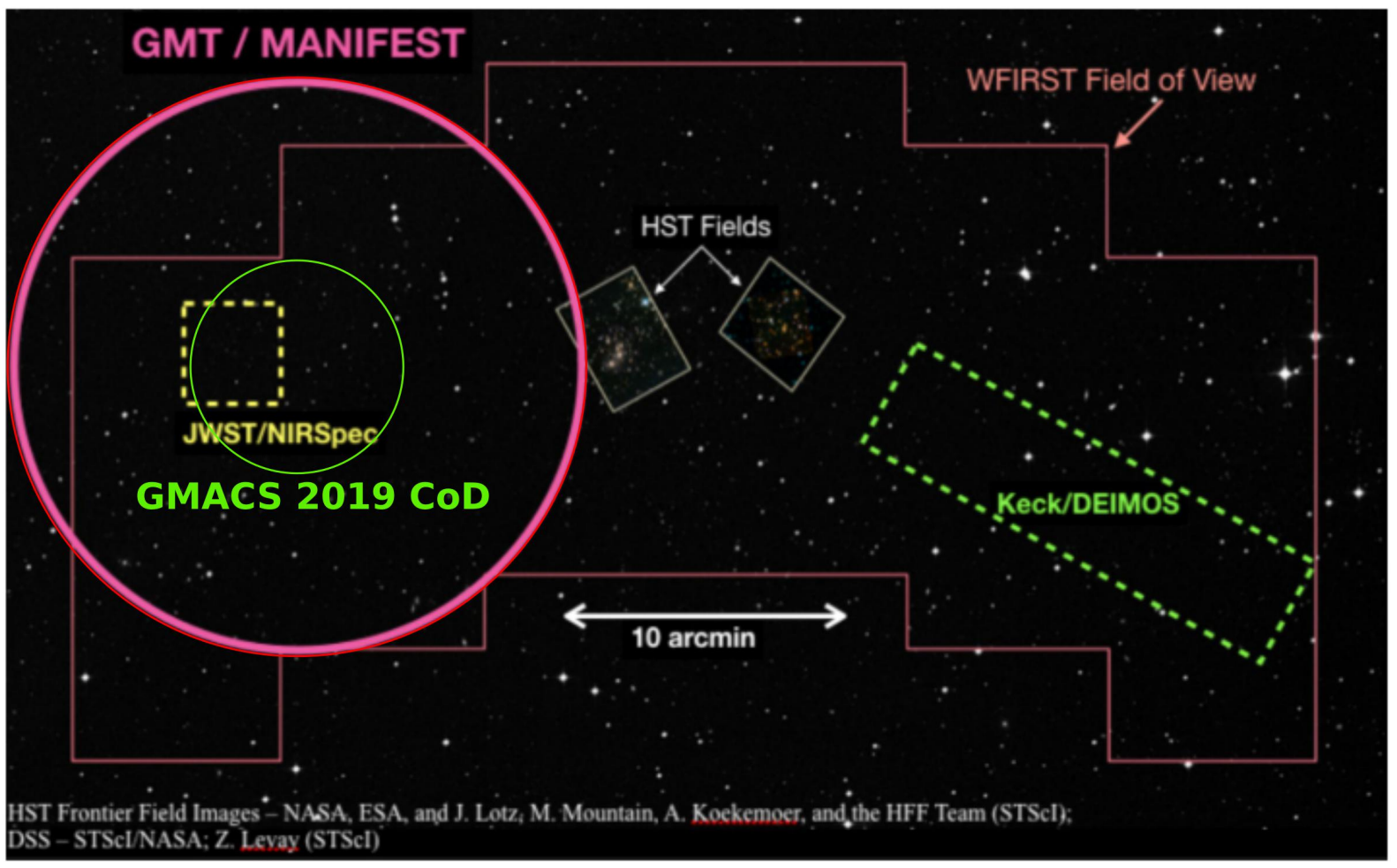

Figure 1.3: Digitized Sky Survey image of the region around Abell 2744, inset with deep images from the HST Frontier Fields. The overlays show the fields from MANIFEST's 20 arcmin field of view, WFIRST, HST, Keck/Deimos, JWST/NIRSpec and GMACS 2019 CoD. Source: adapted from GMT Science Book (2018, p.172) and Colless (2018, p.3).

GMACS Installed in the Direct Gregorian Focus:

1. Mass accretion rates onto forming stars, brown dwarfs, and planets will be detected with GMACS, and where extinction allows, GMACS will also be used to measure accretion-dominated UV continuum emission down to the atmospheric cutoff $(\lambda \approx$ $350 \mathrm{~nm}$ ) (Zhou et al., 2014);

2. GMACS will be especially powerful in the study of supernova and constraining their physical models, contributing with observations in the phases shortly after or long after the explosion, when they are too faint to be observed by 8-10 $\mathrm{m}$ telescopes. GMACS will be crucial in the identification and study of the hosts of core-collapse supernova and superluminous supernova. These latter objects are critical to understanding the first stars in the universe - they are commonly observed in low-metallicity, star-forming galaxies and they are especially bright in the UV (Branch and Wheeler, 2017). Here, there is a major synergy with The Large Synoptic Survey Telescope 
(LSST) where LSST may find these objects that can then be characterized with GMACS spectroscopy;

3. By using GMACS, it will be possible to determine the composition and flow of disrupted stars ripped apart by supermassive black holes (SMBHs) tidal forces (Chornock et al., 2013);

4. GMACS will directly detect the Lyman continuum flux of $L^{\star}$ galaxies at $\mathrm{z}=3$, allowing a direct measurement, at high $\mathrm{S} / \mathrm{N}$ for single objects, of the fraction of the Lyman continuum radiation that can escape star forming regions, providing characterization of the clumpiness of the interstellar medium (Barros et al., 2016);

5. GMACS will also allow the study of UV emission lines of galaxies with $z>7$. This will give invaluable insight about their physical properties, e.g. one may be able to distinguish between different possible origins of some of the lines (Amorín et al., 2017);

6. GMACS spectroscopy of faint galaxies may allow a unique view of the large-scale distribution of hydrogen that forms the cosmic web. Spectroscopy of a large sample of background sources, such as quasars or faint star forming galaxies (Zhang et al., 2016), will allow measurements of the absorption lines formed when the light of these sources pass through gas clouds. Moreover, measuring direct diffuse gas emission around galaxies will provide a tracer of the gas density independent of background sources. These observations, only possible due to the large gathering power of the GMT, will allow determination of temperature of the gas and density variations, improving models and providing a unique 3D view of the gaseous cosmic web; and

7. GMACS will probe the line-of-sight of the many hundreds of new galaxy-scale strong lens systems with variable sources that are expected to be found by LSST, with the goal of detecting other intervening or adjacent mass structures to obtain accurate models and cosmological solutions (GMT Science Book, 2018).

\section{GMACS+MANIFEST:}

1. GMACS+MANIFEST will play a key role in the analysis of streams and satellites of our galaxy as well as around other galaxies of the Local Group by using selected 
candidates from GAIA and other surveys based on their spectroscopic properties. This will enable determination of memberships, kinematics and ages of stars in streams and satellites, allowing a full view of the formation history of the Milky Way (Freeman and Bland-Hawthorn, 2002). Repeated observations may be done to study binaries and variables stars;

2. In the study of the new possible satellites of the Galaxy and other nearby groups, GMACS+MANIFEST will also have important synergy with LSST, confirming the nature of the Ultra-Faint Dwarf (UFDs) galaxies satellites of the Milky Way that may be discovered from imaging alone, and allowing measurements of their stellar dynamics and dark-matter content (Bechtol et al., 2015);

3. By using GMACS+MANIFEST to obtain spectroscopy of low-luminosity galaxies (down to $0.1 L^{\star}$ at $\mathrm{z} \approx 2$ or even going fainter, to $0.01 L^{\star}$ at $\mathrm{z} \approx 2$ when combined with gravitational lensing), we will learn about the internal physical processes happening in them, measure stellar metallicities, dynamics and ionization states, consequently mapping the distribution of galaxy properties. This is crucial to understanding how the general population of galaxies evolved in the early universe and how these building blocks grew to form the more massive systems we observe today. This will also tell us about the evolution of star formation activity from the early universe to its peak (GMT Science Book, 2018);

4. GMACS+MANIFEST will observe rest frame UV spectra of high redshift galaxies $(2<z<5)$, where emission is dominated by massive stars with unprecedented sensitivity. For a $z \approx 5$ galaxy, the Si IV and C IV stellar wind features are located at 8,000-9,000 , well situated for deep spectroscopy with GMACS. GMACS+MANIFEST will allow the study of how the properties of massive stars vary with galaxy properties, providing constraints for models of galaxy evolution (DePoy et al., 2012);

5. GMACS+MANIFEST will allow measuring the interstellar medium covering fraction at high redshift, through observations of large samples of high quality ultraviolet spectra of $z \approx 5-6$ galaxies that today is impractical due to the high opacity of the Intergalactic Medium (IGM) to Lyman Continuum (LyC) photons (GMT Science 
Book, 2018);

6. GMACS+MANIFEST will provide wide-field spectroscopy, which will allow identification of objects in the epoch of reionization $(6<z<9)$, measuring their redshifts and UV emission over large areas. Correlating the locations of these faint objects with maps of the intergalactic medium opacity bubbles produced by the next-generation radio telescopes, such as the Square Kilometer Array, will allow better understanding for how reionization happened (Davies and Furlanetto, 2016); and

7. Through the spectroscopy of faint galaxies, GMACS+MANIFEST will also allow a complete mapping of the spatial distribution of the gas that surrounds disk galaxies for a much larger number of objects than it is possible today (Chen, 2012; Huang et al., 2016). The observed sample will be pairs of galaxies apparently close on the sky, where one is a foreground and the other is a background galaxy (using the background object to probe the gas surrounding the foreground galaxy).

\subsection{GMT Brazil Office}

The São Paulo Research Foundation (FAPESP) is a Brazilian public foundation, subsidized by taxpayer in the state of São Paulo, with the mission to support research projects in higher education and research institutions in all fields of knowledge. The FAPESP process number 11/51680-6 "Explorando o universo: da formação de galáxias aos planetas tipo-terra, com o Telescópio Gigante de Magalhães" (Agência FAPESP, 2015) describes a plan of adhesion of the state of São Paulo to the GMT consortium with 5\% participation, which was equivalent to 40 million dollars at the time, with budgets divided in 10 years from December, 2014. This initiative will bring opportunities for scientific discoveries for Brazilian scientists, bringing new talent, implying a quantitative and qualitative leap for our science, and attracting innovation to our industry through international partnerships, which will establish Brazil's position as a full participant in the world's astronomy (Steiner, 2014). The present research is within the context of this FAPESP granting.

The agreement ${ }^{4}$ amends a prior GMTO agreement, from March 20, 2009, reflecting the admission of new partners, among other issues. The GMT Brazil Office (GMTBrO)

\footnotetext{
${ }^{4}$ The founders' agreement, signed on December 5, 2014, is available at http://www.fapesp.br/en/ 11939. Accessed on 2020-03-15.
} 
assists the resource management, administrative and financial support, representation to the GMT Board of Directors and scientific committees comprised of Brazilian researchers, and provides the contact with national institutes and private companies that might supply services and products for both the GMT project and its instruments. Finally, a long list of several researchers, professors, and undergraduate and graduate students work directly in subareas of the GMT instrumentation community, such as the current research about the GMACS optical system development, which is facilitating the insertion of Brazilian institutes and companies into ELT instrumentation, one of the main objectives of GMTBrO (GMT Brazil Office, 2016).

\subsection{Thesis' Objectives and Outline}

The objectives of this research are divided into two priority levels:

\section{A. Primary}

A.1 Elaboration of a concise methodology for the design, modeling, and analysis of an optical system for a low to mid-resolution MOS for the GMT;

A.2 Description of the Python scripts, macros, and custom functions in Zemax, support worksheets, and any other tools used for the optical design development;

A.3 Elaboration of a review of low and mid-resolution optical spectrographs which have similar performance requirements to GMACS; and

A.4 Development of the optical system of GMACS based on the latest instrument requirements in compliance with the GMT-SOW-01091, Rev. A and B - Statement of Work: GMACS Conceptual Design Study, and attachment (Jacoby, 2016; Contos, 2016, 2017).

Our aim is that the design resulted from this research is accepted in the GMACS Conceptual Design Review (CoDR), which means that, according to Jacoby (2016, p.16), the design "supports the determination that the proposed system meets the stakeholder needs, and has sufficient quality and merit to support proceeding with system development". By the end of the Conceptual Design stage, there should be substantial confidence so that the 
instrument meets all technical, functional, and operational requirements. The design must be able to be manufactured without the need for high-risk technical development (Jacoby, 2016).

The secondary objectives of this research are in the context of strengthening the IAG instrumentation group through the insertion of Brazilian researchers in the development of instrumentation projects in astronomy:

\section{B. Secondary}

B.1 Development of Brazilian human resources specialized in optical design applied to astronomical ground-based instrumentation following principles of system engineering and project management; and

B.2 Improvement of know-how capabilities in Brazil for optical subsystem development of projects in astronomical instrumentation.

In order to accomplish these objectives, the thesis is organized as follows: Chapter 2: Low- and Mid-Resolution Multi-Object Transmissive Grating Spectrographs describes the theoretical background of a VPHG astronomical spectrograph in the optical design perspective and Chapter 3: Review of MOS Optical Designs for Large Telescope Instrumentation describes a brief overview of the optical designs of MOS which share similar requirements to GMACS.

In Chapter 4: Methodology, we present the methodology we used for the development of GMACS, from the stages of interpreting the system requirements and determining the main parameters to the preliminary analyzes of tolerance and flexure due to gravity.

Chapter 5: GMACS Optical System Design presents the core of this research, obtained with the application of the proposed methodologies and that resulted in the conceptual design of the GMACS optical system.

Finally, we conclude this research within the context of the conceptual design recommendation for GMACS and briefly describe the next steps of the project in Chapter 6: Conclusion.

Appendix A: GMT Optical Design shows the GMT concept and its optical characteristics which are important for the development of the GMACS optical system within the context of the GMT instrumentation; Appendix B: List of Zemax Macros and Python 
Scripts presents a few of the macros for OpticStudio Zemax V16.5 software and Python scripts developed for this research and Appendix C: CoD Split Collimator Wavefront Performance shows the wavefront heat maps of the CoD Split Collimator. 
Chapter 2

\section{Low- and Mid-Resolution Multi-Object Transmissive Grating Spectrographs}

This chapter presents the theoretical principles behind the optical design of a multiobject visible astronomical spectrograph for an ELT based on a plane transmissive grating as a dispersing element.

Although the theoretical principles behind this type of spectrograph are widely available in books and papers (Hearnshaw, 2009; Palmer and Loewen, 2014; Eversberg and Vollmann, 2015), the objective of this chapter is to present them in the optical design perspective, valuable to the determination of the instrument parameters and trades. The resulting approach and outcomes help in the elaboration of an electro-optical imaging model of GMACS for the Exposure Time Calculator (ETC), similar to those employed in the aerospace and military areas. This chapter is an overview and potential guide for the essential concepts used in this research, in which we aim to explore the optical design capabilities and characteristics of an ELT visible MOS.

For a deeper understanding of spectographs, their theoretical background and application, the literature provides excellent sources that describe spectrograph varieties classified by the type of dispersive elements, such as prism, reflective grating or grism; the location the instrument is installed on the telescope, which includes Cassegrain, coudé and prime focus; the spectrograph concept, such as échelle, fiber-fed and slitless; and the environment operation, such as ground- and space-based, on rockets and balloons (James and Sternberg, 1969; Hearnshaw, 2009; James, 2007; Palmer and Loewen, 2014). 


\subsection{General Principles of a Multi-Object Optical Spectrograph}

\subsubsection{Spectrograph Layout}

A spectroscopy instrument, such as a spectrograph, a spectrometer and a spectroscope, is a device based on the spectroscopy technique for the measurement of radiation intensity as a function of wavelength. This technique endeavors to study the interaction between matter and electromagnetic radiation, and is concerned with the absorption, emission or scattering of electromagnetic radiation by atoms or molecules within the framework of quantum mechanics (Hollas, 2004).

Spectrographs allow the use of spectroscopy techniques for astronomical applications in order to measure the spectrum of electromagnetic radiation that comes from planets, nebulae, stars, galaxies, Active Galactic Nuclei (AGNs), clusters of galaxies and any other celestial body. These instruments have revolutionized the knowledge of the Sun, the planets, stars, gaseous nebulae, the interstellar medium, galaxies, and quasars (Hearnshaw, 2009). Spectroscopy is one of the most used techniques for astronomy and cosmology.

A spectrograph receives light from a source, disperses it according to its wavelength by a dispersive element into a spectrum, and focuses it onto a detector, which records the spectral image. According to James and Sternberg (1969) and Keller et al. (2015), the main components of this kind of instrument are:

- An entrance aperture, the telescope field stop (i.e., the image plane) at which the resolution element is located (which can be a long slit, slits, fiber or even without any mechanical obstruction);

- A collimator, which generates a collimated, quasi-parallel beam;

- A disperser, an optical element that disperses the incoming light from the collimator;

- A camera, which focuses the dispersed light onto the exit aperture and provides the dispersed image of the entrance apertures on the detector; and

- An exit aperture, the detector.

Figure 2.1 illustrates the optical layout and schematic diagram of an astronomical slit spectrograph. The telescope generates a real image of an object located at infinity (a star, 
a galaxy, or any other object), i.e., an object whose wavefront incident at the telescope entrance pupil can be considered flat. The flux of light of an object located at infinity is made of parallel rays incident on the telescope entrance pupil. Although an optical designer's rule of thumb assumes that any object located at a distance more than ten times the effective focal length is considered located at infinity, a more conservative way would be to multiply that factor by two to five. In any case, any astronomical object can be considered located at infinity.

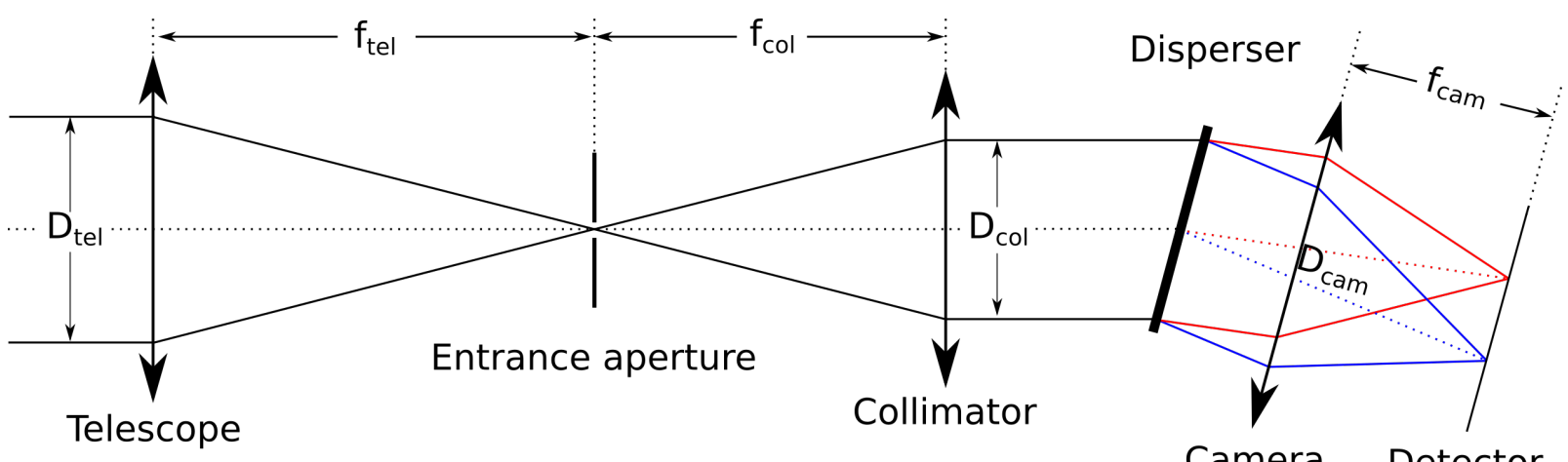

Camera Detector

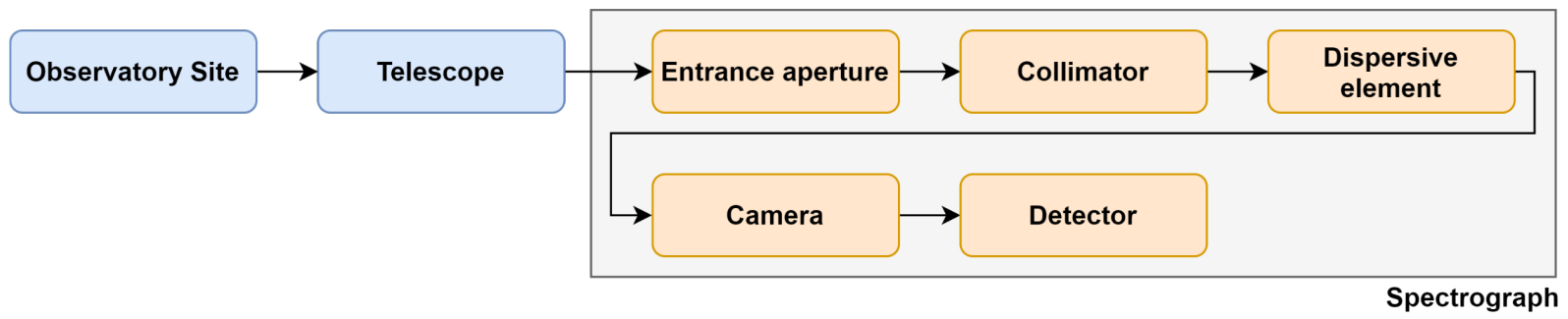

Figure 2.1: Optical layout of a slit transmissive grating spectrograph with the marginal rays and the subsystems schematic diagram.

The real image is located at the telescope focal plane, which is also the entrance aperture plane of the spectrograph. In the case of a slit-based spectrograph, this entrance aperture consists of a slit or a mask with several slits, whose purpose is to select the object's light that will enter the spectrograph. The slit width, among other spectrograph variables such as Effective Focal Length (ELF) and the dispersive element parameters, defines both the element and the wavelength spectrum resolution. The slit width is typically matched in size to the resolution of the telescope or seeing disk in order to maximize the light collected from the objects, the contrast of the object relative to the sky background and wavelength resolution (Oswalt and McLean, 2013). The collimator generates the image 
of the telescope's entrance pupil at the plane named exit pupil where the dispersing element will be ideally located for the optimization of mechanical dimensions and resolution homogeneity. From a raytracing perspective, the collimator projects the image of the slits at infinity, in which the objects' parallel rays cross a particular plane that defines the exit pupil of the Telescope + Collimator subsystem. Eversberg and Vollmann (2015) describes the reasons for the necessity of having a collimator in an optical spectrograph, such as plate scale complexity and degradation of the resolution. The dispersing element, located in this position to minimize its size, disperses the collimated light from the object. The diffracted angle depends on the incident angle and wavelength on the object spectrum (section 2.3). Finally, the camera generates spectral images for each resolution element at the focal plane.

Based on the general design of spectrographs and the theory of gratings, Bingham (1979) and Hearnshaw (2009) identified sixteen parameters for the spectrograph and telescope combination and seven relationships between these variables. It results in nine free variables for designing; according to them, some are determined by the telescope and observatory site parameters, science requirements, and detector characteristics, while the remaining are defined as the spectrograph functional parameters. We have expanded this number to incorporate others commonly used in astronomical spectrograph design, based on their roles in spectrograph development, as shown in table 2.1. The definitions and relationships of the variables are explored further in this chapter.

Table 2.1 - Astronomical spectrograph parameters from the perspective of optical design. Their definitions and relationships are explored further in this chapter.

\begin{tabular}{lcccc} 
& Requirements & Constraints & Design & Configuration \\
\hline Telescope & - & $D_{\text {tel }}, f_{\text {tel }}, \theta_{\text {seeing }}$ & - & $\#$ mirrors, AO \\
Spectrograph & $\mathrm{R}, \lambda, \Delta \lambda, \delta \lambda$, & - & - & - \\
& $\theta, \mathrm{P}, r_{\text {ele }}$ & & & \\
Entrance Apert. & - & - & $w_{s}$ & - \\
Collimator & - & $F_{c o l}$ & $D_{c o l}, f_{c o l}$ & - \\
Disperser & - & - & $n_{i}, \mathrm{~d}, \mathrm{~L}$ & $\alpha, \beta \gamma, \mathrm{m}, r_{a n}$ \\
Camera & - & $F_{c a m}$ & $D_{c a m}, f_{c a m}$ & - \\
Detector & - & - & $p_{x}, p_{p t}, N_{x}, N_{\lambda}$ & \\
\hline
\end{tabular}

One of the principal spectrograph design tasks is to determine the specifications of the 
spectrograph's subsystems that meet the instrument requirements.

\subsubsection{Figure of Merit}

According to Bernstein (2015), the "collected area (telescope entrance pupil area) $\mathrm{x}$ telescope solid angle (limited by the seeing)" factor is directly related to the throughput of the telescope. Equation 2.1 defines a useful merit function to compare telescope performance,

$$
\mathrm{MF}_{t e l}=\frac{A \Omega \gamma}{F_{t e l}^{2} \theta^{2}}
$$

where $A$ is the collected area, $\Omega$ is the available field of view, $\gamma$ is the mirrors' reflectivity and $\theta_{\text {seeing }}$ is seeing size (flux concentration). We have adapted the merit function to include in the denominator the square of the telescope f-number $\left(F_{t e l}\right)$ in accordance with the expression of the flux on the detector (see section 2.3.5.2).

All of the ELT have unique strengths, and their detailed designs hugely impact this metric. Considering the ELTs data in table A.1 and Keck data $(\mathrm{EFL}=175 \mathrm{~m}, \mathrm{~A}=78$ $\mathrm{m}^{2}, \mathrm{M} 1$ aperture $=10 \mathrm{~m}$, seeing $=1^{\prime \prime}$ and 2 mirrors optical design), the relative GMT's,

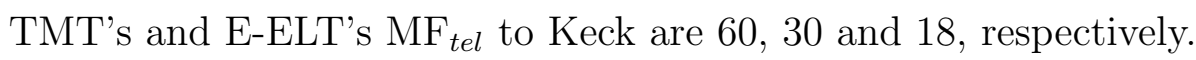

The spectrograph figure of merit is a parameter that expresses the instrument's ability to acquire spectral information. Two critical factors emphasized by Jacquinot (1954) that form the merit function are the resolving power $(R \equiv \lambda / \delta \lambda)$ and throughput (or the luminosity, which means the value of the energy or the flux collected by the receiver).

The resolving power is a dimensionless quantity. The angular dispersion of a dispersive element is defined by $d \alpha / d \lambda$, where $\alpha$ is the angle of diffraction. The angular separation of two monochromatic lines at the resolution limit is $\delta \alpha=\frac{d \alpha}{d \lambda} \delta \lambda$; for a dispersive element of aperture $A$,

$$
R=A \frac{d \alpha}{d \lambda}
$$

This equation, derived first by Rayleigh (1879), represents the general diffraction-limit resolving power. Its strong physical concept relies on the fact that it makes no assump- 
tions concerning the nature of the dispersive element, nor the design parameters of the spectrograph other than the dispersive aperture and the angular dispersion.

For astronomical slit spectrographs, the resolving power will typically be slit-limited rather than diffraction-limited. A wider slit width admits more light for a given object, and hence increasing throughput. In contrast, the resolution is inversely proportional to the slit width. Schroeder (1987) shows the importance of concepts of the luminosity-resolution product in evaluating spectrometer performance. Bingham (1979) shows that R $\theta_{\text {slit }}$ (the product of the resolving power and the entrance slit width along the spectral direction) is regarded as a figure of metric involving diffraction grating, while $\mathrm{N} \theta_{\text {slit }}$ (the product of the number of resolved spectral elements and the entrance slit width along the spectral direction) is a figure of metric involving cameras. Futhermore, two useful metrics to include in a spectrograph's merit function are related to the incident flux in the resolving element at the detector: the flux per resolved spectral element depends on $\theta_{\text {slit }} / R$, and the total flux detected depends on $N \theta_{\text {slit }} / R$. These values are calculated in the Graphic User Interface (GUI) for Spectrograph Simulator developed in this research, described in section 4.2.2.

\subsection{Spectrograph Optical Relationships}

\subsubsection{Spectrograph Optical Principles}

This section describes the paraxial optical relationships of the elements that comprise a Volume Phase Holographic Grating (VPHG) spectrograph. The simple lens equation for an arbitrary optical system is

$$
\frac{1}{f}=\frac{1}{s}+\frac{1}{s^{\prime}}
$$

where the signal convention is that $s$ and $s^{\prime}$ are positives in the object and image regions, respectively, and $f$ is positive for positive lens. The transverse magnification $M$ is given by

$$
M=\frac{s^{\prime}}{s}=\frac{i}{h}
$$


where $s$ is the distance between the object and the first principle surface, $s^{\prime}$ is the distance between the second principle surface and the image, $h$ is the object height, and $i$ is the image height, assuming unity index of refraction in the object and image planes.

The f-number is the ratio of the effective focal length to the entrance pupil's diameter. The notation varies according to the author and context, for instance, $f_{\# i}, f /$ or only $F$. In this work, we use the notation $F_{i}$ or $f /$ for math and text expressions, respectively. Therefore,

$$
F_{i} \equiv \frac{f_{i}}{D_{i}}
$$

where the index $i$ can be any optical subsystem, such as the telescope, collimator and camera, with the indexes "tel", "col", and "cam", respectively. In the spectrograph optical design context, $D_{t e l}$ is the entrance pupil diameter of the telescope, normally located at or close to the primary mirror for Gregorian and Schmidt-Cassegrain telescopes, and usually its value is equal to the primary mirror diameter; $D_{\text {cam }}$ is the entrance pupil diameter of the camera; and $D_{c o l}$ is the exit pupil diameter of the collimator. We will adopt the notation to describe any optical design similar to that of lens manufacturers. For instance, a $594 \mathrm{~mm} \mathrm{f} / 2.2$ camera means a camera with $594 \mathrm{~mm}$ of ELF at a speed of 2.2. If the speed is not expressed, the number represents the EFL; therefore, a $594 \mathrm{~mm}$ camera is a camera with $594 \mathrm{~mm}$ of ELF.

\subsubsection{Telescope + Collimator afocal subsystem}

The subsystem "telescope + collimator" images the entrance pupil at its exit pupil. In other words, it images the primary mirror surface in the collimator's exit pupil plane. They are conjugate planes, similar to the object and image surfaces for an imaging system. We can consider it as finite-finite conjugate optics, also called as afocal optics, with the object's plane located in the entrance pupil and the image's plane located in the collimator exit pupil. In this case, the object distance is $s=f_{c o l}+f_{\text {tel }}$, and considering the effective focal length $f=f_{\text {col }}$, we get $s^{\prime}=f_{\text {col }}\left(f_{\text {col }}+f_{\text {tel }}\right) / f_{\text {tel }}$ from equation 2.3. Therefore, the transversal magnification $\left(M \equiv s^{\prime} / s\right)$ of the telescope + collimator is, considering it as a finite-finite conjugate optics, 


$$
M_{\text {col-tel }}=\frac{f_{\text {col }}}{f_{\text {tel }}}=\frac{\tan \theta_{c o l}}{\tan \theta}
$$

where $\theta$ is the field as seen by the telescope and $\theta_{\text {col }}$ is the incident principal ray angle at the collimator exit pupil. As expected, the collimator exit pupil position, $s^{\prime}$, is approximately $f_{\text {col }}$, because $f_{\text {tel }}>>f_{\text {col }}$.

In contrast to the linear magnification in equation 2.4, the angular magnification, $M_{\theta}$, cannot be generally solved in the form of $\theta_{c o l}=M_{\theta} \theta$, since

$$
\theta_{c o l}=\arctan \left[\frac{\tan (\theta)}{M_{\theta}}\right]
$$

where $\theta_{c o l}$ is the maximum ray angle at the disperser plane and $\theta_{t e l}$ is the telescope object field of view. For a small $\theta$, it can be simplified to $\theta_{c o l}=\theta / M_{c o l-t e l}$. Figure 2.2 illustrates both the cases for $f_{t e l}=205.6 \mathrm{~m}$ and $f_{\text {col }}=2.2 \mathrm{~m}$.

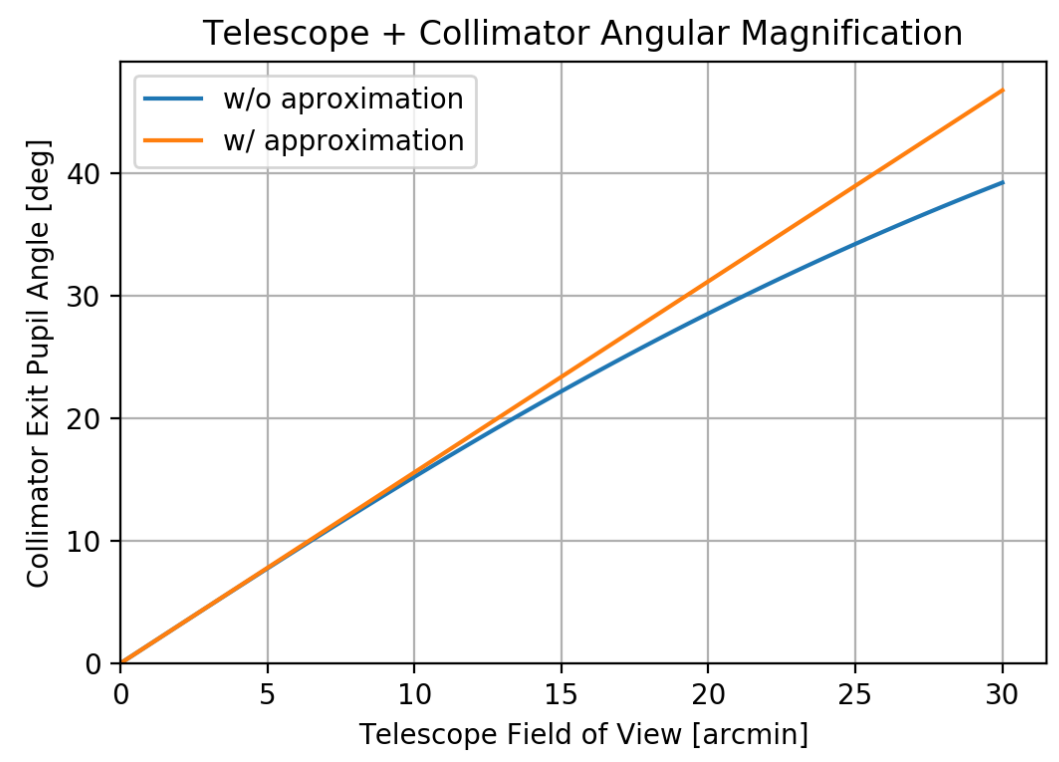

Figure 2.2: Collimator exit pupil ray angle of incidence as a function of the telescope field of view with and without the small telescope angle approximation for $f_{\text {tel }}=205.6 \mathrm{~m}$ and $f_{c o l}=2.2 m$. The linear coefficient is given by $1 / M_{\text {coltel }}$.

The quality of the wavefront generated by the telescope + collimator afocal subsystem is not directly proportional to the image quality of the slits generated at the focal plane of 
the camera, since any aberrations in the exit pupil can, in principle, be corrected by the camera. On the other hand, since the efficiency of the VPH grating is a function of the angle of incidence, an exit pupil with significant aberrations may generate a more complex and inefficient resolution and spectral resolving power responses for high-resolution modes, in addition to extending the instrumental profile of the spectrograph. Hence, ideally, the exit pupil wavefront should be as flat as possible for all the fields and wavelengths, while the residuals aberrations should be balanced through the spectrograph subsystems. Expressed in terms of Zernike polynomial, the wavefronts should have only the X-and Y-tilt components referring to the angles of the chief rays to the normal of the exit pupil.

An essential design constraint is that the $F_{c o l}$ must be the same, or as close as possible, as the $F_{t e l}$ to avoid light loss. Therefore, using the equation 2.5, the following identity is valid:

$$
\frac{f_{t e l}}{D_{t e l}}=\frac{f_{c o l}}{D_{c o l}} \rightarrow D_{c o l}=\frac{f_{c o l}}{f_{t e l}} D_{t e l}=\frac{f_{c o l}}{F_{t e l}}
$$

Assuming that the collimator exit pupil is at the same position of the camera entrance pupil, their diameters are the same, $D_{c o l}=D_{\text {cam }}$. So,

$$
F_{c a m}=F_{t e l} \frac{f_{c a m}}{f_{c o l}}
$$

where $f_{\text {cam }}$ is the camera effective focal length and $f_{\text {col }}$ is the collimator effective focal length.

\subsubsection{Slit Image}

According to geometrical optics, the image size of any finite conjugate optics from an object of angular size is given by

$$
I^{\prime}=2 f_{t e l} \tan \left(\frac{\theta}{2}\right)
$$

where $I^{\prime}$ is the image size on the telescope focal plane and $\theta$ is the object angular size. This equation is also applied to determine the image position in absence of any field aberration 
which changes the chief ray height, such as distortion. Although the geometrical distortion for a small angular object is negligible, even a small geometrical distortion can result in a few pixel shifts on the telescope focal plane.

When the spectrograph is inserted in the telescope optical path as shown in figure 2.1, the image size from equation 2.10 is magnified by two factors: the collimator-camera finite-finite conjugate subsystem $\left(f_{\text {cam }} / f_{\text {col }}\right)$; and the magnification generated by the disperser $\left(r_{a n}\right)$, also called as anamorphic magnification, which depends on the incident and diffraction angles (see section 2.3.3.1). Thus, the resulting image size is

$$
I=\frac{2 r_{a n} f_{\text {tel }} f_{\text {cam }}}{f_{\text {col }}} \tan \left(\frac{\theta}{2}\right)
$$

This equation is useful to calculate the image size of a slit on the spectrograph focal plane when the slit width is given in angular units by replacing $\theta \rightarrow \theta_{\text {slit }}$ (the angular size of the slit projected back onto the sky) in Eqs.2.10 and 2.11. If we define the physical size of the slit at the telescope focal plane as $w_{s}$, we get

$$
w_{s}=2 f_{\text {tel }} \tan \left(\frac{\theta_{\text {slit }}}{2}\right)
$$

$\theta_{\text {slit }}$ is obtained by solving 2.12

$$
\theta_{\text {slit }}=2 \arctan \left(\frac{w_{s}}{2 f_{\text {tel }}}\right)
$$

According to the equations 2.10 and 2.11, the resulting slit image size, $w_{s}^{\prime}$, at the spectrograph focal plane is

$$
w_{s}^{\prime}=\frac{r_{a n} f_{c a m}}{f_{c o l}} w_{s}=\frac{2 r_{a n} f_{\text {tel }} f_{c a m}}{f_{c o l}} \tan \left(\frac{\theta_{\text {slit }}}{2}\right)
$$

where $w_{s}^{\prime}$ is the slit image size at the spectrograph focal plane. For a small $\theta_{\text {slit }}$, equation 2.14 results in

$$
w_{s}^{\prime} \approx \frac{r_{a n} f_{\text {tel }} f_{c a m}}{f_{\text {col }}} \cdot \theta_{\text {slit }}
$$

where $\theta_{\text {slit }}$ units must be in radians. 


\subsubsection{Spectrograph Field of View}

The spectrograph FoV is a region projected in the sky in which the spectrograph generates an image of any object within it. The shape of the collimator exit pupil will be precisely the shape of the telescope entrance pupil. Although the telescope, collimator, and camera generate a circular symmetric shape FoV, the detector might limit (or even define) the spectrograph FoV since it usually presents a rectangular shape.

The expression for a spectrograph $\mathrm{FoV}$ as a function of the principal parameters is obtained by solving $\theta$ in equation 2.11 and considering the detector size along the field dimension as the product of $N_{x} p_{x}$, where $N_{x}$ is the number of pixels in the detector field direction and $p_{x}$ is the detector pixel size, as follows:

$$
\theta=2 \arctan \left(\frac{N_{x} p_{x}}{2 r_{\text {an }}} \frac{f_{\text {col }}}{f_{\text {tel }} f_{\text {cam }}}\right)
$$

Figure 2.3 illustrates a case in which the spectrograph FoV is limited by the spatial dimension of the detector (vertical axis).
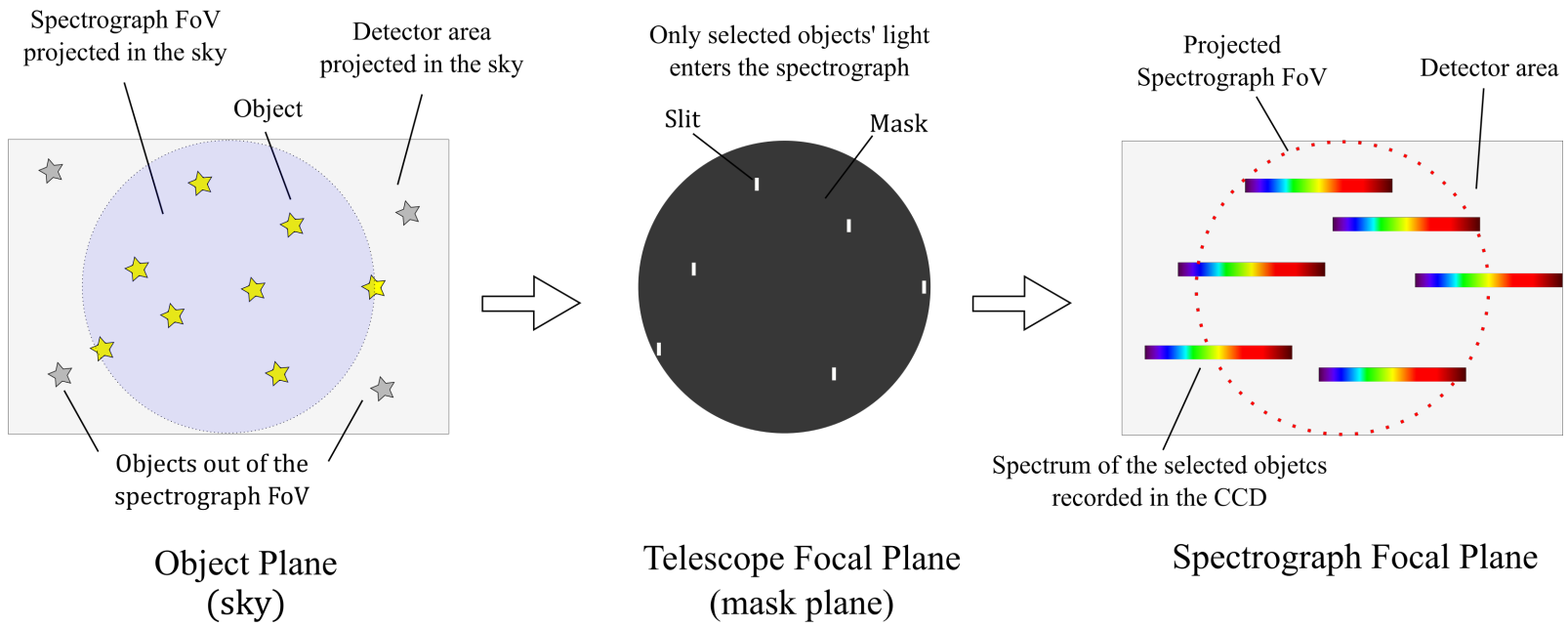

Figure 2.3: Detector projection in the sky and the spectrograph FoV limited by the spatial dimension of the detector. Any object located within the region bounded by the spectrograph field of view will be imaged in the detector. The illustration shows a case in which the bandwidth dispersion is entirely within the projected area of the detector. 


\subsubsection{Plate Scale}

The Telescope Plate Scale $\left(\Gamma_{t e l}\right)$ is the ratio between the object angular size to its image size at the telescope focal plane. For a small angle, the plane scale is given by the reciprocal of the $f_{\text {tel }}$ in $\mathrm{mm}$, which results in units of $\mathrm{rad} / \mathrm{mm}$.

$$
\Gamma_{t e l}=\frac{\arctan \left(\frac{D_{t e l}}{f_{t e l}}\right)}{D_{\text {tel }}}[\mathrm{rad} / \mathrm{mm}] \simeq \frac{206,265}{f_{\text {tel }}}[\operatorname{arcsec} / \mathrm{mm}]
$$

where $f_{\text {tel }}$ and $D_{t e l}$ units are given in $m m$, and $1 \mathrm{rad}=206.265^{\prime \prime}$.

The Spectrograph Plate Scale $\left(\Gamma_{\text {spec }}\right)$ follows the same definition, but including the spectrograph magnifications parameters. It is the ratio of the object angular size to its size at the camera's focal plane,

$$
\Gamma_{\text {spec }}=206,265 \frac{f_{\text {col }}}{r_{\text {an }} f_{\text {tel }} f_{\text {cam }}}=\Gamma_{\text {tel }} \frac{f_{\text {col }}}{r_{\text {an }} f_{\text {cam }}}[\operatorname{arcsec} / \mathrm{mm}]
$$

where all the focal length units are given in $\mathrm{mm}$. Similar to the expression before, this one is also only valid for a small $\theta_{\text {slit }}$.

\subsubsection{Components' Principal Parameters}

\subsubsection{Collimator}

The incident chief ray angle at the collimator exit pupil, $\left(\theta_{\text {col }}\right)$ is a function of the telescope+collimator angular magnification and the spectrograph FoV, as shown in equation 2.19:

$$
\theta_{c o l}=2 \arctan \left[\frac{f_{\text {tel }}}{f_{\text {col }}} \tan \left(\frac{\theta}{2}\right)\right]=2 \arctan \left[M_{\text {pupil }} \tan \left(\frac{\theta}{2}\right)\right]
$$

where $M_{\text {pupil }}$ (see equation 2.6) is the pupil (de)magnification. This result is useful to determine the light incidence maximum angle at the disperser plane.

The collimator EFL $\left(f_{\text {col }}\right)$ is one of the spectrograph parameters that defines slit width and the minimum grating size. According to Eqs.2.12 and 2.58, increasing the $f_{\text {col }}$ for a fixed slit image size $w^{\prime}$ will also allow the slit width to increase, which in turn affects 
positively the throughput of the system. On the other hand, equation 2.44 shows that the minimum size of the grating increases linearly with the $f_{c o l}$, which in turn is a constraint for the value of $f_{c o l}$, as we will describe further in equation 2.45.

\subsubsection{Camera}

The camera's field of view $\left(\theta_{\text {cam }}\right)$ is defined by the camera focal length and the detector size. If $p_{x}$ is the pixel size, $p_{p t}$ is the pixel pitch and $N_{i}$ is the number of pixels in the spatial, $N_{x}$, or spectral, $N_{\lambda}$, direction of the detector:

$$
\theta_{c a m}=2 \arctan \left[\frac{p_{p t}\left(N_{i}-1\right)+p_{x}}{2 f_{c a m}}\right]
$$

In this context, the spectrograph FoV is represented by the camera FoV, although it is known it is also a function of the telescope, collimator and camera EFLs, and the detector size.

If we assume that the FoV generated by the spectrograph is circumscribed in the detector (i.e., there is spectra field loss - see figure 2.4), the camera FoV can be rewritten by the detector's diagonal considering its aspect ratio, defined as $N=N_{\lambda} / N_{x}$. It means that the number of pixels along the spectral direction is $N$ times higher than the number of pixels along the spatial direction. The diagonal of the detector in pixels is then given by $D_{\text {detec }}=\sqrt{N_{x}^{2}+N_{\lambda}^{2}}$, and, therefore, $D=N_{x} \sqrt{\left(1+N^{2}\right)}$. Hence, equation 2.20 is rewritten as

$$
\theta_{\text {cam }}=2 \arctan \left[\frac{p_{p t}\left(N_{x} \sqrt{\left(1+N^{2}\right)}-1\right)+p_{x}}{2 f_{c a m}}\right]
$$

In the case of FFa $\approx 1, p_{p t} \approx p_{x}$,

$$
\theta_{\text {cam }} \approx 2 \arctan \left[\frac{N_{x} p_{x} \sqrt{\left(1+N^{2}\right)}}{2 f_{\text {cam }}}\right]
$$

The inferior limit case happens when $\theta_{\text {cam }}$ is inscribed in the detector. It is an inefficient situation, as the camera would not be able to image the spectrum of objects located in the edge of the spectrograph FoV. This limitation occurs in the spatial direction of the 
detector since the FoV along the dispersion will always be higher than the spatial one. Therefore, the camera FoV would be, for $p_{p t} \approx p_{x}$ and $N=0$,

$$
\theta_{\text {cam }} \approx 2 \arctan \left[\frac{N_{x} p_{x}}{2 f_{\text {cam }}}\right]
$$

A trade-off analysis is commonly performed to determine an intermediate solution in order to increase the $\theta_{\text {cam }}$ without compromising the spectrograph image quality and design complexity.

An intermediate case happens when the $\theta_{\text {cam }}$ is limited by the larger size of the detector. In this case, the equation is rewritten as

$$
\theta_{\text {cam }}=2 \arctan \left[\frac{p_{p t}\left(\max \left(N_{x}, N_{\lambda}\right)-1\right)+p_{x}}{2 f_{c a m}}\right]
$$

A numerical example for $N_{x}=8,192, N=3: 2, p_{x}=15 \mu \mathrm{m}, p_{p t}=15 \mu \mathrm{m}$ and $f_{\text {cam }}=594 \mathrm{~mm}$ results in camera $\mathrm{FoV}$ of $11.8^{\circ}, 17.6^{\circ}$, and $21.1^{\circ}$ for the cases illustrated in figure 2.4.

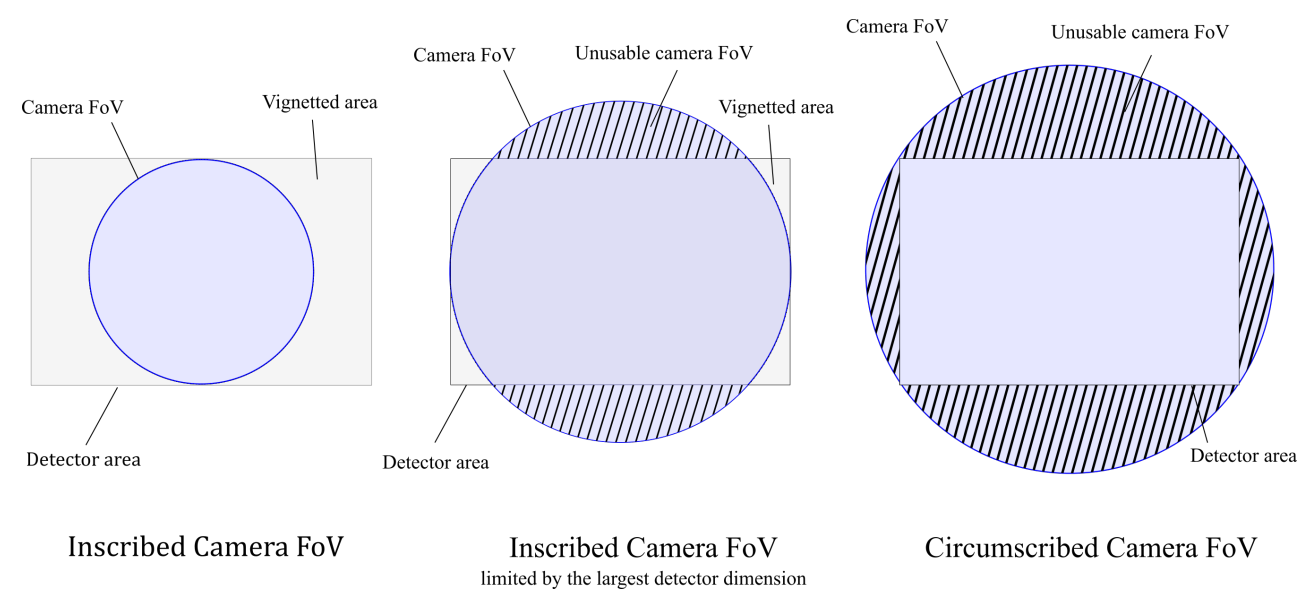

Figure 2.4: The inscribed FoV is limited by one of the detector dimensions, usually the spectral one, to minimize the vignetting and the unusable FoV. The unvignetted circumscribed camera FoV projection is limited by the diagonal of the detector, but it produces the most significant loss of the camera FoV.

In order to satisfy the Nyquist theorem, the spatial density of pixels must be at least $2 /\left(D_{x} \delta \lambda\right)$ pixels $\cdot m m^{-1}$ or each pixel must be of size $p_{x} \leq w_{s}^{\prime} / 2=r_{\text {ele }} / 2$, where $p_{x}$ is the 
pixel size in the direction of dispersion and $w_{s}^{\prime}$ is the image of the slit on the spectrograph focal plane (defined as resolution element, $r_{\text {ele }}$ ). Rewriting equation 2.14,

$$
\frac{w_{s}^{\prime}}{w_{s}}=\frac{r_{a n} f_{c a m}}{f_{c o l}}
$$

$f_{\text {cam }}$ must meet the following conditions:

$$
f_{\text {cam }} \geq \frac{p_{x} f_{\text {col }}}{r_{\text {an }} f_{\text {tel }} \tan \left(\theta_{\text {slit }} / 2\right)} \approx \frac{2 p_{x} L_{\text {grat }} \cos \beta}{\theta_{\text {slit }} D_{\text {tel }}}
$$

where $L_{\text {grat }}$ is the maximum grating length along the spectral direction (see equation 2.44).

In general, this limit for $f_{\text {cam }}$ is inferior to the one resulting from the constraints of a typical camera speed or the required aperture since designs faster than $\mathrm{f} / 1$ are impractical for designs with FoV larger than $15^{\circ}$. Equation 2.5 determines that $f_{\text {cam }}=F_{\text {cam }} D_{\text {col }} \geq D_{\text {col }}$, and, according to equation 2.9,

$$
f_{\text {cam }}=f_{\text {col }} \frac{F_{\text {cam }}}{F_{\text {tel }}} \geq \frac{f_{\text {col }}}{F_{\text {tel }}}
$$

\subsubsection{Detector}

The usual detector model considers a plane staring array detector with $N_{x}$ and $N_{\lambda}$ numbers of pixels along the spatial and spectral direction, respectively. In many staring arrays, the sensors do not completely fill the cell area of the detector. The ratio of the active element area to the cell is defined as Fill Factor $(\mathrm{FFa})$; then, generically, FFa $=$ $p_{x} p_{y} / p_{p t x} p_{p t y}$. It is assumed in this work that both the detector element and the pixel size are squared, therefore, $p_{x}=p_{y}$ and $p_{p t x}=p_{p t y}$. Binning changes the effective size of the pixels. The active element area of the detector, whose size is the pixel $p_{x}$ in this work, is located where the photons effectively generate the charge in the semi-conductor. The element detector $p_{x}$ is used for radiometric purposes; while the cell area defines the spatial element of the system and is used to calculate the contrast of the image as a function of the spatial frequency (the Modular Transfer Function, MTF). The distance between the centers of two cell areas is known as pixel pitch $p_{p t}$. Figure 2.5 shows the geometrical model of a starring detector. 
The number of pixels along the spatial direction $\left(N_{x}\right)$ is directly obtained from equation 2.11 by replacing the image size with the product of $N_{x}$ and $p_{x}$. Solving the resulting equation for $N_{x}$ and assuming that there is no anamorphic magnification,

$$
N_{x}=\frac{2 f_{\text {tel }} f_{\text {cam }}}{p_{x} f_{\text {col }}} \tan \left(\frac{\theta_{x}}{2}\right)
$$

where $\theta_{x}$ is the camera $F_{0} V$ in the spatial direction.

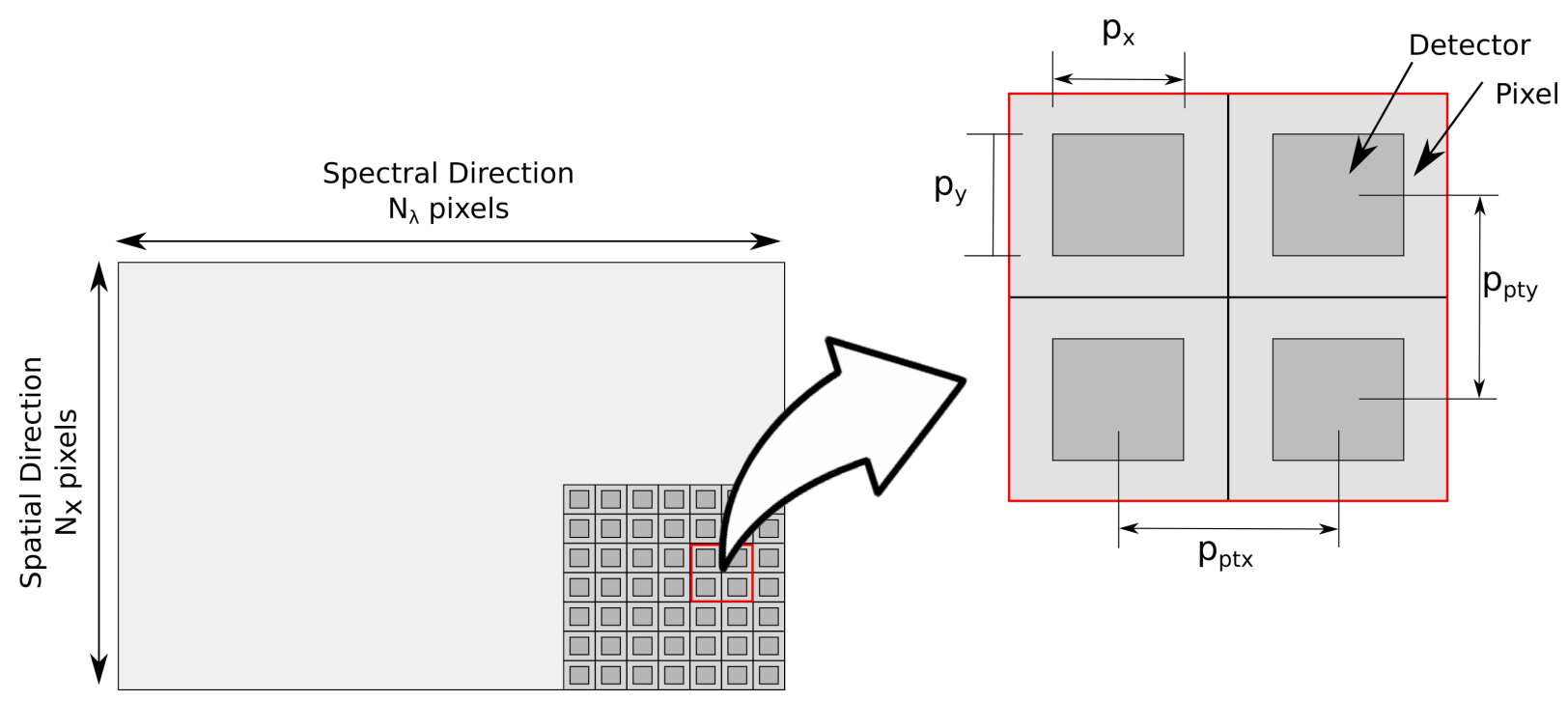

Figure 2.5: Detector's principal parameters. The detector has $N_{x} \mathrm{x} N_{\lambda}$ pixels with a detector element size of $p_{x}$ and a pixel pitch of $p_{p t}$.

As we will show in section 2.2.2.2, the assumption that a feasible camera must be slower than $f / 1$ results in the condition $f_{\text {cam }} / f_{\text {col }} \geq F_{\text {tel }}^{-1}$ (equation 2.27). In this case, equation 2.28 can be altered to

$$
N_{x} \geq \frac{2 f_{t e l}}{p_{x} F_{t e l}} \tan \left(\frac{\theta_{x}}{2}\right)=\frac{2 D_{t e l}}{p_{x}} \tan \left(\frac{\theta_{x}}{2}\right)
$$

\subsection{General VPH Transmission Grating Equations}

In a VPH transmission grating, light is diffracted as it passes through a thin layer $(\approx$ 3-30 mm) of a dichromated gelatin (DCG) in which the material's refractive index presents 
an approximately sinusoidal modulated profile. The general VPH grating equation is given by

$$
m \lambda=n_{i} d_{g}\left(\sin \alpha_{i}+\sin \beta_{i}\right) \cos \gamma_{i}
$$

where $m$ is the grating order, $\lambda$ is the light wavelength, $n_{i}$ is the index of refraction of the medium where the grating is immersed, $d_{g}$ is the projected grating spatial period (i.e., the projected separation between the fringes in the plane of the grating, equivalent to the groove spacing on a ruled grating), $\alpha_{i}$ is the angle between the incident beam and the plane perpendicular to the grating grooves, $\beta_{i}$ is the diffraction angle from the grating normal and $\gamma_{i}$ the angle between the incident light path and the plane perpendicular to the grooves at the grating center, also known as off-plane angle (James and Sternberg, 1969; Baldry et al., 2004; Palmer and Loewen, 2014). A useful term to represent the concept of grating spatial period, $d$, is its reciprocal value, called line density $(\nu=1 / d)$. The grating equation can apply to angles in the DCG layer $(i=2)$, in the glass substrates $(i=1)$, or in the air $(i=0)$ as long as the air-glass boundaries are parallel to the DCG layer (Baldry et al., 2004). The sign convention is such that $\beta=-\alpha$ means no diffraction; i.e., zeroth order) for order $m$.

The off-plane angle becomes important in some special spectrograph layouts (such as white pupil echelle). When $\gamma=0$ and the index of refraction is $n=1$, the system is called classical or in-plane diffraction, also known as Fraunhofer's diffraction-grating equation, which results from the analysis of the properties of diffraction gratings involving Fourier transform (Hearnshaw, 2009),

$$
m \lambda=d(\sin \alpha+\sin \beta)
$$

Figure 2.6 shows a diagram of a VPH grating with the definition of the variables.

In the simplest VPH transmission grating, according to Baldry et al. (2004), the plane of the fringes is perpendicular to the plane of the grating. This case, referred to as unslanted fringes, is similar to the distance between fringes in a ruled grating, $d$. In the general case, 


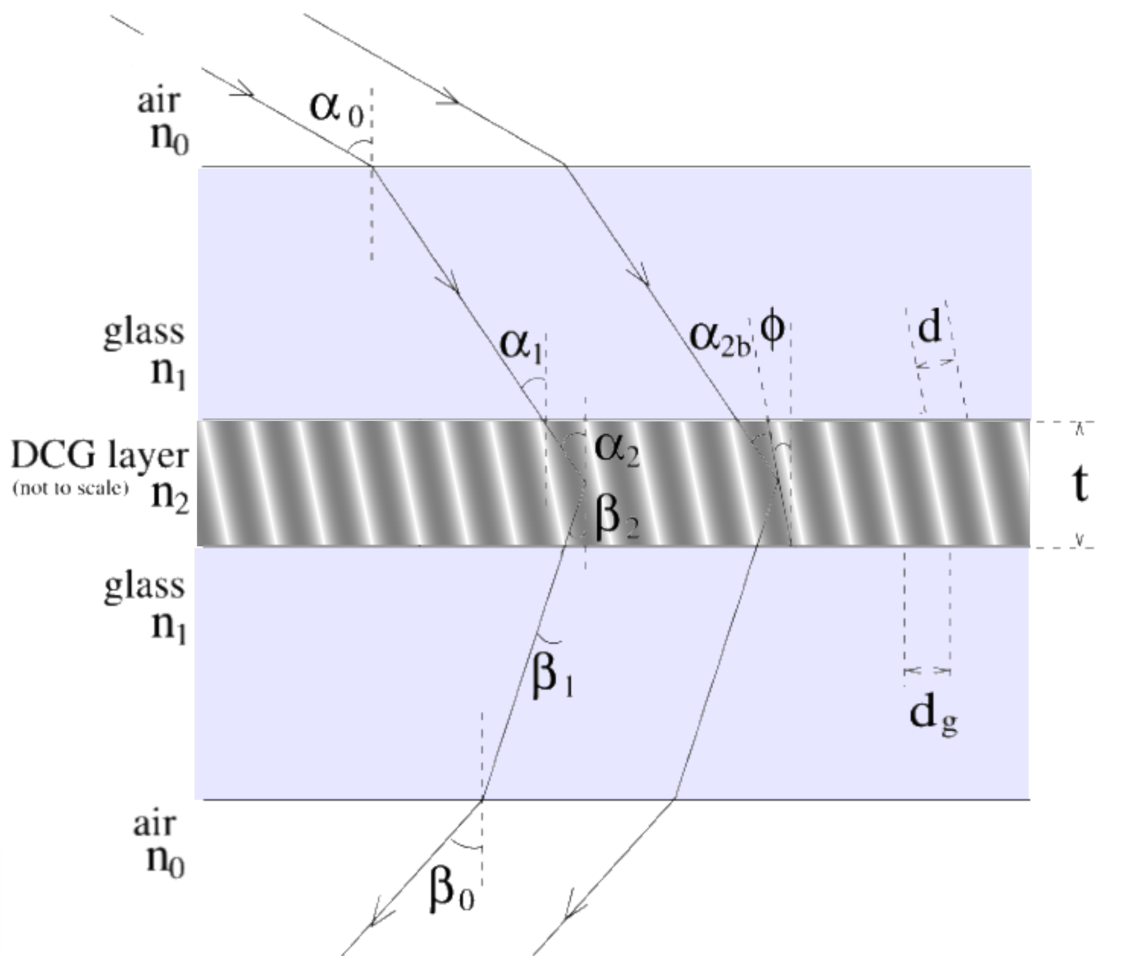

Figure 2.6: Diagram of a VPH grating. Source: adapted from Baldry et al. (2004).

$$
d_{g}=\frac{d}{\cos \phi}
$$

where $\phi$ is the "slant" angle between the grating normal and the plane of the fringes.

The DCG has an average index of refraction of 1.5. It is usually manufactured with an index of refraction modulation ranging from 0.02 to about 0.10 in layers as thick as 4 to greater than $20 \mu \mathrm{m}$ and line densities of 300-6000 lines $\mathrm{mm}^{-1}$. In general, the index of modulation produced in the DCG layer is assumed to be sinusoidal and can be modeled by the following equation (Barden et al., 2000):

$$
n_{2}(x, z)=n_{g}+\Delta n_{g} \cos \left[\frac{2 \pi}{d}(x \sin \phi+z \cos \phi)\right]
$$

Figure 2.7 illustrates a VPH grating with the resulting index of refraction modulation for $n_{g}=1.5, \Delta n_{g}=0.07, d=600$ lines $\cdot \mathrm{mm}^{-1}$. 


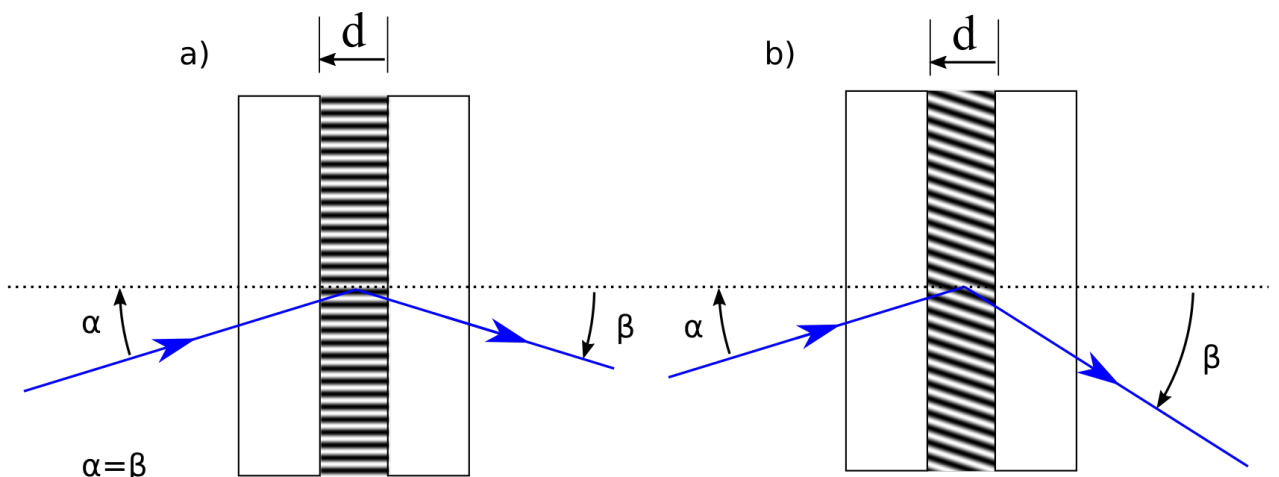

c)

d)

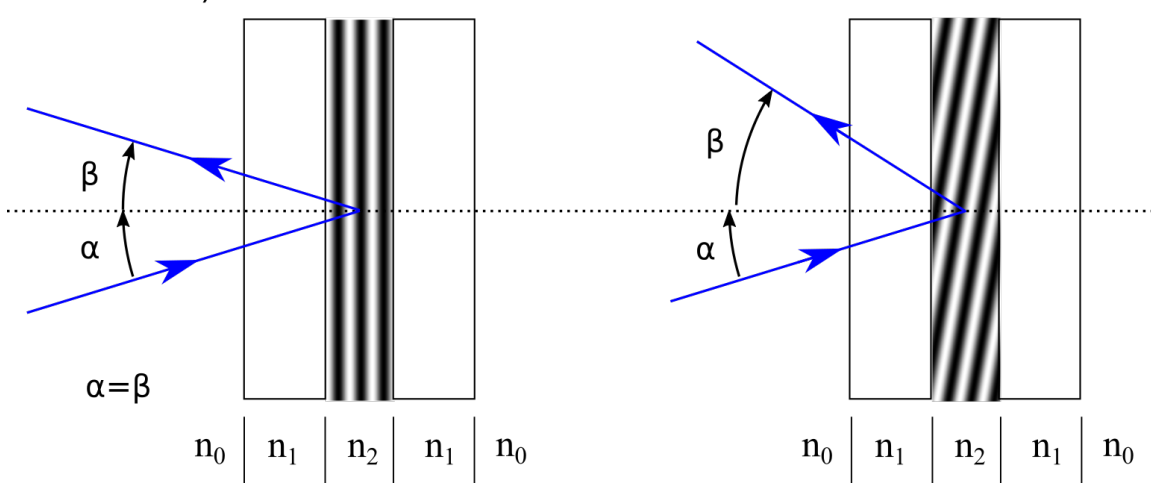

Figure 2.7: Fringe structures for VPH gratings for a) Littrow transmission grating, b) non-Littrow transmission grating c) nondispersive reflection grating (notch filter) and d) dispersive reflection grating. VPH grating has the index of refraction modulation with $n_{g}=1.5$, $\Delta n_{g}=0.07, d=600$ lines $\cdot \mathrm{mm}^{-1}$. The VPH grating in this research is shown in case A. Source: adapted from Barden et al. (2000).

\subsubsection{Blaze grating and Littrow condition}

The blaze angle $\left(\theta_{B}\right)$ refers to the uniformly angled facets tilted at a specific angle to the grating normal for high efficiency at single order. According to Palmer and Loewen (2014), although holographic gratings do not have a triangular groove profile as do the ruled gratings, they still present blazing characteristics. Therefore, the term blazing refers to high diffraction efficiency, regardless of the groove profile or the method used to generate them. According to the same authors, holographic gratings are usually blazed by ion bombardment (ion etching).

The blaze phase-matching angle condition for best performance is given when the incident and diffraction angles are equal, $\theta_{B}=\alpha_{2}-\phi=\beta_{2}+\phi$, where $\alpha_{2}$ is the angle of incidence and $\beta_{2}$ is the angle of diffraction from the grating normal in the DCG layer. It is an important parameter for diffraction by VPH gratings because it directly affects 
efficiency and bandwidth, and indirectly affects the resolving power (Baldry et al., 2004).

Substituting this condition in equation 2.30 for a slanted fringes VPH grating on-plane,

$$
\lambda_{B}=\frac{2 n_{2} d \sin \theta_{B}}{m}
$$

where $\lambda_{B}$ is the blaze wavelength. Solving it for $\theta_{B}$,

$$
\theta_{B}=\arcsin \left(\frac{m \lambda_{B}}{2 n_{2} d}\right)
$$

The blazed diffraction grating has become the standard type of reflection grating used in recent instruments.

The Littrow configuration is a particular geometry in which the blaze angle is such that the incidence and diffraction ray angles are identical, enhancing the diffraction efficiency. Hence, it holds that $\alpha=\beta=\theta_{B}$.

\subsubsection{First-Order Diffraction Efficiencies}

The diffraction efficiency of VPH gratings is a function of the Bragg conditions, the semi-amplitude of the refractive index modulation $\left(\Delta n_{2}\right)$ and $D C G$ thickness $(t)$, and the incidence and diffracted angles. The Rigorous Coupled-Wave Analysis (RCWA) provides an exact formulation of the VPH grating diffraction efficiency without approximations (Moharam and Gaylord, 1981; Gaylord and Moharam, 1985). Although a robust efficiency modeling of VPH grating is essential for the development and characterization of a spectrograph, its detailed prior knowledge does not influence the optical design process since it only requires the raytracing diffracting behavior of the light. However, we will briefly describe Kogelnik's formulation, a useful first-order theory of diffraction efficiency as a function of VPH grating variables in the Bragg condition (Kogelnik, 1969). His theory uses an approximation that is accurate to within $1 \%$ when the following condition is satisfied (Baldry et al., 2004):

$$
\rho=\frac{\lambda^{2}}{d^{2} n_{2} \Delta n_{s}}>\rho_{\min }
$$


where $\rho_{\min } \approx 10$. Substituting the blaze wavelength (see equation 2.35) for the first order and solving it for $\alpha_{B}$,

$$
\sin \alpha_{B}>\sqrt{\frac{\rho_{l i m} \Delta n_{2}}{4 n_{2}}}
$$

However, Kogelnik's theory is only accurate for a given refractive-index modulation, i.e., for Bragg angles above a certain value. For example, the minimum blaze angle for $n_{2}=1.5$ and refractive-index modulation $\Delta n_{2}=0.07$ is $\approx 20^{\circ}$, which compromises the efficiency calculation for low-resolution modes. For unpolarized light, the Kogelnik efficiency is given by

$$
\eta=\frac{1}{2} \sin ^{2}\left(\frac{\pi \Delta n_{2} t}{\lambda \cos \alpha_{B}}\right)+\frac{1}{2} \sin ^{2}\left[\frac{\pi \Delta n_{2} t}{\lambda \cos \alpha_{B}} \cos \left(2 \alpha_{B}\right)\right]
$$

where the first term is for s-polarized light (i.e., the electric vector is perpendicular to the fringes), and the second term is for p-polarized light (i.e., the electric vector is parallel to the fringes). Figure 2.8 shows the variation of efficiency versus grating thickness for two different Bragg angles for fixed $\Delta n_{2}=0.07$ and $\lambda=600 \mathrm{~nm}$.

According to Kogelnik (1969) and Barden et al. (2000), light that nearly satisfies the Bragg condition can also be diffracted with good efficiency depending on the grating parameters only. As the angle of incidence changes, the diffraction efficiency for transmission grating with a given fixed wavelength can be approximated by

$$
\Delta \alpha_{\mathrm{FWHM}} \propto \frac{d}{t}
$$

As the wavelength deviates from the Bragg wavelength condition for a fixed grating angle, the diffraction efficiency is approximated by

$$
\Delta \lambda_{\mathrm{FWHM}} \propto \lambda \frac{d}{t} \cot \alpha_{2}
$$

where the terms $\Delta \alpha_{\mathrm{FWHM}}$ and $\Delta \lambda_{\mathrm{FWHM}}$ are called the angular Bragg envelope and spectral Bragg envelope, respectively (Kogelnik, 1969). 

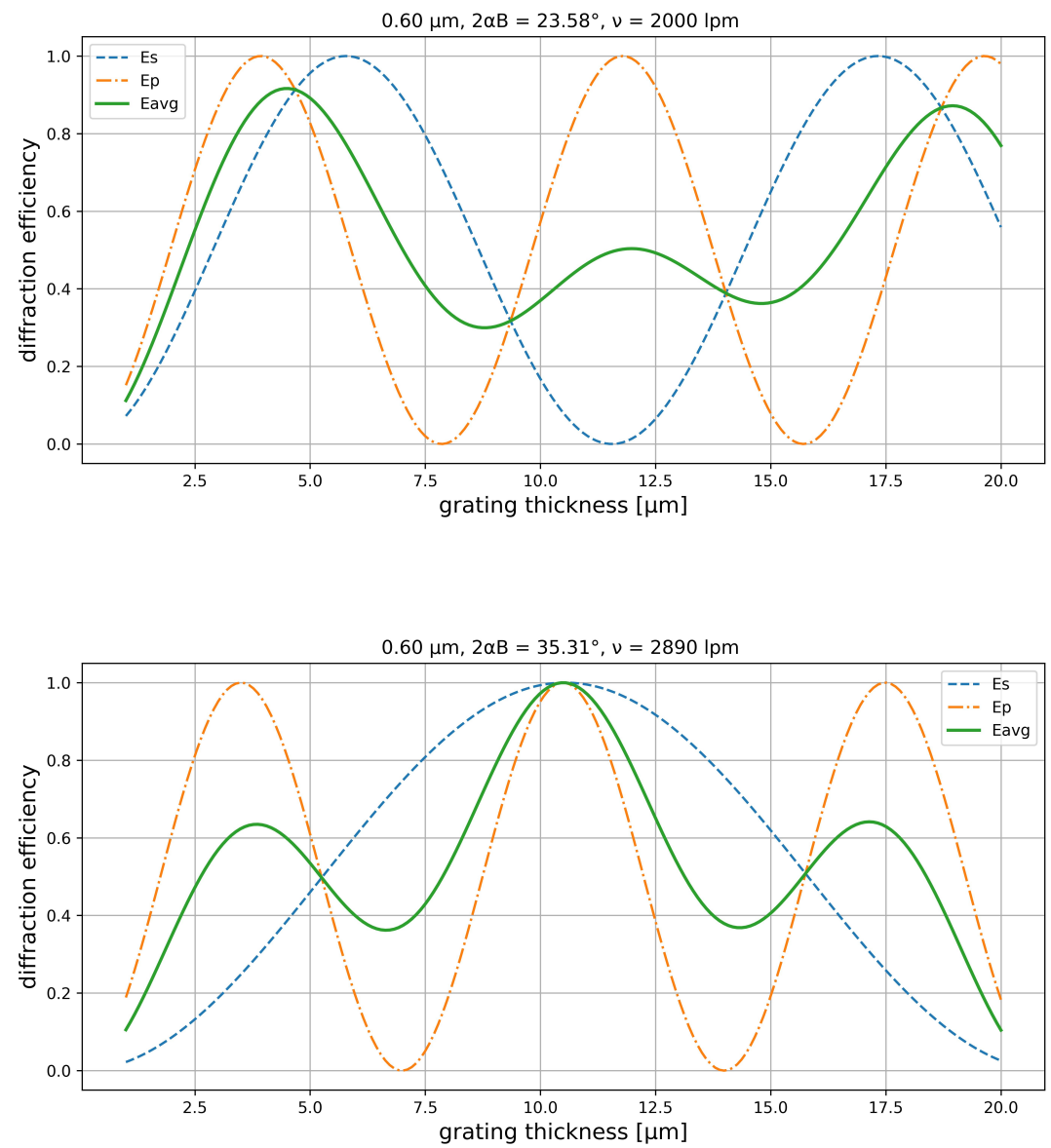

Figure 2.8: Variation of diffraction efficiency versus grating thickness for two different Bragg angles a) $2 \alpha_{B}=23.58^{\circ}$ and b) $35.31^{\circ}$. The solid green lines represent the efficiency of unpolarized light, while the dashed blue and orange lines represent the s- and p-polarization states, respectively. Source: Adapted from Baldry et al. (2004).

\subsubsection{Spectrograph Principal Parameters}

\subsubsection{Grating Parameters}

The definition of the anamorphic magnification factor $\left(r_{a n}\right)$ is the "ratio of change in angle of camera beam in direction of dispersion to change in angle of collimator beam" (Schweizer, 1979, p. 150). Konidaris (2014) uses ray tracing and general grating equations to illustrate the effects of $r_{a n}$ and the "rotation anamorphism" caused by the angle projected along the line rulings in the grating, indicated as $\gamma$ in equation 2.30 and figure 2.9. Rearranging equation 2.31 and differentiating in $\alpha$ and $\beta$,

$$
\delta \beta=-\frac{\cos \alpha}{\cos \beta} \delta \alpha
$$



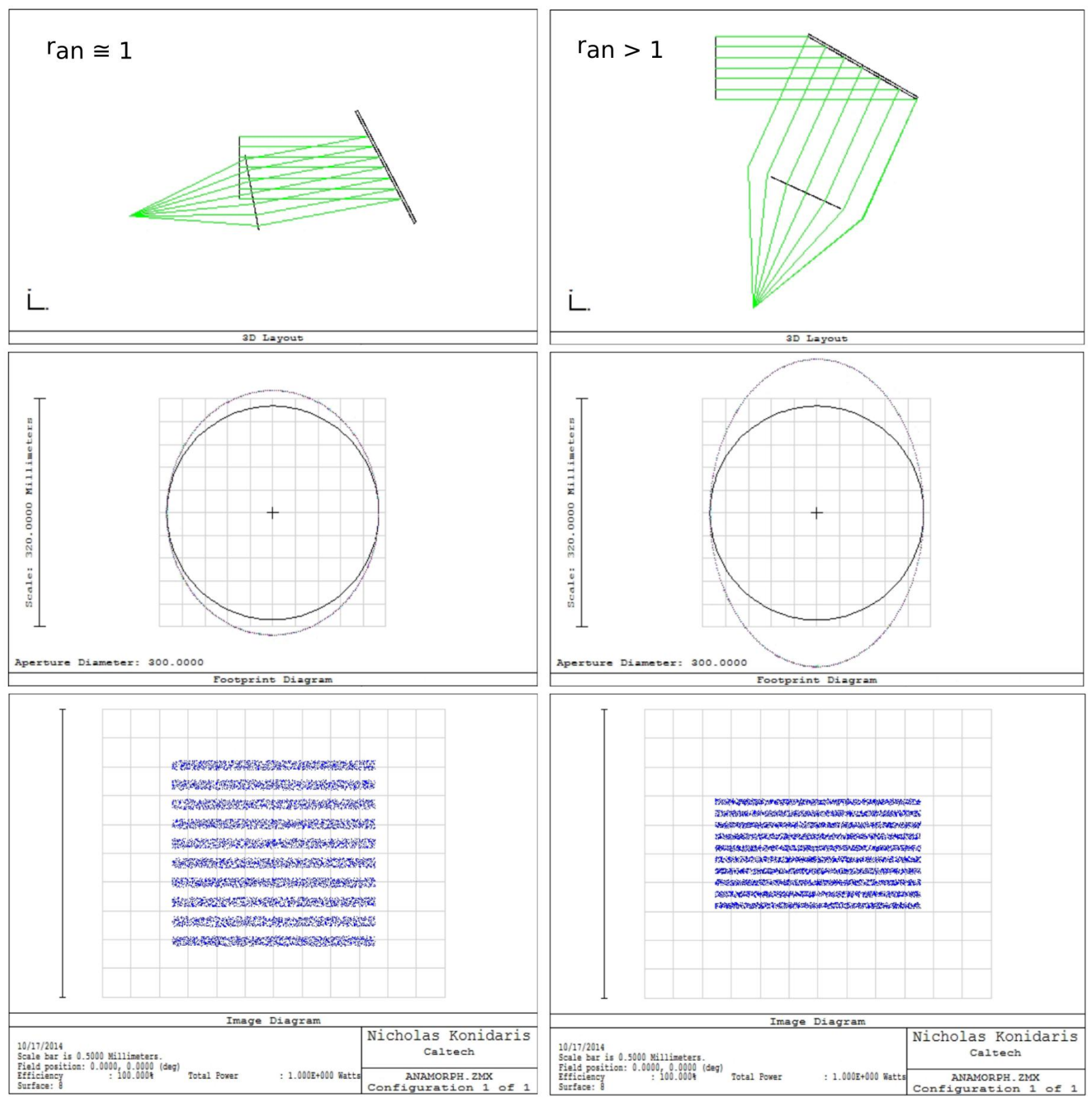

Figure 2.9: Effect of the anamorphic magnification of a simplified system consisting of a collimated beam focusing on a reflection diffraction network and a paraxial camera. The top frames show the layout and ray traces for $r_{a n} \approx 1$ and $r_{a n}>1$. As the grating is tilted, the pupil on the camera increases in the dispersion direction. The middle frames show the footprint diagrams of the beam on the camera. The bottom frames show the image, comprised of line bars, on the detector. As the grating is tilted, the image of the object shrinks in the vertical direction (dispersion direction). Source: Konidaris (2014). 
defining $r_{a n}$ as

$$
r_{\text {an }} \equiv-\frac{\delta \beta}{\delta \alpha}=\frac{\cos \alpha}{\cos \beta}=\frac{D_{\text {in }}}{D_{\text {out }}}
$$

where $D_{\text {in }}$ and $D_{\text {out }}$ are the incident and diffracted beam diameters, measured transversely to the optical axis, according to figure 2.10.

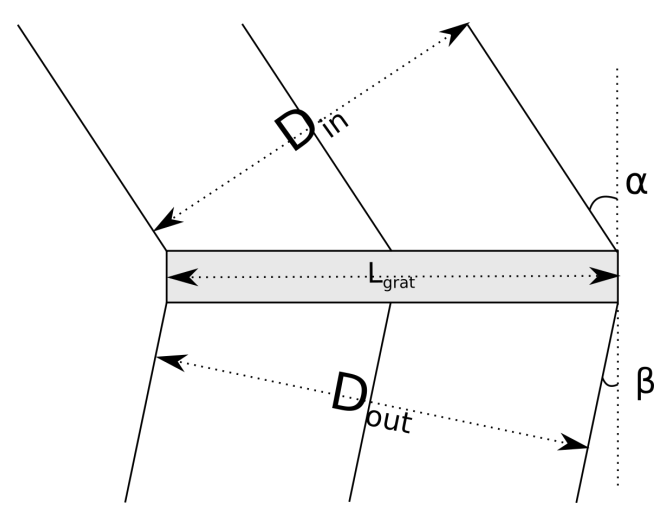

Figure 2.10: Variables for defining the maximum value for the size of the dispersive element. $D_{\text {in }}$ and $D_{\text {out }}$ are the incident and diffracted beam diameter, respectively, measured transversely to the optical axis, $L_{\text {grat }}$ is the grating size, $\alpha$ and $\beta$ are the incident and diffracted angles, respectively, according to the grating equation.

For a given value of $\alpha$, according to the general diffraction equation (equation 2.31), the value of $\beta$ is a function of the dispersive element parameters and the wavelength. The dependence of $r_{a n}$ on the wavelength, grating parameters and incident angle can be found by inserting equation 2.43 in the equation 2.42 .

$$
\beta=\arcsin \left[\left(\frac{m \lambda}{n d \cos \gamma}\right)-\sin \alpha\right]
$$

The consequence of anamorphic magnification is the change in the plate scale along the dispersion direction. For a fixed object, as the diffraction angle $\beta$ for a grating also is also proportional to the wavelength, the $r_{a n}$ is proportional to wavelength. Hence, the resolution element is larger for redder wavelengths.

The cross-section of a tilted grating located in a collimated beam has a generic elliptical shape, in which the maximum axis (along the dispersion direction) is the projected collimator exit pupil subtended by the grating incident angle, and the minimum axis is the 
exit pupil size in the spatial direction, as shown in figure 2.10 . The grating size $\left(L_{\text {grat }}\right)$ is given by

$$
L_{\text {grat }}=\frac{D_{c o l}}{\cos \alpha}=\frac{f_{c o l}}{f_{\text {tel }}} \frac{D_{\text {tel }}}{\cos \alpha}=\frac{f_{c o l}}{F_{\text {tel }} \cos \alpha}
$$

For instance, considering a maximum angle $\alpha=45^{\circ}$, the following condition would be valid for the grating size:

$$
\frac{f_{c o l}}{F_{t e l}}<L_{\text {grat }} \leq \sqrt{2} \frac{f_{c o l}}{F_{t e l}}
$$

\subsubsection{Dispersion and reciprocal dispersion}

The definition of the dispersion $(D)$, in the context of the optical design, is the ratio of a given parameter variation to the radiance bandwidth. For spectrographs, the parameter might be the incident angle at the grating, the diffraction angle, the projected image size or the height at the focal plane. Therefore, the dispersion unit is usually given by "dispersive angle range/wavelength range" or "focal plane range/wavelength range". The reciprocal dispersion is defined as $P \equiv D^{-1}$. Commonly used units for $\mathrm{P}$ are $\AA / \mathrm{mm}$ or $\mathrm{nm} / \mathrm{mm}$. This parameter is sometimes referred to as the plate factor, a reference to the use of photographic plates to record spectra (Hearnshaw, 2009).

Dispersion can be written in terms of angular dispersion $\left(D_{\theta}[\operatorname{arcsec} / \AA]\right)$, linear distance on the focal plane $\left(D_{x}[\mu \mathrm{m} / \AA]\right)$ or in pixel units $\left(D_{x_{p}}[\right.$ number of pixels $\left./ \AA]\right)$. Their definitions are presented in the equations below,

$$
\begin{aligned}
D_{\theta} & \equiv \frac{d \beta}{d \lambda} \\
D_{x} & \equiv \frac{d x}{d \lambda} \\
D_{x_{p}} & \equiv \frac{d p_{x}}{d \lambda}
\end{aligned}
$$

and their relations are defined as 


$$
\begin{aligned}
D_{x} & =f_{\text {cam }} D_{\theta} \\
D_{x_{p}} & =\frac{D_{x}}{p_{x} B_{x}}=\frac{f_{c a m}}{p_{x} B_{x}} D_{\theta}
\end{aligned}
$$

Although the dispersion $D_{x}$ is dimensionless, it is commonly expressed in $[\mu m / \AA]$ by multiplying the equation $2.46 \mathrm{~b}$ by a factor of $10^{-4}$.

The angular dispersion $\left(D_{\theta}\right)$ is obtained by differentiating the equation 2.31 with respect to the angle of diffraction $\beta$,

$$
D_{\theta}=\frac{m}{n d \cos \beta} \frac{1}{\cos \gamma}=\frac{\sin \alpha+\sin \beta}{\lambda \cos \beta}
$$

For a small $\beta$, the approximation $d x=f_{\text {cam }} d \beta$ is valid, where $d x$ is the transversal coordinate on the focal plane resulted from the diffracted angle $\beta$, where $\beta$ is in radians. The value of $d x$ may be expressed in pixels through the equation $d x \rightarrow d p_{x}=\frac{f_{c a m} d \beta}{p_{x} B_{x}}$, where $p_{x}$ is its pixel size in the direction of dispersion and $B_{x}$ is the binning factor on the detector plane. Thus,

$$
D_{x_{p}}=\frac{f_{c a m} d \beta}{p_{x} B_{x}} \frac{1}{d \lambda}=D_{\theta} \frac{f_{c a m}}{p_{x} B_{x}}
$$

The binning $B_{x}$ is a characteristic of staring array detectors that acts as an integer factor that increases the pixel size along the spectral direction. It results in an effective larger pixel that does not have to be necessarily symmetrical along the spectral and spatial directions. It can offer benefits in faster readout speeds and improved signal to noise ratios, albeit at the expense of reduced spatial and/or spectral resolution.

Inserting equation 2.48 in equation $2.49, D_{x_{p}}$ is also expressed as

$$
D_{x_{p}}=\frac{m n}{d \cos \beta} \frac{1}{\cos \gamma} \frac{f_{\text {cam }}}{p_{x} B_{x}}=\frac{(\sin \alpha+\sin \beta)}{\lambda \cos \beta} \frac{f_{c a m}}{p_{x} B_{x}}
$$

when divided by $1 \AA$, the units of $D_{x_{p}}$ are number of pixels $/ \AA$. 
The equation 2.50 and its reciprocal value are very useful for the determination of the resolution of a spectrograph based on a 2D array type of detector such as CCD:

$$
N_{\lambda} \propto \frac{\Delta \lambda}{\lambda}
$$

\subsubsection{Resolving Power and Resolution}

The spectral resolving power $(R)$ of a grating is the measure of its ability to separate adjacent spectral lines of an average wavelength $\lambda$. It is a characteristic of the grating and the angles at which it is used (Palmer and Loewen, 2014), usually expressed in a dimensionless unit value,

$$
R \equiv \frac{\lambda}{\delta \lambda}
$$

According to Palmer and Loewen (2014) and Hutley (1982), while the word power has a specific meaning of "unit of energy per time", the term resolving power does not involve this concept of power, but "the ability to resolve".

The ability to resolve two wavelengths $\lambda_{1}$ and $\lambda_{2}=\lambda_{2}+\delta \lambda$ does not involve only the grating, but also the dimension and location of the entrance and exit slits (detector elements), the aberrations in the image and the magnification of the images. The minimum difference $\delta \lambda$ (called resolution or spectral resolution) between two wavelengths that can be resolved is the convolution of the image of the entrance aperture with the exit aperture (Palmer and Loewen, 2014). The spectroscopic resolution is defined as the Full Width at Half Maximum (FWHM) of the one-dimensional Point-Spread Function (PSF), in wavelength units.

Substituting the linear distance with the slit image size on the detector plane (equation 2.14 ) in the definition of the linear dispersion (equation 2.46b), and assuming small $\theta_{\text {slit }}$,

$$
d \lambda=\frac{d x}{D_{x}} \equiv \frac{w_{s}^{\prime}}{D_{x}}=\frac{r_{a n} w_{s}}{D_{x}} \frac{f_{c a m}}{f_{c o l}} \approx \frac{r_{a n} \phi_{s}}{D_{x}} \frac{f_{c a m} f_{t e l}}{f_{c o l}}
$$

or, expressing it as a function of dispersion, in pixel units, 


$$
d \lambda=\frac{r_{a n} w_{s} p_{x} B_{x}}{D_{p_{x}}} \frac{f_{c a m}}{f_{c o l}}
$$

The resolving power can be presented in different ways, each one showing the relations with the functional parameters of a spectrograph,

$$
\begin{aligned}
R & =\frac{D_{x} \lambda}{w_{s}^{\prime}} \\
& =\frac{D_{x} \lambda}{r_{a n} w_{s}}\left(\frac{f_{c o l}}{f_{c a m}}\right) \\
& =\left(\frac{\sin \alpha+\sin \beta}{\cos \alpha}\right) \frac{D_{c o l}}{D_{\text {tel }}}\left(\frac{1}{2 \tan \left(\theta_{\text {slit }} / 2\right)}\right) \\
& =\left(\frac{m \lambda}{d}\right) \frac{D_{\text {col }}}{D_{\text {tel }}}\left(\frac{1}{2 \tan \left(\theta_{\text {slit }} / 2\right)}\right) \\
& =\left(\frac{m \lambda}{d}\right) \frac{f_{\text {col }}}{w_{s}} \\
& \approx\left(\frac{m n \lambda}{d r_{a n} \phi_{s}}\right)\left(\frac{f_{\text {col }}}{f_{\text {tel }}}\right)\left(\frac{1}{\cos \beta \cos \gamma}\right)
\end{aligned}
$$

For Littrow configuration, assuming $\theta_{\text {slit }}$ small (in radians), equation 2.44 and equation $2.55 \mathrm{c}$ can be rewritten as

$$
R \approx\left(\frac{2}{\theta_{\text {slit }}}\right)\left(\frac{D_{\text {col }}}{D_{\text {tel }}}\right) \tan \theta_{B}=\left(\frac{2}{\theta_{\text {slit }}}\right)\left(\frac{L_{\text {grat }}}{D_{\text {tel }}}\right) \sin \theta_{B}
$$

A limit case happens when the field is observed with a diffraction-limited telescope, which means that $\phi_{s} \approx \frac{\lambda}{D_{t e l}}$. Substituting this expression in equation 2.55f, using the relation of the telescope and collimator f-number (equation 2.8) and the grating equation (equation 2.31) for in-plane and Littrow configuration,

$$
R=\frac{2 D_{\text {col }}}{\lambda} \tan \theta_{B}
$$

The image of the slit on the detector plane, known as resolution element $\left(r_{\text {ele }}\right)$, can be expressed as a function of the physical width of the slit image on the detector plane along 
the dispersive axis (as shown in equation 2.58a in units of length) or resolution element as a function of the number of pixels ( $r_{\text {elep }}$ ) (as shown in equation $2.58 \mathrm{~b}$ ). The image $I^{\prime}$ is replaced by the slit width multiplied by $M_{\text {pupil }}$ (see equation 2.6) and $r_{a n}$.

$$
\begin{aligned}
& r_{\text {ele }} \equiv w_{s}^{\prime}=r_{a n} \frac{f_{\text {cam }}}{f_{\text {col }}} w_{s}=r_{a n} \frac{f_{\text {tel }} f_{\text {cam }}}{f_{\text {col }}} 2 \tan \left(\frac{\phi_{s}}{2}\right) \\
& r_{\text {elep }}=\frac{r_{\text {ele }}}{p_{x}}
\end{aligned}
$$

Generally, the telescope spatial resolution is limited by the seeing and/or telescope quality, not by diffraction. Similarly, the spectral resolution of a spectrograph is limited by the slit width or seeing (the larger of the two), the detector sampling and spectrograph optics.

The bandwidth per resolution element $\left(\delta \lambda_{\text {slit }}\right.$, [ $\AA$ or $\left.\left.\mathrm{nm}\right]\right)$, given by the slit width, is actually the smallest bandwidth resolvable for a slit-limited spectrograph (see equation 2.52 , where $\delta \lambda_{\text {slit }} \equiv \delta \lambda$ ). It is given by the ratio of the wavelength resolution element and the corresponding dispersion,

$$
\delta \lambda_{\text {slit }}=\frac{r_{\text {ele }}}{D_{x}}=\frac{r_{\text {elep }}}{D_{x_{p}}}
$$

Replacing the variables in the equation 2.59, we find useful relationships to express $\delta \lambda_{\text {slit }}$.

$$
\delta \lambda_{\text {slit }}=r_{a n} B_{x} \frac{\cos \beta}{m n} \frac{f_{\text {tel }}}{f_{\text {col }}} 2 \tan \left(\frac{\phi_{s}}{2}\right)=\frac{w_{s}^{\prime} B_{x} \cos \beta}{m n f_{c a m}}
$$

\subsubsection{Spectral coverage}

A detector for a spectrograph has two distinct orientations: one for the field, parallel to the diffraction grating lines, and the other for the dispersion, perpendicular to the diffraction grating lines.

Similar to any imaging system, the practical FoV is a combination of the limitation from the detector dimensions and optics FoV. The concept of the FoV in the dispersive direction 
is translated to spectral coverage for spectrograph. Therefore, excluding the influence of the optical system quality or any field obstructions, the sensor size in the spectral direction is the limiting parameter for the spectral coverage.

A simple way to calculate the spectral coverage is to determine how many resolution elements fit in the spectral direction of the detector and multiply it by its average resolution $\delta_{\lambda}$. Hence,

$$
\Delta \lambda=\frac{\delta_{\lambda} N_{\lambda}}{r_{\text {elep }}}
$$

where $N_{\lambda}$ is the number of pixels of the detector in the spectral direction.

An alternative way is to calculate the limits of the spectral range, which can be done by solving $\lambda$ in equation 2.31 and inserting the camera FoV:

$$
\lambda_{ \pm}=\frac{n d}{m}\left[\sin \alpha+\sin \left(\beta \pm \frac{\theta_{\text {cam }}}{2}\right)\right] \cos \gamma
$$

where $\lambda_{ \pm}$represents the $(+)$redder and (-) bluer wavelength, and $\theta_{\text {cam }}$ is the camera FoV in the spectral direction. Therefore, $\Delta \lambda=\lambda_{+}-\lambda_{-}$.

\subsubsection{Spectrograph System and Subsystems Requirements}

As suggested by Fischer et al. (2008, p. 9), "it is important at the outset of a design project to compile a specification for the desired system and its performance." The functional requirements of a spectrograph do not necessarily have a sufficient level of detail to generate a set of quality criteria for the design and evaluation of the optical design. Consequently, the functional requirements are expanded in order to incorporate restrictions and conditions that can directly or indirectly impact the optical design, which also enables the elaboration of the related optical quality and evaluation criteria. The activity must be supervised by systems engineers, optomechanics, and optics engineers since there are several interrelationships. Section 4.4 .3 presents the proposed methodology for defining requirements and performance criteria for GMACS.

Table 2.2, adapted from Rayner (2010), shows the most common top-level requirements flow down derived from the science cases that summarize the essential characteristics of a 
general spectrograph.

Table 2.2 - Spectrograph design top-level requirements. Source: adapted from Rayner (2010).

\begin{tabular}{ll}
\multicolumn{2}{c}{ System Requirements } \\
\hline Resolving power & Sensitivity \\
Resolution & Central Wavelength \\
Continuous wavelength range & Throughput \\
Simultaneous wavelength range & Pixel field of view or plate scale \\
Slit width and length & Image quality at the spectrograph detector \\
Spectrograph FoV & Sampling \\
S/N limit (includes stray light) & Stray light at the spectrograph detector
\end{tabular}

Spectrograph subsystems must meet the spectrograph top-level requirements. However, unless explicit and justified, the subsystems requirements are design variable and liable to optimization aiming for high efficiency, risk reduction, and complexity.

To simplify the notation in this work, we redefined the GMACS Product Breakdown Structure to turn the optical subsystem spectrograph as a system-level (see section 4.3.1). Therefore, the GMACS optical design is divided into subsystems and assembly levels. Table 2.3 shows the proposed performance specification and requirement topics that must be included in the optical design.

\subsubsection{Spectrograph Performance Criteria}

Although there are peculiarities for optical evaluation criteria of spectrographs that differ from a conventional imaging system, the set of image quality metrics covered by the literature on optical design is an essential reference. They can be divided into geometrical and wavefront image quality metrics, i.e, non-diffractive and diffraction regimes, respectively.

Since most of the optical spectrographs are not considered diffracted-limited imaging systems, the most commonly used image quality metrics for the design are based on geometrical aberrations, for example, (i) ray aberration curves, such as ray fan and longitudinal aberration plots; (ii) spot diagrams, which are related to the encircled (or ensquared) energy, RMS spot size and the Airy Disc for diffraction reference; and (iii) Seidel aberrations for first-order aberrations. Although the MTF is usually used for general imaging systems, a slit optical spectrographs performance is limited by the slit width since its image 
Table 2.3 - Specification and requirements for a spectrograph's optical system basic operation. Source: adapted from Fischer et al. (2008).

\section{Subsystems Parameters}

\section{Telescope:}

Design Version

Effective focal length

Entrance pupil diameter and f-number

Entrance pupil position

\section{Spectrograph Entrance:}

Type (long slit, slitlets, fiber-fed)

Surface size and shape

Apertures size (width and heigth)

Collimator(s):

Type (dioptric, reflexive, catadioptric)

Effective focal distance

Exit pupil diameter and f-number

Exit pupil relief

Front focal distance

Dichroic transition wavelength

Vignetting

\section{Disperser(s):}

Type of disperser

Maximum size

Dispersiver parameter

Stray light and ghosts

Camera(s):

Type (dioptric, reflexive, catadioptric)

Effective focal distance

Entrance pupil diameter and f-number

Vignetting

Back focal distance

\section{Sensor:}

Sensor type

Full diagonal

Number of pixels (horizontal $\mathrm{x}$ vertical)

Pixel pitch (horizontal x vertical)

Nyquist frequency at sensor, line pairs/mm

\section{Usual Optical Design Requirements}

\section{Lens System:}

Number of elements

Aspheric surfaces BF and Slope

Optical Performance:

Transmission

Encircled energy

$\mathrm{MTF}$ as a function of line pars $/ \mathrm{mm}$

\section{Environmental:}

Thermal soak range to perform over

Thermal soak range to survive over

Vibration

Shock

Other (condensation, humidity, sealing)

\section{Packaging:}

Object to image total track

Maximum element diameter

Maximum length

Weigth

\section{Schedule and cost:}

Initial delivery date

Elements lead time

Target cost goal 
is usually larger than the diffraction limit spot size.

The most commonly used diffraction-based image quality metrics, based on the wavefront characteristics, as a deviation from the perfect reference sphere, also known as wavefront aberration, are (i) wavefront deviation; (ii) RMS wavefront error; (iii) Modulation Transfer Function (MTF); and (iv) Strehl Ratio.

The RMS spot size gives a statistical spot size that represents the average distance of a large number of individual rays from the central spot. It is only reliable when the optical design is not considered diffracted-limited, such as for MOS in ELT.

The causes of optical performance degradation in a spectrograph are distinguished between intrinsic and induced aberrations. The intrinsic are those inherent to the RoC, conical surfaces, glass medium, and fabrication errors, while the induced are caused by any external factors such as alignment errors, surface deformations generated by thermal variations, self-induced weight (variations of the gravity-vector due to telescope pointing), improper mounting, and air mass turbulences. The tolerance analysis combines the fabrication aberrations and external factors to estimate the expected optical performance.

\subsubsection{Encircled or ensquared energy}

Encircled or ensquared energy is the fraction of the energy or power within a circle of specific radius $\mathrm{r}$ or square detector of side l, respectively, centered on the chief ray reference point, the beam centroid, or a surface vertex reference. This criterion metric is commonly used for imaging systems, especially the ones that need high SNR. The encircled or ensquared energy is

$$
E E(r)=\frac{\int_{0}^{r} I(r) d r}{\int_{0}^{\infty} I(r) d r}
$$

where $I(r)$ is the intensity of the ray that hits the focal plane (Zemax LLC OpticStudio, 2016) and $0 \leq E E(r) \leq 1$.

A standard quality criterion for astronomical spectrograph is the maximum spot radius or diameter and the RMS of these values. The adopted notation is EEDX, representing the diameter of a circle of which it has X\% of the total energy of the field. For instance, EED80 is for the diameter of a circle that contains $80 \%$ of the energy in it. Numerically, 
the radius of a circle for a given encircled energy is determined by finding the $r$. EED100 is analogous to the geometrical spot size.

\subsubsection{Throughput}

System basic throughput $T$, or étendue $\xi$, is an indication of the total flux that can pass through the system and can be evaluated over any surface that intersects all the rays passing through the system. It depends on the field of view and the aperture of the system. In any loss-less system, it is defined by

$$
\xi=\iint d^{2} \xi=n^{2} \iint \cos \theta d A d \Omega \rightarrow n^{2} A \Omega
$$

where $n$ is the refractive index at the location where the solid angle $\Omega$ is defined, and $A$ is the area of the aperture at the location where the solid angle is defined. In terms of the optical system of figure 2.11, the étendue is given by $n_{0}^{2} A_{0} \Omega_{0}$. Willers (2009) describes in detail the definition of the geometric and projected solid angle.

Optical designers define a quantity called the optical invariant or Lagrange invariant or Helmholtz invariant, $\mathrm{H}$, which is invariant in any given optical system and defines the throughput of the system. $\mathrm{H}$ is constant throughout the entire system, on all the surfaces and spaces between them, and for paraxial approximation is given by

$$
H=n\left(y_{p} u-y u_{p}\right)
$$

where the subscript $p$ refers to the principal (chief) ray, no subscript refers to the marginal ray, and $\mathrm{n}$ is the refractive index of the surface medium. When this formula is used to calculate the optical invariant in the object plane and image plane where the marginal height is zero, equation 2.65 is simplified to $H=h n u=h^{\prime} n^{\prime} u^{\prime}$ where $h, n$ and $u$ are the height of the object, the index of refraction, and the angle of the marginal ray in the object plane, and $h^{\prime}, n^{\prime}$ and $u^{\prime}$ are the height of the object, the index of refraction, and the angle of the marginal ray in the image plane. A sufficiently accurate approximation is obtained by replacing the angles with their sines.

The Lagrange invariant is directly proportional to the square root of the throughput. 


$$
T=\pi^{2} H^{2}
$$

The oblique total flux incident on a detector $\phi_{d}$ for finite conjugate optics is

$$
\phi_{d}=\frac{\pi L A_{d} \cos ^{4} \alpha^{\prime}}{4 F^{2}(1+|M|)^{2}}+\phi_{\text {stray }}
$$

where $L$ is the object's spectral radiance, $A_{d}$ is the detector area, $\alpha^{\prime}$ is the angle from normal object and focal planes to the respective chief ray, $M$ is the transverse (or lateral) magnification, assuming a unity index of refraction in the object and image planes, $f_{\#}$ is the f-number and $\phi_{\text {stray }}$ is any noise stray light incident on the $A_{d}$. This equation assumes that no vignetting, central obscuration or attenuation takes place in the lens. Analysis of any real system would require that these factors be taken into account. The spectral radiance, $L$, is the density of flux per source surface area per solid angle and per wavelength $\left[W \cdot m^{-2} \cdot s t r^{-1} \cdot \lambda^{-1}\right]^{1}$. Willers (2009) describes in detail the physical and mathematical background of an eletro-optical radiometry.

The radiometry of the oblique system, explicitly given by the $\cos ^{4}$ term, is justified thought the following: (i) the oblique view of the pupil increases its ellipticity, resulting in a reduction in projected area by $\cos \alpha$; (ii) the distance between the two elemental areas increases by $1 / \cos \alpha$, but note that the distance occurs in the square, resulting in a $1 / \cos ^{2} \alpha$ factor; and (iii) provided that object, image and pupil planes are parallel, the projected area of the elemental area in the object plane decreases by $\cos \alpha$.

The effective or total throughput accounts for any causes that might decrease the flux on the detector, such as atmospheric extinction, seeing and slit losses, any vignetting factors due to the telescope aperture and obscuration, AR coatings on refractive surfaces and reflectivity of the mirrors, internal material absorption of materials, disperser efficiency, and detector properties, such as quantum efficiency, readout and dark noise. Many of these parameters are wavelength dependent. Concerning the dispersing element, the throughput has a more complex relationship due to its dependence on efficiency with both

\footnotetext{
1 The term "spectral radiance", although not commonly used in the context of astrophysics, is used in radiometry for electro-optical systems (Willers, 2009; Palmer and Grant, 2009).
} 


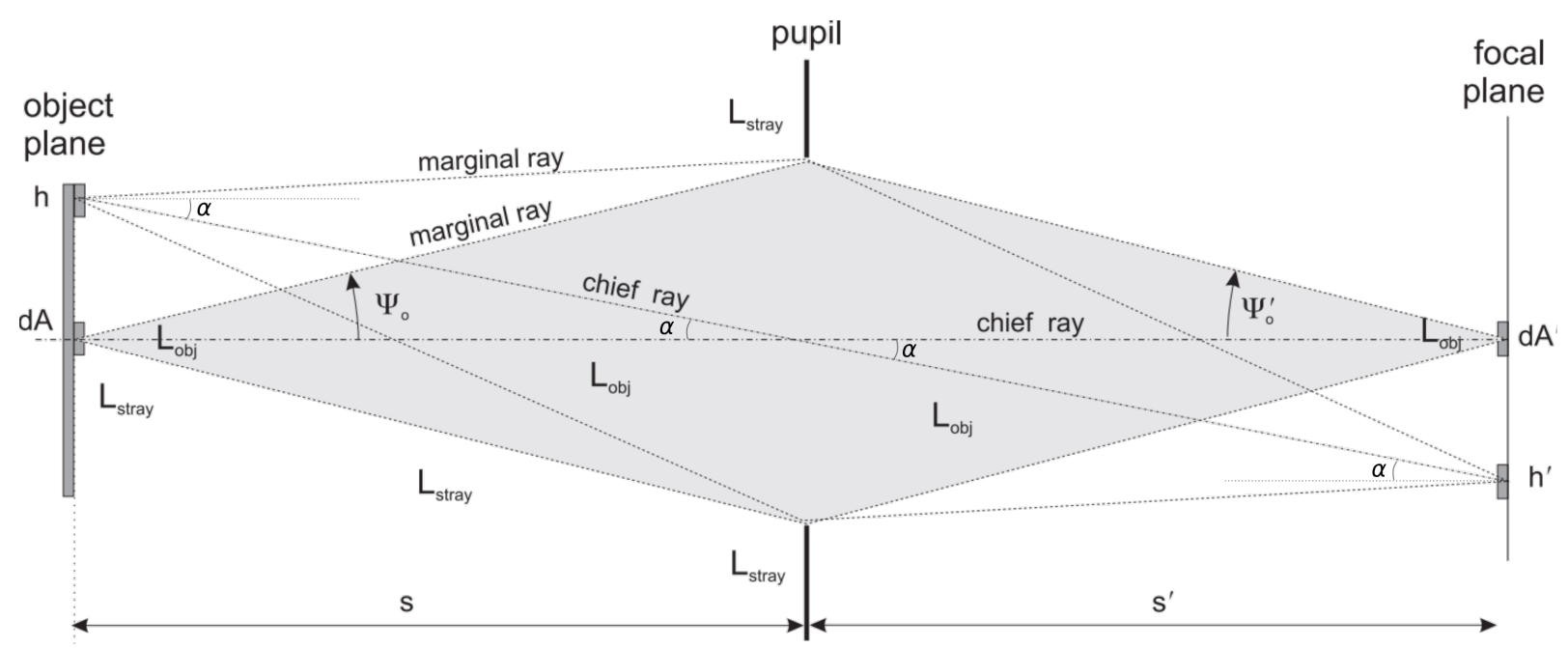

Figure 2.11: Optical parameters for radiometry in a finite conjugate imaging system. A spectrograph can be modeled as a finite conjugate imaging system when $s \rightarrow f_{c o l}$ and $s^{\prime} \rightarrow f_{\text {cam }}$. The object plane is the aperture plane (mask slit) and the pupil is the collimator exit pupil, where the grating is located. Source: Willers (2009).

angles of incidence (i.e., the position of the object) and diffraction, and wavelength. A proper throughput model is the core of an Exposure Time Calculator (ETC), an end-to-end component model that accounts for every parameter described above.

\subsubsection{Depth of Focus}

The depth of focus (DoF) is the maximum axial range of motion of the camera focal plane from which the image performance is not deteriorated according to a pre-established criterion as a function of the current system's performance regime. It is also used to estimate the first-order tilt tolerance of the focal plane based on pixel pitch, camera speed, and central wavelength. For a mosaic focal plane, the tilt tolerance is a function of the detector's size and location at the mosaic.

The DoF, represented by $\delta$, for a diffraction-limited system and non-diffraction-limited system are given by Fischer et al. (2008) and Edmunds Optics (2018),

$$
\begin{aligned}
& \delta= \pm \frac{1}{4} \mathrm{OPD}= \pm 2 \lambda F^{2} \\
& \delta= \pm F p_{p t}
\end{aligned}
$$


The DoF for $\lambda=500 \mathrm{~nm}$ and $\mathrm{f} / 2.2$ is $\delta \pm 4.8 \mu \mathrm{m}$ for a diffraction-limited system. For a pixel-limited system, $p_{p t}=15 \mu \mathrm{m}$ and $\mathrm{f} / 2.2, \delta \pm 66 \mu \mathrm{m}$.

Considering a detector tilt $\epsilon$ and the pixel size as a metric for the estimation of the DoF, the focal plane sagitta $z$ is given by $z=\left(p_{p t} \tan \epsilon\right) / 2$ and assuming that $\delta>2 z$, see figure 2.12.

$$
\epsilon<\arctan \left(\frac{2 F}{D_{\text {detector }}}\right)
$$

where $D_{\text {detector }}=\sqrt{N_{x}^{2}+N_{\lambda}^{2}}$ is the diagonal of the detector. If the detector is comprised of a mosaic, the tilt tolerance can be calculated for each detector individually.
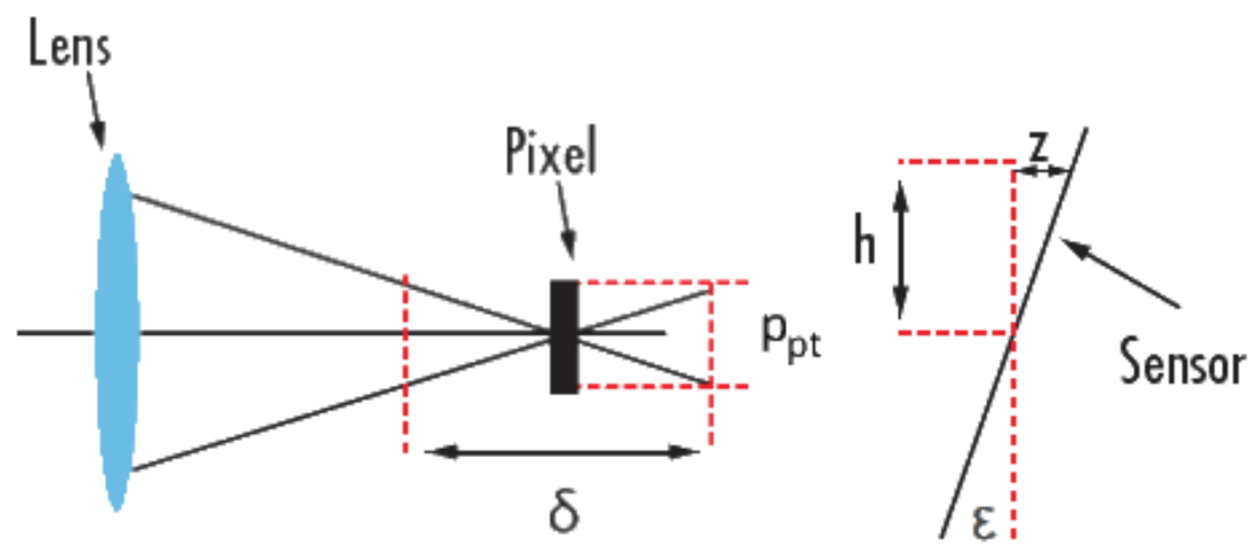

Figure 2.12: Depth of focus and detector tilt first-order tolerance. Source: Edmunds Optics (2018).

\subsubsection{Effective f-number}

The f-number is a radiometry parameter of the system (Fischer et al., 2008; Palmer and Grant, 2009). The effective f-number, $F_{\text {eff }}$, is computed by:

$$
F_{e f f}=\sqrt{\frac{\pi}{4 n^{2} \Omega_{p}}}
$$

where $\Omega_{p}$ is the Projected Solid Angle of the pupil in cosine space weighted for a transmission system. According to Siew (2005), the $F_{\text {eff }}$ for arbitrary exit pupil shapes is different even through their areas are the same. However, this difference becomes significant only 
if the working f-numbers are f/1 or less, which suggests that, under conditions where the precise magnitude of image brightness is not a major concern, the $F_{\text {eff }}$ for a system with a non-circular stop is approximately that with a circular. Therefore, the equation 2.70 is valid for most astronomical telescopes and it can be a useful metric for comparing the brightness of the image formed by them.

In the case of finite conjugate systems, such as a spectrograph, the calculation of the f-number must be corrected by the object-image magnification, $m=i / o$, where $i$ is the image height, and $o$ the object height and the pupil magnification, $m_{p}=$ EXPD/ENPD, is the ratio of the diameter of the exit pupil to the diameter of the entrance pupil,

$$
F_{e f f}=F\left(1+\frac{m}{m_{p}}\right)
$$

The T-number, $T_{\#}$, combines the f-number and the system transmittance, $T$, into a single quantity,

$$
T_{\#}=\frac{F}{\sqrt{T}}
$$

\subsubsection{Degradation of the Spectra Quality}

\subsubsection{Aberrations}

Transverse Chromatic Aberration: The Transverse Chromatic Aberration (TCA), also known by the term "lateral color", is the result of the variation of the transversal magnification as the function of the wavelength caused by the wavelength dependence on the index of refraction of the lens material. This effect arises when the stop is shifted relative to the lens, which is always the case for spectrographs (Geary, 2002). TCA is proportional to the field and f-number, and inversely proportional to the Abbe number $T C A \propto H \cdot F / v$. The principal dependence is that the effective focal length is a function of the wavelength and field $f \rightarrow f(\lambda, H, P)$, where $H$ and $P$ are the fields and pupil coordinates. TCA results in image colors being focused on slightly different heights for the same object.

Mirror-based telescopes do not generate chromatic aberrations and, therefore, do not contribute to the TCA. Although the collimator's objective is to provide a wavelength- 
independent collimated beam at any exit pupil transversal position, a refractive collimator may generate a significant amount of TCA. In this case, the effect of the TCA at the exit pupil is to turn the incident angle on the grating into being dependent on the wavelength $(\alpha \rightarrow \alpha(\lambda))$. It may result in a combination of collimator dispersive effects with the grating dispersion, more complex wavelength dependence on the resolution, and the image color-dependent magnification. Therefore, the diffracted angle from a grating $\beta$, and consequently the resolving power, has a more complex dependence on the field and pupil coordinates, and wavelength $(\beta \rightarrow \beta(H, P, \lambda))$.

This effect is also summed by any TCA resulting from the camera. Although the TCA spectrograph effect can be mitigated by spectral calibration, any attempt to include an image mode can be ineffective in the case of a large spectrograph TCA.

If an imaging mode is requested, the spectrograph must have an acceptable TCA correction only for the field of view corresponding to the imaging mode, which is analogous to the field of view of the collimator. Therefore, a solution is to insert, in the merit function, parameters to correct the TCA within the field of view of the collimator alone, through, for example, the use of a custom ZPLM function.

Distortion of line shapes: The distortion spectral lines along the field direction results from the dependence of $\beta$ on $\gamma$. Gil and Simon (1985) describe it in terms of optical aberrations and distortion effect, while James (2007) describes it by Fraunhofer or "farfield" diffraction for two-dimensional apertures (which is effectively the slit) and oblique incidence. Simply, equation 2.73 shows that the incident ray vector is described by the angles $(\alpha, \gamma)$ and the diffraction ray vector is $(\beta, \gamma)$. As they share the same angle $\gamma$, the diffraction angle in spectral direction is a function of the incidence coordinates, $\beta(\alpha, \gamma)$. Hence, equation 2.31 may be rewritten as

$$
\beta=\arcsin \left[\frac{m \lambda}{n d \cos \gamma}-\sin \left(\alpha-\alpha_{B}\right)\right]
$$

that shows that, for a given fixed $\alpha, \beta$ is proportional to $\gamma$ or $\lambda$. This effect is analogous to conceiving it as a paraxial grating magnification: larger off-axis angle and redder wavelengths will cause larger shape and position magnification.

Figure 2.13 shows two simulations using Zemax that illustrate this distortion on the 
detector plane. The grating lines orientation and the spatial direction is in the vertical axis of the illustration. The dispersion direction is along the horizontal axis - blue to red spectrum from left to right. Each dot in figure 2.13a represents a single-wavelength object field position along the vertical axis (in this example, the object filed is along a central long slit). The distortion of the lines are visible along the field direction, parallel to the grating lines. Figure 2.13b shows the same example for multi-wavelength objects.

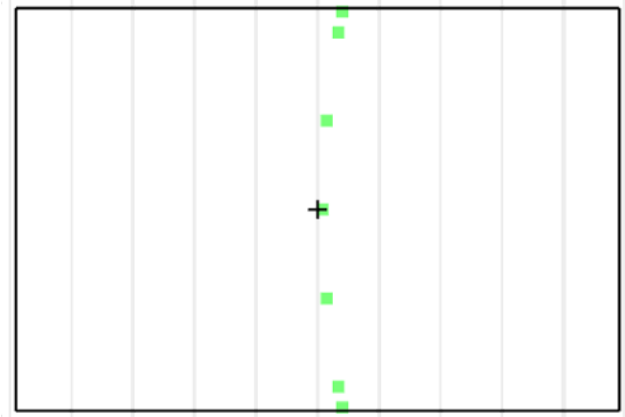

(a) For a single wavelength and several fields.

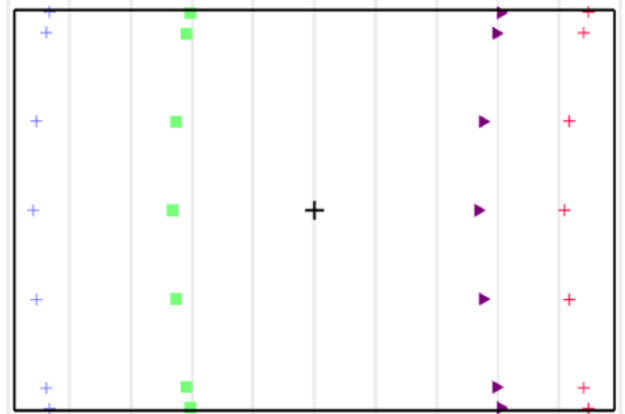

(b) For several fields and wavelengths

Figure 2.13: Example of the detector footprint for a high-resolution mode simulated in Zemax. The horizontal axis is in the spectral direction, and the vertical one in field direction. Colors represent wavelength.

This effect is observed in the grating equation (equation 2.30) by plotting the diffraction angle, $\beta$, as a function of the incident orthogonal angle, $\gamma$, for a fixed set of diffraction order, grating groove density, and $\alpha$. Figure 2.14 shows an example of the spectra of several objects that illustrates the effects of the value of $\gamma$ in the image position. The longer the wavelength and the higher the value of $\gamma$, the more evident the apparent distortion. The configuration in this example is $d=357.19 \mathrm{~nm}(\approx 2,800$ lines per $\mu m), m=1, n=1$ and $\left.\alpha=\beta_{B}=29.34^{\circ}\right)$. 


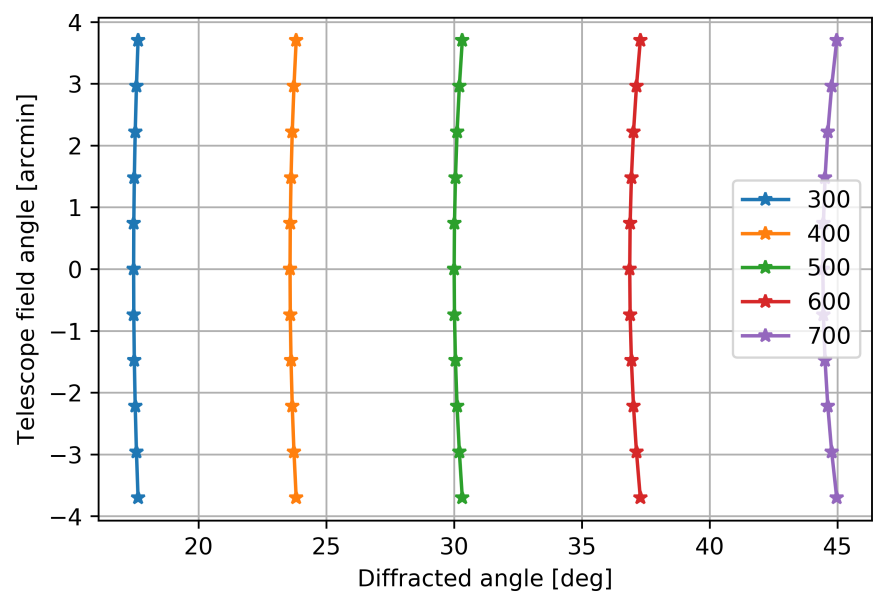

Figure 2.14: Distortion of the image spectra for several objects. The colors represent wavelengths in nanometers and the lines are wavelength-constant curves which illustrate the distortion effect.

\subsubsection{Efficiency Loss}

Vignetting: Many optical systems employ deliberate vignetting by clipping the rays by apertures other than the stop surface, aiming for a better image quality performance. It is a common trade-off among reducing the throughput of the system, getting a better optical image by removing an excessively aberrated portion of the beam, and decreasing the size of the lenses, particularly in wide-angle lenses. Vignetting usually increases the f-number as a function field angle, which in turn darkens the image, but the image quality may improve if the most severely aberrated rays are clipped (Zemax LLC OpticStudio, 2016). The reduction of the size of the optical elements is always desired for application in ELT instrumentation, especially when the element has aspherical surfaces.

For a spectrograph, vignetting causes an additional dependence on the throughput as a function of the diffracted angle $\beta$, according to equation 2.30 , if it is generated after the dispersive element, for example, at the camera. The effects of vignetting in the camera are illustrated in figure 2.15 . 


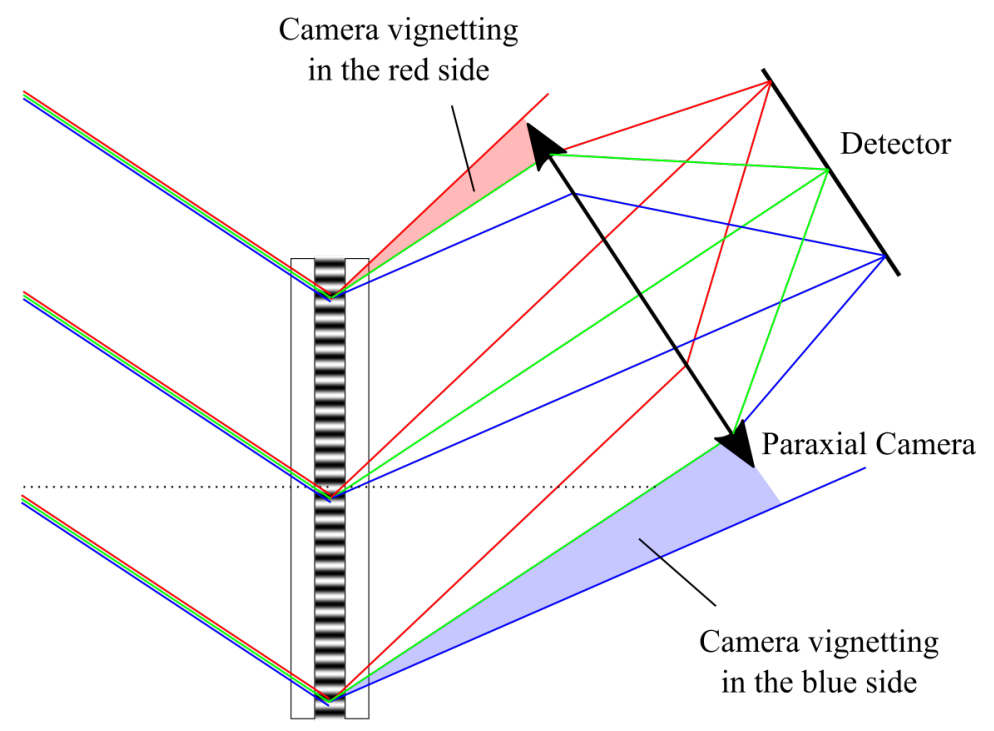

Figure 2.15: The spectral vignetting effect caused by obstruction of the diffracted light by the lens aperture for better image quality performance.

Slit Transmission Factor: The slit transmission factor along the x-direction is given by

$$
T_{x}=\operatorname{erf}\left[\frac{\frac{\theta_{\text {slit }}}{2}}{\sqrt{2} \frac{\theta_{\text {seeing }}}{2.35482}}\right]
$$

where $\theta_{\text {slit }}$ is the slit width in arcsec, seeing is the effective seeing in $\operatorname{arcsec}, T_{x}$ is the slit transmission along the $\mathrm{x}$-direction and $L_{x}=1-T_{x}$ is the slit loss along the $\mathrm{x}$-direction ${ }^{2}$. $\operatorname{erf}(x)$ is the error function, defined as

$$
\operatorname{erf}(x)=\frac{2}{\sqrt{\pi}} \int_{0}^{x} e^{-t^{2}} d t
$$

Figure 2.16 shows the map of the slit transmission factor as a function of the seeing FWHM size and slit width. The dotted curve is the $50 \%$ transmission.

${ }^{2}$ Available at https://www.eso.org/observing/etc/doc/formulabook/node18.html. Accessed on 2020-05-12. 


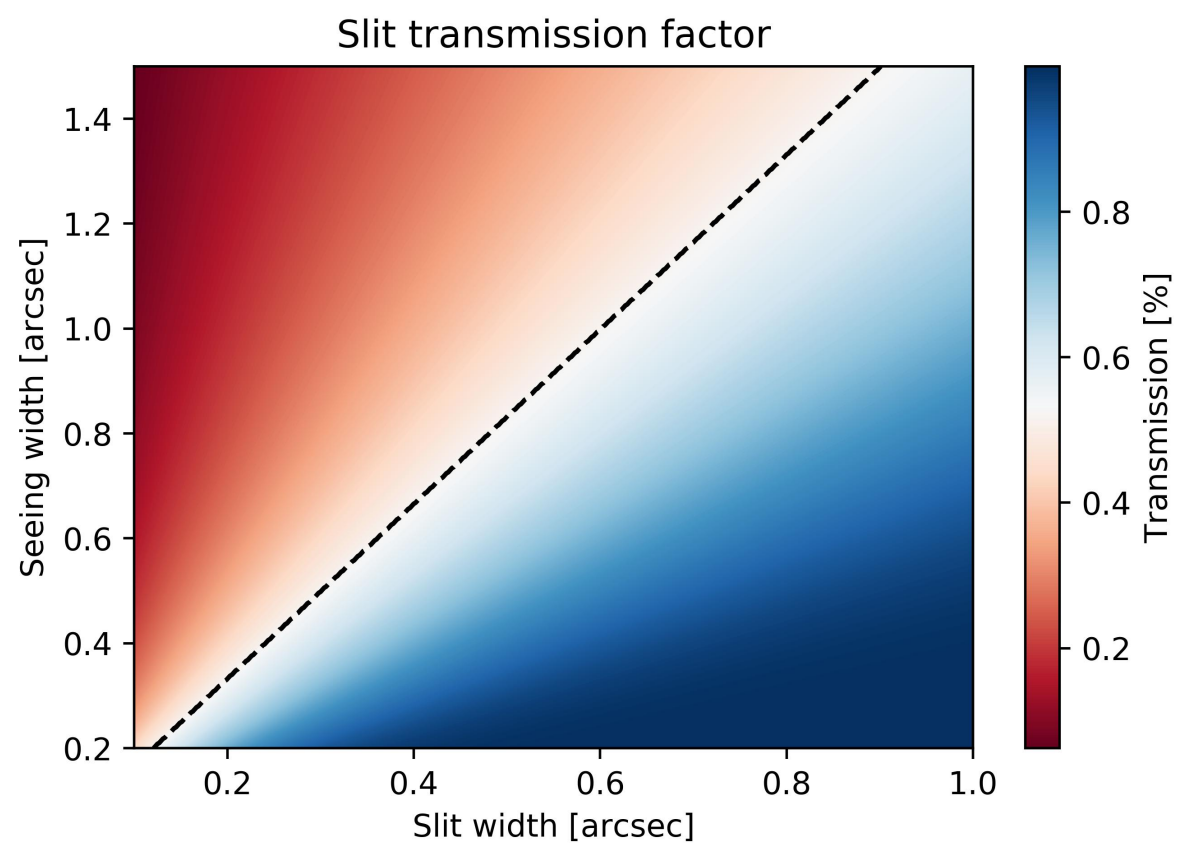

Figure 2.16: Slit transmission factor as a function of seeing and slit width. The dotted curve is the $50 \%$ transmission.

\subsubsection{Opto-mechanical Influences on the Spectrograph Quality}

The typically large sizes required for ELT spectrographs is perhaps one of the most challenging issues that the design must address since it affects the manufacturing, assembling, alignment, and operation. The collimator's exit pupil size must be proportional to the opening of the telescope, thereby ELT instruments can be considerably larger than those in the 8 to 10-meter class telescopes given similar requirements. Furthermore, Gregorian focus instruments are subject to mechanical deformations due to the gravity vector variations caused by the telescope pointing, which is also intensified due to its high weight. Although thermal variation might cause additional deformations, their effects are considered secondary to those caused by gravity. Therefore, in addition to the conventional tolerance analysis for optical systems, a preliminary integrated analysis of the flexure effects on image quality must be performed for a large instrument installed on the Gregorian focus. The integrated analysis takes into account optical, optomechanical, and mechanical aspects of the project. Heijmans et al. (2018, p. 107052D-1) states that "by applying an integrated analysis process early in the project, the risk of finding surprises and design flaws in the hardware are reduced, avoiding delays and additional costs, or failure." 
Except for extreme limits of failure modes, which include yielding, buckling, ultimate failure, fatigue, and fracture, the spectral stability requirement is the one directly affected by any source of flexure. Since the causes of this effect are purely mechanical, its analysis must originate from mechanical modeling that includes information about the instrument and telescope interfaces, principal instrument structure, and optical subsystems mounts. The output is usually based on finite element analysis, whose data must be interpreted and inserted into the optical design software to quantify the resulting image degradation. Depending on the severity of these effects on image quality and spectral stabilization, it is necessary to refine the mechanical model in case the compensators are not sufficient.

There is a large number of possible combinations of the configurations of the spectrograph installed on the Gregorian plane as a function of telescope pointing angle (Zenith angle), instrument rotations, and the spectrograph operating modes. Therefore, the mechanical modeling must certify which are the plausible configurations to generate the most significant effects of structural deformation, either through previous experience of other instruments with a similar operation, foreknowledge, and, of course, the aid of several simulations. Usually, the mechanical models are analyzed by finite element analysis (FEA) techniques performed by the mechanics and optomechanics group of the project, assisted and supervised by the optics group.

The FEA results are comprised of a set of coordinates before and after the deformation, either employing new coordinates or by the differential vectors of each of the nods. Usually, at least three points are used for each reference plane. The locations of the planes are agreed by the mechanics and optics teams, which must be consistent with the available structure and mounting conceptions. The interpreted and converted data are inserted in the optical design software so that the degradation of the image quality and the spectral stability are quantified. For this, it is necessary to follow a rigorous methodology to transfer the mechanical results of finite elements to the optical design software.

The optical designer must employ compensators in the perturbed system, similar to conventional tolerance analyzes, but restricted to the DoF of the elements as a solid-body, and reoptimize the system. If the compensators are not sufficient to reduce the effects of flexure on image quality and stability within the requirements, it is the necessary to review the structure mechanical design in order to reduce the passive flexure and to identify and balance the optical sensitivity throughout the elements. 
The proposed study for conceptual flexure analysis for GMACS considers that the optical system is arranged into groups of elements or assemblies. The primary approximation is that these groups are considered as rigid bodies whose internal elements are not influenced by flexure. It also does not include any information about surface deformations. A more significant number of nods are viable if the methodology has automated algorithms to read, interpret, and insert the results of FEA in the optical design software in order to reduce errors in the interpretation of the results.

According to Heijmans et al. (2018), a solution proposed in the literature to integrate optomechanical analysis, which involves the coupling of the structural, thermal, and optical simulation tools in a multi-disciplinary process, is commonly referred to as StructuralThermal-Optical Performance or STOP analysis. Customized STOP analysis is currently used in large aerospace projects (Fransen et al., 2011; Gracey et al., 2016), but there are also commercial solutions, such as Sigfit, FRED Optical Engineering Software, Lensmechanix among others (Heijmans et al., 2018; Genberg et al., 2017). Although the STOP analysis is part of the optomechanical engineering responsibilities (optomechanical engineering is an application of mechanical engineering principles to design, fabricate, assemble, test, and deploy an optical system that meets operational performance requirements), we decided to develop an in-house methodology for FEA-Zemax integration to satisfy the immediate needs and budget constraints for the conceptual design of GMACS. For the following project phases, we are anticipating that more complete solutions, which include stress or temperature-related refractive index changes, must be used.

\subsection{Spectrograph Subsystems Optical Design}

\subsubsection{Collimator}

This subsection focuses on refractive collimator design ${ }^{3}$ by exploring the alternative of field lens configuration, dichroic and pupil image quality.

\footnotetext{
3 The reasons for the emphasis on the discussion about the components of a refractive collimator is that this concept was adopted for the optical design of GMACS (see section 5).
} 


\subsubsection{Field Lens}

According to Riedl (2001, p. 34), field lens is a "lens placed at or near the focal plane of the objective lens to reduce the needed detector size for a given field coverage. The power of the lens is chosen to image the aperture stop of the objective into the detector plane". For collimator application for a spectrograph, the field lens represents almost the totality of the collimator's power and, therefore, its primary aberrations must be reduced as much as possible. The correction of other residual aberrations, including chromatic ones, must be performed by another group of lenses with weaker focal power.

For a thin lens, the third-order aberrations equations can be simplified and written as a function of the lens bending parameter or shape factor $q$, equation 2.76 , which describes what the lens looks like (defined by the two curvatures).

$$
q=\frac{c_{1}+c_{2}}{c_{1}-c_{2}}=\frac{r_{2}+r_{1}}{r_{2}-r_{1}}
$$

where $c_{i}$ and $r_{i}$ are the center of curvature and radius of curvature for the surface $i$, respectively, and $c=1 / r$. Figure 2.17 shows the corresponding lens shape for a given shape factor.

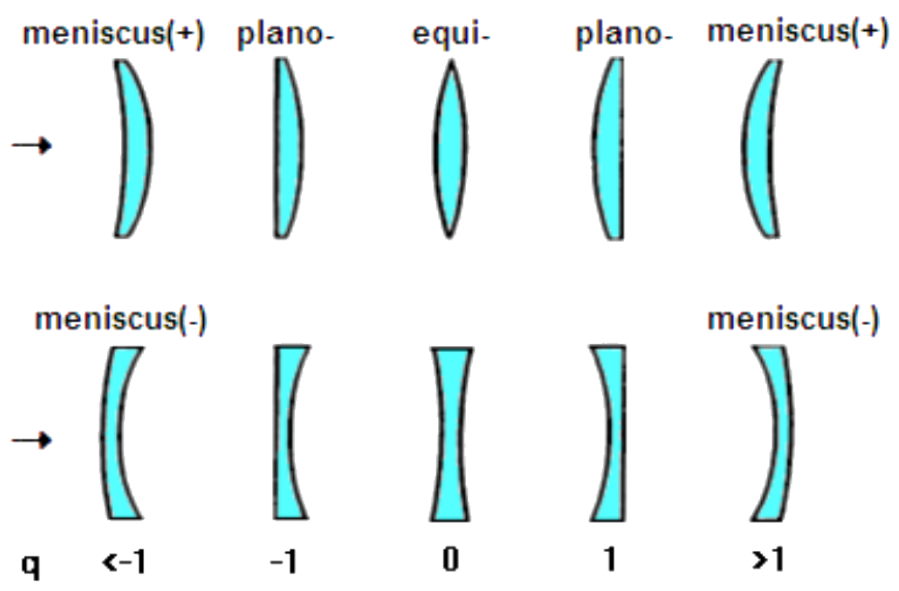

Figure 2.17: Thin lens shape factor " $q$ ", with $r_{1}$ and $r_{2}$ being the front and rear lens radius of curvature, respectively. It changes with the lens form and orientation (according to the sign convention, the surface radius of curvature $r_{i}$ is negative for the center of curvature to the left from the surface $i$ ). Source: adapted from Sacek (2006).

The lens position (or conjugate) factor, $p$ in equation 2.77, describes the magnification at which the lens is used, defined by the focal length and lens-to-image separation, or the 
input and output marginal ray angles.

$$
p=1-\frac{2 f}{i}=\frac{u+u^{\prime}}{u-u^{\prime}}
$$

where $f$ is the focal length, $i$ is the lens-to-image separation, $u$ is the object margin ray angle with the optical axis and $u^{\prime}$ is the image margin ray angle (Bentley and Olson, 2012; Sacek, 2006) $)^{4}$ Figure 2.18 shows some examples of the lens shape as a function of $p$.

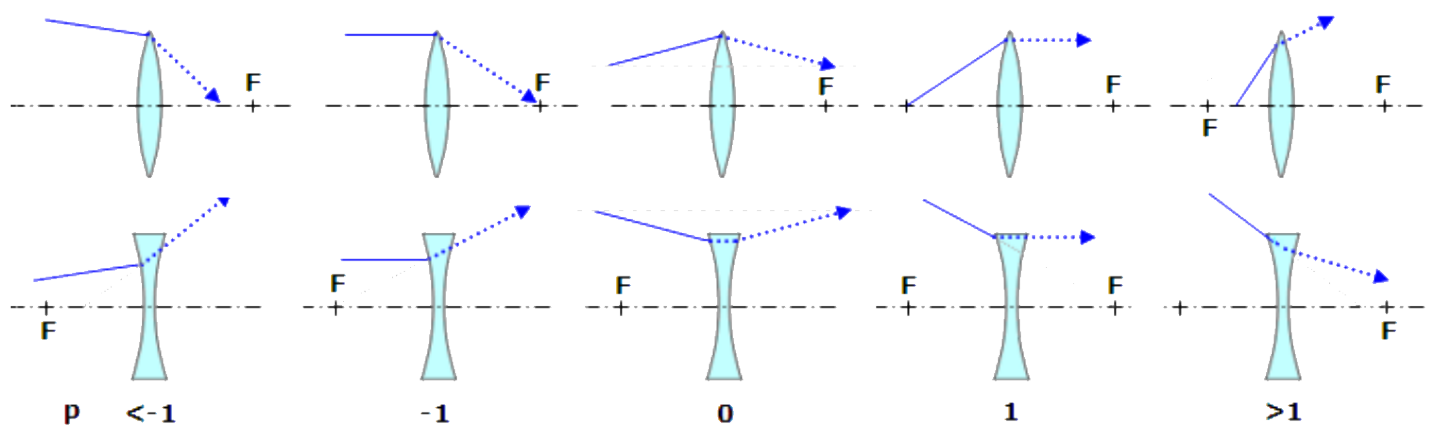

Figure 2.18: Position factor " $p$ " of a thin lens changes with the lens type (positive on the top and negative on the bottom) and the properties of light cone incident to it, with respect to the lens' effective focal length and the resulting image separation $s^{\prime}$, as given by $p=1-2 / s^{\prime}$. Source: adapted from Sacek (2006).

Figure 2.19 illustrates the relationship between both spherical and coma aberration with lens shape and position factors for a lens with refractive index $n=1.5$.

Minimum spherical aberration for any given lens shape $q$ varies with the position factor $p$ and follows a parabolic curve, with the point of minimum aberration shifting along a much milder curve as a function of lens position factor. Variation in the index value does not affect the form of the graph, which remains parabolic for spherical aberration, only causing relatively small changes in its vertical position (Sacek, 2006). The negative lens has a negative signal for the spherical aberration.

For thin lens, coma changes linearly with $\mathrm{q}$ and, unlike spherical aberration, can be zero for an actual object and image. Moreover, unlike spherical aberration, the coma plot has similar orientation for both, positive and negative lens, and it is identical for given $n$, $p$ and $q$.

\footnotetext{
${ }^{4}$ Vladmir Sacek, an optical theorist, is the author of the excellent online optics resource "Amateur Telescope Optics". Available at https://www.telescope-optics.net/. Accessed on 2020-01-27.
} 
Concerning the aperture stop position, it does affect coma only with field lens not corrected for spherical aberration. With an aplanatic (corrected for spherical aberration and coma for infinity) lens system, stop position affects neither coma nor astigmatism and field curvature.

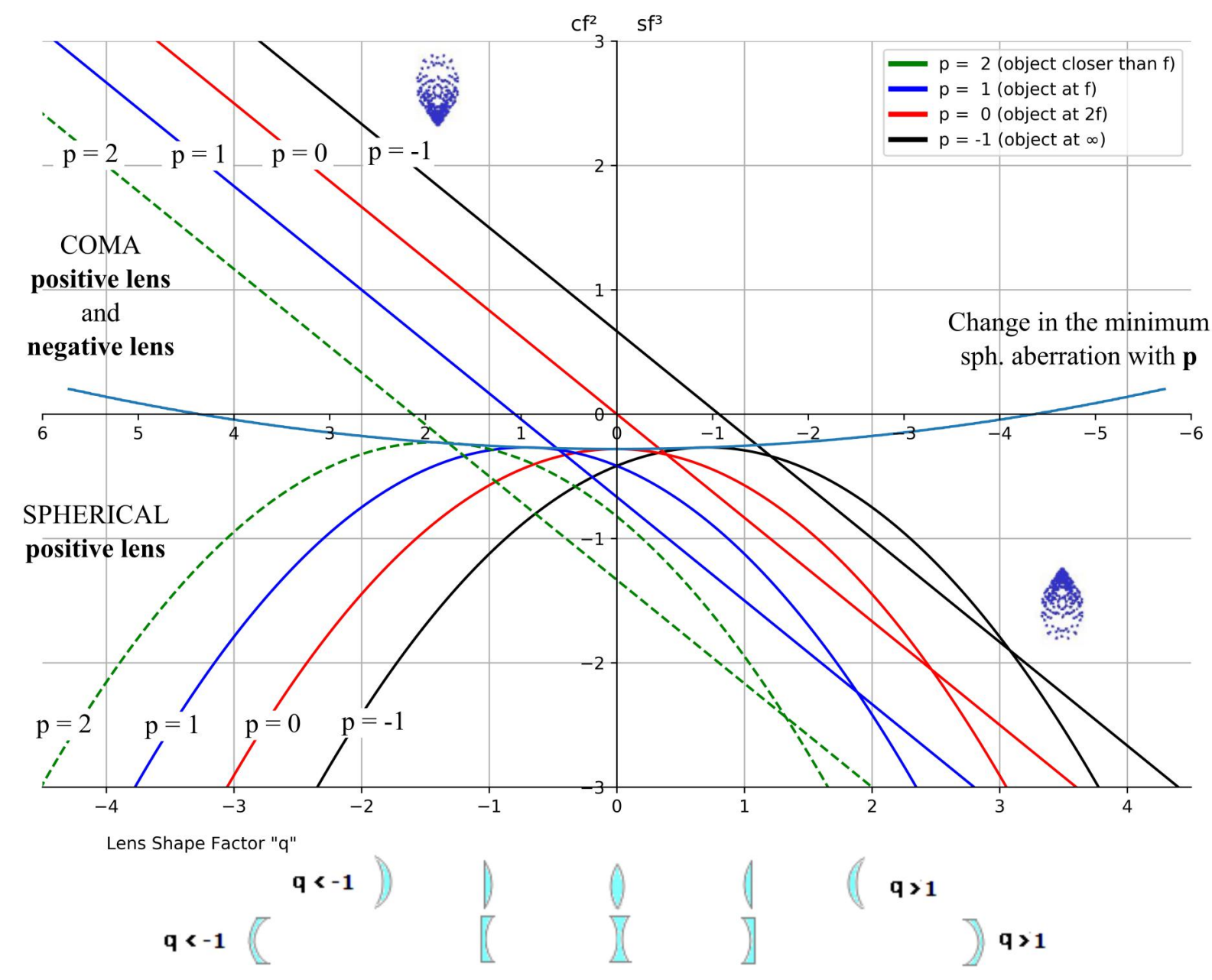

Figure 2.19: Primary spherical aberration and coma of a thin lens as a function of the lens shape factor $q$ and position factor $p$. Source: adapted from Sacek (2006).

The following two interpretations, regarding where the aperture stop of the field lens is, lead to distinct shape factors.

Considering that the field lens generates a conjugated system between the telescope entrance pupil and the collimator exit pupil, we can assume the distance between the entrance pupil and field lens as infinite comparing it with the distance between the field lens and the exit pupil. Therefore, the aperture stop is the entrance pupil, and the rays hit the field lens almost parallel, so the field lens position factor is $p=-1$. This shape 
factor is following the telescope field curvature.

However, an alternative interpretation is based on the assumption that the telescope focus plane is the "image plane". It means that the exit pupil is an entrance aperture of a finite-infinite system, and the focal plane of the telescope is the focal plane ("the detector plane", according to the definition of Riedl (2001)). The image is located very close to the field lens at a much smaller distance than the focal length, which leads to $p>1$.

As expected, the spherochromatism aberration is the most significant for such a large field lens required for an ELT collimator. The spherical aberration profile in figure 2.19 is thickened when considering the dispersive property of the lens material. The best field lens shape is the one which decreases the total amount of spherochromatism and depends on the aberration corrections of the foreoptics collimator.

\subsubsection{Dichroic}

A dichroic is an optical component that can split a beam of light into two beams with differing wavelengths bandwidth, which include mirrors and filters treated with optical coating designed to transmit light over a certain range of wavelengths and reflect light that is outside that range. Usually, large bandwidth spectrographs have more than one dichroic to increase the overall efficiency and reduce risks for the AR coating. Dichroic coatings use the principle of thin-film interference with a series of optical coatings (Fischer et al., 2008).

These devices are commonly used in large bandwidth astronomical spectrographs because splitting the light spectral range into two or more bands helps the correction of chromatic aberrations in the optical design, and increase the efficiency of the spectrograph since the diffraction efficiency gratings are more homogeneous and the requirements on the AR coating are relaxed, and allow for large wavelength range.

As the dichroic filters are usually comprised of a plane-plane window, it is a potential source of many effects, such as (i) axial displacement (defocusing); (ii) lateral displacement; and (iii) spherical, coma and astigmatism aberration (Greivenkamp, 2004; Fischer et al., 2008; Keller et al., 2015; Laikin, 2006).

Consider a plane-parallel window of thickness $t$ made of glass with index of refraction $n$ and tilted by an angle $\theta$ from the optical axis. A perfect window surface placed in a noncollimated beam, i.e., a converging beam, introduces geometrical and chromatic aberrations 
into the system, the last due to intrinsic wavelength dependence on the window's material index of refraction. The absolute power of those aberrations are a function of the material's index of refraction, the rays incidence angle and the window tilt with respect to the optical axis (Geary, 2002).

The major effects for on-axis are defocus (axial displacement) and spherical aberration. They also appear in the general off-axis beam incidence. The axial displacement, $\Delta z$, the lateral spherical aberration, $\Delta l_{\text {spha }}$, and the lateral astigmatism, $\Delta l_{\text {asti }}$, are expressed, according to Keller et al. (2015), by

$$
\begin{aligned}
\Delta z & \approx \frac{t(n-1)}{n} \\
\Delta l_{s p h a} & =\frac{1}{8 F^{2}} \cdot \frac{t\left(n^{2}-1\right)}{n^{3}} \\
\Delta l_{\text {asti }} & =\theta^{2} \cdot \frac{t\left(n^{2}-1\right)}{n^{3}}
\end{aligned}
$$

Once the index of refraction is wavelength dependent, $n \rightarrow n(\lambda)$ might be used in all the equations above.

For a window tilted by $\theta=30^{\circ}$ with an $\mathrm{f} / 8$ optical system, the astigmatism is about 250 times larger than the spherical aberration. Keller et al. (2015) show that temperature non-uniformity and stress birefringence in a window will introduce complex aberrations.

The more generic approach for determining the window aberration is achieved from the Seidel aberration formula, illustrated in figure 2.20.
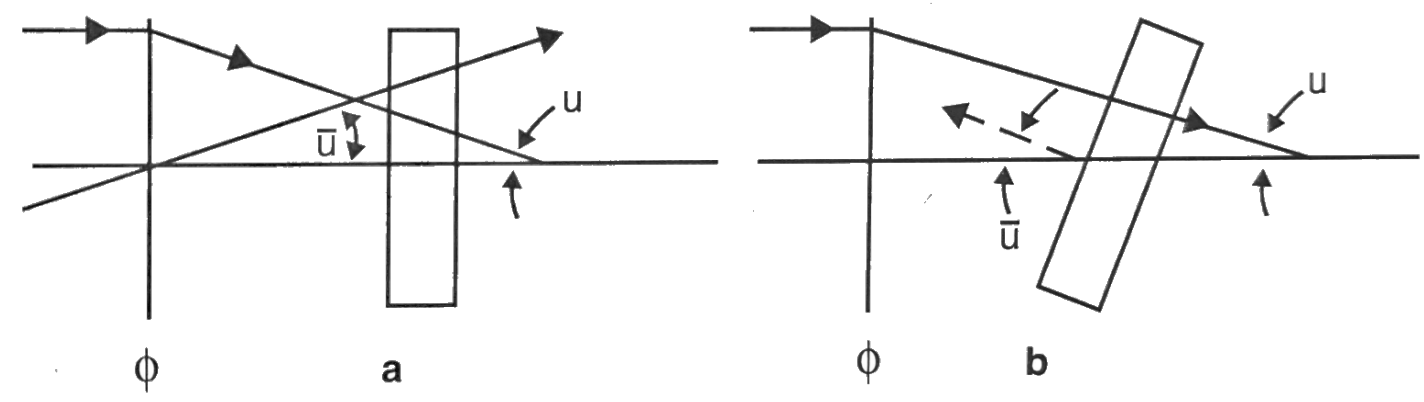

Figure 2.20: Window aberration as a function of the tilt to the optical axis: (a) normal to the optical axis; (b) tilted with respect to the optical axis. Source: Geary (2002).

The Seidel formulae for the window aberration are shown in table 2.4. These equations 
are proportional to the wavefront aberration coefficients.

Table 2.4 - Seidel Coefficients for a plano-plano window

\begin{tabular}{|cc|}
\hline Aberration & Seidel Coefficient \\
\hline Spherical & $S_{I W}=N u^{4}$ \\
Coma & $S_{I I W}=N u^{3} \bar{u}$ \\
Astigmatism & $S_{I I I W}=N u^{2} \bar{u}^{2}$ \\
Petzval Curvature & $S_{I V W}=0$ \\
Distortion & $S_{V W}=N u \bar{u}^{3}$ \\
\hline
\end{tabular}

where $\bar{u}$ and $u$ are the angle normal to the window and the marginal ray angle with respect to the optical axis, respectively. $N$ is defined as

$$
N=\left(\frac{1-n^{2}}{n^{3}}\right) t \Rightarrow \delta N=\left(\frac{n^{2}-3}{n^{4}}\right) t \delta n-\left(\frac{n^{2}-1}{n^{3}}\right) \delta t
$$

A window tilt on the optical path causes field aberrations, such as coma and astigmatism, which affect the quality of a point image, and distortion. The spherical aberrations do not depend on the window tilt, but they are highly dependent on the marginal ray angle. Astigmatism is highly dependent on both the marginal ray angle and the angle of the window tilt with respect to the optical axis. In fact, astigmatism and coma are the most severe aberrations introduced by a tilted window on a converging beam.

According to table 2.4, all the aberration coefficients are proportional to the window thickness and window angle with respect to the system optical axis.

As we will discuss in section 5.7.3.1, the coma and astigmatism caused by a tilted window in a converging beam can be reduced and eliminated by using a wedge plate instead of plano-plano window. According to Laikin $(2006)^{5}$, for zero astigmatism and substantially reduced coma based on the third-order theory, the wedge angle $\theta_{\text {wedge }}$ shall be, in radians,

\footnotetext{
${ }^{5}$ Laikin (2006) cites "de Lang, Philips Res. Rep. 12, 181-189, 1957 (No. 3)", but we could not find the original source.
} 


$$
\theta_{\text {wedge }}=\frac{\sin \beta\left(\cos ^{2} \beta\right) T}{2\left(n^{2}-\sin ^{2} \beta\right) P}
$$

where $t$ is the plate thickness with index of refraction $n$, located at a distance of $P$ to the image with a wedge angle of $\theta_{\text {wedge }}$ in a converging beam, at an angle $\beta$ to a normal of the optical axis, see figure 2.21.

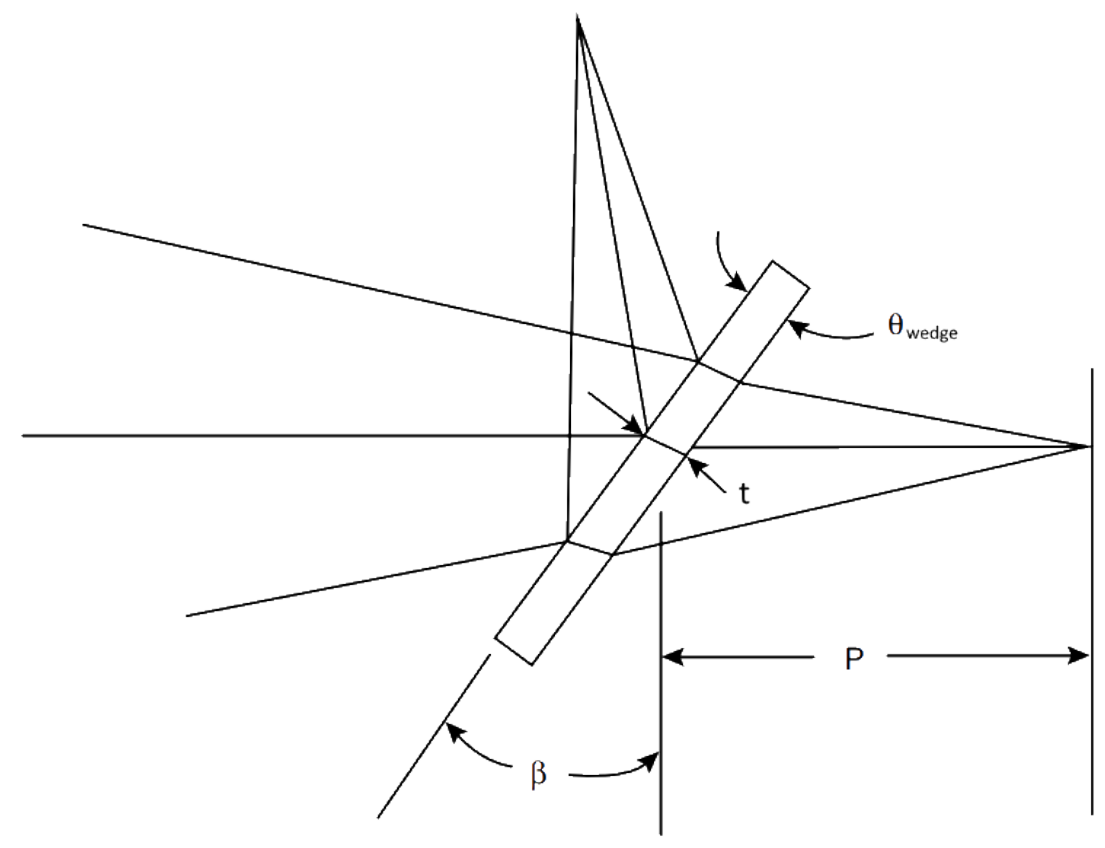

Figure 2.21: Laikin's model of a plate of thickness $t$ with a wedge angle of $\theta$ in a converging beam is at a distance $P$ from the image. The plate is at an angle $\beta$ to a normal to the optical axis. Source: adapted from Laikin (2006).

A $45^{\circ}$ tilted window of thickness $t=30 \mathrm{~mm}$ at $2,200 \mathrm{~mm}$ from the image plane and $n=1.51$ should have a wedge angle of $4.65^{\prime}$ to minimize coma and astigmtism.

The lateral displacement of the beam principal ray is given by

$$
\delta=t \sin \beta \cdot\left[1-\frac{\cos ^{2} \beta}{\sqrt{n^{2}-\sin ^{2} \beta}}\right] \approx t \beta \cdot \frac{(n-1)}{n}
$$

where $\beta$ is the window tilt. The first order approximation is valid for small angles, $\sin \beta \approx \beta$ and $\cos \beta \approx 1$, in radians. 


\subsubsection{Exit pupil quality}

One of the most important optical parameters for the collimator performance evaluation criteria is the quality of the exit pupil. The collimator's function is to generate a real image of the telescope's entry pupil at the position determined by the telescope and collimator EFLs. The entrance and exit pupil planes are combined. Since the pupil and image aberrations are connected (Fischer et al., 2008), it is reasonable to select one of these criteria for the collimator merit function.

For the collimator design, the pupil image quality was controlled by setting all the chief and margin rays of the sampled fields and wavelengths to cross the exit pupil plane on their respective height. The circle of confusion for the chief rays was less than $10 \mathrm{~mm}$, centered on the pupil plane. The collimator performance was quantified using a paraxial $594 \mathrm{~mm}$ focal length camera. A good pupil image is important to control the stray light of the instrument; a single aperture placed on the pupil plane is enough to decrease it.

Considering the collimator EFL, $f_{\text {col }}$, the image height for an incident collimated beam angle $\theta$ is

$$
\tan \theta=\frac{h}{2 f_{\text {col }}}
$$

The differential of equation 2.82 in terms of $\theta$ and $h$ is

$$
\delta h=2 f_{\operatorname{col}}\left(\sec ^{2} \theta\right) \delta \theta
$$

One way to determine the quality of the wavefront in the exit pupil is to consider the difference between the sag of two wavefronts, which cause a variation of a given pixel value in the focal plane of the spectrograph,

$$
\operatorname{sag}=\frac{D_{c o l}}{2} \sin \left(\Gamma_{\text {spec }} N \frac{f_{t e l}}{f_{c o l}}\right)=\frac{D_{c o l}}{2} \sin \left(\frac{N p_{p t}}{r_{a n} f_{c a m}}\right)
$$

where $N$ is the distance in number of pixels of the images and $\Gamma_{\text {spec }}$ is the Spectrograph Plate Scale. For a numerical example, considering $N=0.5, p_{p t}=15 \mu \mathrm{m}, r_{a n}=1$ and $f_{\text {cam }}=594 \mathrm{~mm}$, the wavefront error should be $\approx 1.7 \mu \mathrm{m}$, or $2.7 \lambda$ for $\lambda=632.8 \mathrm{~nm}$. 


\subsubsection{Cameras}

\subsubsection{Field Flattener}

The Field Flattener, as its denomination implies, is an optical element used to correct the residual curvature of the field in the image plane since most sensors are flat. The Petzval sum, which is more significant for fields away from the optical axis, must be small for flat field applications (Geary, 2002). One way to correct the Petzval curvature is to insert a negative component close to the focal plane to counteract the field-angle dependence on the focal length of a system, which causes shifts of the focal points of the Petzval surface to lie on the same plane. The required thickness, $t$, of a flat window that causes an axial focal shift, $\delta_{s}$, is given by

$$
t=\left(\frac{n}{n-1}\right) \delta_{x}
$$

It can be shown from a sag based approach, according to Geary (2002), that the radius of curvature for the lens, $R_{f}$, which would flatten out the field, described by the radius of curvature of the Petzval surface, $R_{p}$, is given by

$$
R_{f}=\left(\frac{n-1}{n}\right) R_{p}
$$

Therefore, only the field flattener glass thickness plays a role in flattening the Petzval image field. 
Chapter 3

\section{Review of MOS Optical Designs for Large Telescope}

\section{Instrumentation}

This chapter presents a brief optical design critical review of a selected list of astronomical multi-object spectrographs that have comparable GMACS science and instrument requirements, which are among the highest-ranked instruments. The main objective is to extract the general and particular optical design solutions that can be adapted for the GMACS optical design.

We decided to limit this review to GMACS-like instruments solely to avoid an excessively long section, see table 3.1. Nevertheless, we cite a few of the significant instruments explored in this research, such as MODS (VLT); EFOSC2 and EMMI (NTT); ISIS (WHT), GOODMAN, and survey telescopes, for instance, SDSS.

The instrument data come from published papers, proceedings, web pages of the instruments, datasheets, slides presented in congresses, events, and documents developed by team members and researchers during the instrument's design, commissioning and operation phases. The lessons learned from them are an essential piece of information to avoid design errors and reduce the project risks.

As demonstrated in chapter 2, since the optical design methodology cannot be considered disconnected from the other areas of the instrument, the instrument's final layout is the result of a complex combination of optical, mechanical, structural, thermal, and operational requirements and constraints. Therefore, a one-by-one comparison among the instrument capabilities is not adequate or appropriate, especially their differing specific scientific purposes. However, even admitting the peculiarities of the instruments' purposes and design choices, it is possible to identify, through a careful analysis of instrument 
design, the distinct solutions adopted for problems that are usually encountered in the development of astronomical MOS. In this chapter, we assume that the designs' optical performance meets their particular requirements and, therefore, the imaging and spectrograph performances are not presented, although they are available in the references. As part of the literature review methodology proposed in this research, we are in constant construction of an optical design Zemax library of telescopes and instruments.

We divided this chapter into two parts: the first describes the earlier GMACS version and the second lists the instruments, organized by the telescope where they are or were installed.

Table 3.1 - Principal parameters of the selected instruments

\begin{tabular}{|c|c|c|c|c|c|}
\hline Instrument & $\begin{array}{l}\text { Telescope } \\
\left(D_{t e l} \text { in } \mathrm{m}\right)\end{array}$ & Focus & MOS FoV & $\begin{array}{c}D_{\text {col }} \\
{[\mathrm{mm}]}\end{array}$ & $\begin{array}{c}\text { Imaging } \\
F_{\text {cam }}\end{array}$ \\
\hline GMOS & Gemini (8.1) & Cassegrain & $5.5^{\prime} \times 5.5^{\prime}$ & 98 & $\mathrm{f} / 4.6$ \\
\hline DEIMOS & Keck II (10) & Nasmyth & $16.5^{\prime} \times 16.5^{\prime}$ & 150 & $\mathrm{f} / 2.75$ \\
\hline \multirow{2}{*}{ IMACS } & \multirow{2}{*}{ Magellan (6.5) } & \multirow{2}{*}{ Cassegrain } & $27.2^{\prime} \times 27.2^{\prime}$ & 150 & $\mathrm{f} / 2.3$ \\
\hline & & & $15.5^{\prime} \times 15.5^{\prime}$ & 150 & $\mathrm{f} / 4.3$ \\
\hline OSIRIS & GTC (10.4) & Nasmyth & $7.8 \times 8.5^{\prime}$ (vign) & 75 & $\mathrm{f} / 2.3$ \\
\hline BINOSPEC & $\operatorname{MMT}(6.5)$ & Cassegrain & $2 \times\left(8^{\prime} \times 15^{\prime}\right)$ & 98 & $\mathrm{f} / 1.8$ \\
\hline VIMOS & \multirow{3}{*}{ VLT (8.4) } & Nasmyth & $4 \times\left(7^{\prime} \times 8^{\prime}\right)$ & 138 & $\mathrm{f} / 1.8$ \\
\hline \multirow{2}{*}{ FORS } & & \multirow{2}{*}{ Cassegrain } & $4.25^{\prime} \times 4.25^{\prime}$ & 90 & $\mathrm{f} / 3.1$ \\
\hline & & & $6.8^{\prime} \times 6.8^{\prime}$ & 45 & $\mathrm{f} / 3.1$ \\
\hline FOCAS & Subaru (8.2) & Cassegrain & $7^{\prime} \times 7^{\prime}$ & 90 & $\mathrm{f} / 3.6$ \\
\hline
\end{tabular}

\subsection{GMACS 2011 CoDR}

The GMACS presented in the 2011 CoDR is an ultra-wide field MOS with four arms and two channels each, as shown in figure 3.1 (DePoy et al., 2011, 2012, 2014; GMTO Coorporation, 2013d). The concept requires the GMT wide-field corrector, followed by the slit mask and a low expansion tent mirror that splits the $9^{\prime} \times 18^{\prime}$ incident field into four separate segments to individual arms, each comprising a double-beam spectrograph. Each $4.5^{\prime} \times 9^{\prime}$ off-axis field arm consists of a refractive collimator, followed by a dichroic 
to split the beam into red and blue channels. This GMACS design provides simultaneous wavelength coverage between $\approx 360-1,020 \mathrm{~nm}$, limited in the bluer side by the $\mathrm{BAK}_{2}$ glass in the collimator design.

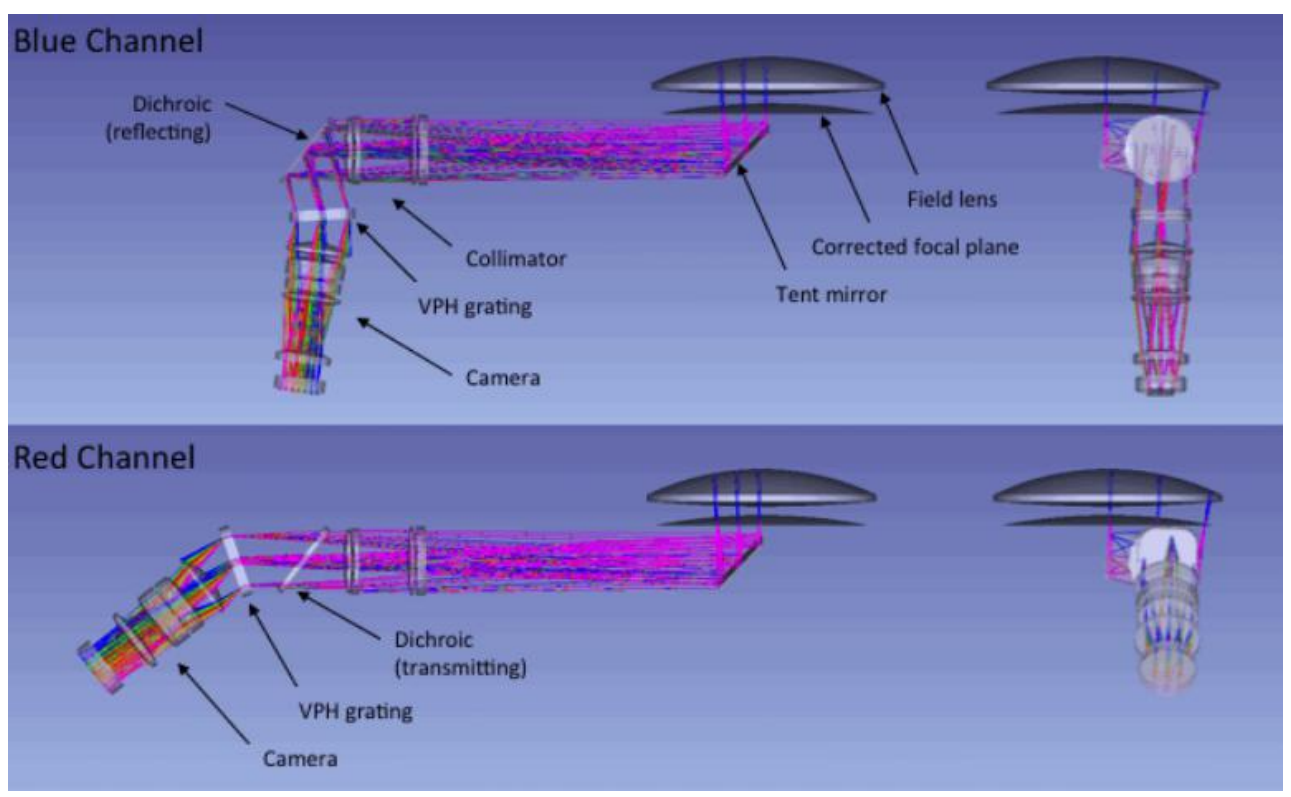

Figure 3.1: Layout of each individual channel of GMACS shown from two orthogonal orientations. The large initial lens is the final element of the GMT wide field corrector, which is $\approx 1.5 \mathrm{~m}$ in diameter. Source: DePoy et al. (2012).

The 2012 GMACS broadband collimator is based on IMACS (see section 3.2.3.1), as shown in figure 3.2. It is comprised of two Fused Silica (FS) lenses followed by a fluid couplet $\mathrm{BAK}_{2}$ and $\mathrm{CaF}_{2}$ doublet. The Schott $\mathrm{BAK}_{2}$ limits the deep UV-Blue transmittance since the $320 \mathrm{~nm}$ internal transmittance of this glass is about $70 \%$ for a $25 \mathrm{~mm}$ sample thickness (Schott, 2014).

The optomechanical articulated camera's layout are illustrated in figure 3.3, which details the $675 \mathrm{~mm} \mathrm{f} / 2.25$ refractive cameras' designs. The blue camera has seven elements, including three aspheric surfaces. The materials are $\mathrm{CaF}_{2}, \mathrm{FS}, \mathrm{BSM} 51 \mathrm{Y}$, and BSL7Y. The blue camera works well to at least $350 \mathrm{~nm}$. The red camera also has seven elements, again with three aspheric surfaces. We could not find in the references which surfaces are aspheric. The materials are $\mathrm{CaF}_{2}$, FS, PBL6Y, PBL35Y, and BSM51Y (DePoy et al., 2012). Vignetting in the camera first lens is visible in both of the resolution modes, as can be seen in figure 3.1. The highly aberrated rays are clipped to get better image performance, an usual optical design tradeoff. 


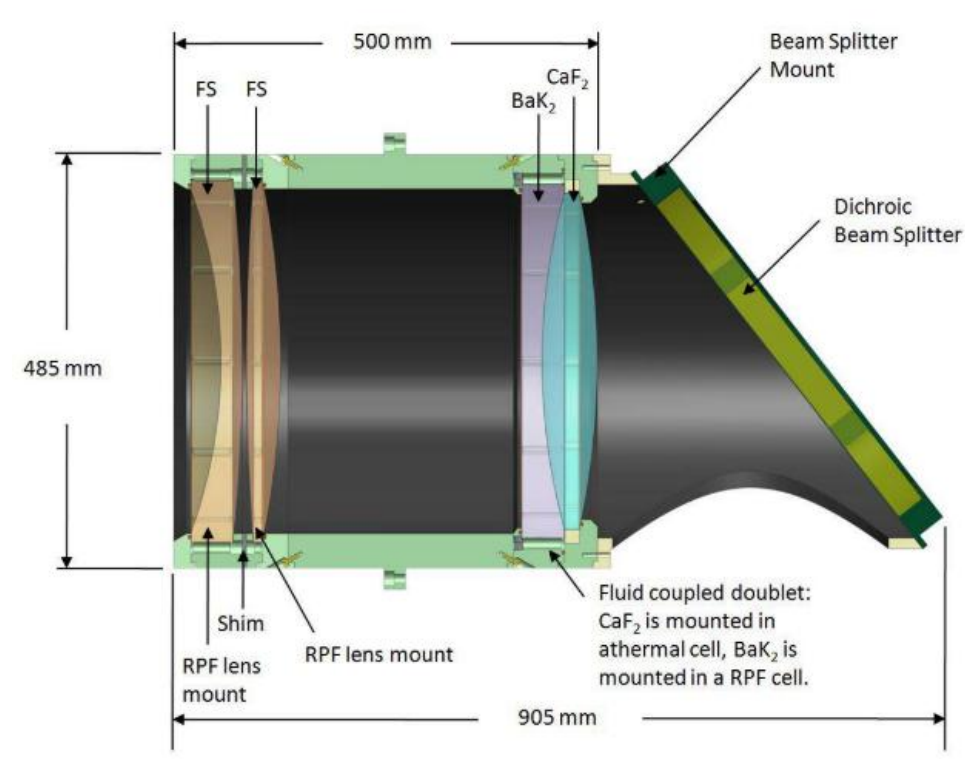

Figure 3.2: 2012 GMACS collimator cell and structure. Source: DePoy et al. (2012).

\subsection{GMACS-like Multi-object spectrographs}

\subsubsection{Gemini Observatory}

\subsubsection{GMOS}

The two Gemini Multi-Object Spectrographs, GMOS-N and GMOS-S, installed at the Cassegrain focus of the Gemini North and South Observatory, respectively, are long-slit, and multi-slit spectrographs over a $5.5^{\prime}$ squared FoV, with wavelength coverage from 360 $\mathrm{nm}$ to $1,030 \mathrm{~nm}$. Each spectrograph is equipped with a Integral Field Unit, IFU. The science field of view of the IFU is $35^{\prime \prime}$ squared, with a sampling of $0.2^{\prime \prime}$ and spectral resolving power raging from 500 up to 8,000 for the widest slits used on extended objects and 0.25" slit width, respectively (Davies et al., 1997; Crampton et al., 2000; Hook et al., 2004; Gemini Observatory, 2020).

GMOS was planned to have an ADC + field corrector Lens, which would partially flatten the field to improve the image quality in both imaging and spectrograph modes, mainly for high precision velocities mode (Szeto et al., 2003); however, it has not been implanted. The field corrector allows the collimator to be refractive instead of reflexive, as expected for a Ritchey-Chrétien telescope type. Similar to the GMACS current requirements, the high UV-blue throughput was a significant driver for the design. The optical layout, including the ADC + Field Corrector, is shown in figure 3.4. Oil was used instead 


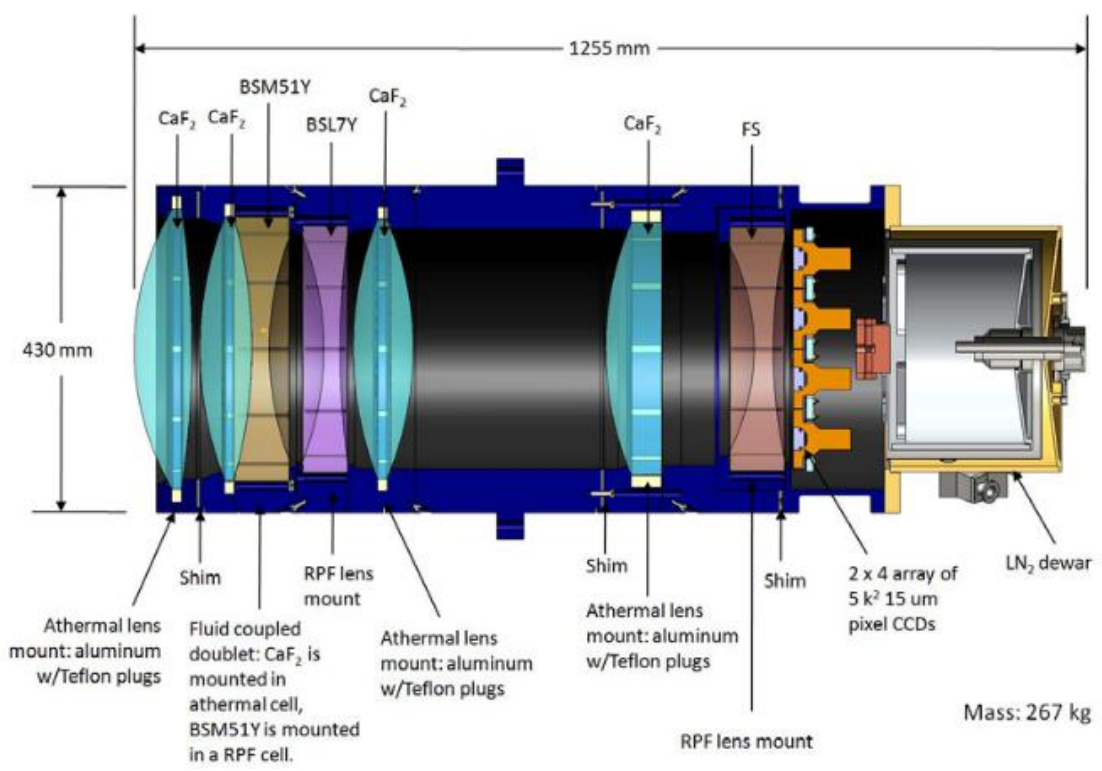

(a) Blue camera

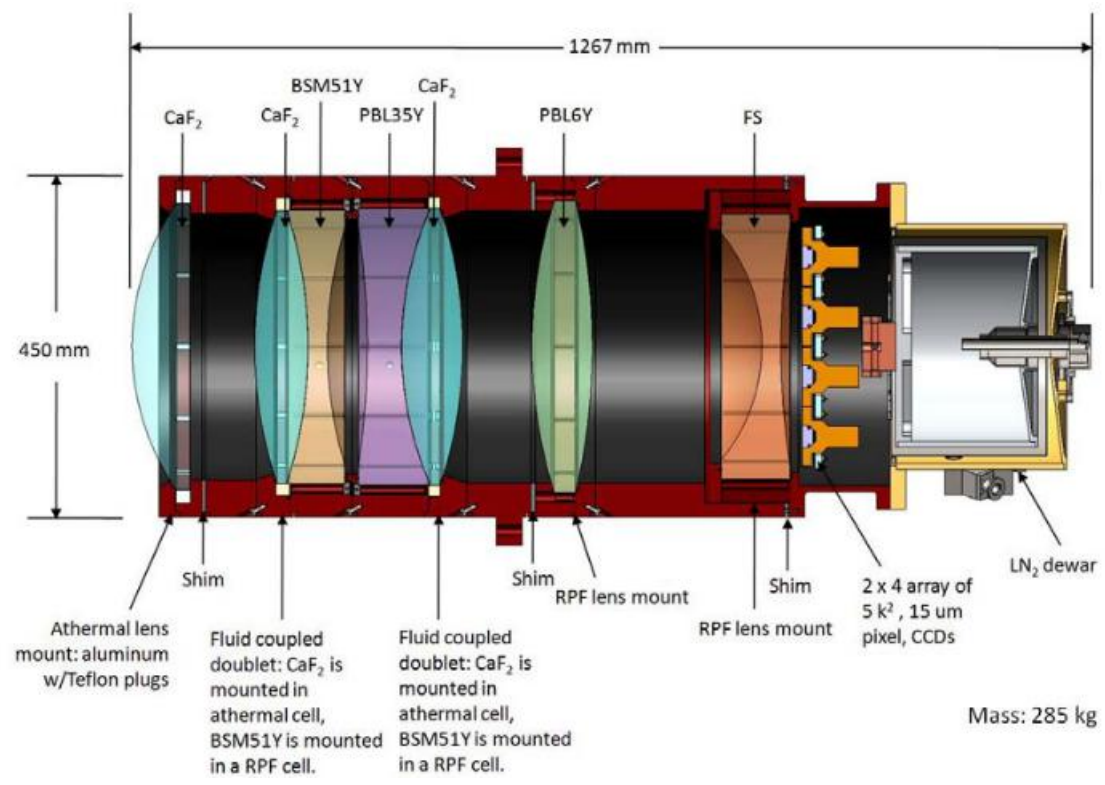

(b) Red camera

Figure 3.3: 2012 GMACS blue (top) and red (bottom) cameras' layouts with the structure and design details. Source: DePoy et al. (2012).

of cementing optics due to the large CTE difference between the lens materials. The lenses are mounted in rings that have similar CTE to the glass itself (Crampton et al., 2000).

The refractive collimator, shown in detail in figure 3.5, is a telephoto lens comprised of a strong positive triplet, followed by a negative triplet and a four-lens element. Since the system is intrinsically unsymmetrical concerning the stop position, each component must be individually achromatized for lateral and axial lateral color correction. The filter 


\section{GMOS Optical Train}

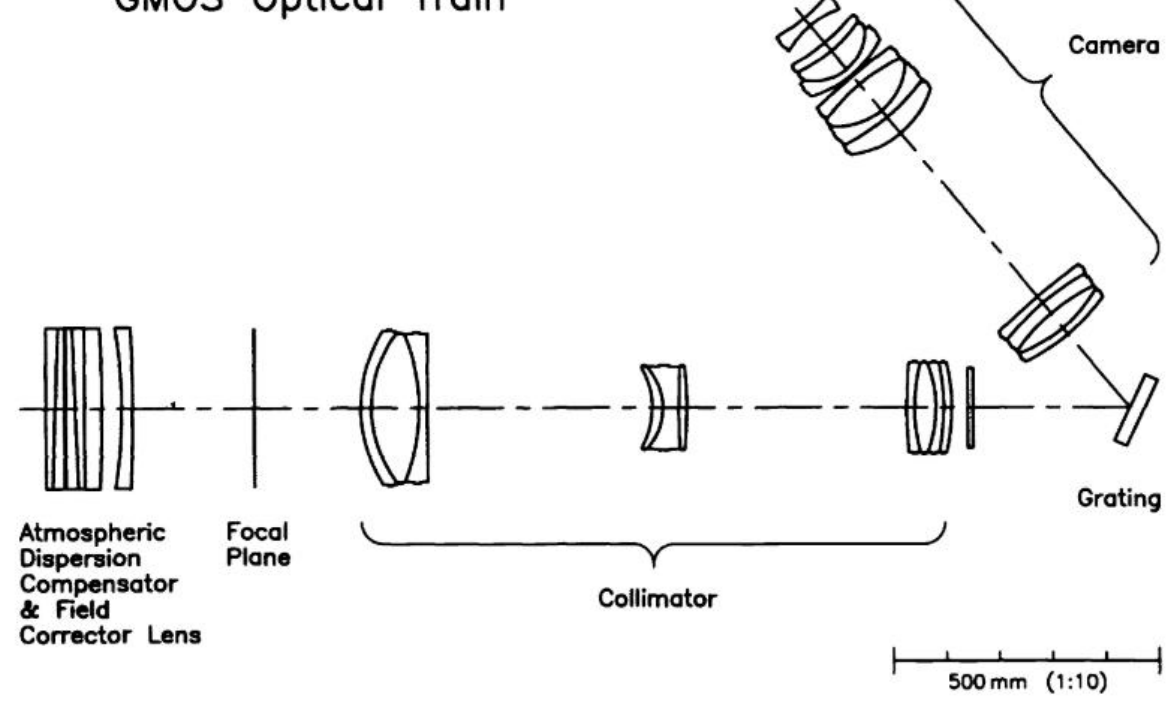

Figure 3.4: GMOS optical layout showing the ADC with a field corrector lens, a three element collimator and a three element camera. Source: Davies et al. (1997).

is located at a collimated beam region before the collimator exit pupil. The pupil size is $98 \mathrm{~mm}$ in diameter.

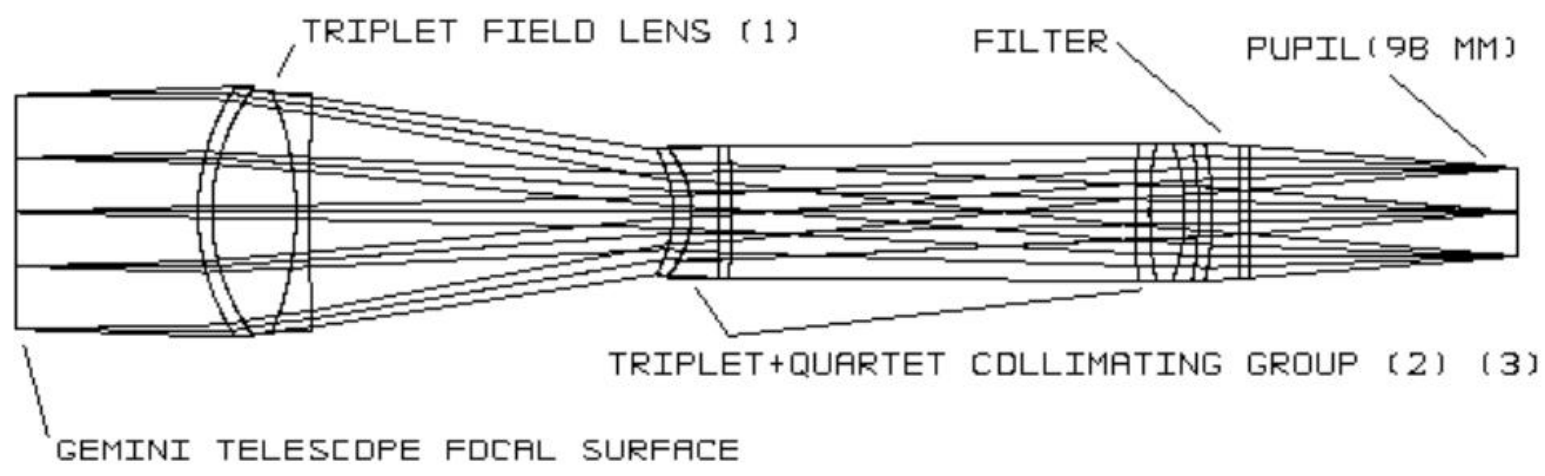

(1) BSLTY/CAFL/BRL $15 Y$

(Z) 5 -日SL7/CAFL/PBL $1 Y$

(3) BAL $15 Y / C A F L / B A L F 5 / P B L I Y$

Figure 3.5: GMOS collimator design. Source: Murowinski et al. (2003).

The refractive camera, shown in figure 3.6, has a first four-lens positive element followed by a four-lens element, a triplet, and the field flattener. The $465 \mathrm{~mm} \mathrm{f} / 4.6$ camera entrance pupil is $98 \mathrm{~mm}$ in diameter. The large number of different materials employed in the lenses is an indication of the efforts to control color aberrations such as axial colour and the spherochromatism. The choice for compound lens instead of singlets is to achieve 
achromatic groups, as expected in Petzval lenses and for alignment purposes.

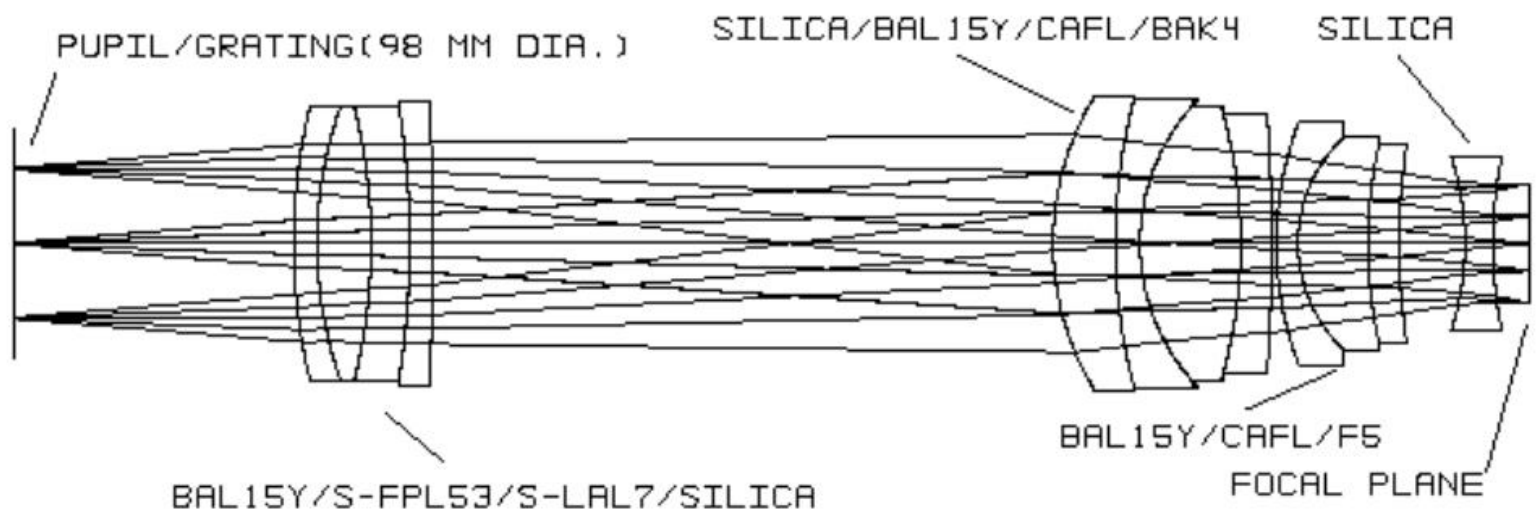

Figure 3.6: GMOS camera design. Source: Murowinski et al. (2003).

The optics manufacturing is based on the as-built approach, in which the camera design is reoptimized based on the as-built collimator (Murowinski et al., 1998).

Excluding the ADC + Field Corrector, filter, and grating, GMOS has almost thirty surfaces from the Gemini focal plane to the GMOS focal plane, which is a crucial drawback for achieving high throughput. Considering an AR-coating with 99\% transmittance in average, the loss in throughput is $0.99^{30} \approx 0.74$, although Murowinski et al. (2003) have reported an average throughput up to $80 \%$.

\subsubsection{Keck II}

\subsubsection{DEIMOS}

The Deep Imaging Multi-Object Spectrograph (DEIMOS), at Keck II, is a faint-object, multi-slit imaging spectrograph with slit length spanning $16.6^{\prime}$ on sky (Cowley et al., 1997; Faber et al., 2003), capable of gathering spectra from 130 galaxies or more in a single exposure (Keck Observatory, 2020). It is one of the world's most sensitive optical spectrographs, which achieves typical SNR $\approx 3$ per spectral resolution element for 1-hour exposures with $\mathrm{R} \approx 2,000$ for objects of $\mathrm{i}^{\prime}=24$ AB mag (DePoy et al., 2011).

DEIMOS has a reflective ellipsoidal collimator with 1.17 meters in diameter, followed by a tent mirror that feeds the reflective grating and the refractive camera (Cowley et al., 1997). The design allows for a twin camera by including an additional tent mirror followed by a similar grating and camera setups. Figure 3.7 shows the major optical and mechanical components. 


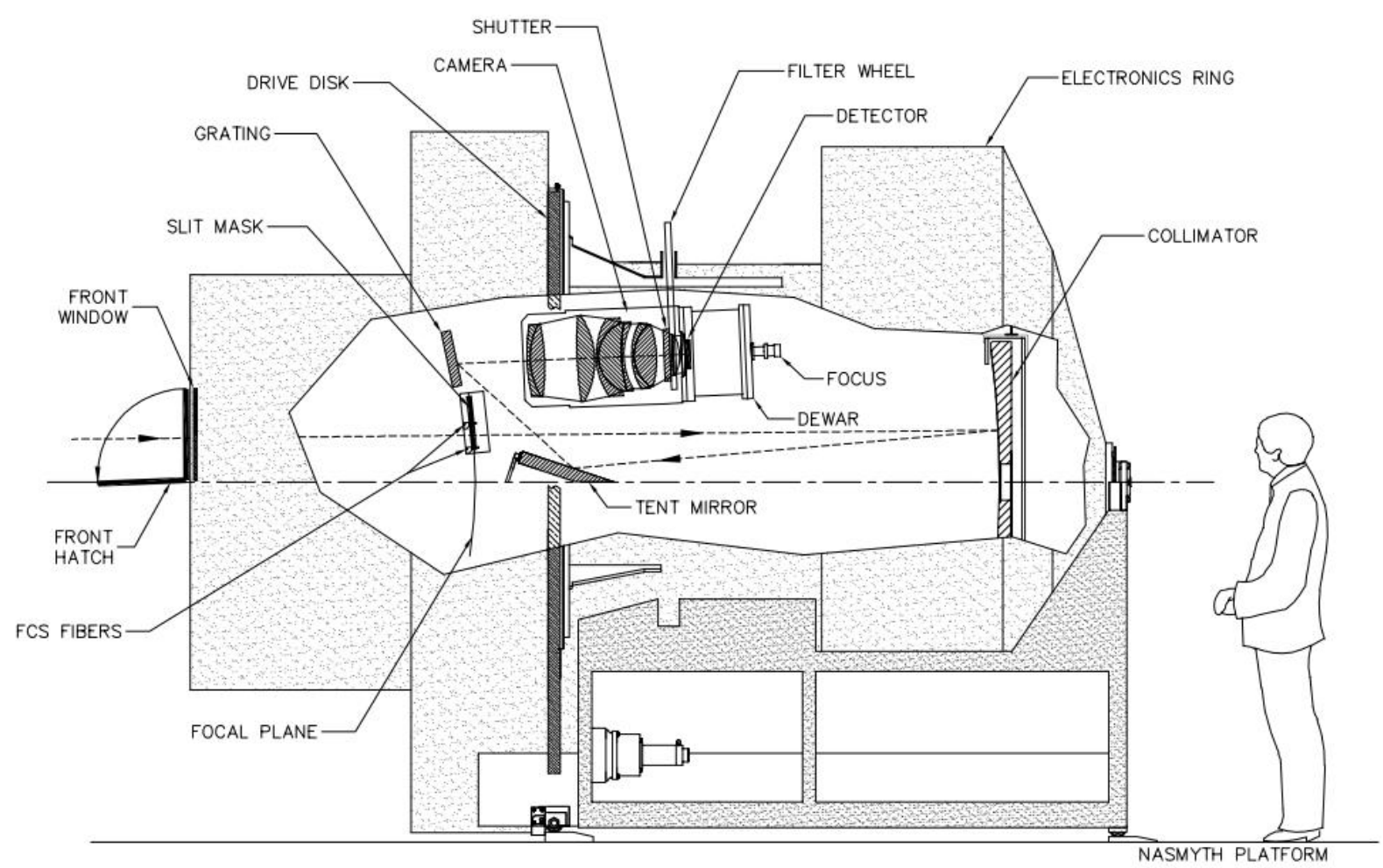

Figure 3.7: DEIMOS major optical and mechanical components. Source: Faber et al. (2003).

DEIMOS $380 \mathrm{~mm} \mathrm{f} / 2.75$ camera, shown in figure 3.8, is comprised of an LAK, $\mathrm{CaF}_{2}$ doublet, a $\mathrm{CaF}_{2}$ singlet followed by an SSK5, $\mathrm{CaF}_{2}$ and KZSF01 triplet, an LAK01, FK01 doublet, and an LAK09 as a field flattener. Three aspherical surfaces are located at the rear negative element of the first doublet (LAK convex surface) and in the rear and front surfaces of the last doublet (LAK01 and FK01 convex surfaces).

\subsubsection{Las Campanas Observatory, Magellan 1}

\subsubsection{IMACS}

The Inamori-Magellan Areal Camera and Spectrograph (IMACS), built at Carnegie Observatories, is a wide-field, multipurpose imaging spectrograph on the Magellan-Baade telescope at Las Campanas Observatory, LCO. The instrument is mounted on the right Nasmith instrument rotator, where it will operate with the f/11 Gregorian configuration + ADC. IMACS offers two cameras, but unlike the classical double spectrograph divided by spectral range, it offers different imaging scales (LCO, 2020). According to Dressler et al. (2011), IMACS is the most versatile and powerful spectrograph wide-field optical spectroscopy. It provides the following modules: (i) wide-field imaging at two scales, $0.11^{\prime \prime} /$ pixel 


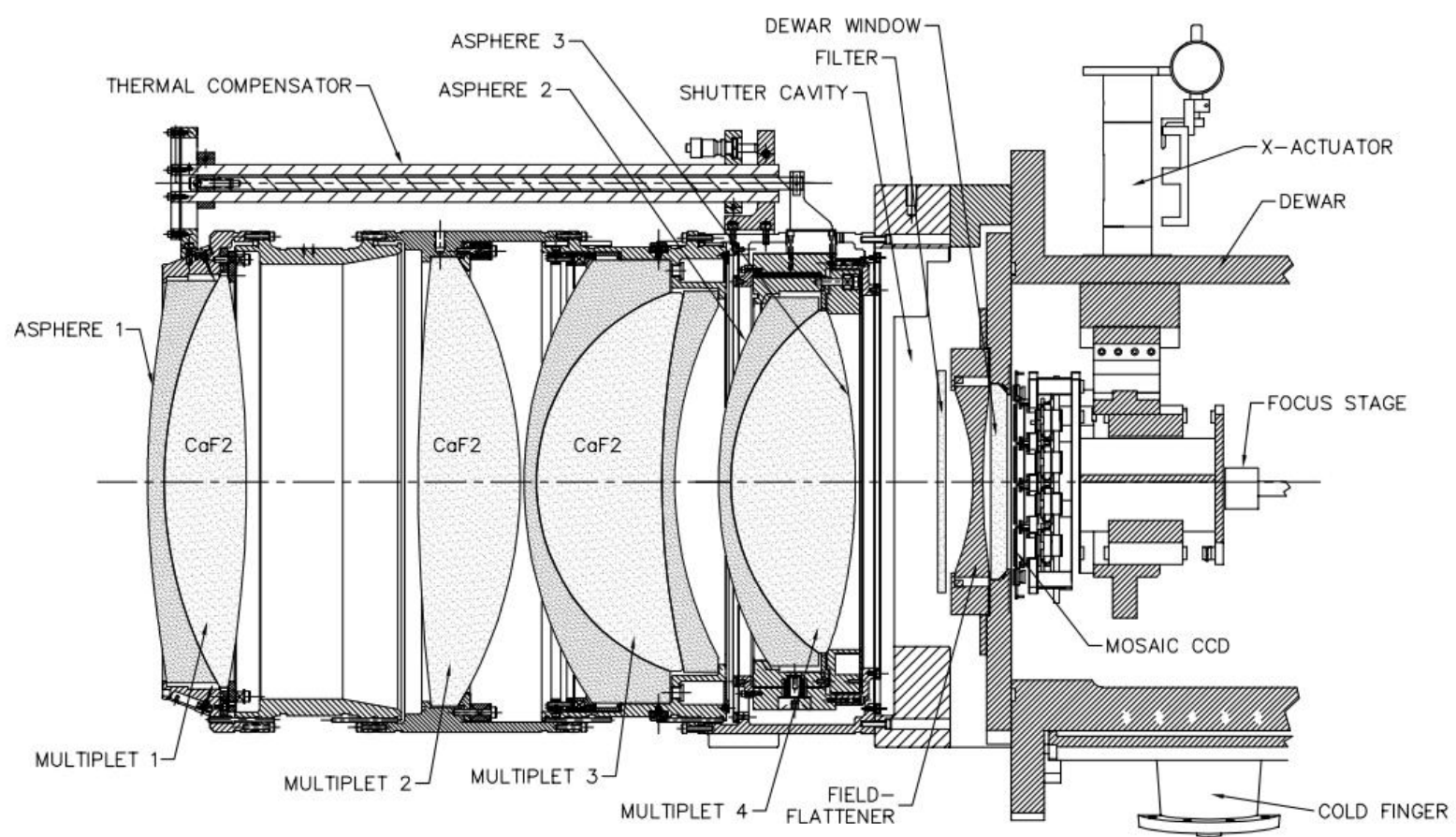

Figure 3.8: DEIMOS optics, camera cell, shutter, filter wheel, field flattener, dewar window and detector. Source: Faber et al. (2003).

and $0.20^{\prime \prime} /$ pixel; (ii) single-object and multislit spectroscopy that covers spectral resolutions $20<\mathrm{R}<20,000$ through a combination of prisms, grisms, and gratings; (iii) a $2 \mathrm{x}$ 1000 fiber-fed integral field unit (IFU) with $5^{\prime \prime} \times 7^{\prime \prime}$ areas each sampled at $0.2^{\prime \prime} /$ pixel; (iv) a Multi-Object Echelle mode (MOE) capable of $\mathrm{N} \approx 10$ simultaneous full-wavelength $\mathrm{R} \approx$ 20,000 spectra; (v) a Full-Field Tunable Filter for both spectroscopy and narrow-band imaging for the faster camera (MMTF); and (vi) an image-slicing reformator for dense multislit coverage over a $4^{\prime} \times 4^{\prime}$ field (GISMO). The covering spectral resolutions are 20 $<\mathrm{R}<20,000$ through a combination of prisms, grisms, and gratings, and most modes are instantly available in any given IMACS configuration (Dressler et al., 2006, 2011). The IMACS optical layout is shown in figure 3.9.

The IMACS optical design is all-dioptric. The collimator is made of two FS singlets and an air-spaced $\mathrm{CaF}_{2}$ /BAL35Y doublet and produces a $150 \mathrm{~mm}$ diameter exit pupil, approximately $275 \mathrm{~mm}$ beyond the last surface vertex (Epps and Sutin, 2003).

The six-lenses all-spherical "long" $641.8 \mathrm{~mm} \mathrm{f} / 4.3$ camera operates in the $365-1,000$ $\mathrm{nm}$ spectral range and provides a $15.5^{\prime} \times 15.5^{\prime}$ image $\mathrm{FoV}\left(\approx\right.$ in $21.9^{\prime}$ diameter $)$ or $15.5^{\prime}$ long slit. The eight lenses (with two aspheric surfaces) "short" $355.6 \mathrm{~mm} \mathrm{f/2.3} \mathrm{camera,}$ optimized for $390-1,050 \mathrm{~nm}$ spectral range, provides a $27.2^{\prime} \times 27.2^{\prime}$ image FoV $\left(\approx 38.8^{\prime}\right.$ in 


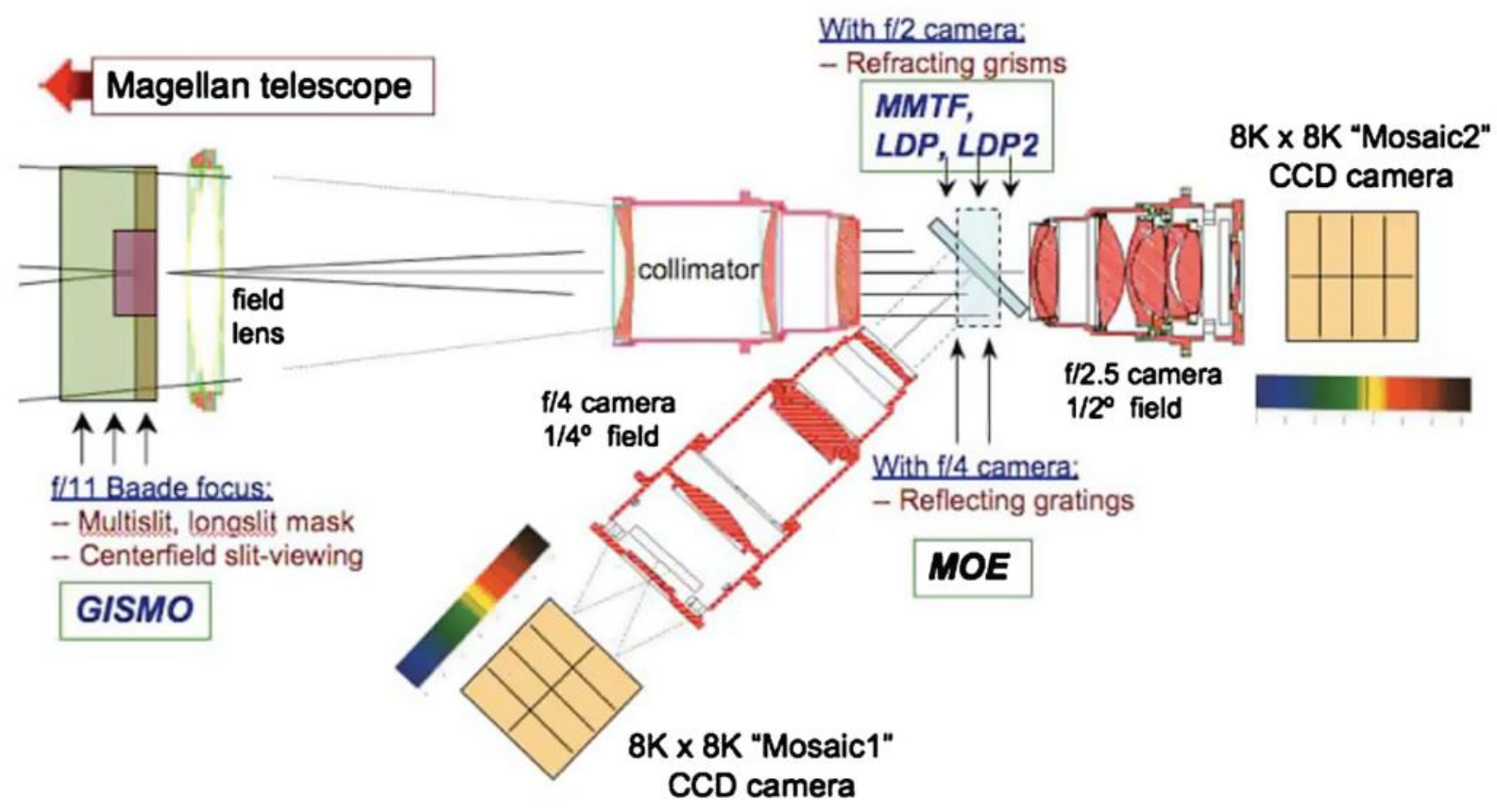

Figure 3.9: IMACS optical system layout. This original illustration has no scale information. Source: Dressler et al. (2011).

diameter, with slightly vignetting by the collimator) or long slit spectrograph of $27^{\prime}$ FoV with a variety of standard $150 \mathrm{~mm}$ aperture grisms. Both cameras feed a $8 \mathrm{~K}$ x 8K CCD array and have oil-coupled doublets (Epps and Sutin, 2003; Sutin, 2003; Dressler et al., 2006, 2011).

Figure 3.10 shows details of the faster camera optical design, which shares design similarities with the DEIMOS camera shown in figure 3.8.

\subsubsection{Gran Telescopio Canarias, GTC}

\subsubsection{OSIRIS}

The Optical System for Imaging and low-Intermediate-Resolution Integrated Spectroscopy (OSIRIS), located at the Nasmyth-B focus of GTC, is an imager and grism spectrograph for the optical wavelength range from 365 to $1,050 \mathrm{~nm}$ with a total field of view of $7.8^{\prime} \times 8.5^{\prime}\left(7.8^{\prime} \times 7.8^{\prime}\right.$ unvignetted $)$, and $7.8^{\prime} \times 6.0^{\prime}$, for direct imaging and multi-object spectroscopy, respectively. OSIRIS also provides additional capabilities such as the narrowband tunable filters imaging, charge-shuffling, and multi-object spectroscopy (GTC, 2020). According to Cobos et al. (2002), OSIRIS is compact enough to fit at the Cassegrain focus envelope. 


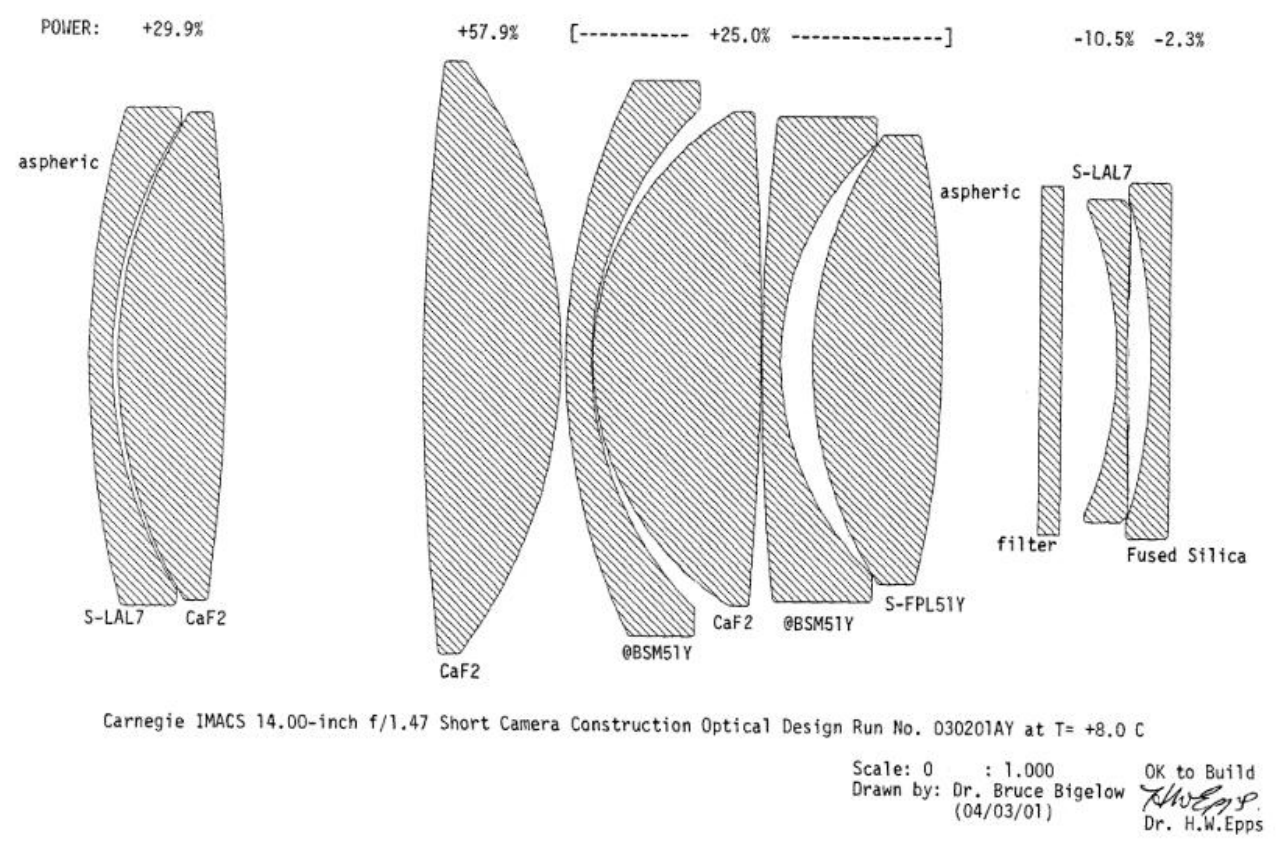

Figure 3.10: IMACS fast camera layout. Source: Epps and Sutin (2003).

The off-axis ellipsoidal mirror collimator has an EFL of 1,240 mm, while the all-spherical refractive f/2.47 camera has an EFL of $180.5 \mathrm{~mm}$ (Cobos et al., 2000, 2002). To the best of our knowledge, we could not find an updated illustration of the OSIRIS optical design better than the one presented by Cobos et al. (2002). Cobos et al. (2000) presented a concept illustration of the instrument and camera optical layout in figures 3.11 and 3.12, respectively. The camera's optical design has two doublets, followed by a strong positive lens, a low dispersion doublet, and two field flatteners.

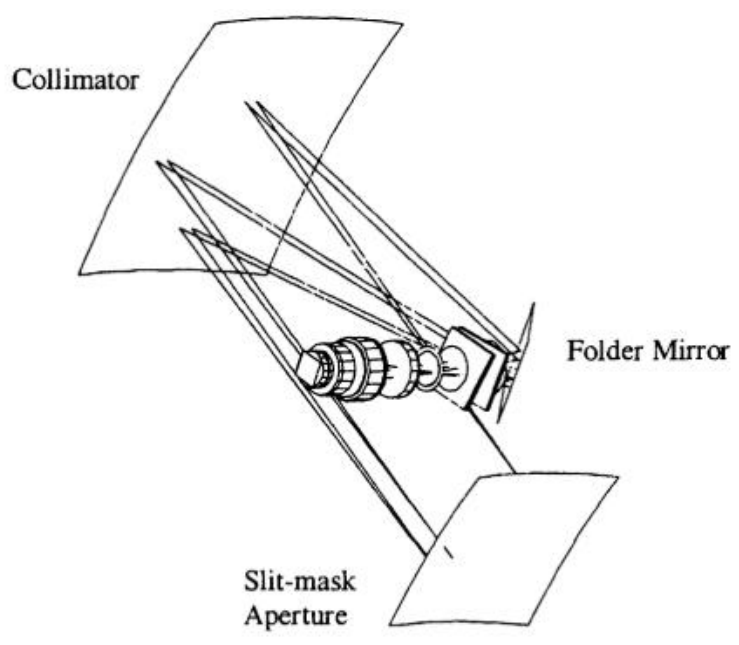

Figure 3.11: OSIRIS optical layout for the high and standard resolution mode. Source: Cobos et al. (2000). 


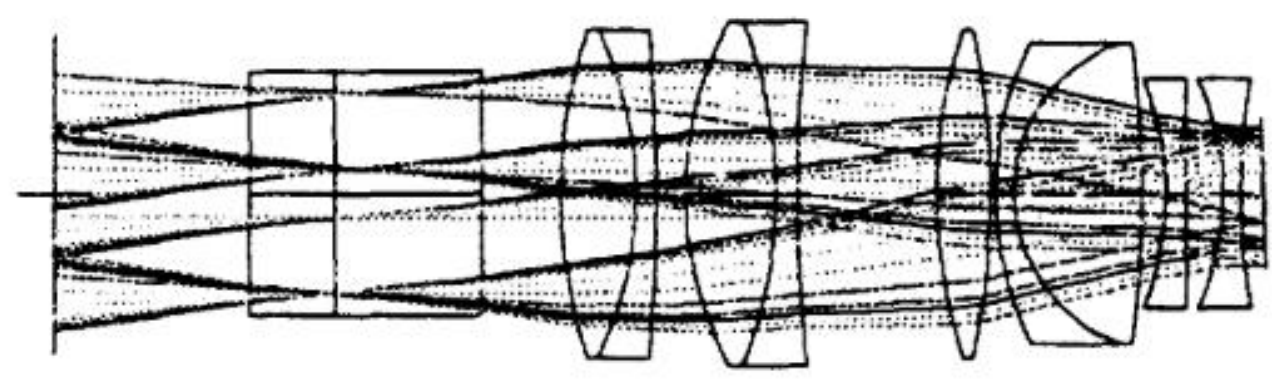

Figure 3.12: OSIRIS camera optical layout with the tunable filter. Source: Cobos et al. (2000).

\subsubsection{Multiple Mirror Telescope, MMT}

\subsubsection{BINOSPEC}

Binospec, commissioned in late 2017 at the $\mathrm{f} / 5$ Cassegrain focus of the $6.5 \mathrm{~m} \mathrm{MMT}$, is a high throughput imaging spectrograph that addresses two adjacent $8^{\prime} \times 15^{\prime}$ fields of view, operating in a $370 \mathrm{~nm}$ to 1,000 nm spectral range. Each Binospec beam includes a refractive collimator and a refractive camera, with focal lengths $1097 \mathrm{~mm}$ and $404 \mathrm{~mm}$, respectively, producing a scale at the detector of $0.24^{\prime \prime}$ per $15 \mu \mathrm{m}$ pixel size. Three reflection gratings, duplicated for the two beams, provide resolutions between 1,300 and > 5,000 with a $1^{\prime \prime}$ wide slit. Binospec's collimated beam diameter is $\approx 208 \mathrm{~mm}$. Figure 3.13 shows a 3D solid model of one of Binospec's two beams. The MMT focal plane's large distances from the first collimator surface and between the second and third collimator groups were necessary to accommodate the fold mirrors.

Figure 3.14 shows the collimator and camera optical designs without the fold mirrors and grating (Fabricant et al., 2019). The $1097 \mathrm{~mm}$ EFL collimator, comprised of three lens groups, has the lens materials BAL15Y/S-FSL5Y followed by a five-elements of PBM2Y, PBL6Y, BAL35Y, $\mathrm{CaF}_{2}$ and PBL6Y, and a doublet BSM51Y and $\mathrm{CaF}_{2}$. The $404 \mathrm{~mm}$ f/1.8 camera, comprised of four lens groups including the field flattener/dewar window lens, is BAL35Y and $\mathrm{CaF}_{2}$ followed by a quadruplet $\mathrm{CaF}_{2}, \mathrm{BAL} 35 \mathrm{Y}, \mathrm{CaF}_{2}, \mathrm{PBM} 2 \mathrm{Y}$, a triplet FPL51Y, NaCl and CaF, and BSM51Y as the field flattener. The LL5610 coupling fluid is used for all the compound elements (Fabricant et al., 1998, 2019). Active flexure control keeps the spectrograph alignment and the focus under varying gravity and thermal conditions. 


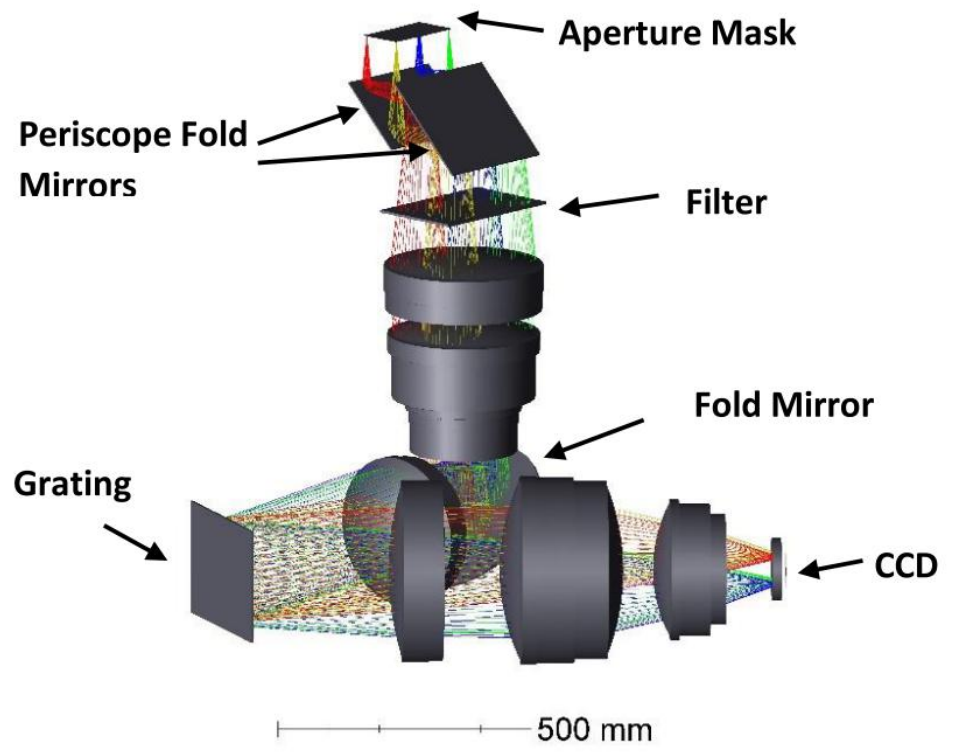

Figure 3.13: A 3D solid model of one of Binospec's two beams.

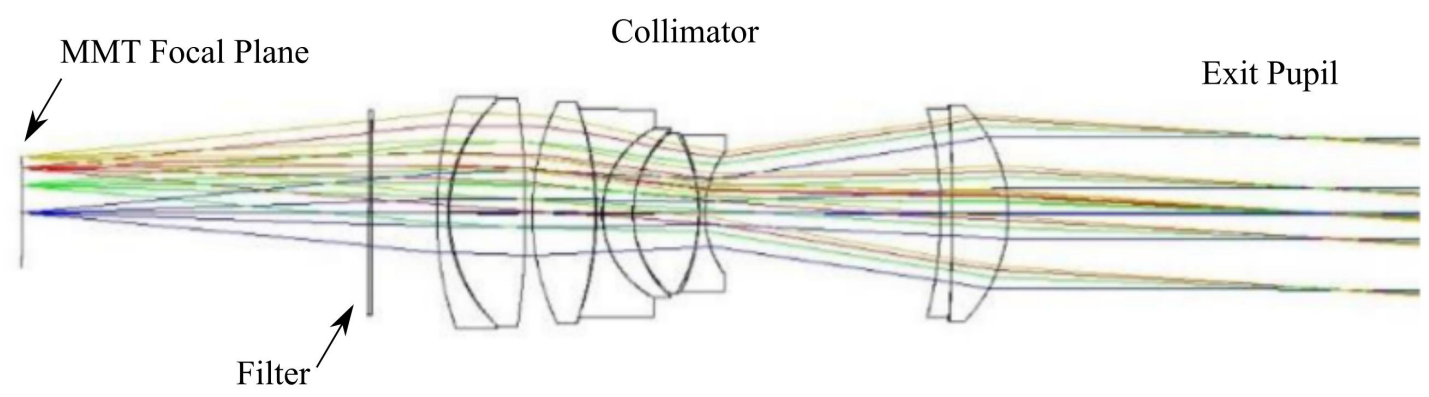

(a) The Binospec collimator.

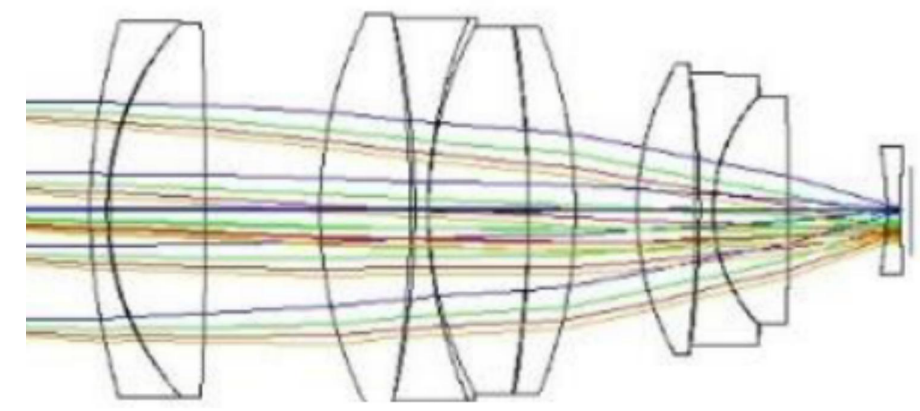

(b) The Binospec camera.

Figure 3.14: Binospec optical layout. Source: adapted from Fabricant et al. (2019). 


\subsubsection{Very Large Telescope, VLT}

\subsubsection{FORS}

The visual and near UV Focal Reducer and low dispersion Spectrograph (FORS), installed at the Cassegrain focus of the Very Large Telescope (VLT) of the European Southern Observatory (ESO), is an all-dioptric MOS, operating in the $330 \mathrm{~nm}$ to 1,100 nm spectral range, for spectral resolving power of 260-2,600, and two image scales of $0.25^{\prime \prime} /$ pixel (for the standard resolution collimator mode) and $0.125^{\prime \prime} /$ pixel (with the highresolution collimator mode). The corresponding field sizes are $6.8^{\prime} \times 6.8^{\prime}$ and $4.25^{\prime} \times$ $4.25^{\prime}$, respectively. FORS allows imaging, long-slit and multi-object spectroscopy, imaging polarimetry, and multi-object polarimetry (Appenzeller and Rupprecht, 1992; Seifert et al., 1994; ESO, 2020).

The main instrument optics consists of two remotely exchangeable f/15 collimators of EFL 1,233 $\mathrm{mm}$ and $616 \mathrm{~mm}$ for the standard and high resolution, respectively, giving $\approx$ $90 \mathrm{~mm}$ and a $44.5 \mathrm{~mm}$ diameter pupil. Figure 3.15 shows the schematic layout of a focal reducing optomechanical system. The single-camera is $280 \mathrm{~mm} \mathrm{f/3.1} \mathrm{(ESO,} \mathrm{2020).} \mathrm{Figure}$ 3.15 shows the optical layout of the standard and high resolution mode (Nicklas, 2005; ESO, 2020).

Although both collimators are telephoto lenses, they present different designs. The collimator for the high-resolution mode has a large positive field lens, so it contributes the most to the total optical power, followed by a positive singlet, negative doublet, and a strong positive singlet. In the standard resolution collimator, the first doublet, which does not contribute to focal power and is probably responsible for correcting field aberrations and contributing to achromatizing, is followed by another high positive doublet and a negative singlet. The camera has three groups: the first is a weak four-lens element, a strong positive lens, and the bi-concave field flattener. The four-lens group is responsible for the correction of remaining aberrations of the collimator's exit pupil and the other camera elements. We have not been able to find detailed information about the optical system, such as materials and the existence of aspherical surfaces. 


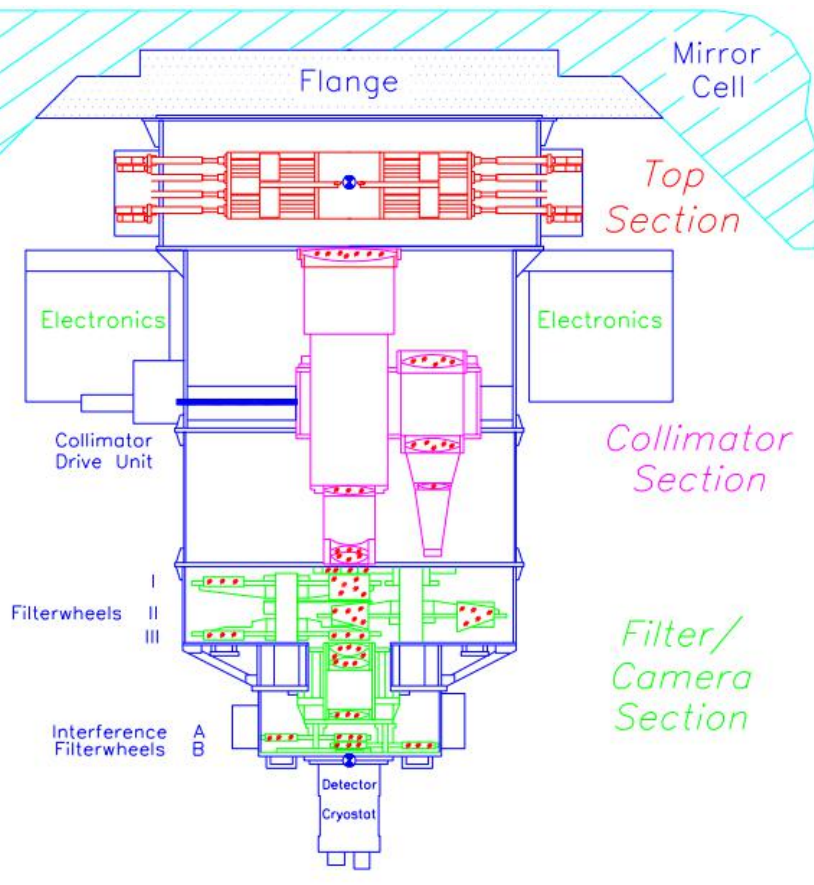

Figure 3.15: FORS's three main sections: the top or VLT's focal plane section, the central collimating section with the two collimator designs and the filter/grisms/camera section. Source: Nicklas (2005).
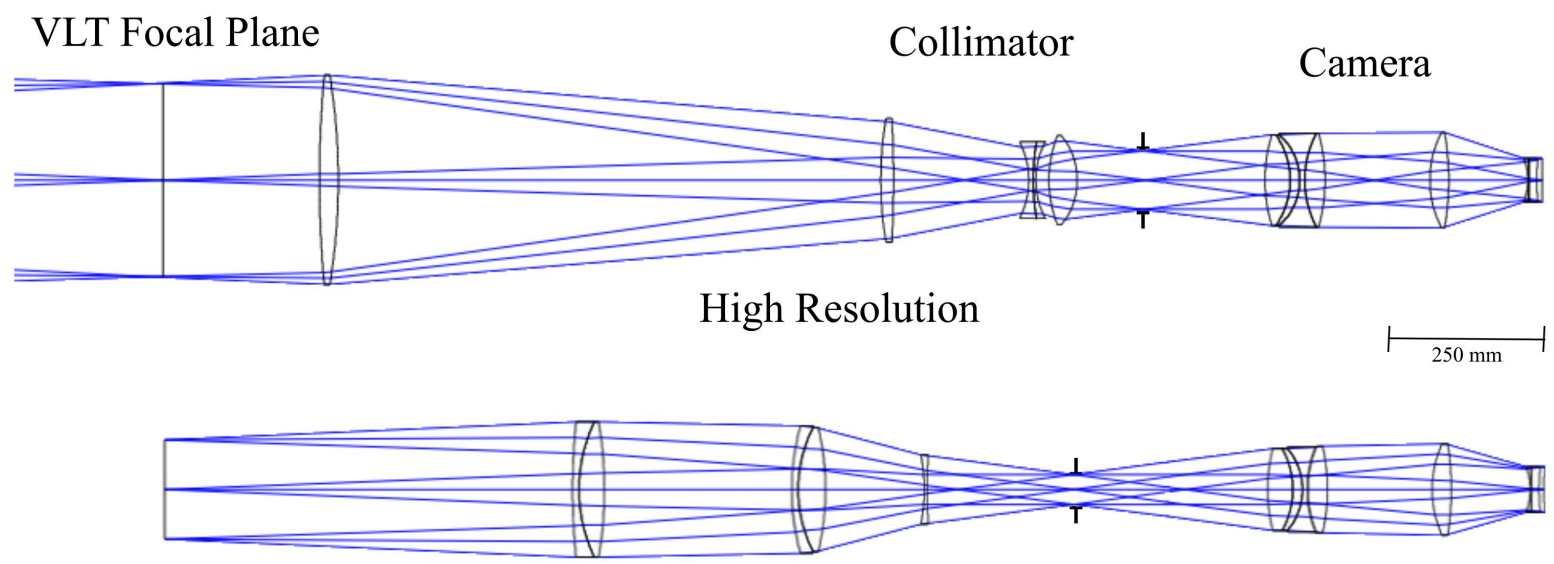

Standard Resolution

Figure 3.16: FORS optical layout for the high and standard resolution mode. The VLT focal plane and the cameras remain in the same location while the high and standard collimators are interchangeable. Source: adapted from Nicklas (2005).

\subsubsection{VIMOS}

The Visible Multi-Object Spectrograph (VIMOS), currently decommissioned, was a seeing-limited wide-field imager and MOS with IFU capabilities installed at the Nasmyth 
focus of the VLT UT3. The instrument contains four identical arms, each with a field of view of $7^{\prime} \times 8^{\prime}$ with a plate scale of $0.205^{\prime \prime} /$ pixel; the IFUs have pixels down to $0.33^{\prime \prime}$ covering up $13^{\prime \prime} \times 13^{\prime \prime}$ up to $54^{\prime \prime} \times 54^{\prime \prime}$ on sky depending on the spectral resolution and spatial magnification choice (LeFevre et al., 2003b). Each arm equipped with grisms provided a power resolution of $\approx 200-2,500$ with wavelength coverage from $360 \mathrm{~nm}$ to $1000 \mathrm{~nm}$. The spectral resolution and coverage are similar to multi-object spectroscopy (ESO, 2020).

VIMOS optical system is the combination of four identical optical channels, which includes a Focal Plane Adaptation Lens (FPAL), to correct telescope aberrations at the Nasmyth focus and reduce the field curvature to allow the use of flat masks (see figure $3.17)$.

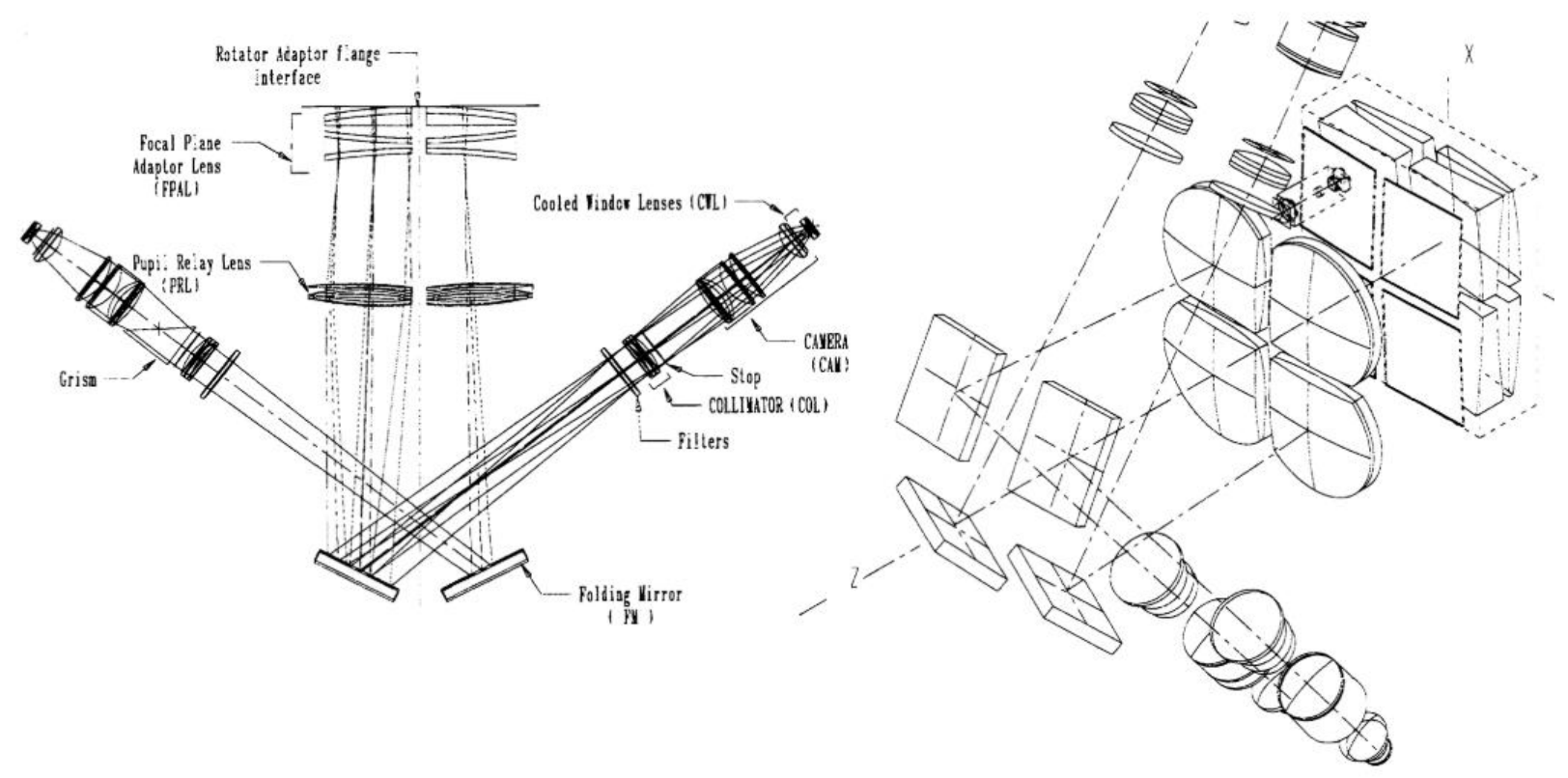

Figure 3.17: VIMOS optical layout in projection (right) and axonometric view. Source: LeFevre et al. (1998).

The 1970 mm refractive collimator has two groups (see figure 3.18): an SFSL5 and F2 field doublet lens, which contributes most to the collimator power, is followed by a negative air-doublet for aberration control close to the $180 \mathrm{~mm}$ exit pupil (LeFevre et al., 1998). The collimator corrects pupil aberrations and focus while the camera compensates for the residual field curvature. A fold mirror compacts the system, and the filter is located close to the second group, in a region with quasi-collimated beam.

The $248.4 \mathrm{~mm} \mathrm{f/1.8} \mathrm{camera} \mathrm{has} \mathrm{four} \mathrm{groups:} \mathrm{the} \mathrm{cemented} \mathrm{four-lenses} \mathrm{element,} \mathrm{FK54,}$ LF, K5 and FK54 (according to LeFevre et al. (1998), flexible types of cement such as 


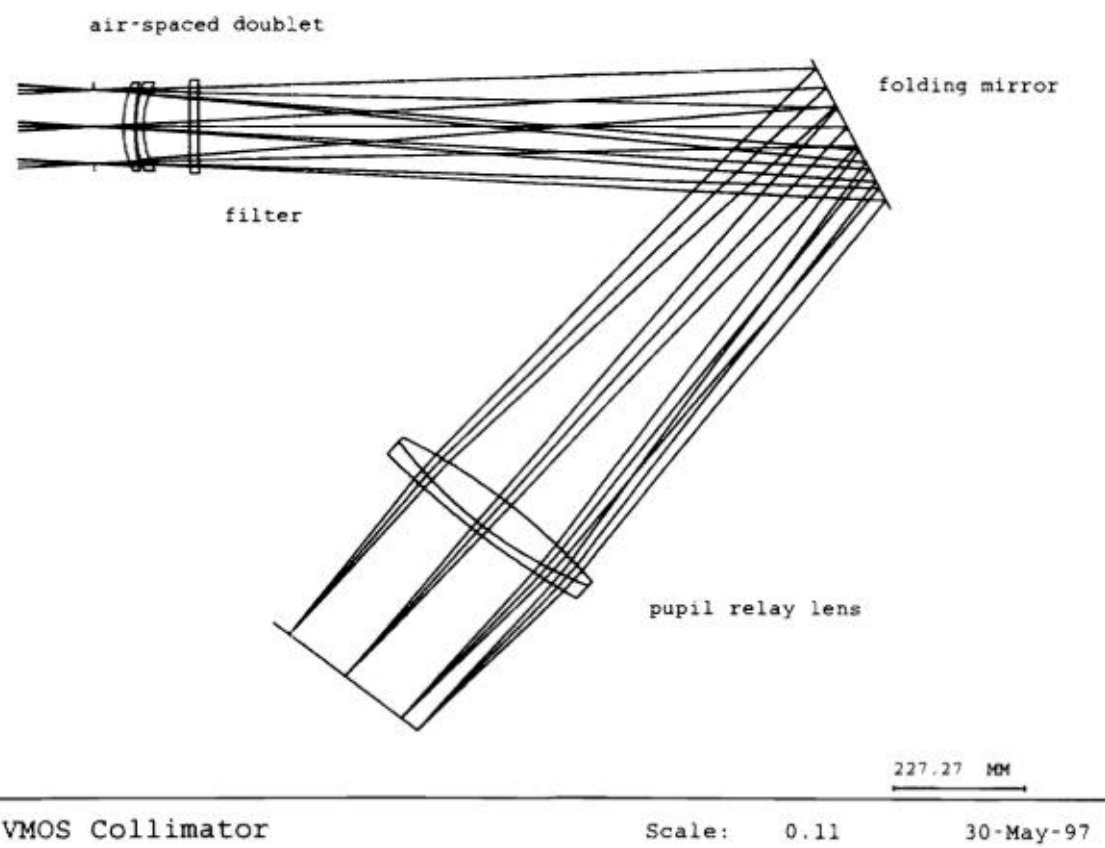

Figure 3.18: VIMOS collimator layout. Source: LeFevre et al. (1998).

RTV 141 can be used to mitigate the issues of CTE differences), is followed by an FK54 and CaF2 singlets, and an SF5-Silica doublet as cryostat window (see figure 3.19). The last lens rear surface is aspheric.
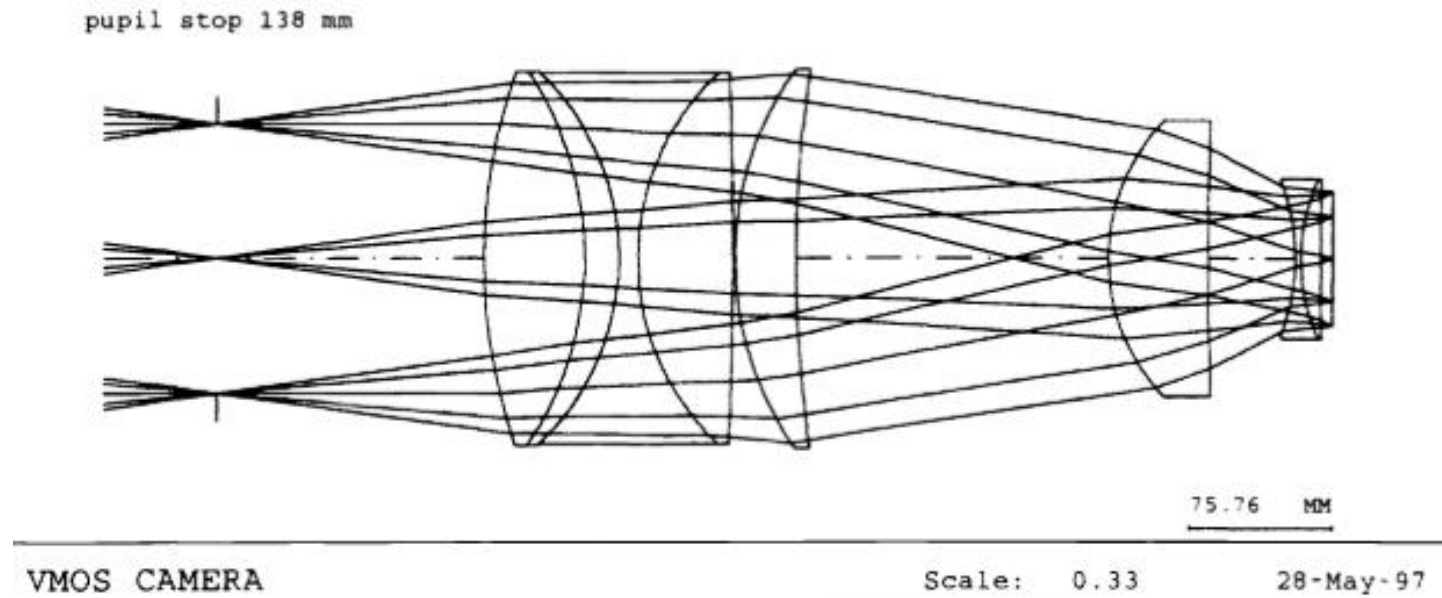

Figure 3.19: VIMOS' camera layout. Source: LeFevre et al. (1998). 


\subsubsection{Subaru}

\subsubsection{FOCAS}

The Faint Object Camera and Spectrograph for the Subaru Telescope (FOCAS) installed at the Cassegrain focus of Subaru, provides direct imaging, long-slit, and multi-slit grism spectroscopy, polarimetric imaging and spectropolarimetry modes. This versatile instrument covers the entire $6^{\prime}$ in diameter unvignetted FoV region of the Subaru Cassegrain. The resolution power ranges from $R=250-2,000$ for a $0.4^{\prime \prime}$ slit width for the spectral range of $380 \mathrm{~nm}$ to 1,000 $\mathrm{nm}$ (Kashikawa et al., 2000, 2002). The ADC installed at the telescope Cassegrain unit is used in most FOCAS observations (Subaru Telescope, 2019). Figure 3.20 shows the instrument structure attached to the Subaru.

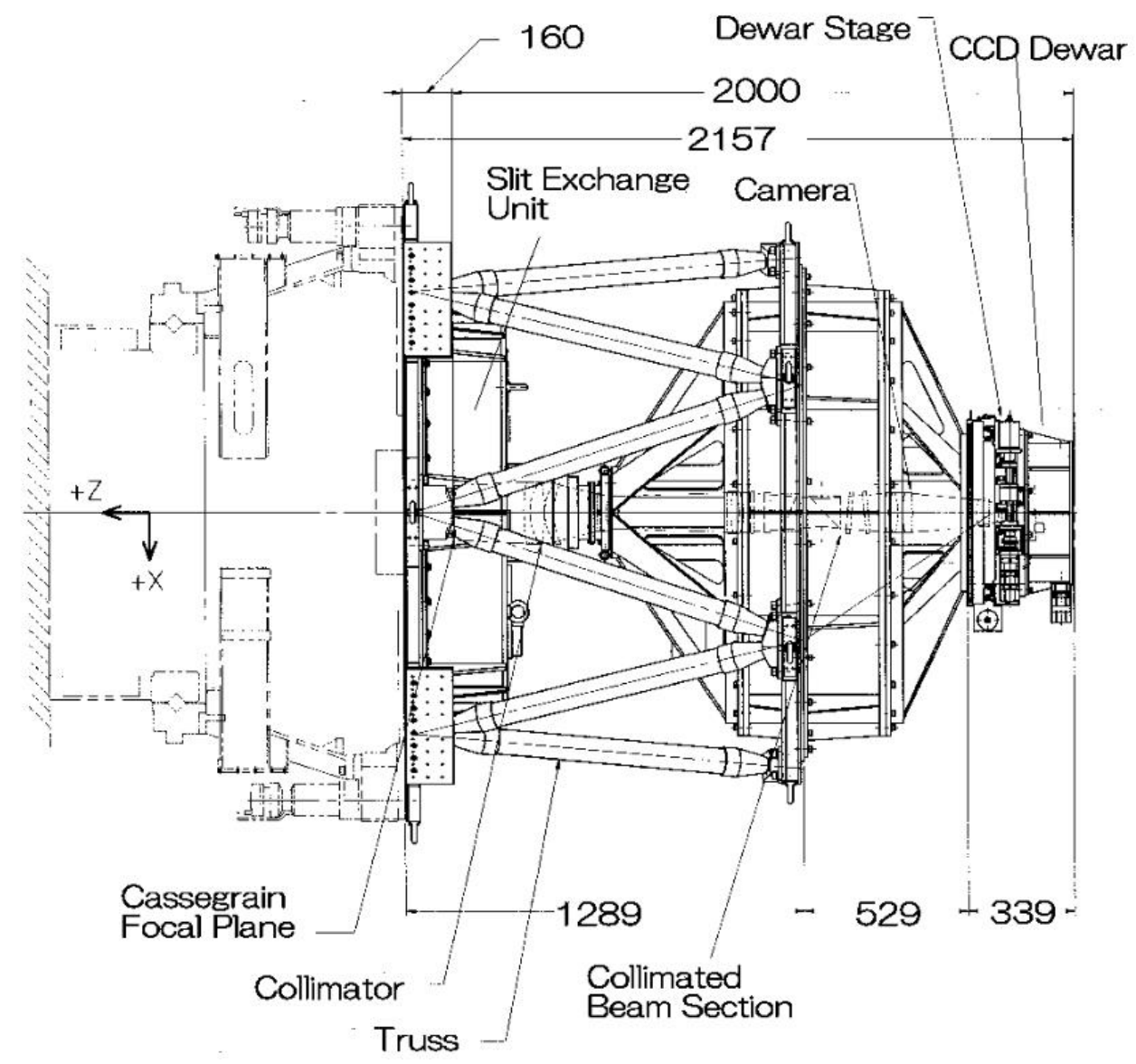

Figure 3.20: Overview of FOCAS and structure. The Subaru Telescope is to the left. The unit is millimeter Source: Kashikawa et al. (2002).

The $450 \mathrm{~mm}$ total collimator exit pupil and camera entrance pupil reliefs are larger than observed for the other instruments to allow the insertion of the elements for polari- 
metric modes and filters. The $1098 \mathrm{~mm}$ refractive telephoto collimator has the positive groups comprised of a doublet and meniscus, followed by the negative groups composed of a negative doublet and a positive triplet, close to the exit pupil. The $324 \mathrm{~mm} \mathrm{f} / 3.6$ camera has a general Petzval camera layout with a positive triplet and singlet followed by a low dispersion positive lens and the field flattener (Kawabata et al., 2003). Figure 3.21 shows the FOCAS instrument optical layout. We have not been able to obtain detailed information on the materials and properties of the FOCAS optical system.

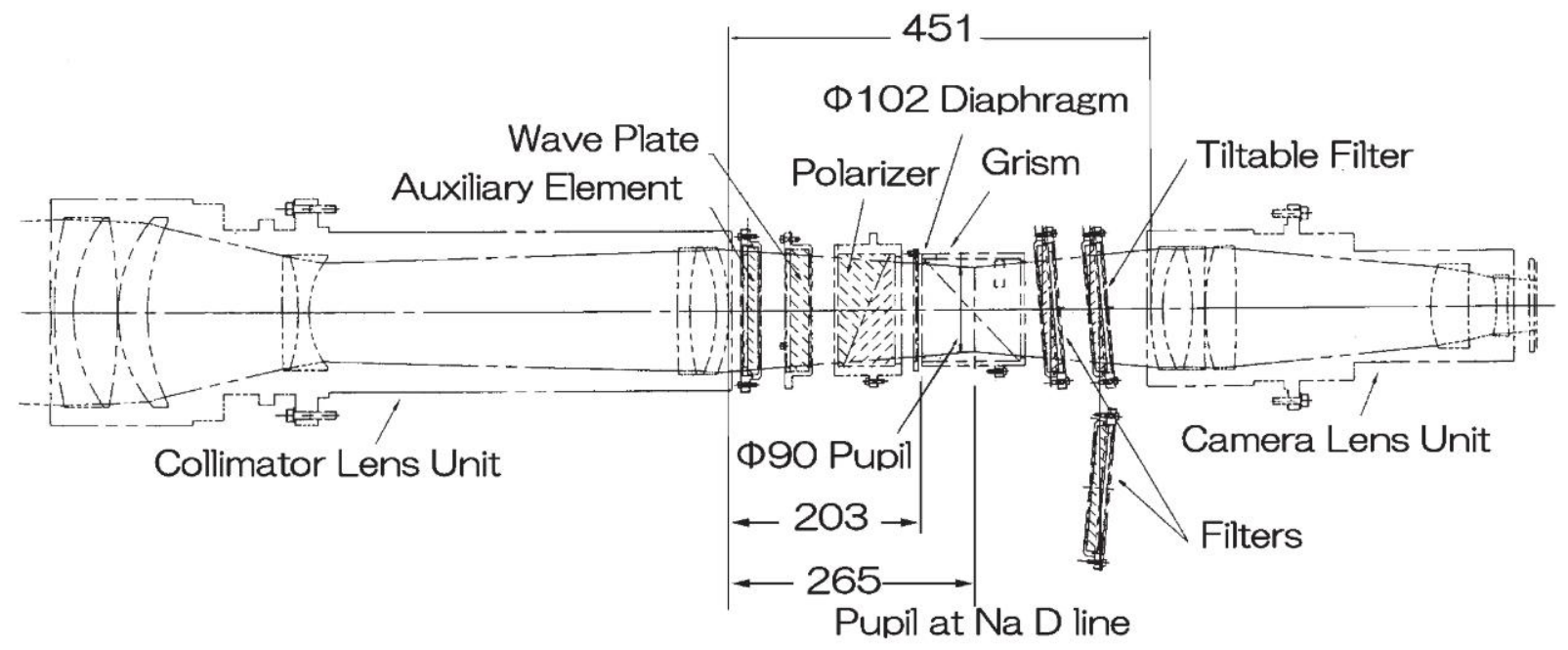

Figure 3.21: FOCAS optical layout. The unit is millimeter. Source: Kashikawa et al. (2002). 
Chapter 4

\section{Methodology}

This section details the methodologies used in this work to develop the CoD GMACS optical system, from earlier phases, such as the determination of the spectrograph functional parameters to the description of the supporting computational tools for the optical and optomechanical performance analyzes. Although motivated and applied for the development of the GMACS optical system, this set of methodologies can be adapted to any similar optical designs for low-resolution astronomical spectrographs.

\subsection{Optical Design as Part of the Project}

The interpretation of the high-level instrument specifications is one of the early phases of an optical design, which may include any areas that directly or indirectly impact the instrument's optical performance. This phase involves scientific and technical iterations between astronomers, instrumentalists, optical, mechanical and optomechanical engineers, managers, and stakeholder needs. The central role of the optical engineering group is not only to assist in generating a set of initial optical requirements, but also to make sure that the requirements are coherent with each other, technically cohesive, realistic with the most commonly adopted practices for optical design, manufacturing and alignment processes. We emphasize that it is necessary to keep a constant follow-up with suppliers to check their capabilities on providing crucial materials, such as optical blanks for lens manufacturing, and optical and optomechanical components and services. Although this obligation is usually shared with the systems engineering team and the project management, the optical group should lead this task and provide the project management with full information, in constant communications, for refining the project budget, schedule, and risks. 
In parallel, the optical engineering group begins the literature review phase, a common practice adopted in any optical system development project. This step consists of a detailed review of the lens design literature from general articles, white papers, books, and patents related to the field of application of the optical system. This review should include state-ofthe-art engineering and optical physics capabilities, lens design techniques for instrument application, and vendors' feedback for a constant update about technical limitations in optical element manufacturing, subsystem assembly, alignment, and testing.

The subsequent steps include (i) elaboration of the preliminary paraxial drafts; (ii) earlier identification of the potential tradeoff studies; (iii) definition of the most appropriate optical performance criteria for the application, such as encircled energy, MTF, depth of focus, throughput, image stability, among others; (iv) identification of the possible optical design architecture; and (v) development of a detailed plan for tolerances analyzes for assembly mounting and system integration that includes error budget and mitigation plan.

The transition between one project phase and the next must be assessed by the quality criteria defined by the project management, supervised and assisted by the systems engineering group, and supervised by the manager. Since the optical system activities are under the project schedule, the optical design group is responsible for supplying updated information on the progress of each internal task, identifying risks, elaborating mitigation plans and recommendations to assess their impact on cost and schedule (Jacoby, 2016). Another crucial element is an effective communication between the optical group and all the others, such as the mechanical, optomechanical, structural, and thermal groups.

One of the primary motivations of this work, besides a complete CoD GMACS optical design, is to provide to the astronomical instrumentation community a detailed description of the methodologies and tools. The CoD optical design methodology, far from being a linear development process, has undergone adjustments and improvements throughout the last four years of intermediate phases, such as kick-off meetings, mid-point review, and inperson and remote meetings with those involved in the optical design. The methodology incorporates design peculiarities that require revisions, adaptations, and inclusion of steps in the usually adopted procedures for optical design.

The GMACS optical system design is incorporated in the concept, development, manufacture, assembly, integration, test and commissioning of the GMACS project. Therefore, the methodologies developed must be included in the project management context, in 
which the activities and milestones shall be synchronized with other areas of the project, especially those that interface with the optical system, such as mechanics and optomechanics. It is essential, particularly in the initial phases of the project, which those responsible for the optical system actively participate in major decisions taken by the management and systems engineering teams regarding the definitions of the technical requirements as well as the scope and schedule of the project.

\subsection{Design Tools}

\subsubsection{Optical Design Software}

The optical designs of this work were elaborated using the commercial software OpticStudio Zemax V16.5, a well-established software for the design of optical systems (Zemax Development Corporation, 2011). Two principal reasons motivated this decision: (i) GMTO requires that all the instrument development groups use it for the formal milestone deliveries, such as final optical models, performance and analysis reports (in particular the SOW: GMACS Conceptual Design Study (Jacoby, 2016)); therefore, using the same software facilitates the elaboration of reports and design exchange; and (ii) the author has more than ten years of design experience with this commercial software, dramatically reducing the required learning time.

OpticStudio Zemax, or simply Zemax, output files are .ZMX, which contains all the information necessary to build the optical design, and .ZDM, which records the windows layout for analysis purposes. Although these files are essential to run an optical design in Zemax, the most appropriate format to exchange Zemax files among team members must be the compacted file. $Z A R$, which contains the glass data, filters data and any other file required to run Zemax, in addition to the.$Z M X$ and.$Z D M$ files.

\subsubsection{OpticStudio Zemax macros}

OpticStudio Zemax allows the user to interact with the program through additional formats of the standard GUI to customize applications and automate actions and analysis. According to Zemax LLC OpticStudio (2016), the available OpticStudio programming forms are: 
- ZPL (Zemax Programming Language): interpreted macro language which runs within the GUI;

- ZOS-API (Application Programming Interface): control a lens design with an external programming language, such as $\mathrm{C}++$, Matlab and Python;

- DLL (Dynamic Link Library):

1. UDS (User-Defined Surface): Implementing surfaces not already built into OpticStudio;

2. UDO (User-Defined Object): Non-sequential sources, scattering functions, physical optics propagation source, etc.

All custom applications created for the development of GMACS were designed through ZPL and data visualized by Python scripts. The most significant motivation for choosing these two tools is, besides the author previous experience, the ease of scripting, debugging, implementation, and sharing with the rest of the GMACS development team, who also have experience in ZPL.

We are envisaging the implementation of UDO for the more accurate sequential and non-sequential modeling of VPH grating to refine the throughput and stray light analyzes of the integrated spectrograph for future work. For this, the efficiency of the VPH grating must be previously known for different angles of incidence, diffracted, and spectral band. Due to modeling complexity of the efficiency of VPH grating, we considered using, for the next GMACS project phases, the G-Solver analysis tool ${ }^{1}$, which provides grating numerical efficiency calculation by using RCWA.

\subsubsection{Graphical User Interface for Grating Spectrographs Simulator}

A Graphical User Interface (GUI) simulator was developed to allow quick calculation of the fundamental characteristic for a grating spectrograph. We elaborated an Excel based database that has the principal parameters of several telescopes and spectrographs parameters (including all the instruments described in chapter 3) from which the Simulator automatically reads. Some of the telescope and spectrograph properties considered as

\footnotetext{
${ }^{1}$ G-Solver diffraction grating analysis tool is developed by Grating Solver Development Company (P.O. Box 353, Allen, TX 75013). Available at https://www.gsolver.com/. Accessed on 2020-03-24.
} 
design parameters are user-defined, such as Blaze wavelength, slit width, telescope, collimator and camera EFLs, and detector parameters, such as pixel pitch and detector size. Figure 4.1 presents the software GUI: the cells in yellow background color are the ones that the user can change while the white background parameters are automatically calculated based on the yellowish ones.

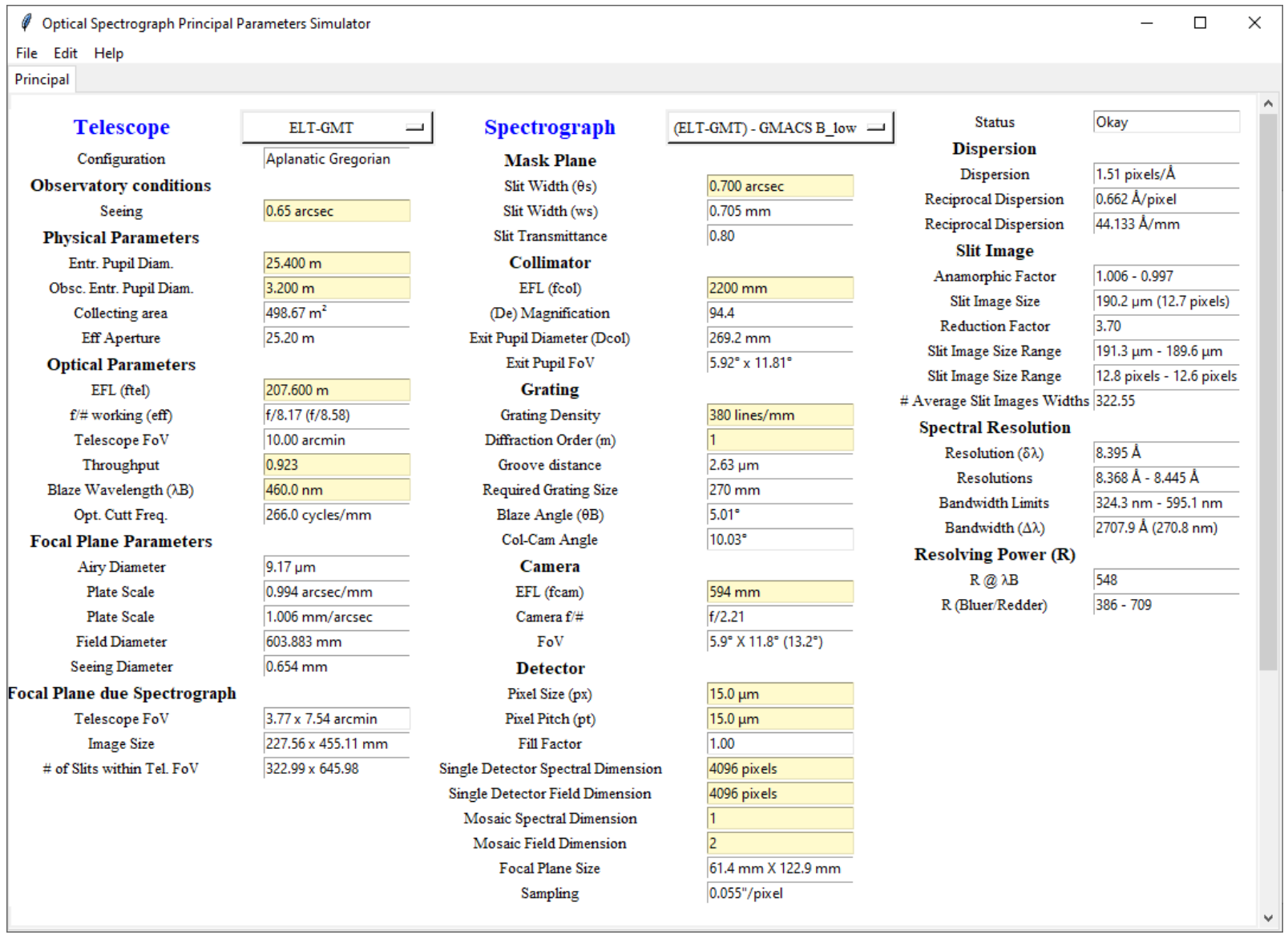

Figure 4.1: Graphical User Interface for the modeling of an optical spectrograph principal parameters. The yellowish banks are user defined and the white ones are calculated in real time by the software.

The GUI Simulator includes the useful Python package astropy ${ }^{2}$, which contains functionalities aimed at aiding professional astronomers and astrophysicists, including the valuable astropy.units, which handles unit conversions and performs arithmetics with physical quantities

Although a spectrograph can be modeled in spreadsheets, the motivations for the development of this script are to prevent accidental changes to the formula equations, allow

\footnotetext{
${ }^{2}$ Available at https://www. astropy.org/. Accessed on 2020-01-24.
} 
the insertion of a database for quick search and comparison between combinations of telescopes and instruments and eventually create a stand-alone executable. The database is made via a spreadsheet that is automatically read by the Python script through the Pandas package. The user can select any combinations of the telescopes and instrument parameters, as well as modify any of them.

\subsubsection{Computational Infrastructure}

The computational tools used for the elaboration of this project were:

- Hardware: PC-XPS Dell Intel(R) Core (TM) i7-6700 @ 3.40Ghz, 16.0 Gb RAM with Windows 10;

- Optical Design Software: OpticStudio Zemax V16.5 SP3 Network Premium Edition (64-bit);

- Programming: Python 2 and 3 (Anaconda);

- Meeting: RingCentral and Hangout;

- General Drawing: DrawIO, Inkscape.

Institutional OpticStudio Zemax licenses (a total of 10) bought by the University of São Paulo, yet open for use of any interested Brazilian optical groups, is hosted by the Institute of Physics (IF). Any interested group can access the program remotely via IP from the server located at IF. This solution proved to be valuable since it allowed the author to access both the IAG infrastructure, through desktop remote control software, and Zemax licenses with a very low incidence of connection errors or server failures.

\subsubsection{Optical Design Configuration Manager}

The development of an optical system as complex as GMACS usually requires refining several versions of the optical drawings to explore different solutions and compare performances, whether at the level of systems, subsystems, or for specific solutions. Therefore, it was urgent to implement a kind of configuration management of optical drawings and data backup from the beginning of the development phase. The Optical Design Configuration Manager (ODCM) is a database developed for version control that contains information such as design dates of elaboration, subsystem description, a summary of the objectives for the study, the performance achieved and conclusions, whether the subsystem constitutes a 
baseline, and the links to the optical design files. Although essential for the management and control of project documents, as the Product Data Management is for SolidWorks, in practice, ODCM was used on an individual level, and therefore it is not justified to acquire specific software for application in optical systems. In fact, to the extent of our knowledge, there is no specific commercial software for managing optical designs version. Therefore, the most straightforward yet effective individual ODCM is a spreadsheet (see figure 4.2) including links for accessing drawings directly through the OpticStudio Zemax pre-installed on the computer.

\begin{tabular}{|c|c|c|c|c|c|c|c|c|c|}
\hline Date $=$ & Subsystem = & $\bar{\gamma}$ & & $\bar{F}$ & ID $=$ & Comments & Baseline? $=$ & Baseline Date $=$ & Link \\
\hline 2018-05-08 & Collimator & $\boldsymbol{v}$ & Split Collimator & 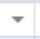 & 2 & Released SPIE version & $\square$ & 2018-05-08 & \\
\hline 2018-05-08 & $\begin{array}{l}\text { Collimator + } \\
\text { Blue Camera }\end{array}$ & $\checkmark$ & Split Collimator & $\boldsymbol{r}$ & 2 & Released SPIE version & $\square$ & 2018-05-08 & Drive Link \\
\hline 2018-05-08 & $\begin{array}{l}\text { Collimator + } \\
\text { Red Camera }\end{array}$ & . & Split Collimator & 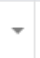 & 2 & Released SPIE version & $\square$ & 2018-05-08 & Drive Link \\
\hline 2018-05-08 & Collimator & 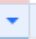 & Split Collimator & 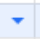 & & SPIE Version (improved) & $\square$ & & \\
\hline 2018-05-13 & $\begin{array}{l}\text { Collimator + } \\
\text { Blue Camera }\end{array}$ & 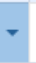 & & $\boldsymbol{v}$ & 3 & Pre-midpoint review (with tolerance analysis) & $\square$ & & \\
\hline 2018-06-02 & Collimator & $\vec{r}$ & Single Collimator & 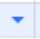 & & KoM & $\square$ & 2018-06-02 & Drive Link \\
\hline 2018-06-02 & Collimator & $\vec{r}$ & Single Collimator & $\vec{r}$ & & Achromatic & $\square$ & & \\
\hline 2018-06-02 & Collimator & 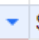 & Single Collimator & $\boldsymbol{r}$ & & Baseline no Cemented & $\square$ & & \\
\hline 2018-06-03 & $\begin{array}{l}\text { Collimator + } \\
\text { Red Camera }\end{array}$ & 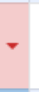 & & 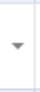 & 3 & $\begin{array}{l}\text { Pre-midpoint review (with tolerance analysis) } \\
* 6 \text { lenses } \\
{ }^{*} \mathrm{f} / 2.2\end{array}$ & $\square$ & & \\
\hline 2018-11-05 & $\begin{array}{l}\text { Collimator + } \\
\text { Blue Camera }\end{array}$ & . & Split Collimator & 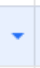 & 1 & $\begin{array}{l}{ }^{*} 8 \text { lenses } \\
{ }^{*} \mathrm{f} / 2.2\end{array}$ & $\nabla$ & 2018-11-05 & \\
\hline 2018-11-05 & $\begin{array}{l}\text { Collimator + } \\
\text { Red Camera }\end{array}$ & $\checkmark$ & Split Collimator & 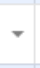 & 1 & $\begin{array}{l}{ }^{*} 6 \text { lenses } \\
{ }^{*} / 2.2\end{array}$ & $\checkmark$ & 2018-11-05 & \\
\hline 2018-11-17 & Blue Camera & $r$ & $\mathrm{n} / \mathrm{a}$ & $\checkmark$ & & $\begin{array}{l}{ }^{*} 8 \text { lenses } \\
{ }^{*} / 2.2\end{array}$ & $\square$ & & \\
\hline 2018-11-19 & Red Camera & $r$ & $\mathrm{n} / \mathrm{a}$ & 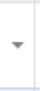 & & $\begin{array}{l}{ }^{*} 6 \text { lenses } \\
{ }^{*} \text { f } / 2.2 \\
{ }^{*} \text { better performance than the previous }\end{array}$ & $\square$ & & \\
\hline $2019-01-10$ & Collimator & 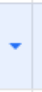 & Split Collimator & 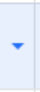 & & $\begin{array}{l}{ }^{*} \text { Distance of } 650 \mathrm{~mm} \text { between the blue and red } \\
\text { collimating groups } \\
{ }^{*} \text { Wedge Compensator }\end{array}$ & $\square$ & $2019-01-10$ & Drive Link \\
\hline 2019-01-16 & Collimator & 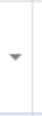 & Split Collimator & 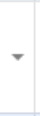 & 1 & $\begin{array}{l}\text { * Distance of } 650 \mathrm{~mm} \text { between the blue and red } \\
\text { collimating groups } \\
\text { * Lateral Colour Corrected } \\
{ }^{*} \text { Wedge Compensator }\end{array}$ & $\checkmark$ & 2019-01-16 & Drive Link \\
\hline 2019-01-22 & Red Camera & $\checkmark$ & $\mathrm{n} / \mathrm{a}$ & $\checkmark$ & & $\begin{array}{l}{ }^{*} 6 \text { lenses } \\
{ }^{*} / 1.6\end{array}$ & $\square$ & & \\
\hline $2019-02-25$ & Red Camera & $r$ & $\mathrm{n} / \mathrm{a}$ & 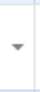 & & $\begin{array}{l}{ }^{*} 6 \text { lenses } \\
{ }^{*} \text { f/2.2 } \\
\text { * thicker lenses }\end{array}$ & $\square$ & & \\
\hline
\end{tabular}

Figure 4.2: Example of the Optical Design Configuration Manager window, ODCM.

The University of São Paulo and Google signed a cooperation agreement that allows the university community, students, alumni, teachers, and employees to use the resources of the G Suite for Education tool (USP Alumni, 2017). In addition to the unlimited use of email, contacts, and calendars, the cooperation term includes the document storage and sharing service. The latter, through Google Drive and the automatic synchronization tool via Drive File Stream and Google Sync, offers a helpful and essential tool to optical design backup and to recover any prior design. Any accidental changes to a file that have 
already been synchronized can be easily reversed online. Finally, the service offers specific Drives for team access. This functionality has been used successfully among the institutes participating in the development of GMACS, led by TAMU.

\subsubsection{Communication, Meetings, Data sharing and Reviews}

GMACS optics team meetings took place weekly since mid-2015 and proved to be essential for the definition of activities, exchange of experiences and information, presentation and discussion of results, and the maturing of personal relationships between team members. The combination of experienced members with those less experienced was an effective way of quickly leveling the concepts and techniques related to astronomical instrumentation and, more specifically, optics instrumentation. In the CoDR's meeting (September 2019), the GMACS optics group counted two astronomers, one being the GMACS Instrument Scientist (Dr. Luke Schimidt) and the other an experienced professional in astronomical instrumentation (Dr. Keith Taylor), and two optical designers (Prime Optics, represented by Dr. Damien Jones) and the author of this thesis.

The use of online shared presentations proved to be an efficient solution and has become essential to enable quick information exchange and storage of previous chronological results given the international character of the development team. The access link was available in the weekly Hangout invitation. The entire GMACS team met during the conceptual design period in bi-weekly videoconferences, to which GMTO personnel were also invited.

There is a consensus among groups of astronomical instrumentation that the execution of large projects, such as those for ELT instrumentation, requires a high degree of internationalization aiming at the diversification of sources for financing and the aggregation of capacities that experienced groups can provide for the project, which in turn reduces the overall technical risks and costs. Therefore, the adopted protocol of communication must promote a proper environment for information exchange. For this, the functionalities of online collaborative tools for sharing and generating documents, and communication platforms are beneficial to enabling the internationalization of projects.

The GMACS group, as described above, has access to Google Docs through G Suite for Education services. Google Drive provides text, Google Docs, and presentation editors, Google Sheets, among other tools. Although the most widely adopted technical and scientific communication happens via writing, a very effective way of communicating and 
exchanging information in an agile and fast way between team members of an ongoing project is through online shared presentations, in this case, via Google Slides. We observed that the most productive approach is to add chronological slides in the same presentation, similar to a logbook, to avoid the generation of numerous documents about the same subject. It proved beneficial for sharing the results of discussions, concentrating the information in the same document, and producing chronological results for the studies carried out. Additionally, it provides data for the project milestones deliverable.

In addition to the G Suite for Education services for internal communication, TAMU suggested the use of the Ringcentral ${ }^{3}$, a publicly-traded provider of cloud-based communications and collaboration solutions for businesses.

\subsubsection{Literature Resources}

One of the indispensable steps for any $R \& D$ activity is the technical bibliographic review for a broad range of objectives, such as the identification of the available solutions for similar requirements, the methodology, software, and any tools which were previously adopted. It is necessary to collect the lessons learned and the references previously used and finally compile any design questions that are still open.

Similar to other areas, the general sources for astronomical instrumentation are papers and proceeding papers, books, and instruments' websites. Although there are few explicit patents concerning astronomical instrumentation issues, we have included them in the list of technical literature resources. They are valuable review sources since they usually describe the theory, methodologies, and optical design solutions for the most diverse applications. The results can be used as a starting point for optical design, although it is essential to avoid patent infringement.

Below is a list of the technical literature resources we used in this research:

\footnotetext{
${ }^{3}$ Available at https://www.ringcentral.com/. Accessed on 2016-03-15.
} 
1. Papers;

2. Instruments' websites (Phases Review Documents);

3. Optical design and astronomical instrumentation books;

4. Starting point design sources:

(a) Zebase, a collection of optical design forms that are pre-built as OpticStudio models that provides a starting point for a design;

(b) Optical Design Books with design examples cases (Smith, 1992; Laikin, 2006);

5. Patents:

(a) Google Patents Search (https://patents.google.com);

(b) United States Patent and Trademark Office (USPTO) (https://www. uspto. gov $)^{4}$

(c) European Patent Search (EPO)(https://www.epo.org);

(d) National Institute of Industrial Property (INPI-Brazil)(http://www . inpi .gov . br);

\subsection{System Engineering and Management Tools}

According to Faes et al. (2018), Systems Engineering (SE) proposes a series of methodologies and practices to ensure the successful development and operation of systems. The GMTO strongly encourages the instrument development teams to fully integrate into the GMTO SE (Johns, 2001). Considering this request and the current trend of using these tools in the development of complex projects, the GMACS development team has applied SE methodology since the beginning of the conceptual phase dated 2016. Similar to GMACS, a novel systems engineering approach is being applied to the GMT-Consortium Large Earth Finder (GCLEF) (Podgorski et al., 2014).

This section presents the SE concepts and methodologies used directly in the execution of the research.

\subsubsection{Product Breakdown Structure}

The proposed hierarchical division of the Product Breakdown Structure (PBS) used for the optical design is:

\footnotetext{
4 The patents were within the classification "G02B - Optical elements, systems, or apparatus", more specifically the "G02B 13/00 - Optical objectives specially designed for the purposes specified below".
} 
1. System Level

1.1. Subsystem Level

1.1.1. Assembly Level

1.1.1.1. Subassembly (or groups) Level

1.1.1.1.1. Component (or element) Level

In this work, the telescope and the spectrograph are independent systems. According to SE definitions, a system is an integrated set of elements that accomplishes a given objective or collection of objectives. The SE classification shows that GMACS is a GMT subsystem. However, we will raise the level of GMACS in this work to simplify terms. Thus, GMACS has the subsystems shown in the diagram of figure 2.1, entrance aperture, collimator, disperser, camera, and exit aperture.

\subsubsection{Process Flowchart}

The flowchart, also called a process map, is a data representation technique widely used in project management to describe procedures and processes. It shows the sequence of steps and the branching possibilities that exist for a process that transforms one or more inputs into one or more outputs and enables a quick understanding of the functioning of the process (PMBOK, 2017). This tool is used to describe the methodologies explored for the development of the GMACS optical system.

The general guideline to make an effective flowchart is (i) define the process; (ii) define the scope of the process and the level of details that will be included in the diagram; (iii) include the activities that take place during the process; (iv) organize activities in an appropriate sequence; (v) draw the symbols for the activities and; (vi) draw arrows to show the process flow.

All the flowcharts were generated by "Draw.io" ${ }^{5}$, a free online diagram software for making flowcharts, process diagrams, org charts, UML, ER, and network diagrams.

\subsubsection{Decision Analysis - Tradeoff Studies}

According to Cilli and Parnell (2014, p. 313), "tradeoff studies are a critical tool to provide information to support decision making for discipline engineers, systems engineers, and program managers throughout the system life cycle". The objective is to allow an

\footnotetext{
${ }^{5}$ Available at https://about.draw.io/. Accessed on 2016-06-20.
} 
"in-depth understanding of the complex relationship between requirements, the design choices made to address each requirement, and the system level consequences of the sum of design choices across the full set of performance requirements, as well as other elements of stakeholder value to include cost and schedule". Tradeoff studies are an SE tool that allows multidisciplinary teams to identify the most balanced technical solution amongst a set of proposed viable solutions (Johns, 2001).

The tradeoff analysis was widely used in this research for quantitative and qualitative design choices. The adopted quantitative one, detailed in Johns (2001), comprises the following stages: (i) define the objectives; (ii) establish trade parameters; (iii) identify viable alternative; (iv) establish scoring criteria; (v) establish weighting; (vi) score each option for each of the trade parameters; (vii) calculate the total score for each option; (viii) eliminate low scoring outliers; (ix) evaluate weighting sensitivity of remaining options; and $(\mathrm{x})$ document the trade matrix and final selection. A qualitative methodology shares the same stages, except the ones related to score, weight and trade matrix ones.

The expected output of the tradeoffs is a recommended course of action and associated implementation plan provided in the form of a high-quality decision report (Cilli and Parnell, 2014).

\subsection{Optical Spectrograph Design}

\subsubsection{Basic Principles for General Optical Design}

The optical design development used in this work follows the general methodologies for optical imaging system development. Shannon (1997), Smith (2007) and Fischer et al. (2008) are indispensable references for an extensive understanding of optical design development, including tolerance analysis, mounting, alignment and case studies. Although applied to infrared systems, Holst (2008) and Willers (2009) are indispensable references for a better comprehension of electro-optical system modeling, design, and analysis, which is valuable for MOS optical design. In addition, Zebase, Smith (1992) and Laikin (2006) provide a comprehensive list of optical design starting points.

A fundamental part of the optical design is the determination of the best Merit Function (MF) for a given set of requirements. MF is a function that measures the agreement between the data and the fitting model for a particular choice of predetermined parame- 
ters. Since the MF, by convention, is small when the agreement is good, the goal of all optimization methods is to reduce this MF as much as possible. A proper MF definition is essential to achieving the design goal and speeding up the optimization processes.

The starting design for any optical design, as described by Malacara-Hernández (2004), may be set up in several different ways: (i) select similar existing designs in the technical literature (refer to section 4.2.6); (ii) scale of an existing design that shares similar f-number and principal features with GMACS, such as entrance pupil size and location, EFL, and spectral bandwidth; (iii) substantially modify a design with characteristics at least close to the ones desired; and (iv) first-order design the system from scratch and later use third-order aberration theory to obtain an approximate design.

\subsubsection{Glass Selection for ELT Instrumentation}

Glass selection is a fundamental beginning part of any development of optical systems. The selected glass characteristics such as dispersion, internal transmission, thickness and diameter of available blanks, costs, lead time, ease of fabrication, resistance to environmental conditions, such as radiation, humidity, and thermal variation, must be under the instrument requirement. Oswalt and McLean (2013) states that this process is highly iterative since the suppliers' properties cited above may change during the project duration.

Glass information is frequently updated and made available by manufacturers, such as Schott, Ohara, and Nikon, on their web pages. Manufacturers also provide periodic updates of glass catalogs in a specific file format for use in the most known optical design software. In the case of Zemax, these file extension are ".agf" (Zemax LLC OpticStudio, 2016). Zemax allows the customization of private glass catalogs from the original suppliers in the software, which empowers the designers to create specific catalogs. Below we describe the Glass Analysis Python Script developed which reads the Zemax ".agf" files for several data processing purposes.

\subsubsection{Glass Analysis Python Script}

Although the management, access, and visualization of glass information can be done within the Zemax GUI environment, it has stringent limitations for more complex and broad studies and analyses. In order to mitigate this issue, we developed a Python script 
based on the Nathan Hagen algorithm (MIT/X11 License) ${ }^{6}$, which has a set of utilities for reading the Zemax ".agf" glass catalogs, correlating glass properties, and data displaying. This tool allows quick implementation of several methodologies available in the literature for glass selection, such as Hariharan (1997); Gruescu et al. (2008); Albuquerque et al. (2012); Jones (2014). We used this tool to select the most suitable combinations of glasses for achromatism as a function of the spectral bandwidth, internal transmittance, suppliers' status (such as standard, preferential, and obsolete) directly from the data from manufacturers via Zemax Glass Catalogs.

\subsubsection{Requirement Definition and Flow Diagram}

Similar to other GMT instrument developments, the GMACS team was first charged with developing the science requirements and then deriving the technical, functional, and operational requirements for the GMACS concept (GMTO Coorporation, 2013e). According to Faes et al. (2018), the requirements flow-down for GMACS starts from the identification of the scientific cases, operational aspects, and constraints imposed by the observatory, which are the input for the first draft flow-down that guides the development activities of the technical team.

However, due to the large number of external factors that influence the decision-making process, such as the innovative design feature, the high degree of instrument complexity and interfaces, the budgetary limitations, and the stakeholders' expectations, we conceived a cyclical approach top-down requirements, see figure 4.3. The players can iteratively influence science and technical decisions that will guide the execution of the project. The participation of the optical design group, which makes part of the Instrumentation Group, is essential in this cyclical phase of elaboration of requirements and preliminary performance studies. Moreover, the instrumentation group's experience is essential to making decisions that will reduce the possibilities and narrow down the requirements.

Despite not being the idealized top-down approach for generating the instrument requirements, the cyclical approach applied to this research proved to be effective based on the following. The changes in specifications that occurred during the project's execution demanded that critical functional parameters be revised in relatively advanced phases of

\footnotetext{
${ }^{6}$ Open source under the MIT/X license. Available at https://github.com/nzhagen/zemaxglass. Accessed on 2016-02-10.
} 


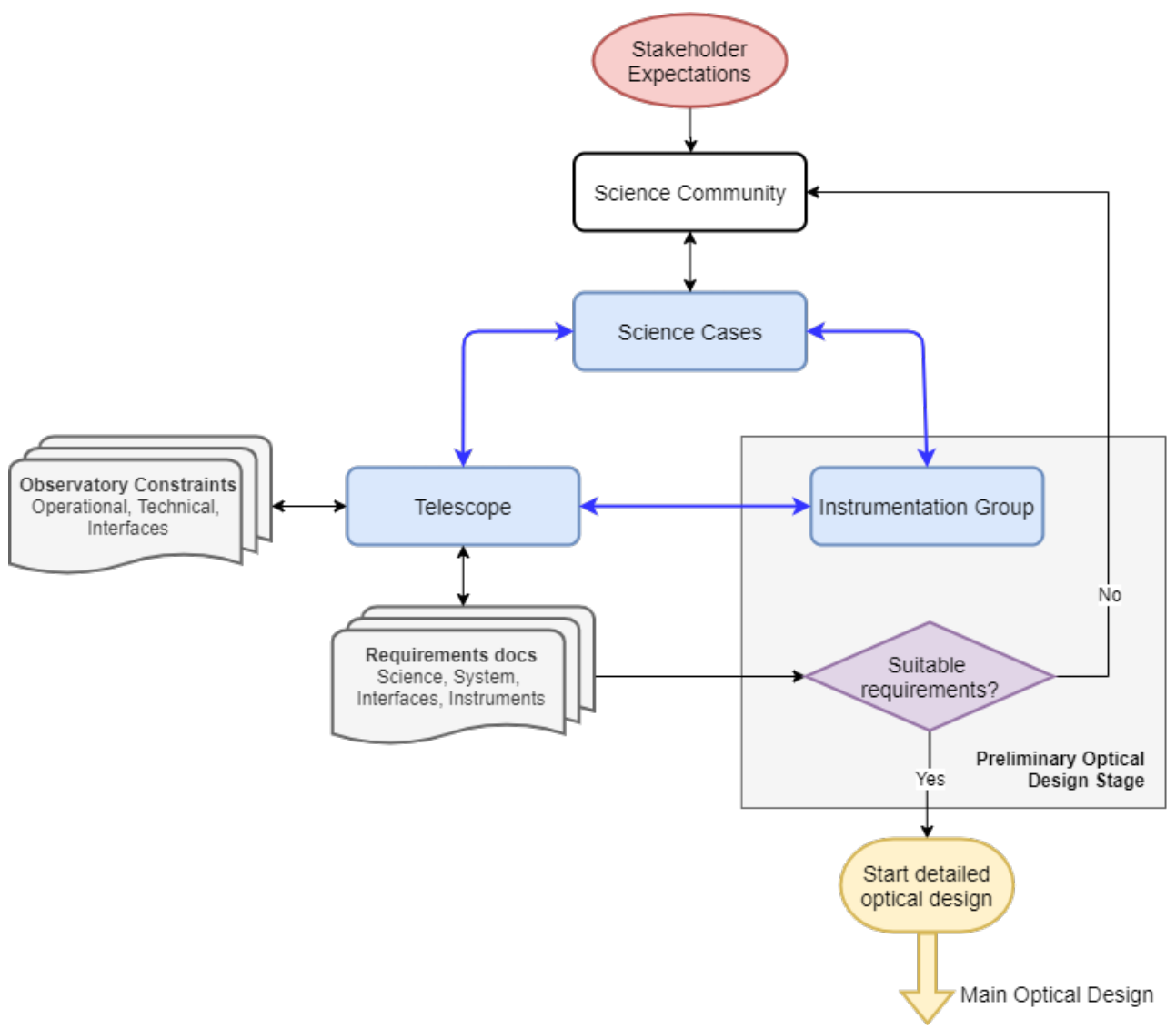

Figure 4.3: GMACS Requirements cyclical flow diagram.

the optical project, which in turn required the elaboration of several alternative of the drawings at the same or similar design maturity to compare performances. This approach is similar to the term "Design Loop" used by Keller et al. (2015) for a technical requirement definition process. Therefore, the proposed optical design methodology anticipates and adapts to the execution conception of this astronomical instrumentation project.

\subsubsection{Preliminary Phase}

\subsubsection{Principal Parameters Trade Study}

The principal parameters trade study goal is to establish the range of the subsystems' optical parameters that meet the instrument's requirements and allows for realistic manufacturing, assembly, and operation.

The first phase is the definition of the optical subsystem's specifications to meet the high-level requirements of the instrument. The system is split into essential components: the slit, collimator, disperser, and camera. 
The inputs for this phase are the instrument requirements, suppliers' capabilities for high-risk components (for both manufacturing and lead time), design constraints, and prior designers' experience with similar instruments.

The first-order theoretical basis is the imaging optical systems and VPH grating spectrograph equations, as described in chapter 2. The parameter constraints originate from different sources, such as (i) instrument requirements; (ii) manufacturing limits of components (e.g., the maximum diameter of the diffractive element); (iii) suppliers' capabilities for high-risk components (for both manufacturing and lead time purposes); (iv) design complexity (faster viable optical systems); and (v) design restrictions. Indirect constraints, such as subsystem compactness, are left for future tradeoff analyses. As mentioned above, it is imperative to consider the lessons learned from the prior experience of the development team members in similar instruments.

The expected result is a list of first-order optical parameter ranges for each subsystem, which will guide the following optical design processes.

\subsubsection{Paraxial Modeling}

According to Fischer et al. (2008, p. 781), the term paraxial is related to "the region where the angles between the rays and the optical axis are small, and the approximation that the sines and tangents of the angles can be represented by their values, in radian, is valid. This makes computations fast and easy, and provides for a convenient means of location, for example, the image positions without regards to aberrations." The paraxial model of the optical system, assisted by optical design software, is one of the first phases of the optical design. It aims to (i) verify that the functional parameters, previously defined in the requirements and specifications phases, are not in conflict, (ii) certify the positions and dimensions of the pupils; and (iii) confirm the maximum performance of the system, since the performance of the paraxial modeling is, by definition, at the diffraction limit.

The paraxial modeling of the telescope must be incorporated into the instrument's paraxial design. The spectrograph must contain all its components: collimator (including dichroic(s)), dispersing element(s), camera(s), and detector size. Most of the available commercial optical design software have multi-configuration capabilities, useful for including the parameters for each resolution mode such as collimator-camera angle, bandwidth, and field data. The performance evaluation criteria and the elaboration of the MF are also 
started during the paraxial phase.

\subsubsection{High-Level Design Trade Studies}

The next objective is to compare the estimated optical subsystem performance of several high-level design concepts. A straightforward starting reference point is instruments that share requirement similarities which were approved by the astronomical community. The key output is any earlier identification of any technical challenges for design, fabrication, and assembly for each proposed concept. It is usually done by tradeoff analysis of the selected subsystems. This phase is crucial for the following steps because it deals with the interaction of the subsystem and takes into account the mechanical, electrical, and, most importantly, operational issues. Usually, the tradeoff subjects are defined during the execution of the project. However, some of them are foreseen since the beginning of the project or required by the stakeholders.

Similar to other large projects related to scientific instruments, GMACS will have only one unit fabricated and commissioned. It is usual to occur changes in the science requirement during the development due to several reasons, e.g., in light of the trade analysis results, feedback from suppliers, or stakeholders' requests. In any case, they must be identified as soon as possible to avoid any crucial late changes in the optical design, which might compromise the project schedule and budget.

\subsubsection{GMACS Optical Design Methodology}

The proposed GMACS optical design methodology is described in the GMACS Optical Design Flowchart, see figure 4.4.

The input is the preliminary optical design phase resulting from the requirements definition flowchart of figure 4.3. The design methodology has independent subsystems and integrated system optimizations, of which each of the optimization blocks assigns to the traditional optical design processes followed by the dedicated requirement verification. The tolerance analyses are performed after an internal review by the optics group so that any design idea can be incorporated into the optical design. Any restrictions from mechanics are considered as requirements and can be subject to review. 


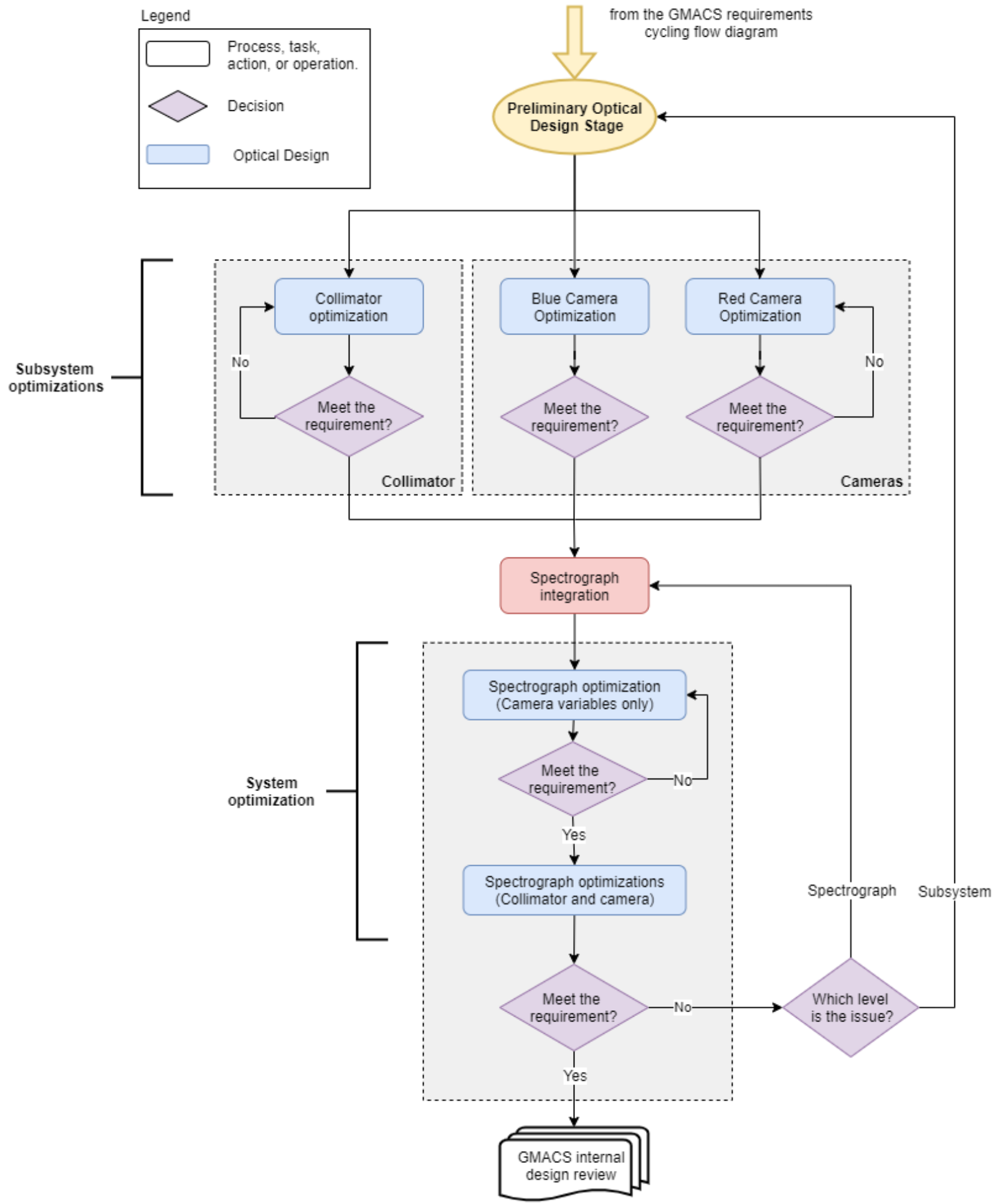

Figure 4.4: Flow diagram of the methodology for the GMACS optical system design, continuation of figure 4.3 .

\subsubsection{Subsystem Optimization Phase}

The next step is related to the first round of the subsystem optimization. The best design solutions that resulted from the tradeoff analysis are explored in parallel. In this 
section, we explore the details of the GMACS optical subsystem optimization.

In the case of the two-channel design, the collimator and cameras share similar MFs, quality criteria, and overall design optimization strategy, although the spectral range and, consequently, the glass selection are individualized. The refractive element constraints are the glass type, element center thickness, and edge thicknesses. Considering that we use the multi-configuration optimization approach, the center glass and air thickness can be set up only for one single-mode, while the edge must be set up for each configuration individually, given that the marginal ray height is a function of field and wavelength.

The RoC must satisfy the conditions for decreasing the AOI as much as possible to avoid significant sensitivities. The plate fit analysis is not performed, since we are expected to fabricate only one GMACS unit.

The use of aspherical surfaces in ELT instrumentation is inevitable for the correction of high aberrated rays, even if considering the risks regarding manufacturing and testing. Fischer et al. (2008) states that an aspheric surface can replace up to five surfaces, which is decisive for image quality and high throughput. Two criteria to quantify the complexity and risk of manufacturing such surfaces are the Best Fit Spherical Deviation (BFSD) and the slope. Both parameters are provided by Zemax's Best Fit Sphere (BFS) Data function, BFSD, and must be inserted in the configuration that requires the largest lens dimensions since they are a function of the lens radial diameter. The surfaces chosen to be aspherical should be located as close as possible to the pupils to avoid that localized figure errors compromise the quality of the final image. In other words, the aspherical surfaces should be located as far as possible from the focal plane of the telescope and the focal plane of the spectrograph camera. Finally, based on manufacturing feedbacks, the general recommendation is that only the concave surfaces should be aspherical (source: suppliers' emails).

Collimator: We decided to incorporate the GMT telescope model in the collimators' optimization process to guarantee that the incoming beam to the collimator has a realistic GMT performance, including the telescope focal ratio, field curvature, and any other field aberrations. The same approach is used in the integrated phase.

The collimator optimization steps depend on the previously selected architecture, which in turn is supported by trade-off studies that include any constraints from optical perfor- 
mance, optomechanical design, manufacturing, flexure and stability, and budget.

The main objective of a spectrograph's collimator is to collimate the incident radiation at the dispersive element plane for the spectrograph field of view and bandwidth. This will ensure that, for a grating-based spectrograph, the entire grating, regardless of the mode of operation, is evenly illuminated by a flat wavefront.

Although the afocal approach is the most obvious to use since the GMT+collimator is an afocal system, we decided to use a paraxial surface to simulate the camera located just after the exit pupil plane; therefore, we could use the default Zemax MF to minimize the RMS spot radius with reference to the centroid. We noticed that the wavefront based MF is inefficient for the earlier design phase. The image quality criteria commonly used for imaging systems, such as microscope lenses and eyepieces, can be directly adopted. However, it is necessary to include additional criteria and metrics for the position, diameter, and quality of the exit pupil in the MF as a function of field of view and wavelength.

Based on the relationship between the image quality of a paraxial surface and the wavefront flatness incident on this surface, both the approaches are valid for the collimator optical quality metrics. The result of these metrics is what defines, then, the optical quality of a collimator.

From a ray tracing perspective, a low-quality collimator causes the rays coming from the same object to hit the plane of the diffraction grating with a broad range of incident angles, which in turn diffracts with different angles. Thus, the spectrograph spectral resolving power presents a complex relationship with field and wavelength. According to Palmer and Loewen (2014), the quality of the collimated beam in the direction orthogonal to the grating lines may be lower than in the perpendicular direction, as there is no diffraction in the first situation. However, since the system is radially symmetrical, we have no distinction between the tangential and sagittal directions.

Since the ultimate spectrograph goal is to generate clear images of the slits in the camera focal plane, any collimator residual field and chromatic aberrations can be balanced with the camera. This strategy must be considered in the design process of an ELT spectrograph.

Camera: The camera entrance pupil location must be at the camera's pivot center with a suitable relief to allow the grating mount to rotate without mechanical interference. The required entrance pupil diameter and the FoV result from the principal parameters study. 
The FoV, since it is proportional to the diffracted angle range, must be calculated for the higher resolution mode. The adopted MF shares similarities with the one used for imaging camera that minimizes the RMS spot radius with reference to the centroid and, since the camera is not diffraction-limited, the performance metrics are:

1. Spot diagram as a function of the resolution size $\left(\mu m^{2}\right.$ or $\left.\operatorname{arcsec}^{2}\right)$;

2. Ensquared or encircled energy for $80 \%$ of energy;

3. Spherochromatism;

4. Field curvature (Petzval);

5. Lateral color (for imaging); and

6. Vignetting in spatial direction.

\subsubsection{Integrated Optimization Phase}

The output of the previous step is acceptable collimator and camera designs. The next step is to integrate them into a GMT and grating model, keeping only the camera's elements as variables. The MF and multi-configuration editors must be adapted to the spectrograph image performance metrics.

The spectrograph model has essential differences compared to the previous subsystem optimization processes due to the incorporation of the dispersive element. For the same object field, the diffracted rays are focused along the spectral direction of the detector. From a ray tracing perspective, the grating generates bundles of rays whose exit angles depend on the entrance ray angle, wavelength, and the grating parameters. Any lateral color constraints available on the Zemax default MF must be removed since the grating dispersion effect is considered similar to an asymmetrical lateral color.

The multi-configuration approach is used to model the image and spectrograph modes. The grating and configuration parameters set the blaze wavelength, which is considered the central wavelength for each spectrograph mode. Finally, their respective spectral ranges are set according to the blaze wavelength and the camera's principal parameters, such as EFL and detector size, in the dispersive direction. All the configurations are optimized together, although the unique MF can be customized for each configuration by using the flag "CONF" in Zemax. We created macros to automate the process of insertion data in the multi-configuration editor to reduce errors. 


\subsubsection{Tolerancing}

The objective of the tolerance analysis of an optical system is to distribute and error-bud the manufacturing and mounting tolerances of the optical and optomechanical elements or groups throughout the system to ensure the manufactured system will meet the required level of performance at a reasonable cost and time scale. Fischer et al. (2008, p. 348) say that "the key point is that manufacturing errors in the form of fabrication, assembly, and alignment errors can be extremely important and are a major contributor to the overall level of performance of an optical system, even if the paper design is excellent." According to Zemax Development Corporation (2011, p. 531), "ZEMAX provides a flexible and powerful tolerance development and sensitivity analysis capability. The tolerances available for analysis include variations in construction parameters such as curvature, thickness, position, index of refraction, Abbe number, aspheric constants, and much more. ZEMAX also supports analysis of decentration of surfaces and lens groups, tilts of surfaces or lens groups about any arbitrary point, irregularity of surface shape, and variations in the values of any of the parameters or extra data. Since the parameter and extra data terms may describe aspheric coefficients, gradient index coefficients, and more, any of these values may also be made part of the tolerance analysis. The various tolerances may be used in any combination to estimate alignment and fabrication error effects on system performance". Figure 4.5 shows the flowchart for the tolerancing process proposed by Landau (2007).

OpticStudio Zemax's tolerance comprises sensitivity, inverse sensitivity, and MonteCarlos analysis. Sensitivity is an optical design tool used to measure the sensitivity of each of the user-defined parameters by perturbing it by a given value and calculating the degradation of predetermined performance criteria. In contrast, inverse sensitivity is the determination of the maximum perturbation that a determined parameter can stand for a fixed performance degradation. Sensitivity and inverse sensitivity analyses consider the effects on system performance for each tolerance individually. Monte-Carlo analysis considers the combined effects of the individual perturbations by randomly generating perturbed optical designs (Zemax LLC OpticStudio, 2016).

There are potential tradeoff studies between operational performance and tolerance sensitivity, given that relaxed tolerances help mitigate the risk of problems during instrument alignment, integration, and test. 


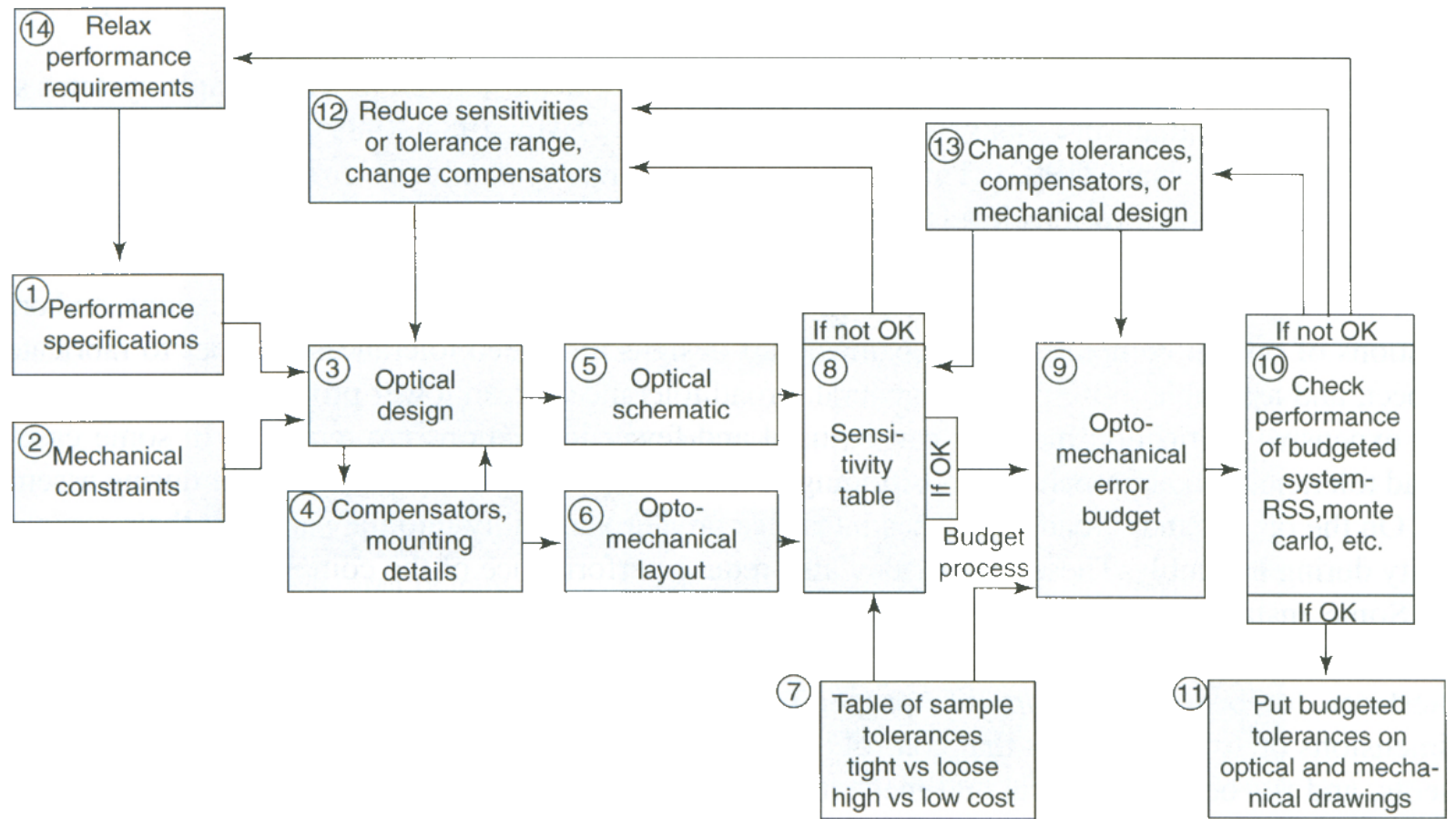

Figure 4.5: Flowchart for Tolerancing Process. Source: Landau (2007).

Although Zemax has a build-in tolerance function called TOLR (an optimizing tolerance sensitivity) that relaxes the design sensitivity during the optimization process according to the predefined Tolerance Data (Zemax LLC OpticStudio, 2016) and uses the user-provided tolerance data to minimize all the possible combinations of these parameters, it requires too much computational processing that inevitably takes longer to optimize. Hence, we decided to avoid using TORL. In addition, there are methods which constrain the system's parameters that are sensitive to disturbances (Albuquerque et al., 2016); we decided not to use them given the time required to learn and adapt the concept to the GMACS optical design. Therefore, we decided to adopt a methodology based on conventional optical design best practices, such as reducing AOI at optical interfaces, controlling the diameter/thickness ratio, restricting the minimum distance between lenses and the minimum thickness of lens edges, balancing lens power, experience, and common sense. 


\subsubsection{Sensitivity Analysis}

The sensitivity equation, Eq. 4.1, shows the degradation of a performance criteria, $M$, for the parameters $x$ and $y$.

$$
\begin{aligned}
& M=\frac{\partial M}{\partial x} \partial x+\frac{\partial M}{\partial y} \partial y+\cdots \\
& M=S_{x} \partial x+S_{y} \partial y+\cdots
\end{aligned}
$$

where $S_{i}$ is defined as the sensitivity of the $i$ parameter. The signal depends on the quality criteria adopted. For instance, decreasing the spot size or increasing the MTF makes the MF decrease.

The Zemax Tolerance Output file (.TOL) is the text file that resulted from the Tolerance Script. It contains the tolerance operand with a four-letter mnemonic, such as TRAD for tolerance on a surface radius of curvature in lens units, TTHI for tolerance on thickness or position in lens units, and so on (see Zemax LLC OpticStudio, 2016, Chap: The Tolerance Tab). These output sensitivity results are usually laborious to carry out the reading, so we propose an alternative visual sensitivity analysis resulting from the Sensitivity Diagram Script Tool. The Sensitivity Diagram Script reads the .TOL file, and all the tolerance operand data recorded in a Pandas Dataframe for Python ${ }^{7}$. Figure 4.6 illustrates the output which is based on the Seidel Diagram available in Zemax. The diagram shows each optical design element as a progressive column and explicitly highlight the aspheric surfaces with a red line.

\subsubsection{Flexure Analysis}

Although flexure analysis is part of the tolerance study, we dedicate a separate subsection to discuss this topic due to its relevance in the GMACS development.

\subsubsection{Preliminary Flexure - Group Tolerances}

The first objective of the preliminary flexure analysis is to determine which of the groups and elements cause considerable spectral displacement in the focal plane due to tip

\footnotetext{
${ }^{7}$ Python Data Analysis Library. Available at https://pandas.pydata.org/. Accessed on 2016-02-02.
} 


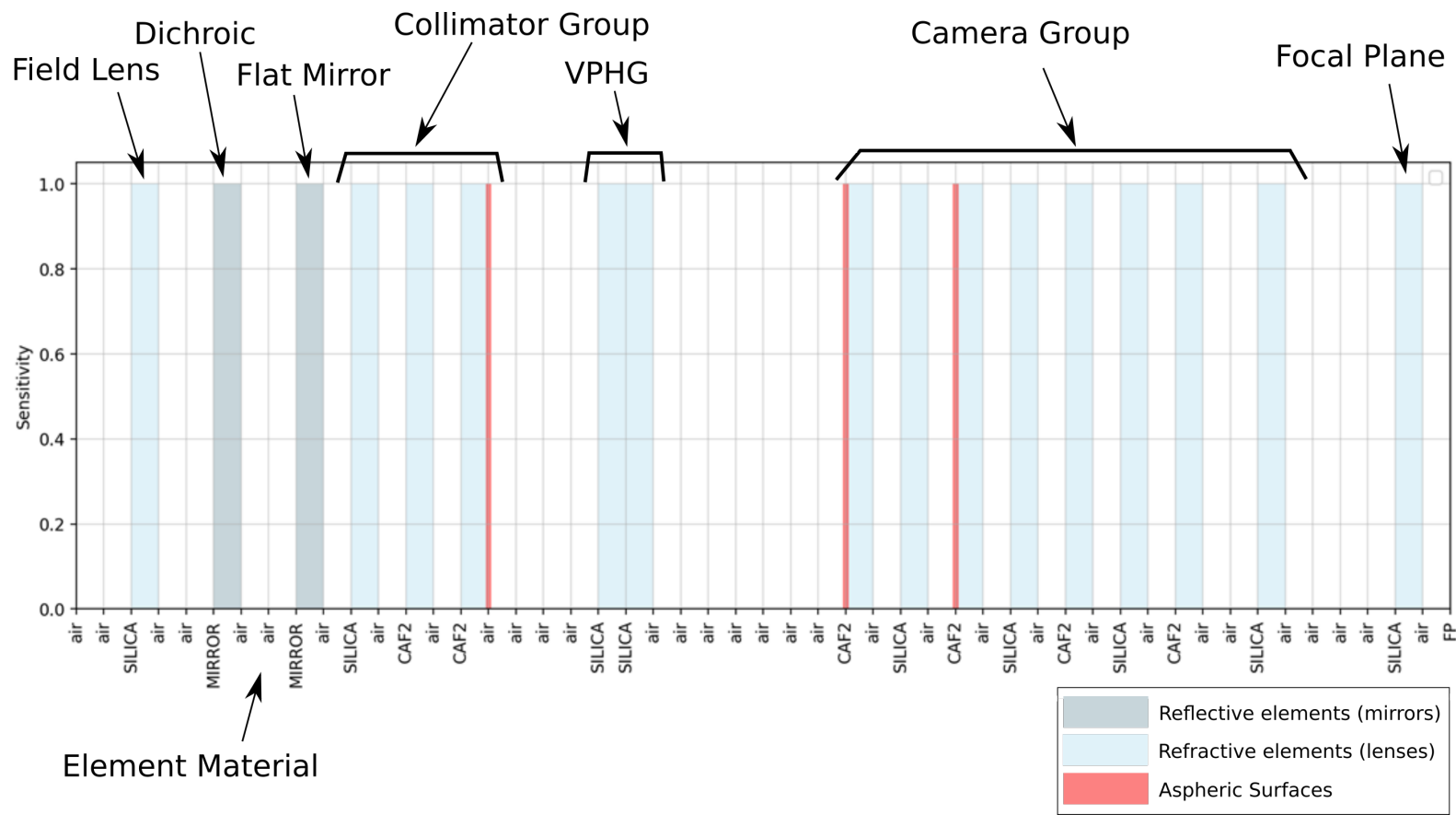

Figure 4.6: Sensitivity Diagram example generated by the Sensitivity Diagram Script.

and tilt perturbations. The secondary is to quantify which optical element or group is the best candidate for active control to compensate for this flexure. The principle of the flexure MF is to track the displacement of the spots centroid or the chief rays of the image slits on the focal plane. The choice between the spot centroid or chief ray to be the parameter must take into account the time required to run the scripts and whether the flexure causes a significant image degradation. Therefore, the flexure study should incorporate metrics related to image quality since, at first, it is unknown whether the image degradation caused by flexure is restricted to spectral stability only. Thus, the flexure MF must also incorporate the parameter EEDxx (parameter of Image Quality, IQ) and spectral stability (SS) given by the vectorial difference between the nominal and perturbed chief rays or centroids as a function of field and wavelength. The results are compared to the maximum allowed displacement given by the requirements. We noticed that the flexure caused by the gravity vector is not sufficient to create aberrations on the spot such that the coordinates from centroid or chief ray parameters are significantly different. Therefore, we adopted the chief ray as the flexure parameter, although the IQ was always tracked.

The central assumption for the flexure in this research is the consideration that the optical groups or elements act as solid bodies. Therefore, the effects of the flexure are 
described by 3-D rigid body transformation, called the Euclidean or Rigid transform, and it preserves the shape and size. Since Eggert et al. (1997) conclude that the only truly distinguishing factor among the algorithms to compute 3-D rigid-body transformation is the execution time, we have adopted the Singular Value Decomposition (SVD) of a matrix derived from the standard $[\mathbf{R}, \mathbf{T}]$ representation. The Euler angle computation and analysis used are described by Slabaugh (2000) and Day (2010), and the translation to Zemax as described by Cheng and Humphreys (2017), Day (2010) and Jones (2018). Ribeiro et al. (2018a) describe the preliminary results from the application of this methodology using FEA results of flexure simulations of the GMACS structure due to the gravity vector influence.

The flexure analysis has the following progressive steps for the FEA-Zemax integration. In Zemax, a database, called Nominal Database, gathers the information of all Chief Ray (CR) coordinates as a function of field and wavelength. After inserting the FEA processed results in the optical design software (see section 4.4.7.2), a new database is calculated, named Perturbed Database. The CR coordinates are calculated thought a ZPL script based on the function. The vectorial displacement is calculated as the difference from the nominal to the perturbed coordinates. A Python script is used to process the raw FEA data into the format required to import to Zemax. We describe the steps in detail in the next section.

\subsubsection{FEA-Zemax Integration}

The integration between the FEA results and Zemax is composed of steps to determine the solid-body rotation Euler angles and coordinate changes. Table 4.1 shows an example of the raw FEA data coordinate nodes for three planes and the corresponding offsets. 
Table 4.1 - FEA results data example for three planes, identified as A, B, and C, each with three nodes. Source: GMACS Team Internal Report.

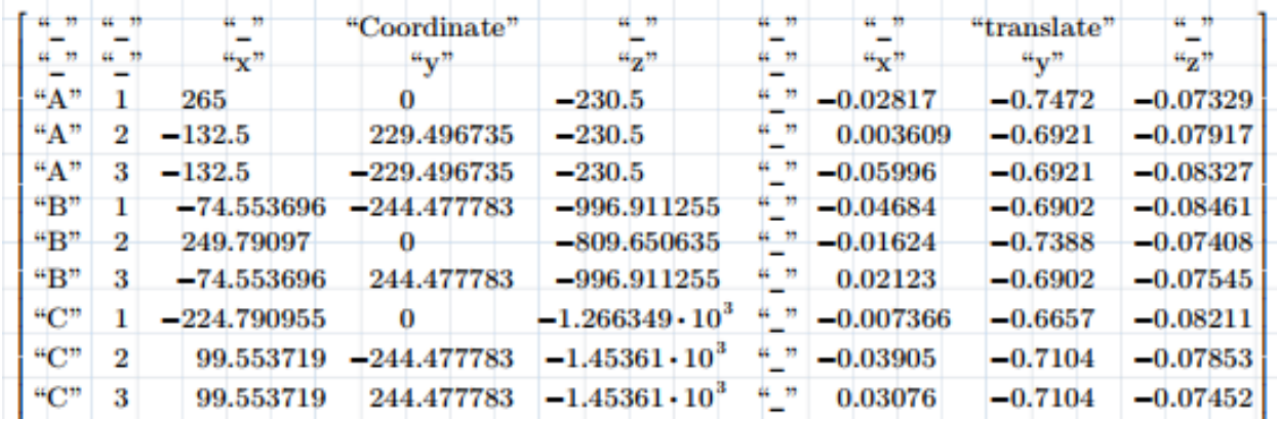

Since Zemax's sequential mode works only with local coordinates, it is necessary to switch each of the optical groups from local to global coordinates. Although Zemax has an automatic inbuilt tool to switch from Local to Global and Global to Local coordinates, it showed to be inaccurate with existing CBK in the LDE. Therefore, we created a Zemax macro that automatically inserts the CBK and the decenter and tilt values with the reference plane provided by user input by following the steps: (i) the user is asked for which surface must be changed from a local to the global coordinate; (ii) the macro automatically identifies the reference surface (the comment must be "FEA REFERENCE") and sets it as Global Coordinate; (iii) it automatically inserts three CBK surfaces for the decenter and tilt; (iv) it calculates the Euler angles based on the rotation matrix and inserts the results, as well as the decenters, into the corresponding CBK parameters ${ }^{8}$; and (v) it automatically renames the comments sequentially, considering all the existing local-to-global surfaces.

The resulting LDE layout is illustrated in figure 4.7 .

\begin{tabular}{|r|r|r|r|}
\hline & & \multicolumn{3}{|c}{ Order } \\
\hline Coordinate Break - & NODE 01: return & $-0,000$ R & 1 R \\
\hline Coordinate Break - & NODE 01: decenter & 0,000 & 0 \\
\hline Coordinate Break - & NODE 01: tilt & 0,000 & 0 \\
\hline
\end{tabular}

Figure 4.7: Zemax coordinate breaks to move from local to global coordinates.

\footnotetext{
${ }^{8}$ During the next GMACS project phase, we will implement the option to calculate the Euler angles and offsets with reference to any unperturbed FEA plane. Therefore, from the optical design perspective, it will be possible to isolate the outside flexure (generated, for instance, from the instrument mounting structure, which can be compensated by the telescope guiding system) from the inside one (which effectively affects the image stabilization and must be active or passive compensated).
} 
This macro does not work for both modes simultaneously, because it requires additional changes in the MCE. Figure 4.8 shows an example of the LDE with three nodes. Another macro was developed to delete all the CBK in order to recover the previous LDE.

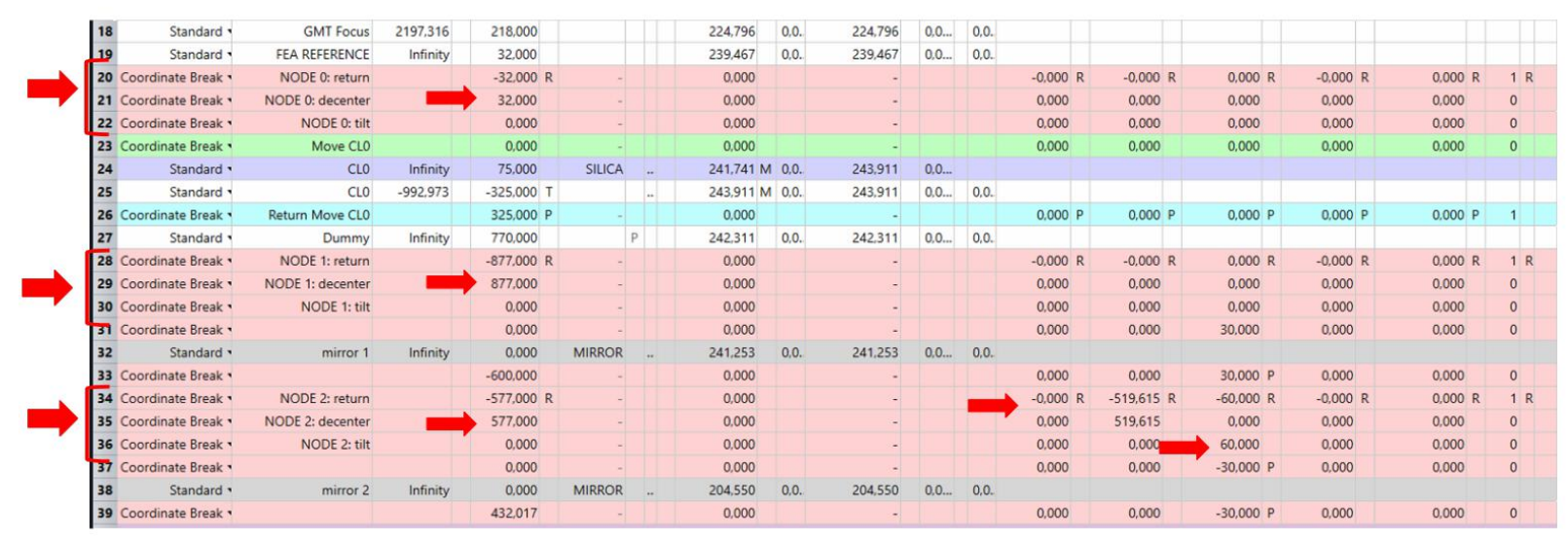

Figure 4.8: Example of the LDE with three nodes after applying the local-to-global macro.

The general workflow process is as follows (see figure 4.9):

1. Gather the FEA reference plane location for each group under analysis;

2. A dummy surface is added in Zemax at the location of the FEA reference planes before each lens group surface. In case the FEA pivot point is not located at the first surface of the optical group, it is necessary to include two sequentially CBKs separated with a dummy surface to move the pivot point. The thickness of the 2 nd CBK must be picked-up with factor -1 . The dummy surface is for local-to-global coordinate changes;

3. The FEA output is recorded in a text file with all the nominal coordinate "tab spaces" in individual lines followed by their respective variation;

4. The text file is read by a Python script that applies the 3-D rotation matrix to convert the FEA coordinate plane system to the Zemax coordinate by calculating the translation vector $\mathbf{T}$ and $3-\mathrm{D}$ rotation matrix $\mathbf{R}$. The user can choose to perform the order of the matrix,; however, the default is the one used for Zemax. From R, we can calculate the corresponding Euler angles. The translation vector $\mathbf{T}$ is calculated based on the displacement of the centroids (center of gravity) between the two planes. This step is done by a Python Script: a prompt window is opened for the user to 
indicate the text file with the coordinates. A text file is automatically generated, with the addition of "_R_and_t". This script also takes into consideration the axis orientation of the FE model and the Zemax model by a user-defined matrix or Euler angles; and

5. A script sets the origin of the global coordinate system (center of the slit mask) and adds three surfaces before and after each optical group to return to the global reference surface, introduce the coordinate translations and rotations, and finally return to the original coordinate system. A support script allows the user to remove these surfaces for debugging purposes.

The rigid transformation methodology and ideas for the elaboration of the scripts was highly inspired in the works from Nghia $\mathrm{Ho}^{9}$, Satya Mallick ${ }^{10}$, Olga Sorkine-Hornung and Michael Rabinovich ${ }^{11}$. All the Zemax macros and Pythons scripts results were debugged and compared with the results from Jones (2018).

\footnotetext{
${ }^{9}$ Available at http://nghiaho.com/?page_id=671. Accessed on 2020-03-24

${ }^{10}$ Available at https://www.learnopencv.com/rotation-matrix-to-euler-angles/. Accessed on 2020-03-24

${ }^{11}$ Available at https://igl.ethz.ch/projects/ARAP/svd_rot.pdf. Accessed on 2020-03-24
} 


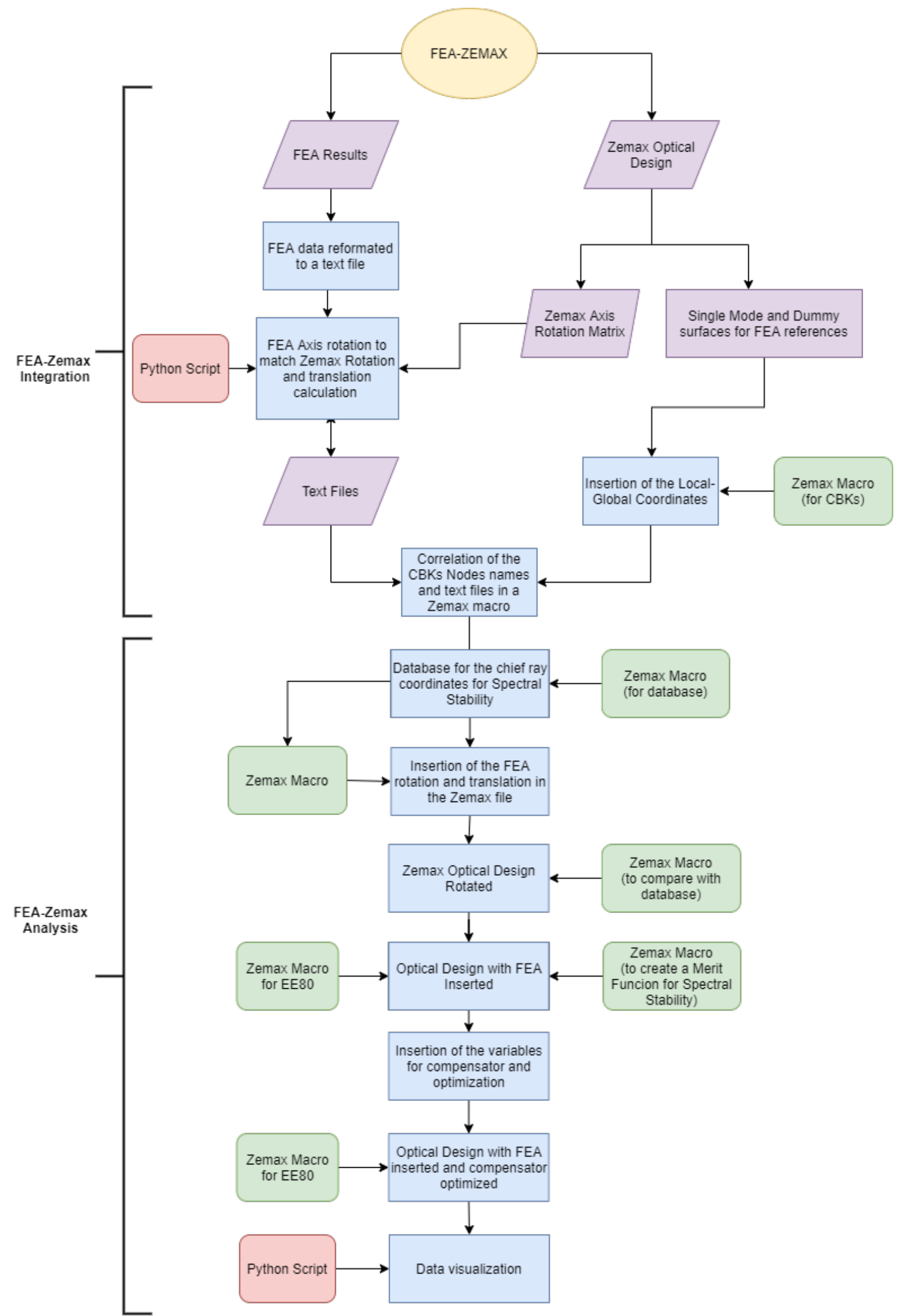

Figure 4.9: FEA to Zemax integration and spectral stability analysis diagram process. 


\subsubsection{FEA-Zemax Analysis}

The optical analysis is completely done with Zemax by customized macros (to insert the FEA data into the optical design and to calculated the CR coordinates) and custom MF (to optmize the IQ and SS for the compensators). The steps are:

1. The unperturbed CR coordinates and EED80 image quality for all fields/wavelengths are recorded in a text file;

2. The output (rotations and translations) from the Python script is loaded via a macro into Zemax with the surfaces comments in accordance with the macro;

3. The perturbed CR coordinates and EED80 image quality for all fields/wavelengths are recorded and the change from the unperturbed state is calculated;

4. A custom MF to minimized the spectral displacement and the RMS spot size for a given compensator is loaded. The objective is to determine which optical groups are best suited to compensation and the range of movement required; and

5. The correct CR coordinates and EED80 image quality for all fields/wavelengths are recorded and the change from the unperturbed state is calculated.

Finally, the CR displacement and EED data visualization (for instance, the EED histogram) is done with a Python script. 
Chapter 5

\section{GMACS Optical System Design}

This chapter describes the results of the proposed methodology applied to the conceptual optical system design of GMACS, from its conception to the performance analyses and the details of the computational support tools generated throughout the research.

Since the GMACS optical system design is embedded in the GMACS project framework, the input and output results, milestones, schedules, and proposed activities agree with the project management schedule and objectives. Therefore, several of the results presented in this chapter were made available in internal documentation for the GMACS and GMTO teams. These results, unless explicitly reported, were generated by the author of this thesis. Although the GMACS optical design development may seem pure optical engineering applied to ELT instrumentation, the optical design has shown unique challenges that justified dedicated research and development activities.

\subsection{Design Chronology}

This section briefly describes the GMACS optical design highlights since the GMT Conceptual Design Document in 2006 to the present stage.

2011 December: GMT Wide Field Optical Spectrograph: The ultra-wide field concept for GMACS was presented in the Proc. SPIE 8446, Ground-based and Airborne Instrumentation for Astronomy IV in July, 2011 (DePoy et al., 2012, 2014; GMTO Coorporation, 2013d). The concept employs a Zerodur ${ }^{\circledR}$ tent mirror placed after a Field Lens (FL) that splits the incident field into four separate segments across the field of the telescope (Oswalt and McLean, 2013). The multiple fly's eye collimator is based on a design strategy to increase the field of view by deploying multiple collimators followed by the gratings and 
cameras. The four-fold mirrors at the GMT focal plane redirected a $9 \times 18$ arcmin Field of View (FoV) to four individual arms and each comprised a double-beam spectrograph, resulting in a $162 \operatorname{arcmin}^{2}$. Hence, each arm has a $4.5 \times 9.0$ arcmin off-axis field. Figure 5.1 shows the optical layout. Section 3.1 provided details of the design.

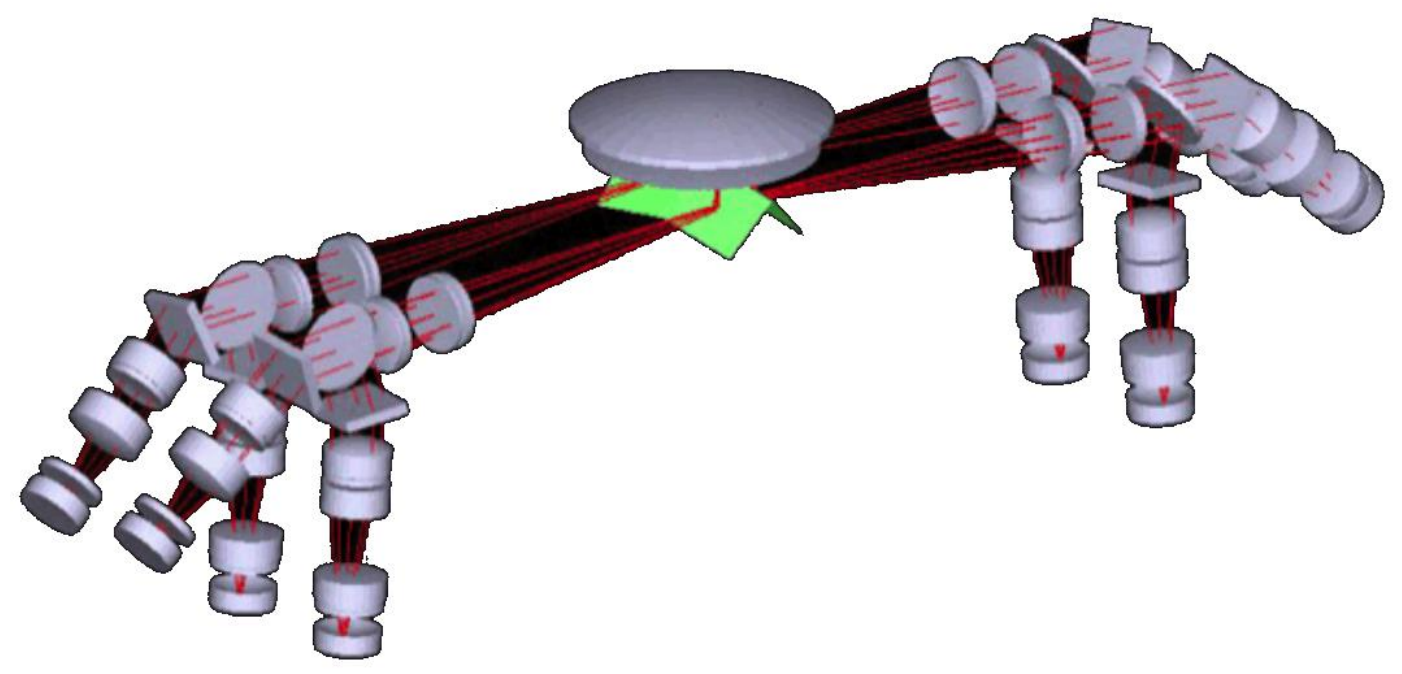

Figure 5.1: General optical layout of the GMT Wide Field Optical Spectrograph. The large lens in the middle is the last element of the GMT wide field corrector. The green "tent" looking reflections are the four mirrors that direct the quadrants into the individual spectrograph arms. Each arm consists of a two-channel spectrograph. Source: Adapted from DePoy et al. (2012).

Although the optical performance is acceptable for the required spot size and encircled energy, the design was not developed due to the high complexity, expected overall cost of the architecture and mechanical stability issues. The instrument selector panel recommended re-scope the design to an on-axis single spectrograph to reduce cost, complexity and risks.

2016 July: SPIE Design: A preliminary design was presented in July, 2016, during the Proc. SPIE 9908, Astronomical Telescopes \& Instrumentation, described by Schmidt et al. (2016) and Prochaska et al. (2016), in response to the GMTO Conceptual Design Study Statement of Work, SOW (Jacoby, 2016; Contos, 2016). The research activities of this thesis started in mid-2015, and the first results were in response to this SOW. Its main objective was to de-scope the Proc. SPIE 8446 design to an on-axis single collimator according to the functional parameters of GMTO Coorporation (2013d), shown in table 5.1. 
The fundamental optical architecture is based on a single panchromatic on-axis collimator with large pupil relief to accommodate both the $45^{\circ}$ tilt dichroic and tilt grating, followed by two spectral blue and red $\mathrm{f} / 2.2$ cameras.

2017 March: Kick-off Meeting: The KoM GMACS optical design, presented in March, 2017, consists of an upgrade of the 2016 SPIE design and retained the same architecture concepts. The glasses used are available from standard vendors such as Schott and Ohara, both known to provide the blank sizes and quality required for astronomical instrumentation application.

2017 July: 2017 Baseline: The 2017 Baseline GMACS optical design presented in July, 2017 had the main objective of increasing the throughput for wavelengths less than 350 nm. Although the fundamental architecture remained the same as the KoM design, the throughput was optimized by the replacement of some glasses by the Nikon NIGS family catalog, which presents high internal transmittance in UV spectral band and excellent alternative for color correction.

2017 September: Optical Design Baseline: The September 2017 design was discussed during the Technical Interchange Meeting, TIM. Several design recommendation and considerations were gathered, such as the concessions for using more $\mathrm{CaF}_{2}$ elements, air-spaced doublets and triplets to avoid unmatched Coefficient of Thermal Expansion (CTE) issues, the dichroic wavelength transition overlap between the channels and the trade for hexagonal collimator FoV. The throughput cutoff requirement was set to $320 \mathrm{~nm}$ because, despite atmospheric losses, it is a potential unique feature of GMACS with respect to other ELT MOS.

2018 July: Optical Design SPIE: The July 2018 design is the compilation results of the TIM and the outcomes from the trade studies for the collimator architecture in response to the SOW for Split Collimator Design (Contos, 2017). The design is comprised of a two arm split collimator for $320 \mathrm{~nm}$ throughput enhancement, with an astigmatism compensator for the red arm, followed by the two blue and red optimized cameras (DePoy et al., 2018; Ribeiro et al., 2018a,b). 
2019 July: Conceptual Design Review: The July 2019 Conceptual Design is the latest proposed design for GMACS, presented in details in this chapter and also presented in the CoDR held at TAMU on September 4-5th, 2019.

\subsection{GMACS Requirements}

Through collaboration with the GMTO, its partners and other representatives from the scientific community, a set of principal functional requirements has been developed, see table 5.1. On March 18-19, 2014, at Texas A\&M University, in College Station, a GMACS community workshop included more than 50 participants with interests in a broad range of research topics including (i) stars, star-formation, and planets; (ii) resolved galaxies (including dwarf galaxies) and near-field cosmology and (iii) distant galaxies (including reionization and first light science) (DePoy et al., 2014; Schmidt et al., 2016).

Although the main objective is to design a GMACS optical system that meets the requirement, we aimed to satisfy the goals as much as possible without significant design compromises, small increase in cost or schedule. In general, the goals are subject to technical and management trade since cost, risk and schedule might be affected. According to the GMTO, the supplier is only held to the requirement not the goal since the last are not contractually binding. The instrument requirements define the actual needs and the goals indicate desires.

\subsection{Preliminary Design Trade Study}

This section describes the results of the first high-level tradeoff studies, which makes part of the functional parameter definition phase according to the methodology described in section 4.4.5. In some cases, such studies have required significant advancement in optimizing steps to provide enough information on performance, complexity, cost and risk estimations to the decision-making GMACS team (composed by the Principal Investigator, PI, project manager, instrument scientist, and the interested technical teams). For example, within the context of the split collimator development (as described in section 5.7.3), it was needed to explore in detail a few alternative to reduce astigmatism caused by the dichroic located in a converged beam so that the result performances could be compared in a common level for tradeoff purposes. 
Table 5.1 - Summary of the principal functional parameters. Source: Jacoby (2016) and Jacoby et al. (2016).

\begin{tabular}{|c|c|c|c|}
\hline Item & Parameter & Requirement/Goal & Comments \\
\hline 1 & Field of View & $30 / 50$ arcmin sq & $\begin{array}{l}\text { Roughly equivalent to diameters } \\
\text { of } 8-10^{\prime} \text {. Interplay with items } 2 \\
\text { and } 3 \text { must be considered. }\end{array}$ \\
\hline 2 & $\begin{array}{l}\text { Wavelength } \\
\text { coverage }\end{array}$ & $\begin{array}{l}\text { Blue limit: } 350 / 320 \mathrm{~nm} \\
\text { Red limit: } 950 / 1000 \mathrm{~nm}\end{array}$ & $\begin{array}{l}\text { "Limit" refers to wavelength } \\
\text { where throughput drops to } 25 \% \\
\text { (blue) and } 25 \% \text { (red) of peak. In- } \\
\text { terplay with items } 1 \text { and } 3 \text { must } \\
\text { be considered. }\end{array}$ \\
\hline 3 & $\begin{array}{l}\text { Spectral } \\
\text { resolution }\end{array}$ & $\begin{array}{l}\text { Blue limit: } 1000-6000 \\
\text { Red limit: } 1000-6000\end{array}$ & $\begin{array}{l}\text { For a slit width of } 0.7 " \text { and the } \\
\text { R } \phi \text { product is constant. At the } \\
\text { higher resolutions, wavelength co- } \\
\text { verage may be sacrificed, yet full } \\
\text { coverage is required at lower re- } \\
\text { solutions. Interplay with items } 1 \\
\text { and } 2 \text { must be considered. }\end{array}$ \\
\hline 4 & Image quality & $80 \%$ EED at $0.30^{\prime \prime} / 0.15^{\prime \prime}$ & $\begin{array}{l}\text { This parameter may be linked } \\
\text { to resolution, use of MANIFEST, } \\
\text { and the GLAO observing mode. }\end{array}$ \\
\hline 5 & Throughput & $\begin{array}{l}\text { Desired }>40 \% \\
\text { at all wavelengths }\end{array}$ & $\begin{array}{l}\text { This parameter may be linked } \\
\text { to resolution, use of MANIFEST, } \\
\text { and the GLAO observing mode. }\end{array}$ \\
\hline 6 & $\begin{array}{l}\text { Spectral } \\
\text { stability }\end{array}$ & $\begin{array}{l}0.3 / 0.1 \text { spectral } \\
\text { resol. elements/hour }\end{array}$ & $\begin{array}{l}\text { Affected, for example, by flexure } \\
\text { or temperature change. }\end{array}$ \\
\hline 7 & $\begin{array}{l}\text { Grating } \\
\text { exchange }\end{array}$ & $1 />2$ & $\begin{array}{l}\text { Implies manual or remote grating } \\
\text { exchange operations. Interacts } \\
\text { with items } 1,2,3 \text {. }\end{array}$ \\
\hline 8 & $\begin{array}{l}\text { Slit mask } \\
\text { exchange }\end{array}$ & $12 />20$ & $\begin{array}{l}\text { The mechanism for exchanging } \\
\text { slit masks may be simplified with } \\
\text { a more complex slit mask design } \\
\text { concept. }\end{array}$ \\
\hline
\end{tabular}

\subsubsection{Principal Parameters Trade Study Results}

The assumptions and constraints for the principal parameters trade study is based on the instrument requirements from table 5.1 and the GMT optical properties, summarized in Appendix A (see table A.2 and table A.3). 
The first design constraint is that $f_{\text {col }}$ is limited by the maximum available grating size and the assumption of a maximum collimator-camera angle, $2 \alpha$, is less than $90^{\circ}$. Figure 5.2 shows that, according to equation $2.45, f_{\text {col }}$ shall be $\leq 2,600 \mathrm{~mm}$ in order to maintain the grating maximum allowed size at a maximum grating tilt for all the required range of the collimator-camera angle. The curvature of the GMT focal plane, which is $\approx 2,200$ $\mathrm{mm}$, is an important starting point to define the optimized $f_{c o l}$.

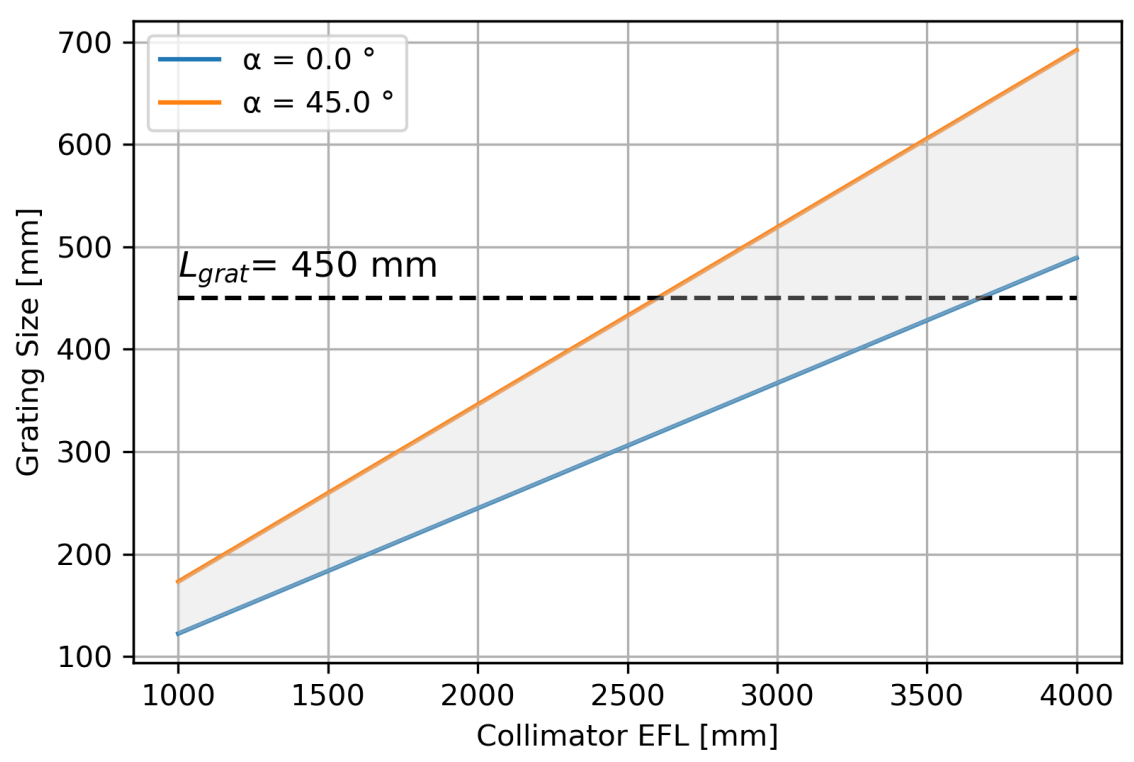

Figure 5.2: Grating size, $L_{\text {grat }}$, as a function of the collimator EFL for image mode $\left(\alpha=0^{\circ}\right.$ and maximum collimator-camera angle $2 \alpha=90^{\circ}$. The greyish area is the allowed region according to equation 2.45 . The $L_{\text {grat }}$ upper limit is provided by suppliers, and we assumed that $L_{\text {grat }} \approx 450 \mathrm{~mm}$, shown as the black dotted horizontal line. Therefore, the maximum allowed $f_{\text {col }}$ is $\approx 2,550 \mathrm{~mm}$.

Furthermore, $f_{\text {col }}$ is proportional to $f_{\text {cam }}$ for a given telescope speed. Figure 5.3 shows the relationship between $f_{c o l}$ and $f_{\text {cam }}$ that considers the conservation of the telescopecollimator etendue. A collimator with $f_{\text {col }}$ around $2,200 \mathrm{~mm}$ will require a $f_{\text {cam }}=550 \mathrm{~mm}$ or $650 \mathrm{~mm}$ for $\mathrm{f} / 2$ or $\mathrm{f} / 2.5$ camera, respectively.

The value of $f_{\text {col }}$ constrains the maximum resolving power since the maximum grating density is known. Based on suppliers' feedback, we assumed that a reasonable maximum grating groove density is about 3,000 lines/mm. Figure 5.4 illustrates the resolving power as a function of the $f_{c o l}$ for three grating grooves density and four blaze wavelengths within the GMACS spectral range, $0.7^{\prime \prime}$ slit width and first diffraction order. The bluer spectral 


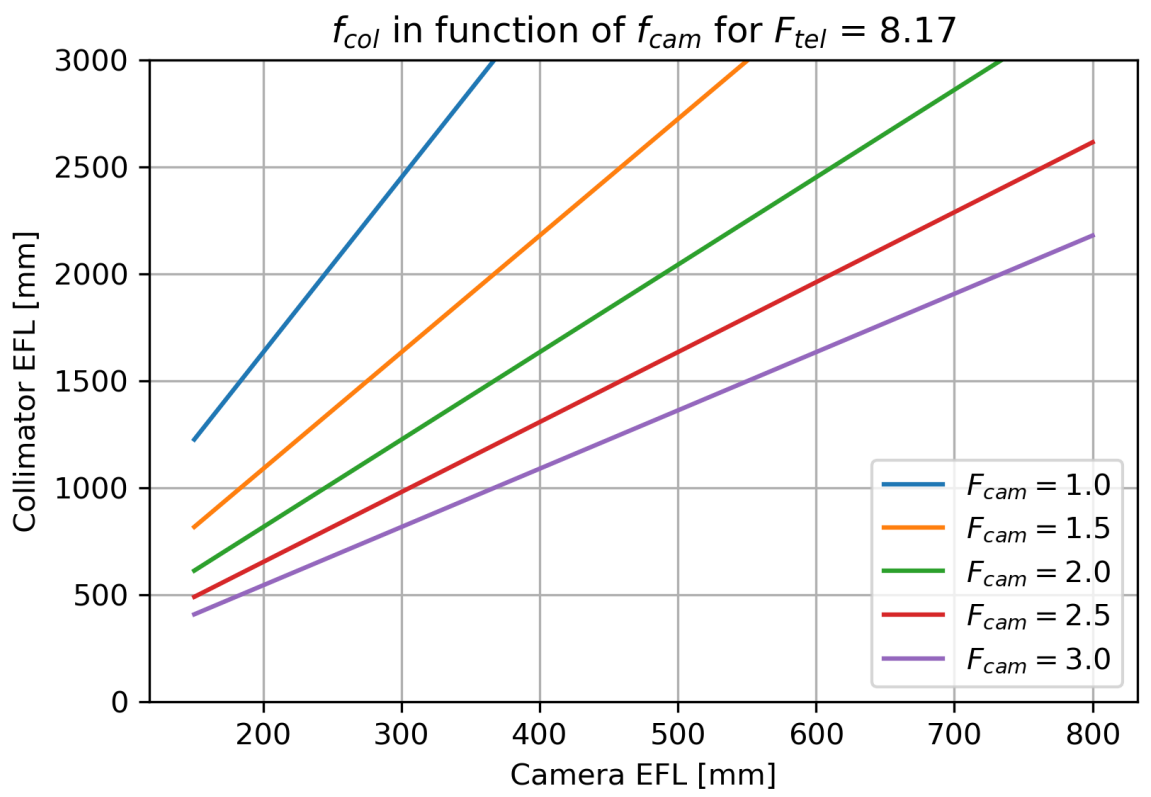

Figure 5.3: Collimator EFL as a function of the camera EFL for telescope f/8.16 and several camera speeds.

side is the region that presents the minimum resolving power since it is also proportional to the wavelength. A $f_{\text {col }}$ around 2,200 $\mathrm{mm}$ results in $R=3,000$ for the edge blue wavelength.

The baseline pixel size is $15 \mu \mathrm{m}$. The required 30 to 50 arcmin square FoV is similar to a long slit of $6.2^{\prime}$ to $7.9^{\prime}$, respectively, for a circular FoV shape. Considering equation 2.29, the minimum number of pixels in the spatial direction is $N_{x} \approx 3.0 \mathrm{k}$ and $3.8 \mathrm{k}$, respectively, for a f/1 camera. A f/3 camera would result in $N_{x}$ three times these values, $9.0 \mathrm{k}$ and $11.4 \mathrm{k}$.

The simultaneous wavelength coverage requirement also imposes several constraints in the principal parameters. For example, if we set $\mathrm{R}=1,000$ as the lowest resolution for all the required spectral range (320 to 1,000 nm), the number of pixels in spectral direction, $N_{\lambda}$, for a slit width of $0.7^{\prime \prime}$ and a $\mathrm{f} / 1$ camera is $\approx 3,000$ pixels. Assuming no spectral loss of the objects in the edge of the field, the total pixels in the spectral size must be at least three times this value, $\approx 9 \mathrm{k}$ pixels. This last assumption assumes that the spectrograph FoV is inscribed in the detector area, and consequently, the detector presents a rectangular shape. A more realistic camera speed, for instance, f/2.5, would result in $17.7 \mathrm{k}$ pixels along the spectral direction. Therefore, considering design arguments such as better grating efficiency and AR coating performances, it is reasonable to split the design into two arms spectrally. For example, a spectral bandwidth of 320-550 nm and 550-1,000 $\mathrm{nm}$ for the same slit width, spectral resolving power, and f/2.5 cameras would 

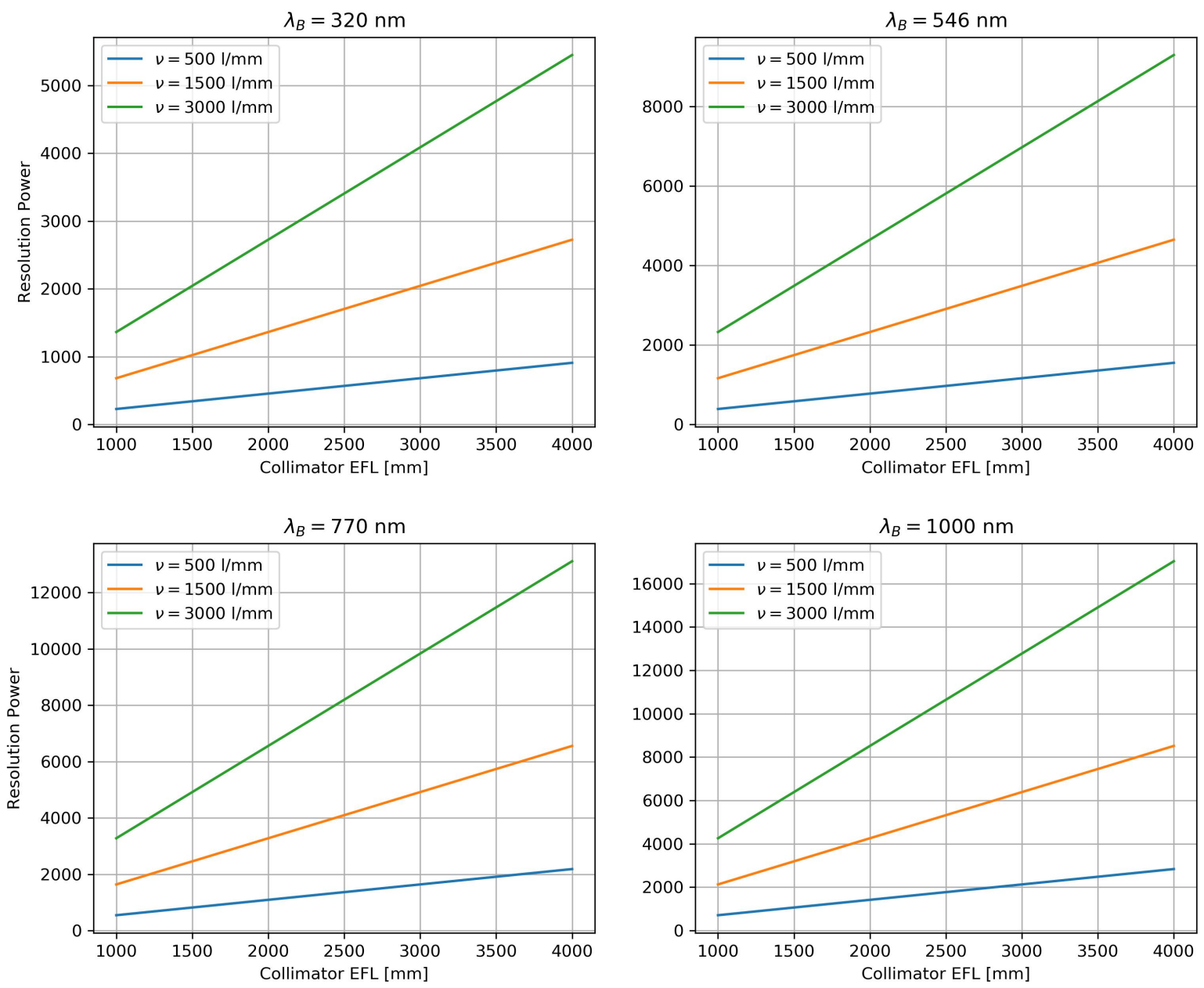

Figure 5.4: Resolving power as a function of the $f_{c o l}$ for three grating grooves density, four blaze wavelengths, $0.7^{\prime \prime}$ slit width and first diffraction order.

require $9 \mathrm{k}$ and $10 \mathrm{k}$ pixels in the spectral direction, respectively. Since the CCD detectors are available in sizes of $1 \times 1 \mathrm{k}^{2}$, or $2 \times 2 \mathrm{k}^{2}$ or $4 \mathrm{x} 4 \mathrm{k}^{2}$, it is usual to adopt these unit sizes to define the resulting focal plane. Therefore, the example above would require three $4 \mathrm{k}^{2}$ detectors along with the spectral range.

The camera FoV required as a function of the $f_{c a m}$, given the $N_{x m i n}$ and $F_{c a m}$, is shown in figure 5.5. The effective detector size for aspect ratio $N=1.5$ and $\mathrm{f} / 2$ camera would be $\approx 7.7 \times 11.6 \mathrm{k}^{2}$, or a $8 \mathrm{k} \mathrm{x} 12 \mathrm{k}^{2}$ considering the available detector sizes. It results in a $f_{\text {cam }}$ around $600 \mathrm{~mm}$.

A $f_{\text {col }} \approx 2,200 \mathrm{~mm}$ results in a $270 \mathrm{~mm}$ exit pupil size. A realistic $\mathrm{f} / 2.5$ camera results in a $f_{\text {cam }}=675 \mathrm{~mm}$. Table 5.2 shows the relationships between the camera's speed (from an optimistic $\mathrm{f} / 1.7$ to a realistic $\mathrm{f} / 2.2$ camera), the collimator ELF, the sizes of the exit pupil, and the spectrograph FoV for an 8k detector size along the spatial direction. 


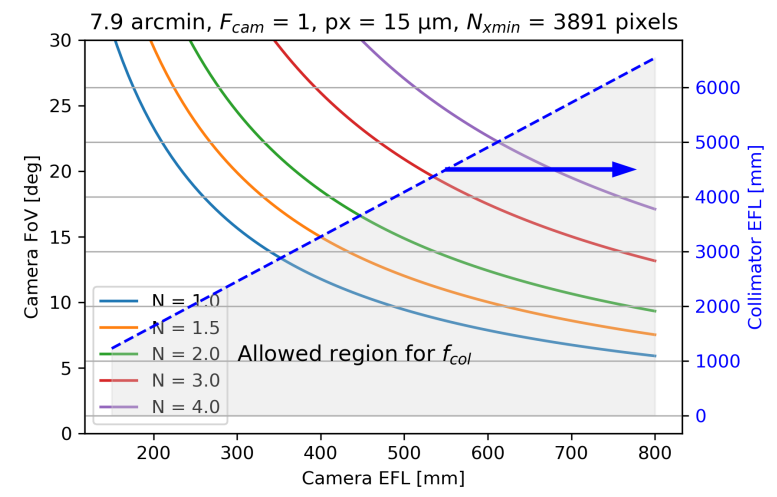

(a) $F_{c a m}=1$

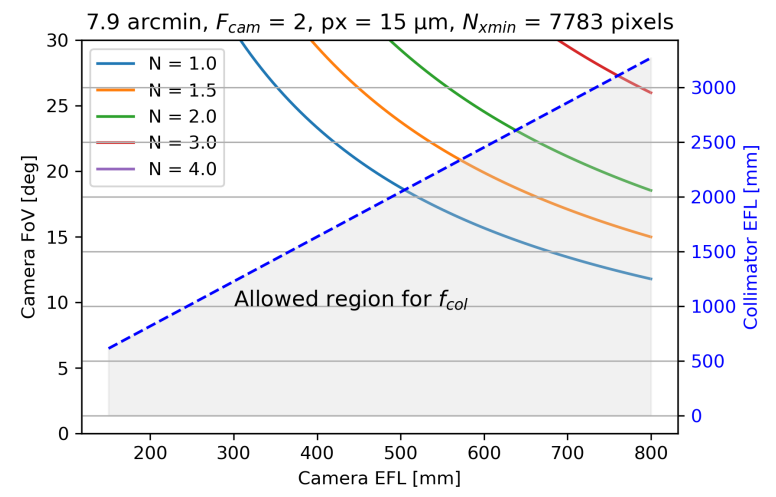

(b) $F_{c a m}=2$

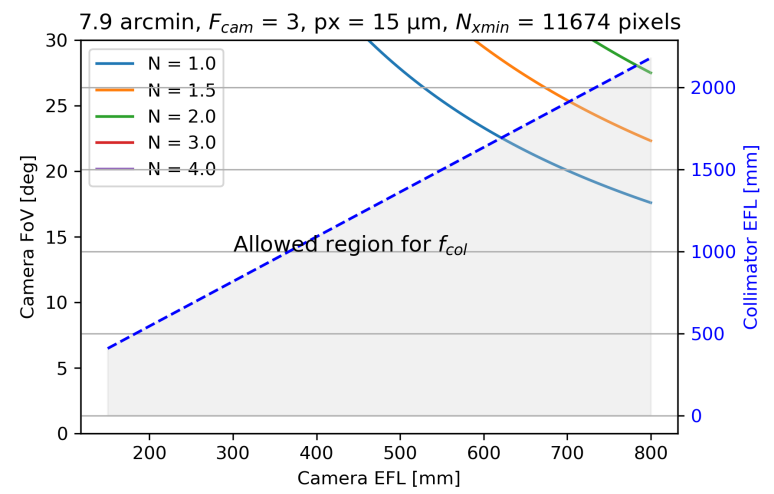

(c) $F_{\text {cam }}=3$

Figure 5.5: Camera FoV as a function of the camera EFL for several detector aspect ratios for $F_{c a m}=1,2$ and 3 . The minimum value of $N_{x}$ is also re-calculated as a function of $F_{\text {cam }}$.

Table 5.2 - Simulation of the system parameters as a function of the camera speed.

\begin{tabular}{ccccc}
\hline $\boldsymbol{F}_{\text {cam }}$ & $\begin{array}{c}\boldsymbol{f}_{\text {col }} \\
{[\mathbf{m m}]}\end{array}$ & $\begin{array}{c}\text { Exit Pupil } \\
\text { Size }[\mathbf{m m}]\end{array}$ & $\begin{array}{c}\boldsymbol{f}_{\text {cam }} \\
{[\mathbf{m m}]}\end{array}$ & $\begin{array}{c}\text { GMACS FoV } \\
{[\mathbf{a r c m i n}]}\end{array}$ \\
\hline 1.70 & 2,500 & 306 & 522 & 9.36 \\
1.87 & 2,382 & 292 & 547 & 8.52 \\
2.03 & 2,285 & 280 & 570 & 7.92 \\
2.20 & 2,194 & 268 & 592 & 7.32 \\
\hline
\end{tabular}

Since we assume a conservative design approach for the GMACS conceptual design, the adopted baseline principal optical parameters for GMACS is $f_{c o l}=2,200$, resulting in a $270 \mathrm{~mm}$ exit pupil (maximum grating size of $380 \mathrm{~mm}$ in diameter) and $594 \mathrm{~mm} \mathrm{f} / 2.2$ camera for a long slit of $7.4^{\prime}$. The $0.7^{\prime \prime}$ image size width is $190 \mu \mathrm{m}$, which represents 12.6 pixels for a focal plate scale of $0.055^{\prime \prime} /$ pixel. The detector is a mosaic of $2 \times 34 \mathrm{k}^{2}$ 
detectors, pixel pitch of $15 \mu \mathrm{m}$, resulting in an effective detector of $8 \times 12 \mathrm{k}^{2}$. Although the inter-chip between the detectors causes small gaps in the wavelength interval covered by a single observation, we expected to reduce this issue by using customized one-side readout detector. The unvignetting camera $\mathrm{FoV}$ is $17.6^{\circ} \mathrm{x} 11.8^{\circ}\left(21.2^{\circ}\right.$ in diagonal), which can be changed according to the achieved performance during the preliminary stages of optical design. The GUI for Spectrograph Simulator (section 4.2.2) was used to confirm the spectrograph parameters.

Dichroic transition wavelength: The dichroic is an optical element that splits the incoming ray into two spectral ranges. In general, it is a flat window coated with an interferometric coating on one of the surfaces. The dichroic transition wavelength should be made as narrow as possible and centered in a sky background emission line to avoid spectral information loss from the object. The GMACS team showed that a potential dichroic transition wavelength is $557.7 \mathrm{~nm}$, which is a bright night sky from the terrestrial airglow (GMACS Team, 2018b). Although the resulting blue and spectral range are not balanced, and considering that the dispersion gradient of the glass materials used for large refractive optics is high below $400 \mathrm{~nm}$, the unbalanced spectral range is compensated by the challenges to correct chromatic aberrations in the bluer spectral range.

Disperser Element: Ruled grating has relatively wide bandwidth and flat efficiency curve, yet it usually is expensive for the required size and generally has long lead time for production. On the other hand, VPH grating has a relatively higher throughput (although lower if large bandwidth is required (Barden et al., 1998)), it is cheaper than the ruled ones and can be manufactured within a few months compared to the ruled grating (Barden et al., 2000). This type of disperser has an encapsulated grating structure with AR coatings that allows cleaning and handling of the grating assembly, and it has potential for the fabrication of very large grating assemblies, an essential need for ELT instrumentation (National Optical Astronomy Observatories, 1999; Bernstein et al., 2002). Moreover, the ability of a VPH grating to be tuned in blaze wavelength, by changing the grating tilt, results in VPH multi-slit spectrographs exhibiting a shift of the blaze wavelength for objects that are off-axis in the spectral direction (Baldry et al., 2004). Therefore, we have adopted transmission VPH grating as default disperser element for GMACS. 
The adopted VPH grating model for the optical design is based on the "Difraction Grating" surface in Zemax, which has the RoC, groove density "lines/ $\mu \mathrm{m}$ " and "Difraction Order" as input parameters. The model consists of the non-thickness surface sandwiched by two $30 \mathrm{~mm}$ flat Fused Silica (FS) windows.

\subsubsection{GMACS Paraxial Modeling}

This section presents the results of the GMACS paraxial model. According to the conclusions presented in section 4.4.4.2, the adopted principal parameters are $f_{\text {col }}=2,200$ $\mathrm{mm}, f_{\text {cam }}=594 \mathrm{~mm}$, grating density groove of 380 lines $/ \mathrm{mm}$ and 3,300 lines $/ \mathrm{mm}$ for the low- and high-resolution modes, respectively, with first diffraction order. The lower grating density value was chosen based on the no spectral loss requirement and detector size. The higher resolution mode is based on the maximum $90^{\circ}$ collimator-camera angle of rotation. We included the image mode by setting the collimator-camera angle zero and diffraction order zero. The presented paraxial model is for the blue arm only, although the red arm follows similar modeling by changing the wavelength range, grating density groove and blaze angles.

We tested two alternative for the GMACS paraxial model, also aiming at defining the strategy for the real optics design. The first consists of a set of configurations for each mode of operation. Each of the intra-configurations has a unique wavelength, while the diffraction grating and field parameters are kept the same. For example, for three modes of operation with six wavelengths each, results in eighteen different configurations. The advantage of this type of approach is that the merit function for imaging does not need to ignore the Transverse Chromatic Aberration, TCA (also known as lateral color), once the spot size is calculated individually for each wavelength of each operating mode. The second alternative condenses the wavelengths of each operating modes and controls (or eliminates the restriction) the TCA correction since spectrographs intrinsically generate TCA. The last option proved to be more efficient and straightforward for the optimization steps since it allows cleaner presentation of the results, the merit function is explicit and of easy comprehension. The first alternative was used for the KoM and 2017 Baseline designs, while the second was used from the Optical Design Baseline design ahead. We describe next only the second approach for the elaboration of the paraxial model.

Figure 5.6 (left) shows the Lens Data Editor (LDE) used to model GMACS. The 
distance from the paraxial telescope surface to the telescope focal plane (row \#2) is the same from the telescope EFL (by using the pickup solve). The collimator exit pupil is determined by solving the next surface from the collimator for chief ray at zero height, where the grating surface is located. The dummy surface \#9 is the camera relief and it is followed by the paraxial camera. The distance from the camera to the spectrograph focal plane is the same from the camera EFL. The surface \#12 is a small focus axial shift for spot footprint visualization. Figure 5.6 (right) shows the Multi-Configuration Editor (MCE) for three configurations: image, low- and and high-resolution modes. The differences among them are the blaze angle for Littrow configuration, grating groove density, diffraction order, wavelengths and fields. The input data were obtained with the GUI Spectrograph Simulator, described in section 4.2.2. Figure 5.7 shows the overlay optical layout for the low- and high-resolution modes.

\begin{tabular}{|c|c|c|c|c|c|c|c|}
\hline 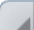 & Surf:Type & Comment & Radius & Thickness & Semi-Diamete & Lines/ $/ \mu \mathrm{m}$ & Diffract Order \\
\hline 0 & Standard $\mathbf{v}$ & & Infinity & Infinity & Infinity & & \\
\hline 1 & Paraxial . & GMT & & 0,000 & 12744,000 & 207588,800 & 1 \\
\hline 2 & Standard & & Infinity & $207588,800 P$ & 12744,000 & & \\
\hline 3 & Standard & GMT Focal Plane & Infinity & 2200,000 & 224,633 & & \\
\hline 4 & Paraxial - & Collimator & & 0,000 & 362,073 & 2200,000 & 1 \\
\hline 5 & Standard & & Infinity & $2223,315 \mathrm{C}$ & 362,073 & & \\
\hline 6 & Coordinate Break - & Element Tilt & & 0,000 & 0,000 & 0,000 & 0,000 \\
\hline 7 & Diffraction Grating & DIFF & Infinity & 0,000 & 190,429 & 3,300 & 1,000 \\
\hline 8 & Coordinate Break & Element Tilt:return & & $0,000 \mathrm{~T}$ & 0,000 & 0,000 & $0,000 F$ \\
\hline 9 & Standard & Dummy & Infinity & 250,000 & 161,935 & & \\
\hline 10 & Paraxial & Camera & & 0,000 & 206,115 & 594,000 & 0 \\
\hline 11 & Standard & & Infinity & $594,000 \mathrm{P}$ & 206,115 & & \\
\hline 12 & Standard & & Infinity & 2,000 & 115,728 & & \\
\hline 13 & Coordinate Break V & & & 0,000 & 0,000 & 0,000 & 0,000 \\
\hline 14 & Standard & Detector & Infinity & - & 116,318 & & \\
\hline
\end{tabular}

\begin{tabular}{|c|c|c|c|c|}
\hline 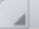 & Active : $2 / 3$ & Config 1 & Config 2* & Config 3 \\
\hline 1 & MOFF $=$ & IMAGE & SPEC & SPEC \\
\hline 2 & MOFF - - & & LOW & HIGH \\
\hline 3 & PRAM $=6 / 3$ & 0,000 & $-5,000$ & $-44,530$ \\
\hline 4 & PRAM $=7 / 1$ & 0,000 & 0.380 & 3.300 \\
\hline 5 & PRAM $=7 / 2$ & 0.000 & 1.000 & 1.000 \\
\hline 6 & WAVE $=1$ & 0,324 & 0.324 & 0,390 \\
\hline 7 & WAVE $=2$ & 0.378 & 0.378 & 0.403 \\
\hline 8 & WAVE -3 & 0.432 & 0.432 & 0,416 \\
\hline 9 & WAVE $=4$ & 0,486 & 0.486 & 0.429 \\
\hline 10 & WAVE $=5$ & 0,540 & 0.540 & 0.442 \\
\hline 11 & WAVE $=6$ & 0.594 & 0.594 & 0.455 \\
\hline 12 & MOFF - - & & & \\
\hline 13 & $X F I E=1$ & 0.000 & 0.000 & $-0,062$ \\
\hline 14 & $X F I E=2$ & 0,000 & 0.000 & 0.062 \\
\hline 15 & $X F I E=3$ & 0,062 & 0,062 & $-0,030$ \\
\hline 16 & $X F I E=4$ & $-0,062$ & -0.062 & 0.030 \\
\hline 17 & YFIE $=1$ & -0.062 & -0.062 & 0.000 \\
\hline 18 & YFIE $=2$ & 0,062 & 0,062 & 0,000 \\
\hline 19 & YFIE $=3$ & 0.000 & 0.000 & 0.000 \\
\hline 20 & YFIE $=4$ & 0,000 & 0.000 & 0.000 \\
\hline 21 & MOFF - & & & \\
\hline
\end{tabular}

Figure 5.6: (left) Lens Data Editor (LDE) and (right) Multi-Configuration Edito (MCE), for the GMACS paraxial model. The power surface types are "paraxial" and the grating is "Diffraction Grating". The LDE shown refers to a low-resolution mode and first diffraction order. The collimator and camera EFL are 2,200 $\mathrm{mm}$ and $594 \mathrm{~mm}$, respectively. Three configurations were simulated for the image mode, the low- and the high-resolution modes.

The detector footprint diagram is shown in figure 5.8 for each mode. The bandwidth resulted from the Spectrograph Simulator is only unvignetted in the center of the field. 


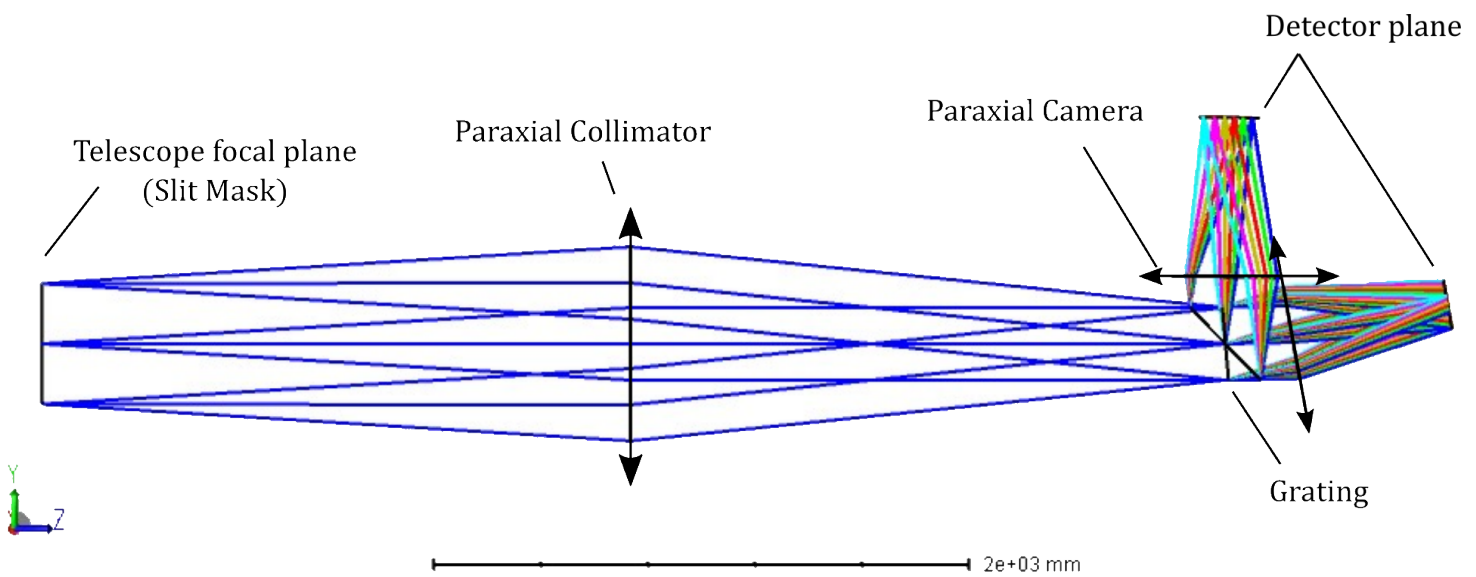

Figure 5.7: Optical layout for the paraxial model with a low- and high-resolution modes superposed. The grating grooves are in the $\mathrm{x}$-direction (orthogonal to the page).

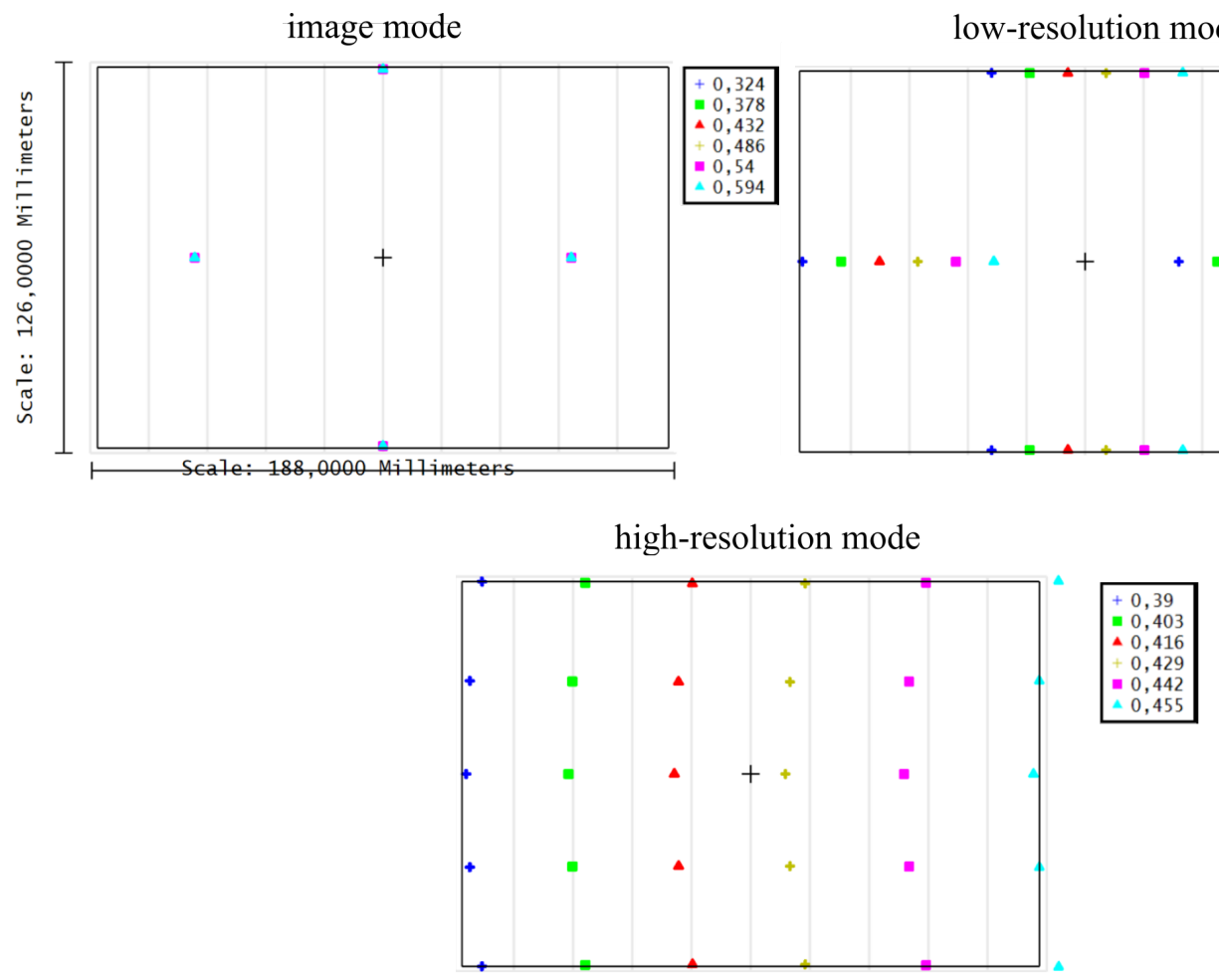

Figure 5.8: Detector footprint diagram for the imaging, low- and high-resolution mode paraxial models. There is no spectral loss in the low-resolution mode for any field within the spectrograph FoV. Similary for the high-resolution mode, the central field presents no vignetting; however, the edge objects present vignetting due to the intrinsic distortion of the spectral images. The legend is wavelengths in $\mu \mathrm{m}$ units.

\subsection{Definition of Optical Concepts and Architectures}

The first tradeoff analysis consists of determining the advantages and disadvantages of optical system concepts based on the results from the principal parameters study described 
in section 5.3.1. The selected concepts are purely reflexive, refractive, and catadioptric. Table 5.3 shows a general description of the characteristics of purely refractive and reflective optical designs.

Table 5.3 - General characteristics of refractive and reflective optical designs for ELT instruments.

\begin{tabular}{|l|l|}
\hline \multicolumn{2}{|c|}{ Refractive systems } \\
\hline \multicolumn{1}{|c|}{ Advantages } & \multicolumn{1}{c|}{ Disadvantages } \\
\hline Surfaces can mainly be spherical & $\begin{array}{l}\text { The index of refraction of the lens ma- } \\
\text { terials is wavelength dependent }\end{array}$ \\
\hline On-axis design (axial symmetry) & $\begin{array}{l}\text { Spherochromatism is significant for } \\
\text { large lens }\end{array}$ \\
\hline Compactness & $\begin{array}{l}\text { Athermalization is generally more com- } \\
\text { plex }\end{array}$ \\
\hline High field of view can be archived & $\begin{array}{l}\text { Broadband AR coating may be costly } \\
\text { and difficult to achieve }\end{array}$ \\
\hline Stray light easier to manage & $\begin{array}{l}\text { Limited availability for large optical } \\
\text { blanks }\end{array}$ \\
\hline Full aperture is available & \\
\hline
\end{tabular}

\begin{tabular}{|c|c|}
\hline \multicolumn{2}{|c|}{ Reflective systems } \\
\hline Advantages & Disadvantages \\
\hline Intrinsically achromatic & Limited field of view \\
\hline General less spherical aberration & Large aspherical mirrors \\
\hline $\begin{array}{l}\text { Naturally athermalized if the surfaces } \\
\text { are in the same material as the housing }\end{array}$ & $\begin{array}{l}\text { More sensitive to irregularity and align- } \\
\text { ment errors }\end{array}$ \\
\hline Fewer surfaces needed & $\begin{array}{l}\text { Packaging restrictions due to off-axis } \\
\text { configurations }\end{array}$ \\
\hline $\begin{array}{l}\text { Integrated mounting and alignment fe- } \\
\text { atures }\end{array}$ & Stray light is harder to manage \\
\hline Mirror mass reduction & $\begin{array}{l}\text { Central obscuration reduces through- } \\
\text { put }\end{array}$ \\
\hline $\begin{array}{l}\text { Compact: design can be folded to meet } \\
\text { packaging constraints }\end{array}$ & $\begin{array}{l}\text { Complex off-axis configuration requires } \\
\text { additional (flat) mirrors }\end{array}$ \\
\hline $\begin{array}{l}\text { High throughput due to few optical sur- } \\
\text { faces }\end{array}$ & $\begin{array}{l}\text { Per-element fabrication is more costly } \\
\text { and takes longer to manufacture }\end{array}$ \\
\hline
\end{tabular}

A useful method to arrange and distinguish the different types of design correlates their typical field of view and speed ranges. Figures 5.9 and 5.10 illustrate this relationship 
(half-FoV and Numerical Aperture NA $\left.=1 /\left(2 f_{w}\right)\right)^{1}$ ) for an arbitrary set of refractive and reflective design-form, respectively. According to Smith (1992) and Bentley and Olson (2012), the boundary of each area should not be considered as definitive and does not necessarily represent diffraction-limited performance. However, this classification is a quick starting point step for designers based only on the required FoV and speed.

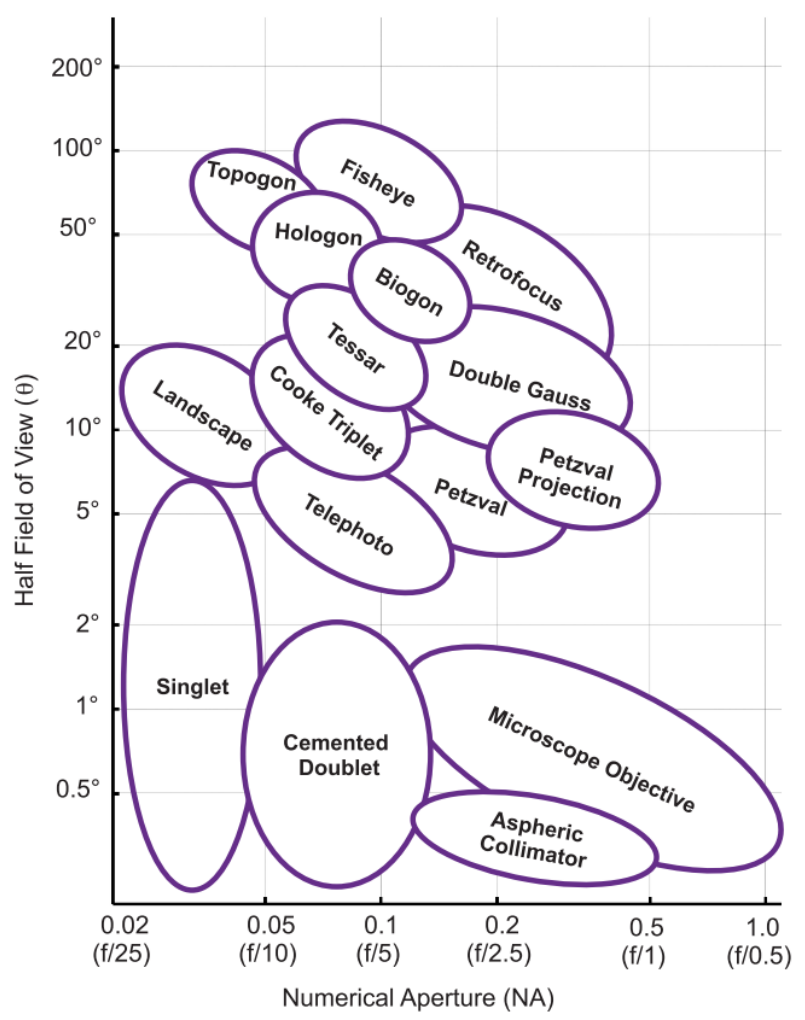

Figure 5.9: Aperture and field size for classical refractive design-form. Source: Bentley and Olson (2012).

\subsubsection{Glass Selection for ELT Instrumentation}

The production challenges for large optical blanks with high homogeneity is the principal reason for the low availability of glasses for large refractive optical systems (Jedamzik and Hartmann, 2004; Jedamzik et al., 2008, 2016; Hartmann and Jedamzik, 2006; Oswalt and McLean, 2013). The GMACS system requirement for high UV-Blue throughput is an aggravating factor which further reduces the range of glass options and, consequently, compromises the optical design since it reduces the means for the optical designer to reduce

\footnotetext{
${ }^{1} f_{w}$ is the Working f-number, defined as $f_{w}=1 /(2 n \sin \theta)$, where $\theta$ is the real marginal ray angle in image space and $\mathrm{n}$ is the index of refraction of image space (Zemax LLC OpticStudio, 2016).
} 


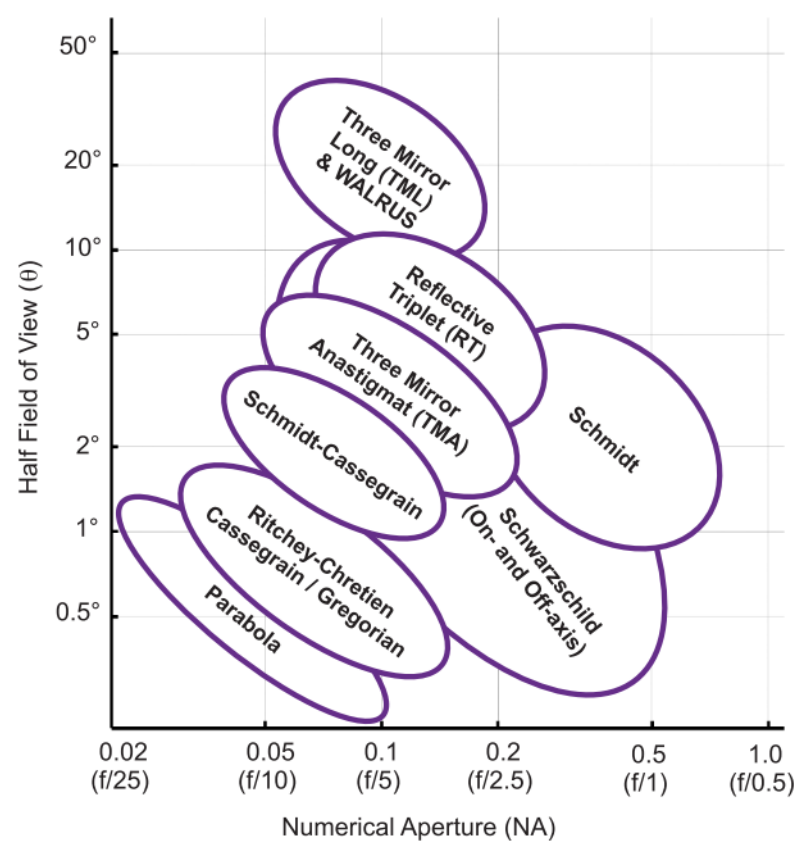

Figure 5.10: Aperture and field size for classical reflective design-form. Source: Bentley and Olson (2012).

and control chromatic aberrations, such as transversal and spherochromatism, which are the main design challenges for large refracting systems. According to Oswalt and McLean (2013, p. 595), the "only materials that will not attenuate significantly down to the atmospheric cutoff (300-320 nm, depending on altitude) are calcium fluoride and fused silica. Because of its high transmission and low dispersion, calcium fluoride is a critical material for cameras operating at any wavelength". Table 5.4 shows a list of large optical glass blanks ordered descending from $320 \mathrm{~nm}$ and $330 \mathrm{~nm}$ internal transmittance.

In addition to the limited number of optical glasses, the restriction of the maximum blank diameters considerably reduces the optical design options for GMACS, particularly for the required fast camera designs $(\mathrm{f}-$ number $\leq 2.5$. A faster camera would require deep aspherics (Saunders, 2016)). For example, designs that have negative rear elements and large intermediate lenses, such as reverse telephoto, may be prohibitive.

Nikon NIGS i-line glass family increased the number of glass types available for astronomical instrumentation since it offers two notable feature materials. The first is NIGS 4786, which has an intermediate refractive index and dispersion values between $\mathrm{CaF}_{2}$ and S-FPL51Y, and high internal transmittance in the UV-Blue region, making it an excellent alternative for achromatizing lenses. The second, NIGS 7054, at the other end of the Abbe 
Table 5.4 - List of large optical glass blanks. "DIA" is diameter; "THK" is thickness; "IT" is internal transmittance for a $10 \mathrm{~mm}$ thickness sample. This list is ordered from higher to lower $320 \mathrm{~nm}$ internal transmittance glasses and have been constantly updated. Source: Schott $(2005,2006)$ and suppliers' e-mails.

\begin{tabular}{|c|c|c|c|c|c|}
\hline Manufacturer & Glass Material & DIA (mm) & THK (mm) & $\mathrm{IT}(320 \mathrm{~nm})$ & $\mathrm{IT}(330 \mathrm{~nm})$ \\
\hline Nikon & NIGS 4786 & 368 & 41 & 0.999 & \\
\hline Hellma Materials & $\mathrm{CaF}_{2}$ & 440 & 80 & 0.995 & 0.993 \\
\hline Nikon & $\mathrm{CaF}_{2}$ & 435 & 240 & 0.995 & 0.999 \\
\hline Nikon & Silica & 1100 & 115 & 0.995 & 0.991 \\
\hline Nikon & NIGS 5859 & 290 & 53 & 0.986 & \\
\hline Ohara & S-FSL5Y & 500 & 100 & 0.984 & \\
\hline Schott & N-FK5 & 180 & 140 & 0.98 & 0.999 \\
\hline Nikon & NIGS 7054 & 245 & 35 & 0.975 & 0.984 \\
\hline Ohara & S-FSL5 & 300 & 60 & 0.961 & 0.983 \\
\hline Ohara & S-FPL51Y & 280 & 55 & 0.943 & 0.971 \\
\hline Ohara & BSL7Y & 800 & 80 & 0.932 & 0.978 \\
\hline Ohara & BAL35Y & 300 & 80 & 0.92 & 0.966 \\
\hline Ohara & BSM51Y & 550 & 60 & 0.88 & \\
\hline Nikon & NIGS 5165 & 230 & 35 & 0.82 & \\
\hline Ohara & PBL6Y & 300 & 40 & 0.79 & \\
\hline Schott & N-PSK3 & 160 & 40 & 0.77 & \\
\hline Schott & $\mathrm{N}-\mathrm{BK} 7$ & 1000 & 300 & 0.77 & \\
\hline Schott & LLF1 & 1000 & 300 & 0.618 & \\
\hline Ohara & S-FPL51 & 400 & 60 & 0.6 & \\
\hline Nikon & NIGS 5742 & 290 & 53 & 0.55 & \\
\hline Schott & LF5 & 1000 & 300 & 0.32 & \\
\hline Schott & $\mathrm{F} 2$ & 1000 & 300 & 0.08 & \\
\hline Schott & SF6 & 1000 & 300 & 0 & 0.95 \\
\hline Schott & N-FK51A & 360 & 60 & & 0.947 \\
\hline Schott & LAK8 & 160 & 43 & & 0.77 \\
\hline Schott & LAK9 & 160 & 43 & & 0.935 \\
\hline Schott & KZFSN4 & 160 & 43 & & 0.88 \\
\hline
\end{tabular}

Diagram, has a high refractive index and dispersion value, can be used as a strong negative element, and is an excellent choice for the design of an apochromatic triplet formed by $\mathrm{CaF}_{2}$ and PBL6Y. Despite the significant dispersive advantages, the company offers limited blank sizes as per Table 5.5, and the evidence of previous benefits of these glasses in ELT instrumentation is still insufficient. Therefore, although such glasses were used 
for designing a broadband collimator in the KoM, not shown herein, the current optical systems do not contain any Nikon material. However, given the potential advantages, the evolution of Nikon's productive capacities and glasses performances by case studies will be monitored in the upcoming years.

Table 5.5 - Nikon NIGS i-line glass family blanks dimensions. Source: supplier's e-mail.

\begin{tabular}{|c|c|c|}
\hline NIGS Glass & Diamenter $[\mathrm{mm}]$ & Thickness $[\mathrm{mm}]$ \\
\hline 4786 & 368 & 41 \\
5165 & 230 & 35 \\
5742 & 290 & 53 \\
5859 & 290 & 53 \\
7054 & 245 & 35 \\
\hline
\end{tabular}

Figure 5.11 shows the Abbe Diagram and the corresponding CTE for the glasses with blanks larger than $250 \mathrm{~mm}$ and more than $88 \%$ internal transmittance at $320 \mathrm{~nm}$ for a sample thickness of $10 \mathrm{~mm}$. Among them, the highest $320 \mathrm{~nm}$ internal transmissions materials are $\mathrm{CaF}_{2}$, NIGS 4786, Silica and NIGS 5859. This last glass has similar optical parameters as BAL35Y and BSM51Y, yet higher internal transmittance for bluer wavelengths.

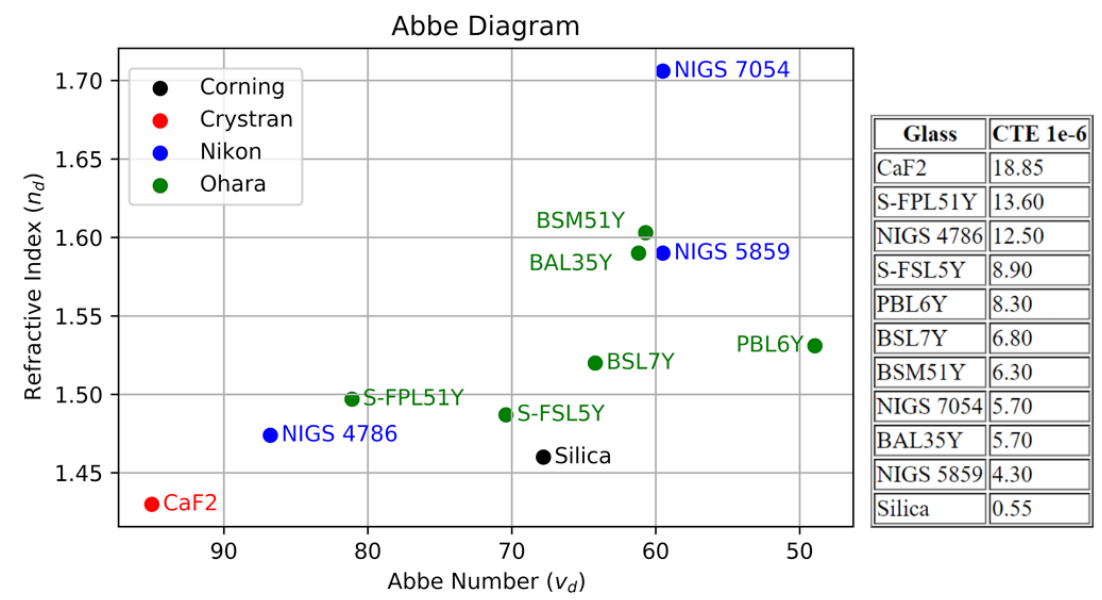

Figure 5.11: Abbe Diagram for GMACS glasses and the corresponding CTEs $\left[1 \cdot K^{-1}\right]$. 


\subsection{Initial Considerations for the Optical Design}

\subsubsection{Telescope Optical Model}

The GMACS sequential optical design incorporates the GMT optical model version "GMT DGNF non-segmented 120206" provided by the GMTO (GMT-SE-DOC-A-00012). More information is available in Appendix A. The importance of using an accurate telescope model in the optical design is to guarantee that all the telescope optical features are taken into account in the optical design, such as telecentricity, exit pupil aberrations and locations, as a function of field. Although the correct entrance aperture geometry is essential for the PSF optical characterization and SNR estimate, the symmetrical circle aperture is employed for the $\mathrm{CoD}$ without design compromises.

\subsubsection{Collimator Optical Quality Metrics}

The metrics adopted to measure the performance of the collimator is a combination of wavefront and geometrical criteria. Although the wavefront performance metric is more appropriate for the telescope + collimator subsystem design since it is afocal, we have included a paraxial camera to simulate the complete spectrograph. Therefore, particularly during the initials design steps, we used the standard performance metrics for imaging systems.

The collimator exit pupil quality is a critical feature of a spectrograph since the image spectra quality is strongly dependent on the quality of the incident wavefront on the dispersing element. Jones (2016) shows that a well-corrected collimator is essential for GMACS to have all spectra images focused instead of only the central wavelength. Although the camera might provide some wavefront correction, the first methodology proposed for GMACS has parallel optimization steps for the collimator and the cameras, in which the pupils' positions and dimensions are constrained in the merit functions. Therefore, we concluded that the paraxial camera's approach is a useful tool for any earlier stages in a collimator design. Later, the following integrated optimization step aims only at correcting residual aberrations.

The metrics used for the collimator + paraxial-camera design are the EED80 and EED100, MTF for Nyquist frequency equivalent to a resolution element, exit pupil chromatic circle of confusion, which is calculated by the ray coordinates at the exit pupil 
plane employing the functions REAX (or REAY) for all the wavelengths and edge of fields. Since the chief rays control the chromatic pupil position by forcing them to cross the optical axis for every field and wavelength data, we incorporated the Zemax functions REAX and REAX for $\left(P_{x}, P_{y}\right)=(0,0)$, well-sampled through the field into the design Merit Function, MF. The marginal rays control the pupil size. We tried to constrain the chromatic pupil size by using the Diameter Maximum Value function (DMVA); however, it was ineffective compared to controlling the height of the marginal ray. We also used the ENPP and EXPP Zemax functions to constrain the pupil positions.

The wavefront merit function is used only when a good EED100 performance is achieved. The wavefront aberrations are measured by the wavefront peak to valley and RMS for the model without the paraxial cameras and the Zemax aperture parameters set to afocal image space.

\subsection{Collimator Concept Tradeoff Analysis}

The tradeoff analysis for the collimator has two progressive levels. The first defines the collimator concept that mainly addresses the mechanical envelope constraints and allows a satisfactory optical quality for the consequent imaging by the cameras. The concepts explored are reflective and refractive systems. Once the concept is defined, the second level is for the determination of the best optical architecture. Figure 5.12 shows a diagram for the concepts and architectures of collimator optical systems considered in this research.

The attributes for comparison consisted of a combination of image quality performances, design capabilities, material restrictions for fabrications, and assembly, expected flexure sensitivity, mechanical envelope constraints within the IMF, operation risks and previous design experience. Their most important characteristics were described in table 5.3.

The reflective concepts are divided into two types concerning the absence or presence of central obscurations. The principal advantages are intrinsic achromaticity and high throughput due to the reduced number of optical surfaces compared to the refractive concepts. However, we identified the following disadvantages of a reflective collimator for GMACS: (i) in the case of obscured ones, such as Cassegrain, Schmidt, Gregorian, Schmidt-Cassegrain, Ritchey-Chretien, the reduction of system throughput due to the obscuration (it acts as a neutral filter); (ii) the large FoV required for the collimator $(\approx$ 


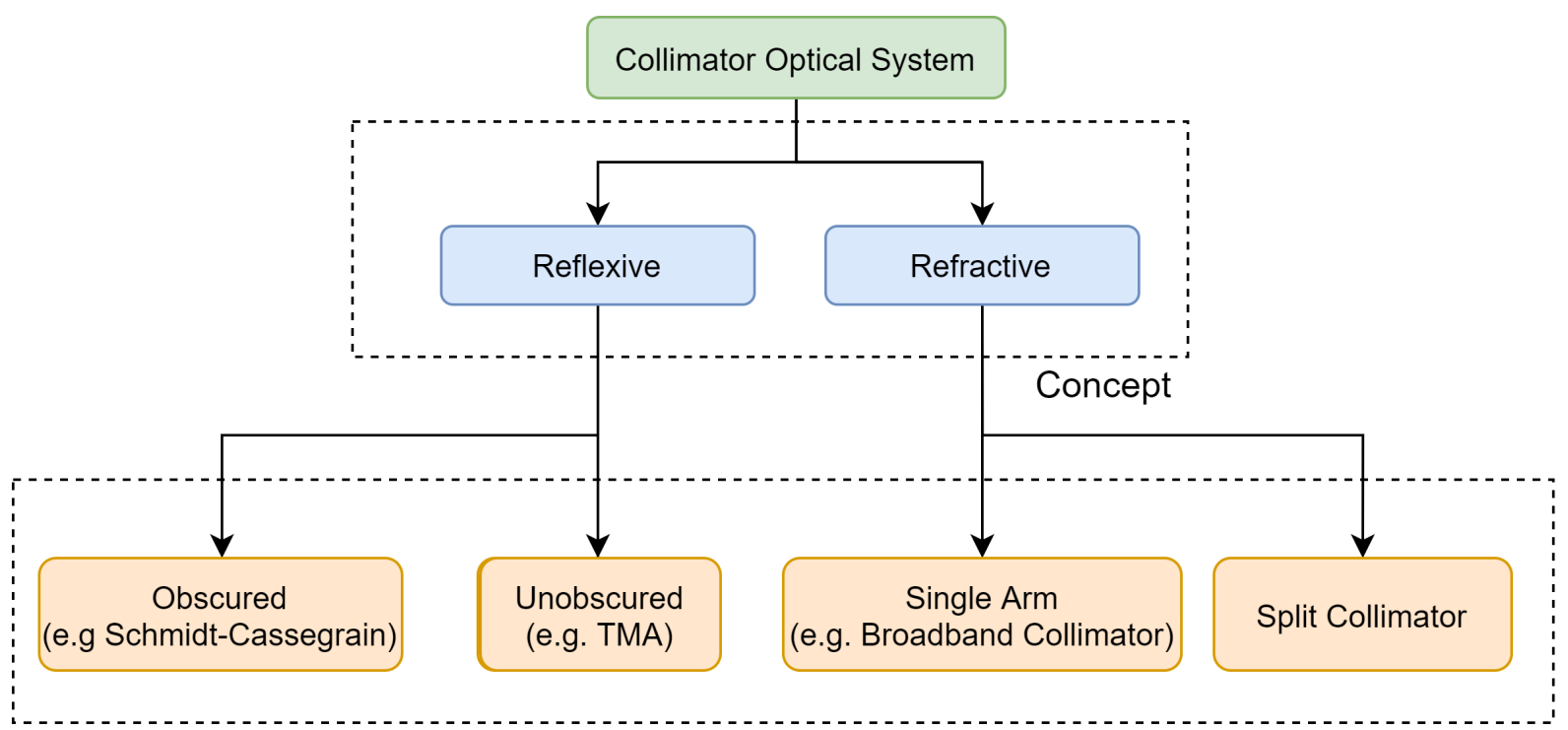

Architecture

Figure 5.12: Collimator concepts, architectures and design examples.

$11^{\circ}$ ) requires an off-axis design, which in turn demands a large mechanical envelope, and (iii) the size of the image generated by the telescope, which is approximately $440 \mathrm{~mm}$ for the field of $7.4^{\prime}$, would require an obscuration diameter in the primary mirror of similar value.

These three disadvantages argue against further exploration of the obscured reflexive concept for the collimator. Although non-obscured reflective systems provide solutions for the disadvantages mentioned above, such as off-axis TMA and reflective triplet, their most striking drawbacks are packaging restrictions and the need for complex assembly and alignment processes. For example, figure 5.13 shows a TMA collimator draft design within the IMF. Although the design presents an EED100 less than $0.35^{\prime \prime}$ over all the FoV and it is intrinsically achromatic, these disadvantages are decisive in excluding the reflexive option for the GMACS collimator optical system.

On the other hand, refractive systems can afford large FoV, generate good image pupil, and allow external aperture stop flexibility. The axial symmetry facilitates the fabrication of the elements and the processes of assembly and alignment. Since the GMT field of curvature at the focal plane is concave towards the instrument, refracting collimators are particularly well-matched to Gregorian telescopes because of their intrinsic Petzval curvature. This particular advantage avoids excessive negative power for field curvature control (Oswalt and McLean, 2013). However, the significant challenge, similar to every 

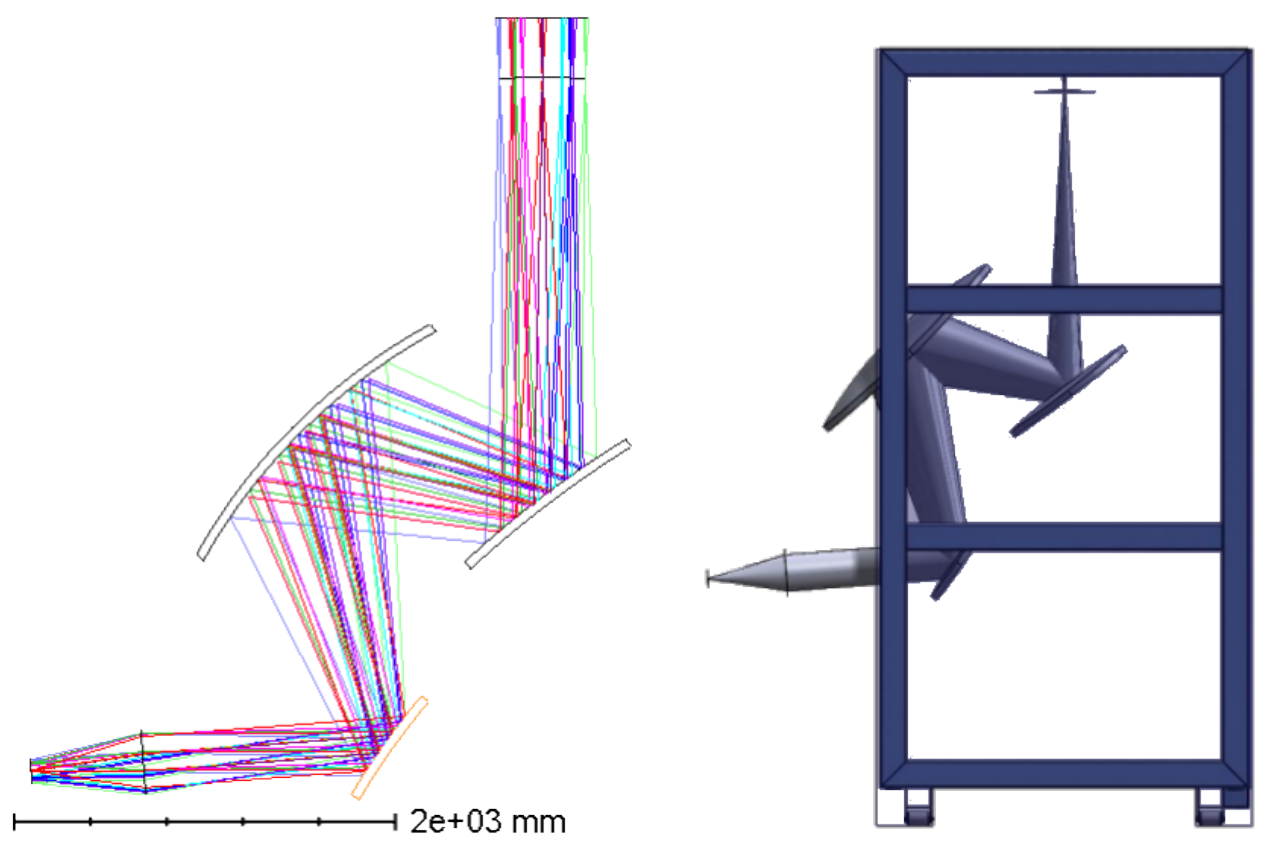

Figure 5.13: TMA Collimator layout and a mechanical design draft showing the packaging restriction within the IMF. Source: GMACS Team (2018a).

refractive ELT instrumentation, is the limited availability of large optical blanks, mainly those with high internal transmittance in the UV-Blue spectral range.

In conclusion, both the reflective and refractive architectures are compliant with the GMACS image quality requirements. However, the required size, packaging constraints, throughput losses, alignment challenges, and stability requirements are strong negatives for reflective architectures. Therefore, the selected concept for the GMACS collimator is refractive. The next section describes a tradeoff study for two potential refractive collimator designs.

\subsection{Refractive Collimator Architecture Tradeoff Analysis}

Refractive collimators can generate external exit pupils without the need for off-axis configurations, thus enhancing total throughput. The combination of optical glasses and the addition of aspherical surfaces near the exit pupil support the controlling of spherochromatism, lateral color, astigmatism, and coma. A refractive collimator has the potential to have a well-controlled achromatic exit pupil and suitable image quality.

The two explored collimator architectures have similar layouts: a Field Lens, FL, followed by a collimating group. The FL, located near the telescope's focal plane, mostly 
controls pupil imagery and contributes to the collimator power, although it has little control over the other system aberrations. The collimating group, placed near the exit pupil, makes a relatively small contribution to the overall power; however, it is essential for correcting the spherochromatism, field aberrations, and residual aberration caused by the FL. Any aspheric surfaces must be located in the collimating group. The location of the dichroic is the characteristic that differentiates the two concepts explored.

The first refractive architecture, referred to as "single collimator", has the dichroic located in the collimated beam before the grating. This architecture feeds both cameras; therefore, it must be capable of operating over the entire GMACS spectral range. The alternative architecture has the dichroic located between the FF and the collimating group, requiring two independent collimator subsystems, one for each spectral band. This layout is referred to as "Split Collimator". Both are illustrated in figure 5.14, which shows single (top) and split (bottom) collimator layouts.

The single collimator concept presents challenges due to (i) the limited choice of glasses that have $\approx 420 \mathrm{~mm}$ diameter and acceptable internal transmittance at $320 \mathrm{~nm}$, which is the GMACS goal for UV-Blue limit cutoff for the conceptual design and (ii) the large exit pupil relief in order to accommodate the dichroic and grating. According to section 5.4.1, the only materials which satisfy these requirements are FS and $\mathrm{CaF}_{2}$. The latter is not an optical glass, but a crystal whose primary use is in the semiconductor industry, where it is employed in the optics of the wafer stepper for micro-lithography chip production (Rueger, 2002). A design with such glass option limitation presents primary and secondary color aberrations that are difficult to correct. Although alternative glasses from the Nikon catalog could be added to the glass selection, the viability of these glasses, according to the supplier, is risky for the required dimensions and homogeneity. Therefore, the Nikon glasses were excluded from the CoD GMACS optical design.

The principal characteristic of the Split Collimator is that the dichroic is located between the FL and the collimator group. Consequently, there are two independent collimator groups, one for each spectral range. This layout allows for better pupil and color correction since the collimator groups are optimized for a smaller bandwidth. Additionally, the dichroic is located before the collimator group and then the pupil relief can be smaller than for the single collimator, which in turn also reduces the diameter of all the camera elements. The disadvantages, however, are the need for additional optical elements, the 

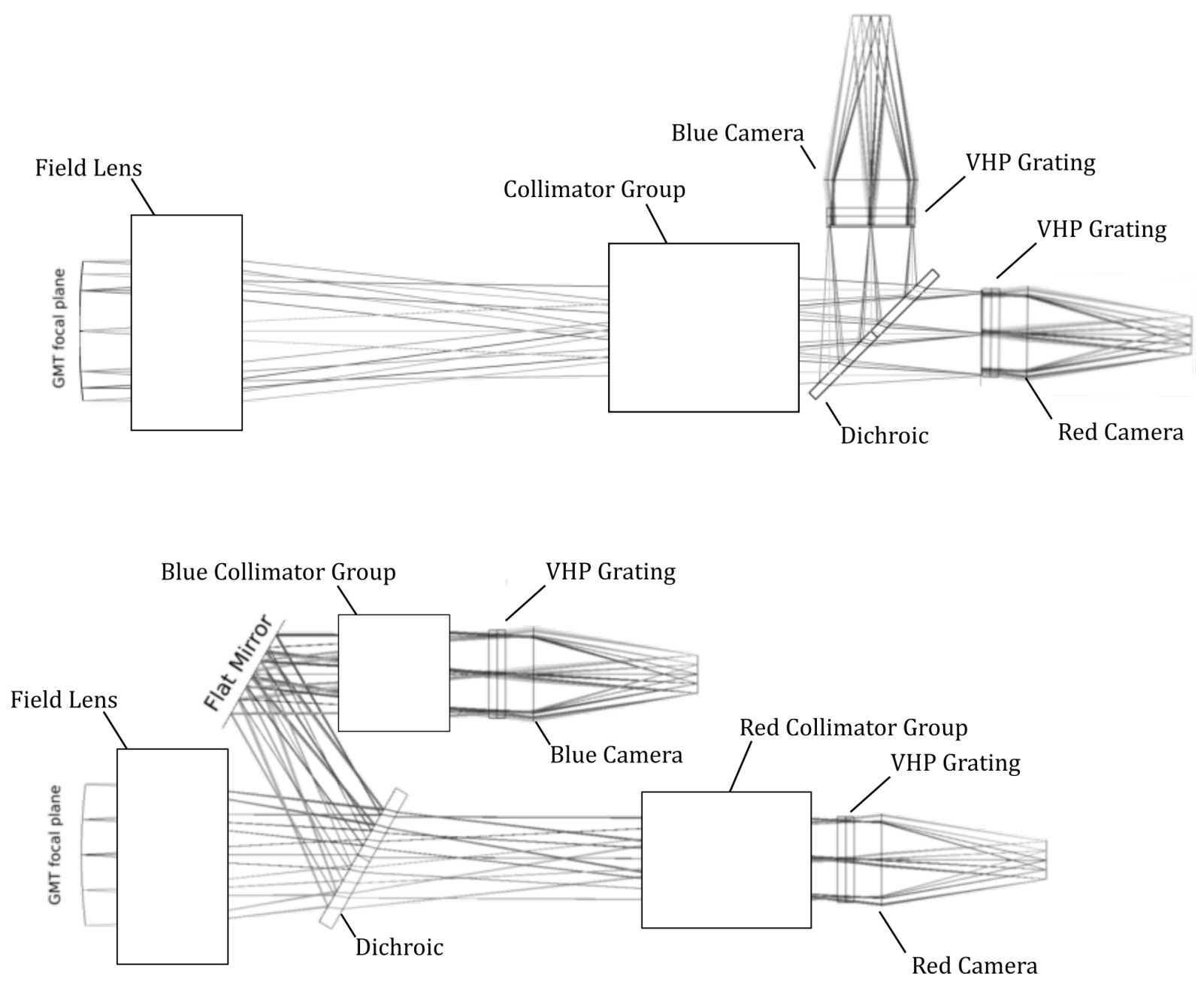

Figure 5.14: General optical layouts for the refractive collimator architectures. Both the collimators have similar layouts: a field lens, located near the focal plane of the telescope, which mostly controls the pupil imagery and is followed by a collimating group, essential for aberration correction. The location of the dichroic differentiates the two concepts explored: while the single collimator (top) has the dichroic placed in a collimated region, next to the collimator exit pupil, the two arm split collimator (bottom) has the dichroic at a converging beam region, next to the field lens.

necessity for a flat mirror due to mechanical limits, a component for correcting astigmatism caused by the dichroic location in the convergent beam region and a new mechanical structure design.

The collimator architecture decision is of paramount importance because it will impact the rest of the project and directly influence the maximum performance achieved by GMACS. Figure 5.15 shows the diagram of the refractive collimator architectures that we developed and explored in this research. In the next sections, we explore both designs' 
common elements.

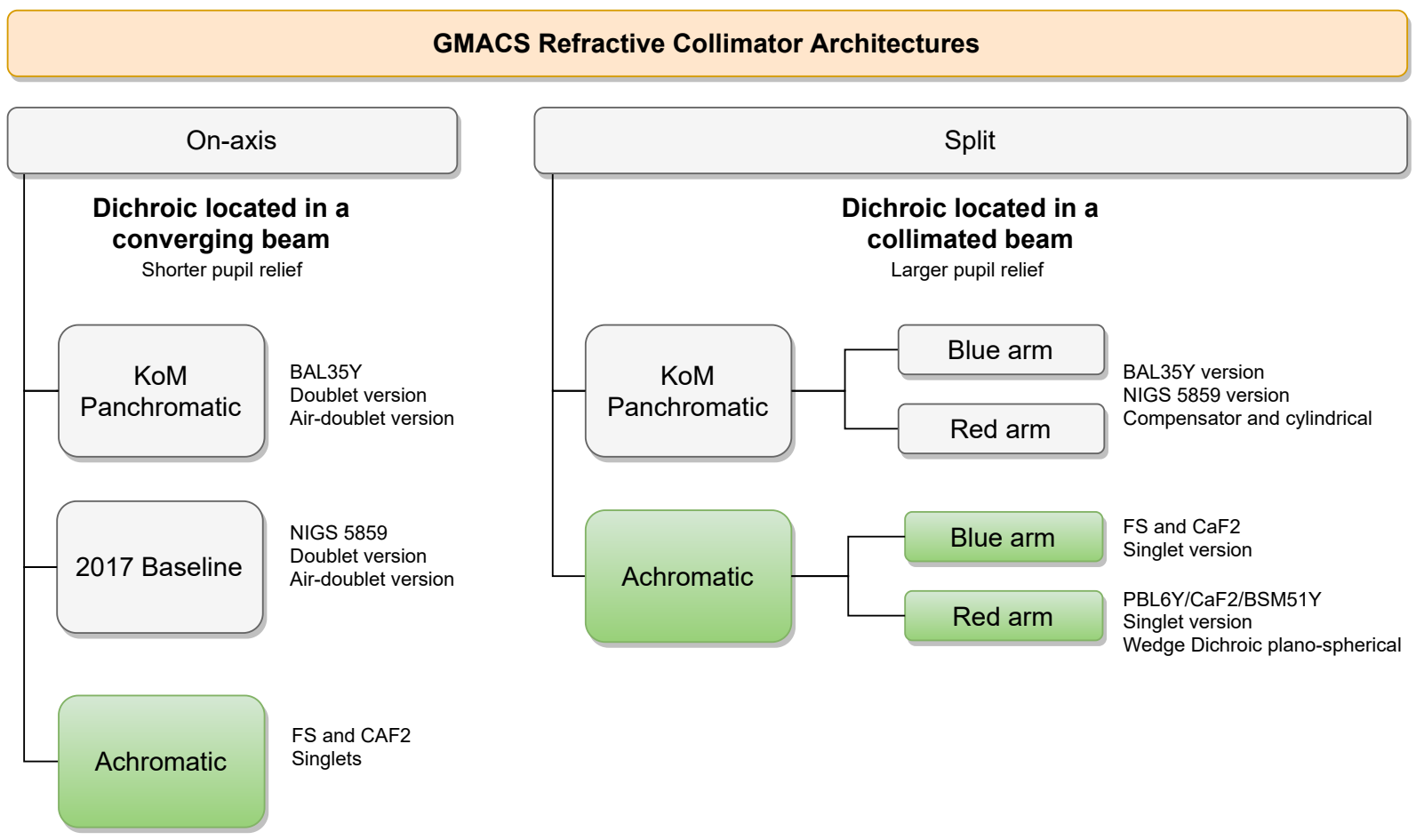

Figure 5.15: GMACS collimator refractive architectures explored in this work.

\subsubsection{Common Elements}

\subsubsection{Field Lens}

The primary purpose of an FL is to image the telescope entrance pupil. This element (or group of elements) has little control over system aberrations. According to section 2.4.1.1, which reviews the relationship between the shape factor of a lens and the aberration correction, we can consider that the FL generates a real image of a far-conjugate, which is the telescope entrance pupil, onto an available conjugate. It means that the FL optimum orientation is the spherical surface to face the long or infinite conjugate. The alternative interpretation is that the images of the slits are projected into infinity, which consequently reverses the conclusion above. Although the spherical aberration might remain at the same value, the coma signal is inverted (see figure 2.19). Therefore, the choice for the FL shape depends on the collimating group capabilities to correct the coma introduced by the FL and the spherochromatism expected for any FL choice orientation. We did several local and global optimizations to find the best shape factor for the FL, considering 
it as the principal power provider for the collimator and for several collimating group design concepts. In addition, an important feedback to define the best FL shape was the Finite Element Analysis (FEA) of the gravity vector influence on two possible FL shape factors, biconvex and plano-convex field lens. Figure 5.16 summarizes the results. The plano-convex FF shows a better performance for the normal and $60^{\circ}$ gravity vector.

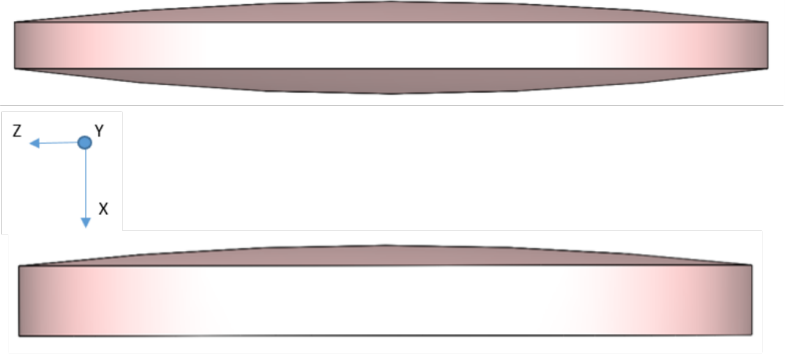

\begin{tabular}{|c|c|c|}
\hline & Normal Along X (mm) & 60 about $Y(\mathrm{~mm})$ \\
\hline Max Res. Displacement & $1.3630 \mathrm{E}-04$ & $6.8470 \mathrm{E}-05$ \\
\hline Max X Displacment & $1.3630 \mathrm{E}-04$ & $6.8170 \mathrm{E}-05$ \\
\hline Max Y Displacment & $1.8790 \mathrm{E}-05$ & $9.3980 \mathrm{E}-06$ \\
\hline Max Z Displacment & $1.8790 \mathrm{E}-05$ & $1.3900 \mathrm{E}-05$ \\
\hline & Normal Along $\mathrm{X}(\mathrm{mm})$ & 60 about $Y(\mathrm{~mm})$ \\
\hline Max Res. Displacement & $8.656 \mathrm{E}-05$ & $4.366 \mathrm{E}-05$ \\
\hline Max X Displacment & $8.6560 \mathrm{E}-05$ & $4.3290 \mathrm{E}-05$ \\
\hline Max Y Displacment & $1.3530 \mathrm{E}-05$ & $6.7680 \mathrm{E}-06$ \\
\hline Max Z Displacment & $1.3530 \mathrm{E}-05$ & $1.0920 \mathrm{E}-05$ \\
\hline
\end{tabular}

Figure 5.16: Finite element results due to gravity for two FL shape factors - biconvex and planoconvex. The maximum resulting displacements for each axis for the gravity vector oriented along $\mathrm{x}$-axis and $60^{\circ}$ about $\mathrm{y}$-axis are shown in the left tables. The planoconvex FF shows a better performance for the normal and $60^{\circ}$ gravity vector. Source: TAMU internal reports.

\subsubsection{Filters Location}

The two possible locations for the filters are within the collimator or the camera barrels. If the filters are located within the camera barrel and close to the focal plane, they can originate instrument stability issues since the flexure is more aggravated at the ends of the instrument. This factor is aggravated because the required filter selector mechanism would considerably affect the camera barrel stiffness and stability. Local surface irregularities or filter coating strain may also provoke stray light, transmittance losses, and throughput dependence on the field. The filter located right after the diffraction grating next to the camera entrance, despite being a valid solution, would require the lens dimensions to increase to avoid vignetting and longer entrance pupil relief. This effect is similar in the case of the filter positioned in the collimator, before the grating. The collimator exit pupil relief would increase, which requires larger lenses and more complex collimator designs.

The ideal position for a flat window is a region with smaller AOI value distribution to avoid the complexity of filter coatings, polarization-dependent effects, and aberrations. Since the camera design would be severely limited by increasing the entrance pupil relief, 
the filters shall be located in the collimator. Therefore, considering the refractive collimator composed of an FL followed by a collimating group, the most suitable place for the filters that causes a minimal disturbance in the optical design, reduce the complexity of the lenses coating, the required size of the filters, and potential effects of flexure, is just before the group of collimators. Since we conclude it after the CoDR, however, the designs we presented in this research present the filter and shutter within the camera barrel.

\subsubsection{Single On-axis Collimators}

The single on-axis collimator layouts that we designed are the KoM, 2017 Baseline, and Broadband. Their designations refer to the project milestones in which they were introduced, which were motivated by the required tradeoff studies. Schmidt et al. (2016) shows the previous single-arm collimator for UV-Blue cutoff at $370 \mathrm{~nm}$, in which the glass catalog was expanded to include flint glasses such as BAL35Y, FK5, and FK54.

The baseline refractive collimator design, similar to the concept design for an imaging spectrograph proposed by Shectman (2004), is comprised of an FL followed by a correcting group as close as possible to the exit pupil, yet far enough to allow the grating mechanism. The KoM and 2017 Baseline layouts are very similar, except for the material of one of the lenses. Both designs are a $7.4^{\prime}$ on-axis refractive design with a single FL followed by a reverse telephoto group comprised of one doublet and two singlets with one asphere surface located at the last optical surface, shown in 5.17.

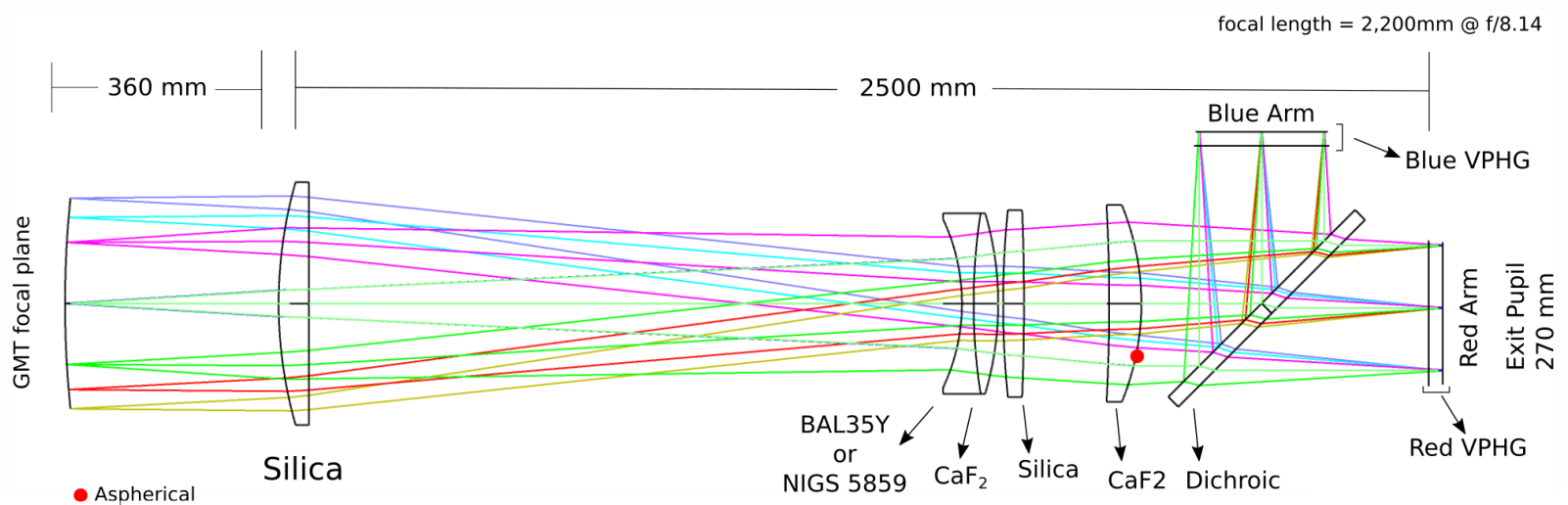

Figure 5.17: KoM and 2017 Baseline collimator optical layout. The first has the BAL35Y and the second has the NIGS 5859 glass material in the first negative lens.

The on-axis collimator is a simple and efficient collimator for GMACS. This architecture 
allows minimum intrusion from the available instrument volume into the adjacent volume. Although the Broadband Anti-Reflection (BBAR) coating is one of the major risks for this design, the feedback from coating suppliers shows that the current technology for BBAR coatings is suitable for providing the required throughput over all the instrument spectral range and supports the decision to adopt this design over the split collimator, described during the Kickoff Meeting.

The collimator concept has a relatively high complexity, in terms of the number of lenses and an aspheric surface, compared to the similar wide-field collimators, mainly because of the high pupil image quality and large exit pupil relief required by ELT spectrographs. On the other hand, the field aberrations, spherochromatism, and lateral and transverse color aberrations can be highly minimized.

The optical data for the Baseline panchromatic collimator is shown in table 5.6. The Best Fit Spherical Deviation, BFSD, is defined as the difference of the aspherical surface from the best spherical curvature.

Table 5.6 - Lens Data for the KoM and 2017 Baseline Collimators.

\begin{tabular}{|c|c|c|c|c|}
\hline & ID & Glass Material & Opt. Diameter [mm] & Asp. surf. $\backslash$ BFSD \\
\hline & CL0 & FS & 510 & - \\
\hline \multirow{4}{*}{ DB1 } & CL1 & BAL35Y/NIGS 5859 & 390 & - \\
\hline & CL2 & $\mathrm{CaF}_{2}$ & 390 & - \\
\hline & CL3 & Silica & 430 & - \\
\hline & CL4 & $\mathrm{CaF}_{2}$ & 430 & $2^{\text {nd }} / 282 \mu \mathrm{m}$ \\
\hline
\end{tabular}

The main disadvantages of the Baseline design are the required NIGS5859 blank dimensions. Although the thickness issue might be solved by cementing two parts of the same material, the diameter is a technical obstacle. Three possible solutions are (i) returning to the KoM panchromatic design, which would decrease considerably blue throughput; (ii) replacing NIGS5859 for another glass, which will require a new phase of re-optimization; and (iii) funding Nikon to develop the technology to increase the current blank diameter. Since the current design includes bonded elements and to assess true performance and avoid any use of solid or oil cemented optics, the doublets, and triplets must be air-space; consequen- 
tly, the system must be re-optimized. The lenses will get stronger and probably require increment in thickness that, in turn, might make glasses more difficult to obtain at the needed size. Since the dichroic is between the collimator and the exit pupil, the pupil relief length increases, resulting in larger collimator lens diameters.

The following results were generated using a paraxial f/2.2 camera placed on the VPHG location to solely account for the collimator performance. The spot diagram for the panchromatic collimator is shown in figure 5.18. The box size is $0.3^{\prime \prime}$ width, and the fields cover $7.4^{\prime}$ collimator FoV. The maximum RMS diameter is $0.08^{\prime \prime}$ and GEO diameter, EED100, is $0.40^{\prime \prime}$ with the centroid as the reference.

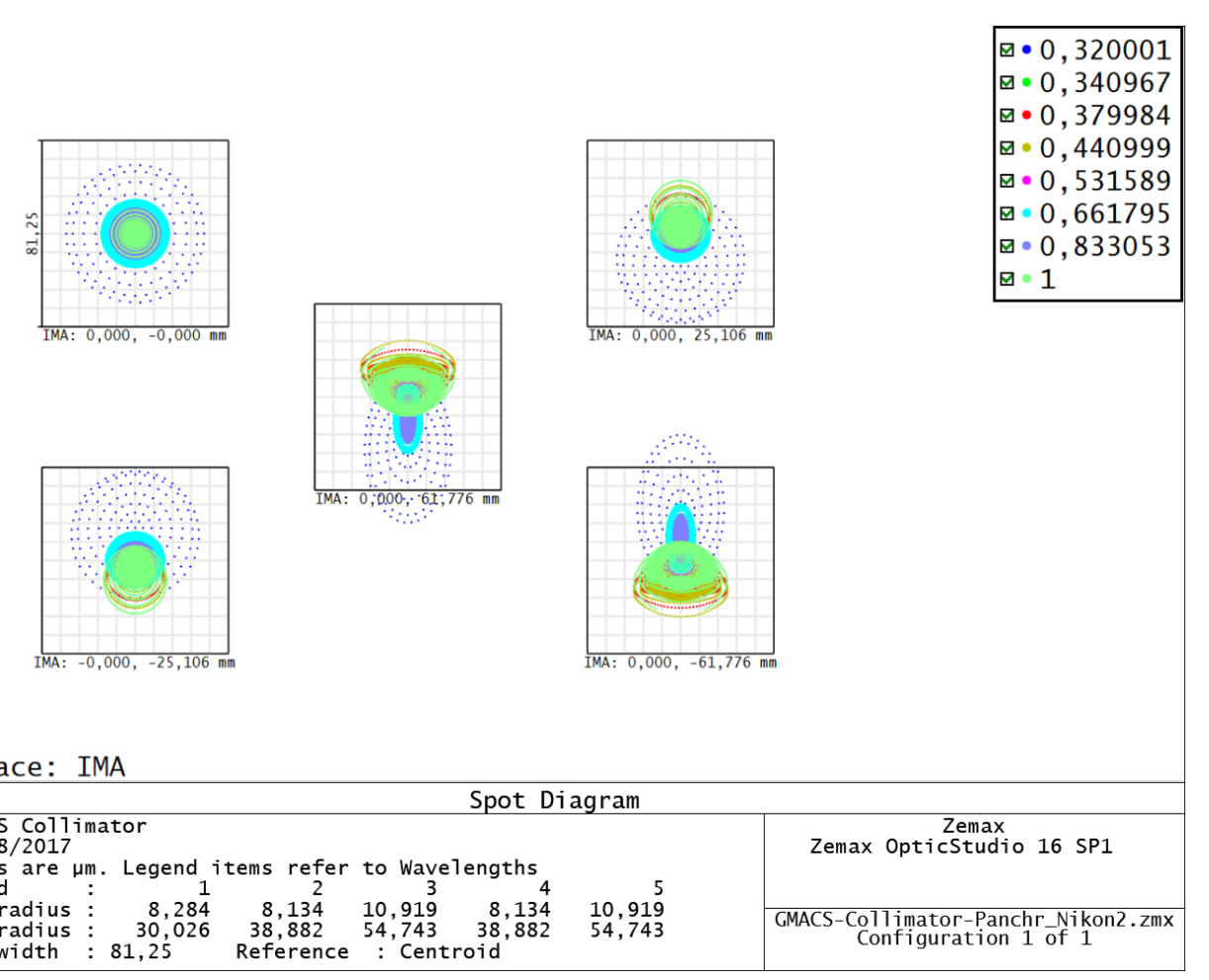

Figure 5.18: Baseline Panchromatic collimator spot diagram. The box size is $0.3^{\prime \prime}$.

The fraction of enclosed energy as a function of the width from centroid is shown in 5.19. The lines are the average over the wavelength for all the fields considered in the simulation. The average wavefront RMS is $\leq 1 \lambda @ 455 \mathrm{~nm}$, with the worst performance on the bluer side. 


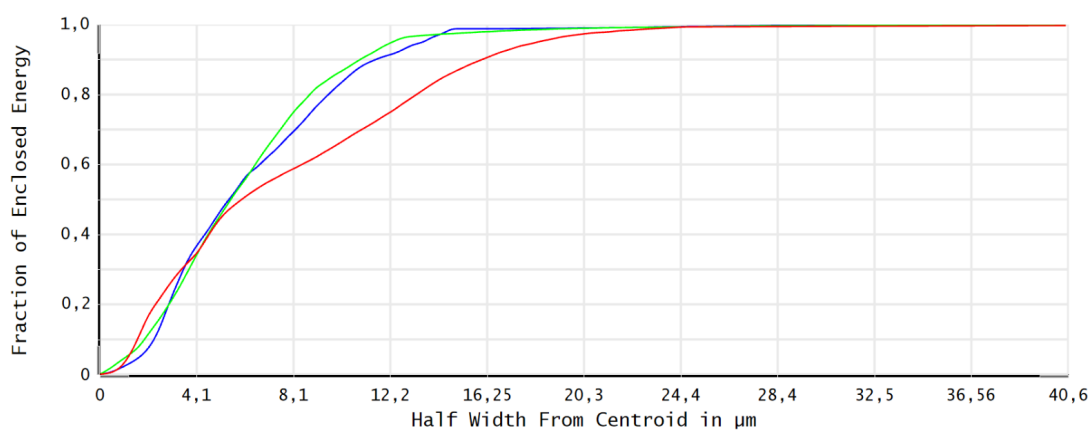

$-0,00,0,00 \mathrm{~mm}-0,00,25,08 \mathrm{~mm}-0,00,61,44 \mathrm{~mm}$

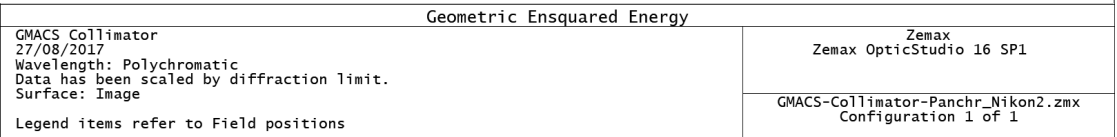

Figure 5.19: Baseline Panchromatic collimator geometric ensquared energy.

The Nikon glass NIGS5859 considerably increased the 320-350 nm throughput compared to the KoM design which used BAL35Y.

The chromatic focal shift of the collimator is shown in figure 5.20. The correction has three waves at the same focal length over the GMACS spectral range; two centered in the corresponding blue and red spectral range, and the third around the dichroic cut-off. The maximum focus shift range is $182 \mu \mathrm{m}$.

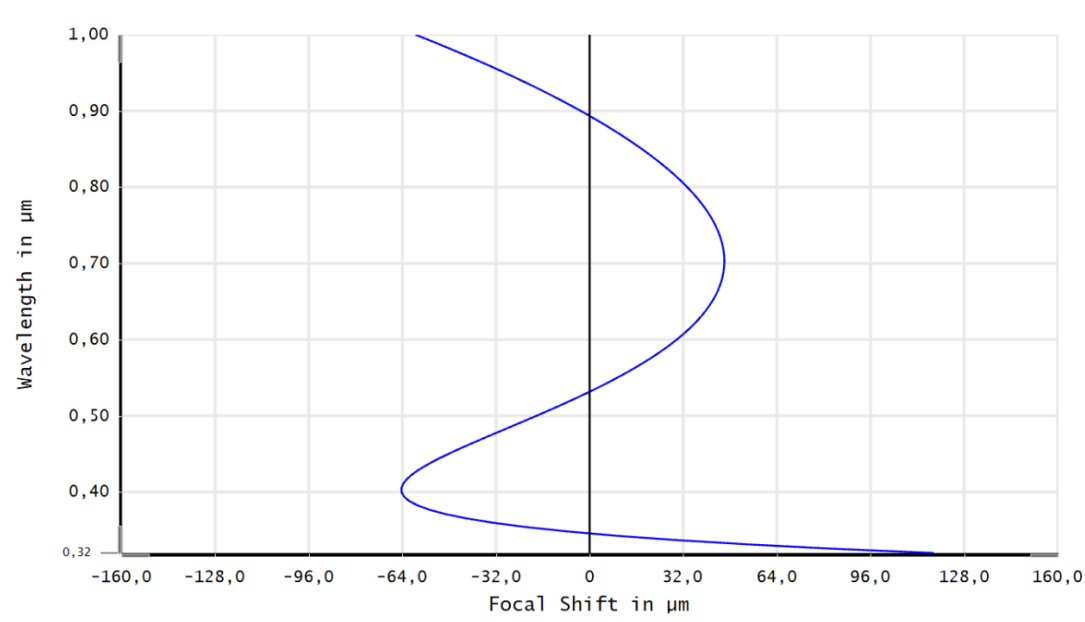

\begin{tabular}{|c|c|}
\hline \multicolumn{2}{|c|}{ Chromatic Focal Shift } \\
\hline $\begin{array}{l}\text { GMACS Collimator } \\
28 / 08 / 2017 \\
\text { Maximum Focal Shift Range: } 182,0114 \mu \mathrm{m} \\
\text { Diffraction Limited Range: } 10,765 \mu \mathrm{m}\end{array}$ & $\begin{array}{l}\text { Zemax } \\
\text { Zemax OpticStudio } 16 \text { SP1 }\end{array}$ \\
\hline Pupil Zone: 0,0000 & $\begin{array}{l}\text { GMACS-Collimator-Panchr_Nikon2.zmX } \\
\text { Configuration } 1 \text { of } 1\end{array}$ \\
\hline
\end{tabular}

Figure 5.20: Panchromatic collimator chromatic focal shift. 
Figure 5.21 shows the Broadband collimator layout conception. The key highlights are simplicity and high throughput for the deep UV-Blue spectral range. It is a single on-axis optical system with the only glasses with good internal transmittance below $350 \mathrm{~nm}$ : FS and $\mathrm{CaF}_{2}$. The principal disadvantages are the large spherochromatism due to the required broad spectral range, which cannot be well-balanced with only two glass types. Aspheric surfaces cannot satisfactorily compensate for it. The $\mathrm{BB} F \mathrm{~S} / \mathrm{CaF}_{2}$ collimator performance is the worst among the other architectures. Table 5.7 summarizes the performance of the single on-axis collimator designs.

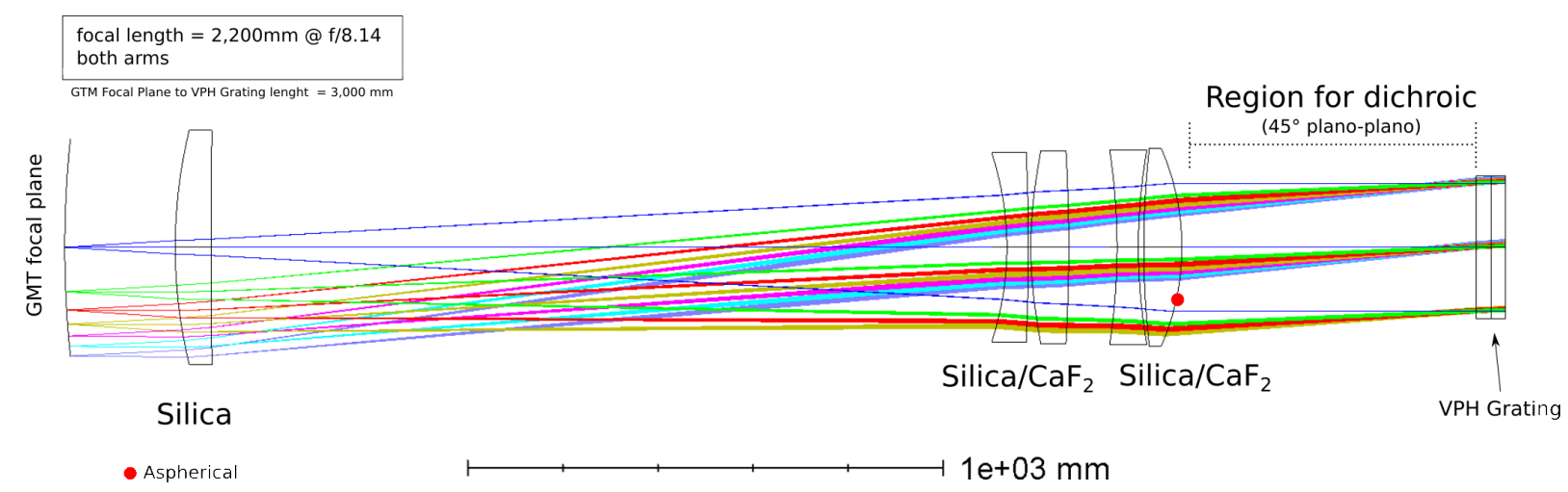

Figure 5.21: Broadband Collimator Optical Layout.

\subsubsection{Split collimators}

The Split Collimator is a compact on-axis two-arms collimator with the dichroic placed in a converging beam region. The dichroic reflected beam is redirected by a fold mirror towards a collimator group while the transmitted one is followed by the other collimator group.

The principal driver for this layout is to reduce the magnitude of the expected flexure, but likely not completely eliminate it. Intrusion into adjacent instrument volumes is more substantial compared to the single on-axis collimator (GMACS Team, 2019) since the optical axis for the reflected beam is offset from the main telescope axis.

The split collimator architecture has additional design constraints compared to the onaxis version, for instance, the distance between the axis of the arms, the dichroic tilt, and the need for a transversal displacement of the collimator group in the transmitted beam arm. Figure 5.22 shows the design layout and the variables concerning the axial distance 
Table 5.7 - Performance of the single on-axis collimators.

\begin{tabular}{|c|c|c|c|}
\hline & $\begin{array}{c}\text { KoM } \\
\text { Panchromatic }\end{array}$ & $\begin{array}{l}2017 \text { Baseline } \\
\text { Panchromatic }\end{array}$ & $\begin{array}{c}\text { FS-CaF } 2 \\
\text { Achromatic }\end{array}$ \\
\hline Collimator Concept & Single on-axis & Single on-axis & Split \\
\hline Wavelength range & 320-1000nm & 320-1000nm & $300-600 \mathrm{~nm}$ \\
\hline Focal Length $[\mathrm{mm}]$ & 2,200 & 2,200 & 2,200 \\
\hline \# Lenses & 5 & 5 & 4 \\
\hline Material & $\begin{array}{l}2 \mathrm{x} F S \\
2 \mathrm{x} \mathrm{CaF}_{2} \\
1 \mathrm{x} \text { BAL35Y }\end{array}$ & $\begin{array}{l}2 \mathrm{x} F \mathrm{~S} \\
2 \mathrm{x} \mathrm{CaF}_{2} \\
1 \mathrm{x} \text { NIGS } 5859\end{array}$ & $\begin{array}{l}2 \mathrm{x} F \mathrm{~S} \\
2 \mathrm{xaF}_{2}\end{array}$ \\
\hline Cemented optics & $\begin{array}{c}\text { Yes } \\
\mathrm{FS} / \mathrm{CaF}_{2}\end{array}$ & $\begin{array}{c}\text { Yes } \\
\mathrm{FS} / \mathrm{CaF}_{2}\end{array}$ & No \\
\hline $\begin{array}{c}80 \% \text { Ensquared } \\
\text { Energy Diameter } \\
\text { (field, value }[\text { arcsec]) }\end{array}$ & $\begin{array}{c}\text { on-axis: } 0.060 \text { arcsec } \\
0.7 \text { pupil: } 0.124 \operatorname{arcsec} \\
1 \text { pupil: } 0.145 \operatorname{arcsec}\end{array}$ & $\begin{array}{l}0.078 \text { arcsec } \\
0.097 \text { arcsec } \\
0.113 \text { arcsec }\end{array}$ & $\begin{array}{l}0.349 \text { arcsec } \\
0.320 \text { arcsec } \\
0.305 \text { arcsec }\end{array}$ \\
\hline $\begin{array}{c}\text { Throughput } \\
\text { (wavelength, value [\%]) }\end{array}$ & $\begin{array}{l}300 \mathrm{~nm}: \leq 10 \\
320 \mathrm{~nm}: 15 \\
350 \mathrm{~nm}: 40 \\
400 \mathrm{~nm}: 85 \\
600 \mathrm{~nm}: 85 \\
800 \mathrm{~nm}: 85 \\
1000 \mathrm{~nm}: 85\end{array}$ & $\begin{array}{l}70 \\
80 \\
85 \\
85 \\
85 \\
85 \\
85\end{array}$ & $\begin{array}{l}85 \\
85 \\
85 \\
85 \\
85 \\
85 \\
85\end{array}$ \\
\hline $\begin{array}{l}\text { Lateral colour } \\
\text { corrected }\end{array}$ & Yes & Yes & No \\
\hline $\begin{array}{l}\text { Vignetting } \\
\text { field or spectral }\end{array}$ & none & none & none \\
\hline $\begin{array}{c}\text { \# Asph. surf. } \\
\text { (surf, ID, BFSD) }\end{array}$ & $\begin{array}{c}1 \\
\text { 2nd, CL5, } 300 \mu \mathrm{m}\end{array}$ & $\begin{array}{c}1 \\
\text { 2nd, CL5, } 300 \mu \mathrm{m}\end{array}$ & 0 \\
\hline $\begin{array}{l}\text { On-axis total length } \\
\text { (GMT Focal plane } \\
\text { to Grating) }[\mathrm{mm}]\end{array}$ & $2,300 \mathrm{~mm}$ & $2,300 \mathrm{~mm}$ & $2,300 \mathrm{~mm}$ \\
\hline Grating Relief [mm] & 620 & 620 & 450 \\
\hline $\begin{array}{l}\text { Max. Opt. Lens Diam. } \\
\text { (material, diam.) }\end{array}$ & $\begin{array}{l}\text { FS, } 550 \mathrm{~mm} \\
\mathrm{CaF}_{2}, 250 \mathrm{~mm} \\
\text { BAL35Y, } 230 \mathrm{~mm}\end{array}$ & $\begin{array}{l}\mathrm{FS}, 550 \mathrm{~mm} \\
\mathrm{CaF}_{2}, 250 \mathrm{~mm} \\
\text { NIGS } 5859,230 \mathrm{~mm}\end{array}$ & $\begin{array}{l}\mathrm{FS}, 550 \mathrm{~mm} \\
\mathrm{CaF}_{2}, 250 \mathrm{~mm}\end{array}$ \\
\hline $\begin{array}{l}\text { Spectral Beamsplitter } \\
\text { Solution }\end{array}$ & $\begin{array}{l}45^{\circ} \text { Dichroic in } \\
\text { collimated region }\end{array}$ & $\begin{array}{l}45^{\circ} \text { Dichroic in } \\
\text { collimated region }\end{array}$ & $\begin{array}{c}45^{\circ} \text { Dichroic in } \\
\text { collimated region }\end{array}$ \\
\hline
\end{tabular}


between the arms. The distance between the optical axis of both of the collimator arms is constrained in the MF by the difference of the Global vertex $\mathrm{x}, \mathrm{y}$, and z-coordinate of the first surface followed by the fold mirror and dichroic. Although the realistic value shall be provided by the mechanical constraints, we adopted a baseline distance of $\mathrm{D}=650$ $\mathrm{mm}$ based on a first-order value estimate as a function of the diameters of the elements. Therefore, considering figure 5.22, the remaining variables for optimization are A, B, C, and $\sigma$. Since the challenges for the blue arm are higher compared to the red arm mainly due to the glass limited options, we decided that the reflected beam would be directed towards the blue arm. Therefore, the red arm should provide correction for the aberrations caused by the tilted dichroic located in a converging beam region.

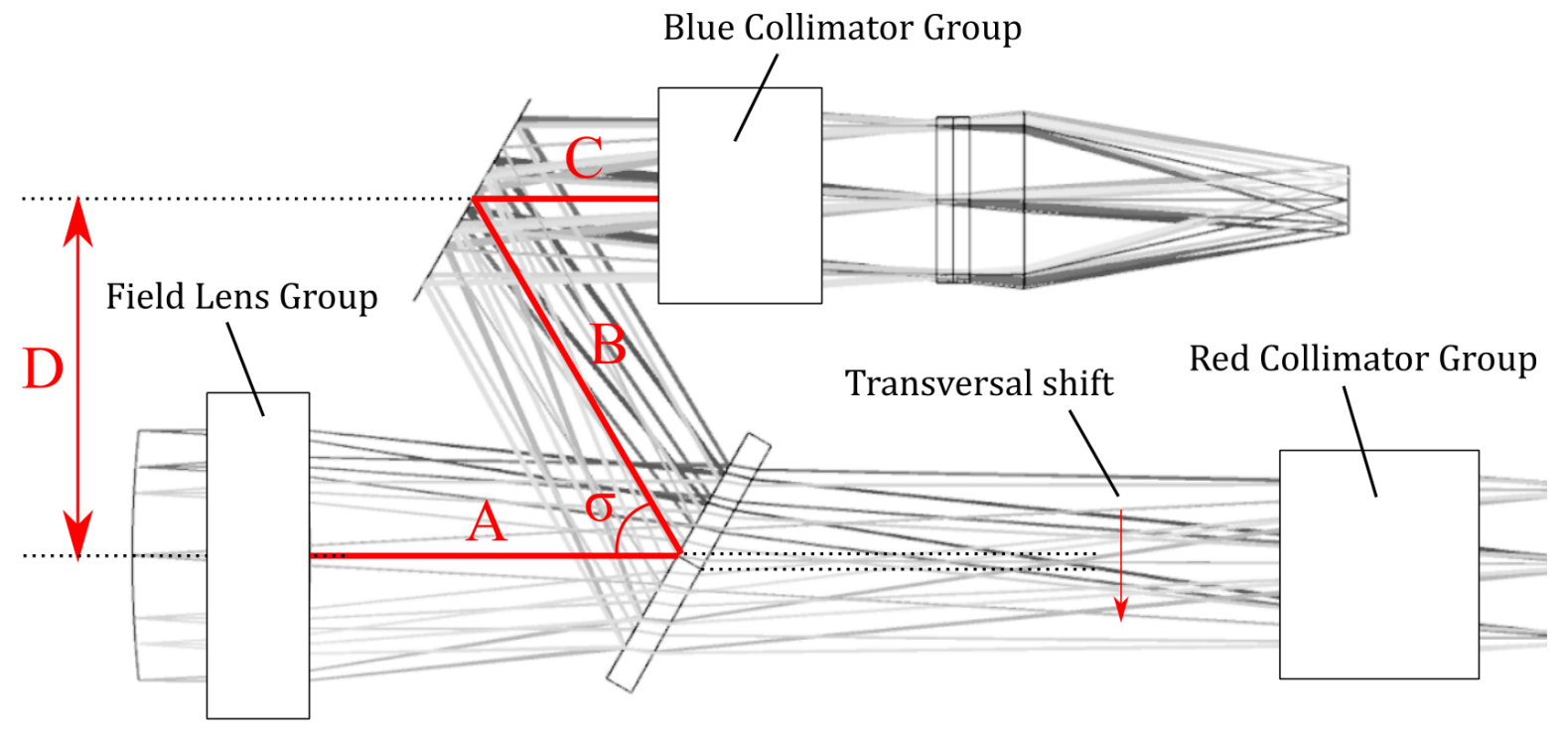

Figure 5.22: Split Collimator general layout and the variables to define the axial distance between the channels.

The positive spherical FS FL is the only optical element shared by both collimator arms. The red collimator group and the following optics are given a transverse displacement in the plane of figure 5.22, set as variable in the MF, to compensate the beam deviation caused by the tilted dichroic, according to section 2.4.1.2. A dichroic splits the beam feeding the two collimator arms. The dichroic acts as a plane mirror in the blue collimating arm. A plane mirror is used to accommodate space constraints, followed by the blue collimating group.

The MCE for the split collimator has a unique configuration for each of the arms. It is based on a set of IGNR functions to ignore surfaces on the LDE to change between the 
blue and red collimator within the same Zemax file and to optimize the collimator arms simultaneously.

\subsubsection{Astigmatism Compensator}

This section presents a brief comparison among the selected alternative for the correction of astigmatism caused by dichroic located in a region of converging beams. The favored solution is the one which satisfies astigmatism corrections, minimizes degradation of the throughput by reducing the elements, and presents low risk for manufacturing.

Compensator: According to the Seidel coefficient in table 2.4, the dependence of the astigmatism is the square of the tilt angle, and therefore it does not depend on the sign of the window tilt. Thus, astigmatism cannot be compensated by a second plate tilted on the opposite direction from the first plate and in the same plane of tilt. Fischer et al. (2008) suggest that the insertion of a second window with the tilt in a plane orthogonal to the plane of tilt of the first window can correct most of part of the astigmatism. However, the sheer size of the collimator field causes large, asymmetric and uncorrectable residual aberrations.

Wedge plano-plano dichroic and compensator: This solution adds a second plate tilted in the opposite signal to the beamsplitter. However, both the elements have a small wedge and act as prisms. The resultant aberrations, including lateral color, are nearly entirely symmetric and thus intrinsically correctable. It also eliminate the manufacturability concerns, although adding elements impacts the throughput. This solution was adopted in the earlier version of the Split Collimator (Ribeiro et al., 2018b).

Cylindrical Compensator: The cylindrical element compensator is the straightforward solution since it gives an additional power in the same direction of the astigmatism. In addition, instead of a new element, the cylindrical shape might be fabricated on the dichroic rear surface. Both solutions work satisfactorily. The resulted cylindrical surface RoC for the red collimator arm is about $\approx 120$ meters. The principal drawbacks are related to (i) the manufacture since suppliers may have difficulties to produce large RoC, and (ii) to alignment since the cylindrical axis must be aligned to the plane generated by the optical axis and the dichroic orthogonal tilt. 
Weak Menisco or altered dichroic: Fischer et al. (2008, p. 76) shows that "a weak meniscus lens close to the image plane acts similar to a tilted plate with a tilt angle which changes from zero on-axis to a certain angle at the edge of the field. This way, astigmatism created by the meniscus can partly or entirely cancel the astigmatism of the rest of the optical system". Although this compensator proposed by Fischer et al. (2008) cannot be located close to the collimator image plane, which would be the mask plane, the weak meniscus approach can be adapted by using a weak spherical surface on the tilted window or a weak wedge instead of a parallel window.

Combination of the solutions: The combination of the above mentioned methods was explored in order to optimize the solution in terms of astigmatism corrections and throughput. For instance, a cylindrical lens decentered acts like a weak power lens with wedge that, in combination with a compensator, might correct the astigmatism and coma. Moreover, a wedge plano-spherical dichroic acts as a weak meniscus.

Wedge plano-spherical dichroic: An optimum solution would be the same element that combines the dichroic with the astigmatism compensator. Therefore, rather than being a plane-plane element, the wedge plano-spherical dichroic consists of a small wedge planeconcave element with the rear surface tilt of $\approx 30^{\prime}$. The front surface of the dichroic is flat and acts as a beamsplitter that reflects the blue spectral range to the blue collimator group and transmits the spectral band to the red collimator group. The RoC of the second surface is $\approx 60$ meters. The optical axis of the transmitted beam must be given an offset of $17 \mathrm{~mm}$. This solution is adequate for a dichroic tilted by a maximum of $30^{\circ}$ with a thickness of $\leq 50 \mathrm{~mm}$ (GMACS Team, 2019). This solution eliminates the need for the introduction of an additional element to compensate for astigmatism, and avoids any manufacturability concerns of the alternative solutions, such as the one which uses cylindrical surfaces with a large radius of curvature. The resulting aberrations, including lateral color, are intrinsically correctable. The transverse displacement of the transmitted beam is compensated by a transverse displacement of the red collimator subsystem. The tilted dichroic, illustrated with exaggerated scale for visualization purposes in figure 5.23, is specially designed to prevent it from generating astigmatism on the red arm of the collimator.

The reflective coating on the flat mirror will be optimized for the blue arm for $30^{\circ}$ central 


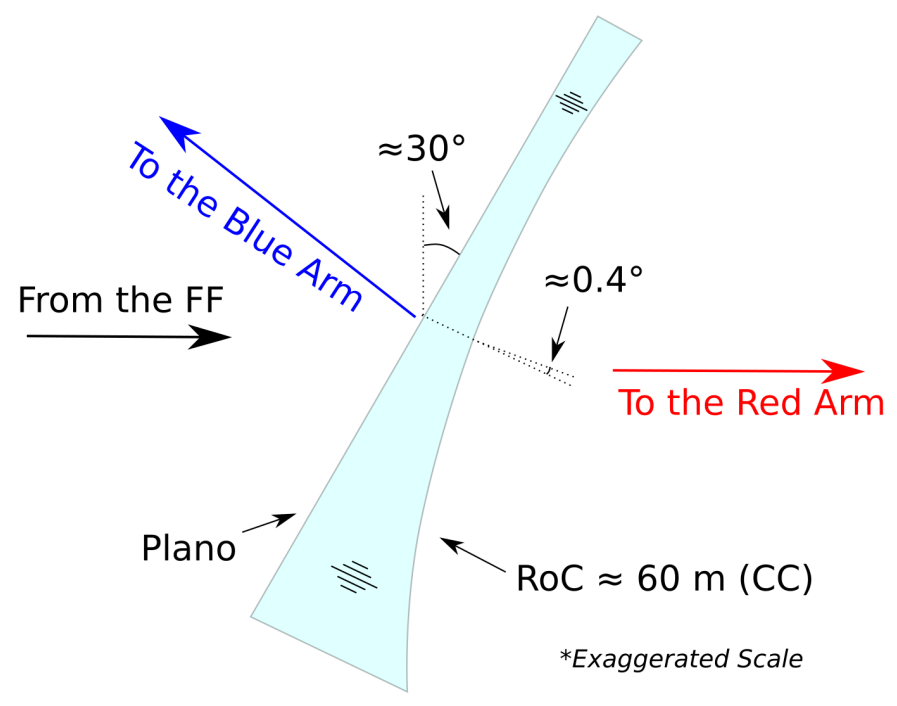

Figure 5.23: Wedged plano-spherical dichroic acting as a beamsplitter and an astigmatism compensator.

AOI. This coating shall be made of UV-enhanced aluminum to optimize the throughput in the range of $320 \mathrm{~nm}$ to $400 \mathrm{~nm}$.

\subsubsection{Split Collimator Design}

Figure 5.24 shows the final solution of the Split Collimator design presented in the GMACS CoD Review. All the glass materials for the blue arm are FS and $\mathrm{CaF}_{2}$. The red arm is comprised of the wedged plano-spherical dichroic and an air-spaced collimator group of PBL6Y, $\mathrm{CaF}_{2}$ and BSM51Y glasses.

Both the collimator groups have one aspheric surface, marked with red dots in figure 5.24 , located in the rear surface of the closest element to the collimator exit pupil, $\mathrm{CaF}_{2}$ and BSM51Y for blue and red collimator arm, respectively. The BFSD and the maximum slope, shown in table 5.8, are less than $200 \mu \mathrm{m}$ and $10 \mathrm{mrad}$. The maximum radial coordinate used for the calculation of the slope is the clear semi-diameter of the surface.

Table 5.8 - Aspheric surface parameters of the blue and red CoDR Split Collimator arms.

\begin{tabular}{|c|c|c|c|}
\hline Collimator Arm & Optical Surface & BFSD $[\mu \mathbf{m}]$ & Max. and RMS Slope $[\mathrm{mrad}]$ \\
\hline Blue & $\mathrm{CaF}_{2}$ 2nd $(\mathrm{CX})$ & $\leq 200$ & $\max =9.2 ; \mathrm{RMS}=3.7$ \\
Red & $\mathrm{BSM} 51 \mathrm{Y}$ 2nd $(\mathrm{CX})$ & $\leq 120$ & $\max =5.7 ; \mathrm{RMS}=2.3$ \\
\hline
\end{tabular}

The collimator subsystem pupil reliefs are $\approx 270 \mathrm{~mm}$, which is enough space to accommodate up to a $45^{\circ}$ rotation grating for the higher resolution modes. 


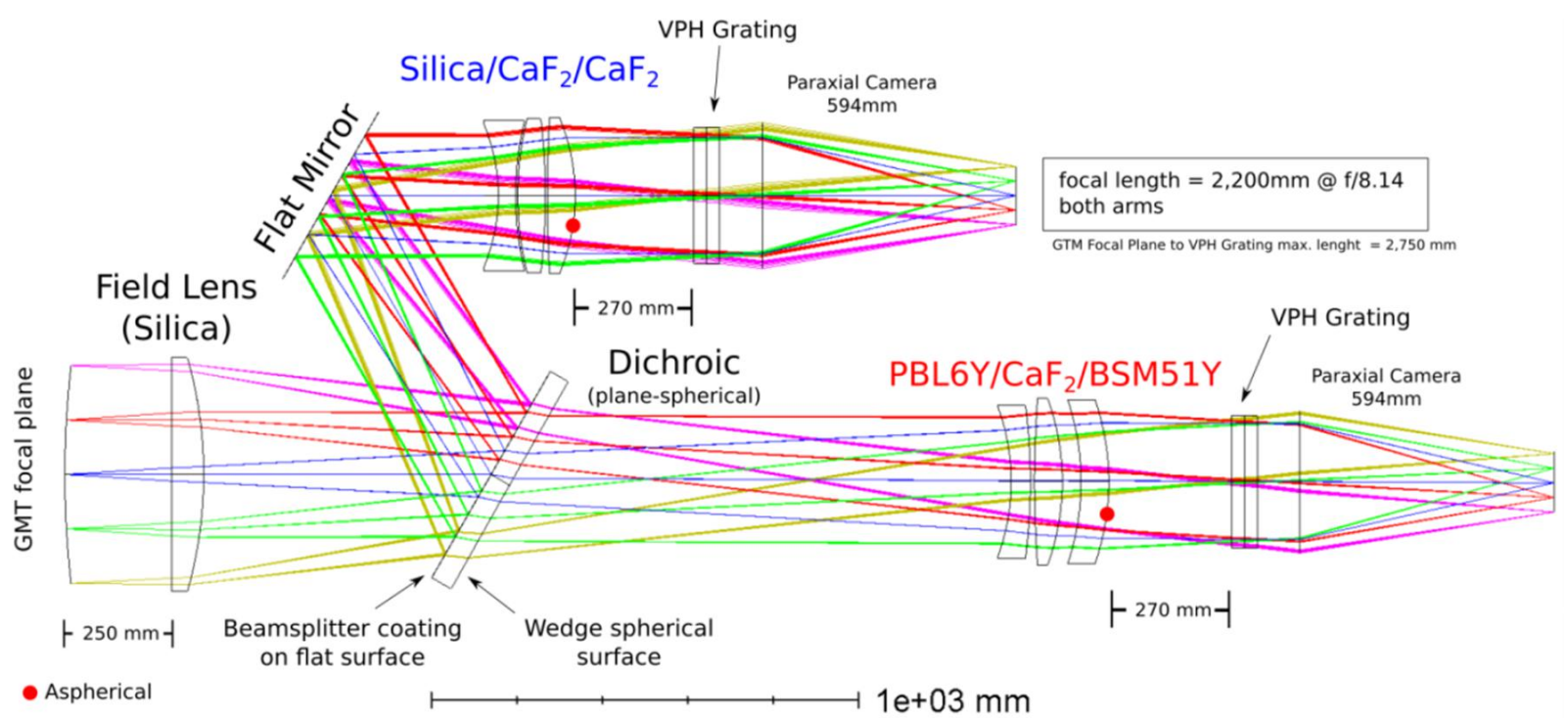

Figure 5.24: CoD Split Collimator 2,200 $\mathrm{mm} \mathrm{f/8.2,} \mathrm{FoV} 7.4^{\prime}$ in diameter, exit pupil of $270 \mathrm{~mm}$ in diameter, with paraxial cameras.

The Zemax total throughput for unpolarized light are $T_{\text {col-blue }} \approx 77.1 \%$ and $T_{\text {col-red }}$ $\approx 86.8 \%$ for the blue and red collimator arms, respectively, and present small dependence on the field and wavelength. We assumed the following considerations: (i) excluding the reflectance of the GMT mirrors and M1 central obscuration; (ii) 99\% efficiency of the dichroic in both transmissive and reflective spectral regions; (iii) 90\% flat mirror surface reflectance (UV Enhanced Aluminum) in the blue arm; and (iv) 99\% transmittance of all AR coatings including those on the FL.

Figure 5.25 shows the spot diagrams of the Split Collimator with a $594 \mathrm{~mm}$ EFL paraxial camera for a circular FoV of $7.4^{\prime}$ diameter. The reference circle is $0.3^{\prime \prime}$ in diameter. The average, RMS and standard deviation EED80 is $0.069^{\prime \prime}, 0.065^{\prime \prime}$ and $0.023^{\prime \prime}$ for the blue arm and $0.071^{\prime \prime}, 0.078^{\prime \prime}$ and $0.021^{\prime \prime}$ for the red arm, respectively.

The wavefront quality on the grating plane is calculated as a function of the field and wavelength. Figures 5.26 and 5.27 show the wavefront PV and RMS for the blue and red GMACS spectral range. The wavefront field dependence for the blue arm presents radial symmetry for the PV and RM while the wavelength dependence is optimized for the internal region of the blue spectral range. The bluer (UV-Blue) and redder (close to the dichroic transition wavelength) range have lower performance. The red arm presents an unsymmetrical profile for the field dependence and better performance of the redder side of the spectrum. Since Zemax does not provide the wavefront PV and RMS as a function 


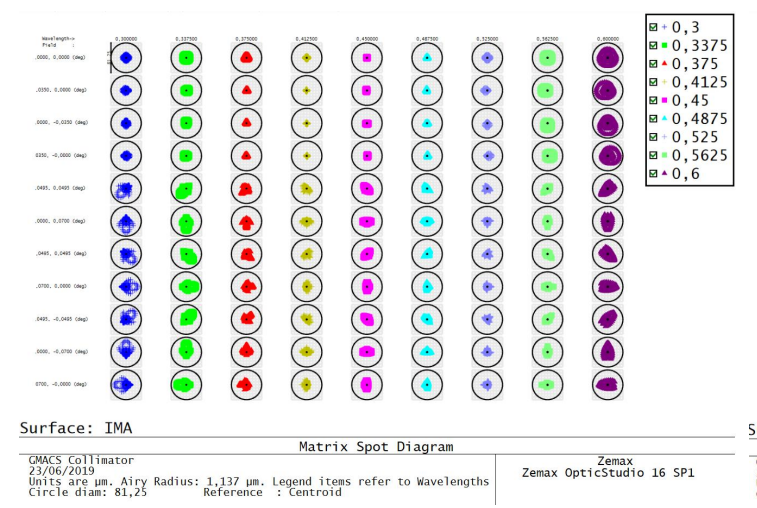

(a) Blue Arm.

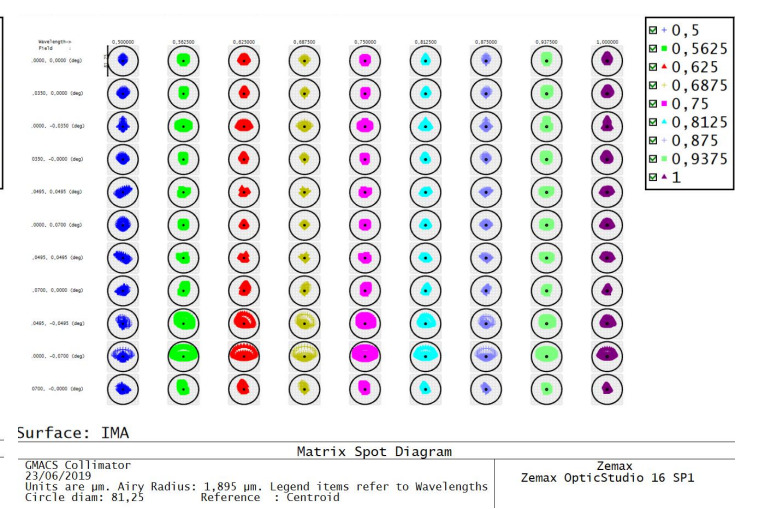

(b) Red Arm.

Figure 5.25: Spot size diagram for the Split Collimator. The box size is $0.3^{\prime \prime}$.

of the field and wave, we developed a Zemax macro that records this data and a Python script for data visualization as a heat map. Appendix $\mathrm{C}$ presents the detailed wavefront PV and RMS heat maps.

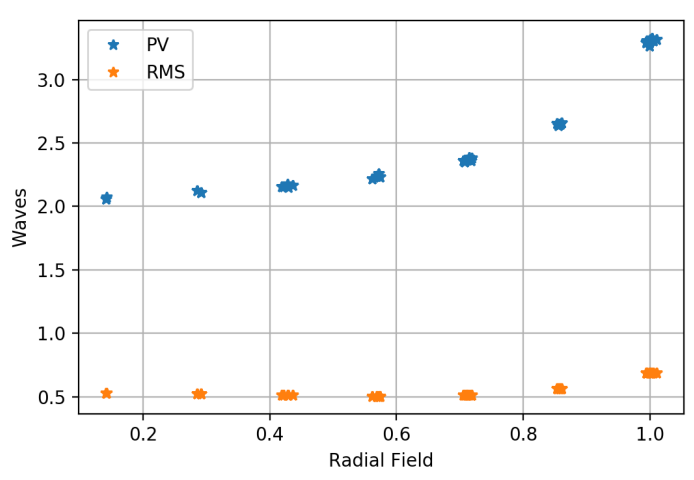

(a) Wavefront as a function of the field.

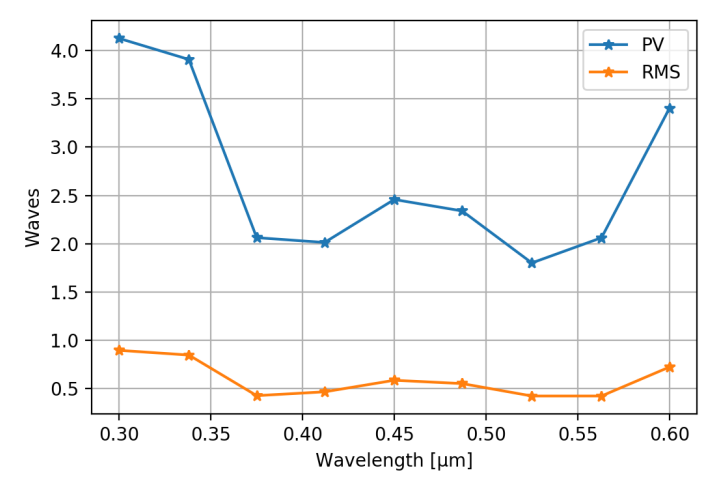

(b) Wavefront as a function of the wavelength.

Figure 5.26: The PV and RMS profile for the Split Collimator in the blue channel.

Since the primary purpose of GMACS is to extract the spectrum of the objects within the FoV, there are no constraints for the correction of the TCA. Disregarding any corrections of TCA by the cameras, large TCA in the collimator implies poor color image contrast for the image mode. Although not explicitly required, we checked the image mode performance of the CoDR Split Collimator since, according to Oswalt and McLean (2013), an image mode is often convenient for aligning the slit mask on the sky and for imaging in general, especially with narrow-band interference filters or Fabry-Perot etalons (Bernstein and Shectman, 2013).

Figure 5.28 shows that the TCA is less than $170 \mu \mathrm{m}$ for blue and $100 \mu \mathrm{m}$ for red for 


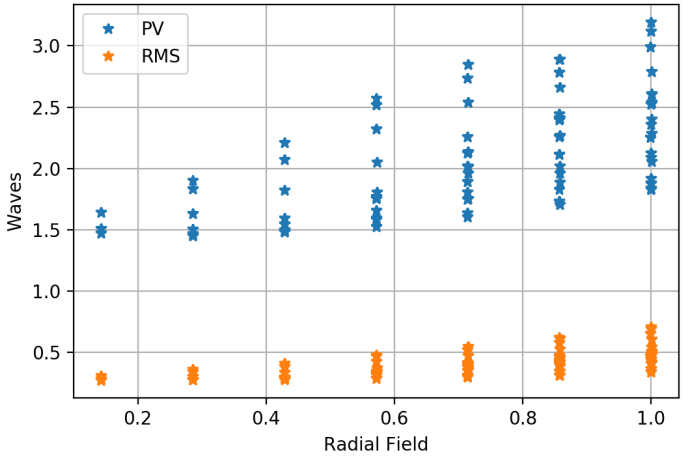

(a) Wavefront as a function of the field.

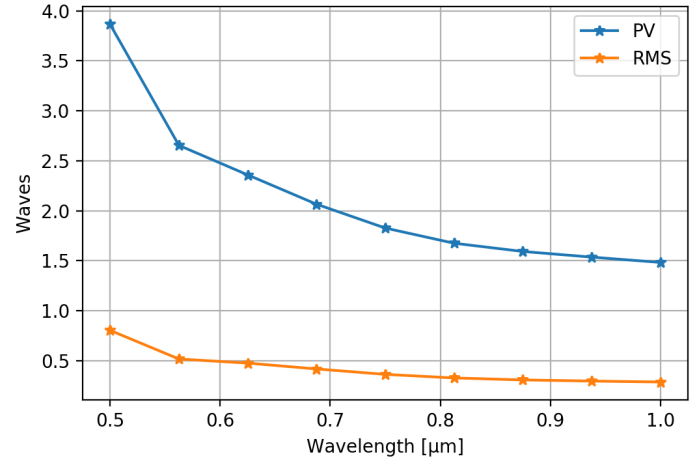

(b) Wavefront as a function of the wavelength.

Figure 5.27: The PV and RMS profile for the Split Collimator in the red channel.

the full field. Considering a maximum transverse distance between two wavelengths set as a pixel of distance (which is $15 \mu \mathrm{m}$ ), the Split Collimator would allow for an image mode with resolution of $\approx 10$ and 7 pixels size for the blue and red arm, respectively. The image sharpness is increased with narrow-band filters, yet limited by the spot size.
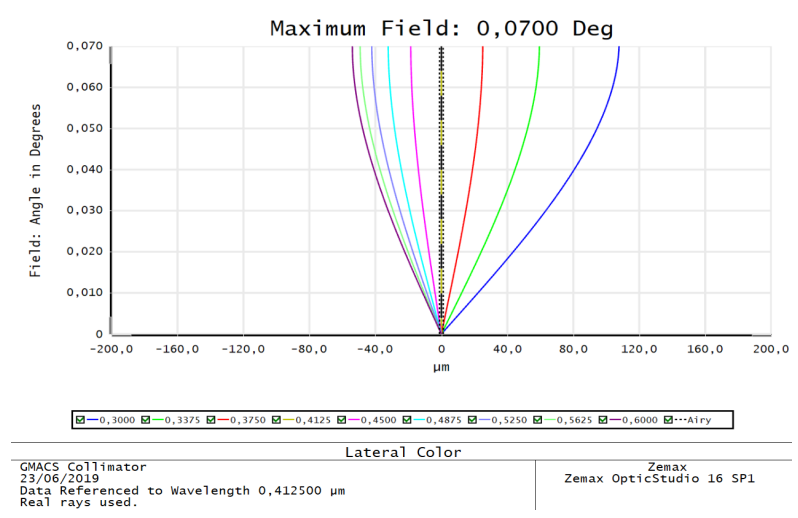

(a) Blue Arm.
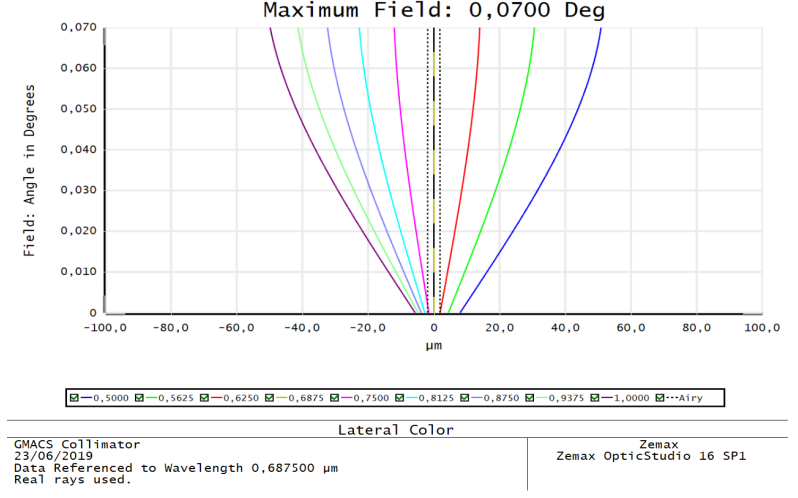

(b) Red Arm.

Figure 5.28: Transverse Chromatic Aberrations for the Split Collimator.

Alternative Split Collimator Design: The telescope, collimator, and camera EFL determine the spectrograph FoV for fixed detector dimensions. Since the spectrograph can be considered as a finite-finite conjugated, the FoV might be limited by the field stops located at the object plane of the spectrograph (focal plane of the GMT, where the mask slit is located) or at the image plane (camera's detector size). Any reduction of the dimensions of these field stops will either reduce the FoV or even change its shape.

The useful area of the detector for objects located at the edge of the field for the 
low-resolution mode might be optimized by changing the telescope field stop shape and dimensions. Consequently, all the remaining elements of the collimator must be increased to avoid vignetting, and the collimator and camera EFL values should be recalculated.

The straightforward FoV shape, which is the default CoD Split Collimator FoV, has circular symmetry. However, assuming the requirement of no spectral loss for the lowresolution mode, the edges of the detector will be unused. In order to best utilize the detector area, two alternative FoV shapes are considered. The first is a hexagonal FoV, as illustrated in figure 5.29, which slightly increases the available FoV by incorporation of the grayish area, although the redder area will present spectral vignetting. The second shape is a rectangular FoV that presents a reasonable choice due to the shape of the detector; however, it requires a significant change in the spectrograph principal parameters and, consequently, collimator and camera redesigns.

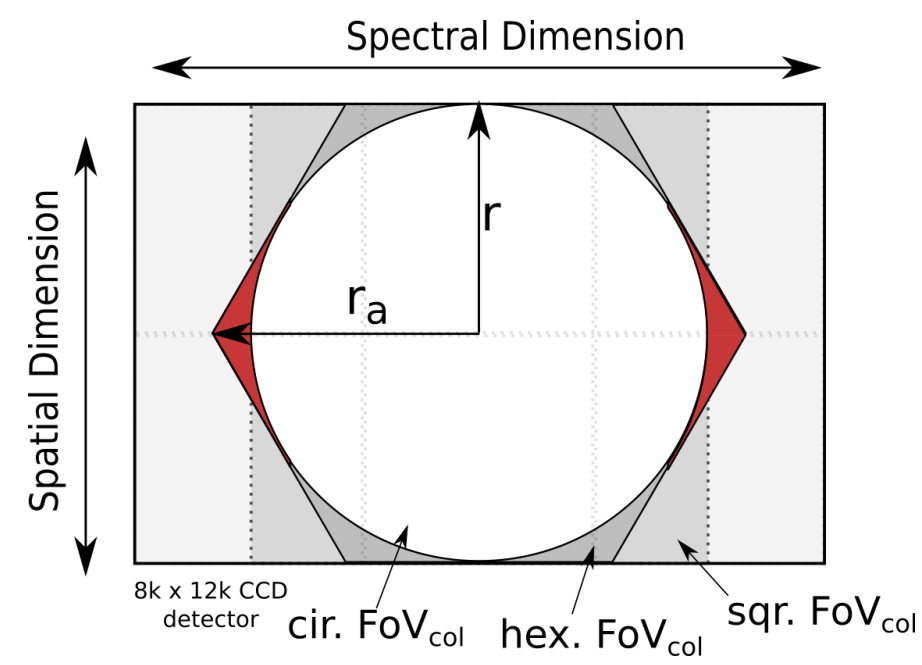

Figure 5.29: Comparison between hexagonal and circular FoV for low-resolution mode projected on the detector plane. The red region is where objects will have spectral loss.

The hexagonal area is given by $A_{\text {hex }}=3 r_{a}^{2} \sqrt{3} / 2=2 r^{2} \sqrt{3}$, so the gain from a circular shape FoV to a hexagonal is $10.2 \%(2 \sqrt{3} / \pi \approx 1.102)$, and to a squared is $27.3 \%(4 / \pi \approx$ $1.273)$.

Squared FoV Split Collimator: An adapted version of the Split Collimator aims to make the most of the detector area by generating a $7.4^{\prime} \times 7.4^{\prime}$ field of view so that the entire detector area can be used for low-resolution modes, which results in a total FoV area of $57.8^{\prime}$ square, $\approx 27 \%$ larger than the current Split Collimator. 
The architecture and lens materials remained the same as the Split Collimator. The FL has dimensions of $540 \mathrm{~mm}$ x $540 \mathrm{~mm}$ for mass reduction. The dimensions of the masks, together with the entire mechanical supporting structure of the optical elements, were redesigned to support the larger components. Mechanical envelope issues should be reviewed. The camera redesign and the spectrograph performance have not been explored since they require project schedule and budget allocations. Figure 5.30 shows a 3D Layout of the Squared Field Split Collimator.

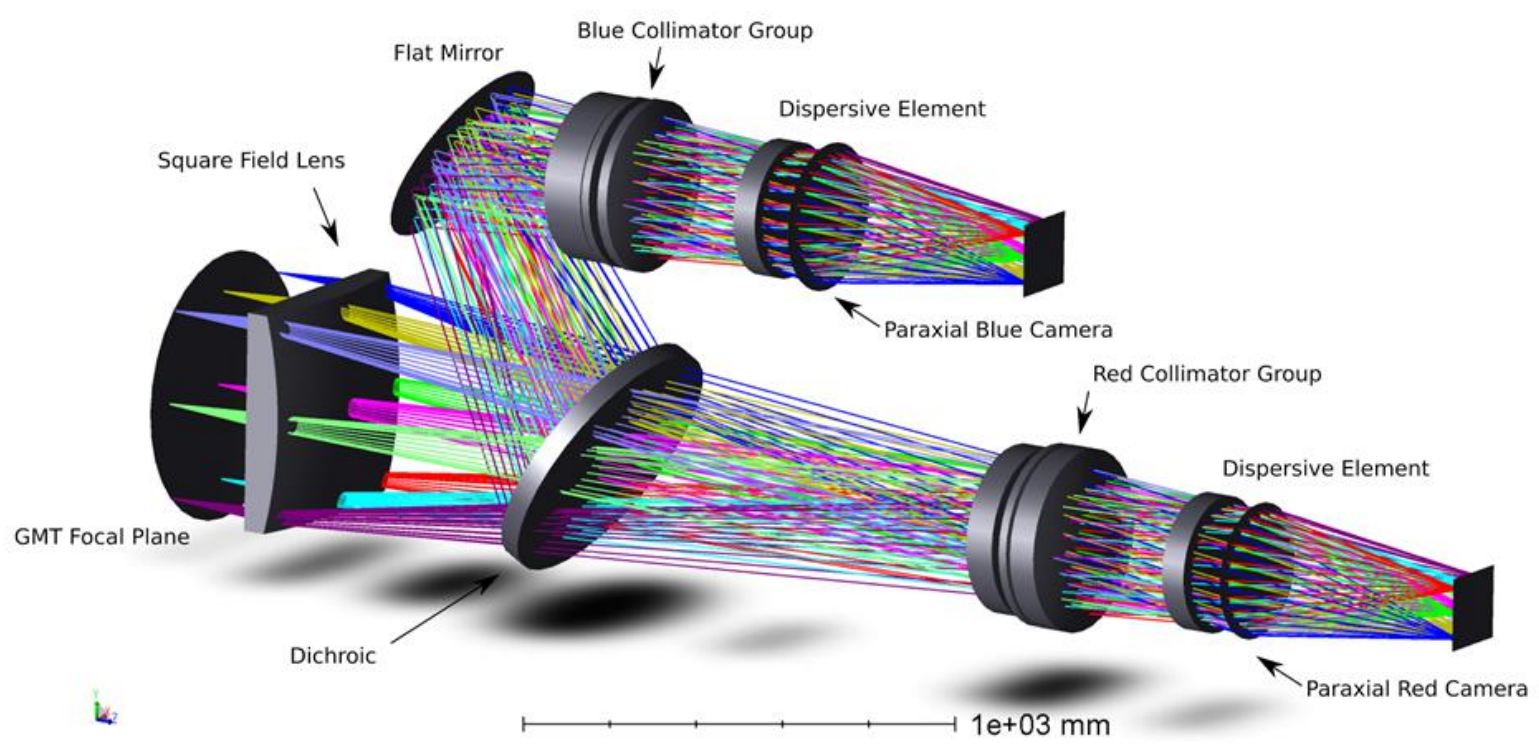

Figure 5.30: 3D Layout of an alternative Squared FoV Split Collimator 2,200 mm f/8.2, FoV 7.4' x 7.4', exit pupil of $270 \mathrm{~mm}$ in diameter, with paraxial cameras.

\subsubsection{Collimator Architecture Trade Conclusion}

The final concept and architecture chosen for GMACS is the refractive Split Collimator since this design enables us to: (i) reduce the UV-Blue wavelength cutoff down to 320 nm; (ii) improve throughput due to better VPH grating efficiencies for low-resolution mode; (iii) provide better spherochromatism correction and overall wavefront quality due to bandwidth split; (iv) allow shorter collimator relief; and (v) present satisfactory solution for the astigmatism correction. 


\subsection{Camera Design}

The major challenge in the design of a conventional wide-field and multi-object spectrograph is the camera design. The principal difficulty is that the entrance pupil of the camera lies on the grating, at some distance in front and outside the camera body; therefore, it requires an external aperture stop. In general, standard imaging systems have an internal stop and would not work otherwise without redesign. This requirement gets harder to satisfy as larger telescopes and multi-object spectroscopy calls for faster cameras to cover wider fields of view (Wall, 1993). The number of required cameras is also an important input parameter for trade subject between cost and instrument complexity. In case the collimator splits the incoming beam into more than one channel, it becomes easier to design more efficient and optimized systems since the bandwidth for each arm is reduced.

The same value of EFL for all the spectrograph cameras is preferred to maintain the same value of the spectrograph plate scale, although it requires different grating parameters given a fixed spectral resolving power.

The external entrance pupil requires a type of camera that also presents an external aperture stop, such as Petzval, telephoto, and inverse telephoto (Fischer et al., 2008). Therefore, the coma, distortion, and lateral color correction are expected to be difficult to minimize. In order to correct both lateral and axial color in such lens types, the components of the camera must be individually achromatized Smith (1992). Similar to the collimator's tradeoff, Schmidt and any derivative reflexive or catadioptric on-axis camera designs would dramatically reduce the throughput due to internal obscuration. Schwarzschild camera or a TMA off-axis designs also suffers from the same disadvantage and packing limitation. According to section 5.4 and figure 5.9, and considering the proposed camera principal parameters, the best starting point for the refractive camera designs are Double Gauss, Petzval, Petzval Projection, and the telephoto family. The Double Gauss lens type is not appropriate since its key feature is the internal and symmetrical stop position. The telephoto lens type, in principle, is not recommended due to the intrinsic telephoto ratio characteristic, that would increase the total camera length, which in turn is a disadvantage for flexure and packaging.

Fischer et al. (2008) provide an interesting visualization of the lens layout as a function 
of the lens type. Apart from the single-element and single achromatic doublet, the lens type with aperture stop located out of the lens elements are Petzval, telephoto (and reverse telephoto), and eyepiece. Although typical applications are not related to CCD's based spectrographs, this last lens type has features that surprisingly match the external aperture stop requirement. For instance, in figure 5.31, the eyepiece's eye relief plays the role of the entrance aperture stop for a spectrograph camera.

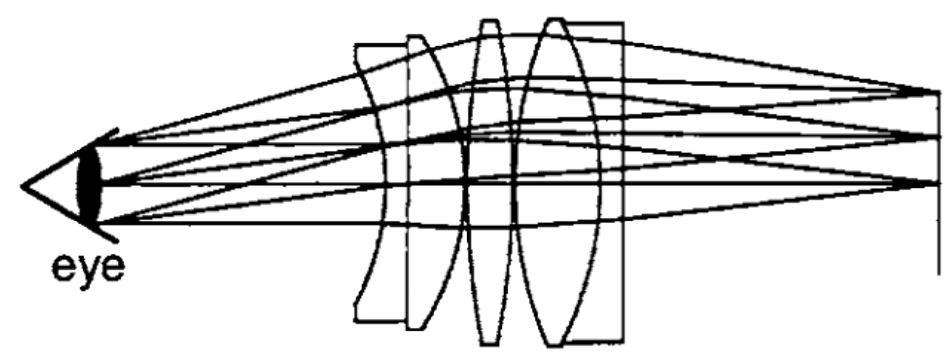

Figure 5.31: An example of the eyepiece optical layout. The eye relief plays the role of the entrance aperture stop for the camera. Source: Fischer et al. (2008).

Furthermore, in figure 5.32, a flat-field objective that corrects the Petzval curvature is a good starting point for the camera design, in which a thick negative achromatic element placed at the entrance aperture helps to correct the Petzval curvature.

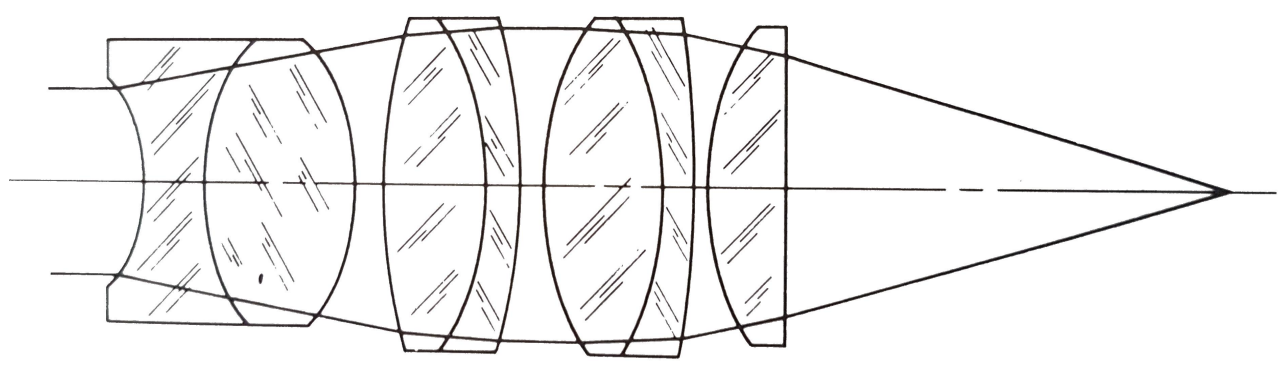

Figure 5.32: An example of the layout of a flat-field microscope objective in which the Petzval curvature is corrected by a thick negative achromatic placed at the entrance aperture. Source: Smith (1992).

In view of the functional parameters defined in section 5.3.1, the considerations above and the general solutions adopted for GMACS-like instruments (see chapter 3), the starting point designs for the cameras are Petzval design and large exit pupil eyepieces. 


\subsubsection{Petzval Lens}

The Petzval objective or Petzval lens is the first photographic portrait objective lens in the history of photography developed by the German-Hungarian mathematics professor Joseph Petzval, Hungarian mathematician and photographic-lens designer, in 1840 (Jan and Schade, 1950; Day and McNeil, 2003).

This Petzval lens is recognized for its excellent axis performance, inward-curving field, large FoV, and high speeds. We focused on adapting the Petzval design to increase the entrance pupil relief to accommodate the grating's rotation mechanisms.

The inward-curving Petzval surface can be corrected by using a thick negative achromatic placed at the entrance aperture or a negative powered Field Flattener (FF) near the focal plane, which can potentially be the window of the dewar. The first option has a drawback necessity for large internal lenses. In the second option, the FF rear surface is made flat (or concave to the detector) to mitigate issues with the back focal distance, BFD. According to Smith (1992), the FF is also known as Piazzi-Smuty FF, a strong negative element placed close to the focal plane, where it has little effect on the focal length or most aberrations, but it corrects the Petzval curvature. Although the stop is originally located inside the lenses to explore the stop symmetry, the Petzval can afford a sort of stop shift. Figure 5.33 illustrates two Petzval lenses with and without the FF.

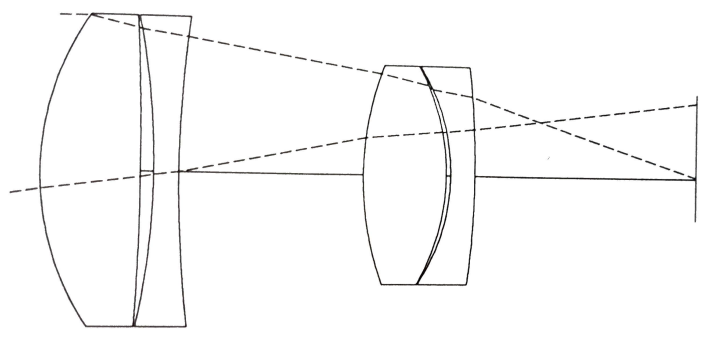

(a) Two achromatic positive air-doublets.

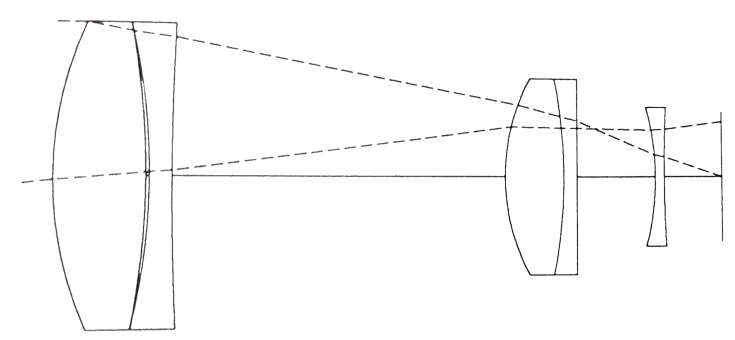

(b) Two achromatic positive doublets with field flattener.

Figure 5.33: Examples of Petzval projection lenses. Source: Smith (1992).

Sandback (1961) patented a high aperture air-spaced Petzval objective type used for projection of moving pictures, yet not limited to such. His designs are corrected for spherical aberration, including those of the third and higher order, chromatic aberrations, coma, astigmatism, field curvature, and distortion. It is an optimized version of the Smith (1962) designs, which is the standard of the Petzval objective lens comprised of two positive ele- 
ments followed by a negative field flattener. The common feature of these designs is that the power is well-balanced along with the elements, and the use of air-doublets and airtriplets aids aberration correction due to more variables available for optimization (Smith, 1992).

The FF is a solution for field correction used in Deimos (Keck II), GMOS (Gemini), FEROS and UVES (ESO), and VIMOS (VLT) (see chapter 3). The drawback is that the FF decreases the camera's working distance, which can generate stray light and undesirable imaging of its surface defects or contamination (Smith, 1992). The conventional approach to avoid such disadvantages is to use the $\mathrm{FF}$ as a dewar vacuum sealing window to allow shorter back focal distances or cement it on the dewar flat window.

As a starting point architecture for camera development, we adopted three achromatic groups with balanced power followed by an FF. We assumed that in the earlier stages of this research that all the composite components would be cemented. However, we have changed this assumption due to two principal reasons (i) potential cementing problems (CTE mismatch, oil cemented maintenance issues, and reduced UV-Blue transmittance); and (ii) increasing the variables for aberration correction, especially spherochromatism. Therefore, since from KoM phase, we decided that every composite elements must be air-spaced

The KoM and Mid-Point Review cameras' designs, performance, and conclusion developed in this research were presented by Schmidt et al. (2016) and Ribeiro et al. (2018b). We will focus the next section on describing in detail the CoD cameras and GMACS optical performance.

\subsubsection{Conceptual Design Cameras}

The two CoD GMACS cameras were designed for the Split Collimator presented in section 5.7.3.2. They have identical optical parameters: $594 \mathrm{~mm} \mathrm{f} / 2.2$, and entrance pupil relief $\approx 270 \mathrm{~mm}$, yet optimized for 320-600 $\mathrm{nm}$ and 500-1,000 $\mathrm{nm}$ for the blue and red arms, respectively. The plate scale is $0.055^{\prime \prime} /$ pixel, which results in a slit image of $190 \mu \mathrm{m}$ (12.6 pixels) for slit width of $0.7^{\prime \prime}$. The filter and shutter mechanisms are placed within the camera's barrels, close to the FF. In post-CoD GMACS design, we moved them before each collimator group since the AOI distribution is broad, and the beam is not concentrated (see section 5.7.1.2). The concave-plane FF act as the dewar window. 


\subsubsection{Blue Camera}

The blue camera, shown in figure 5.34 for a low-resolution mode, has five subassemblies: an entrance air-triplet followed by two air-doublets, the filter and shutter, and the FF. The materials are the crown $\mathrm{CaF}_{2}$ for positive and flint FS for the negative elements, four of each. The element powers are balanced in order to reduce the AOI and consequently avoid high sensitivity elements. The FF is concave-plano to attend the detector design constraints and technical recommendations; therefore, the RoC of the FF rear surface is smaller than a biconcave FF, although the resulting power is similar.

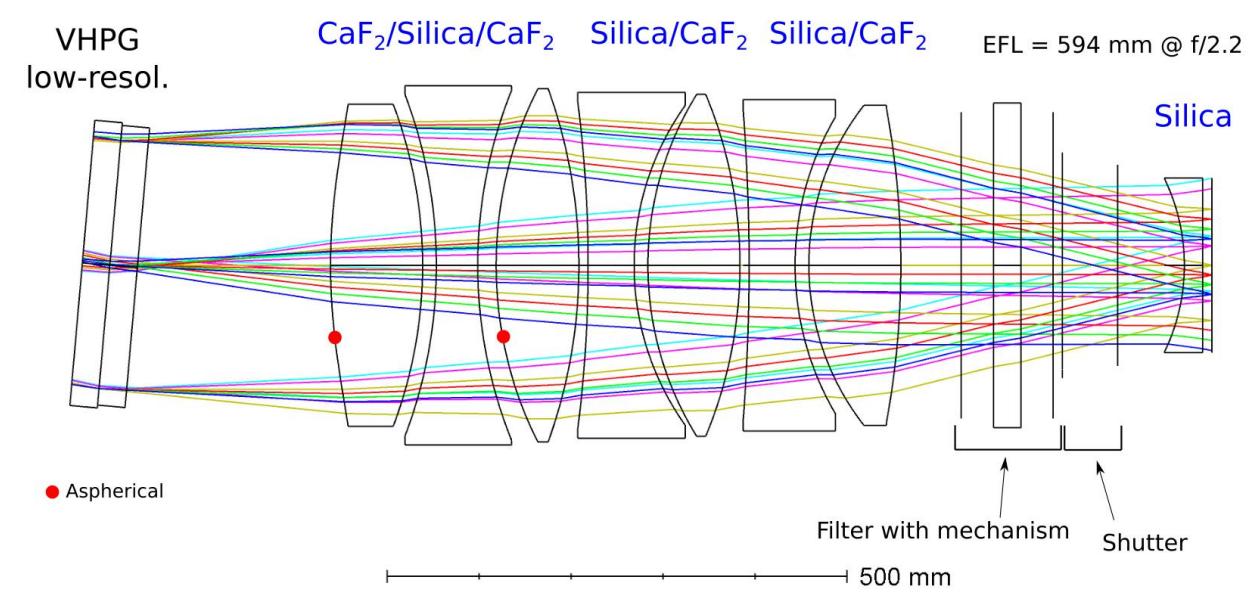

Figure 5.34: CoDR $594 \mathrm{~mm} \mathrm{f} / 2.2$ blue camera with entrance pupil of $270 \mathrm{~mm}$ in diameter.

Two aspheric surfaces on the first two positive $\mathrm{CaF}_{2}$ lenses, placed close to the entrance pupil, help to control the field aberrations. They are marked with red dots in figure 5.34. The maximum deviation from the best spherical fit, RMS, and the maximum slope are presented in table 5.9.

Table 5.9 - Blue camera aspheric surface parameters.

\begin{tabular}{|c|c|c|}
\hline Optical Surface & BFSD $[\boldsymbol{\mu m}]$ & Max. and RMS Slope [mrad] \\
\hline $\mathrm{CaF}_{2}$ 1st $(\mathrm{CX})$ & $<1,000$ & $\max =54 ; \mathrm{RMS}=21$ \\
$\mathrm{CaF}_{2}$ 1st $(\mathrm{CX})$ & $<285$ & $\max =16 ; \mathrm{RMS}=5$ \\
\hline
\end{tabular}

The sixteen surfaces, excluding the VPH grating and filter, give a throughput of $85 \%$ assuming a $99 \%$ transmittance of the AR coatings. Including the internal transmittance data from suppliers, the camera throughput is $\approx 70 \%$ over all the $320-600 \mathrm{~nm}$ spectral 
range. The UV-blue major constraints for the GMACS blue arm are the atmosphere cutoff, grating efficiencies and the detector quantum efficiency.

\subsubsection{Red Camera}

The red camera, shown in figure 5.35 for a low-resolution mode, consists of six lenses of $1 \times$ FS, FPL51, PBL6Y, BSM51Y and $2 \times \mathrm{CaF}_{2}$. The availability of more glass type options allows better chromatic aberration correction with fewer lenses. The design aims for higher throughput mainly by reducing the number of lenses and, consequently, the number of glass-air interfaces. The design is also comprised of four subassemblies: a negative air-doublet followed by two positive elements (singlet and air-doublet), the filter and shutter mechanism, and the FF. The front weak positive air-doublet aims to reduce the total Petzval curvature; however, in order to avoid vignetting, it forces the positive FPL51Y singlet to be the largest element. An alternative design has the FPL51 singlet replaced by $\mathrm{CaF}_{2}$ with the reduction of an aspherical surface. The size, however, is still a concern. The aspherical surfaces are placed on non- $\mathrm{CaF}_{2}$ lenses to avoid any image degradation due to irregularities generated by $\mathrm{CaF}_{2}$ aspherical manufacturing processes by point diamond turning. The PBL6Y lens thickness can be reduced.

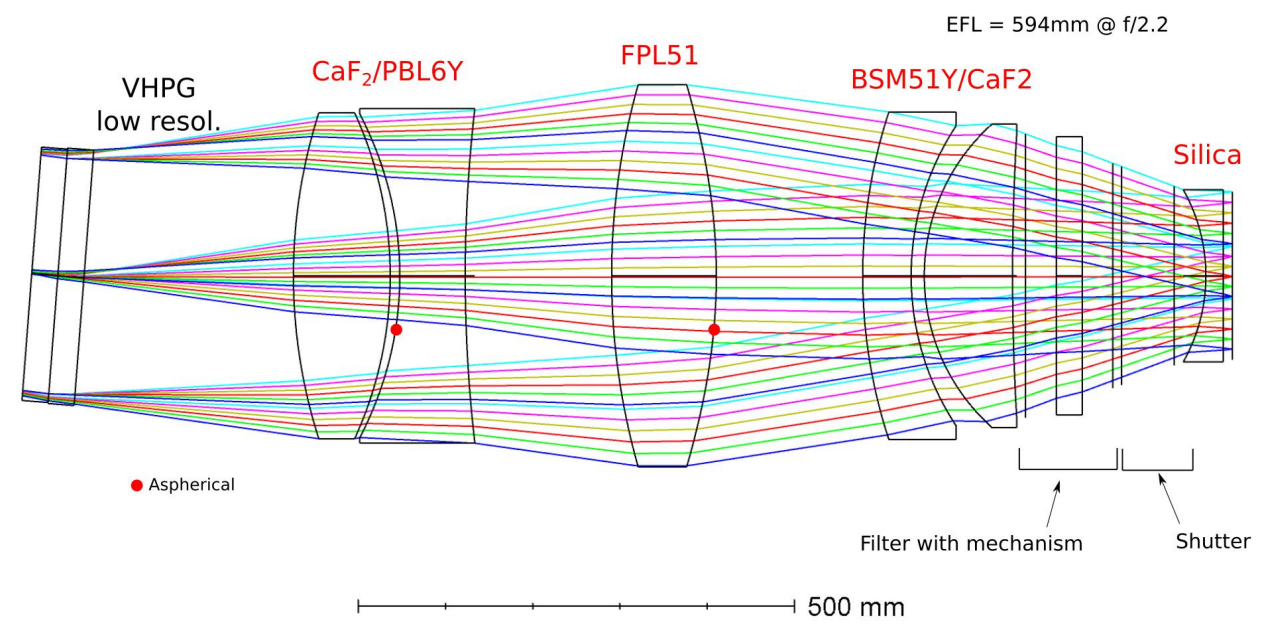

Figure 5.35: CoDR $594 \mathrm{~mm} \mathrm{f} / 2.2$ red camera with entrance pupil of $270 \mathrm{~mm}$ in diameter.

Two aspherical surfaces are located at the front concave surface of the PBL6Y lens and the rear convex surface of the large FPL51. The maximum deviation from the best spherical fit, RMS, and the maximum slope are presented in table 5.10.

The estimated throughput is $\approx 73 \%$ throughout the red spectral range from $550 \mathrm{~nm}$ to 
Table 5.10 - Red camera aspheric surface parameters.

\begin{tabular}{|c|c|c|}
\hline Optical Surface & BFSD $[\boldsymbol{\mu m}]$ & Max. and RMS Slope [mrad] \\
\hline PBL6Y 1st (CX) & $<220$ & $\max =11 ; \mathrm{RMS}=4$ \\
FPL51 1st (CX) & $<820$ & $\max =32 ; \mathrm{RMS}=13$ \\
\hline
\end{tabular}

$1,000 \mathrm{~nm}$.

This red camera, as described in section 5.9 about the GMACS image mode performance, has large TCA that would prevent an image mode with similar blue camera image performance. Therefore, in order to explore better red imaging performance, we presents two alternative designs that have small TCA without reducing the spectrograph image quality. In addition, we present a faster $\mathrm{f} / 1.57$ camera.

TCA Corrected Red Camera: We designed two alternative red cameras, shown in figures 5.36a and 5.36b, which aim to reduce the TCA without compromise the spectral image quality, making them suitable for imaging mode. They present similar optical performance to the CoD red camera and same glass materials; however, additional TCA constraints were used in the merit function. Additionally, the aspherical surfaces were moved to concave surfaces to reduce the risks of fabrication.

The design shown in figure 5.36a has TCA of $25 \mu \mathrm{m}$ between $500 \mathrm{~nm}$ and $1000 \mathrm{~nm}$ and $\mathrm{TCA}=10 \mu \mathrm{m}$ between $600 \mathrm{~nm}$ and $1000 \mathrm{~nm}$, while the one shown in figure 5.36b has $\mathrm{TCA}=25 \mu \mathrm{m}$ between $500 \mathrm{~nm}$ and $1000 \mathrm{~nm}$.

f/1.57 Red Camera: We designed a $425 \mathrm{~mm} \mathrm{f/1.57} \mathrm{red} \mathrm{camera} \mathrm{with} \mathrm{FoV} \mathrm{of} 16^{\circ}$ and 270 mm entrance pupil diameter as a concept demonstration for a faster alternative camera, shown in figure 5.36c. A faster camera allows smaller resolution elements (from 12.6 pixels to 9.1 pixels, for a $0.7^{\prime \prime}$ slit width) and higher throughput for the same bin. It would allow a smaller detector area for the same spectrograph FoV. As expected for such a fast camera with large entrance pupil and pupil relief, this design requires deep aspherical surfaces primarily for field aberration controlling, with BFSD up to $2.4 \mathrm{~mm}$ and $124 \mathrm{mrad}$ slope (visually noticed in the image). 


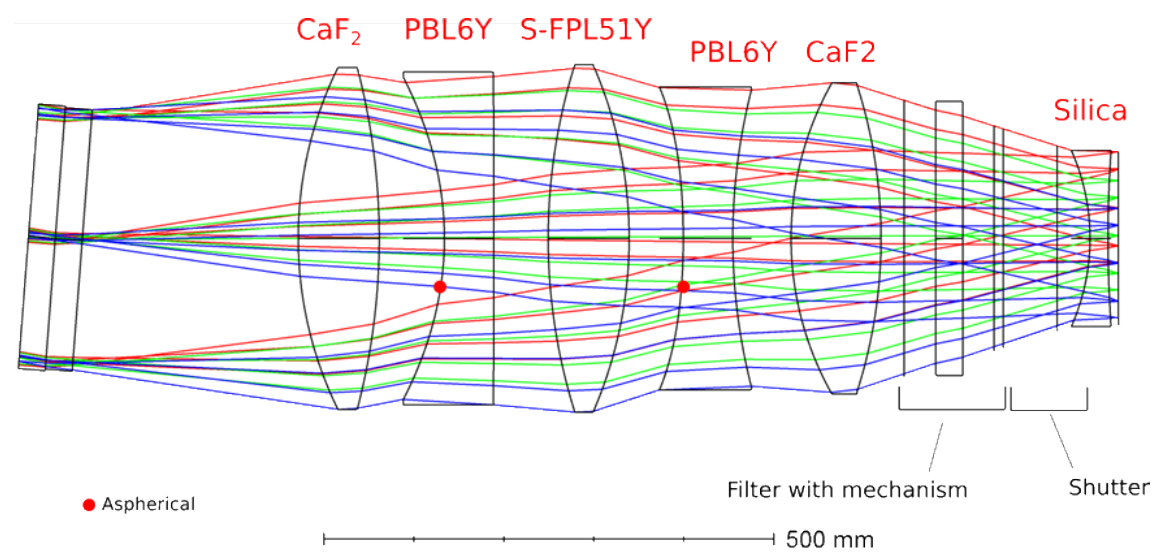

(a) $594 \mathrm{~mm} \mathrm{f} / 2.2$ red camera with TCA $=25 \mu \mathrm{m}$ between $500 \mathrm{~nm}-1,000 \mathrm{~nm}$ and $\mathrm{TCA}=10 \mu \mathrm{m}$ between $600 \mathrm{~nm}-1000 \mathrm{~nm}$.

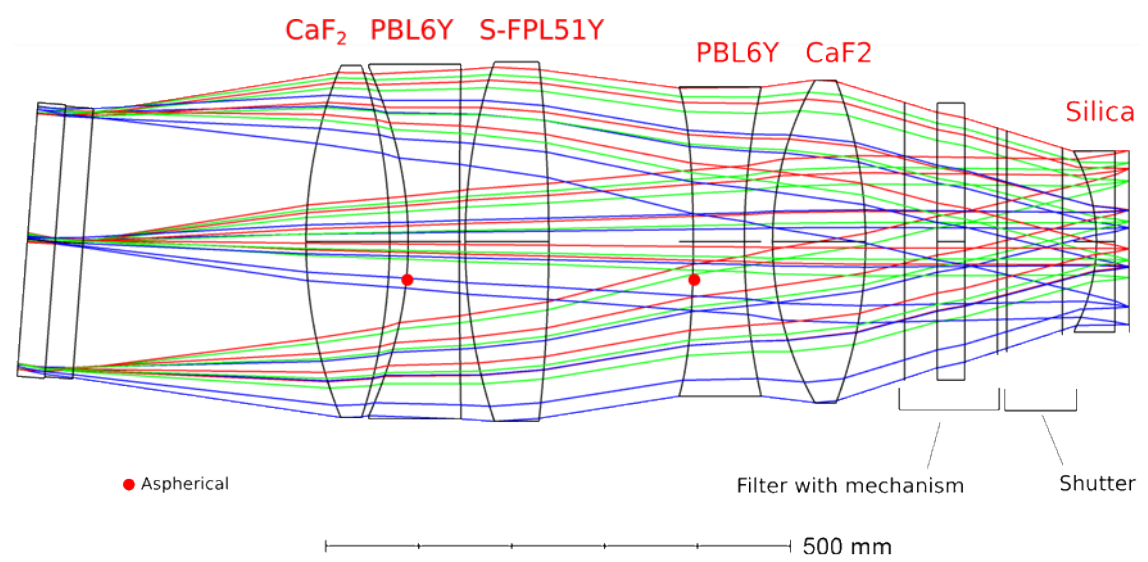

(b) $594 \mathrm{~mm} \mathrm{f} / 2.2 \mathrm{red}$ camera with TCA $=25 \mu \mathrm{m}$ between $500 \mathrm{~nm}-1,000 \mathrm{~nm}$.

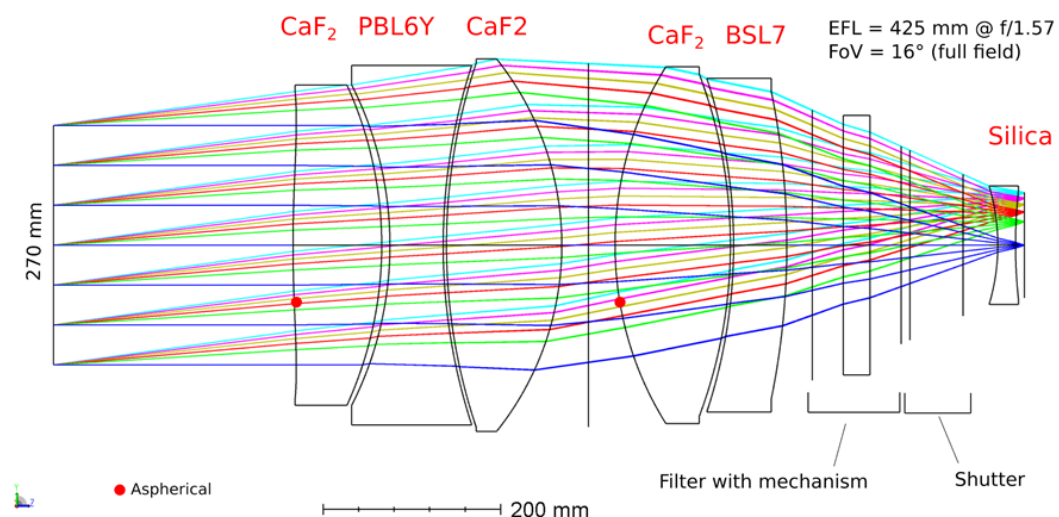

(c) $425 \mathrm{~mm} \mathrm{f} / 1.57$ with $\mathrm{FoV}$ of $16^{\circ}$ and $270 \mathrm{~mm}$ entrance pupil diameter red camera.

Figure 5.36: Alternative CoD red camera designs. 


\subsection{GMACS Conceptual Design}

In this section, we describe in detail the GMACS optical CoD presented in the CoDR held at TAMU 4-5th September 2019. It is the outcome of the 2016 GMACS design rescope requested by the GMTO through the Statement of Work (GMT-SOW-01091) and attachments. We discuss the spectrograph performance, particularities of each channel, tolerance and flexure analysis.

\subsubsection{CoDR GMACS Blue Arm Performance}

The optical layout of the GMACS blue arm, comprised of the Split Collimator (figure 5.24) and blue camera (figure 5.34), is shown in figure 5.37, with the low- and the highresolution spectrograph modes superimposed. The spectrograph configurations used in these results are presented in table 5.11.

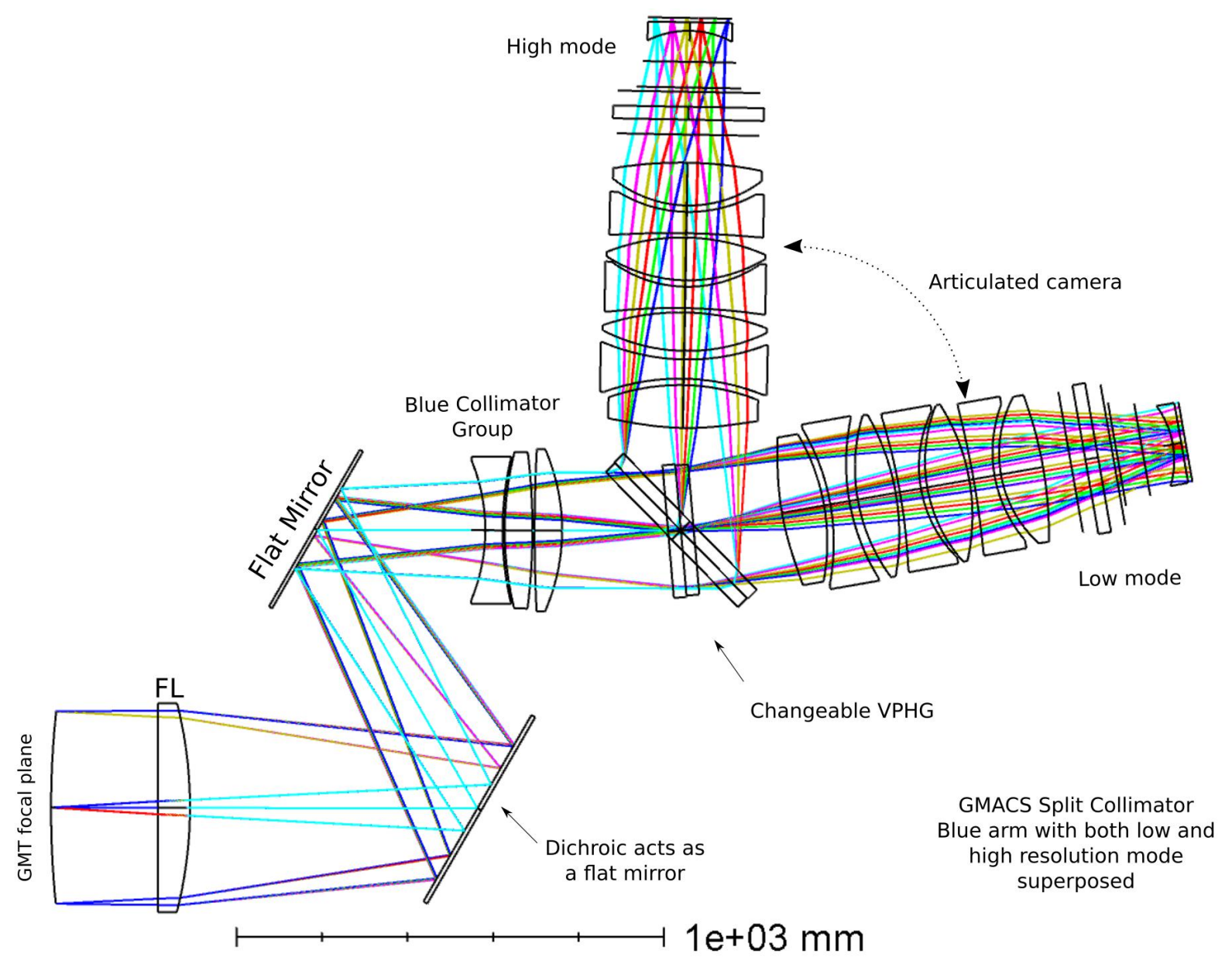

Figure 5.37: GMACS CoDR Blue Arm with a low- and high-resolution modes superposed. The spectrograph configuration is given in table 5.11. 
Figure 5.38 shows the spot diagram for the low-resolution and 320-600 nm bandwidth. We assume that, since the chromatic aberration is well-corrected over all the spectral range, any high-resolution mode will also perform similarly to the low-one presented. However, we created additional configurations in the MCE with several Bragg angles and grating parameters for the highest resolution mode (collimator-camera angle of $45^{\circ}$ ) to certify that we are achieving good spectral performance throughout the required spectral range. In this illustration, the rows represent object fields sampled over the $7.4^{\prime}$, and the columns represent wavelengths. The reference circle is $0.3^{\prime \prime}$ in diameter, the spots are centroid referred, and the legend items refer to wavelengths in micrometers.

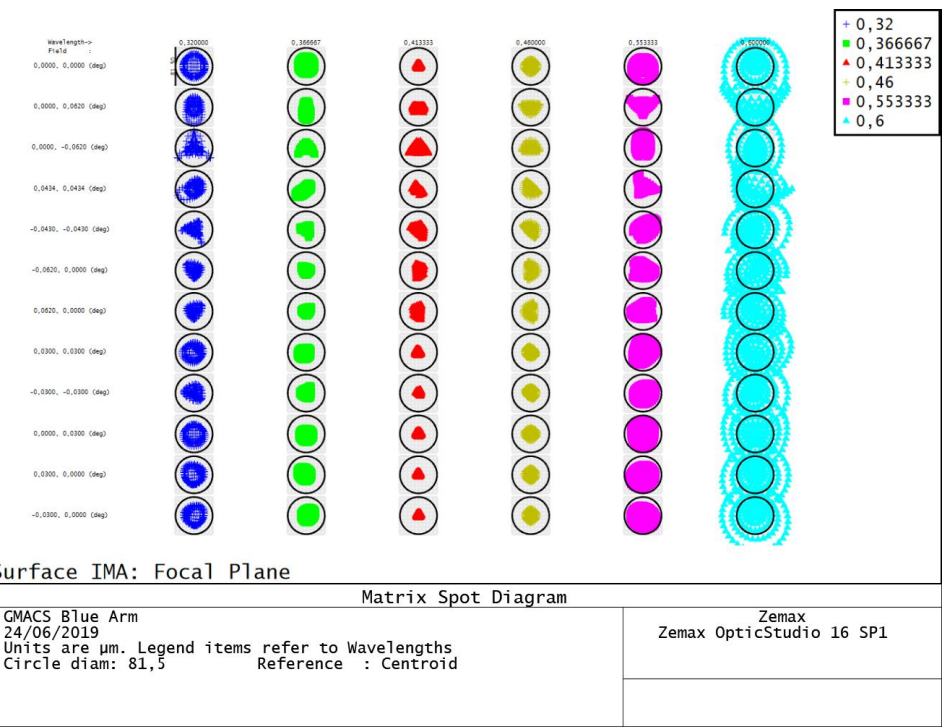

Figure 5.38: GMACS CoDR blue arm spot size diagram. The circle size is $0.3^{\prime \prime}$.

Image quality degradation is acceptable for wavelengths beyond the dichroic transition (wavelength of $557.7 \mathrm{~nm}$ ) for the blue arm. Therefore, the optimization weight was reduced in this region to relax the design's constraints. The RMS EED80 is $<0.16^{\prime \prime}$ for all fields and wavelengths. Table 5.12 describes the performance of the full blue spectral range and a reduced spectral range. 
Table 5.11 - GMACS CoDR Blue Arm spectrograph parameters, resolution and spectral range for figure 5.37 .

\begin{tabular}{c|cc}
\hline Parameter & Low-Resol. Mode & High-Resol. Mode \\
\hline$\theta_{B}$ & $5.0^{\circ}$ & $44.5^{\circ}$ \\
$\nu\left[l \cdot m m^{-1}\right]$ & 380 & 3,300 \\
$R @ \lambda_{c}$ & $550 @ 460 \mathrm{~nm}$ & $6,150 @ 425 \mathrm{~nm}$ \\
$\delta \lambda$ & $8.3 \AA$ & $0.7 \AA$ \\
$\Delta \lambda$ & $270 \mathrm{~nm}$ & $66 \mathrm{~nm}$ \\
\hline
\end{tabular}

Table 5.12 - GMACS CoDR Encircled Energy for Blue Arm Low-Resolution Mode.

\begin{tabular}{ccc} 
& $\begin{array}{c}\text { EED80 [arcsec] } \\
(320 \mathrm{~nm}-600 \mathrm{~nm})\end{array}$ & $\begin{array}{c}\text { EED100 [arcsec] } \\
(320 \mathrm{~nm}-550 \mathrm{~nm})\end{array}$ \\
\hline RMS & 0.198 & 0.295 \\
Average & 0.162 & 0.244 \\
STD & 0.116 & 0.167 \\
Maximum & 0.442 & 0.628 \\
Minimum & 0.044 & 0.056 \\
\hline
\end{tabular}

\subsubsection{Vignetting}

Vignetting, a useful design tool, enables us to reduce the size of the elements while improving imaging performance by blocking highly aberrated marginal rays. Unlike conventional imaging systems, the effect of vignetting generated by any elements after the dispersive element is a function of the diffracted angle from the dispersive element, which is wavelength-dependent. Therefore, camera vignetting in a spectrograph causes throughput losses as a function of field and wavelength.

Figure 5.39a shows the detector footprint for the low-resolution mode (see the parameters in table 5.11) for a set of objects located at the edge collimator FoV. We assumed that the grating lines are parallel to the Y-direction and the dispersion along the $\mathrm{X}$-direction. The objects located in the collimator edge FoV (dotted circle) are represented by green squares, and the colored dots represent the dispersed spectra. Figure 5.39b shows the corresponding vignetting heat map. The data were calculated by Zemax and visualized by a Python script.

Figure 5.40a shows the detector footprint for the high-resolution mode (see the parameters in table 5.11) and a set of objects located at the edges of the collimator FoV. 


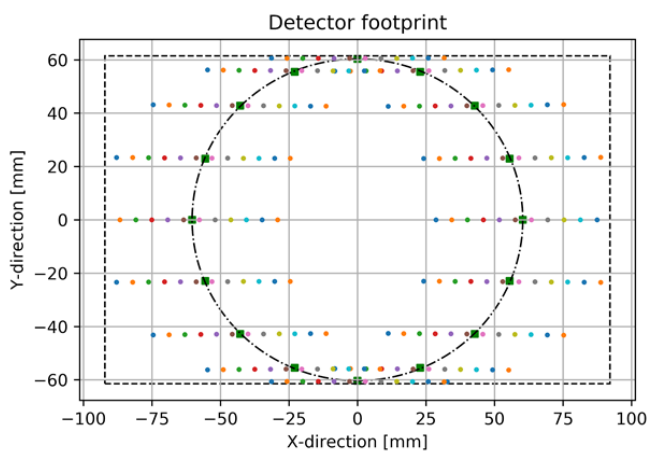

(a) Detector Footprint.

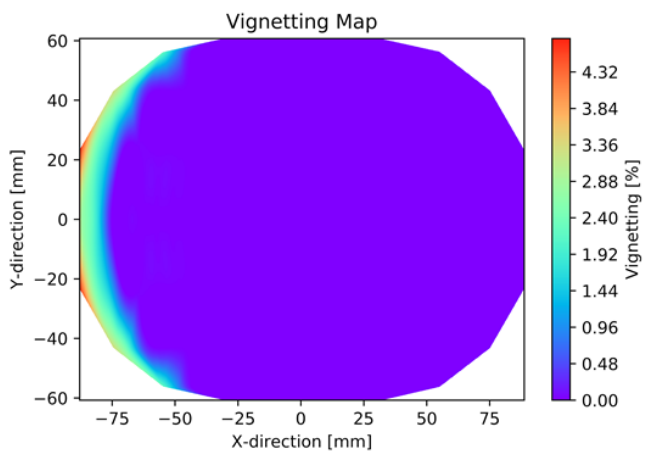

(b) Vignetting Heat Map.

Figure 5.39: (a) Detector footprint diagram for a low-resolution mode and (b) vignetting heat map.

The objects are located in the center of the FoV, similar to a long slit configuration (the objects are the green squares) and the dispersed spectra are represented by the colored dots. Figure 5.40b shows the corresponding vignetting profiles for each of the object fields.

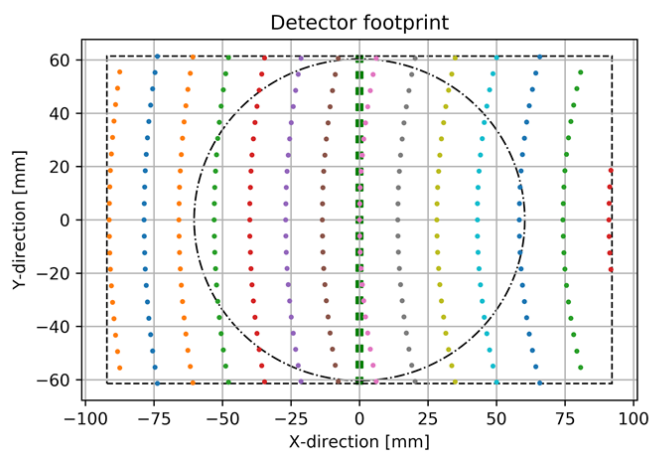

(a) Detector Footprint.

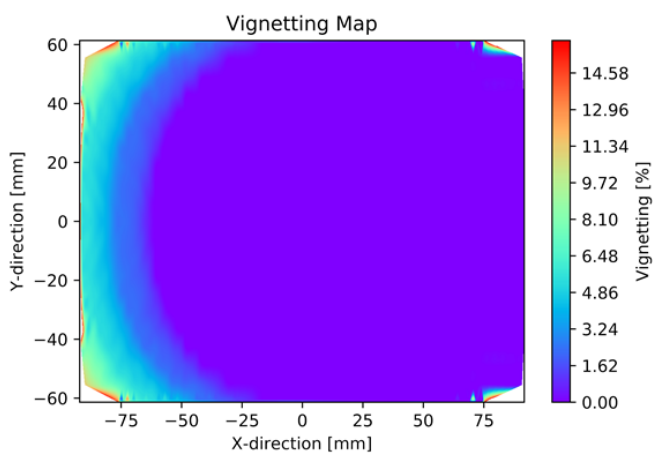

(b) Vignetting Heat Map.

Figure 5.40: (a) Detector footprint diagram for a high-resolution mode and (b) vignetting heat map.

Since the pivot rotation of the camera is the center of the VPHG, the asymmetry of the vignetting is the result of ray clipping in the first lens of the camera due to the greater path length between the camera and the entrance pupil, and to the pupil anamorphism, as shown in figure 5.41. The solution to reducing this asymmetry is to move the camera pivot point from the center to the rear surface of the grating. While it is difficult to accomplish with a coaxial mechanism for the grating and camera, this solution is viable for independent articulation mechanisms. 


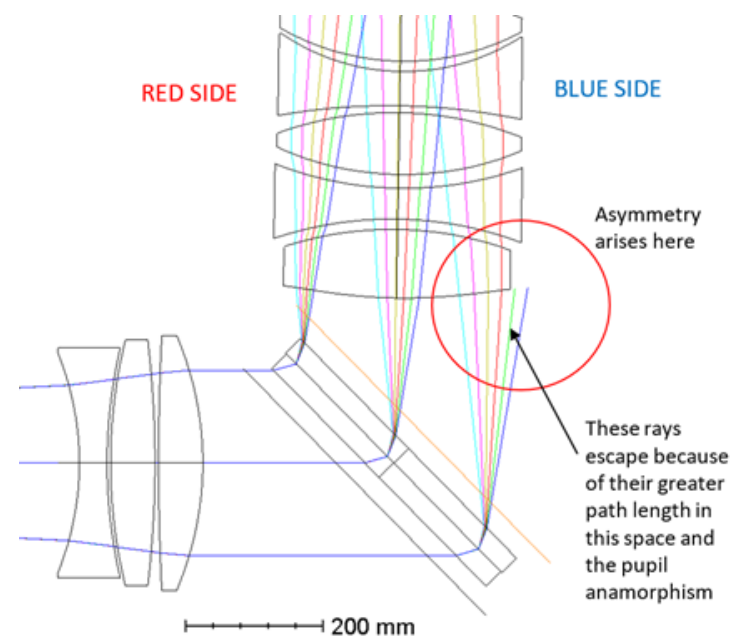

(a) Detector Footprint.

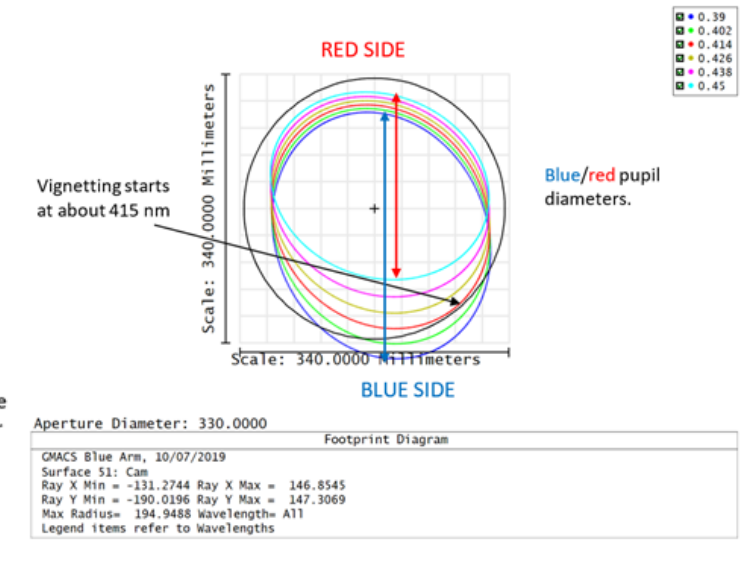

(b) Vignetting Heat Map.

Figure 5.41: Source of asymmetry of the vignetting. Source: GMACS Team (2019) (Damien Jone)

\subsubsection{Image Mode Performance in the Blue Arm}

Although a GMACS image mode is not required, it is prudent to characterize this mode performance. We ensured that any design constraints for the imaging mode could not compromise the spectroscopy image quality. The quality criteria are the ones usually used for optical imagers, such as spot size, MTF, distortion, TCA, PSF, etc.

The Zemax setup for image mode has the grating normal vector oriented along the optical axis, i.e., the respective collimator subsystem and the camera optical axis are parallel to each other. The grating parameters are zero order and null density grooves. In practical terms, the image mode does not require a null flat window since the beam is well-collimated.

The blue arm design has acceptable axial color and spherochromatism aberrations; however, it gives approximately $120 \mu \mathrm{m}$ of TCA for the full blue spectral range, see figure 5.42. Although the TCA can be considered as an additional magnification factor of the slit as a function of field and wave, it causes minor consequences in the spectrograph modes since the observations will have calibration processes. The distortion is less than $0.25 \%$ over the field.

The TCA restricts the spectral bandwidth for the GMACS imaging mode. The spectral bandwidth can be selected by the use of an appropriate optical narrow-band filter located at the filter position of both cameras. 


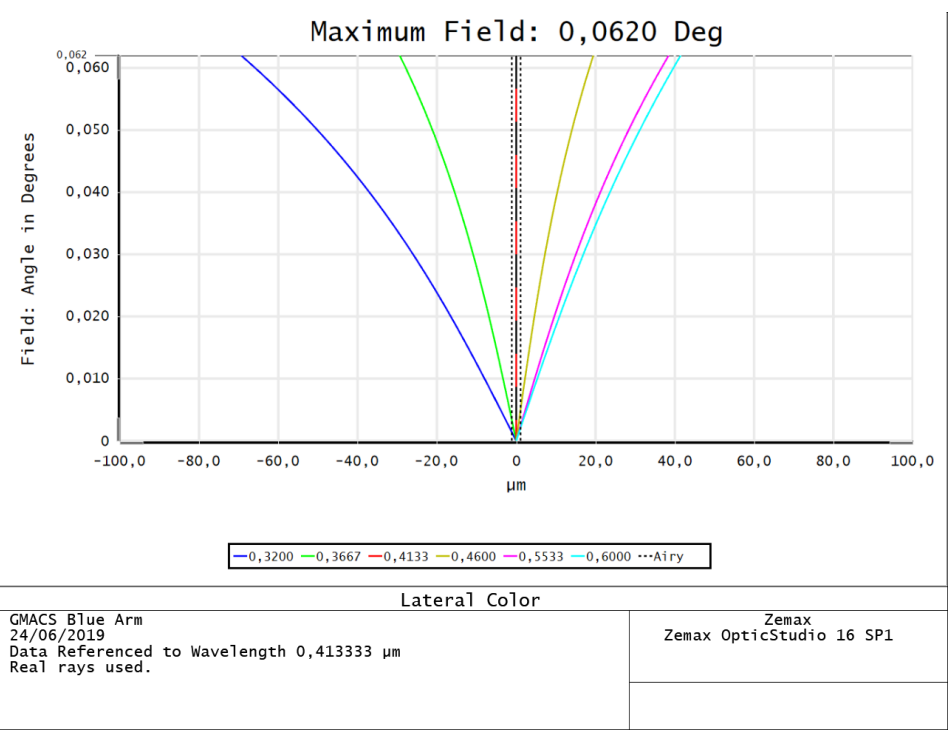

Figure 5.42: Transversal Chromatic Aberrations for the GMACS Blue Arm in the image mode.

Assuming the same performance requirement for spot size, the imaging mode will have a spatial resolution of $81.5 \mu \mathrm{m}$ or $40.7 \mu \mathrm{m}$ for the requirement and goal values, equivalent to approximately 5.4 pixels or 2.7 pixels, respectively. It is considered that the imaging mode with binning of $5 \times 5$ or $3 \times 3$ pixels is reasonable for science images and slit alignment purposes.

Figure 5.43a shows the matrix spot diagram with reference in the chief ray for a box size of $75 \mu \mathrm{m}$, which corresponds to a binning of $5 \times 5$. The spot diagram covers the $7.4^{\prime} \mathrm{FoV}$. Figure $5.43 \mathrm{~b}$ shows the spot diagram with the center wavelength centroid as a reference from the range $320-550 \mathrm{~nm}$. The redder edge was excluded since the requirement is reduced in this region for the blue arm. The box size is $150 \mu \mathrm{m}$, which represents 10 pixels at the focal plane.

Figure 5.44 shows that the polychromatic diffraction MTF is 0.4 to 0.5 at 16,6 cycles/mm, which corresponds to 2 pixel size, from $430-480 \mathrm{~nm}$.

\subsubsection{CoDR GMACS Red Arm Performance}

The optical layout of the GMACS Red Arm is shown in figure 5.45, with the low- and the high-resolution spectrograph modes superimposed. The spectrograph configurations are presented in table 5.13.

The spot diagram for the GMACS red arm from 500 to 1,000 nm is illustrated in figure 5.46. The rows represent object fields and the columns represent the wavelength. The 


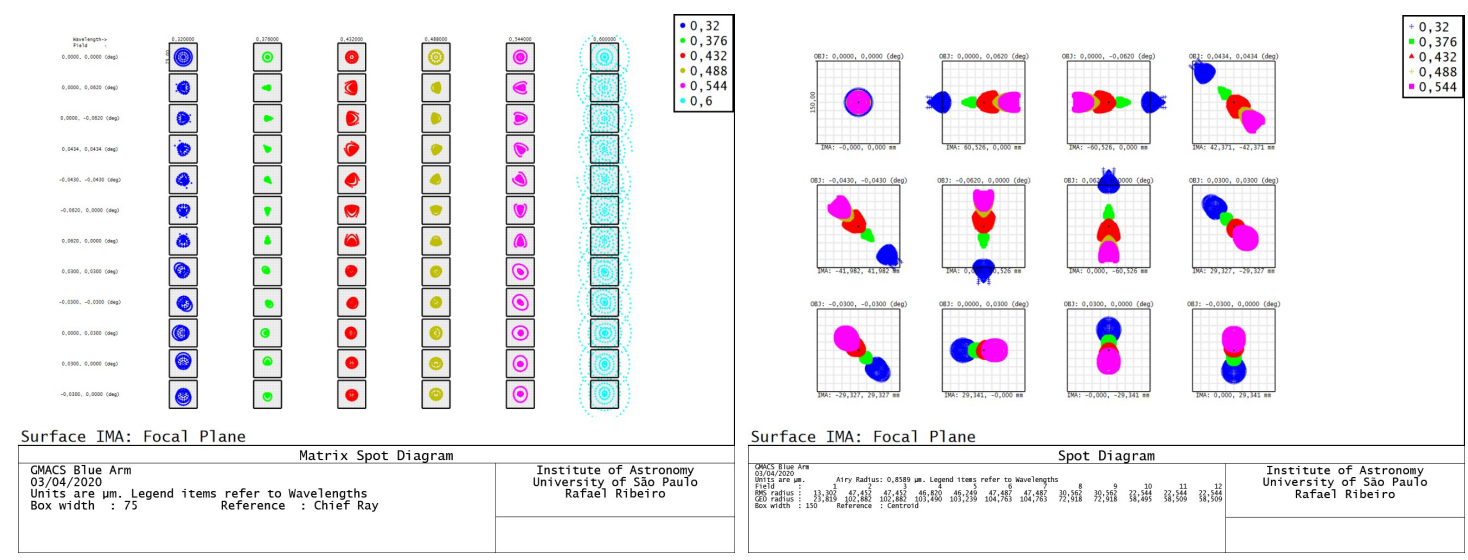

(a) Matrix spot diagram.

(b) Spot diagram.

Figure 5.43: Matrix spot diagram and spot diagram for the blue arm image mode. The box size for the matrix is $75 \mu \mathrm{m}$ and wavelength from 320-600 nm, and for the spot diagram is $150 \mu \mathrm{m}$ for $320-550 \mathrm{~nm}$.
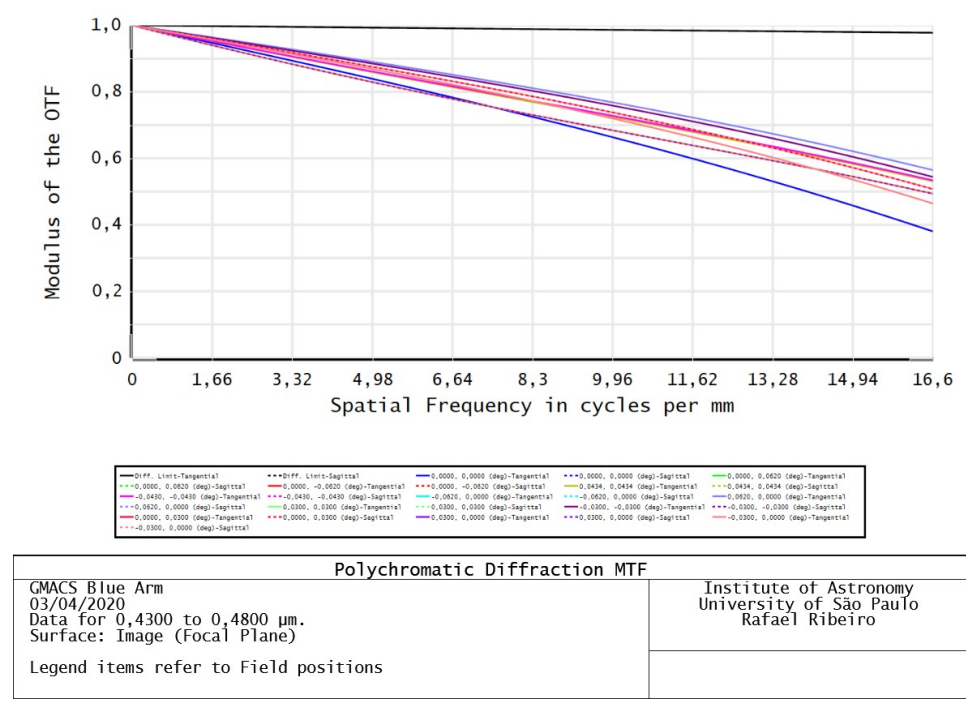

Figure 5.44: Polychromatic diffraction MTF for the blue arm in image mode from 430-480 nm. The frequency 16.6 cycles $/ \mathrm{mm}$ is equivalent to a two $15 \mu \mathrm{m}$ pixel side.

reference circle is $0.3^{\prime \prime}$ in diameter, the spots refer to the centroid and the legend items refer to wavelengths in micrometers.

The overall optimization weight was also reduced for wavelengths shorter to the dichroic transition. Therefore, a reduced optical performance in this region is expected. The RMS EED80 is $<0.16^{\prime \prime}$ for all fields and wavelengths. Table 5.14 describes the performance of the full red spectral range and a reduced spectral range. 


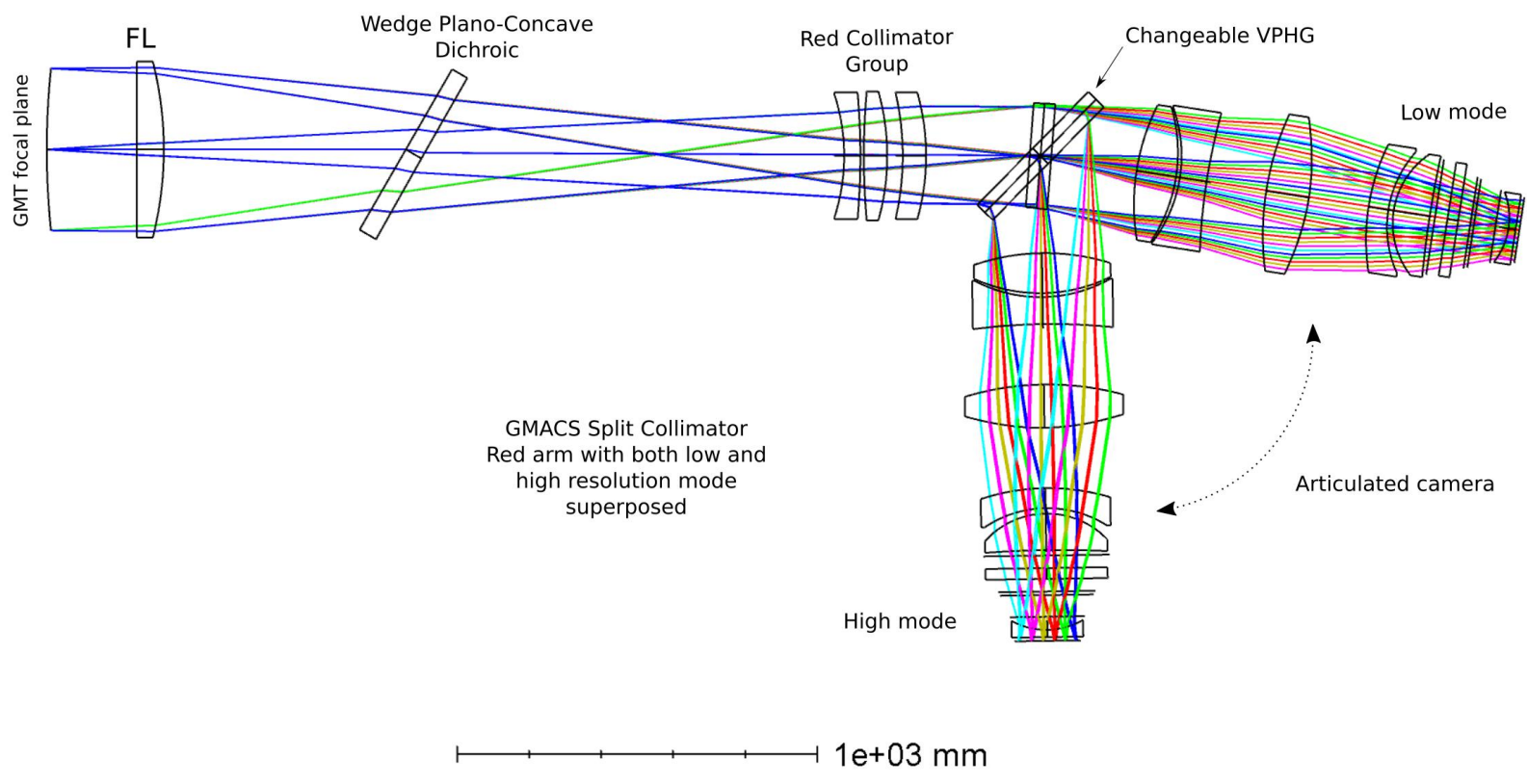

Figure 5.45: GMACS CoDR Red Arm with a low- and high-resolution modes superposed. The spectrograph configuration is given in table 5.13.

Table 5.13 - GMACS CoDR Red Arm spectrograph configuration parameters, resolution and spectral range for figure 5.45 .

\begin{tabular}{c|cc}
\hline Parameter & Low-Resol. Mode & High-Resol. Mode \\
\hline$\theta_{B}$ & $4.4^{\circ}$ & $44.2^{\circ}$ \\
$\nu\left[l \cdot m m^{-1}\right]$ & 205 & 1,800 \\
$R @ \lambda_{c}$ & $550 @ 775 \mathrm{~nm}$ & $6,080 @ 775 \mathrm{~nm}$ \\
$\delta \lambda$ & $8.3 \AA$ & $0.7 \AA$ \\
$\Delta \lambda$ & $460 \mathrm{~nm}$ & $120 \mathrm{~nm}$ \\
\hline
\end{tabular}

Table 5.14 - GMACS CoDR Encircled Energy for Red Arm Low-Resolution Mode.

\begin{tabular}{ccc} 
& $\begin{array}{c}\text { EED80 [arcsec] } \\
(500 \mathrm{~nm}-1,000 \mathrm{~nm})\end{array}$ & $\begin{array}{c}\text { EED100 [arcsec] } \\
(600 \mathrm{~nm}-1,000 \mathrm{~nm})\end{array}$ \\
\hline RMS & 0.155 & 0.313 \\
Average & 0.131 & 0.251 \\
STD & 0.083 & 0.189 \\
Maximum & 0.343 & 0.720 \\
Minimum & 0.046 & 0.102 \\
\hline
\end{tabular}

\subsubsection{Image Mode Performance}

Figure 5.47 shows the lateral color for the GMACS Red Arm in an image mode. The TCA for the red camera is $300 \mu \mathrm{m}$ for the full red spectral range and the edge collimator 


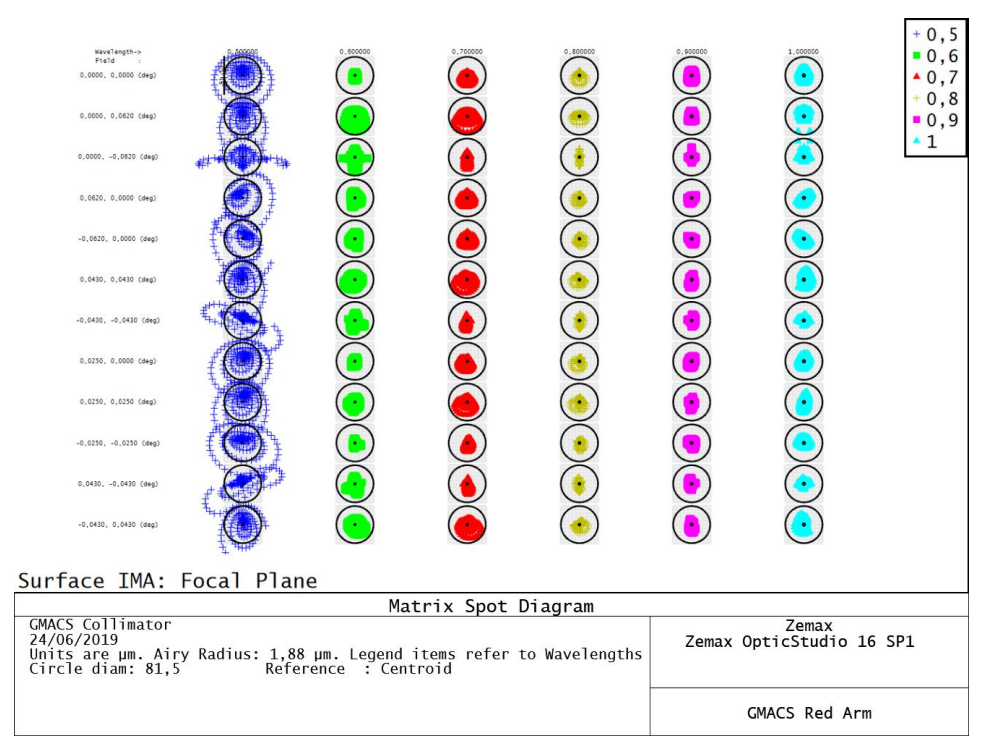

Figure 5.46: GMACS CoDR red arm spot size diagram. The circle size is $0.3^{\prime \prime}$.

field. Considering the same conditions described above for science image quality, the image bandwidth for the central wavelength of $700 \mathrm{~nm}$ would be approximately $35 \mathrm{~nm}$. For 3x3 binning, the value decreases almost linearly to $21 \mathrm{~nm}$. Since this camera has poor TCA correction, we presented two alternative versions of the red camera that have approximately ten times better TCA corrections in section 5.8.2.2. Considering the Split Collimator, the resulting TCA is to the order of $25 \mu \mathrm{m}$.

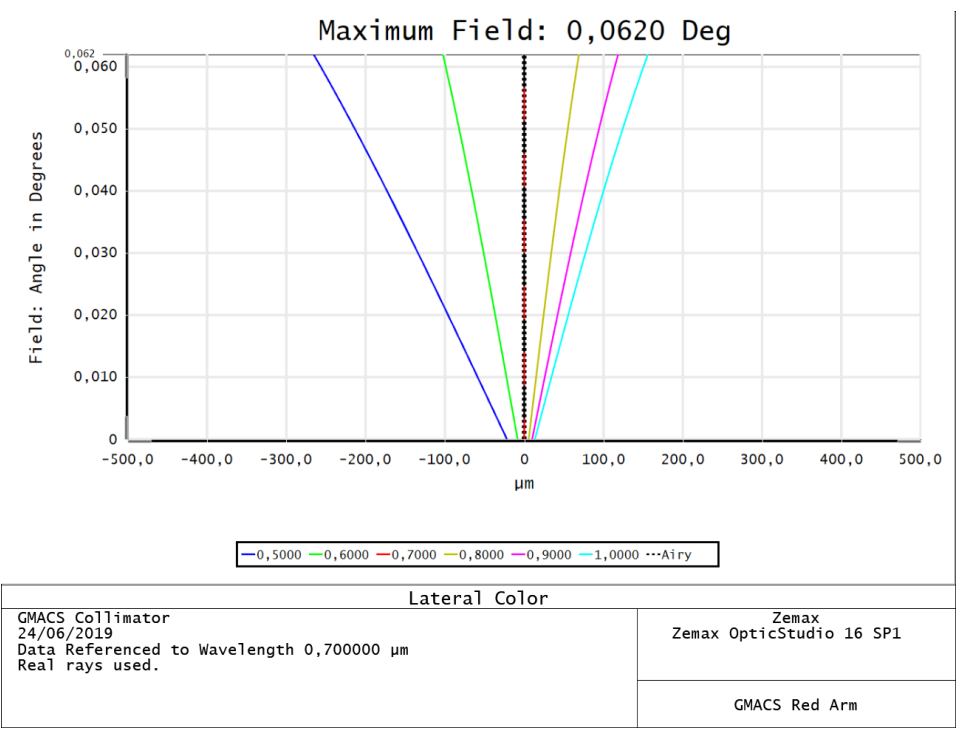

Figure 5.47: Transversal Chromatic Aberrations for the GMACS Red Arm in the image mode. 


\subsubsection{GMACS Configurations}

GMACS is a flexible and configurable spectrograph which provides a large range of resolution and bandwidth configurations. The combination of the grating tilt mechanism, a selectable VPHG, articulated cameras, and possibility for changing the slit width allows many spectrograph configurations to address a broad range of scientific cases. A few examples of grating configurations are illustrated in table 5.15. The mechanical restriction is the maximum of a $90^{\circ}$ tilt between the collimator and the camera optical axis.

\subsubsection{GMACS Optomechanical Design}

Figure 5.48 shows the GMACS optomechanical design presented in the CoDR. The version of the optical design used in this illustration was an early version, which is slightly different from the one presented in this work: a tilted window compensates for the astigmatism caused by the dichroic in the red collimator arm, and the red camera has more lenses then the current design. However, the general optomechanical design concept is maintained for the updated version. Figure 5.49 shows details of the blue camera's articulation mechanisms, with a curved gear rack with break rail, the motor and spur gear, and the pneumatic brake.

\subsection{Tolerance Analysis}

The primary purpose of the tolerance analysis is to determine the manufacturing, assembly, and alignment tolerances of the optical and optomechanical systems, given a predefined maximum allowable degradation of one or a set of optical quality criteria. The tolerance parameters that we used are RoC, element and airspace thicknesses, element wedge, element tilt and decentration, surface irregularity, refractive index, Abbe number, and glass inhomogeneity. Future detailed work will incorporate advanced tolerance parameters such as scratch-dig, lens bubbles, and surface roughness in the analysis since they mainly introduce aesthetic effects and scattered light. Although the tolerance analysis is carried out in a later stage of the optical design, the recommended approach is to reduce any design choices that could result in components, subassemblies, and assemblies with high sensitivity.

As part of the tolerance analysis, the appropriate adjusting parameters and the neces- 
Table 5.15 - Examples of spectrograph configurations for a $0.7^{\prime \prime}$ slit width.

\section{Blue arm}

\begin{tabular}{ccccccc}
$\begin{array}{c}\text { Mode of } \\
\text { operation }\end{array}$ & $\begin{array}{c}\theta_{B}{ }^{*} \\
{\left[^{\circ}\right]}\end{array}$ & $\begin{array}{c}\nu \\
{\left[l \cdot \mathrm{mm}^{-1}\right]}\end{array}$ & $\begin{array}{c}\lambda_{B}^{\dagger} \\
{[\mathrm{nm}]}\end{array}$ & $\begin{array}{c}\text { Resol. } \\
\text { at } \lambda_{B}\end{array}$ & $\begin{array}{c}\Delta \lambda^{\ddagger} \\
{[\mathrm{nm}]}\end{array}$ & $\begin{array}{c}r_{\text {ele }} \\
{[\AA]}\end{array}$ \\
\hline Low-resol. & 5.2 & 410 & 445 & 570 & 250 & 7.8 \\
\hline High-resol. & 44.5 & 2,550 & 550 & 6,140 & 85.7 & 0.895 \\
& 44.3 & 2,800 & 500 & 6,120 & 78.2 & 0.817 \\
& 44.2 & 3,100 & 450 & 6,080 & 70.8 & 0.740 \\
& 44.4 & 3,500 & 400 & 6,120 & 62.5 & 0.653 \\
\cline { 2 - 6 } & 41.3 & 2,400 & 550 & 5,480 & 95.9 & 1.000 \\
& 36.8 & 2,400 & 500 & 4,680 & 102.2 & 1.067 \\
& 32.7 & 2,400 & 450 & 4,000 & 107.5 & 1.123 \\
& 28.7 & 2,400 & 400 & 3,420 & 120.0 & 1.171 \\
\hline
\end{tabular}

\section{Red arm}

\begin{tabular}{ccccccc}
$\begin{array}{c}\text { Mode of } \\
\text { operation }\end{array}$ & $\begin{array}{c}\theta_{B} \\
{\left[^{\circ}\right]}\end{array}$ & $\begin{array}{c}\nu \\
{\left[l \cdot \mathrm{mm}^{-1}\right]}\end{array}$ & $\begin{array}{c}\lambda_{B} \\
{[\mathrm{~nm}]}\end{array}$ & $\begin{array}{c}\text { Resol. } \\
\text { at } \lambda_{B}\end{array}$ & $\begin{array}{c}\Delta \lambda \\
{[\mathrm{nm}]}\end{array}$ & $\begin{array}{c}r_{\text {ele }} \\
{[\AA]}\end{array}$ \\
\hline Low-resol. & 5.0 & 255 & 775 & 550 & 457.3 & 14.179 \\
\hline High-resol. & 43.9 & 1,500 & 925 & 6,020 & 147.2 & 1.538 \\
& 44.3 & 1,600 & 875 & 6,120 & 136.8 & 1.429 \\
& 44.4 & 2,000 & 700 & 6,120 & 109.5 & 1.143 \\
& 44.8 & 2,350 & 600 & 6,208 & 92.5 & 0.966 \\
\cline { 2 - 6 } & 41.3 & 1,200 & 925 & 4,170 & 212.5 & 2.220 \\
& 31.7 & 1,200 & 875 & 3,850 & 217.5 & 2.270 \\
& 24.8 & 1,200 & 700 & 2,890 & 231.9 & 2.422 \\
& 21.1 & 1,200 & 600 & 2,410 & 238.4 & 2.490 \\
\hline
\end{tabular}

* $\theta_{B}$ is the blazed angle for Littrow configuration

$\dagger \lambda_{c}$ is the blaze wavelength

$¥ \Delta \lambda$ is the spectral coverage (equation 2.61)

$\S r_{\text {ele }}$ is the resolution element 


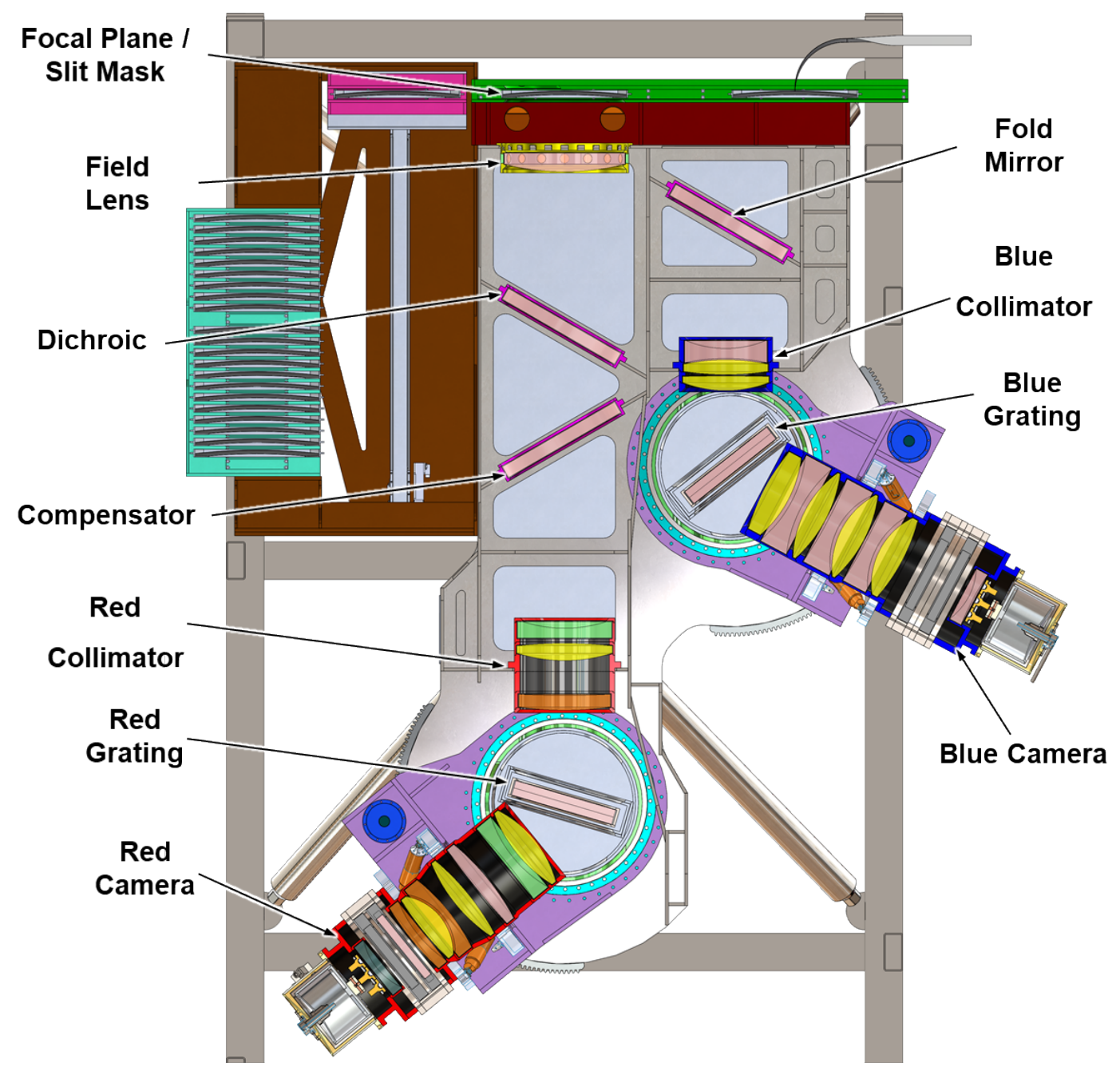

Figure 5.48: Optomechanical Conceptual Design. Source: GMACS Team (2019).

sity for active compensators are discussed (GMACS Team, 2019). The term "compensator" is used do describe any active compensation mechanisms referring to the flexure correction and focusing. The flexure compensator's objective is to provide real-time tracking of the instrument's flexure and the required actuation with, for instance, a closed-loop system. The term "adjuster" is used to describe the small corrections to any particular positioning of the lenses for better system performance, and it is related exclusively to the assembling and aligning processes. Although it is out of the proposed scope of work, we recommend adopting the as-built manufacturing approach for GMACS since only one optical system is expected to be fabricated.

The determination of the type and number of adjusters considers that GMACS is composed of four independent subsystems (two collimators and two cameras) since they 


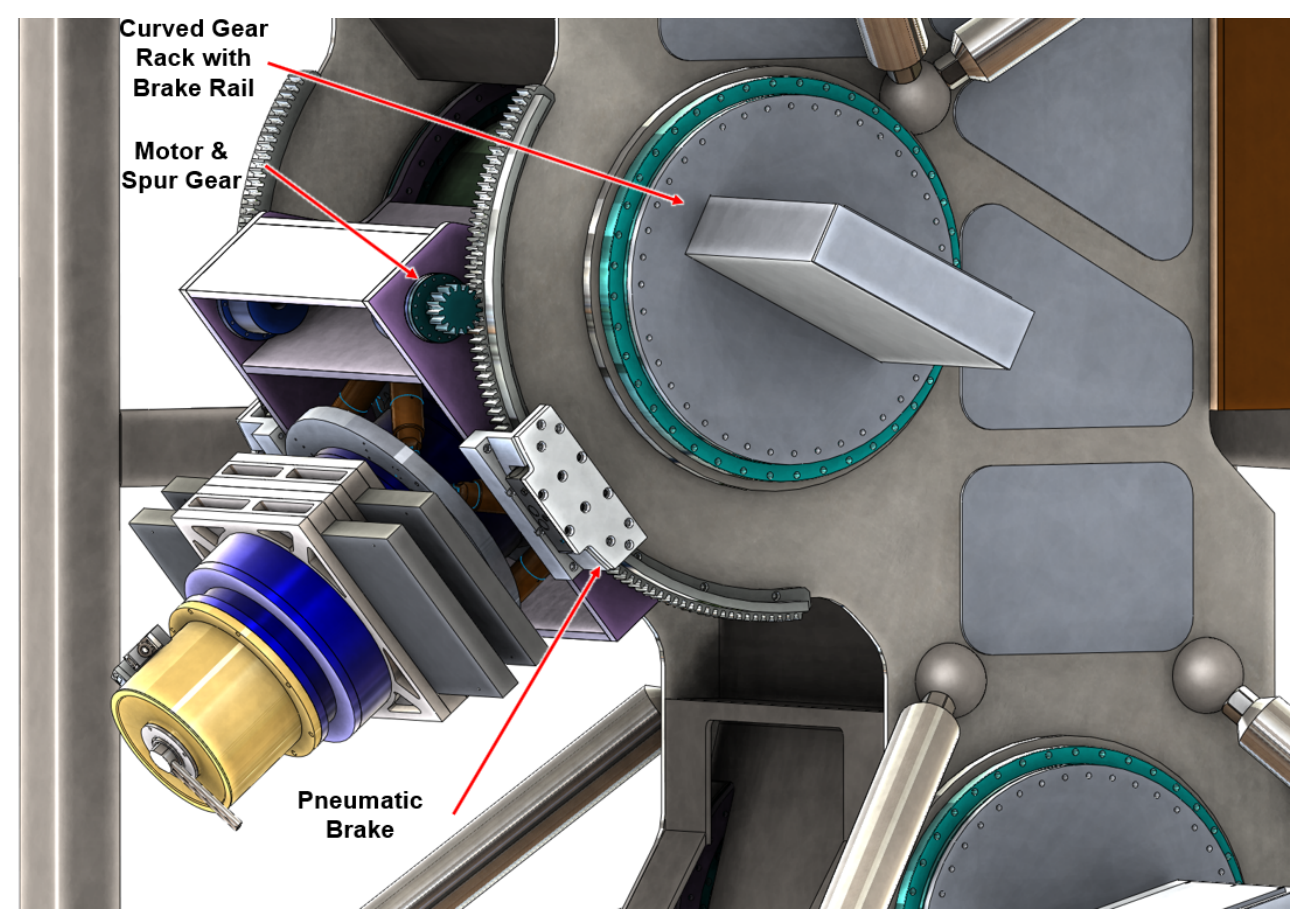

Figure 5.49: Details of the articulation mechanisms for the blue camera. Source: GMACS Team (2019).

will be assembled and aligned individually and independently in their respective optical barrels.

The testplate or tooling for the RoC matching process is not recommended until the present design phase, given the high level of customization and the costs involved in the GMACS optical assembly manufacturing. Nevertheless, in the future project stages, once the company responsible for the lens supply has been defined, the testplate necessity will be reviewed in the case of cost, and lead time reduction could be accomplished.

\subsubsection{Sensitivity}

The objective of the sensitivity analysis is to determine which are the most sensitive optical parameters related to the optical surfaces (radius of curvature, fringes, decenter and tilt) and to the optical elements (thickness, the distance between elements, decenters and tilts, index of refraction and glass homogeneity). These pieces of information is used to define the assembly, mounting and alignment strategies, and even for redesign by iterative optimization process to avoid or mitigate highly sensitive surfaces and elements. Table 5.16 describes the meaning of each sensitivity parameters used in this work. 


\begin{tabular}{cl} 
Acronym & \multicolumn{1}{c}{ Description } \\
TFRN & Tolerance on surface radius of curvature in fringes \\
TIRR & Tolerance on Standard surface irregularity \\
\hline TTHI & Tolerance on position in lens units \\
TTHI_E & Tolerance on thickness in lens units \\
\hline TSDX & Tolerance on Standard surface x-decenter in lens units \\
TSDY & Tolerance on Standard surface y-decenter in lens units \\
TEDX & Tolerance on element x-decenter in lens units \\
TEDY & Tolerance on element y-decenter in lens units \\
\hline TSTX & Tolerance on Standard surface tilt in x in degrees \\
TSTY & Tolerance on Standard surface tilt in y in degrees \\
TETX & Tolerance on element tilt about x in degrees \\
TETY & Tolerance on element tilt about y in degrees \\
\hline TIND & Tolerance on index of refraction of surface \\
TABB & Tolerance on Abbe number of surface
\end{tabular}

Table 5.16 - Adapted Zemax tolerance acronyms used for the sensitivity analysis.

Bentley and Olson (2012) recommend that by examining a lens layout and assessing the margin ROI distribution over its surface gives a quick insight into the performance, manufacturability, and improvement potential for a design. The key recommendation is to minimize ray AOIs throughout the lenses.

The sensitivity factor approach, described in section 4.4.6.1, is a helpful visualization data tool that compares the same parameters for different elements or groups. No adjuster or compensator is employed in this analysis. The average sensitivity was taken between the maximum and minimum sensitivity for each of the parameters.

Although the resolution modes must be considered for a complete system characterization, we focused only on the low-resolution mode. In future phases of the project, the determination of whether a mode is more sensitive to tolerance than another will be performed, as well as for flexure tolerance. The fields are well-sampled through the pupil. Figures 5.50 and 5.51 show the sensitivity diagram for the blue and red arm, respectively. 


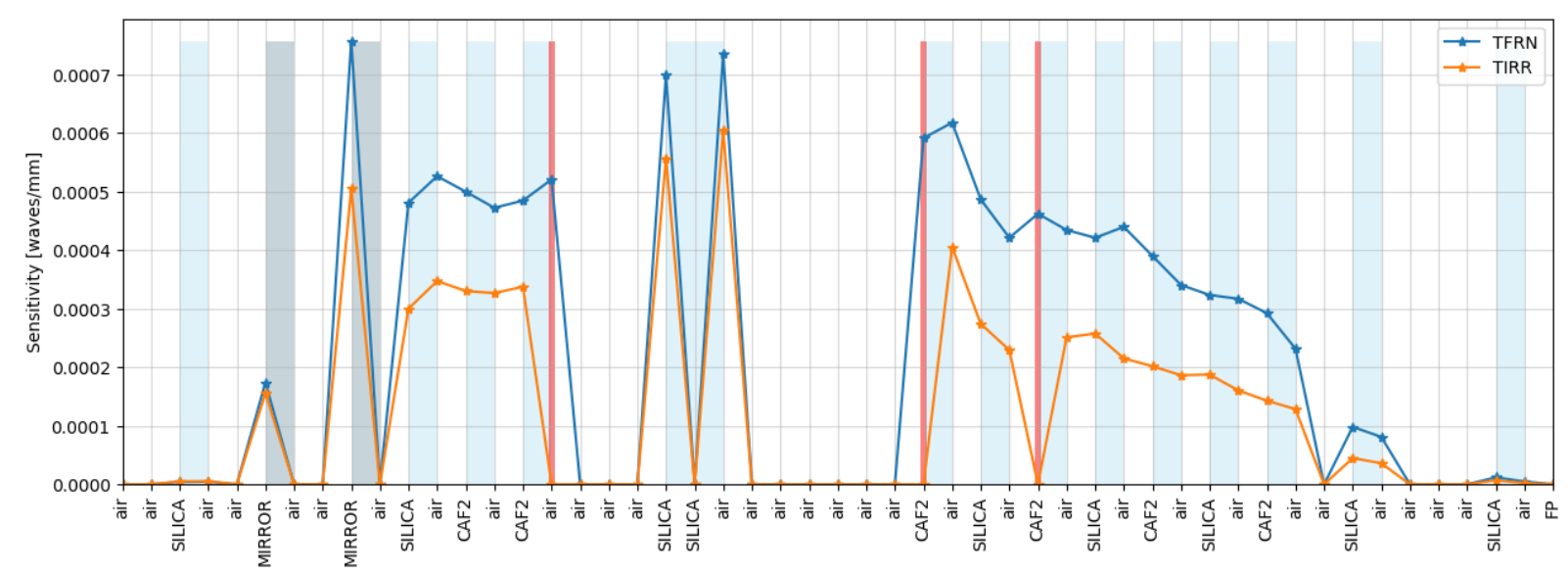

(a) Tolerance on surface radius of curvature in fringes (TFRN) and irregularity (TIRR).

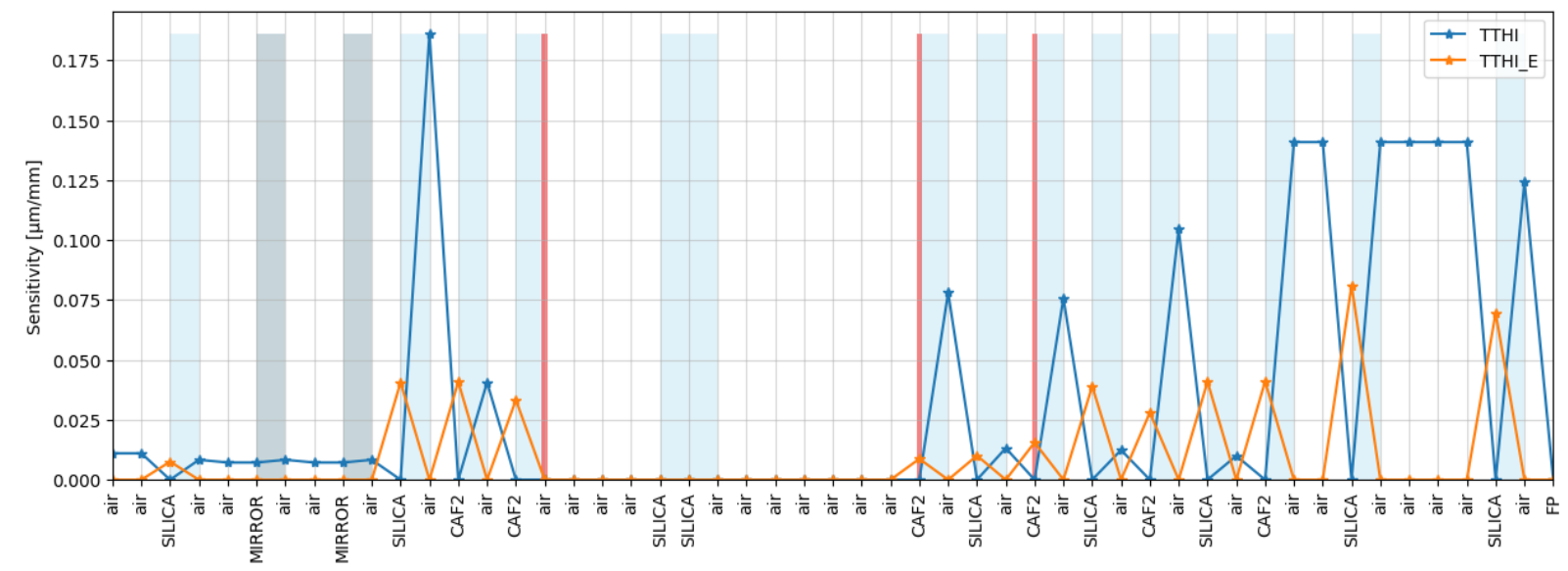

(b) Tolerance on element thickness (TTHI_E) or position (TTHI).

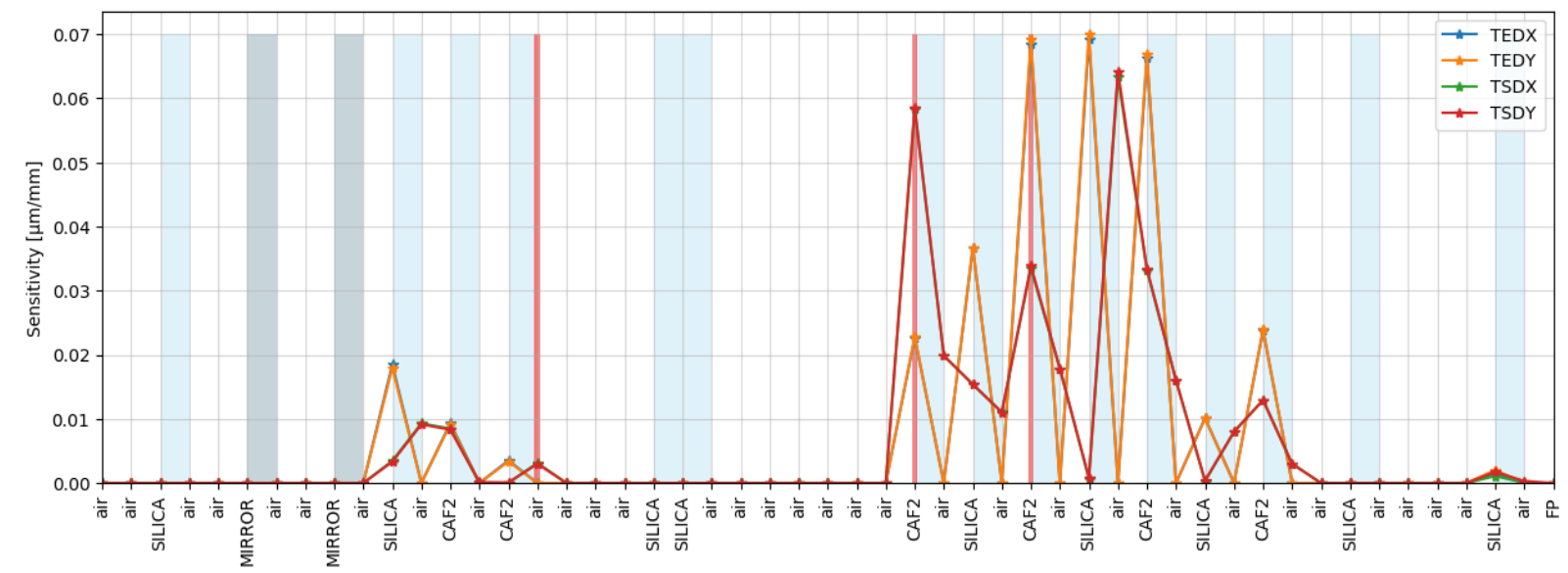

(c) Tolerance on Standard surface $\mathrm{x}-\mathrm{y}$ decenter (TSDX or TSDY) and tolerance on element $\mathrm{x}-\mathrm{y}$ decenter (TEDX or TEDY). 


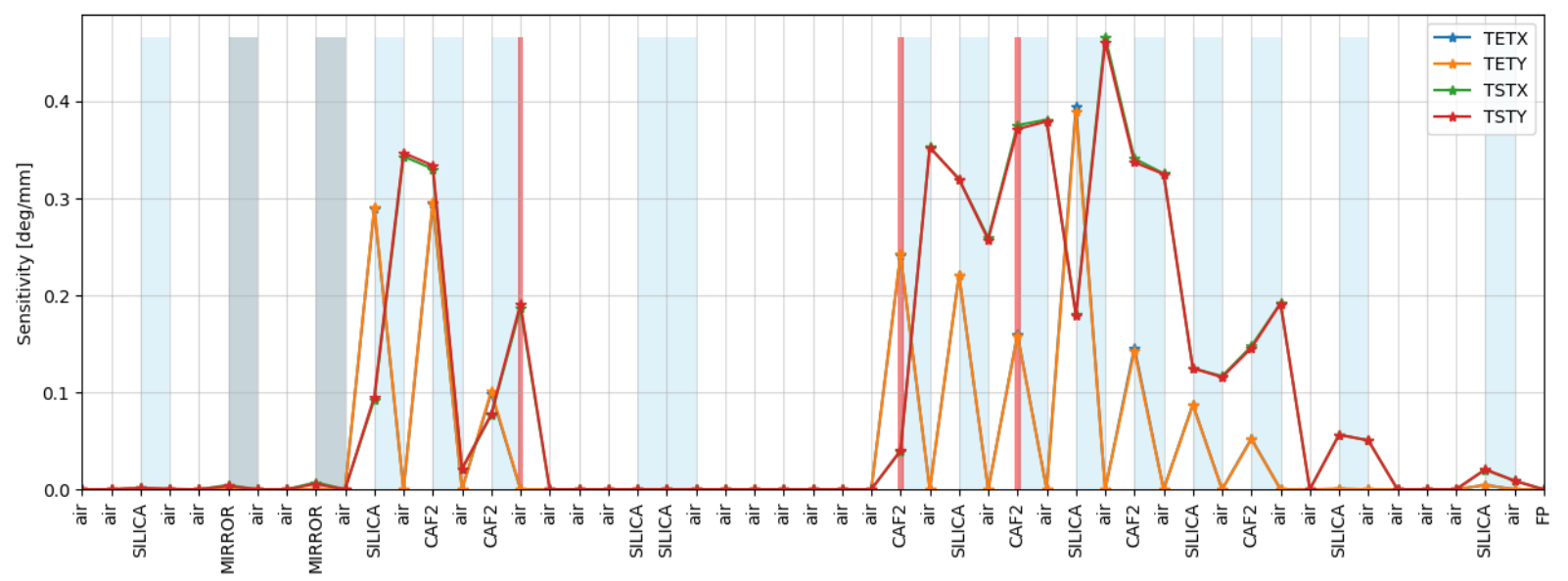

(d) Tolerance on Standard surface $\mathrm{x}-\mathrm{y}$ tilt (TSTX or TSTY) and tolerance on element $\mathrm{x}-\mathrm{y}$ tilt (TETX or TETY).

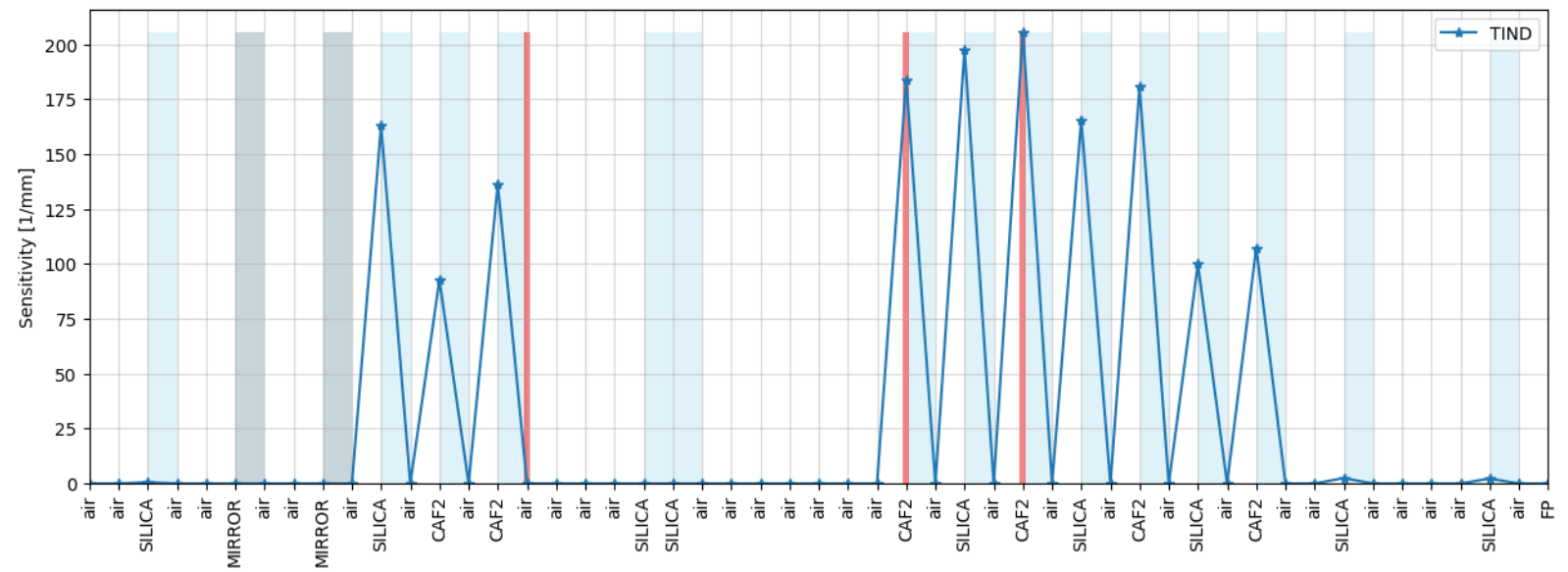

(e) Tolerance on index of refraction of surface (TIND).

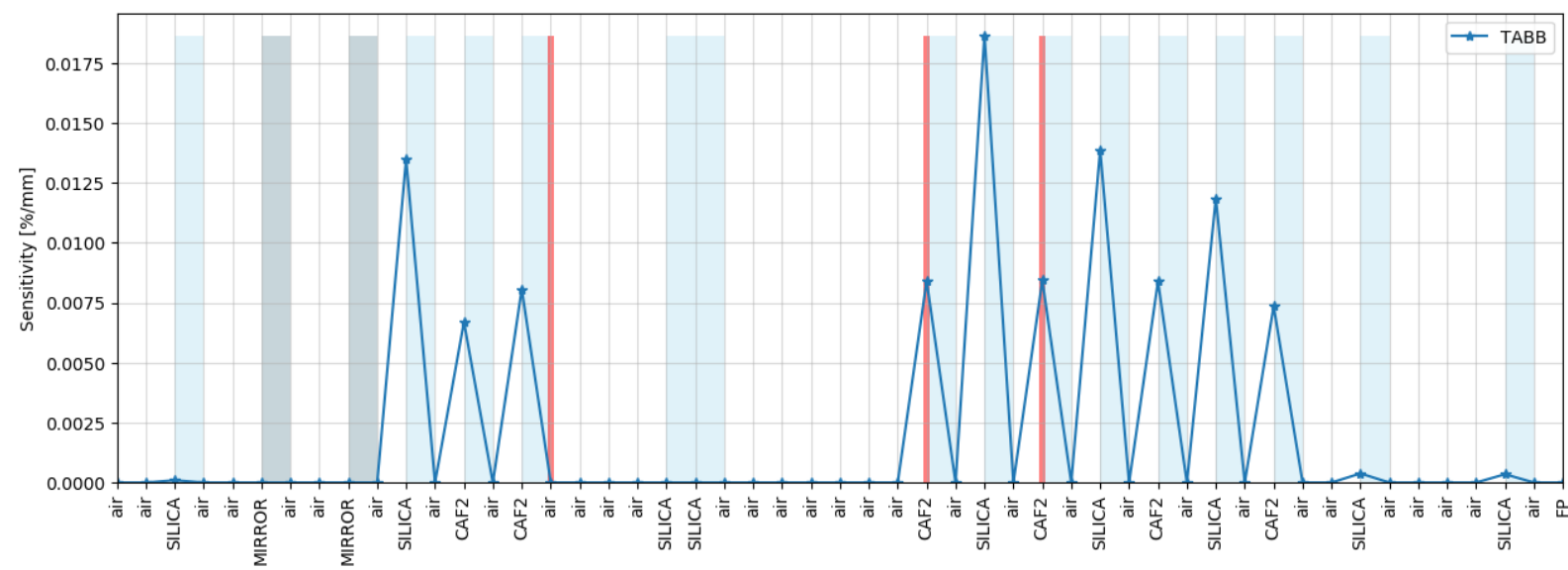

(f) Tolerance on Abbe number of surface in \% (TABB).

Figure 5.50: Image quality sensitivity diagram for the blue arm at low-resolution mode. 


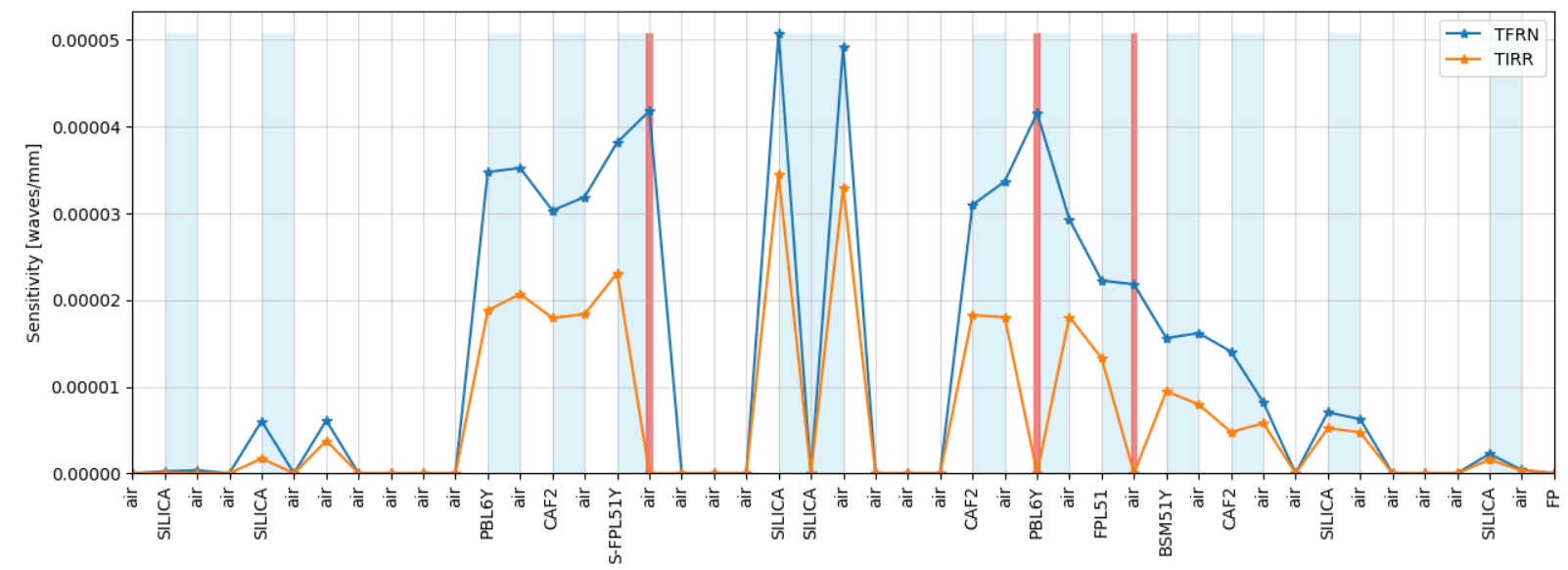

(a) Tolerance on surface radius of curvature in fringes (TFRN) and irregularity (TIRR).

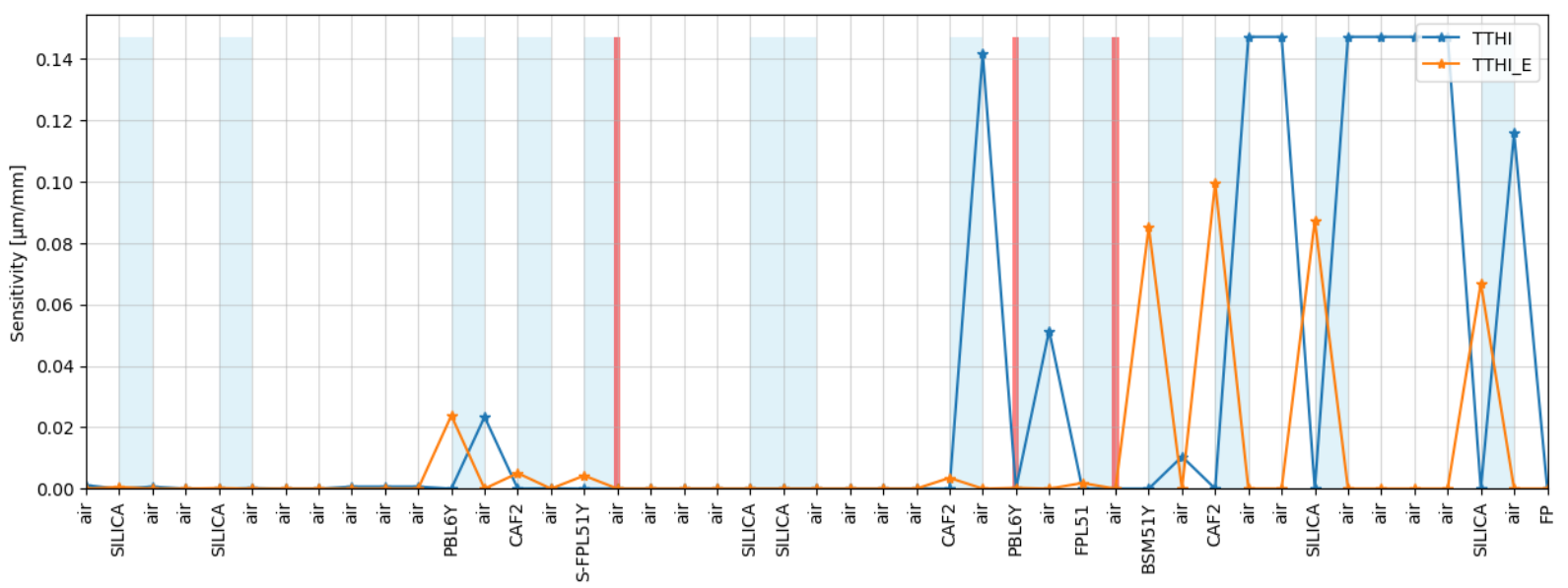

(b) Tolerance on thickness (TTHI_E) or position (TTHI).

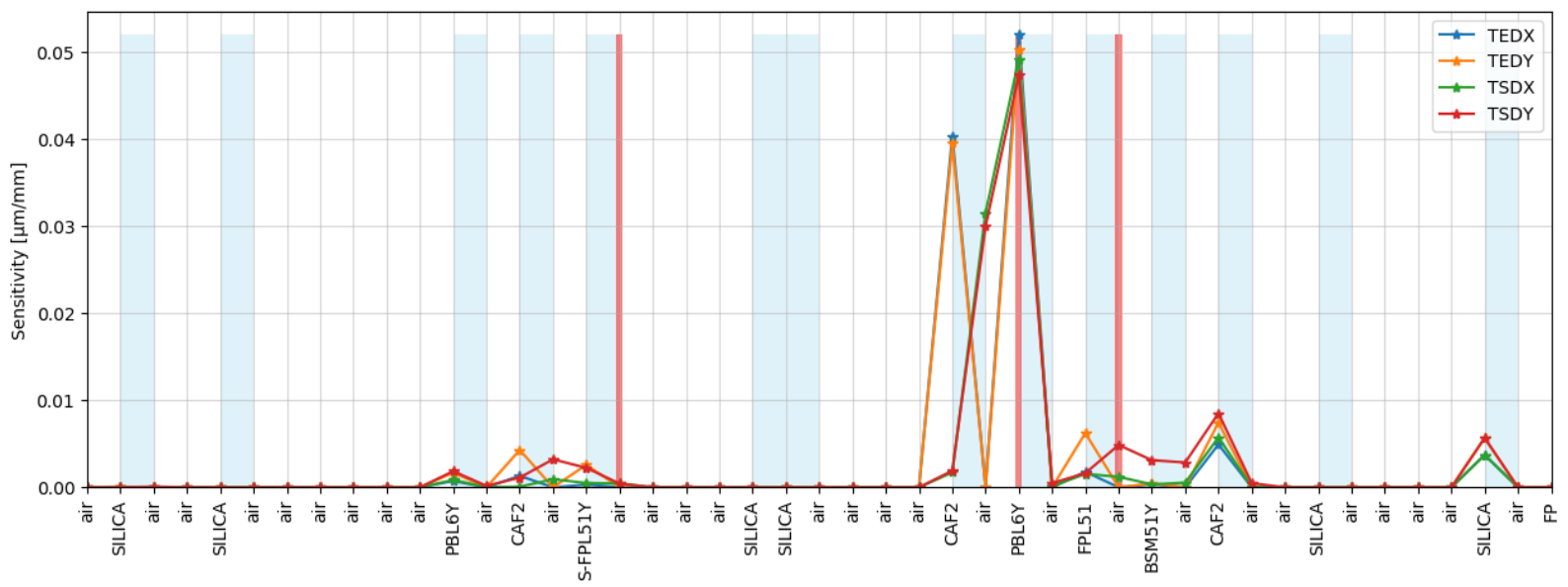

(c) Tolerance on Standard surface x-y decenter (TSDX or TSDY) and tolerance on element x-y decenter (TEDX or TEDY). 


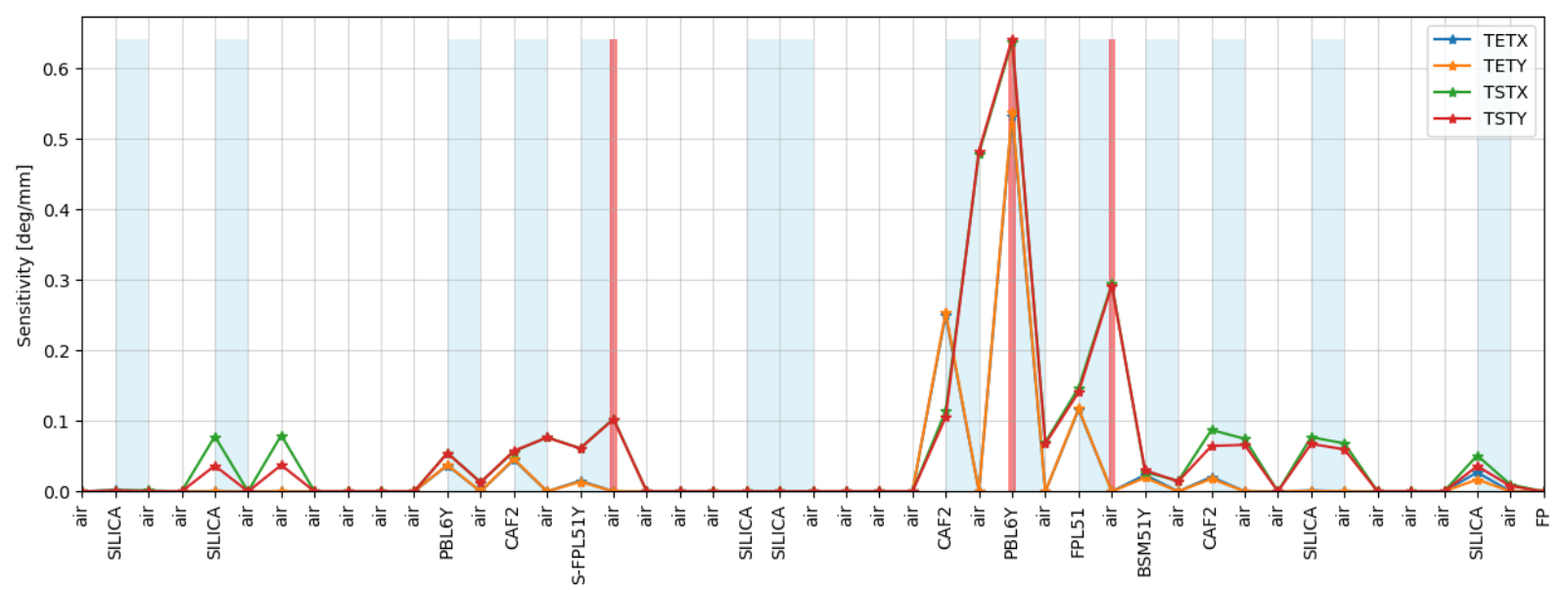

(d) Tolerance on Standard surface $\mathrm{x}-\mathrm{y}$ tilt (TSTX or TSTY) and tolerance on element x-y tilt (TETX or TETY).

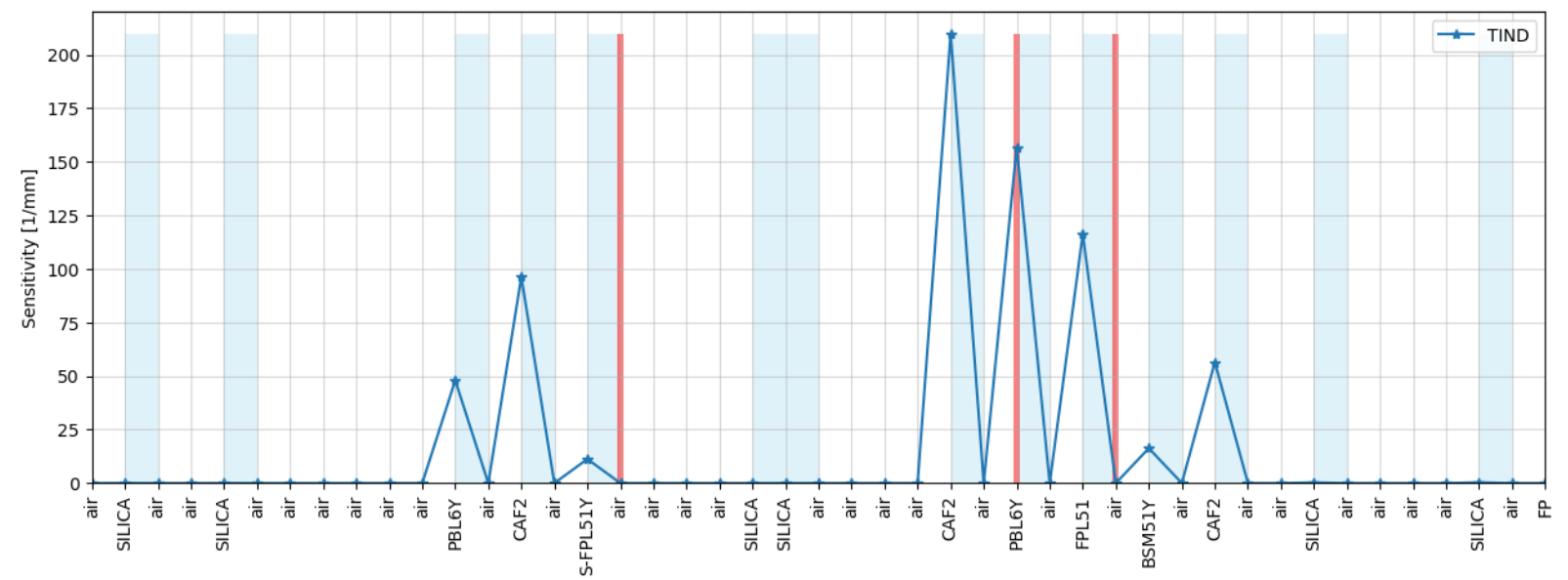

(e) Tolerance on index of refraction of surface (TIND).

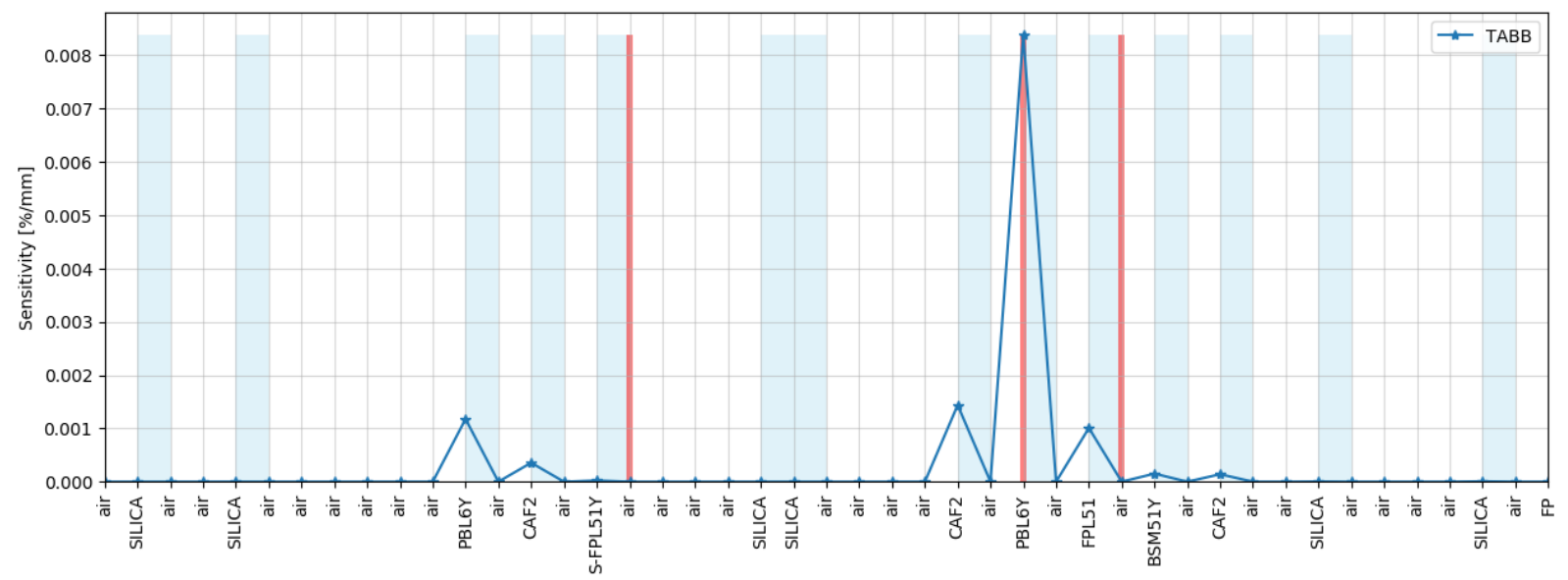

(f) Tolerance on Abbe number of surface in \% (TABB).

Figure 5.51: Image quality sensitivity diagram for the red arm at low-resolution mode.

The most notable curvature and irregularity sensitivities for the blue arm are on the flat surfaces from FM and VPHG. It is imperative that the FM positioning shall be tracked 
and controlled due to its high sensitivity. The effects are reduced close to the focal plane. The collimator's negative lens has the highest positioning sensitivity, even more than the positioning of the FF + Detector. Regarding the decentering sensitivities, the camera is the subsystem which presents the highest value for both manufacturing and alignment. However, the contribution from the collimator to the tilt is significant. The sensitivities for the refractive index and Abbe number are balanced, except for the first positive lens of the blue camera, which has the highest sensitivity for tolerance in dispersion.

The sensitivity of the red channel for curvature and irregularities is similar to the blue channel. On the other hand, the red camera has a high sensitivity for positioning and thickness of the lenses, mainly in the first lens and positioning of the FL + Detector. The significant disadvantage is the decenter and tilt sensitivities of the first negative lens of the red camera, which has an aspherical surface. Moreover, it has a high sensitivity both for variations in refraction and dispersion.

\subsubsection{Tolerance}

Table 5.17 shows the tightest tolerances for the blue arm for $3 \%$ of image quality degradation for each parameter considering axial back focus adjustment (maximum range $760 \mu \mathrm{m})$. These results will be revised after the conclusion of the flexure analysis and the definitions of number and location of the active compensators.

Table 5.17 - Tightest tolerances for the blue arm for 3\% of image quality degradation for each parameter considering axial back focus adjustment (maximum range $760 \mu \mathrm{m}$ ).

\begin{tabular}{cccc}
\hline \multicolumn{2}{c}{ Manufacturing } & \multicolumn{2}{c}{ Assembly } \\
\hline Parameter & Value & Parameter & Value \\
Radius of Curvature & $>1 \mathrm{fringe}$ & Air Space & $\pm 20 \mu \mathrm{m}$ \\
Lens thicknesses Col. & $\pm 60 \mu \mathrm{m}$ & Air Space Cam. & $\pm 30 \mu \mathrm{m}$ \\
Lens thicknesses Cam. & $\pm 60 \mu \mathrm{m}$ & Element Dec. Col. & $\pm 100 \mu \mathrm{m}$ \\
Surface Dec. Col. & $\pm 140 \mu \mathrm{m}$ & Element Dec. Cam. & $\pm 30 \mu \mathrm{m}$ \\
Surface Dec. Cam. & $\pm 40 \mu \mathrm{m}$ & Element Tilt Col. & $\pm 1.4 \mathrm{mrad}$ \\
Surface Tilt Col. & $\pm 180 \mathrm{mrad}$ & Element Tilt Cam. & $\pm 0.3 \mathrm{mrad}$ \\
Surface Tilt Cam. & $\pm 90 \mathrm{mrad}$ & & \\
Irregularity & $>0.2 \mathrm{fringe}$ & & \\
Index of refraction & $10-4$ & & \\
Abbe difference & 0.3 & & \\
\hline
\end{tabular}




\subsubsection{Detector Assy}

Although the detector is very simplified from the point of view of optical design, it is an essential subsystem of GMACS and has a specific group of engineers for its development and manufacture. Thus, the interface between the subsystem tolerances must be managed appropriately. We define Detector Assy as the subsystem comprised of the Field Flattener $(\mathrm{FF})$, which is also the dewar window, and the CCD's planes (2-by-3, 4k $\left.\mathrm{k}^{2} @ 15 \mu \mathrm{m}\right)$, see figure 5.52. The tolerances are divided in (i) assembly, such as FF Decenter and tilt, Axial Distance of the FF to focal plane and CCD assembly planicity (among the CCD planes); (ii) intrinsic to the CCD wafer, such as CCD active surface planicity (info from supplier); and (iii) operational, such as vibration due to the cooling system that would cause PSF blurring. This last is not considered in this analysis.

Assuming GMACS as non-diffraction imaging systems, the Depth of Focus, DoF, is \pm $33 \mu \mathrm{m}$ for an $\mathrm{f} / 2.2$ and pixel size $15 \mu \mathrm{m}$, and the tolerance tilt of $1.2^{\prime}$ and $3.7^{\prime}$ for the entire mosaic and individual detectors.

The FFs for the first camera versions were single CV-CV element and edge distance from the FF to the focal plane is $5 \mathrm{~mm}$ and $10 \mathrm{~mm}$ for the blue and red camera, respectively. Based on the GMACS detector team's recommendation, we constrained the FF to be a CVplano element since this configuration showed to be the best to avoid thermal deformations (GMACS Team, 2019). Flattening the rear FF surface settles pressure on the radius of curvature of the front surface since the sag thickness profile is the parameter that matters in an FF design. The axial distance is set to constant $10 \mathrm{~mm}$ for both cameras.

The analysis is performed for assembling tolerance. The criterion is $3 \%$ of RMS Spot Radius degradation, and the variables are (i) the positioning from the FF to focal plane; and (ii) tilt FF decenter and tilt with reference to the focal plane. The inverse sensitivity for the above criterion was the thickness range of $(-8,30) \mu \mathrm{m}$, minimum decenter of $\approx$ $100 \mu \mathrm{m}$ and minimum tilt of $\approx 3^{\prime}$ without compensator. Including the detector assy as a compensator, the values are $(-450,450) \mu \mathrm{m}$, minimum decenter of $\approx 120 \mu \mathrm{m}$ and minimum tilt of $\approx 4^{\prime}$. 


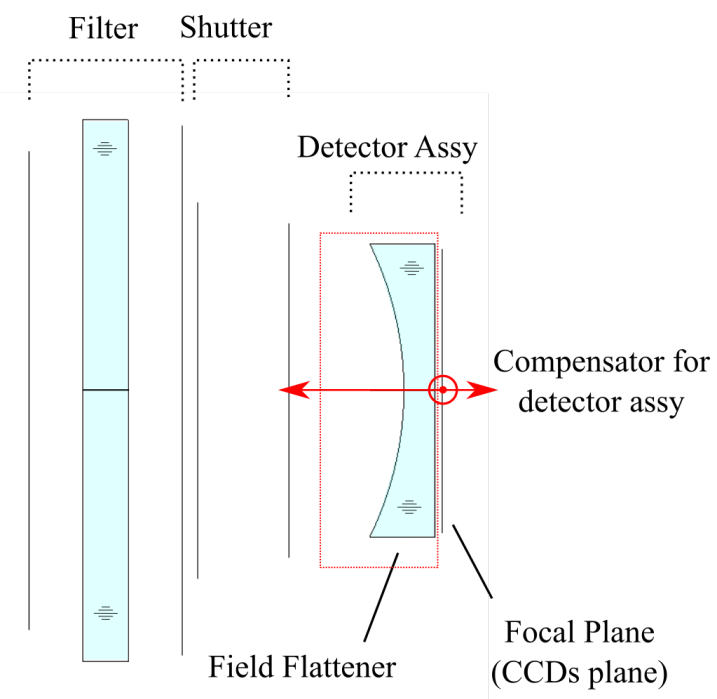

Figure 5.52: Detector Assy tolerance for assembling.

\subsection{Spectral Stability Analysis}

One of the main challenges related to the operability of the GMACS optical system is the spectral stability due to the flexure caused by the variation of the gravity vector. GMACS mounted at the Gregorian focus position of the GMT will experience a continually changing orientation as the telescope tracks an object across the sky during the observation time. Therefore, the gravity-induced flexure of the instrument must be monitored and compensated. A look-up table-based compensation solution shall be difficult to address all the possible flexure due to the reconfigurable nature of GMACS. Thus, the solution points towards an active compensator system. Table 5.1 shows that the spectral stability requirement is equivalent to approximately $56.7 \mu \mathrm{m}$ at the focal plane (i.e., 3.8 pixels), for a slit width of $0.7^{\prime \prime}$. For instance, the tilt of a camera with ELF $594 \mathrm{~mm}$ is $\approx 20^{\prime \prime}$ to reproduce an image shift of $56.7 \mu \mathrm{m}$. The goal reduces this requirement to one-third (GMACS Team, 2019). A preliminary flexure sensitivity and inverse sensitivity analysis of the GMACS optical system is described in this section.

\subsubsection{Flexure Sensitivity Analysis}

Image quality degradation was not analyzed for the flexure sensitivity analysis performed in this section. Therefore, any EED80 variations due to the composition of the perturbations, spectral lines magnification or any effects which might degrade the overall image quality are not taken into account. We assume that such effects are expected to be 
marginal given the small displacements; however, it depends on the intensity of flexure.

The study described below was initially presented by Ribeiro et al. (2018a). Although the presented $\mathrm{CoD}$ optical design version has undergone design modifications, such as the reallocation of the filter in the blue camera and uniting the astigmatism corrector component into the dichroic of the split collimator red arm, the results presented are still valid for the flexure sensitivity $\mathrm{CoD}$ analysis.

Table 5.18 and figure 5.53 show the groups definition for the flexure analysis. The dichroic, CG2, is considered as a plane mirror for the blue arm. The blue collimator group, CG5, contains the air triplet group of the blue Split Collimator arm. CG6 is the blue camera group, and CG7 is the detector assembly group, composed by the filter, shutter, and FF. Even though the FF is part of the camera, for clarity, CG6 is called the blue camera group. CG7 has a minimum of two degree of freedom, DoF: one for focus adjustment (Z-axis) and the other for spectral alignment in detector arrays (about the Z-axis).

Table 5.18 - The optical groups that were tracked in the sensitivity analysis and FEA and had resulting motions input into Zemax for image quality and spectral stability analysis.

\begin{tabular}{cc}
\hline Blue Arm & Red Arm \\
\hline Field Lens & Field Lens \\
Dichroic & Dichroic \\
Fold Mirror & Compensator \\
Blue Collimator & Red Collimator \\
Blue Camera (optics + detector) & Red Camera (optics + detector) \\
\hline
\end{tabular}

The merit function applied to this analysis is proportional to the displacement of the spot centroid on the focal plane due to the group/element decenter and tilt. Before including the perturbation of the system, the spot centroid coordinates are recorded in a reference data file. After the perturbation is included in the optical software, the new set of spot centroid coordinates are recorded and the resulting displacement is calculated as the vectorial composition of the spectral and spatial directions for the unperturbed and perturbed results. This study was performed only for the Blue Arm. 


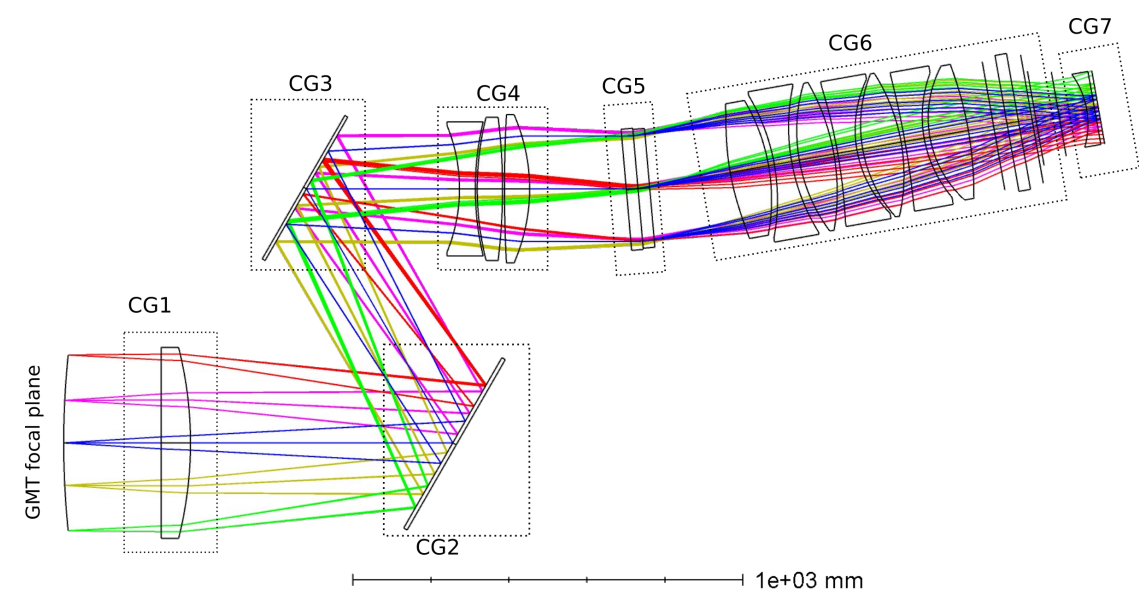

(a) Blue arm.

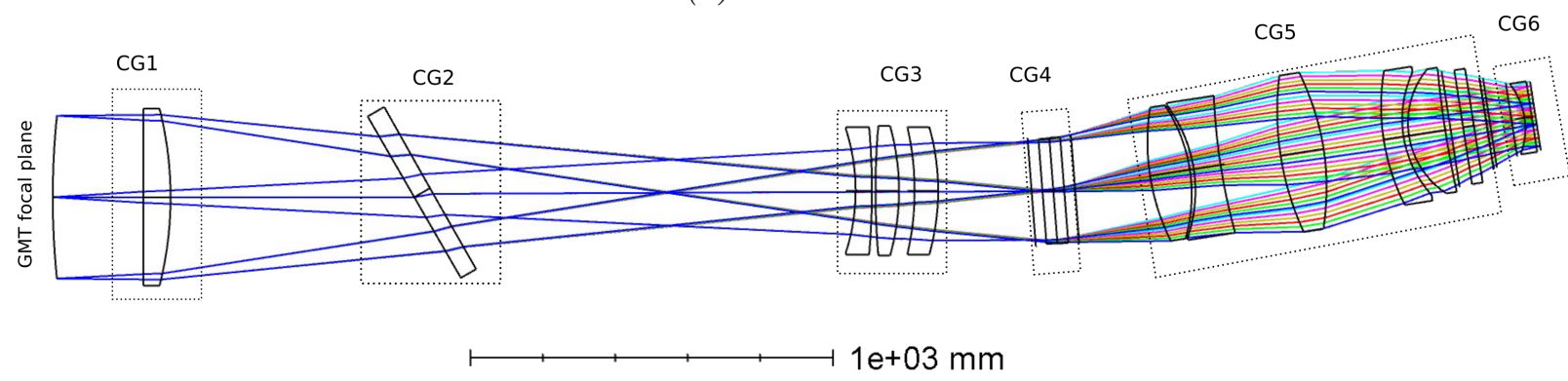

(b) Red arm.

Figure 5.53: Groups definition for the flexure analysis.

Tables 5.19 and 5.20 show the inverse sensitivity analysis for the flexure groups for a 0.3 spectral resolution displacement at the focal plane $(\approx 57 \mu \mathrm{m})$, for a slit width of $0.7^{\prime \prime}$, without any compensator. The x-axis is in the spatial direction and the detector group pivot is at the rear FF surface.

Table 5.19 - Inverse Sensitivity analysis for the GMACS blue arm in low-resolution mode for 0.3 spectral resolution displacement at the focal plane $(\approx 57 \mu \mathrm{m})$.

\begin{tabular}{lccc} 
ID & Description & $\begin{array}{c}\text { Decenter }(\mathbf{X}, \mathbf{Y}) \\
{[\mathrm{mm}]}\end{array}$ & $\begin{array}{c}\text { Tilt }\left(\theta_{x}, \theta_{y}\right) \\
{[\operatorname{arcmin}]}\end{array}$ \\
\hline CG1 & Field Lens & $1.40,1.40$ & $54.2,54.2$ \\
\hline CG2 & Dichroic (acts as a mirror) & n/a, n/a & $0.6,0.6$ \\
\hline CG3 & Fold Mirror & n/a, n/a & $0.3,0.3$ \\
\hline CG4 & Blue collimating group & $0.25,0.25$ & $2.3,2.3$ \\
\hline CG5 & VPHG & n/a, n/a & variable \\
\hline CG6 & Blue camera group & $0.054,0.054$ & $0.45,0.45$ \\
\hline CG7 & Detector Group & $0.058,0.058$ & $8.4,8.4$ \\
\hline
\end{tabular}

The dichroic-fold mirror combination is several times more sensitive to tilt in the blue 
Table 5.20 - Inverse Sensitivity analysis for the GMACS red arm in low-resolution mode for 0.3 spectral resolution displacement at the focal plane $(\approx 57 \mu \mathrm{m})$.

\begin{tabular}{cccc} 
ID & Description & $\begin{array}{c}\text { Decenter }(\mathbf{X}, \mathbf{Y}) \\
{[\mathrm{mm}]}\end{array}$ & $\begin{array}{c}\text { Tilt }\left(\theta_{x}, \theta_{y}\right) \\
{[\text { arcmin }]}\end{array}$ \\
\hline CG1 & Field Lens & $1.5,1.5$ & $55.8,55.8$ \\
\hline CG2 & Dichroic & $23.0,23.0$ & $48.0,48.0$ \\
\hline CG3 & Red collimating group & $0.25,0.25$ & $2.3,2.3$ \\
\hline CG4 & VPHG & n/a, n/a & variable \\
\hline CG5 & Red camera group & $0.054,0.054$ & $0.36,0.36$ \\
\hline CG6 & Detector Group & $0.058,0.058$ & $8.4,8.4$ \\
\hline
\end{tabular}

arm compared to the dichroic in the red arm, as expected for a combination of flat mirrors in the converging or diverging beam of an imaging system. The fold mirror tilt sensitivity is comparable to the camera; therefore it is a key component for passive flexure control in GMACS.

Figure 5.54 shows the effect of the $250 \mu \mathrm{m}$ transversal displacement (plane orthogonal to the optical axis) of the collimator group for the blue arm (similar behaviour for the red arm). The image displacement is represented with a quiver plot, which is basically a type of $2 \mathrm{D}$ plot which shows vector lines as arrows. The effects are similar to any direction in the transverse plane.

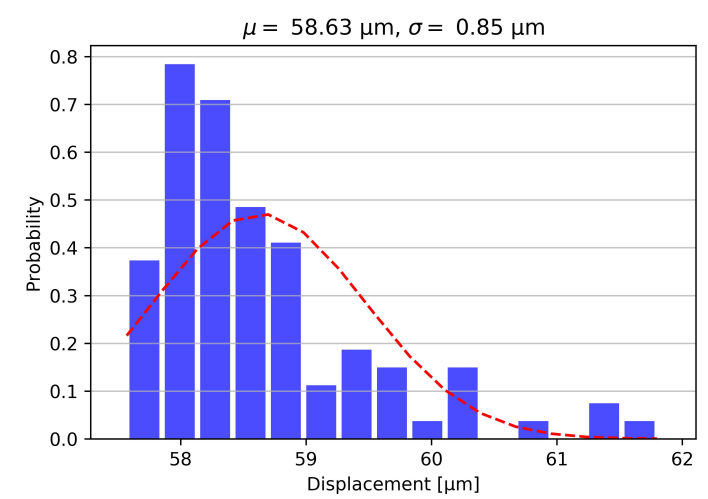

(a) Displacement histogram.

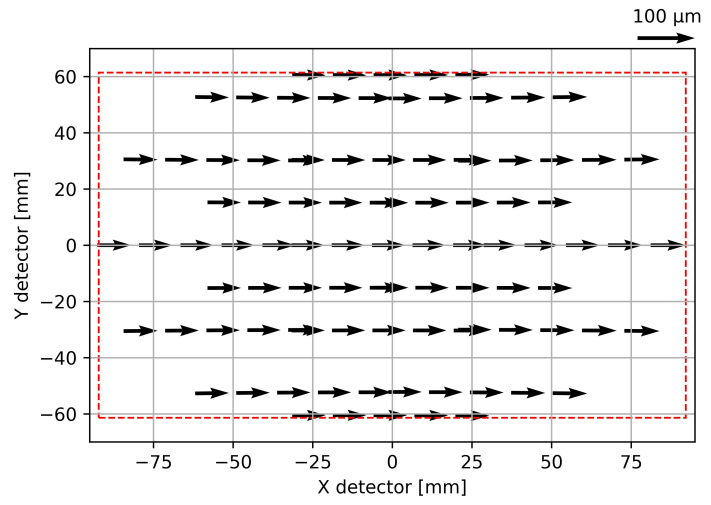

(b) Quiver plot at the focal plane.

Figure 5.54: a) Displacement histogram and b) quiver plot at the focal plane of the perturbed system for $250 \mu \mathrm{m}$ transverse displacement of the collimator group. The spectrum displacements are similar in direction and intensity.

We assume that the less sensitive group is a good choice for being the compensator since larger decenter and tilt variations cause a small change in the spectral centroid position, 
which in turn allows for fine control of the positions of the centroids. Therefore, comparing the collimator and camera groups, we concluded that the former would be the candidate for an active flexure compensator. However, it does not preclude the possibility or the necessity for including additional compensators in the camera and detector groups since this analysis has not yet included image quality metrics. Although the FL is very stable for flexure, this element is not recommended to be the compensator since it is shared for both the arms.

The VPGH sensitivity is explored in figures 5.55 and 5.56, which show the effect of the $0.5^{\circ}$ tilt of the VPHG in the spectral and spatial direction, respectively. The image quality is not affected by the range of displacements used in this inverse sensitivity analysis since the element is located in a collimated beam region.

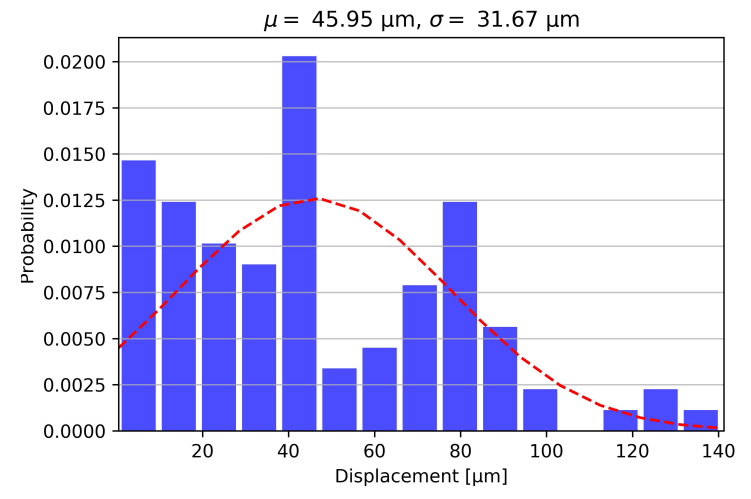

(a) Displacement histogram.

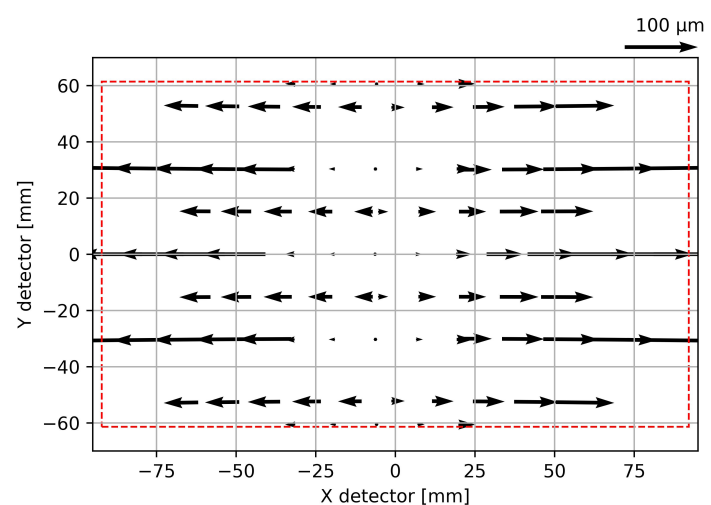

(b) Quiver plot at the focal plane.

Figure 5.55: a) Displacement histogram and b) quiver plot at the focal plane of the perturbed system for $0.5^{\circ}$ tilt of the VPHG in the spectral direction. The spectrum displacements are unsymmetrical as a function of the diffracting exitance angle.

\subsubsection{Flexure Tolerance Analysis}

The object of this study is to determine the best optical group or element to be an active compensator for flexure caused by the variation of the gravity vector and any image degradation residual. The terms used is Spectral Stability (SS) and Image Quality (IQ), respectively. This study incorporates the results from a FEA due to gravity vector perturbation in the optical design software by using Zemax macros and Python scripts, similar to those used by the GMACS Team (2019). For simplicity, only the GMACS blue arm results are shown in this section since we assumed that this arm is more sensitive due to 


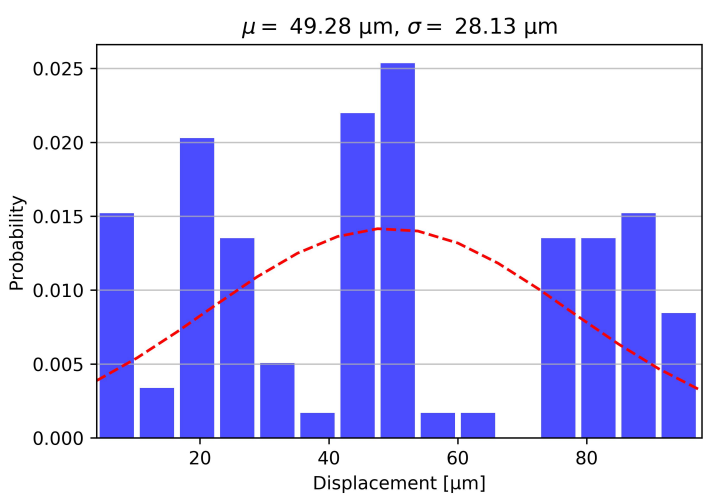

(a) Displacement histogram.

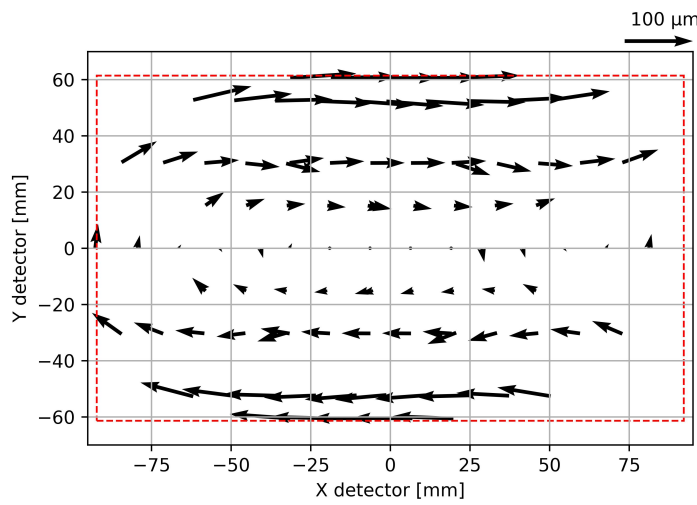

(b) Quiver plot at the focal plane.

Figure 5.56: a) Displacement histogram and b) quiver plot at the focal plane of the perturbed system for a $0.5^{\circ}$ tilt of the VPHG in the spatial direction. The spectrum displacements present a more complex behaviour compared to the previous results.

the combination of the dichroic and flat mirror which are, according to sensitivity analysis, more affected by flexure.

Among the GMT + GMACS pointing configurations, simulated by the optomechanical team, we selected the one that resulted in the highest absolute value of the detector plane flexure. We assumed that intermediate configurations have smaller disturbances. For simplicity, only a few nodes (which resulted in planes) were used for calculating the variation due to gravitational vector perturbation. No twisting or bending of these planes was considered at this stage of analysis. Figure 5.57 illustrates an example of the reference plane located at a mechanical barrel flange for the blue arm collimator group. The same approach was applied to the other groups.

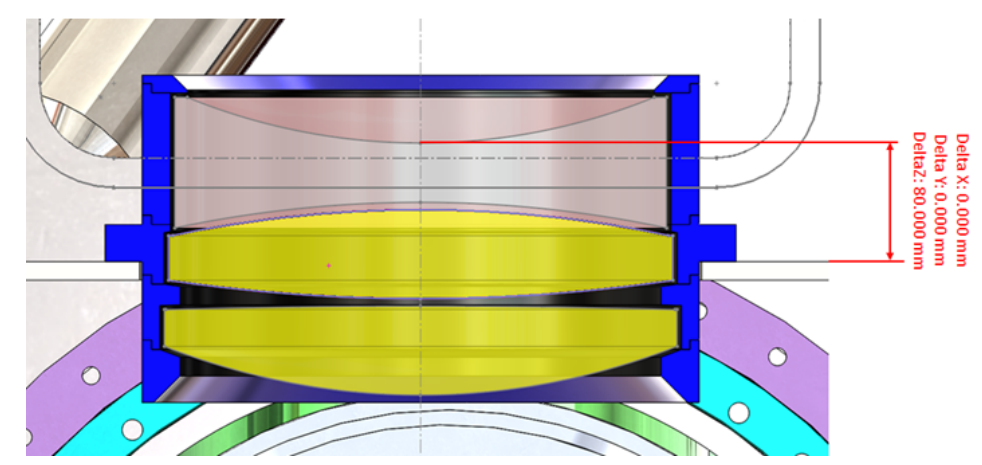

Figure 5.57: Example FEA Reference Plane in the Blue Collimator Group.

Figure 5.58 shows the EED80 distribution resulted for the nominal and perturbed 
models provided by the FEA results. The EED80 RMS and STD was degraded by a factor of three; therefore, the IQ is also affected by the gravitational flexure.

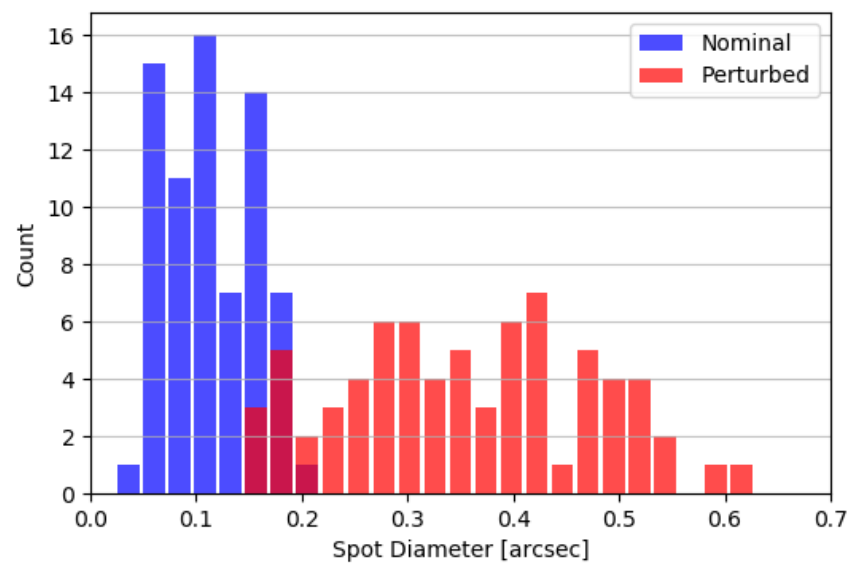

Figure 5.58: EED80 Histogram before $\left(\mathrm{RMS}=0.12^{\prime \prime}\right.$ and $\left.\mathrm{STD}=0.04^{\prime \prime}\right)$ and after flexure (RMS $=0.37^{\prime \prime}$ and $\left.\operatorname{STD}=0.12^{\prime \prime}\right)$.

Figure 5.59a shows the displacements histogram and figure 5.59b the quiver plot at the focal plane of the perturbed system, which informs the direction and intensity of the asymmetrical perturbation and is directed related to the spectral stability. The results are described by RMS and the STD of the vectorial composition of the field centroids in the spectral and spatial directions.

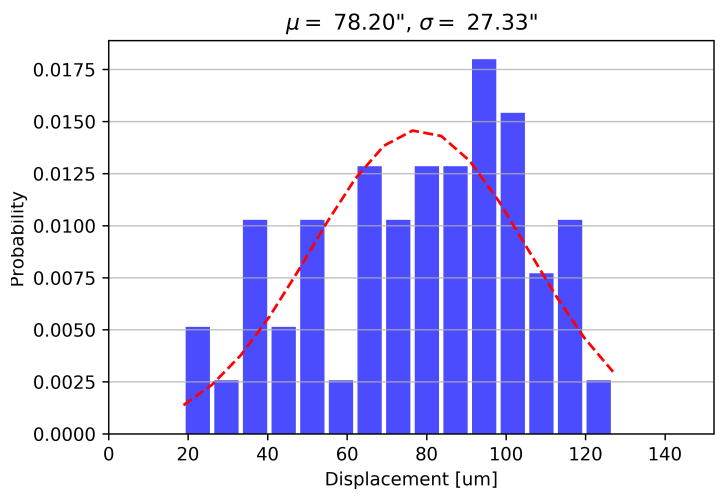

(a) Displacement histogram.

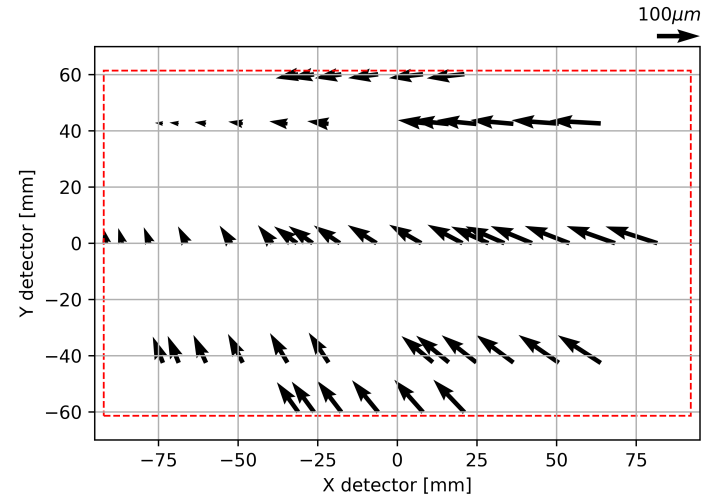

(b) Quiver plot at the focal plane.

Figure 5.59: a) Displacement histogram and b) quiver plot at the focal plane of the perturbed system. The RMS is $\approx 80 \mu \mathrm{m}$ and STD is $30 \mu \mathrm{m}$. Approximately $74 \%$ is out of spec., $24 \%$ is between spec and goal, and only $2 \%$ meet the goal. The quiver plot arrow legend shows the $100 \mu \mathrm{m}$ length reference. The centroid displacements are unsymmetrical in both direction and intensity. 
The strategy aims to define how many and where are the best locations to the IQ and the SS correction. We first checked the individual performance of each group to correct a particular perturbation (IQ or SS) and later measured the effect on the other parameter. For example, let us consider the Field Lens (CG1) to be the compensator. Starting from the optical system disturbed by the flexure, we determine the FL tip, tilt and axial movements that are necessary to correct only the IQ by minimizing the EED80, followed by the SS verification. In the next step, the system is reoptimized for SS by using the specific SS $\mathrm{MF}$ and the macro for generating the database, followed by the IQ verification. The most appropriate compensator is the one that satisfactorily corrects both the IQ and the SS. Figures 5.60 and 5.61 show the EED and the SS when the system is optimized for IQ and SS using the CG1 as a compensator, respectively.

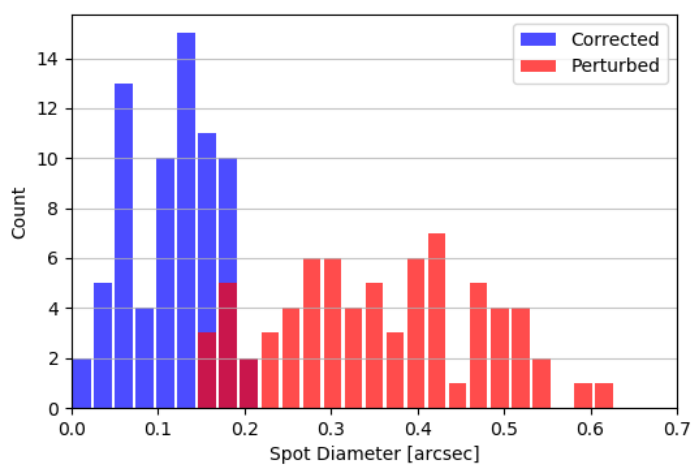

(a) EED80.

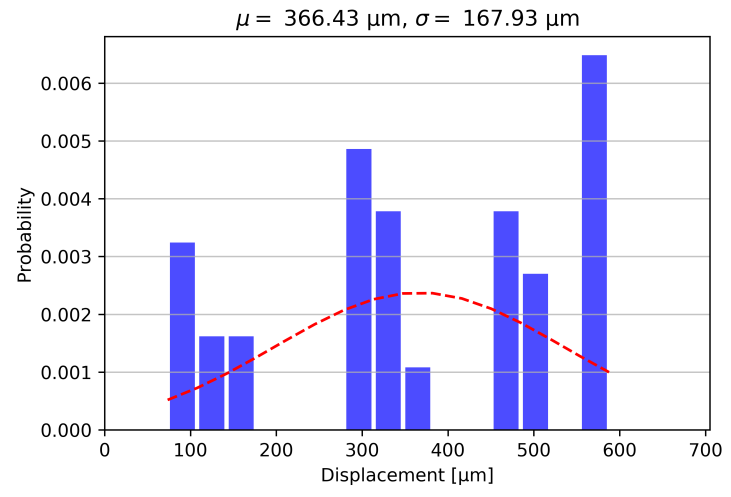

(b) Spectral Stability.

Figure 5.60: EED and SS results for the FL as a compensator when the IQ is optimized in the blue arm.

We noticed that the FL is not sufficient to correct one parameter while the other is minimized, even when both the restrictions are included at the same MF. It means that the IQ and the SS are correlated, and therefore they can not be minimized individually. The FL is indeed not suitable to be a compensator since both channels share it, and since we assumed that the flexure is likely to be different for all the channels, the compensator system shall be unique for each channel.

Since we obtained similar results using the FM as a compensator, we concluded that FM should not be an active compensator since it also makes part of the blue arm solely. We expect to have a common solution to both arms, and consequently reduce cost and risk factors. Hence, the best candidates for flexure compensators are the collimator groups 


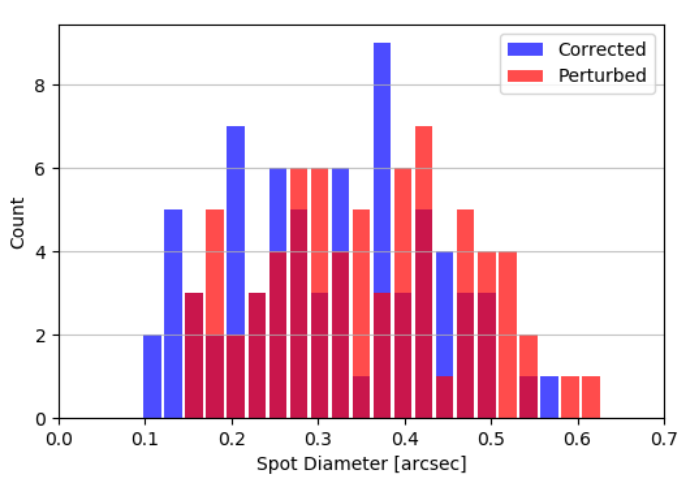

(a) EED80.

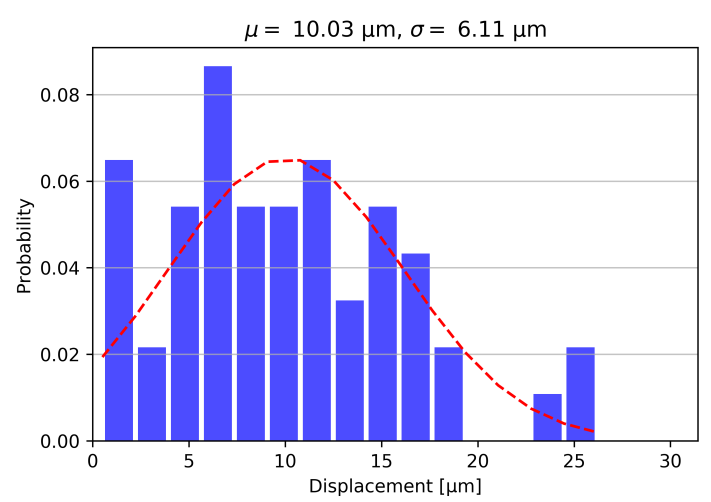

(b) Spectral Stability.

Figure 5.61: EED and SS results for the FL as a compensator when the SS is optimized in the blue arm.

(CG4) and cameras, including the detector. We define "camera" as the combination of CG6 + CG7, considered primarily as a solid body. Figures 5.62 and 5.63 show the EED and the SS with the collimator group as a compensator when the system is optimized for IQ and SS, respectively.

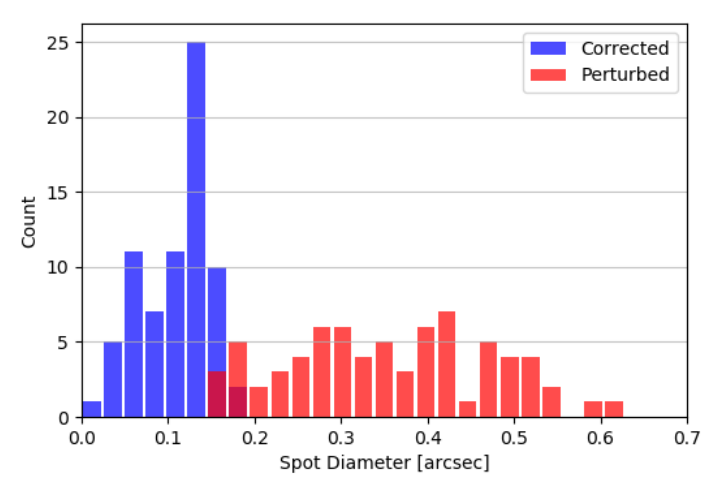

(a) EED80.

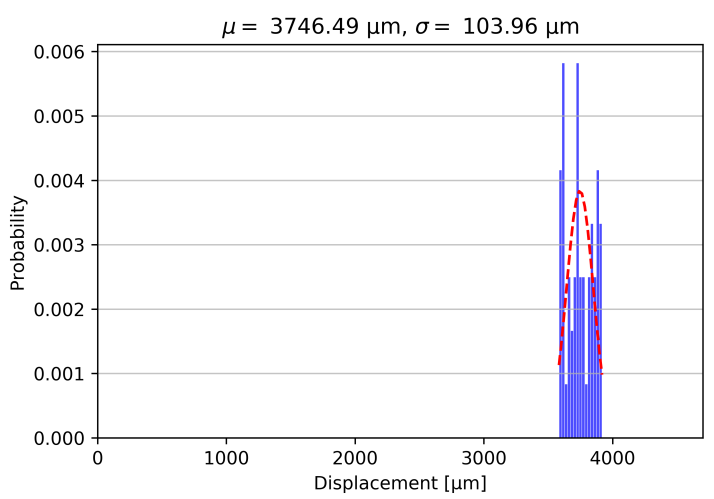

(b) Spectral Stability.

Figure 5.62: EED and SS results for the collimator groups as a compensator when the IQ is optimized in the blue arm.

Although the collimator group could provide a good IQ correction, the SS correction was insufficient. The reverse is also true, and in this case, the SS correction is twice as good as that of the FF and the FM. Since the collimator group is responsible for generating a flat wavefront in the exit pupil, it is a good candidate to compensate for focusing. Therefore, we explored the camera performance to compensate the SS by optimizing the chief ray disturbance. Figures 5.64 and 5.65 show the EED and the SS when the system is optimized 


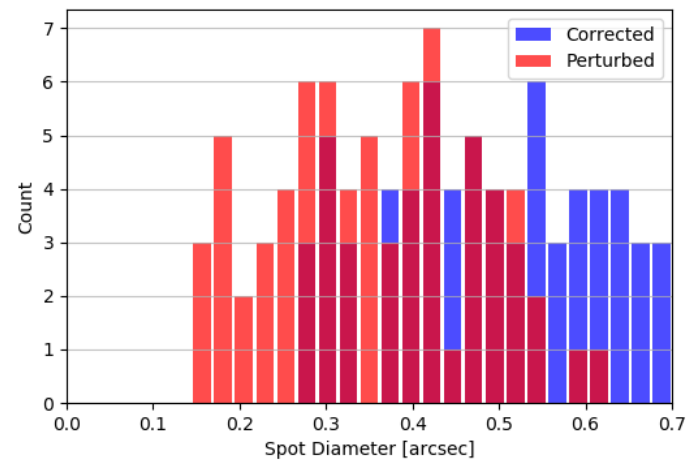

(a) EED80.

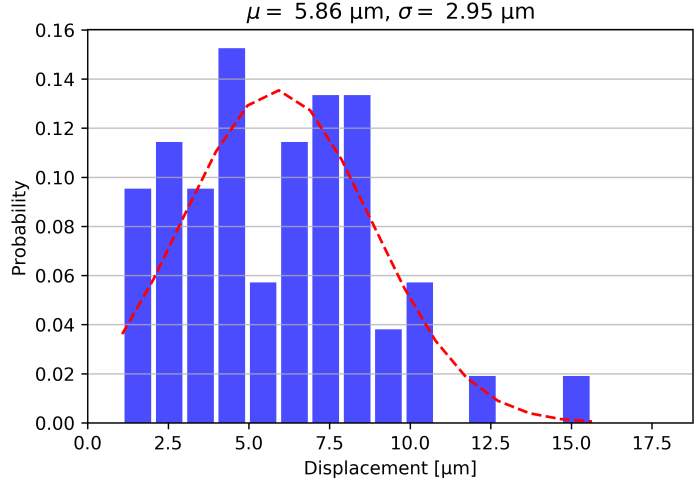

(b) Spectral Stability.

Figure 5.63: EED and SS results for the collimator groups as a compensator when the SS is optimized in the blue arm.

for IQ and SS, respectively, with the camera group as a compensator.

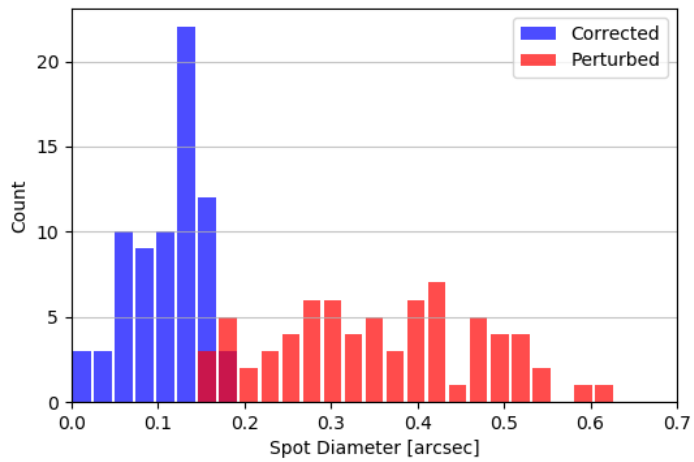

(a) EED80.

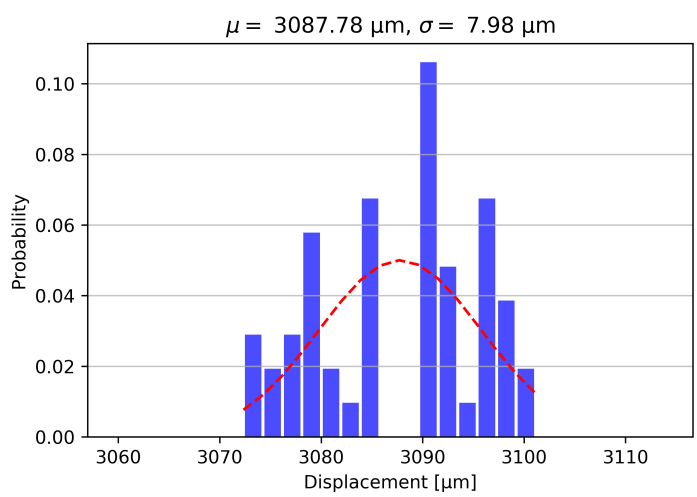

(b) Spectral Stability.

Figure 5.64: EED and SS results for the camera group as a compensator when the IQ is optimized in the blue arm.

The next study aims to explore the combination of the above simulations: the only collimator parameter that is variable is the axial position (Z-axis) while the camera variables are in the axial and transversal directions (X, Y, and Z-axis). Figure 5.66 shows the groups DoF. Only the SS approach was used. Figure 5.67 shows the results.

The collimator is moved by $300 \mu \mathrm{m}$ in Z-axis, and the camera by $\approx 40,70$, and 250 $\mu \mathrm{m}$ in $\mathrm{X}, \mathrm{Y}$, and Z-axis, respectively. We noticed a slightly better performance of IQ correction when both compensators are used instead of a single one. Figure 5.65a shows the IQ correction for the collimator group as a compensator, and figure 5.67a the collimator group and camera as compensators. Although the camera could be the only compensator 


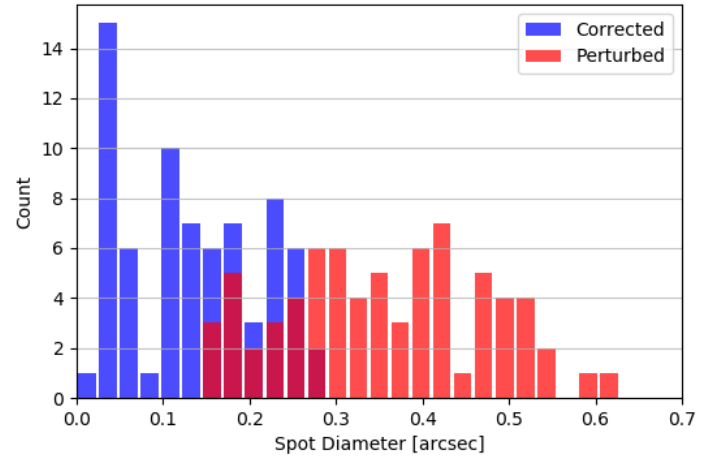

(a) EED80.

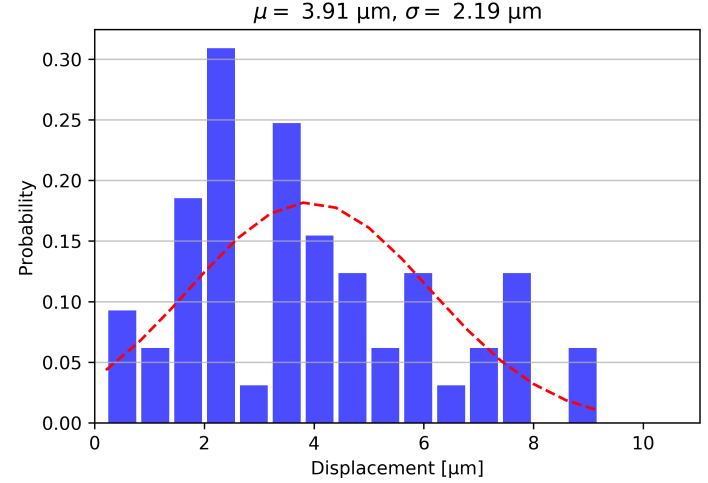

(b) Spectral Stability.

Figure 5.65: EED and SS results for the camera groups as a compensator when the SS is optimized in the blue arm.

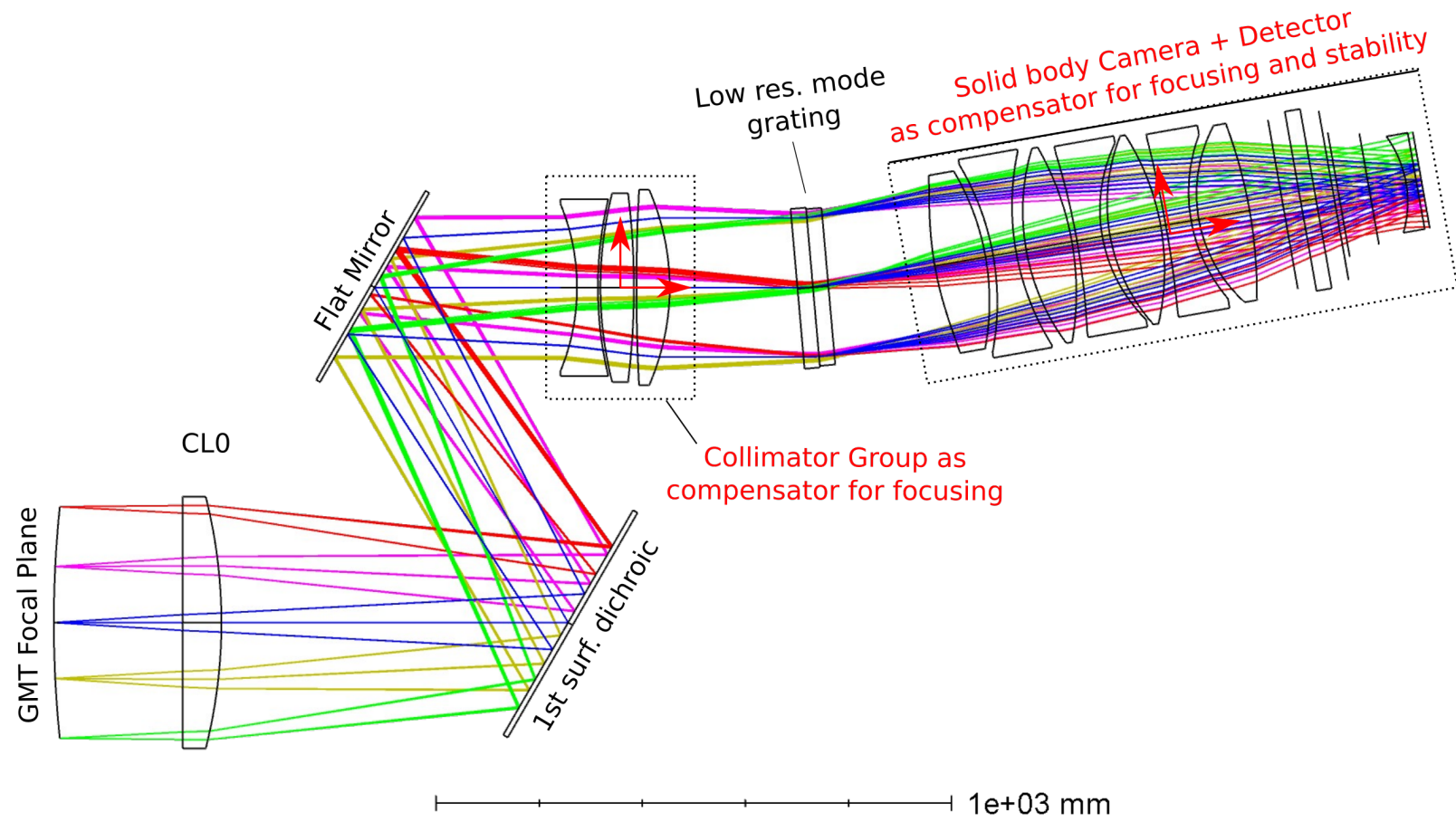

Figure 5.66: Current strategy flexure compensator. The collimator group is the compensator for the focusing while the camera group (camera and detector are considered as a solid body) is the compensator for flexure and focusing.

for the gravitational flexure, we noticed that the axial movement of the collimator group is essential for focusing. In addition, a focusing compensator is essential for any imaging system, even though it does not suffer from gravitational flexure. Therefore, this solution proved to be the most efficient as it satisfies the IQ, SS correction requirements and is an identical solution applicable for both channels since the compensators are located in the collimator groups and cameras. 


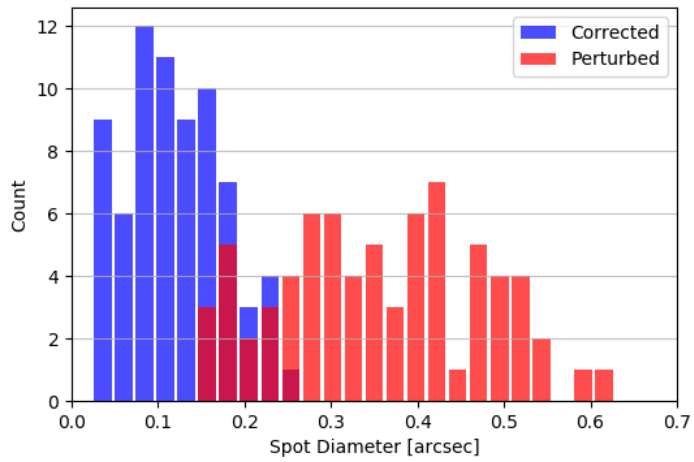

(a) EED80.

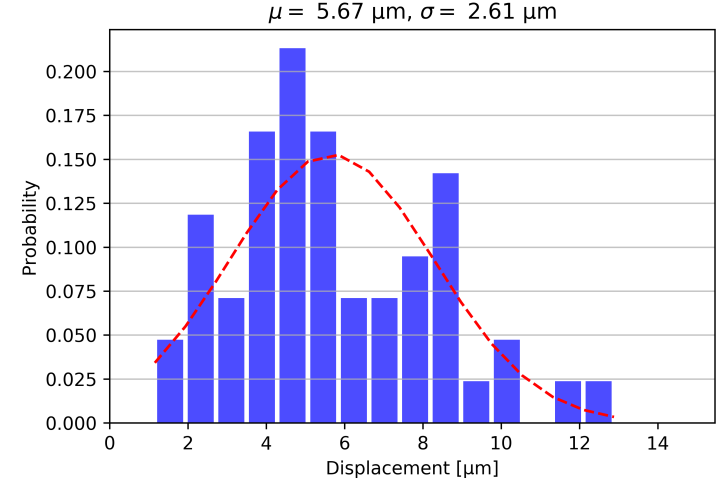

(b) Spectral Stability.

Figure 5.67: EED and SS results for the collimator in the axial position ( $\mathrm{Z}$ axis) and camera groups in the transversal directions ( $\mathrm{X}$ and $\mathrm{Y}$ axis) as a compensator when the SS is optimized in the blue arm.

Since we assumed that the collimator group is used for focusing, we performed a sensitivity analysis for blue arm collimator group in low-resolution mode related to IQ solely for axial movement. A $250 \mu \mathrm{m}$ collimator group axial movement causes about $3 \%$ of the RMS EED80 degradation. The distribution is not symmetric over the central wavelengths: the edge spectra are more degraded. A useful alternative approach is to move the detector plane by a fixed value and optimize the system with the compensator as variable. For instance, a $100 \mu \mathrm{m}$ detector plane defocus requires an $1.9 \mathrm{~mm}$ axial shift of the collimator groups towards the FL to correct it. This alternative will be explored in the next design phase.

Although hexapod compensators are strictly not necessary since linear positioners can supply the requested DoF for IQ and SS correction, the use a six DoF active mechanical positioning with absolute measuring sensors, software, and motion controllers will also be beneficial for the GMACS Assembly, Integration and Tests, AIT phase. Thus, given the high customization of sizes and technical capacities, relatively low cost and versatility for application in AIT and flexure correction, and that unused DoF can be "turned off" by software, the hexapods are highly recommended for the alignment and the flexure compensator system of the GMACS. 
Chapter 6

\section{Conclusion}

We presented the development of the optical conceptual design for the Giant Magellan Telescope Multi-object Astronomical and Cosmological Spectrograph, GMACS, during the years 2015 to 2020. More specifically, it consists of research activities carried out for the GMACS optical project within the context of the 2016 design re-scope requested by the GMT Organization via the Statement of Work (GMT-SOW-01091) and attachments.

We described in detail the theoretical background for a low to mid-resolution VPH grating optical spectrograph for ELTs from the optical design perspective, including a review of pre-existing MOS with similar GMACS specifications, which resulted in a methodology and a set of tools for the optical design, modeling, and analysis applied to GMACS, such as GUI Spectrograph Simulator, Optical Design Configuration Manager and several Zemax macros to automatize tasks and Python scripts for data processing and visualization.

The proposed GMACS conceptual optical design is a seeing limited two-channel VPH spectrograph with a relatively wide field $\approx 7.5^{\prime}$ in diameter, spectral resolving power spanning from 500 to 6,000 , corresponding to a resolution of $\approx 8.5 \AA$ to $0.7 \AA$ for a $0.7^{\prime \prime}$ slit size baseline, high throughput and spectral coverage from $320 \mathrm{~nm}$ to $1,000 \mathrm{~nm}$. The optical subsystems are comprised of a f/8.14 refractive split collimator with an exit pupil of $270 \mathrm{~mm}$ in diameter followed by two f/2.2 refractive cameras based on Petzval objectives optimized for 320-600 $\mathrm{nm}$ and 500-1,000 nm. The spectrograph EED80 is $\leq 0.2^{\prime \prime}$ for all GMACS spectral range. Although the image mode is not a GMACS requirement, the blue channel optical system allows narrow-band imaging mode of about $\approx 50 \mathrm{~nm}$ bandwidth, with $\mathrm{MTF} \approx 0.5-0.6$ for the spatial frequency of 16.6 cycles $/ \mathrm{mm}$ (equivalent to two $15 \mu \mathrm{m}$ pixels). We have developed two alternative red cameras that allow wide wavelength coverage image mode without compromising image quality in spectroscopy modes, in addition 
to improvements in AOI and location of aspherical surfaces to reduce manufacturing risks. The performance is similar to that of the blue channel, but for the entire spectral band of the red channel (500-1,000 nm). The GMACS CoD designs and results were presented in the CoDR in TAMU 4-5th September 2019 and were well received by the committee members.

One of the main challenges related to the GMACS operability, as well for any ELT spectrograph installed at a non-gravity invariant platform, is spectral stability due to the mechanical flexure caused by the variation of the gravity vector. The methodology developed in this research to insert FEA data into the Zemax design revealed that, based on the CoDR FEA of the structure, the expected degradation is also observed in the image quality. The current FEA-Zemax integration only considers solid body movements; therefore, it does not include figure errors, birefringent effects, or temperature-induced index change. Our results suggest that the collimator group and cameras must be actively controlled to achieve both image quality and spectral stabilization correction, for each arm individually. While only the collimator group compensator would be enough for focusing, the correction of the spectral stability requires the collimator and camera compensator acting simultaneously. It also provides a fine focusing. Although hexapod compensators are strictly not necessary since linear positioners can supply the requested DoF for IQ and SS correction, the use of hexapods are recommended because it can be beneficial for the GMACS Assembly, Integration and Tests, AIT phase activities.

The following optics activities of the GMACS project will be based on the results presented in this research. Some of the PDR optical design activities are the refinement of the optical design in the face of the updated demands of the mechanical and optomechanical systems; detailed analysis of assembly tolerance; studies of processes for assembly, alignment and testing of individual and integrated subsystems; assistance with detailed simulations of the performance of the optical system integrated with optomechanical systems and mechanisms in general; and detailing of the interface with MANIFEST. Currently, we are exploring the feasibility of adapting the GMT Atmospheric Dispersion Corrector proposed by Johns and Rakich (2019) in parallel with the development of a particular ADC to meet GMACS requirements, called G-ADC. 


\section{Bibliography}

Agência FAPESP. Parceiros internacionais iniciam a construção do Telescópio Gigante Magalhães (GMT), 2015. Available at http://agencia.fapesp.br. Accessed on 201604-26.

Albuquerque B. F. C. d., de Sousa F. L., Montes A. S., Multi-objective approach for the automatic design of optical systems, Optics Express, 2016, vol. 24, p. 6619

Albuquerque B. F. C. d., Sasián J., Luis de Sousa F., Montes A. S., Method of glass selection for color correction in optical system design, Optics Express, 2012, vol. 20, p. 13592

Amorín R., Fontana A., Pérez-Montero E., Castellano M., Guaita L., Grazian A., Fèvre O. L., Ribeiro B., Schaerer D., Tasca L. A., Thomas R., Bardelli S., Cassarà L., Analogues of primeval galaxies two billion years after the Big Bang, Nature Astronomy, 2017, vol. 1 , p. 52

Appenzeller I., Rupprecht G., FORS - the focal reducer for the VLT, The Messenger, 1992, vol. 67 , p. 18

Baldry I. K., Bland-Hawthorn J., Robertson J. G., Volume Phase Holographic Gratings: Polarization Properties and Diffraction Efficiency, Publications of the Astronomical Society of the Pacific, 2004, vol. 116, p. 403

Barden S. C., Arns J. A., Colburn W. S., Volume-phase holographic gratings and their potential for astronomical applications. In Proc. SPIE 3355, Optical Astronomical Instrumentation, vol. 3, 1998, pp 866-876 
Barden S. C., Arns J. A., Colburn W. S., Williams J. B., Volume-Phase Holographic Gratings and the Efficiency of Three Simple Volume-Phase Holographic Gratings, Publications of the Astronomical Society of the Pacific, 2000, vol. 112, p. 809

Barros S., Vanzella E., Amorín R., Castellano M., Siana B., Grazian A., Suh H., Balestra I., Vignali C., Verhamme A., Zamorani G., Mignoli M., Hasinger G., Comastri A., Pentericci L., Pérez-Montero E., Fontana A., Giavalisco M., Gilli R., An extreme [O III] emitter at $\mathrm{z}=3.2$ : a low metallicity Lyman continuum source, Astronomy \& Astrophysics, 2016, vol. 585, p. A51

Bechtol K., Drlica-Wagner A., Balbinot E., Pieres A., Simon J. D., Yanny B., Santiago B., Wechsler R. H., Frieman J., Walker A. R., Williams P., Rozo E., Rykoff E. S., Queiroz A., Luque E., Benoit-Lévy A., Tucker D., Sevilla I., Gruendl R. A., da Costa L. N., Eight new milky way companions discovered in first-year dark energy survey data, The Astrophysical Journal, 2015, vol. 807, p. 50

Bentley J., Olson C., Field Guide to Lens Design, 2012, 154 p.

Bernstein G. M., Athey A. E., Bernstein R., Gunnels S. M., Richstone D. O., Shectman S. A., A volume-phase holographic spectrograph for the Magellan telescopes. In Optical Spectroscopic Techniques, Remote Sensing, and Instrumentation for Atmospheric and Space Research IV, vol. 4485, 2002, pp 453-459

Bernstein R., Plans for Multi-Object Spectroscopy with the ELTs. Technical report, 2015

Bernstein R. A., Fabricant D. G., Introduction to the Giant Magellan Telescope in , Giant Magellan Telescope Science Book 2018, 2018 pp 1-30

Bernstein R. A., Shectman S. A., Astronomical Spectrographs in Oswalt T. D., McLean I. S., eds, , Planets, Stars and Stellar Systems. Dordrecht, 2013 pp 587-618

Bingham R. G., Grating spectrometers and spectrographs re-examined, Royal Astronomical Society, 1979, vol. 20, p. 395

Branch D., Wheeler J. C., Supernova Explosions. Astronomy and Astrophysics Library, Springer Berlin Heidelberg Berlin, Heidelberg, 2017 
Butcher H., Multi-Aperture Spectroscopy At Kitt Peak. In Proc. SPIE 0331, Instrumentation in Astronomy IV, 1982, pp 296-300

Chen H.-W., The unchanging circumgalactic medium over the past 11 billion years, Monthly Notices of the Royal Astronomical Society, 2012, vol. 427, p. 1238

Cheng M., Humphreys M., 2017. Rotation Matrix and Tilt About X/Y/Z in OpticStudio. Available at https://customers.zemax.com/os/resources/learn/knowledgebase/ rotation-matrix-and-tilt-about-x-y-z-in-opticstudi. Accessed on 2018-11-21.

Chornock R., Berger E., Gezari S., Zauderer B. A., Rest A., Chomiuk L., Kamble A., Soderberg A. M., Czekala I., Dittmann J., Drout M., Foley R. J., Fong W., Huber M. E., Kirshner R. P., Lawrence A., Lunnan R., Marion G. H., Narayan G., Riess A. G., Roth K. C., The ultraviolet-bright, slowly declining transient ps1-11af as a partial tidal disruption event, The Astrophysical Journal, 2013, vol. 780, p. 44

Cilli M. V., Parnell G. S., Systems Engineering Tradeoff Study Process Framework, INCOSE International Symposium, 2014, vol. 24, p. 313

Cobos F. J., Gonzalez J. J., Tejada C., Cepa J., Rasilla J. L., OSIRIS optical design. In Proc. SPIE 4832, International Optical Design Conference 2002, No. December 2002, 2002, p. 249

Cobos F. J., Gonzalez-Hernandez J., Tejada C., Cepa J., Rasilla J. L., OSIRIS optical design, Proc. SPIE 4008, Optical and IR Telescope Instrumentation and Detectors, 2000, vol. 4008, p. 237

Colless M., Key early science with MANIFEST on GMT, 2018, pp 1-4

Contos A., GMT-SOW-01091, Rev. B - Statement of Work: GMACS Conceptual Design Study. Technical report, 2016

Contos A., Attachment to GMACS Conceptual Study GMT- SOW-01091 Rev B for SplitCollimator Design GMT Statement of Work Author. Technical report, 2017

Cowley D. J., Faber S. M., Hilyard D. F., James E., Osborne J., DEIMOS: a wide-field faint-object spectrograph. In Proc. SPIE 2871, Optical Telescopes of Today and Tomorrow, vol. 2871, 1997, pp 1107-1115 
Crampton D., Fletcher J. M., Jean I., Murowinski R. G., Szeto K., Dickson C. G., Hook I., Laidlaw K., Purkins T., Allington-Smith J. R., Davies R. L., Gemini multi-object spectrograph GMOS: integration and tests, Optical and IR Telescope Instrumentation and Detectors, 2000, vol. 4008, p. 114

Davies F. B., Furlanetto S. R., Large fluctuations in the hydrogen-ionizing background and mean free path following the epoch of reionization, Monthly Notices of the Royal Astronomical Society, 2016, vol. 460, p. 1328

Davies R. L., Allington-Smith J. R., Bettess P., Chadwick E., Content R., Dodsworth G. N., Haynes R., Lee D., Lewis I. J., Webster J., Ettedgui-Atad E., Beard S. M., Ellis M. A., Hastings P. R., Williams P. R., Bond T., Crampton D., Davidge T. J., Fletcher J. M., Leckie B., Morbey C. L., GMOS: the GEMINI Multiple Object Spectrographs. In Proc. SPIE 2871, Optical Telescopes of Today and Tomorrow, vol. 2871, 1997, pp $1099-1106$

Day L., McNeil I., eds, Biographical Dictionary of the History of Technology. Taylor \& Francis, 2003, 864 p.

Day M., 2010. Extracting Euler Angles from a Rotation Matrix. Available at https: //pdfs. semanticscholar.org/6681/37fa4b875d890f446e689eea1e334bcf6bf6.pdf. Accessed on 2020-03-25.

DePoy D., Allen R., Barkhouser R., Boster E., Carona D., Harding A., Hammond R., Marshall J. L., Orndorff J. D., Papovich C., Prochaska T., Prochaska K., Rheault J. P., Smee S. A., Shectman S., Villanueva S., GMACS Conceptual Design Report. TAMU Technical report, 2011

DePoy D. L., Allen R., Barkhouser R., Boster E., Carona D., Harding A., Hammond R., Marshall J. L., Orndorff J., Papovich C., Prochaska K., Prochaska T., Rheault J. P., Smee S., Shectman S., Villanueva S., GMACS: a wide field, multi-object, moderateresolution, optical spectrograph for the Giant Magellan Telescope. In Proc. SPIE 8446, Ground-based and Airborne Instrumentation for Astronomy IV, vol. 8446, 2012, p. $84461 \mathrm{~N}$ 
DePoy D. L., Allen R., Li T., Marshall J. L., Papovich C., Prochaska T., Shectman S., An update on the wide field, multi-object, moderate-resolution, spectrograph for the Giant Magellan Telescope. In Proc. SPIE 9147, Ground-based and Airborne Instrumentation for Astronomy V, vol. 9147, 2014, p. 914720

DePoy D. L., Marshall J. L., Schmidt L. M., GMACS Conceptual Design Proposal. Technical report, 2015

DePoy D. L., Schmidt L. M., Ribeiro R. A. S., Taylor K., Jones D., Froning C. S., Bortoletto D., Cook E., Faes D. M., Ji T.-G., Lee H.-I., Marshall J. L., Mendes de Oliveira C. L., Pak S., Papovich C., Prochaska T., Souza A., GMACS: a wide-field, moderate-resolution spectrograph for the Giant Magellan Telescope. In Proc. SPIE 10702, Ground-based and Airborne Instrumentation for Astronomy VII, vol. 10702, SPIE, 2018

Dressler A., Bigelow B., Hare T., Sutin B., Thompson I., Burley G., Epps H., Oemler A., Bagish A., Birk C., Clardy K., Gunnels S., Kelson D., Shectman S., Osip D., IMACS: The Inamori-Magellan Areal Camera and Spectrograph on Magellan-Baade, Publications of the Astronomical Society of the Pacific, 2011, vol. 123, p. 288

Dressler A., Hare T., Bigelow B. C., Osip D. J., IMACS: the wide-field imaging spectrograph on Magellan-Baade. In Proc. SPIE 6269, Ground-based and Airborne Instrumentation for Astronomy, vol. 6269, 2006

Edmunds Optics. Depth of Field and Depth of Focus, 2018. Available at https://www. edmundoptics.com/resources/application-notes/imaging/ depth-of-field-and-depth-of-focus. Accessed on 2018-07-23.

Eggert D. W., Lorusso A., Fisher R. B., Estimating 3-D rigid body transformations: A comparison of four major algorithms, Machine Vision and Applications, 1997, vol. 9, p. 272

Epps H. W., Sutin B. M., Optically-athermalized construction optical design for the IMACS short camera. In Proc. SPIE 4841, Instrument Design and Performance for Optical/Infrared Ground-based Telescopes, vol. 4841, 2003, p. 612

ESO. The Extremely Large Telescope, 2019. Available at https://www.eso.org/public/ teles-instr/elt/. Accessed on 2019-08-27. 
ESO. FORS Instrument Description, 2020. Available at http://www.eso.org/sci/ facilities/paranal/instruments/fors/inst.html. Accessed on 2020-05-16.

ESO. VIMOS - VIsible MultiObject Spectrograph, 2020. Available at https://www. eso . org/sci/facilities/paranal/decommissioned/vimos.html. Accessed on 2020-05-17.

Eversberg T., Vollmann K., Spectroscopic Instrumentation - Fundamentals and Guidelines for Astronomers 1 edn. Springer-Verlag Berlin Heidelberg Berlin, 2015, 677 p.

Faber S. M., Phillips A. C., Kibrick R. I., Alcott B., Allen S. L., Burrous J., Cantrall T., Clarke D., Coil A. L., Cowley D. J., Davis M., Deich W. T. S., Dietsch K., Gilmore D. K., Harper C. A., Hilyard D. F., Lewis J. P., McVeigh M., Newman J., Osborne J., Schiavon R., Stover R. J., Tucker D., The DEIMOS spectrograph for the Keck II Telescope: integration and testing. In Proc. SPIE 4841, Instrument Design and Performance for Optical/Infrared Ground-based Telescopes, No. March 2003, 2003, pp 1657-1669

Fabricant D., Fata R., Epps H., Gauron T., Mueller M., Zajac J., Amato S., Barberis J., Bergner H., Brennan P., Brown W., Chilingarian I., Geary J., Kradinov V., McLeod B., Smith M., Woods D., Binospec: A Wide-field Imaging Spectrograph for the MMT, Publications of the Astronomical Society of the Pacific, 2019, vol. 131, p. 075004

Fabricant D. G., Fata R. G., Epps H. W., Binospec: a dual-beam wide-field optical spectrograph for the converted MMT. In Optical Astronomical Instrumentation, vol. 3355, 1998, pp 232-241

Faes D. M., Souza A., Froning C., Schmidt L. M., Cook E., DePoy D. L., Ji T.-G., Jones D., Marshall J. L., Lee H.-I., Oliveira C. M., Pak S., Papovich C., Prochaska T., Ribeiro R. A. S., Taylor K., Bortoletto D. R., Systems engineering applied to ELT instrumentation: the GMACS case. In Proc. SPIE 10705, Modeling, Systems Engineering, and Project Management for Astronomy VIII, vol. 10705, SPIE, 2018, p. 46

Fèvre O. L., Hill L., Mignant D. L., Maccagn D., OPTIMOS-DIORAMAS - A Wide-field Imaging and Multi-slit Spectrograph for the E-ELT, The Messenger, 2010, vol. 140, p. 34

Fischer R. E., Tadic-Galeb B., Yoder P. R., Optical System Design second edn. McGrawHill Education New York, 2008, 806 p. 
Fort B., Mellier Y., Picat J. P., Rio Y., Lelievre G., Multiaperture Spectroscopy With Rapid Mask Fabrication And Installation, Proc. SPIE 0627, Instrumentation in Astronomy VI, 1986, vol. 0627, p. 321

Fransen S., Doyle D., Catanzaro B., Opto-mechanical modeling of the Herschel Space Telescope at ESA/ESTEC, Integrated Modeling of Complex Optomechanical Systems, 2011, vol. 8336, p. 833604

Freeman K., Bland-Hawthorn J., The New Galaxy: Signatures of Its Formation, Annual Review of Astronomy and Astrophysics, 2002, vol. 40, p. 487

Gaylord T. K., Moharam M. G., Analysis and Applications of Optical Diffraction by Gratings, Proceedings of the IEEE, 1985, vol. 73, p. 894

Geary J. M., Introduction to Lens Design: With Practical ZEMAX Examples. WillmannBell, Inc, 2002, 462 p.

GMOS, 2020. Available at https://www.gemini.edu/instrumentation/ current-instruments/gmos. Accessed on 2020-02-14.

Genberg V. L., Michels G. J., Ave W., Integrated Optomechanical STOP Analysis linking Ansys to Zemax, CODEV, and OSLO with SigFit. Technical Report December, 2017

Gil M. A., Simon J. M., Diffraction grating and optical aberrations: a new and exact formulation, Applied Optics, 1985, vol. 24, p. 2956

GMACS Team , GMACS-PM-01012-Kick-off Meeting Slides - Reflective Collimator. Technical report, $2018 \mathrm{a}$

GMACS Team GMACS-TEC-02003-Dichroic Transition Wavelength. Technical report, $2018 b$

GMACS Team , GMACS-PM-01007-Conceptual Design Report. Technical report, 2019

GMT Brazil Office. GMT Brazil Office Website, 2016. Available at http://www.gmt.iag . usp.br/. Accessed on 2020-03-19.

GMT Science Book Giant Magellan Telescope Science Book 2018, 2018, 208 p. 
GMTO Coorporation Chapter 4: Science Requirements in , GMT-ID-01465. GMTO Coorporation, 2013a Chapt. 4 p. 31

GMTO Coorporation Section 2: High Level Science Goals, Key Science Requirements, Operational Concept in , GMT System Level Preliminary Design Review, 2013b

GMTO Coorporation Section 6: Telescope in , GMT System Level Preliminary Design Review, 2013c p. 478

GMTO Coorporation Section 9: Instrumentation in , GMT System Level Preliminary Design Review, 2013d p. 160

GMTO Coorporation Section 9: Instrumentation in , GMT System Level Preliminary Design Review, 2013e p. 160

GMTO Coorporation GMT-REF-00362, Rev. C - GMT Project Acronyms and Glossary. Technical report, 2016

GMTO Coorporation. Giant Magellan Telescope, 2019. Available at https://www.gmto . org/. Accessed on 2019-08-27.

Gracey R., Bartoszyk A., Cofie E., Comber B., Hartig G., Howard J., Sabatke D., Wenzel G., Ohl R., Structural, thermal, and optical performance (STOP) modeling and results for the James Webb Space Telescope integrated science instrument module, Modeling, Systems Engineering, and Project Management for Astronomy VI, 2016, vol. 9911, p. 99111A

Greivenkamp J. E., Field Guide to Geometrical Optics. Field Guide Series, Society of Photo Optical, 2004

Gruescu C., Nicoara I., Popov D., Bodea R., Hora H., Optical glass compatibility for the design of apochromatic systems, Science of Sintering, 2008, vol. 40, p. 131

GTC. OSIRIS, 2020. Available at http://www.gtc.iac.es/instruments/osiris/ osiris.php. Accessed on 2020-05-20.

Hammer F., Morris S., Kaper L., Barbuy B., Cuby J. G., Roth M., Jagourel P., Evans C. J., Puech M., Fitzsimons E., Dalton G., Rodrigues M., The E-ELT multi-object 
spectrograph: latest news from MOSAIC. In Proc. SPIE 9908, Astronomical Telescopes \& Instrumentation VI, vol. 9908, 2016, p. 990824

Hariharan P., Apochromatic a novel design lens combinations : approach, Optics \& Laser Technology, 1997, vol. 29, p. 217

Hartmann P., Jedamzik R., Large Optical Glass Lenses for ELTs. In Proc. SPIE 6273, Optomechanical Technologies for Astronomy, vol. 6273, 2006, p. 11

Hearnshaw J., Astronomical Spectrographs and Their History. Cambridge University Press, 2009, 228 p.

Heijmans J., Müller M., Holzlöhner R., Combined Opto-mechanical analysis for modern optical instruments, 2018, p. 89

Herschel W., XIII. Investigation of the powers of the prismatic colours to heat and illuminate objects; with remarks, that prove the different refrangibility of radiant heat. To which is added, an inquiry into the method of viewing the sun advantageously, with telesco, Philosophical Transactions of the Royal Society, 1800, vol. 90

Hill J. M., Angel J. R. P., Scott J. S., Lindley D., Hintzen P., Multiple Object Fiber Optic Spectroscopy. In Proc. SPIE 0331, Instrumentation in Astronomy IV, 1982, pp 279-288

Hollas J. M., Modern Spectroscopy 4th edn. Wiley, 2004, 480 p.

Holst G. C., Electro-Optical Imaging System Performance fifth edn. vol. PM187, CD Publishing and SPIE Press, 2008, 538 p.

Hook I. M., Jørgensen I., Allington-Smith J. R., Davies R. L., Metcalfe N., Murowinski R. G., Crampton D., The Gemini-North Multi-Object Spectrograph: Performance in Imaging, Long-Slit, and Multi-Object Spectroscopic Modes, Publications of the Astronomical Society of the Pacific, 2004, vol. 116, p. 425

Huang Y.-H., Chen H.-W., Johnson S. D., Weiner B. J., Characterizing the chemically enriched circumgalactic medium of $\sim 38000$ luminous red galaxies in SDSS DR12, Monthly Notices of the Royal Astronomical Society, 2016, vol. 455, p. 1713

Hughes S., Catchers of the Light: The Forgotten Lives of the Men and Women Who First Photographed the Heavens. vol. 1, ArtDeCiel, 2012, 1612 p. 
Hutley M. C., Diffraction Gratings 1st edn. Academic Press, 1982, 330 p.

Jacoby G., GMT-SOW-01091, Rev. A - Statement of Work: GMACS Conceptual Design Study. GMTO Technical report, 2016

Jacoby G., Bernstein R., Bouchez A., Colless M., DePoy D., Espeland B., Jaffe D., Lawrence J., Marshall J., McGregor P., Sharp R., Szentgyorgyi A., Walls B., Crane J., Uomoto A., Shectman S., Hare T., Instrumentation progress at the Giant Magellan Telescope project. In Proc. SPIE 9908, Astronomical Telescopes \& Instrumentation, vol. 9908, 2016

Jacquinot P., The Luminosity of Spectrometers with Prisms, Gratings, or Fabry-Perot Etalons, Journal of the Optical Society of America, 1954, vol. 44, p. 761

James J. F., Spectrograph Design Fundamentals. Cambridge University Press Cambridge, 2007, 204 p.

James J. F., Sternberg R. S., The design of optical spectrometers 1 edn. Springer US, 1969, 239 p.

Jan F., Schade W., U.S. Patent 2,500,406: Petzval-type photographic objective, 1950

Jedamzik R., Hartmann P., Large Optical Glass Blanks for Astronomy. In Proc. SPIE 5494, Optical Fabrication, Metrology, and Material Advancements for Telescopes, vol. 5494, 2004, pp 382-392

Jedamzik R., Hengst J., Elsmann F., Lemke C., Döhring T., Hartmann P., Optical materials for astronomy from SCHOTT: the quality of large components. In Proc. SPIE 7018, Advanced Optical and Mechanical Technologies in Telescopes and Instrumentation, vol. 7018, 2008

Jedamzik R., Petzold U., Dietrich V., Wittmer V., Rexius O., Large optical glass blanks for the ELT generation. In Proc. SPIE 9912 Advances in Optical and Mechanical Technologies for Telescopes and Instrumentation II, vol. 9912, 2016, p. 99123E

Johns M., GMT-DOC-00002 - Rev2 - Systems Engineering Management Plan, 2001, p. 29

Johns M., The Giant Magellan Telescope (GMT), Proc. SPIE 6267, Ground-based and Airborne Telescopes, 2006, vol. 6267, p. 626729 
Johns M., The Giant Magellan Telescope (GMT), Proc. SPIE 6986, Extremely Large Telescopes: Which Wavelengths?, 2008, vol. 698603, p. 626729

Johns M., Angel J. R. P., Shectman S., Bernstein R., Fabricant D. G., McCarthy P., Phillips M., Status of the Giant Magellan Telescope (GMT) project. In Proc. SPIE 5489, Ground-based Telescopes, vol. 5489, International Society for Optics and Photonics, 2004, p. 441

Johns M., Rakich A., GMT-DOC-00010-G: Optical Design. GMTO Technical report, 2018 Johns M., Rakich A., GMT-DOC-00010-H: Optical Design. Technical report, 2019

Jones D., A Glass selection method for apochromatism: introducing the differential Abbè Number. Prime Optics Technical report, 2014

Jones D., GMACS Panchromatism Discussion. Prime Optics Technical report, 2016

Jones D., GMACS Coordinate System Alignment for Flexure Modelling. Prime Optics Technical report, 2018

Kashikawa N., Aoki K., Asai R., Ebizuka N., Inata M., Iye M., Kawabata K. S., Kosugi G., Ohyama Y., Okita K., Ozawa T., Saito Y., Sasaki T., Sekiguchi K., Shimizu Y., Taguchi H., Takata T., Yadoumaru Y., Yoshida M., FOCAS: The faint object camera and spectrograph for the Subaru telescope, Publications of the Astronomical Society of Japan, 2002, vol. 54, p. 819

Kashikawa N., Inata M., Iye M., Kawabata K. S., Okita K., Kosugi G., Ohyama Y., Sasaki T., Sekiguchi K., Takata T., Shimizu Y., Yoshida M., Aoki K., Saito Y., Asai R., Taguchi H., Ebizuka N., Ozawa T., Yadoumaru Y., FOCAS: faint object camera and spectrograph for the Subaru Telescope, Proc. SPIE 4008, Optical and IR Telescope Instrumentation and Detectors, 2000, vol. 4008, p. 104

Kawabata K. S., Ebizuka N., Sasaki T., Sekiguchi K., Iye M., Aoki K., Asai R., Inata M., Kashikawa N., Kosugi G., Ohyama Y., Okita K., Ozawa T., Saito Y., Shimizu Y., Taguchi H., Takata T., Yadoumaru Y., Yoshida M., Properties of FOCAS optical components. In Proc. SPIE 4841, Instrument Design and Performance for Optical/Infrared Ground-based Telescopes, vol. 4841, 2003, p. 1219 
Keck Observatory. DEIMOS Home Page, 2020. Available at https://www2.keck. hawaii. edu/inst/deimos/. Accessed on 2020-05-21.

Keller C. U., Navarro R., Brandl B. R., Field guide to astronomical instrumentation. vol. FG32, SPIE, 2015, 145 p.

Kogelnik H., Coupled Wave Theory for Thick Hologram Gratings, The Bell System Technical Journal, 1969, vol. 48, p. 2909

Konidaris N., 2014. Classical and $\beta$ Anamorphism in Spectrographs. Available at https: //nickkonidaris.com/2014/10/17/anamorphic-factor. Accessed on 2020-01-18.

Kupke R., Ji H., Nadar S. P., The wide field optical spectrograph (WFOS) for TMT: fiber-WFOS optical design. In Proc. SPIE 10702, Ground-based and Airborne Instrumentation for Astronomy VII2, 2018

Laikin M. M., Lens Design fourth edn. CRC Press, 2006, 487 p.

Landau S., Tutorial : Overview of the Optical and Optomechanical Design Process I . Introduction, 2007, pp 1-8

LCO. IMACS Spectrograph Manuals, 2020. Available at http://www.lco.cl/ telescopes-information/magellan/instruments/imacs/. Accessed on 2020-05-21.

LeFevre O., Saisse M., Mancini D., Brau-Nogue S., Caputi O., Castinel L., D’Odorico S., Garilli B., Kissler-Patig M., Lucuix C., Mancini G., Pauget A., Sciarretta G., Scodeggio M., Tresse L., Vettolani G., Commissioning and performances of the VLT-VIMOS, Instrument Design and Performance for Optical/Infrared Ground-based Telescopes, 2003a, vol. 4841 , p. 1670

LeFevre O., Saisse M., Mancini D., Brau-Nogue S., Caputi O., Castinel L., D’Odorico S., Garilli B., Kissler-Patig M., Lucuix C., Mancini G., Pauget A., Sciarretta G., Scodeggio M., Tresse L., Vettolani G., Commissioning and performances of the VLT-VIMOS. In Proc. SPIE 4841, Instrument Design and Performance for Optical/Infrared Groundbased Telescopes, vol. 4841, 2003b, p. 1670

LeFevre O., Vettolani G., Maccagni D., Mancini D., Picat J. P., Mellier Y., Mazure A., Saisse M., Cuby J. G., Delabre B., Garilli B., Hill L., Prieto E., Arnold L., Conconi 
P., Cascone E., Mattaini E., Voet C., VIRMOS: visible and infrared multiobject spectrographs for the VLT, Proc. SPIE 3355, Optical Astronomical Instrumentation, 1998, vol. 3355 , p. 8

McCarthy P. J., Fanson J., Bernstein R., Ashby D., Bigelow B., Boyadjian N., Bouchez A., Chauvin E., Donoso E., Filgueira J., Goodrich R., Groark F., Jacoby G., Pearce E., Overview and status of the Giant Magellan Telescope Project, Proc. SPIE 9906, Ground-based and Airborne Telescopes VI, 2016, vol. 9906, p. 990612

McCarthy P. J., Fanson J., Bernstein R., Ashby D., Bigelow B., Boyadjian N., Bouchez A., Chauvin E., Donoso E., Filgueira J., Goodrich R., Groark F., Jacoby G., Pearce E., Overview and status of the Giant Magellan Telescope Project. In Proc. SPIE 10700, Ground-based and Airborne Telescopes VII, 2018, p. 990612

Malacara-Hernández D., Handbook of Optical Design 2nd edn. CRC Press, 2004, 522 p.

Moharam M. G., Gaylord T. K., Rigorous coupled-wave analysis of plana-grating diffraction, Journal of Optical Society of America, 1981, vol. 71

Morris S. L., Hammer F., Jagourel P., Myers R., Le Fèvre O., Finogenov A., Kotilainen J., Castilho B., Ostlin G., Afonso J., Dubbledam M., Close M., Parr-Burman P., The ELTMOS (MOSAIC): towards the construction phase. In Proc. SPIE 10702, Ground-based and Airborne Instrumentation for Astronomy VII, No. July, SPIE, 2018, p. 68

Murowinski R. G., Allington-Smith J. R., Crampton D., Davies R. L., Fletcher J. M., Henry D. M., Hook I., Jorgensen I., Juneau S., Morbey C. L., Stilburn J. R., Szeto K., Gemini-north multiobject spectrograph optical performance, Instrument Design and Performance for Optical/Infrared Ground-based Telescopes, 2003, vol. 4841, p. 1440

Murowinski R. G., Bond T., Crampton D., Davidge T. J., Fletcher J. M., Leckie B., Morbey C. L., Roberts S. C., Saddlemyer L. K., Sebesta J., Stilburn J. R., Szeto K., Allington-Smith J. R., Gemini multiobject spectrographs. In Optical Astronomical Instrumentation, vol. 3355, 1998, pp 188-195

National Optical Astronomy Observatories. Volume Phase Holographic (VPH) Gratings, 1999. Available at https://www.noao.edu/nstc/vpgratings/. Accessed on 2020-0113. 
Newton I., Mr. Isaac Newtons answer to some considerations upon his doctrine of light and colors; which doctrine was printed in Numb. 80 of these tracts, Philosophical Transactions of the Royal Society, 1672, vol. 7

Nicklas H., The Focal Reducing Imager and Spectrograph FORS, built for the optical 16-metre 'Very Large Telescope' of the European Southern Observatory, 2005, Ph.D. Thesis, 141

Oswalt T. D., McLean I. S., eds, Planets, Stars and Stellar Systems - Telescopes and Instrumentation. Springer Netherlands Dordrecht, 2013, 643 p.

Palmer C., Loewen E., Diffraction Grating Handbook 7th edn. Newport Corporation, 2014, $265 \mathrm{p}$.

Palmer J. M., Grant B. G., The Art of Radiometry. Press Monograph (Book 184), SPIE Publications, 2009, 386 p.

PMBOK Um Guia do Conhecimento em Gerenciamento de Projetos (Guia PMBOK) sixth edn. Project Management Institute, 2017, 756 p.

Podgorski W., Bean J., Bergner H., Chun M.-Y., Crane J., Evans I., Evans J., Furesz G., Guzman D., Kim K.-M., McCracken K., Mueller M., Norton T., Park C., Park S., Plummer D., Szentgyorgyi A., Uomoto A., Yuk I.-S., A novel systems engineering approach to the design of a precision radial velocity spectrograph: the GMT-Consortium Large Earth Finder (G-CLEF), Proc. SPIE 9147, Ground-based and Airborne Instrumentation for Astronomy V, 2014, vol. 9147, p. 91478W

Prochaska T., Sauseda M., Beck J., Schmidt L. M., Cook E., DePoy D. L., Marshall J. L., Ribeiro R. A. S., Taylor K., Jones D., Froning C. S., Pak S., de Oliveira C. L. M., Papovich C., Ji T.-G., Lee H.-I., Optomechanical design concept for the Giant Magellan Telescope Multi-object Astronomical and Cosmological Spectrograph (GMACS). In Proc. SPIE 9908, Astronomical Telescopes \& Instrumentation, 2016

Rayleigh F., XXXI. Investigations in optics, with special reference to the spectroscope, The London, Edinburgh, and Dublin Philosophical Magazine and Journal of Science, 1879, vol. 8, p. 261 
Rayner J., Spectrograph Optical Design. Institute for Astronomy Technical report, 2010

Ribeiro R. A. S., Jones D., Schmidt L. M., Taylor K., Souza A., Papovich C., Mendes de Oliveira C. L., Froning C. S., Faes D. M., DePoy D. L., Cook E., Lee H.-i., Marshall J. L., Ji T.-g., Prochaska T., Pak S., Optical design for the Giant Magellan Telescope Multi-object Astronomical and Cosmological Spectrograph (GMACS): design methodology, issues, and trade-offs. In Proc. SPIE 10690, Optical Design and Engineering VII, No. June, SPIE, 2018a

Ribeiro R. A. S., Jones D., Schmidt L. M., Taylor K., Souza A., Papovich C., Mendes de Oliveira C. L., Froning C. S., Faes D. M., DePoy D. L., Cook E., Lee H.-i., Marshall J. L., Ji T.-g., Prochaska T., Pak S., The optical design for the Giant Magellan Telescope Multi-object Astronomical and Cosmological Spectrograph (GMACS). In Proc. SPIE 10702, Ground-based and Airborne Instrumentation for Astronomy VII, SPIE, 2018b

Riedl M. J., Optical design fundamentals for infrared systems 2nd edn. SPIE Publications, 2001, 182 p.

Rueger M., "Eyes"for a Telescope, Schott INFO, 2002, pp 18-20

Sacek V., 2006. Amateur Telescope Optics. Available at https://www. telescope-optics.net. Accessed on 2020-01-17.

Sandback I. C., U.S. Patent 2,989,895-1961: Optical Objective, 1961

Saunders W., Very fast transmissive spectrograph designs for highly multiplexed fiber spectroscopy. In Proc. SPIE 9908, Ground-based and Airborne Instrumentation for Astronomy VI, vol. 990896, 2016

Schmidt L. M., Ribeiro R. A. S., Taylor K., Jones D., Prochaska T., DePoy D. L., Marshall J. L., Cook E., Froning C., Ji T.-g., Lee H.-i., Optical design concept for the Giant Magellan Telescope Multi-object Astronomical and Cosmological Spectrograph (GMACS). In Proc. SPIE 9908, Ground-based and Airborne Instrumentation for Astronomy VI, 2016

Schott TIE-35: Transmittance of optical glass. Schott Technical report, 2005

Schott TIE-41: Large Optical Glass Blanks. Schott Technical report, 2006 
Schott Optical Glass - Data Sheets. Technical report, 2014

Schroeder D. J., Astronomical Optics. Academic Press, 1987, 352 p.

Schweizer F., Anamorphic Magnification of Grating Spectrographs - A Reminder, Publications of the Astronomical Society of the Pacific, 1979, vol. 91, p. 149

Seifert W., Mitsch W., Nicklas H. E., Rupprecht G., FORS: a workhorse instrument for the ESO VLT. In Instrumentation in Astronomy VIII, vol. 2198, 1994, pp 213-218

Shannon R. R., The Art and Science of Optical Design. Cambridge University Press, 1997, 608 p.

Shectman S. A., Instrumentation for the Giant Magellan Telescope. In Second Backaskog Workshop on Extremely Large Telescopes, 2004

Siew R. H., f /No. and the radiometry of image forming optical systems with non-circular aperture stops, Optical Modeling and Performance Predictions II, 2005, vol. 5867, p. 586701

Simms W., On the Optical Glass prepared by the late Dr. Ritchie, Memoirs of the Royal Astronomical Society, 1839, vol. 11, p. 165

Slabaugh G. G., 2000. Computing Euler angles from a rotation matrix. Available at https: //www.gregslabaugh.net/publications/euler.pdf. Accessed on 2020-03-25.

Smee S. A., Gunn J. E., Uomoto A., Roe N., Schlegel D., Rockosi C. M., Carr M. A., The multi-object, fiber-fed spectrographs for the Sloan Digital Sky Survey and the Baryon Oscillation Spectroscopic Survey, Astronomical Journal, 2013, vol. 146

Smith W. J., U.S. Patent 3,255,664-1966: Objective of the Petzval Type with field flattner and three or more positive elements, 1962

Smith W. J., Moderns Lens Design: a Resource Manual 1st edn. McGraw-Hill Professional, 1992, $471 \mathrm{p}$.

Smith W. J., Modern Optical Engineering 4th edn. McGraw-Hill Education, 2007, 764 p. 
Spanò P., Zerbi F. M., Norrie C. J., Cunningham C. R., Strassmeier K. G., Bianco A., Blanche P. A., Bougoin M., Ghigo M., Hartmann P., Zago L., Atad-Ettedgui E., Delabre B., Dekker H., Melozzi M., Snÿders B., Takke R., Walker D. D., Challenges in optics for Extremely Large Telescope instrumentation, Astronomische Nachrichten, 2006, vol. 327, p. 649

Steiner J. E., 2014. Explorando o universo: da formação de galáxias aos planetas tipoterra, com o Telescópio Gigante Magellan. Available at http://www.bv.fapesp.br/ pt/auxilios/87808. Accessed on 2016-04-12.

Subaru Telescope. FOCAS Webpage, 2019. Available at https://www.naoj.org/ Observing/Instruments/FOCAS/index.html. Accessed on 2020-06-01.

Sutin B., 2003. IMACS Optical Specifications. Available at https://users.obs. carnegiescience.edu/sutin/imacs/index.html. Accessed on 2020-05-11.

Szeto K., Morbey C. L., Mayer C. J., Crampton D., Fletcher J. M., Murowinski R. G., Stilburn J. R., Taylor P., Wooff R., Design and implementation of an atmospheric dispersion compensator/corrector for the Gemini multi-object spectograph, Instrument Design and Performance for Optical/Infrared Ground-based Telescopes, 2003, vol. 4841, p. 1326

TMT International Observatory. TMT International Observatory, 2019. Available at https: //www. tmt.org/. Accessed on 2019-08-27.

TMT International Observatory. Wide Field Optical Spectroscopy (WFOS), 2020. Available at https://www.tmt.org/page/wfos. Accessed on 2020-03-09.

Tubbs E. F., Goss W. C., Cohen J. G., Multiple Object Fiber Optics Spectrograph Feed For The Hale Telescope, Proc. SPIE 0331, Instrumentation in Astronomy IV, 1982

USP Alumni. USP faz parceria com Google para uso do G Suite for Education - Alumni, 2017. Available at http://www.alumni.usp.br/ usp-faz-parceria-com-google-para-uso-g-suite-education. Accessed on 2020$02-10$.

van Berkel K., van Helden A., Palm L. C., A History of Science in the Netherlands. Brill, 1998 
Wall J. V., ed. Optics in Astronomy. Cambridge University Press, 1993, 300 p.

Watson F. G., Multi-Object Spectroscopy with Optical Fibres, Journal of the British Astronomical Association, 1983, vol. 95, p. 193

Willers C., Electro-Optical System Design 1st edn, 2009, 255 p.

Young T., II. The Bakerian Lecture. On the theory of light and colours, Philosophical Transactions of the Royal Society, 1802, vol. 92

Zemax Development Corporation, Optical Design Program User's Manual, 2011, 789 p.

Zemax LLC OpticStudio , OpticStudio 16.5 SP5 Help Files, 2016, 2435 p.

Zhang H., Zaritsky D., Zhu G., Ménard B., Hogg D. W., Hydrogen emission from the ionized gaseous halos of low-redshift galaxies, The Astrophysical Journal, 2016, vol. 833

Zhou Y., Herczeg G. J., Kraus A. L., Metchev S., Cruz K. L., Accretion onto planetary mass companions of low-mass young stars, The Astrophysical Journal, 2014, vol. 783 
Appendix 

Appendix A

\section{GMT Optical Design}

This chapter presents a brief introduction to the next generation of the ELTs and the GMT's characteristics, with an emphasis on those relevant to the development of the GMACS optical conceptual design.

\section{A.1 Introduction to the Extreme Large Telescopes, ELTs}

The definition of an Extreme Large Telescope (ELT) is an astronomical observatory featuring an optical telescope with an aperture of more than 20 meters in diameter (Spanò et al., 2006). Technological advancement of manufacturing processes and processes, polishing and metrology of large off-axis mirror segments, metrology systems for alignment, mirrors phasing and management of deformations, telescope enclosure, sophisticated applications of computational modeling of fluid dynamics and wind tunnel testing to model the structure's aerodynamics, control, significant advances in multi-conjugate adaptive optics, which is one of the most important technique that allows ELTs for ground-based observation, among others are some of the challenges that the new generation of ground-based optical ELTs will have for their development and construction.

The construction of ELT observatories, which include not only the telescope, enclosure and control but also the instrumentation, is backed by lessons learned from the execution of the projects of the currently largest telescopes in the world: Gran Telescope of the Canaries (GTC), combined Large Binocular Telescope (LBT), Keck 1 and 2, Southern African Large Telescope (SALT), Hobby-Eberly Telescope (HET), Subaru, VLT, Gemini, among others.

Three parallel ELT projects are currently underway: the Giant Magellan Telescope 
(GMT) the Third Meter Telescope (TMT) and the European Extreme Large Telescopes (E-ELT), among which the GMT is in the most advanced stage of construction. Table A.1 presents some of the technical optical specifications of these ELTs.

\begin{tabular}{cccc}
\hline Attribute & GMT & TMT & E-ELT \\
\hline Aperture $[m]$ & 24.5 & 30.0 & 39.3 \\
Collecting Area $\left[\mathrm{m}^{2}\right]$ & 368 & 655 & 978 \\
Final f-number & $\mathrm{f} / 8.2$ & $\mathrm{f} / 15.0$ & $\mathrm{f} / 17.5$ \\
EFL $[\mathrm{m}]$ & 208 & 450 & 682 \\
Number of reflections & 2 & 3 & 5 \\
Focal Plane Scale [mm/arcsec] & 1.0 & 2.2 & 3.6 \\
Field of View [arcmin] & 10 & 10 & 7 \\
Image size of 10' FoV $[\mathrm{m}]$ & 0.6 & $(15 \mathrm{w} / \mathrm{corr})$. & $(10 \mathrm{w} / \mathrm{corr})$. \\
\hline
\end{tabular}

Table A.1 - Technical specifications of the new generation of ELTs. Source: GMTO Coorporation (2019); TMT International Observatory (2019); ESO (2019).

The next section presents the optical and optomechanical GMT characteristics with an emphasis on those relevant to the development of the GMACS optical conceptual design

\section{A.2 The Giant Magellan Telescope, GMT}

According to Bernstein e Fabricant (2018, p. 2), "the GMT partnership formed in 2003 as the members of the 6.5 meter Magellan Telescope consortium and interested scientists from other institutions began developing concepts for a next generation optical and infrared telescope. The conceptual telescope design, released in 2006, capitalized on the technologies and design strategies that had been demonstrated with the exceptional performance of the Magellan Telescopes. The GMTO Corporation (GMTO) is an independent nonprofit organization formed by an international consortium of universities and research institutions to manage the development, construction, and operation of the GMT. As of 2018, the partnership includes Arizona State University, Astronomy Australia Ltd., the Australian National University, the Carnegie Institution for Science, the São Paulo Research Foundation (FAPESP), Harvard University, Korea Astronomy and Space Sci- 
ence Institute, Smithsonian Institution, Texas AM University, The University of Texas at Austin, University of Arizona, and University of Chicago".

Figure A.1 shows the locations of the partners above mentioned.

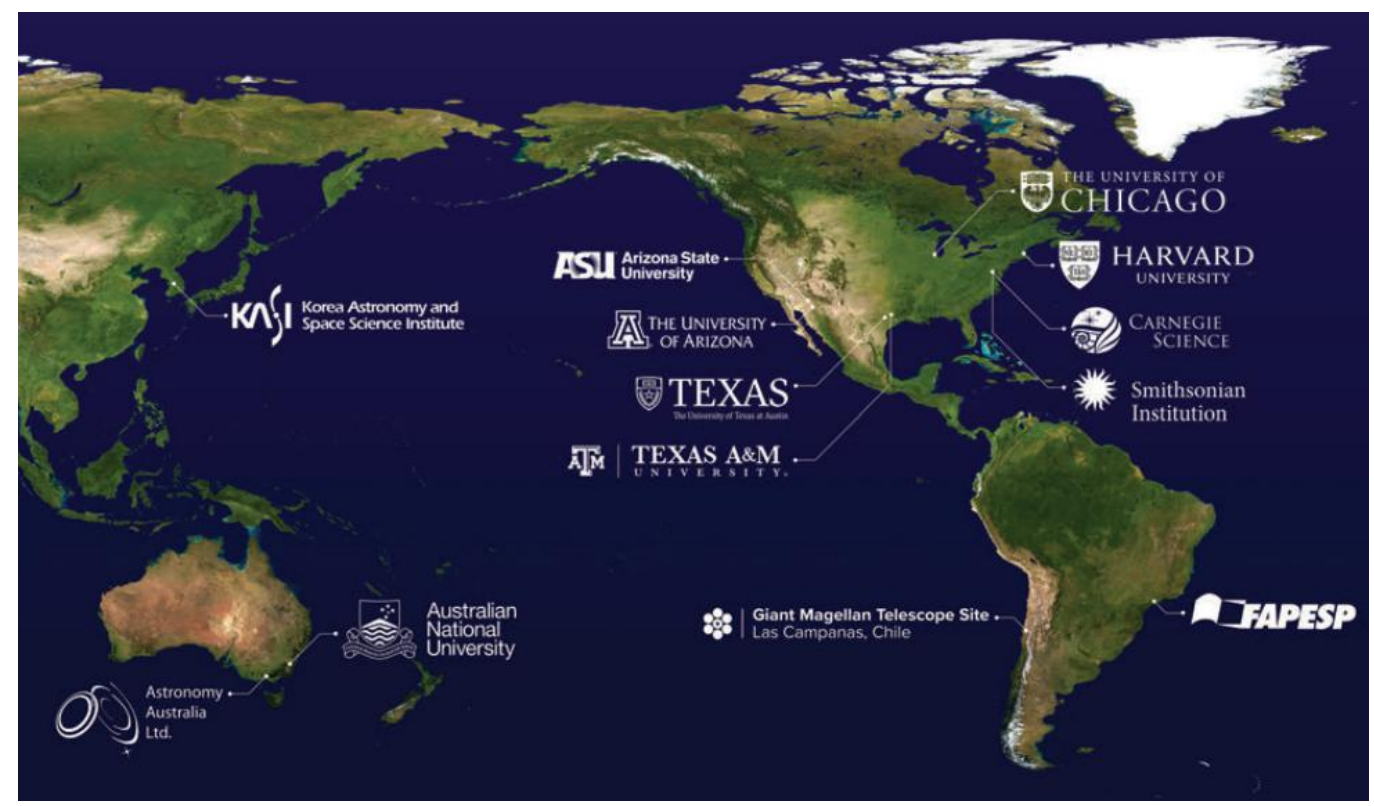

Figure A.1: The founding members of the GMT partnership are an international consortium of universities and research institutions. Source: Bernstein and Fabricant (2018, p.2).

Brazil is represented in the GMT consortion by the São Paulo Research Foundation, FAPESP, with $5 \%$ participation (see section 1.5 ).

\section{A.2.1 GMT Science Goals}

The GMT science case is structured in three parts: discoveries opened by the peculiarities of the GMT optical design, the contemporary science goals with the proposed instrumentation, and the scientific synergies with existing and planned facilities. The combination of these science cases drives the requirements for the facility, scientific instruments, and operations (GMTO Coorporation, 2013b).

Although the science case for the GMT does not diverge substantially from those for other ELTs and has substantial overlap with the science goals for JWST, ALMA, and other general-purpose observatories, the GMT has unique strengths among the ELTs. In essence, its fast focal ratio and Gregorian optical prescription lend to large fields of view in seeing-limited applications, while the reduced number of reflections improves overall 
throughput, including in the UV-blue spectral range. The gain from an increased aperture in seeing-limited applications is characterized as the SNR per unit time, which increases proportionally to the first power of the diameter in background- and source-limited applications, and to the square for detector-limited and other fixed-noise environments. The Adaptive Optics (AO) allows a concentration of the light from a point or compact sources against the foreground sky. In the sky- or background-limited regime, the signal to noise ratio for a point source per unit time increases as $D^{2}$, while the time needed to reach a given signal to noise ratio decreases as $D^{4}$. Two of the powers of $\mathrm{D}$ in the $D^{4}$ argument arise from an increased collecting area; the other two arise from improved image concentration due to diffraction. Therefore, since the effective diameters for collecting area and diffraction differ for the GMT when caompared to an $8 \mathrm{~m}$ aperture, one should consider the GMT AO sensitivity as scaling with $(25.4 / 8)^{2} \cdot(22.0 / 8)^{2}=76.2$.

GMT will address the contemporary science topics that are both of interest and relevant to the ELT user community. We list some of them below.

1. Formation and Evolution of Planetary Systems. The GMT aperture will enable acquisition of spectra of transiting planets 7.5 times faster than current $8 \mathrm{~m}$ telescopes, empowering a new generation of spectroscopic studies of exoplanet atmospheres with an unprecedented spatial resolution;

2. Stellar Populations and Chemical Evolution. GMT will greatly enhance stellar science through the acquisition of high-resolution spectra of targets with SNR ratios that cannot be achieved today and by probing stellar systems at larger distances than possible with 8-10 m apertures. Diffraction-limited imaging provides an additional means for stellar population studies with the GMT;

3. Galaxy Assembly and Evolution. GMT will make unique contributions to our understanding of the formation and evolution of galaxies through its ability to obtain spectra of extremely faint objects and to image galaxies with spatial resolution on the order of 100 pc at large cosmological distances;

4. Dark Matter, Dark Energy, and Fundamental. GMT will improve our understanding of dark matter and dark energy through calibration of large-scale cosmological probes, dynamical studies of dark matter in massive galaxy clusters, and the structure of dark 
matter halos in low mass dwarf galaxies through wide-field optical spectrograph such as GMACS/MANIFEST; and

5. First Light and Reionization. GMT working in conjunction with JWST, SKA, and other facilities, will provide new and powerful observational tools for studies of the reionization epoch and the period of early galaxy growth that followed.

Finally, GMT science impact will be maximized from other sources' inputs. Much new ground- and space-based facilities are planned for the next couple of years and will benefit from spectroscopic follow-up with large aperture telescopes and ELTs in particular, such as ground-based facilities (ALMA and LSST) and space-based missions (JWST, Euclid, and WFIRST).

\section{A.2.2 GMT Optical Design}

The Giant Magellan Telescope (GMT) is a 25 meter altitude-azimuth Gregorian optical/infrared telescope designed for operation over the wavelength range $320 \mathrm{~nm}$ to 25 $\mu \mathrm{m}$. It will be the first member of the next generation of ELTs. The GMT design and implementation plan were firstly presented at a Conceptual Design Review in February, 2006 (Johns, 2006), and since then several design modification and updates were performed (GMT Science Book, 2018; McCarthy et al., 2016, 2018). GMT design adapts and improves the technologies that produced the highly successful Magellan Telescopes, the Multiple Mirror Telescope, MMT, and the Large Binocular Telescope, LBT (GMT Science Book, 2018). GMT is currently unde construction in north-northeast of La Serena, Chile, at Las Campanas Observatory (McCarthy et al., 2018).

GMT is an aplanatic Gregorian configuration optics which provides up to 10 arcmin Field of View (FoV) or 20 arcmin FoV with corrector. An aplanatic design is one that eliminates both spherical aberration and coma, two major aberrations found in telescopes. The Gregorian design has three main advantages (Johns et al., 2004): (i) the secondary mirror is optically conjugated to a position around 160 meters above the ground, which enables excellent ground layer adaptive optics correction over a large field of view; (ii) it allows in-telescope calibration of all the science instruments as well as calibration of the adaptive secondary mirror since the primary focus is real and the telescope exit pupil is close to the secondary mirror; and (iii) the focal plane is concave towards the instruments, 
which is well-matched to the demands of a wide-field collimator for a multi-object seeing limited spectrograph.

The segmented primary mirror is composed of seven $8.4 \mathrm{~m}$ diameter circular segments, the six peripherals are off-axis, and the center has obscuration of 1 meter for access to the Gregorian focus. The six outer primary mirror segments have a completely unobscured view of the sky, which is particularly advantageous for high contrast imaging as it reduces thermal backgrounds and scattered light. The GMT focal plane, due to the Gregorian intrinsic characteristics, is curved towards the instruments, which simplifies the design of wide-field, multi-objects, seeing limited spectrographs, such as GMACS. The segmented mirrors allow a very fast (f/0.7) primary mirror focal ratio that contributes to the overall compactness of the GMT structure and reduces the size of the secondary mirror, correctors, and instrumentation. The final focal ratio (f/8.2) optimizes the collimated beam size for re-imaging spectrographs, such as GMACS. Table A.2 shows the basic GMT properties.

The GMT primary mirror produces the collecting area of the telescope and defines the segmented telescope aperture. The size and shape of the mirror segments drive the phasing strategy for adaptive optics. The most significant practical and state of the art for monolithic mirror size is in the 8.1 to 8.4 meters range. The current generation of $6.5-8 \mathrm{~m}$ optical telescopes uses a single monolithic mirror, e.g., Gemini, Very Large Telescope (VLT) Subaru and Large Binocular Telescope (LBT) for the 8 meter members, and Multiple Mirror Telescope (MMT) and Large Altazimuth Telescope (BTA-6), for the 6 meters ones. Larger telescopes employ a mosaic of small segments of approximately 1.5 meters each, e.g., Keck, Gran Telescopio Canarias (GTC). The Large Binocular Telescope (LBT) with two 8.4.m mirrors, is the only existing telescope which combines multiple large-segments in a common mount. All the members of the next generation of ELTs will have segmented primary mirrors. The European Extreme Large Telescope (E-ELT), and Third Meter Telescope (TMT), use small segments similar in size to Keck, yet requiring approximately 500 and 800 segments for TMT and E-ELT, respectively. The GMT has taken a different approach; its primary mirror is an array of the largest possible segments manufactured at the University of Arizona Steward Observatory Mirror Lab, and each one of the seven segments has 8.4 meters in diameter. In this configuration, the central mirror is surrounded by six off-axis segments, which allows a compact telescope structure (GMTO Coorporation, 2013b). The off-axis for both M1 and M2 segments are tilted by $13.522^{\circ}$. 
Table A.2 - Basic GMT properties. Source: Johns and Rakich (2018).

Optical Prescription:

Mounting type:

Effective diameter (for diffraction):

Total collecting area of mirrors:

Effective diameter (for collecting area):

Primary mirror, M1:

M1 Central mirror obscuration:

Secondary mirror, M2:

Effective Focal length:

Final f/\# (with WFC):

Field of view:

Wavelength coverage:

Plate scale:

Image size:

Diffraction-limited angular resolution:

Size of $20^{\prime}$ field:

Field curvature:
Aplanatic Gregorian

Altitude/Azimuth

$25.448 \mathrm{~m}$

$380.2 \mathrm{~m}^{2}$

$22.002 \mathrm{~m}$

$7 \times 8.365 \mathrm{~m}(\mathrm{f} / 0.71)$

$2.39 \mathrm{~m}$ diameter

$7 \times 1.063 \mathrm{~m}$

$202.745 \mathrm{~m}$

$\mathrm{f} / 8.16(\mathrm{f} / 8.34)$

$10^{\prime}$ diameter

(20' with corrector)

$320 \mathrm{~nm}$ to $25.000 \mathrm{~nm}$

$0.997 \mathrm{~mm} / \operatorname{arcsec}$

1.2 m diameter @ 20'

$0.01^{\prime \prime}$ at $1 \mu \mathrm{m}$

$1.2 \mathrm{~m}$

$2.203 \mathrm{~m}$ concave as seen from the instruments

The secondary mirror segments are conjugated to the primary mirror segments, as shown in figure A.2. GMT will have Adaptive Secondary Mirror (ASM) and a Faststeering Secondary Mirror (FSM) assemblies with similar optical prescription (GMTO Coorporation, 2013b; McCarthy et al., 2016). 

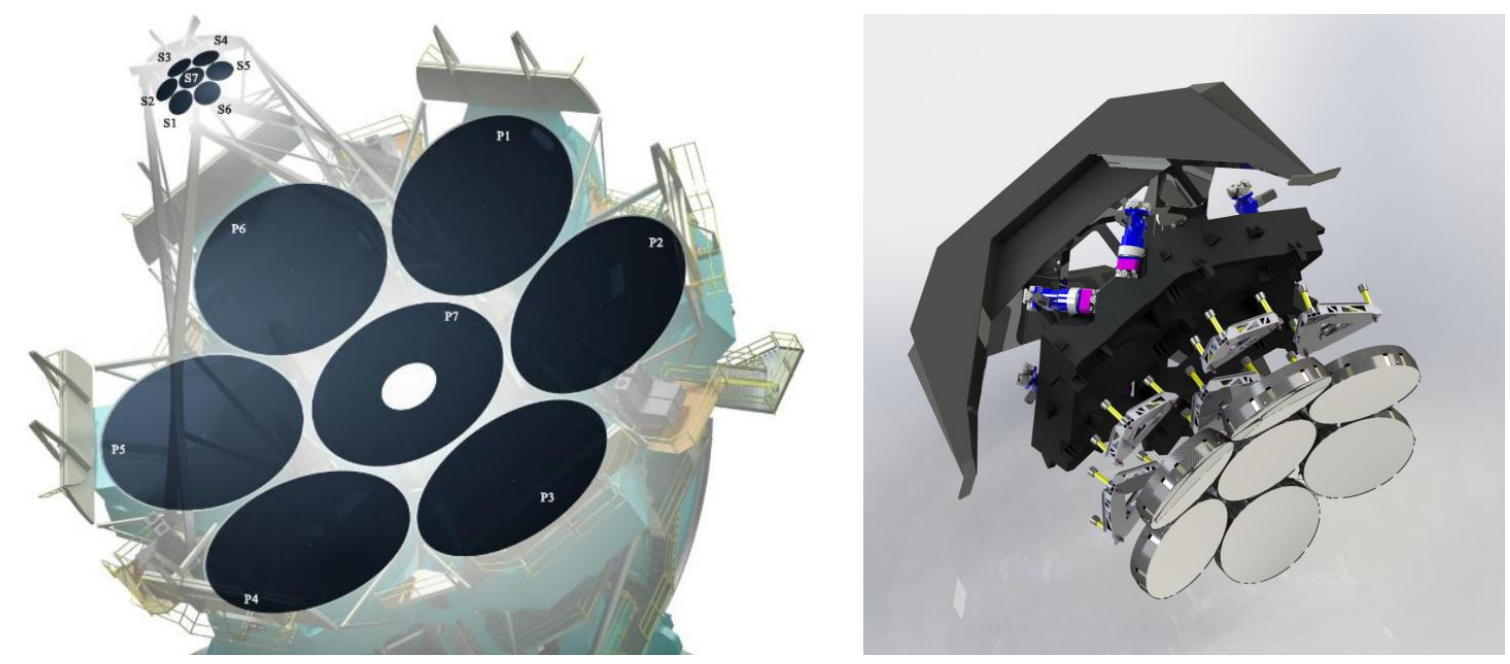

Figure A.2: Left: GMT optical layout showing the conjugation of the primary mirror segments and the secondary segments. Right: The fast-steering mirror system and an adaptive secondary mirror system. Source: McCarthy et al. (2018) and GMT Science Book (2018).

The altitude/azimuth mount provides complete access to the sky above a $30^{\circ}$ elevation angle with a $1^{\circ}$ diameter exclusion zone for tracking at the zenith. Multiple instrument mounting locations accommodate a wide range of scientific requirements. The GMT is designed to operate in both active, through actuators below the segments of the primary mirror, and adaptive optics modes. Figure A.3 shows the GMT in its enclosure. Table A.3 summarizes the GMT prescription data.

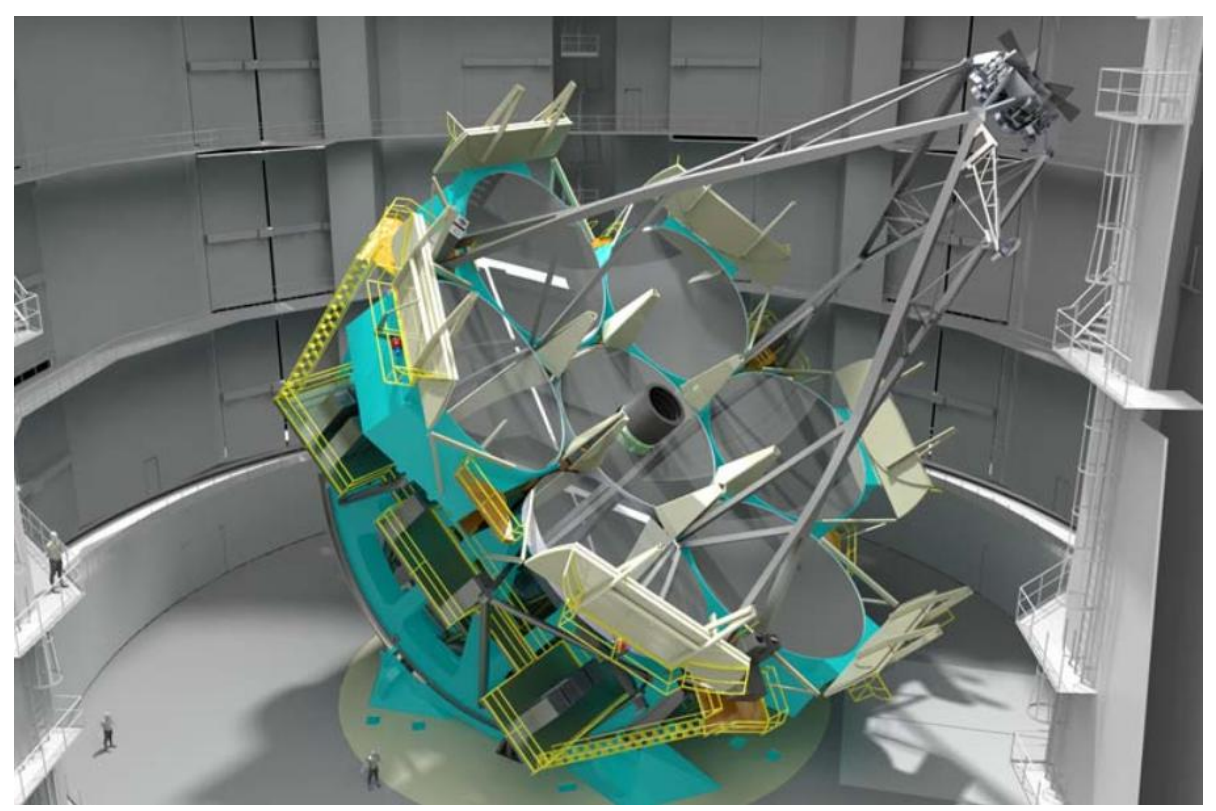

Figure A.3: The GMT illustrative design within the dome. Source: GMTO Coorporation (2013c). 
Table A.3 - GMT Optical Prescription

\begin{tabular}{|c|c|}
\hline \multicolumn{2}{|c|}{ Segmented Primary Mirror, M1 } \\
\hline Configuration & $7 \times 8.365 \mathrm{~m}$ dia. \\
\hline Center hole diameter & $1.78 \mathrm{~m}$ \\
\hline Effective Diameter & $25.448 \mathrm{~m}$ \\
\hline $\mathrm{RoC}$ & $36.000 \mathrm{~m}$ \\
\hline Conic constant $(\mathrm{k})$ & -0.99829 \\
\hline \multicolumn{2}{|c|}{ Segmented Secondary Mirror, M2 } \\
\hline Configuration & $7 \times 1.063 \mathrm{~m}$ dia. \\
\hline Effective Diameter & $3.2 \mathrm{~m}$ \\
\hline $\mathrm{RoC}$ & $4.2058 \mathrm{~m}$ \\
\hline Conic constant (k) & -0.71087 \\
\hline \multicolumn{2}{|c|}{ GMT (M1 and M2 combined) } \\
\hline M1-M2 separation & $20.290 \mathrm{~m}$ \\
\hline M1 to focal plane separation & $5.5 \mathrm{~m}$ \\
\hline
\end{tabular}

Figure A.4 shows the field curvature profiles of the Ritchey-Chrétien and the Gregorian telescope concepts. According to Sacek (2006), as long as the image is observed on the Petzval surface, there is no optical path difference between the chief ray and the other rays, and field curvature does not cause image degradation. The Petzval curvature, $R_{p}$, for two mirrors is given by $R_{p}=\left(2 / R_{2}-2 / R_{1}\right)=2 \phi_{M 2}-2 \phi_{M 1}$, where $R_{1}$ and $R_{2}$ are the RoC of M1 and M2, respectively, and $\phi$ are their power. As long as the M2 is stronger than M1, the Petzval curvature signal is the same as M2.

The Ritchey-Chrétien has no M1 real image, the focal plane is convex towards the instruments, and the chief rays are divergent, which usually requires a mirror-based collimator that matches the pupil curvature signal. Some Ritchey-Chrétien telescopes are TMT, Keck, VLT, and Gemini observatories. On the other hand, in the Gregorian telescope concept, there is a real M1 image, useful for instrument calibrations and flat fielding purposes; the curvature is concave towards the instrument, and the chief rays are conver- 
gent, which are advantages for refractive based wide-field collimators. Some examples are GMT, Magellan, and LBT.

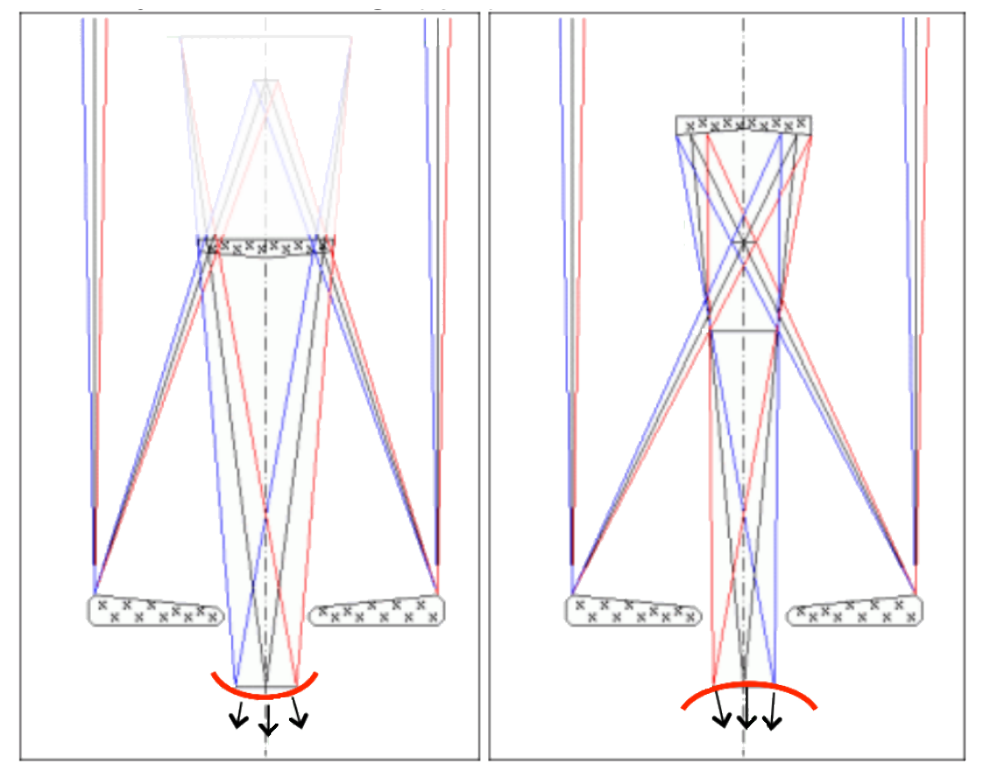

Figure A.4: The field curvature for Ritchey-Chrétien (left) and Gregorian (right) telescopes. Source: Adapted from Bernstein (2015).

\section{A.2.3 GMT's First Generation Instruments}

The initial GMT instrument concepts were presented in the Conceptual Design Review in 2006 (Johns, 2006). Seven of the concepts were further developed for instrument conceptual design review in 2011, and now five of those are under development (see table A.4). The scientific instruments will be installed in the structure of the telescope in different locations that are dependent on the characteristics of each of the instruments. The access to the GMT focal plane can be obtained directly via reflection from the secondary mirror or by reflection of the movable tertiary mirror. The three GMT optical settings, according to the GMT Science Book (2018), are:

- Direct Gregorian Narrow Field (DGNF): The DGNF is the straightforward two mirror combination that focuses the light beam on the nominal Gregorian focus. Vignetting limits the field of view to $20^{\prime}$ and field aberrations become significant outside the central $10^{\prime}$ diameter. 
- Folded Port (FP): The FP configuration uses a tertiary mirror to direct the beam to instruments mounted on the top surface of the Gregorian Instrument Rotator (GIR). The unvignetted field of view is $3^{\prime}$. The tertiary mirror rotates about the reference optical axis to feed individual instruments.

- Direct Gregorian Wide Field (DGWF): In this setting, a 20' well-corrected field is delivered to the direct Gregorian focus with the Corrector-ADC inserted in the beam. Fiber-based instruments might access this broad field.

Table A.4 - GMT's first generation instruments. Source: GMT Science Book (2018).

\begin{tabular}{|c|c|c|c|c|}
\hline Instrument & Description & $\begin{array}{c}\lambda \text { Range } \\
(\mu \mathbf{m})\end{array}$ & Resol. & FoV \\
\hline G-CLEF & $\begin{array}{l}\text { Optical High-Resolution } \\
\text { High-Stability Spectrograph }\end{array}$ & $0.36-1.00$ & $\begin{array}{l}20,000- \\
100,000\end{array}$ & $\begin{array}{l}7 \times 0.23^{\prime \prime} \text { pack } \\
\text { or } 1.2^{\prime \prime} \text { fiber }\end{array}$ \\
\hline GMACS & $\begin{array}{l}\text { Wide-Field Optical } \\
\text { Multi-Object Spectrograph }\end{array}$ & $0.32-1.00$ & $\begin{array}{l}1,500 \\
4,000\end{array}$ & $43 \operatorname{arcmin}^{2}$ \\
\hline GMTIFS & $\begin{array}{l}\text { Near-IR IFU Spectrograph } \\
\text { and Imager }\end{array}$ & $1.00-2.50$ & $\begin{array}{r}5,000 \\
10,000\end{array}$ & 10 or $400 \operatorname{arcsec}^{2}$ \\
\hline GMTNIRS & $\begin{array}{l}\text { Near- to Mid-IR High } \\
\text { Resolution Spectrograph }\end{array}$ & $1.08-5.40$ & $\begin{array}{c}65,000(\mathrm{JHK}) \\
85,000(\mathrm{LM})\end{array}$ & $1.2^{\prime \prime}$ long-slit \\
\hline MANIFEST & $\begin{array}{l}\text { Facility Robotic } \\
\text { Fiber System }\end{array}$ & $0.36-1.00$ & $\mathrm{n} / \mathrm{a}$ & $20 "$ diam. \\
\hline
\end{tabular}

Additional science instruments, not mounted at the principal foci, will be fed with instrument dependent optical relays, including gravity invariant instruments in the azimuth structure.

Figure A.5 shows the mounting locations of the GMT science instruments (Auxiliary Ports are not shown). 


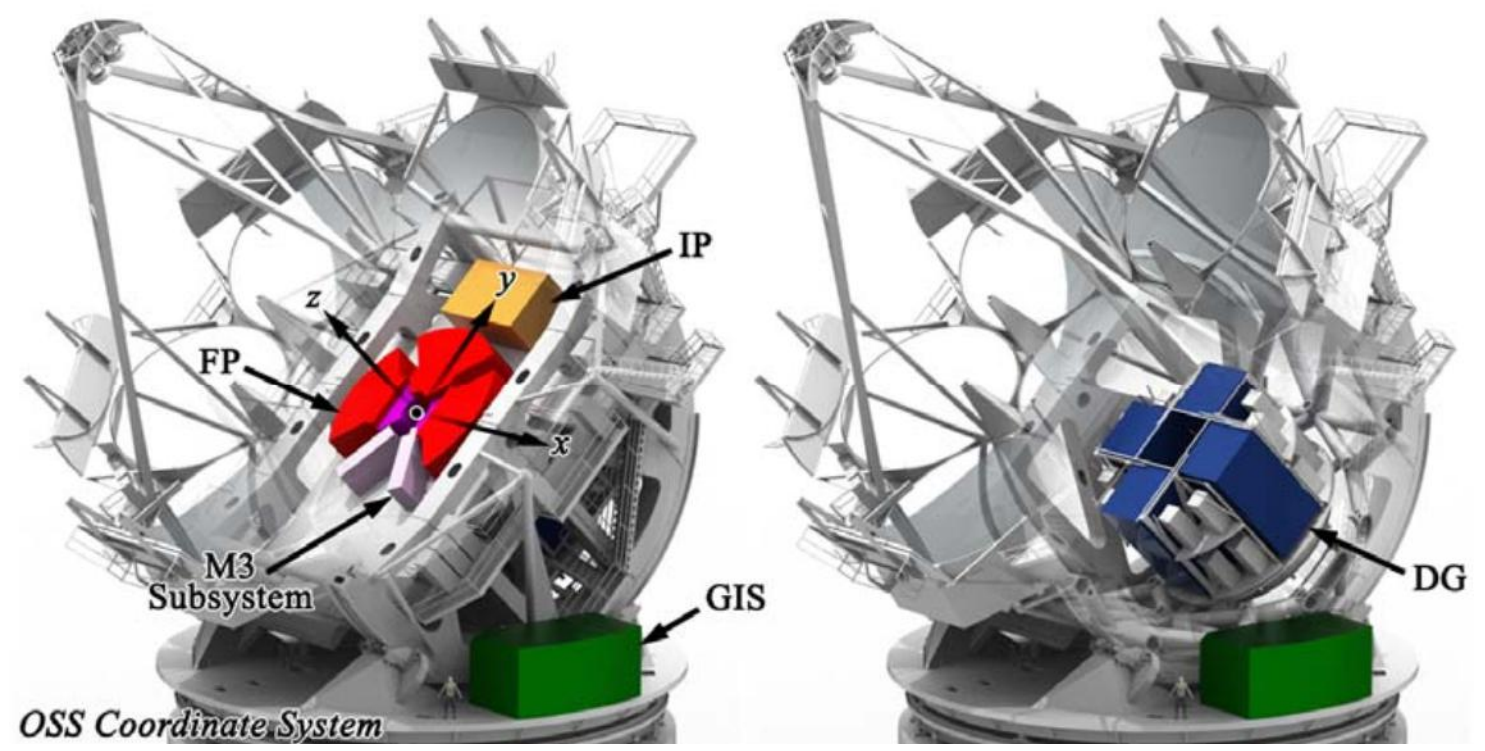

Figure A.5: Instrument mounting. FP: Folded Port; IP: Instrument Platform; GIS: Gravityinvariant Instrument Station; OSS: Optical Support Structure; DG: Direct Gregorian. Source: GMTO Coorporation (2013c).

\section{A.3 GMT Design and Optical Performance}

All the GMACS optical designs developed in this research were based on the GMT sequential optical model version "GMT DGNF non-segmented 120206" provided by the GMTO. The prescription data, spot diagram and encircled energy performances are shown in figures A.7, A.8, and A.6, respectively, for the unsegmented version. More information is available at GMTO Coorporation (2013c); Johns and Rakich (2019). The operational temperature is $\mathrm{T}=11.6^{\circ} \mathrm{C}$ (GMT site nigh-time median), pressure of $742 \mathrm{mmBar}$, GMT site height $=2,516$ meters, humidity $=36 \%$.

\begin{tabular}{|c|c|c|c|c|c|c|c|}
\hline Surf & Type & Radius & Thickness & Glass & Diameter & Conic & Comment \\
\hline OBJ & STANDARD & Infinity & Infinity & & 0 & $\theta$ & \\
\hline 1 & STANDARD & Infinity & -23000 & & 25448 & 0 & Reference surface \\
\hline 2 & STANDARD & Infinity & 23000 & & 25514,88 & $\theta$ & M2 obscuration \\
\hline STO & STANDARD & Infinity & 2248,7 & & 25448 & 0 & \\
\hline 4 & STANDARD & Infinity & 0 & & 25454,54 & 0 & \\
\hline 5 & COORDBRK & - & 0 & & - & - & Misalign PM \\
\hline 6 & STANDARD & -36000 & 0 & MIRROR & 25448 & $-0,9982857$ & PRIMARY MIRROR \\
\hline 7 & COORDBRK & - & 0 & & - & - & Restore PM \\
\hline 8 & STANDARD & Infinity & $-20262,48$ & & 29092,38 & 0 & \\
\hline 9 & STANDARD & Infinity & 0 & & 3745,747 & 0 & \\
\hline 10 & COORDBRK & - & 0 & & - & - & Misalign SM \\
\hline 11 & STANDARD & 4163,901 & $\theta$ & MIRROR & 3167,361 & $-0,7169275$ & SECONDARY MIRROR \\
\hline 12 & COORDBRK & - & 0 & & - & - & Restore SM \\
\hline 13 & STANDARD & Infinity & 20262,48 & & 3279,149 & $\theta$ & \\
\hline 14 & STANDARD & Infinity & 4900 & & 3560 & 0 & REFERENCE SURFACE \\
\hline 15 & STANDARD & Infinity & 620 & & 695,9197 & 0 & IP top surface \\
\hline 16 & STANDARD & Infinity & 310 & & 635,9274 & 0 & Instrument volume top \\
\hline 17 & STANDARD & 2197,316 & 100 & & 604,3724 & 0 & \\
\hline
\end{tabular}

Figure A.6: GMT prescription data. Source: Johns and Rakich (2019). 

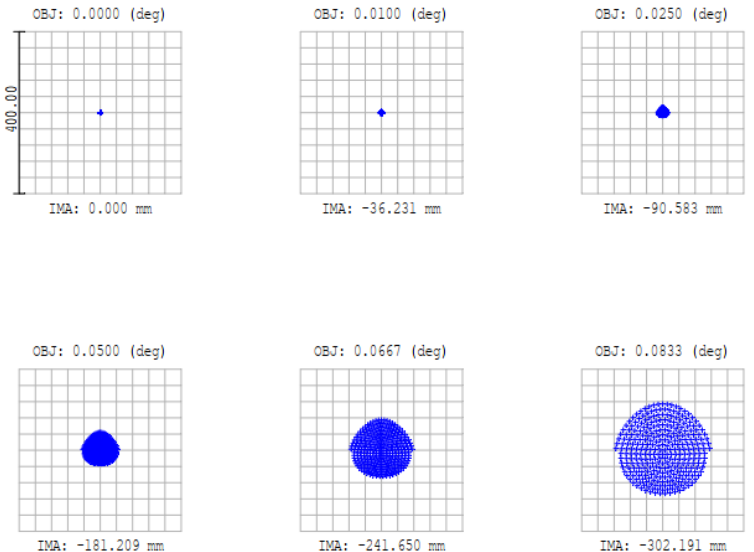

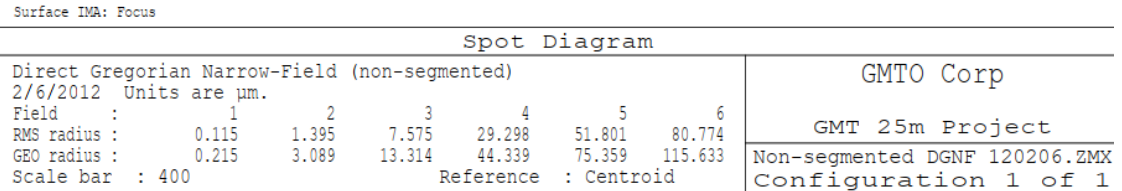

Figure A.7: GMT DGNF non-segmented spot diagram. Source: Johns and Rakich (2019).

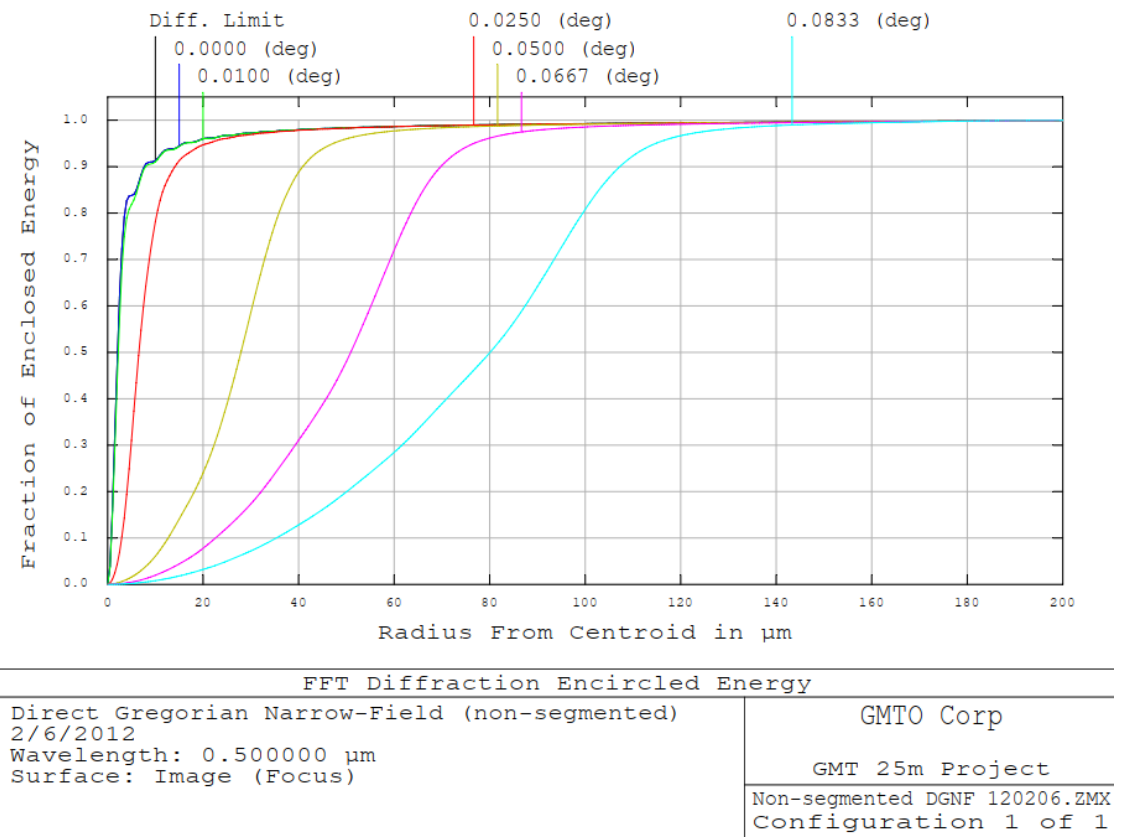

Figure A.8: GMT DGNF non-segmented encircled energy diagram. The box size is $0.4^{\prime \prime}$. Source: Johns and Rakich (2019).

The spot diagram and on-axis point spread function (PSF) for the segmented version is shown in figure A.9 and figure A.10. The PSF at $2.18 \mu \mathrm{m}$ is also shown on a log scale. This pattern rotates on the sky as the telescope tracks in altitude and azimuth. The $80 \%$ encircled energy diameter is 100 mas equivalent to 23 mas at $500 \mathrm{~nm}$ (GMTO 
Coorporation, 2013c; Johns and Rakich, 2019).
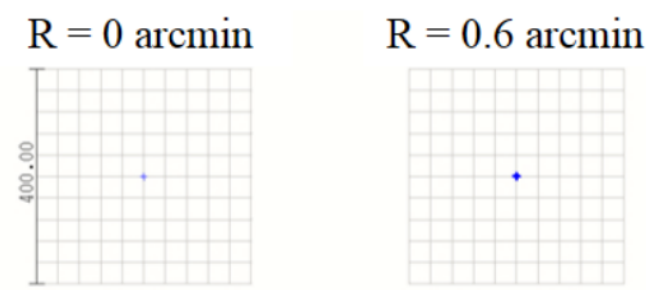

$6 \operatorname{mas} \Phi$

$\mathrm{R}=1.5 \operatorname{arcmin}$

$0.8 \operatorname{mas} \Phi$

$\mathrm{R}=3.0 \operatorname{arcmin}$

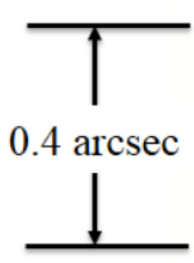

$\mathrm{R}=4.0 \operatorname{arcmin}$

$\mathrm{R}=5.0 \operatorname{arcmin}$

$28 \operatorname{mas} \Phi$

Figure A.9: GMT DGNF segmented spot size diagram on the curved focal surface. The box size is $0.4^{\prime \prime}$ or $400 \mu \mathrm{m}$. Source: GMTO Coorporation (2013c).
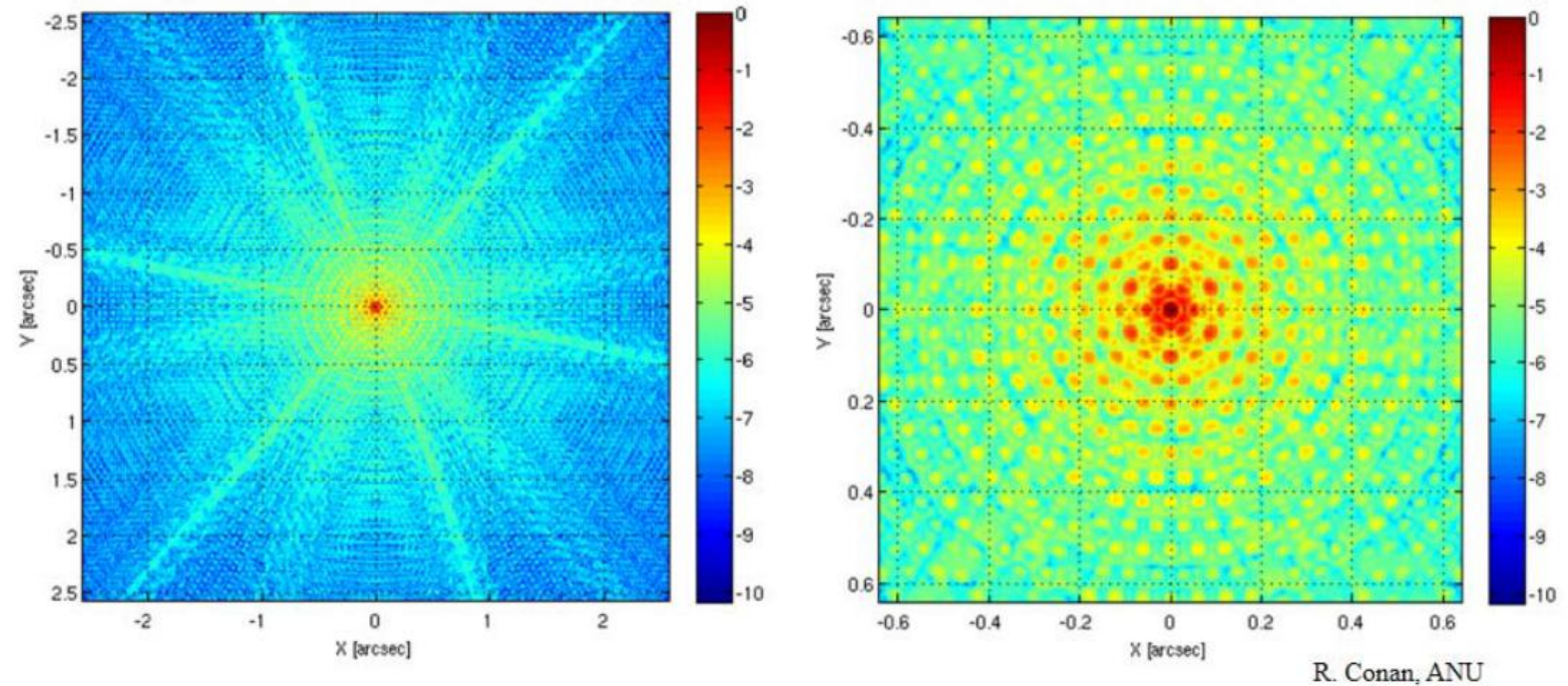

Figure A.10: Center field PSF at the reference wavelength of $2.18 \mu \mathrm{m}$. Log scale intensity. Source: GMTO Coorporation (2013c). 


\section{Appendix B}

\section{List of Zemax Macros and Python Scripts}

This appendix presents some of the macros for the OpticStudio Zemax V16.5 software and the Python 3 scripts that were developed for this research. The organization depends on their application and their source is distinguished by means of the letter "Z" and "P" referring to Zemax and Python, respectively.

The others Zemax macros, Python scripts or stand alone executable developed in this research, for instance, the detector footprint diagram, vignetting map and GUI for Spectrograph Simulator, are available under the author's request ${ }^{1}$.

\footnotetext{
${ }^{1}$ Rafael Ribeiro, rafael.alves.ribeiro@alumni.usp.br.
} 


\section{B.1 Optical Design}

\section{B.1.1 EEDx $(Z)$}

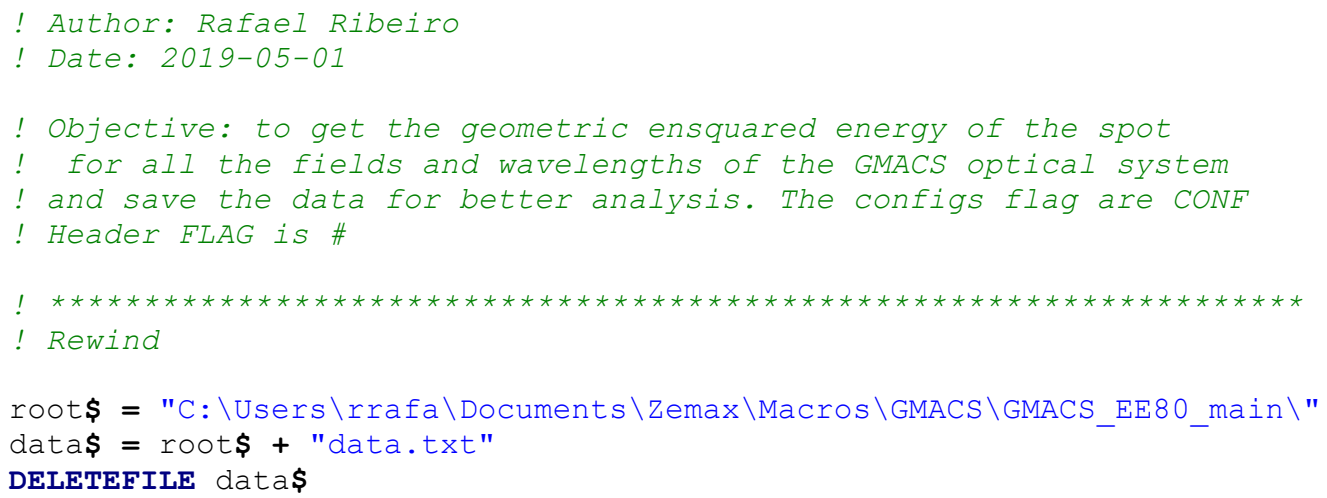




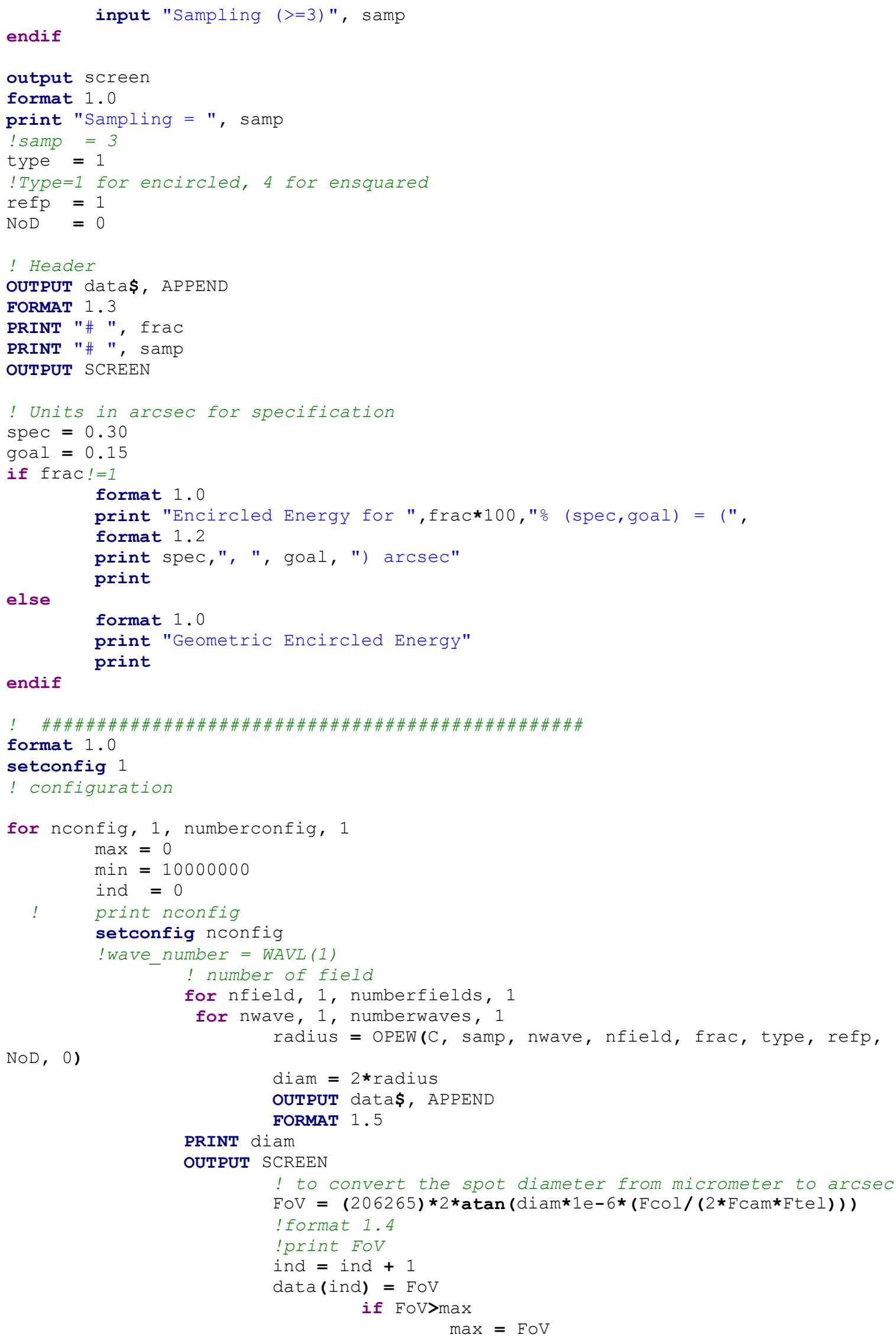




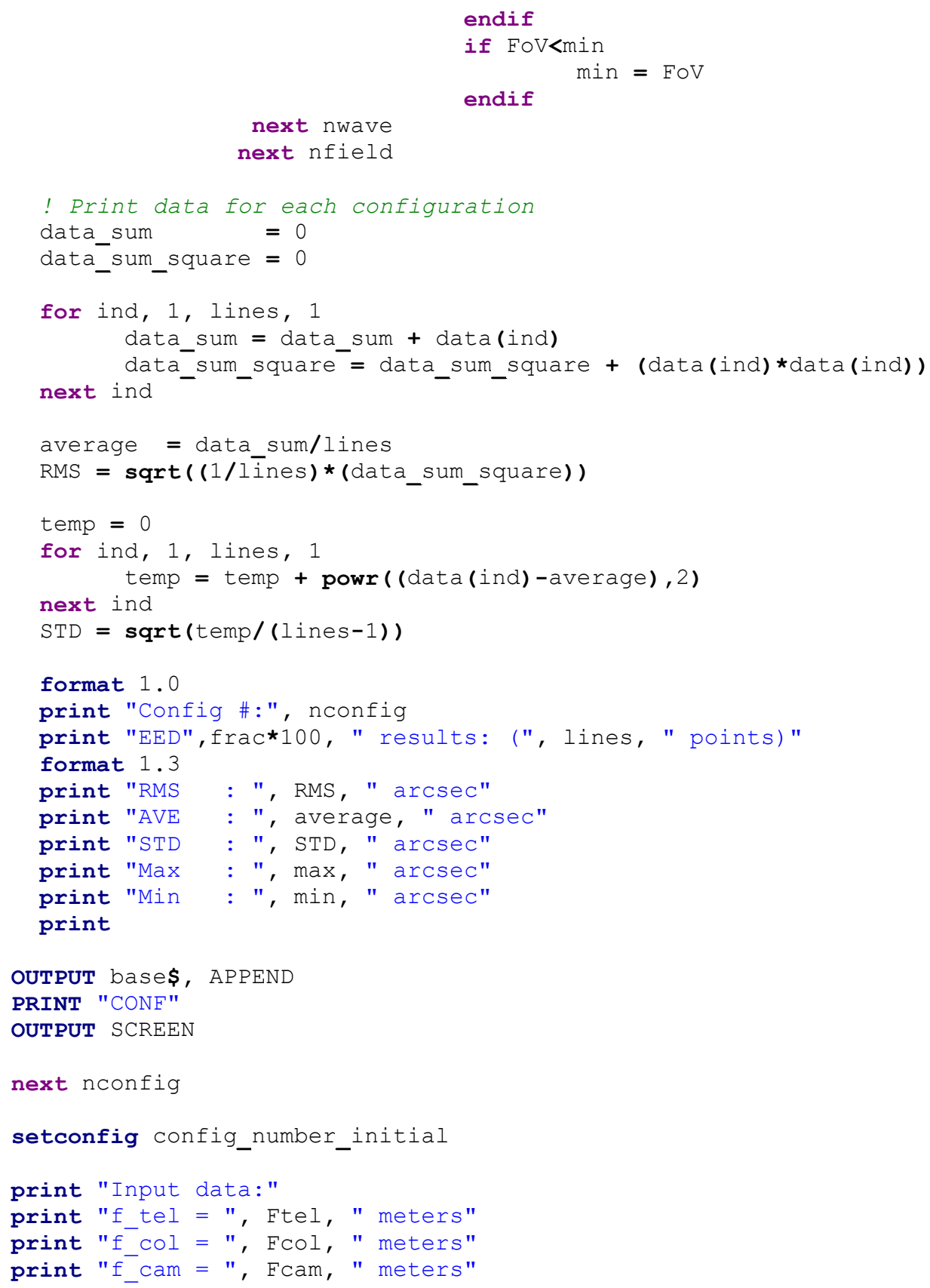




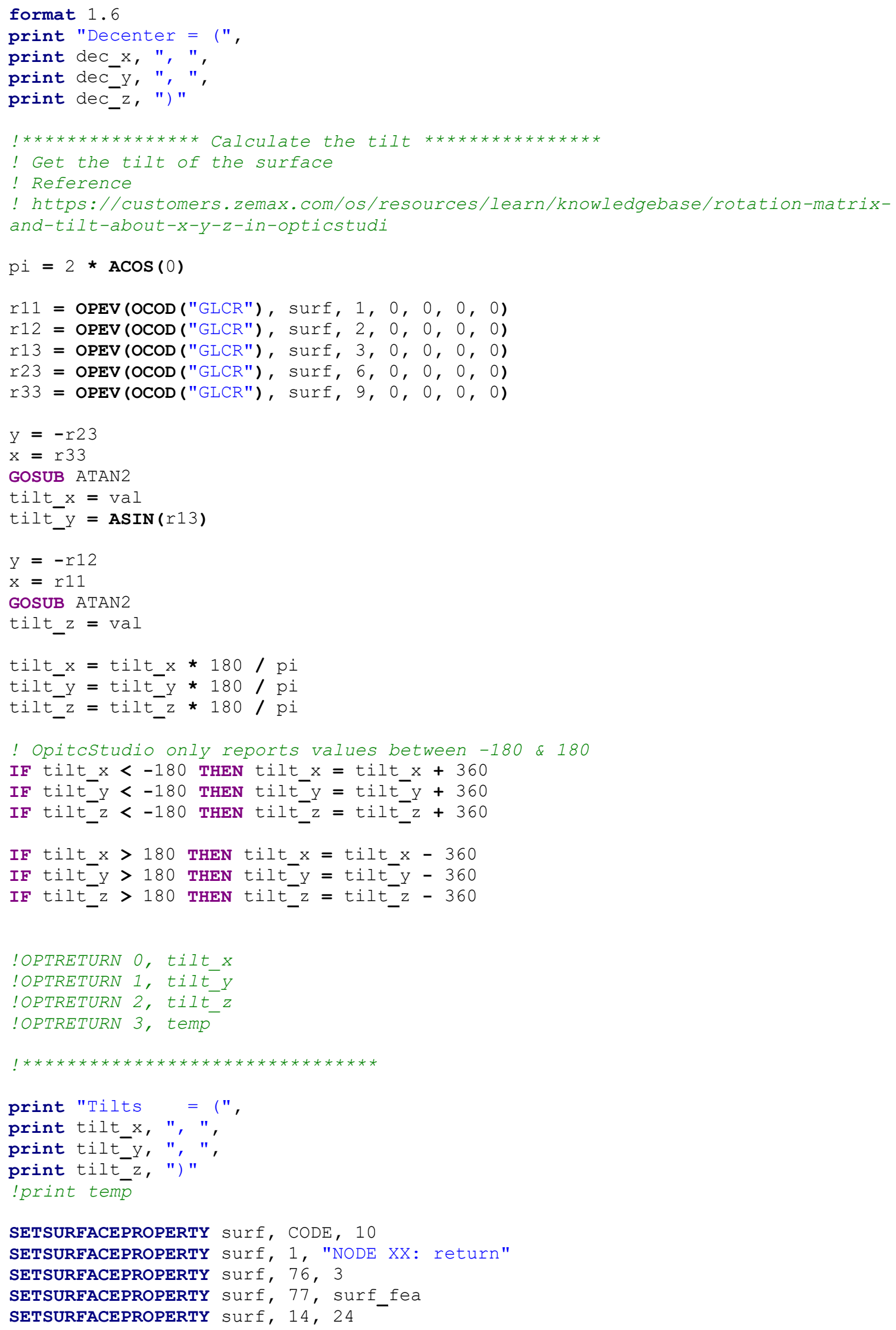




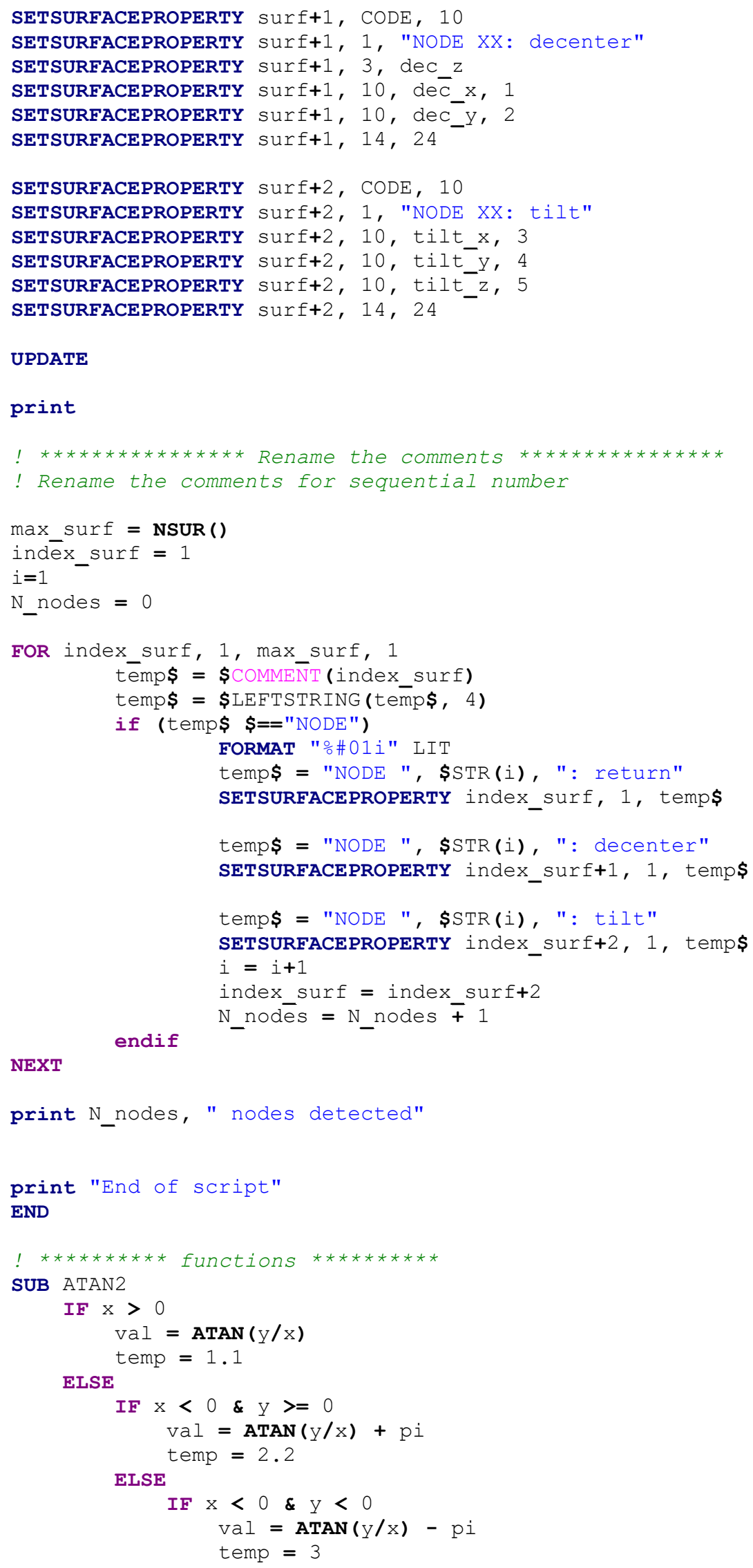




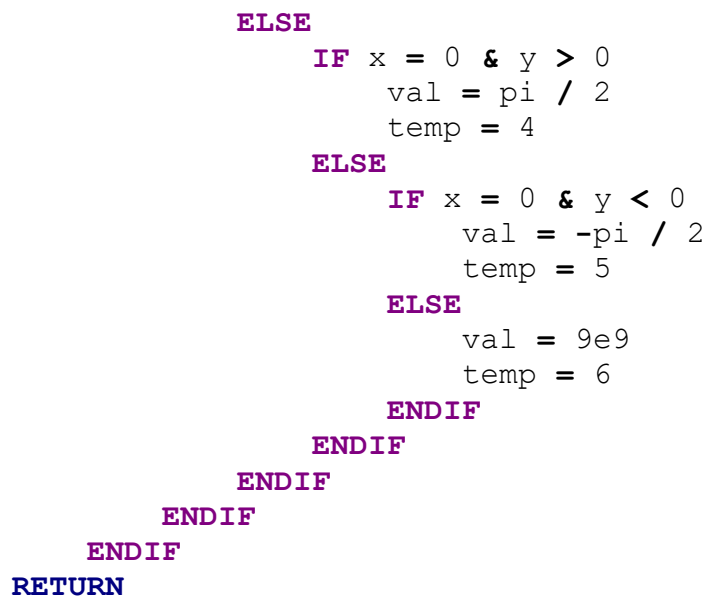




\section{B.2.2 Local to Global - Remove (Z)}

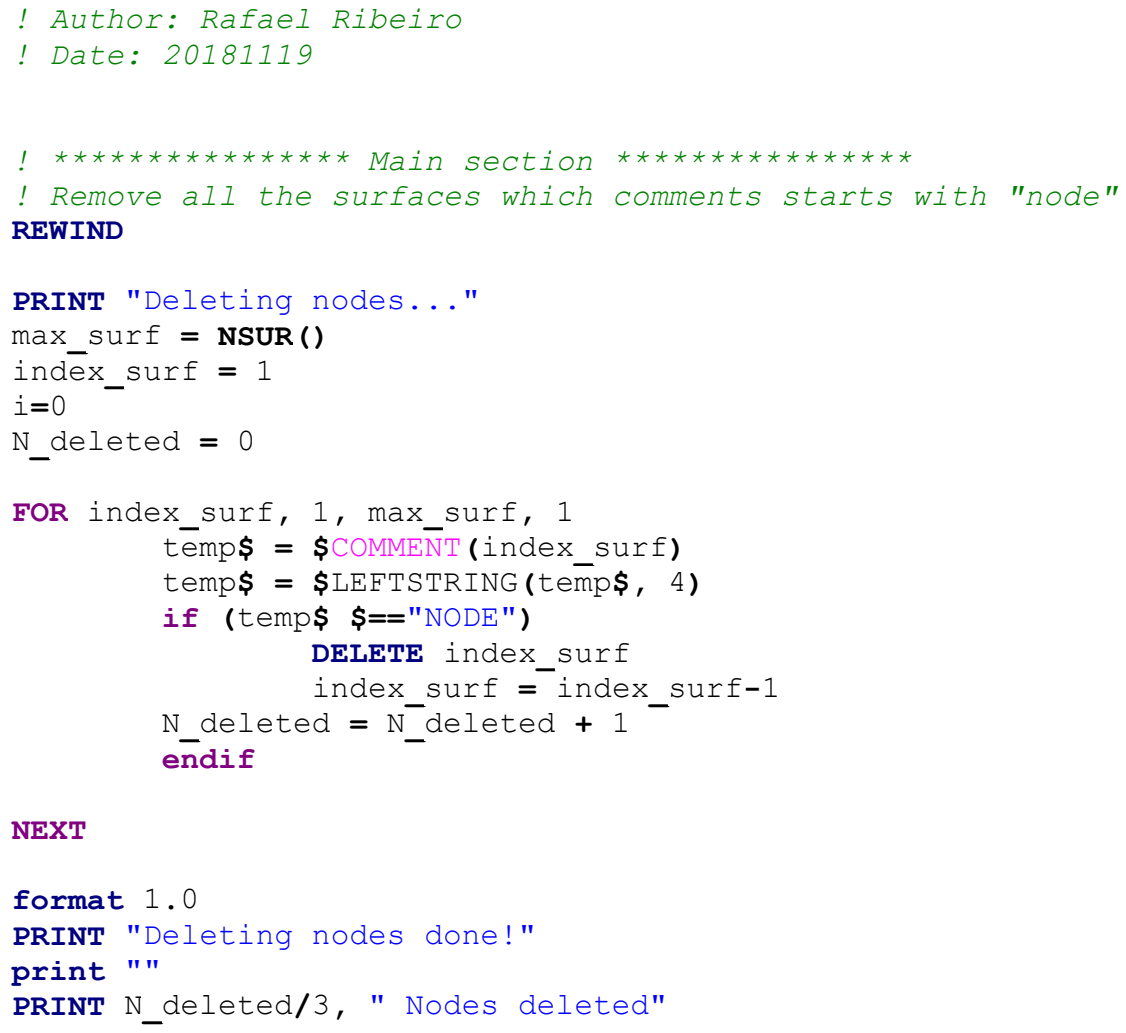




\section{B.2.3 Get Nominal Centroid or Chief Ray (Z)}

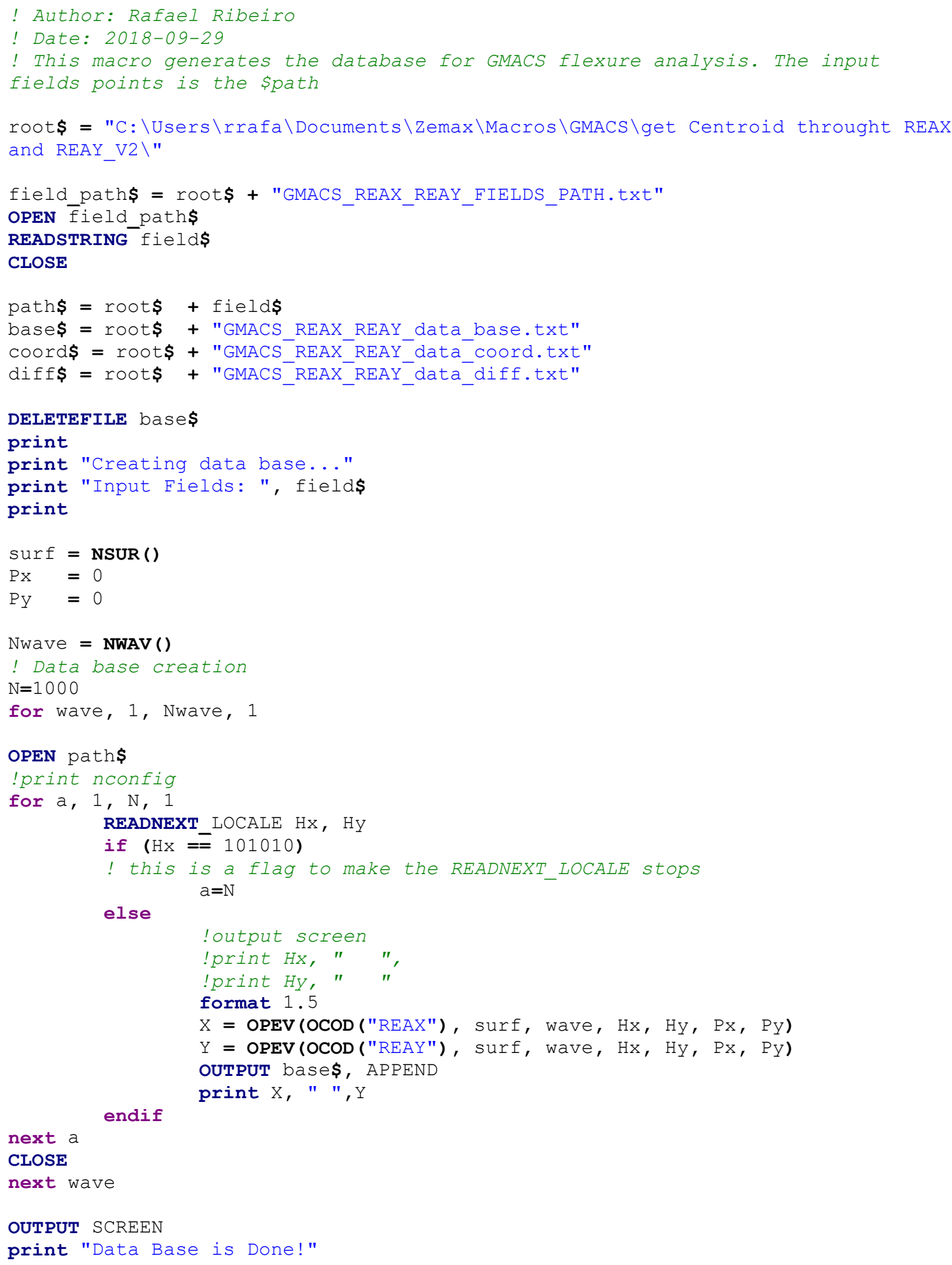




\section{B.2.4 Differential Centroid or Chief Ray (Z)}

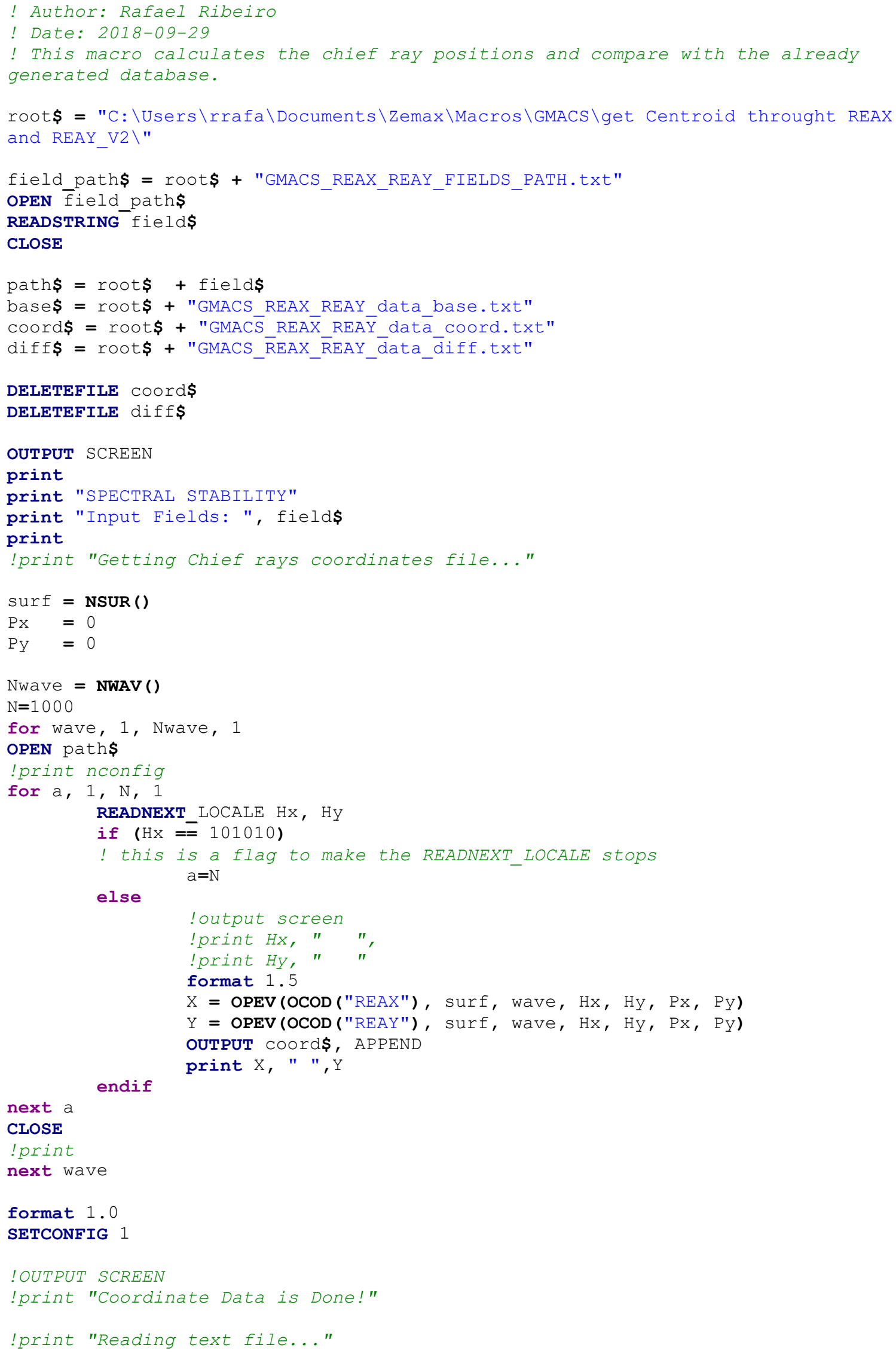




\section{B.2.5 3-D Rigid Transformation $(P)$}

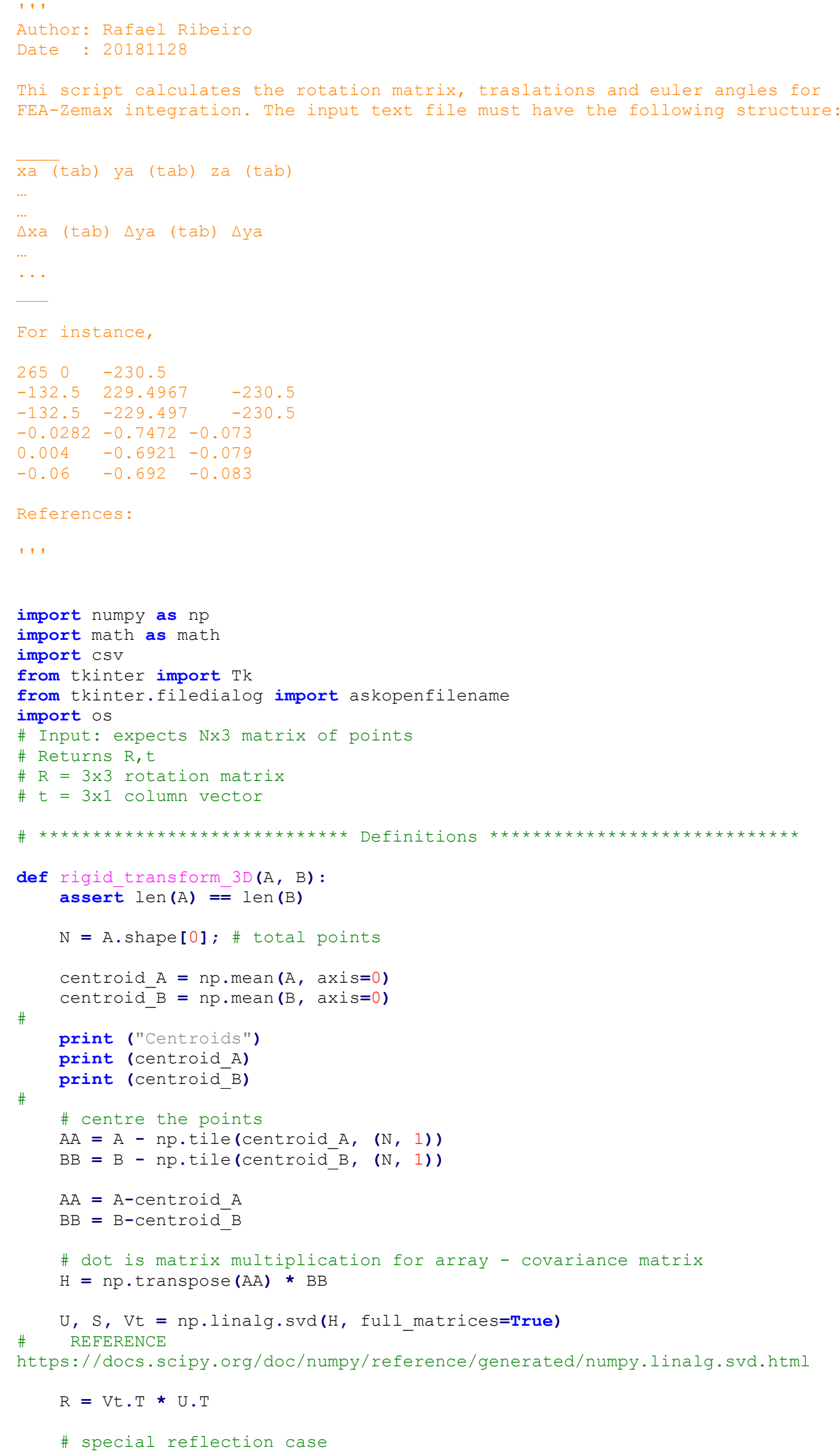




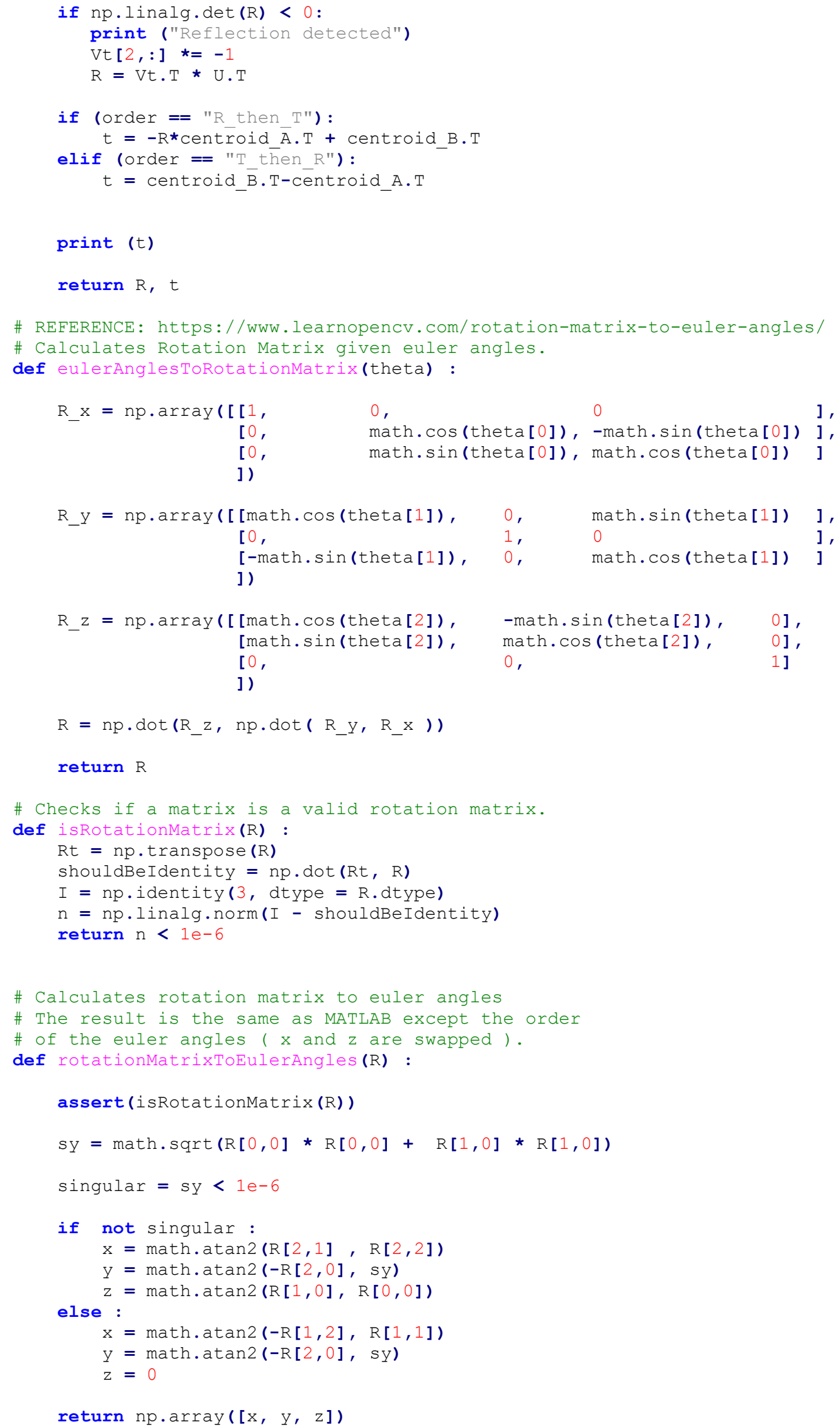


]$-a[0][2] * a[1][1] * a[2][0]-a[0][1] * a[1][0] * a[2][2]-a[0][0] * a[1][2] * a[2][1]$

\#unit normal vector of plane defined by points $a, b$, and $c$

def unit normal $(a, b, c)$ :

$\mathrm{x}=\overline{\operatorname{det}}([[1, \mathrm{a}[1], \mathrm{a}[2]]$,

$[1, \mathrm{~b}[1], \mathrm{b}[2]]$,

$[1, \mathrm{c}[1], \mathrm{c}[2]]])$

$\mathrm{y}=\operatorname{det}([\mathrm{a}[\mathrm{a}], 1, \mathrm{a}[2]]$,

$[\mathrm{b}[0], 1, \mathrm{~b}[2]]$,

$[\mathrm{c}[0], 1, \mathrm{c}[2]]])$

$\mathrm{z}=\operatorname{det}([\mathrm{a}[\mathrm{a}], \mathrm{a}[1], 1]$,

$[\mathrm{b}[0], \mathrm{b}[1], 1]$,

$[\mathrm{c}[0], \mathrm{c}[1], 1]])$

magnitude $=(x * \star 2+y * * 2+z * * 2) * * .5$

return ( $x /$ magnitude, $y /$ magnitude, $z /$ magnitude)

\# dot product of vectors a and b

$\operatorname{def} \operatorname{dot}(a, b)$ :

return $a[0] * b[0]+a[1] * b[1]+a[2] * b[2]$

\#cross product of vectors $\mathrm{a}$ and $\mathrm{b}$

def $\operatorname{cross}(a, b)$ :

$x=a[1] * b[2]-a[2] * b[1]$

$y=a[2] * b[0]-a[0] * b[2]$

$z=a[0] * b[1]-a[1] * b[0]$

return $(x, y, z)$

\#area of polygon poly

def area(poly):

if len $(p o l y)<3$ : \# not a plane - no area

return 0

total $=[0,0,0]$

for $i$ in range (len $(p \circ l y))$ :

vil $=$ poly $[i]$

if $i$ is len (poly)-1:

else:

vi2 $=$ poly[0]

$\mathrm{vi2}=\operatorname{pol}_{\mathrm{y}}[i+1]$

$\operatorname{prod}=\operatorname{cross}(\mathrm{vi1}, \mathrm{vi2})$

total[0] $+=\operatorname{prod}[0]$

total[1] $+=\operatorname{prod}[1]$

total[2] $+=\operatorname{prod}[2]$

result $=\operatorname{dot}($ total, unit_normal $($ poly[0], poly[1], poly[2]))

return abs(result/2)

def file len(fname):

with open(fname) as $f$ :

for $i, l$ in enumerate $(f)$ :

pass

return $i+1$

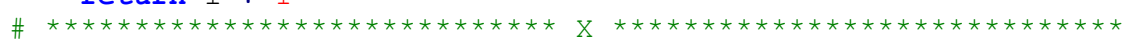

root $=\mathrm{Tk}()$. withdraw() \# we don't want a full GUI, so keep the root window from appearing

filename = askopenfilename() \# show an "Open" dialog box and return the path to the selected file

print (

print(filename)

file_name, ext = os.path.splitext(filename)

order $=$ "R then $T "$

order $=$ "T_then_R"

results $=[]$

with open((file nametext), 'r') as $f$ :

reader $=\mathrm{csv} \cdot$ reader $\left(\mathrm{f}, \operatorname{delimiter} \mathbf{\prime}^{\prime} \backslash t^{\prime}\right)$

for row in reader:

results. append (row)

print ( $r o w$ ) 


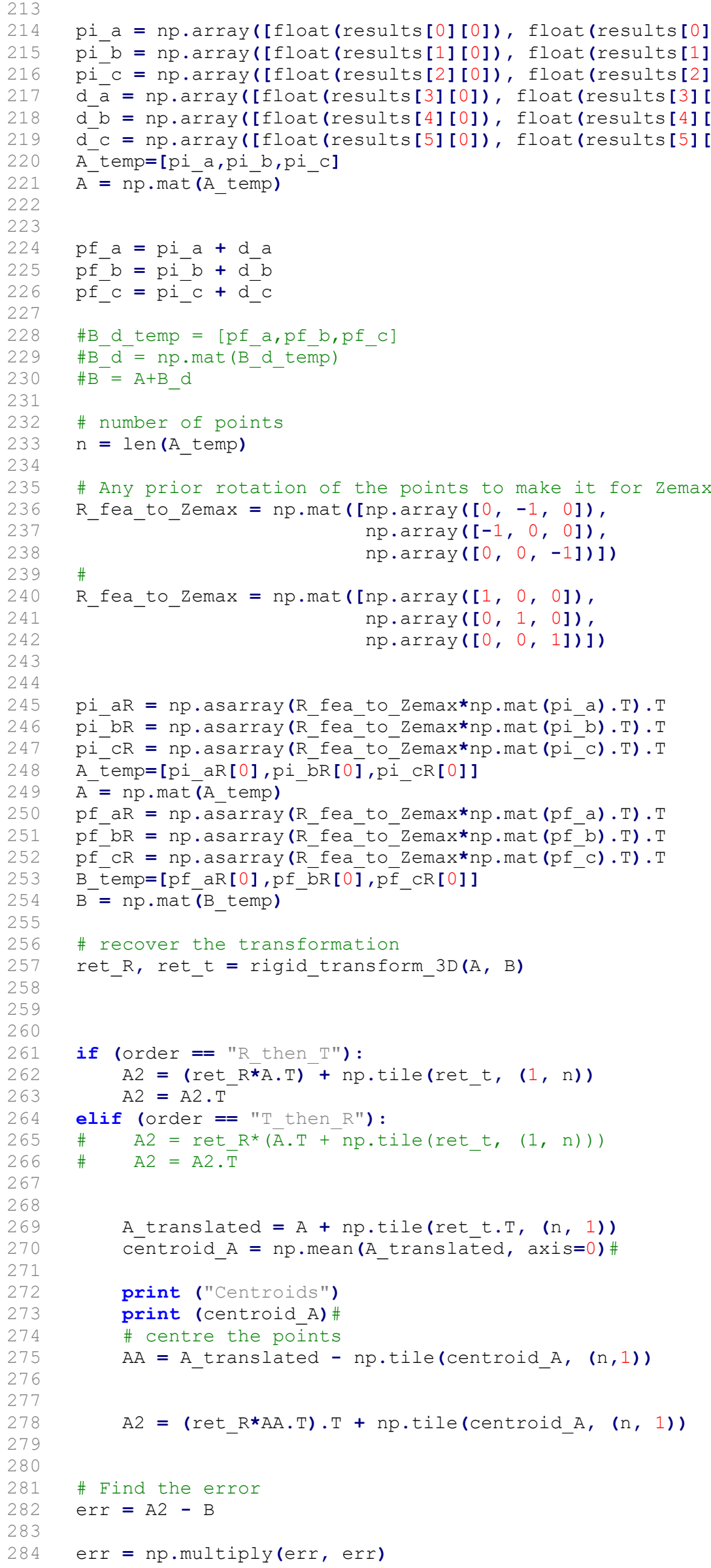




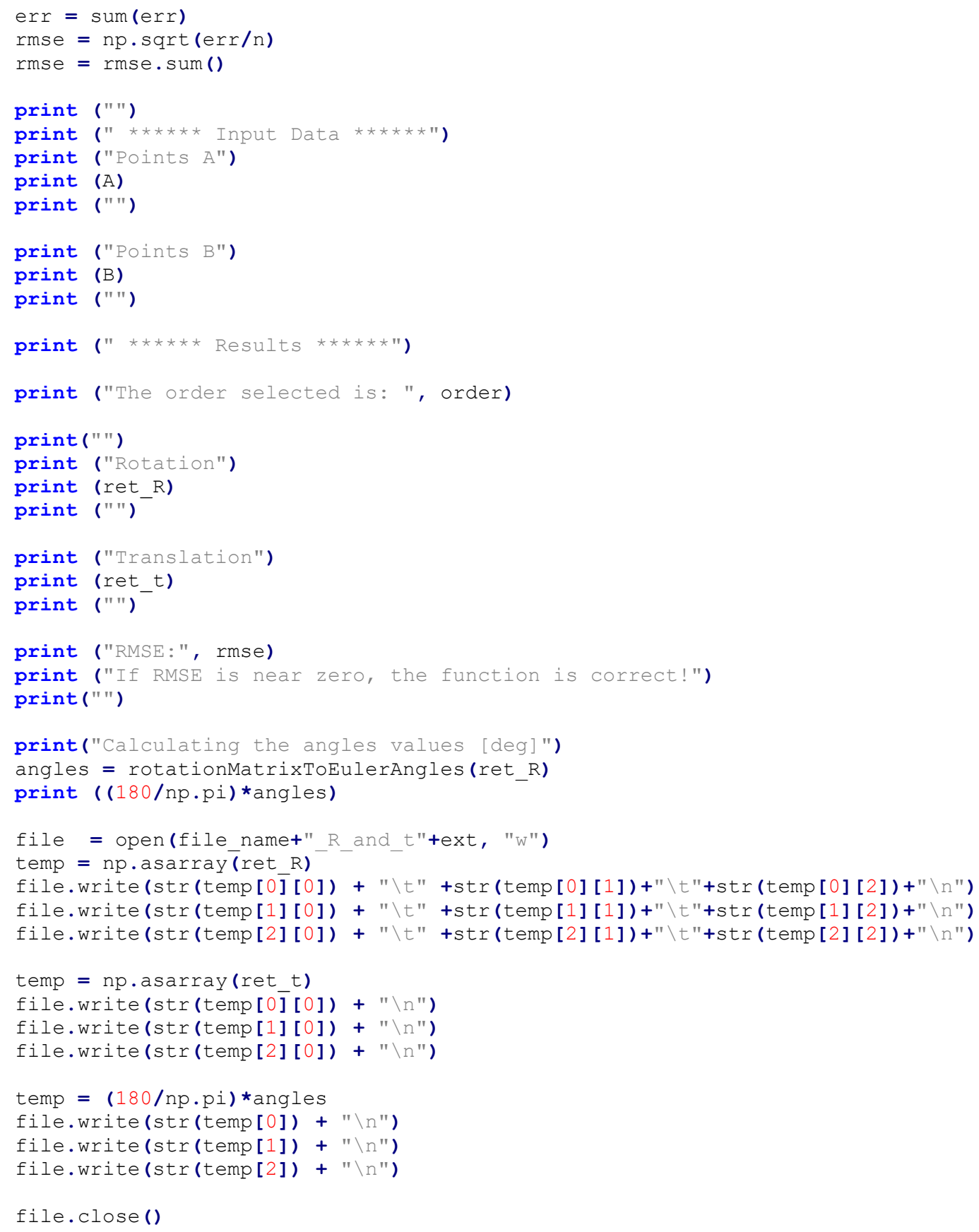




\section{B.2.6 Insert FEA in Zemax (Z)}

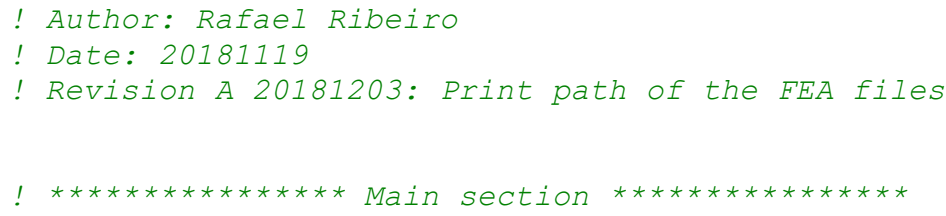




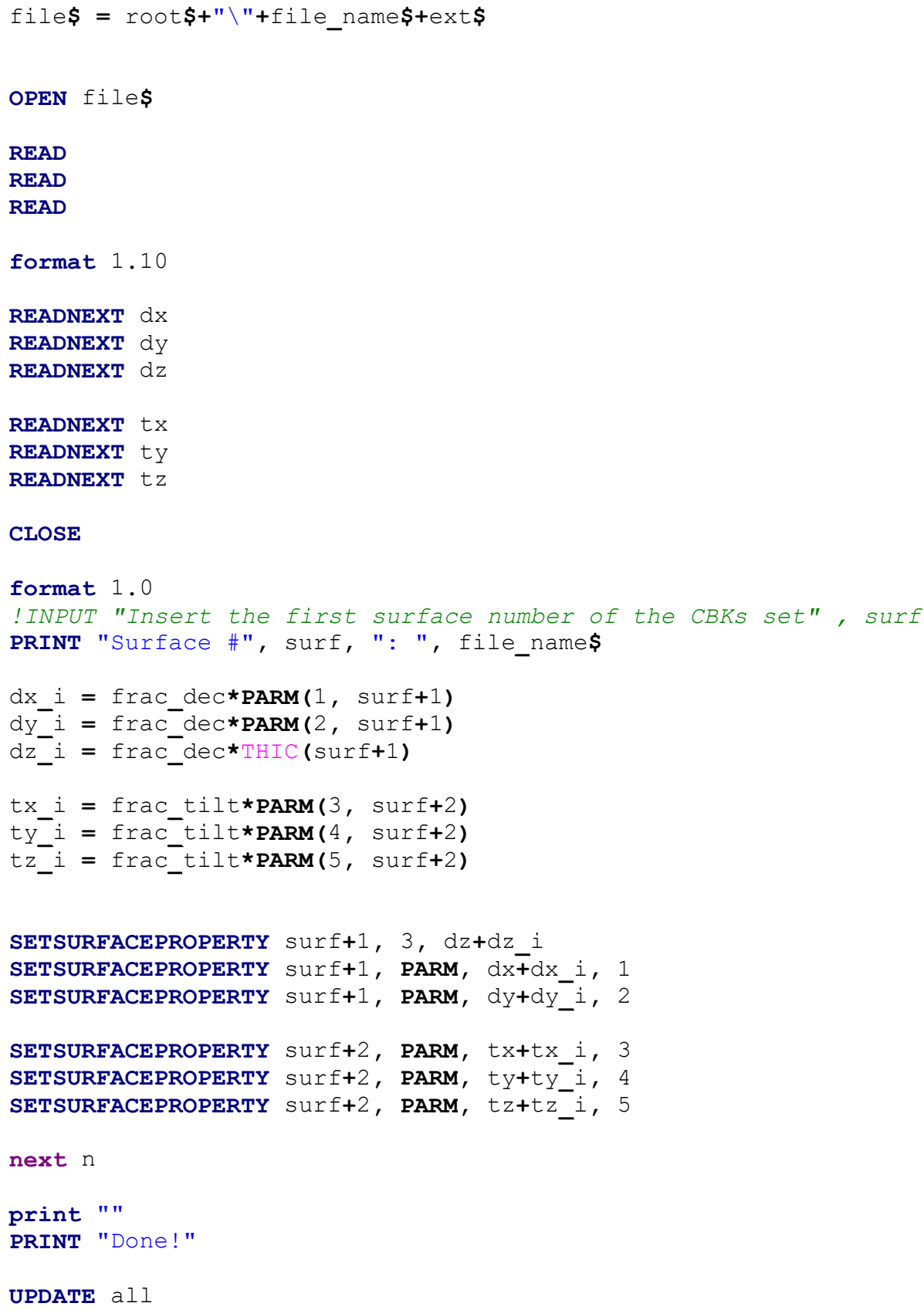


B.2.7 Create Merit Function for Spectral Stability (Z)

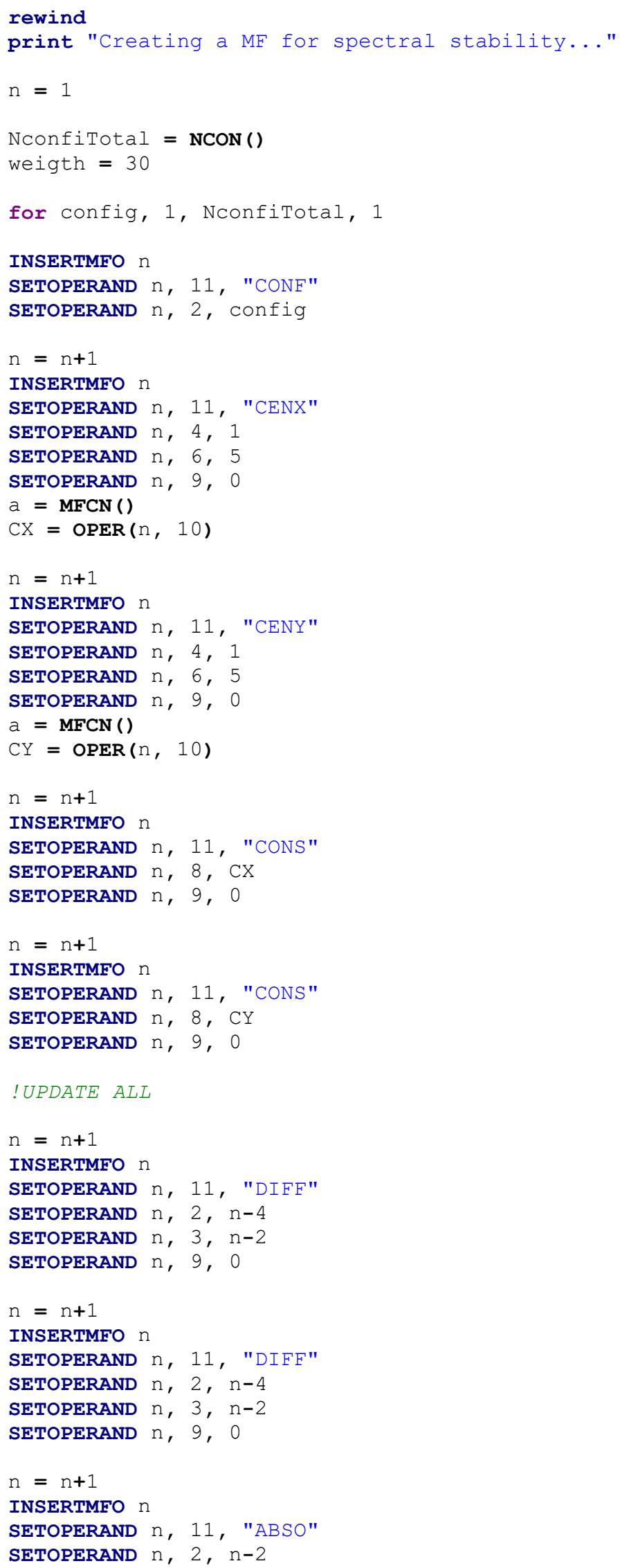




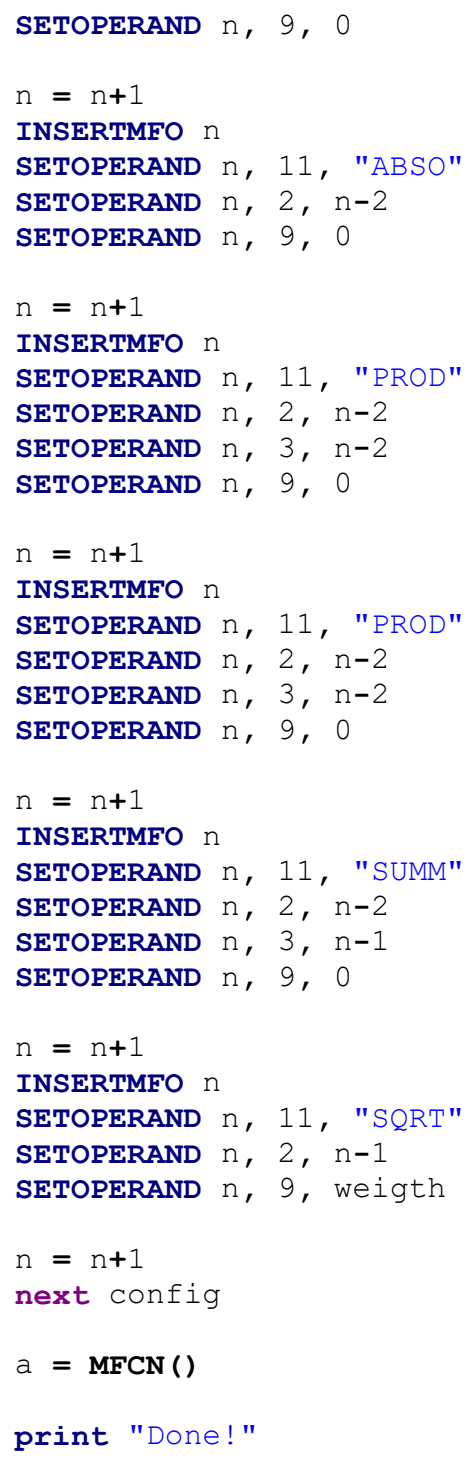




\section{Appendix C}

\section{CoD Split Collimator Wavefront Performance}

The wavefront performance of the CoD split collimator is presented in this appendix.

Figures C.1 and C.2 for the blue arm and figures C.3 and C.4 for the blue arm

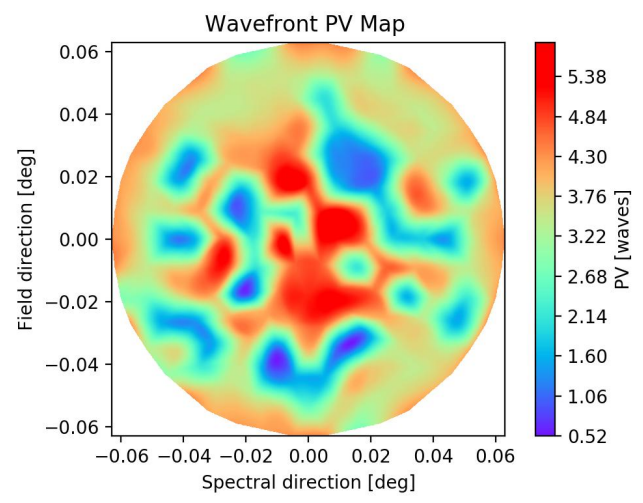

(a) PV 300-600 nm

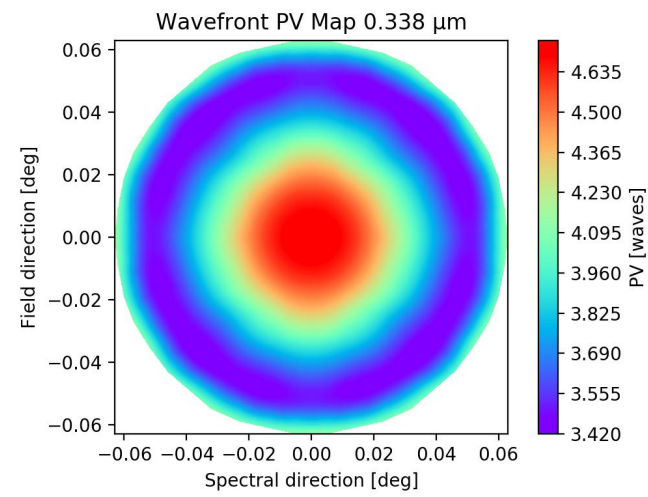

(c) PV@338 nm

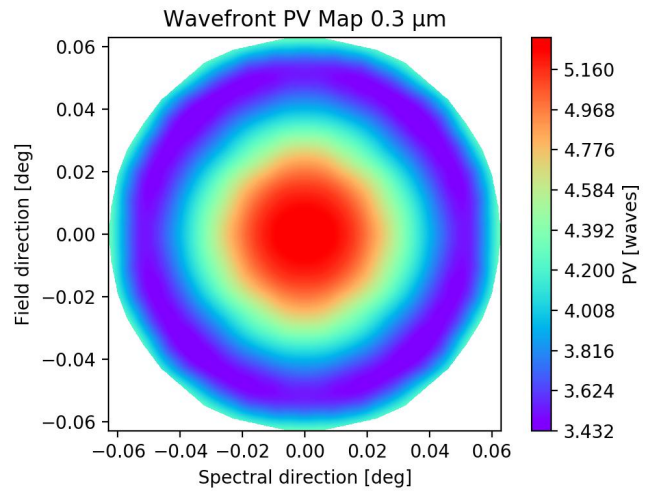

(b) PV@300 nm

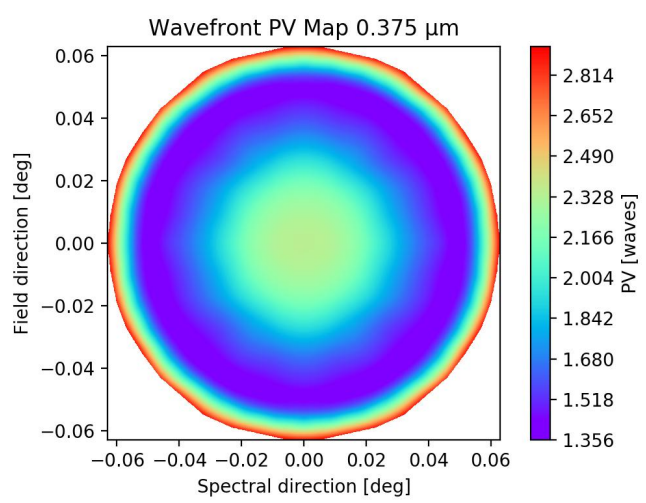

(d) PV@375 nm 


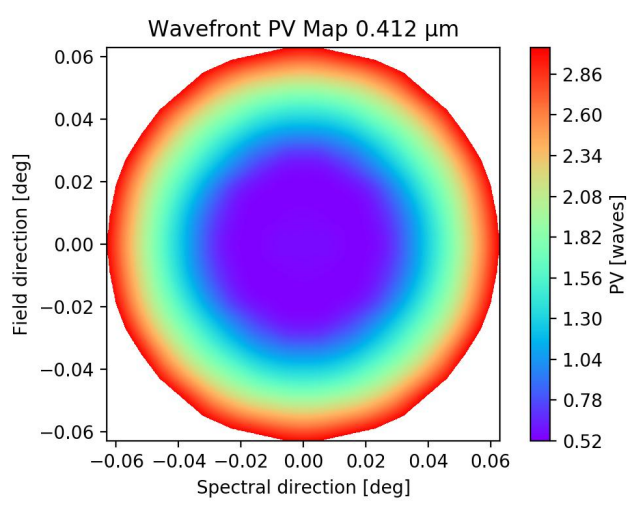

(e) PV@412 nm

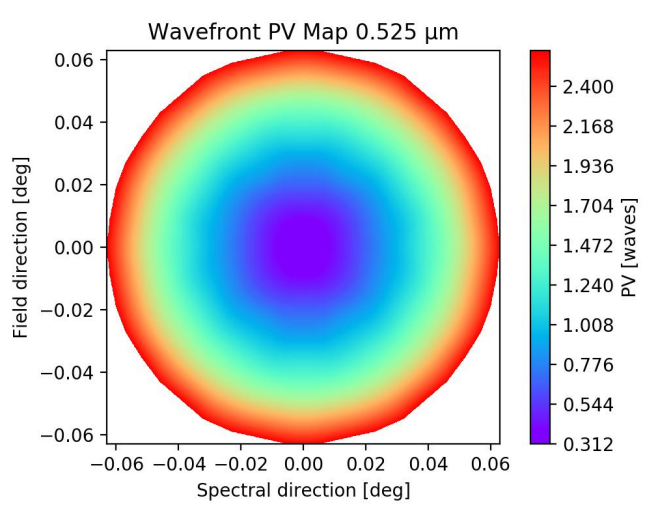

(g) PV@525 nm

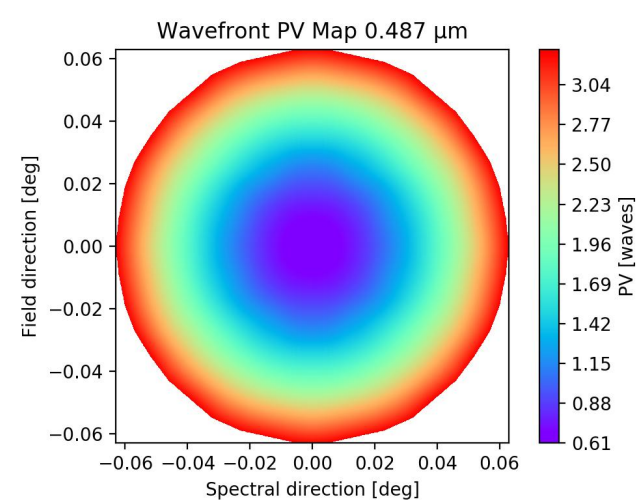

(f) PV@487 nm

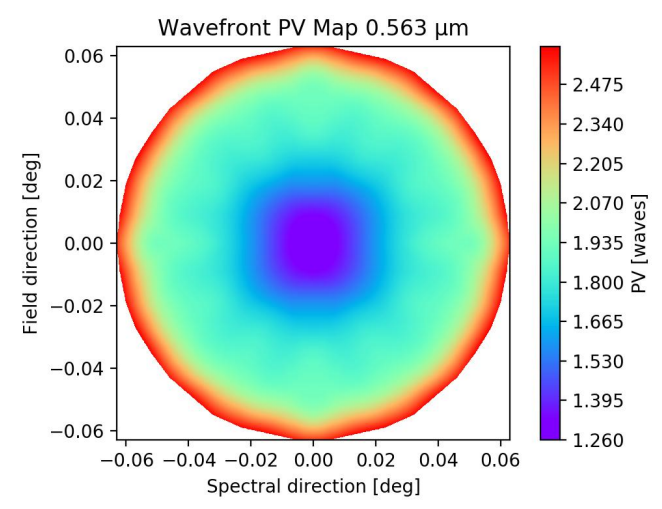

(h) PV@563 nm

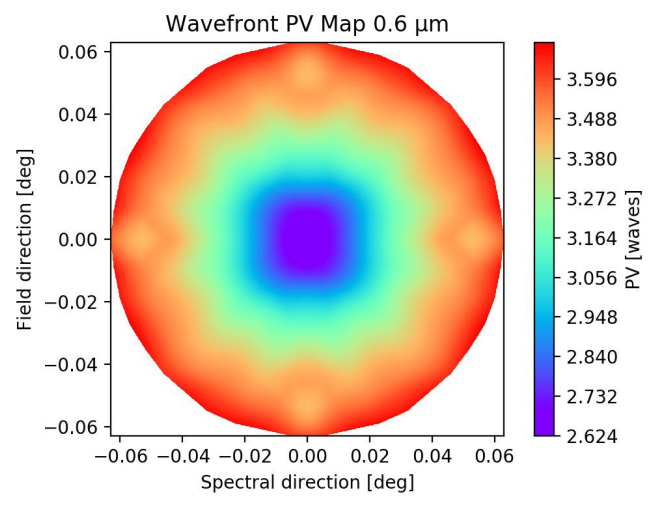

(i) PV@600 nm

Figure C.1: Split collimator blue arm PV wavefront performance at the exit pupil. 


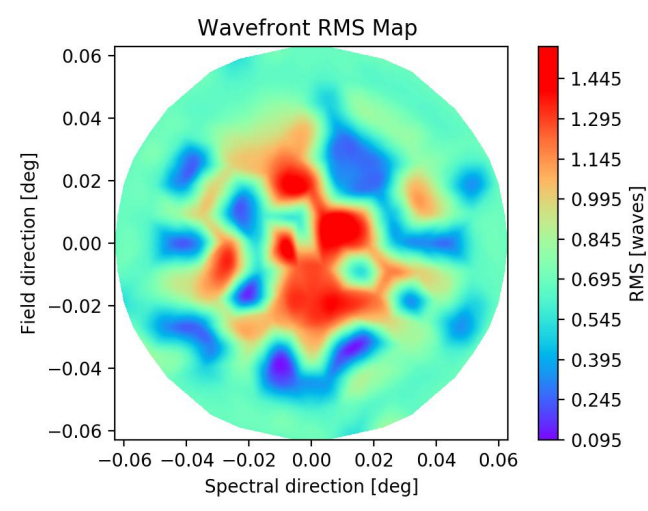

(a) RMS 300-600 nm

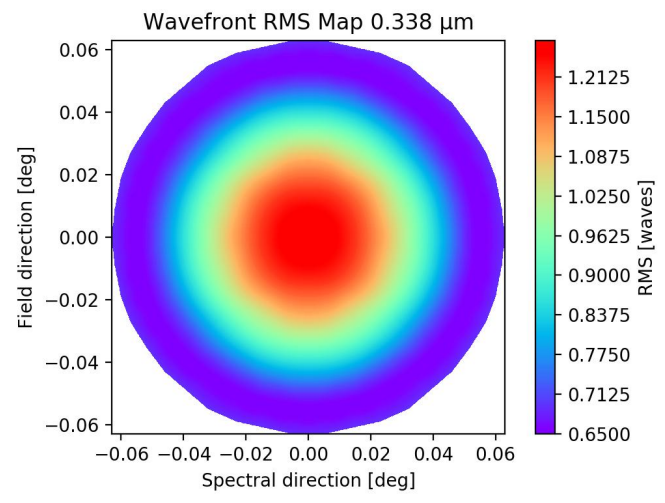

(c) RMS@338 nm

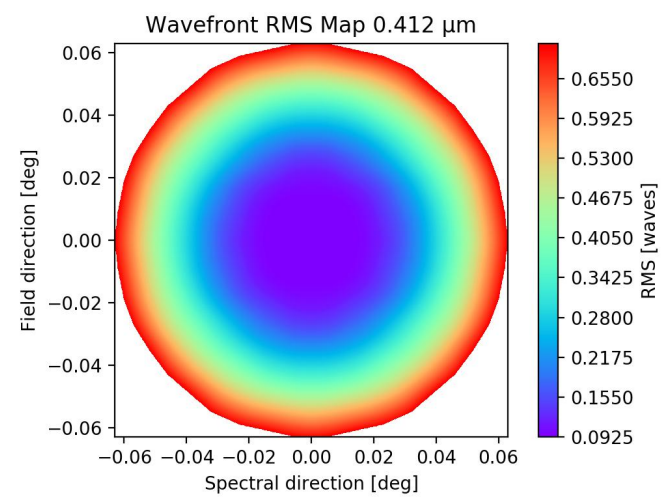

(e) RMS@412 nm

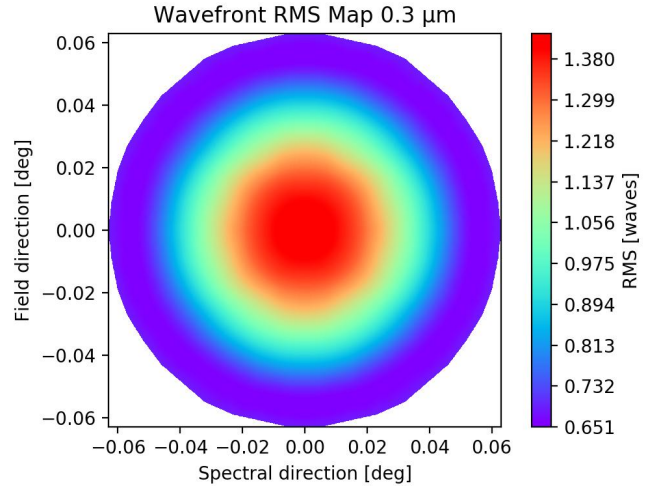

(b) RMS@300 nm

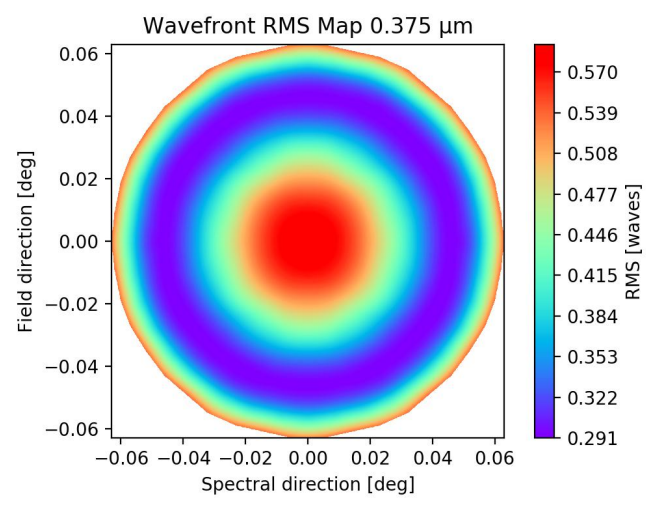

(d) RMS@375 nm

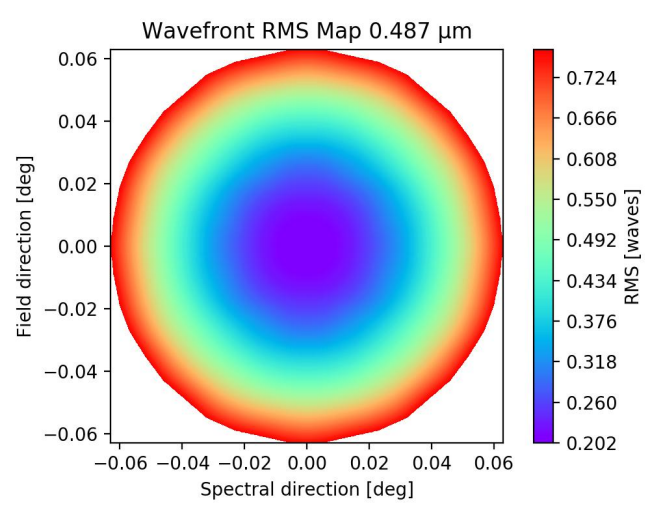

(f) RMS@487 nm 


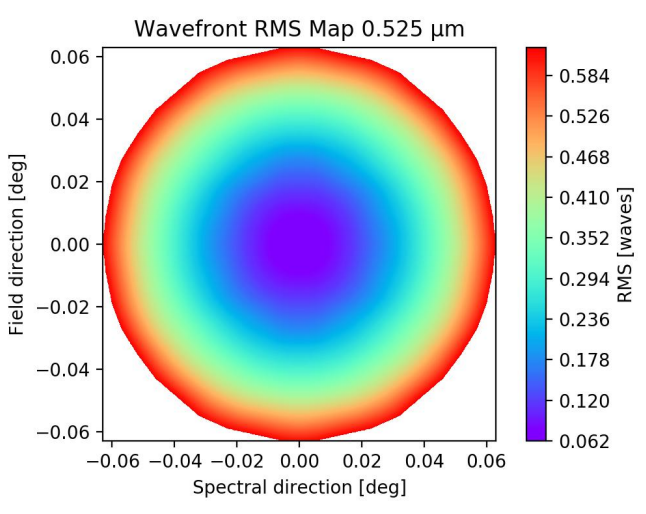

(g) RMS@525 nm

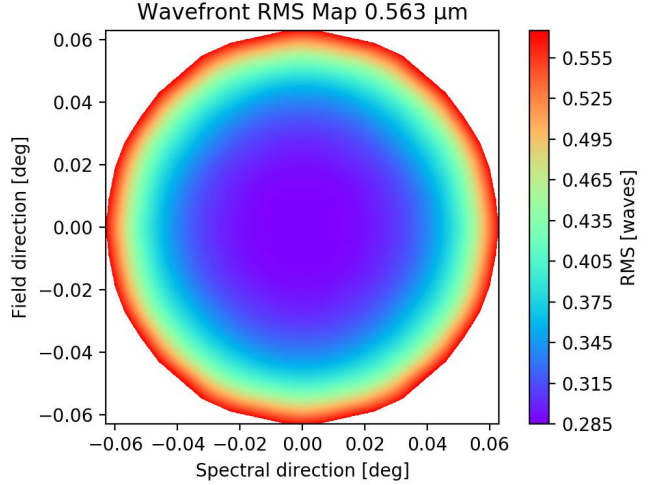

(h) RMS@563 nm

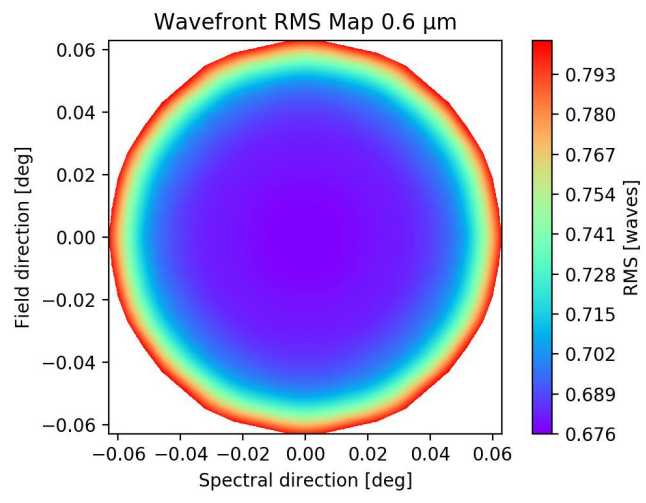

(i) RMS@600 nm

Figure C.2: Split collimator blue arm RMS wavefront performance at the exit pupil. 


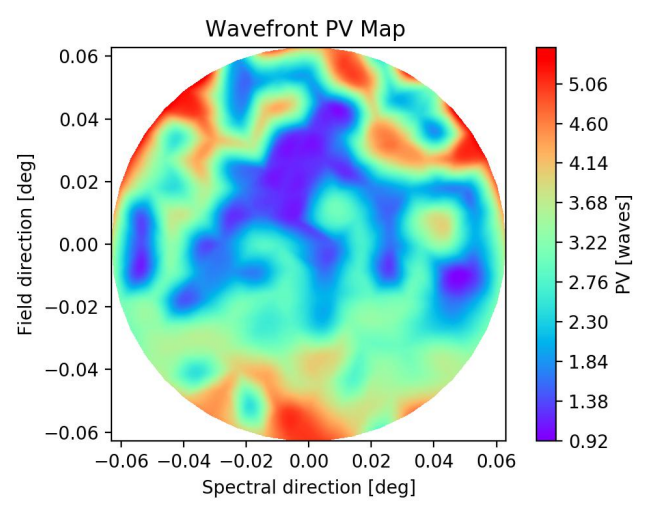

(a) PV 500-1,000 nm

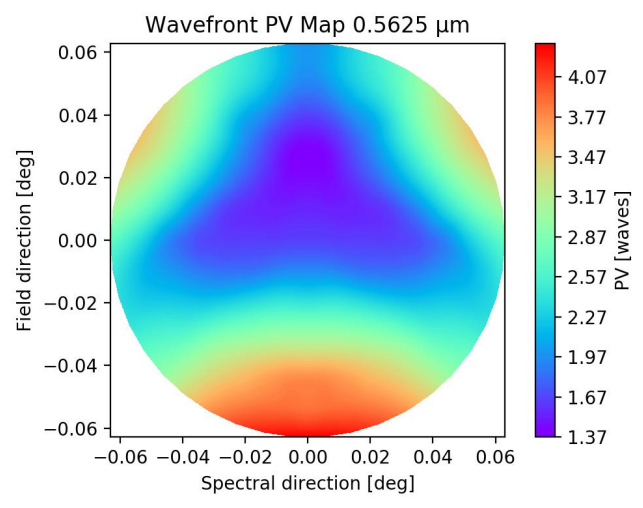

(c) PV@562 nm

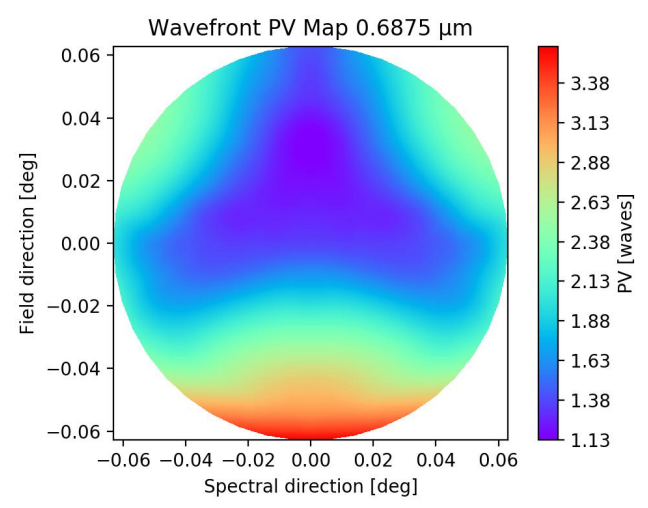

(e) PV@687 nm

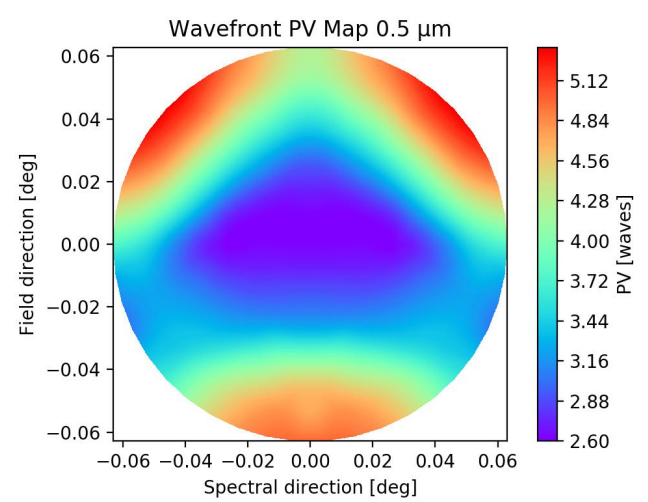

(b) PV@500 nm

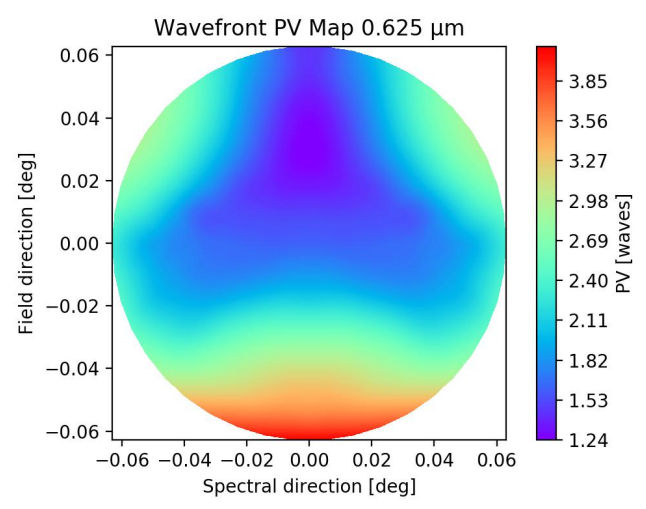

(d) PV@625 nm

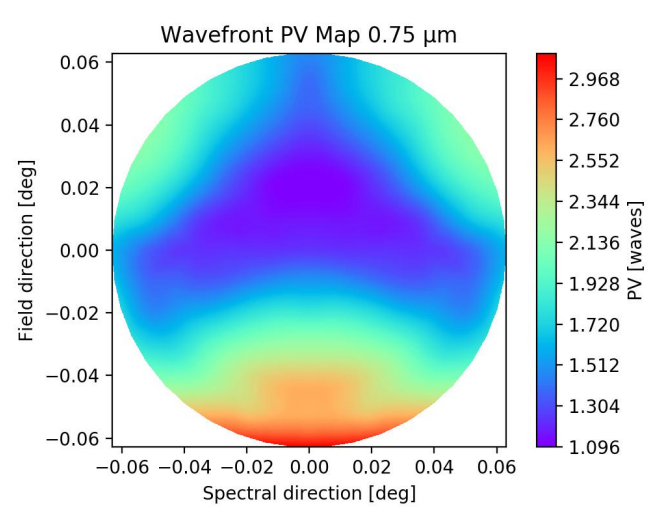

(f) PV@750 nm 


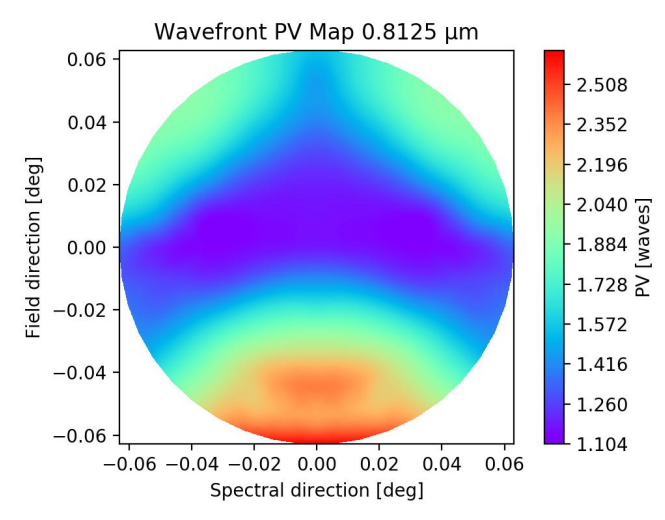

(g) PV@812 nm

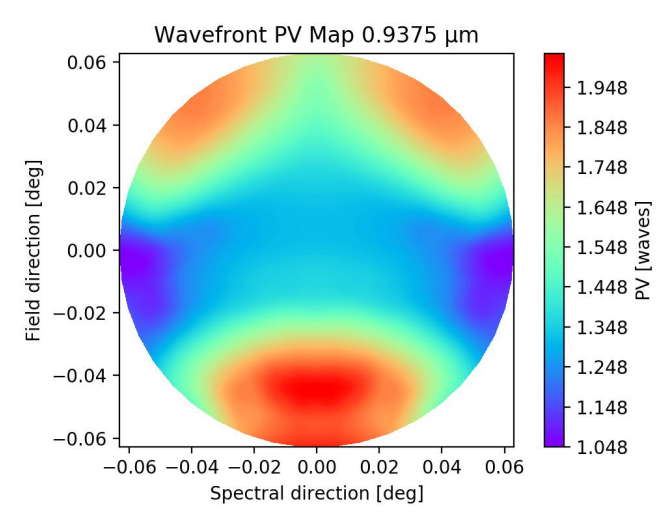

(i) PV@937 nm

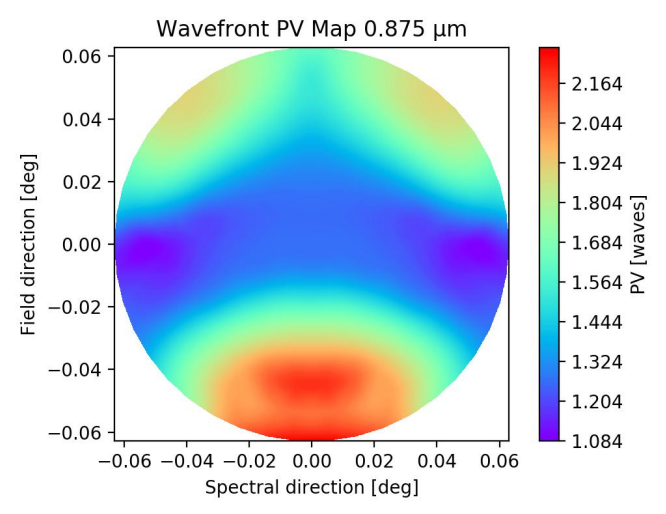

(h) PV@875 nm

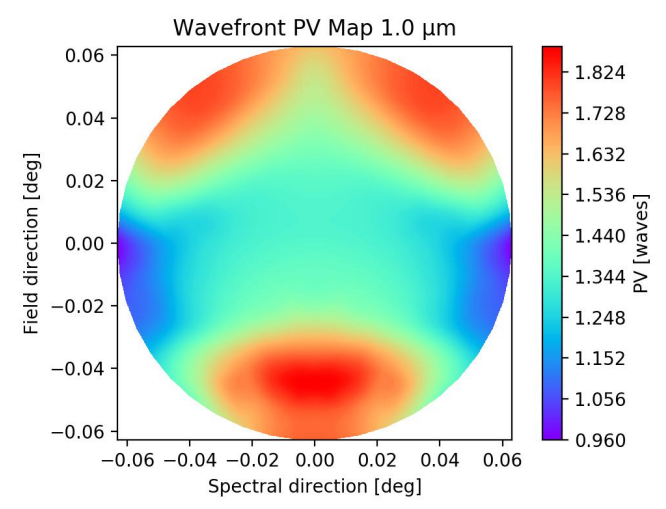

(j) PV@1,000 nm

Figure C.3: Split collimator red arm PV wavefront performance at the exit pupil. 


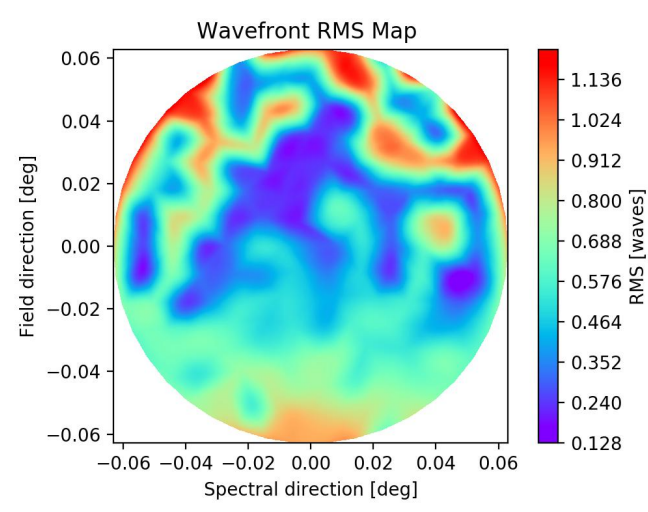

(a) RMS 500-1,000 nm

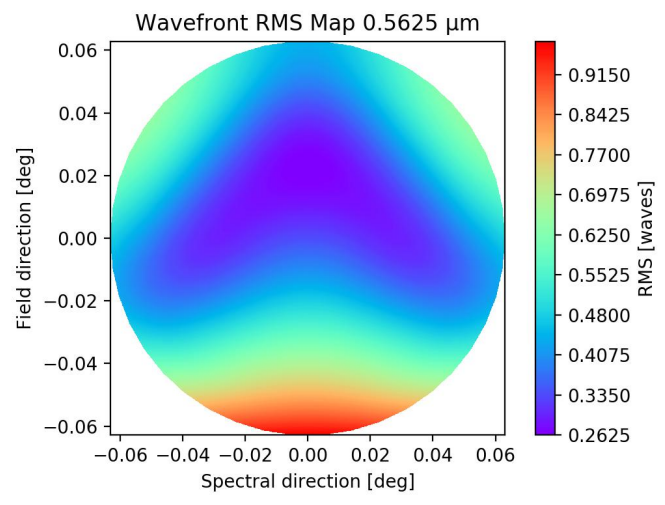

(c) RMS@562 nm

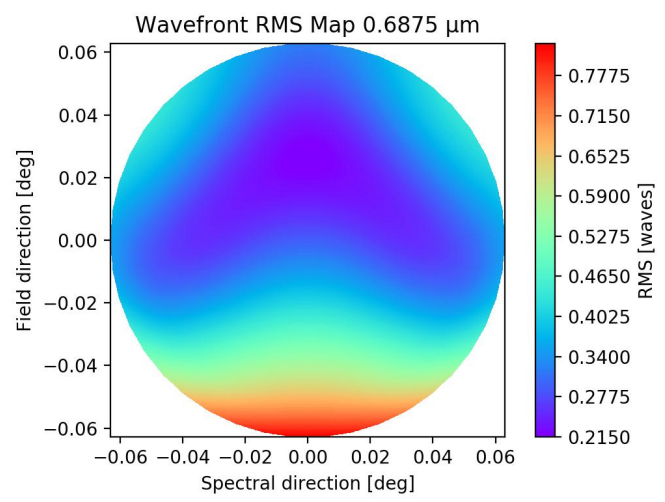

(e) RMS@687 nm

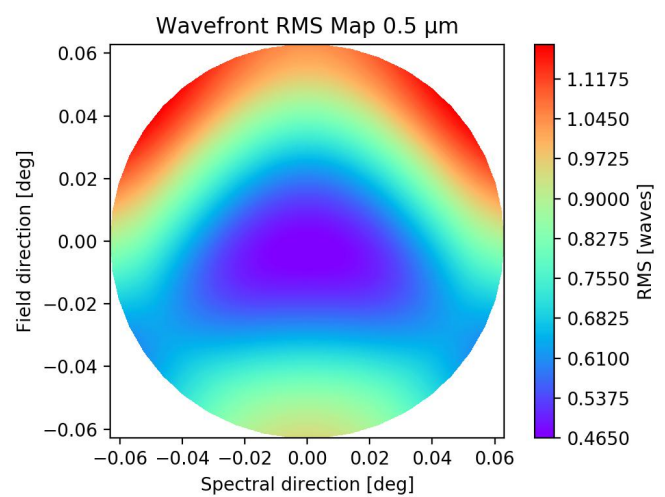

(b) RMS@500 nm

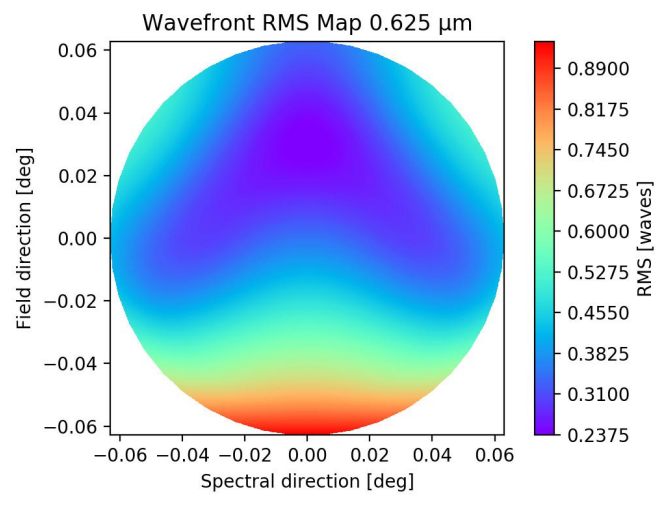

(d) RMS@625 nm

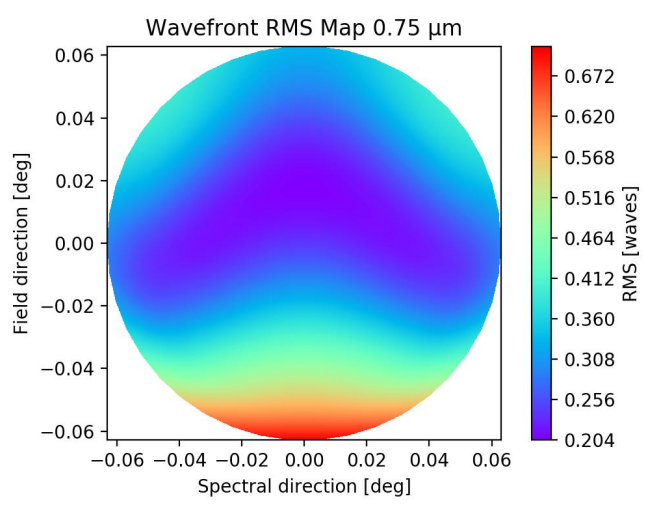

(f) RMS@750 nm 


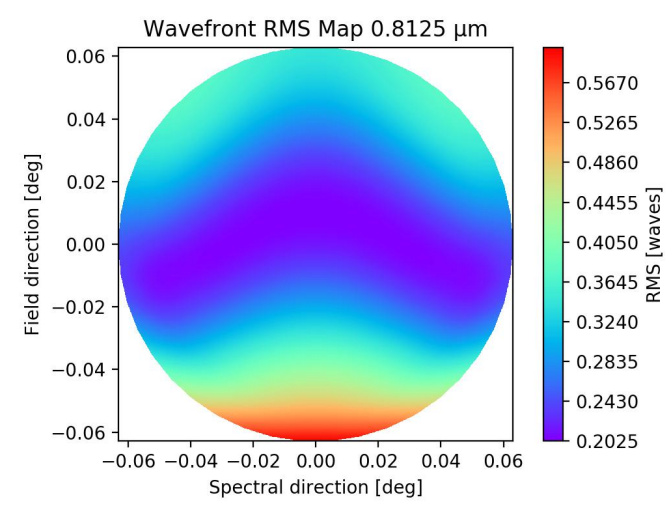

(g) RMS@812 nm

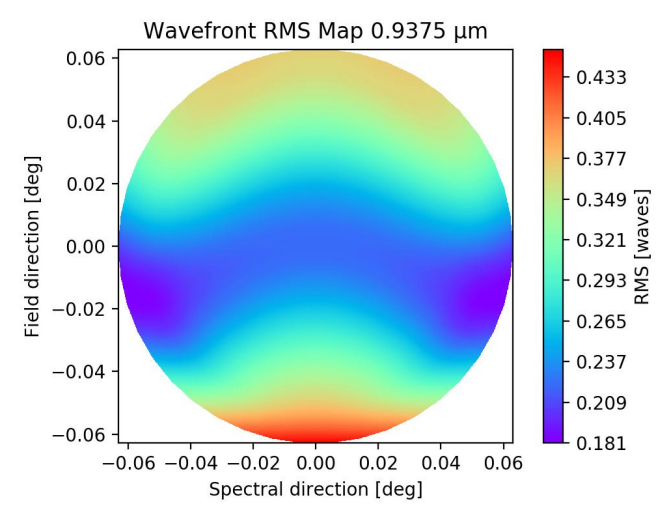

(i) RMS@937 nm

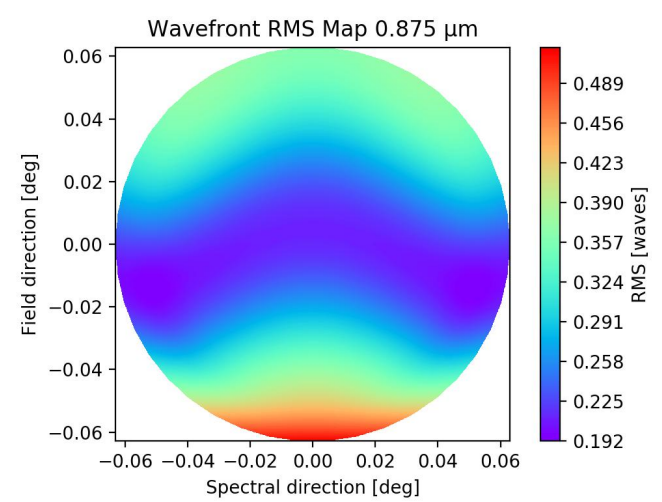

(h) RMS@875 nm

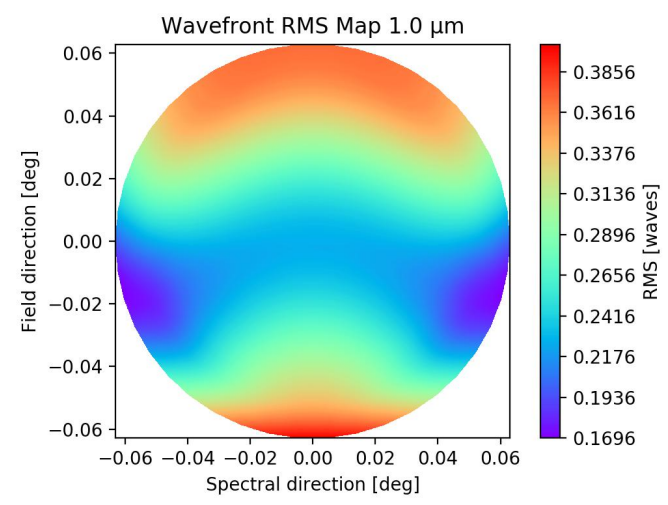

(j) RMS@1,000 nm

Figure C.4: Split collimator red arm RMS wavefront performance at the exit pupil. 